\title{
Monitoring the Systemic Immune System to Understand and Improve the Efficacy of Immunotherapy for Metastatic Osteosarcoma
}

justin edward markel

West Virginia University, jemarkel@mix.wvu.edu

Follow this and additional works at: https://researchrepository.wvu.edu/etd

Part of the Investigative Techniques Commons, Medical Cell Biology Commons, and the Oncology Commons

\section{Recommended Citation}

markel, justin edward, "Monitoring the Systemic Immune System to Understand and Improve the Efficacy of Immunotherapy for Metastatic Osteosarcoma" (2022). Graduate Theses, Dissertations, and Problem Reports. 7770.

https://researchrepository.wvu.edu/etd/7770

This Dissertation is protected by copyright and/or related rights. It has been brought to you by the The Research Repository @ WVU with permission from the rights-holder(s). You are free to use this Dissertation in any way that is permitted by the copyright and related rights legislation that applies to your use. For other uses you must obtain permission from the rights-holder(s) directly, unless additional rights are indicated by a Creative Commons license in the record and/ or on the work itself. This Dissertation has been accepted for inclusion in WVU Graduate Theses, Dissertations, and Problem Reports collection by an authorized administrator of The Research Repository @ WVU.

For more information, please contact researchrepository@mail.wvu.edu. 
Monitoring the Systemic Immune System to Understand and Improve the Efficacy of Immunotherapy for Metastatic Osteosarcoma

Justin E. Markel

Dissertation submitted to the School of Medicine at West Virginia University in partial fulfillment of the requirements for the degree of Doctor of Philosophy in Cancer Cell Biology

\author{
Scott Weed, PhD, Committee Chairperson \\ Ming Pei, MD, PhD \\ Lori Hazlehurst, PhD \\ Ivan Martinez, PhD \\ Michael Ruppert, MD, PhD \\ Brock Lindsey, MD, co-mentor 1 \\ Timothy Eubank, PhD, co-mentor 2
}

Cancer Cell Biology Program

Morgantown, West Virginia

2020

Keywords: osteosarcoma, systemic immunity, immunotherapy, macrophage, $T$ cell, myeloid-derived suppressor cell, immunophenotype

Copyright Justin E. Markel 2020 


\title{
Abstract \\ Monitoring the Systemic Immune Response to Understand and Improve the Efficacy of \\ Immunotherapy for Metastatic Osteosarcoma
}

\author{
Justin E. Markel
}

Osteosarcoma (OS) is a complex tumor with no effective targeted therapies due to its genomic heterogeneity and pleomorphism. The immune response it creates, particularly against metastatic lesions, is considerable; however, various suppressive mechanisms induced by the tumor prohibit its effectiveness. The presence of infiltrating lymphocytes suggests that therapeutic disinhibition through checkpoint blockade could increase antitumor immunity, though none have been successful in clinical trials. The complexities of the immune response to OS tumors have yet to be unraveled; however, there is evidence to suggest that cell-mediated immunity (CMI, specifically T cells, Natural Killer [NK] cells, and myeloid-lineage cells [MLCs]) plays an important role. New technologies have made it possilble to assess large numbers of cell antigens simultaneously, producing detailed information on the immune status of an individual. A snapshot of the immunological status of an organ or tumor can be conveyed by means of an immunophenotype (IPT), which uses cytometry, microarray, or gene set enrichment analysis to determine the relative percent populations and activation states of immune cells. The majority of data collected on the OS IPT are from primary tumors. Unlike metastatic tumros, primary tumors are easily removed by surgeons and do not account for aossicated high mortality rates. As such, we have investigated the systemic immune response to disease progression with and without immunotherapy. Using a highly metastatic luciferase(+) K7M2 (luc-K7M2) orthotopic OS model in an immunocompetent host, it was determined that the introduction of luciferase into tumor cells had little to no effect on the overall antitumor immune response and clinical disease progression. A systems-wide comparison of tumor-bearing and sham (surgery only) mice showed that there are significant changes in the IPTs of tumor-bearing mice at various time points in disease progression, and that these changes are consistent across different tissues. As disease progresses, $\mathrm{T}$ and NK cells are gradually depleted, and macrophages (M $\varphi s)$ adopt intermediate phenotypes through increased expression of both M1 and M2 polarization markers. Moreover, NK cell depletion in blood is specific to disease progression. T cells, under constant exposure to OS tumor antigens, become overstimulated and upregulate exhaustion markers like programmed cell death protein 1 (PD-1) and $\mathrm{T}$ cell immunoglobulin and mucin domain-containing protein 3 (TIM-3). Further, a notable systemic immune biomarker was uncovered using a co-expression ratio of immunosuppressive programmed death ligand 1 (PD-L1) to immunostimulatory major histocompatibility complex class II (MHC-II) on monocytic-myeloid-derived suppressor cells (M-MDSCs), a specific marker of OS disease progression in blood. Monotherapy with monoclocal antibody anti( $\alpha$ )-PD-L1 reversed the malignancy-induced immunological disturbances but did coindice with increased survival. Conversely, treatment of OS tumor-bearing mice with poly(lactic-co-glycolic) acid (PLGA)-encapsulated interleukin(IL)-12 decreased metastatic rate and increased cure rate in a immunophenotype-dependent manner that coincided with treatment-induced NK cell proliferation. These results demonstrate that systemic immunophenotyping can be used to non- 
invasively monitor OS patient disease progression and response to immunotherapeutics, potentially offering clinicians the opportunity to modify treatment regimens in real-time. 


\section{Dedication}

This dissertation is dedicated to my family, including my mother, father, brother, and grandparents. They have continually supported me throughout my entire journey, and for that I am eternally grateful. This dissertation is also dedicated to my mentors, Drs. Brock Lindsey and Timothy Eubank. I first want to extend my sincerest gratitude to Dr. Lindsey for taking a chance on me; he graciously accepted me into his lab after one brief meeting. We had instant chemistry, and he offered me the chance to lead his tumor immunotherapy project. Together, we have developed exciting new nanoscale immunotherapies with an associated flow cytometry-based monitoring platform, and have even filed for a patent on our creations. His clinical expertise gave real-world meaning to each experiment we did, and we never lost sight of the final goal - to help patients fight cancer. I will forever feel like a welcomed member of the Lindsey lab family. Secondly, I would like to thank Dr. Eubank. His PhD perspective was essential for me when preparing experiments and crafting grants and other manuscripts. Further, he taught me how to use Adobe Illustrator to help translate my science in the clearest way possible. Both of my mentors were available to me via text, so I always had instant access to their expertise if I had any questions. Finally, I want to dedicate this dissertation to the other members of my laboratory. I will miss you all, and wish you all the very best. 


\section{Acknowledgements}

Brock Lindsey for his excellent mentorship, T lymphocyte expertise, and clinical experience. I am forever grateful to be a part of the Lindsey lab, and I feel very well prepared to pursue a career as a physician scientist.

Timothy Eubank for his excellent mentorship and macrophage expertise. Dr. Eubank helped me design experiments, interpret data, and present conclusions.

My committee including Scott Weed, Ming Pei, Michael Ruppert, Lori Hazlehurst, and Ivan Martinez, who provided me with excellent guidance throughout my graduate school journey.

Our department statistician Gerry Hobbs, who helped me carry out the proper statistical treatments for our data.

I would also like to acknowledge my high school mathematics teacher, Donald Babbit, who instilled a love of science in me and tragically passed away in 2019.

Finally, I would like to thank the West Virginia University MD/PhD program for allowing me to pursue my goal of becoming a physician scientist. 


\section{Table of Contents}

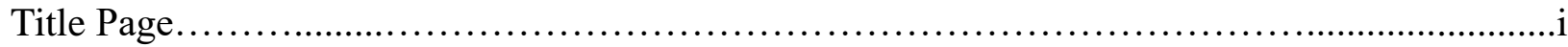

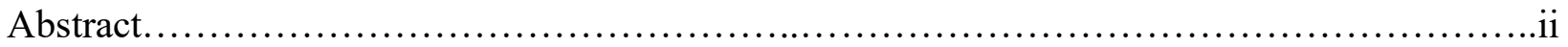

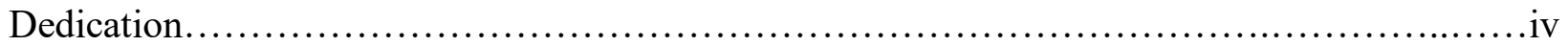

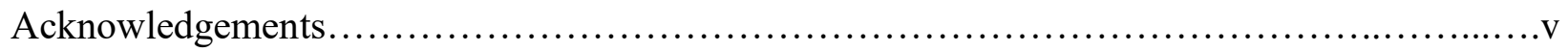

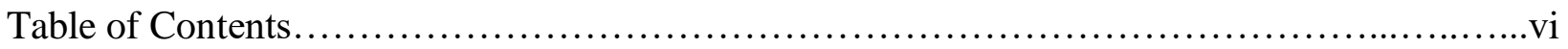

Chapter 1 - Introduction and Literature Review.........................................

Chapter 2 - Using the Spleen as an in vivo Systemic Immune Barometer Alongside

Osteosarcoma Disease Progression and Immunotherapy with $\alpha$-PD-L1 .......................51

Chapter 3 - Systems-Wide Immunophenotyping Defines Distinct Malignancy-Induced Immunological Changes in an Immunocompetent K7M2 Orthotopic Murine Model of Osteosarcoma.

Chapter 4 - IL-12-loaded Poly(lactic-co-glycolic) acid (PLGA) Nanospheres Reduce Metastasis and Increase Cure Rate in a NK cell-dependent Fashion in an Immunocompetent K7M2 Orthotopic Murine Model of Osteosarcoma............................................... 145

Chapter 5 - Overall Discussion and Future Directions......................................179

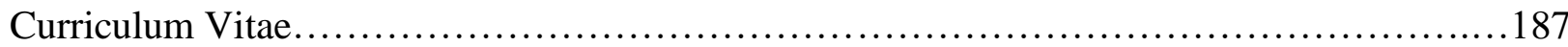

Appendix AI: Lindsey, B. A., Markel, J. E., \& Kleinerman, E. S. (2017). Osteosarcoma overview. Rheumatology and therapy, 4(1), 25-43.

Appendix AII: Grisez, B. T., Ray, J. J., Bostian, P. A., Markel, J. E., \& Lindsey, B. A. (2018). Highly metastatic K7M2 cell line: a novel murine model capable of in vivo imaging via luciferase vector transfection. Journal of Orthopaedic Research®, 36(8), 2296-2304.

Appendix AIII: Markel, J. E., Lacinski, R. A., Lindsey, B. A (in publication). Nanocapsule Delivery of IL-12. In Kleinerman, E. S., Gorlick, R. (Eds.), Clinical Advances in Osteosarcoma. Springer Nature

Appendix AIV: Pratt, H. G., Markel, J.E., Lindsey, B. A. (in publication). Applying Osteosarcoma Immunology to Understand Disease Progression and Assess Immunotherapeutic Response. In Kleinerman, E. S., Gorlick, R. (Eds.), Clinical Advances in Osteosarcoma. Springer Nature 
Appendix AV: Lindsey, B. A., Markel, J.E., Stewart, A. B., Lacinski, R. A., Noore, J. U.S. Provisional Patent Application No. 62/944, 191. Morgantown, WV: U.S. Patent and Trademark Office. 


\section{Chapter 1}

\section{Introduction and Literature Review}




\title{
Osteosarcoma Overview
}

\author{
Osteosarcoma Epidemiology
}

Osteosarcoma (OS) is a rare primary malignancy of bone, with 800-900 new cases occurring in the United States per year with children and young adults accounting for the majority. OS constitutes around $2 \%$ of all childhood cancers; OS in adults over 60 make up about $10 \%$ of cases and is often associated with pre-existing Paget's disease or past exposure to ionizing radiation (1-3). Disease arising in older patients is axial (most commonly the pelvis) in $39.7 \%$ of cases (4). While multiple histological subtypes exist, high-grade osteoblastic OS is the most aggressive and constitutes the majority of cases in children and young adults (5). A classical clinical presentation of OS is a teenager or young adult who fractures a long bone of the extremity with a force that appears disproportionately minor compared to the damage. For patients with localized disease of an extremity, the 5-year relative survival rate is $77 \%$. The overall 5-year survival rate for children and young adults is $60 \%$; however, when metastatic, the 5-year survival decreases dramatically (6). Metastatic disease is characterized as either pulmonary or extrapulmonary, with the lung involved in $80 \%$ of cases (5). At diagnosis, adverse factors include pulmonary metastasis, non-pulmonary metastases, and axial tumor site (7); in these cases, outcome is very poor and no advances in treatment have been made in the last few decades (5).

Osteosarcoma Standard of Care 
Currently, OS standard of care treatment involves combinatorial neoadjuvant chemotherapy, surgery, and post-operative adjuvant chemotherapy. Chemotherapeutics include an alkylating agent such as cisplatin or isofosfamide, anthracycline antibiotics such as doxorubicin, and antimetabolites like methotrexate (with leucovorin rescue). Even if metastases are not detected initially, all cases of OS are considered micrometastatic at diagnosis (approximately 80\% of patients have subclinical pulmonary micrometastases) and are treated with chemotherapy in addition to surgical resection (often limb amputation or limb salvage) with wide margins $(5,8)$. Neoadjuvant chemotherapy has greatly increased the survival rate of OS patients, however, these agents are highly cytotoxic and associated with significant systemic toxicities. Additionally, multidrug chemoresistance (MDR) is a main reason for treatment failure in Stage IV patients (9). Histological response (in terms of primary tumor necrosis) to neoadjuvant chemotherapy was historically considered an important prognostic factor in OS (10). When metastatic to the lung, chemotherapy is only effective when disease burden is low; larger legions (if resectable) must be removed surgically and survival rates are poor (11).

\section{Osteosarcoma Genetics and Epigenetics}

OS tumors show high clonal heterogeneity, both as a consequence of its unstable genome and selective pressures from chemotherapeutic agents. The OS genotype is in a constant state of remodeling, particularly in the case of sarcomagenesis via chromosomal instability, which results in losses and/or gains of entire chromosomes (12). Classically, OS tumors have been associated with mutations in retinoblastoma $(\mathrm{Rb})$ and p53 (5). Mutant p53 has been shown to be a predictor of poor 2-year survival outcomes, and whole genome sequencing has recently revealed that over 
$90 \%$ of OS tumors harbor either sequence or structural TP53 mutations (13). Mutations in RB1 contribute to mitotic missegregation and loss of heterozygosity, and much work has been done to associate particular genotypes with patient outcomes (14-16). Moreover, deregulation of the RBE2F pathway alter the epigenetic landscape of OS tumors and leads to more aggressive phenotypes (17). A meta-analysis published in 2015 concluded that RB1 mutations resulted in a 1.62-fold increase in mortality, with decreased histological response to neoadjuvant chemotherapy and significant increases in metastases (18). Cytotoxic chemotherapies also contribute to the genetic heterogeneity of OS tumors. Cisplatin, one of the main agents used in OS chemotherapeutic therapy, was shown to induce missense mutations of $N F 1$ and activating KIT G565R mutations (19). To date, no gene therapies have been approved for the treatment of OS (14).

\section{Cell-Mediated Immunity}

Innate Immunity: Overview

The human immune system is classified into two arms, namely the innate (early) and adaptive (delayed) immune systems. These two arms can be further subdivided into cell-mediated and humoral immunity. Cell-mediated immunity (CMI) is primarily concerned with the living effector cells that physically amount the response, while humoral immunity includes the various antimicrobial products secreted into the extracellular fluid, including antibodies of adaptive immunity and complement proteins of innate immunity; this manuscript focuses exclusively on CMI, which is most relevant to OS tumor immunology. 
The innate immune system constitutes the first level of response and is composed of a wide variety of myeloid-lineage cells (MLCs) and a subset of lymphoid-lineage cells (LLCs) that are produced in the primary lymphatic organ, bone marrow (BM), in the healthy adult (20). However, both homeostatic and pathogenic processes expand immune cell production to secondary lymphoid organs (i.e., lymph nodes, spleen, Peyer's patches, and mucosal tissues) and liver in a process called extramedullary hematopoiesis (21). Unlike $\mathrm{T}$ and B cell receptors (TCR and BCR, respectively) of the adaptive immune system, innate immune system receptors (e.g., pattern recognition receptors [PRRs]) such as the toll-like receptor (TLR) family, are strictly encoded in germline DNA and respond to nonspecific pathogen- and damage-associated molecular patterns (DAMPs and PAMPs, respectively). Some common PAMPS include gram negative bacterial lipopolysaccharide (LPS), viral RNA and DNA genomes, and gram positive bacterial lipoteichoic acid and peptidoglycan (22-26). DAMPs underly the basis for immunological responses to sterile injury and include High Mobility Group Box 1 (HMGB1), mitochondrial DNA, heat-shock proteins (HSPs), and uric acid among many others (27).

The first immune cells that a non-self entity meets upon entry into (or, in the case of cancer, mutates into) are the resident immune cells of that particular organ. Tissue-resident immune cells vary by organ, however, they most often include a phagocyte subtype (e.g., microglia in the brain, Kupffer cells in the liver, dust cells in the lung, and osteoclasts in the bone) (28-32). In the absence of an apparent threat, tissue-resident phagocytes play a central role in tissue homeostasis by engulfing foreign materials and debris (33-36). When a threat is detected, soluble factors are released throughout the surrounding tissue and sent via the blood to multipotent hematopoietic stem cells (HSCs) of the BM. Normally quiescent in nature, these stem cells then begin proliferating, differentiating, and producing progeny that migrate to target sites (37-39). 
In classical hematopoiesis, one of the earliest processes in HSC differentiation includes commitment to either the myeloid or lymphoid lineage through the common myeloid and lymphoid progenitors (CMP and CLP, respectively), with the lymphoid progenitor class showing greater heterogeneity (40-43). Derived from the CLP, innate lymphoid cells (ILCs) are very similar to antigen-specific $\mathrm{T}$ and $\mathrm{B}$ lymphocytes and mirror $\mathrm{T}$ lymphocyte subsets in form and function but lack somatically-recombined antigen-specific receptors. Normally residing in non-immune tissues, ILCs play important roles in tissue homeostasis and differentiate in response to cytokines such as IL-15, IL-2, Flt3 ligand, and $c$-kit ligand (44-47). Of the signals that induce production of MLCs, colony-stimulating factors (CSFs) are the most well-studied and include CFS-1 (also known as macrophage[M]-CFS), CFS-2 (also known as granulocyte-macrophage[GM]-CSF), CFS-3 (also known as granulocyte[G]-CFS), and interleukin(IL)-3 (also known as “multi"-CFS) (48).

Innate Immunity: Prominent Cellular Constituents

\section{Granulocytes}

Granulocytes are the most abundant leukocyte and include neutrophils, basophils, and eosinophils; neutrophils account for the vast majority of circulating granulocytes, and in the absence of heme malignancy or rare parasitic infections, can roughly be assumed to be equivalent to the granulocyte count (49). Due to their short half-life, neutrophils are constantly replenished by HSCs within the BM $(50,51)$. Along with tissue-resident immune cells, neutrophils are one of the first immune cells encountered by a foreign invader. Due to the presence of vast reserves in the BM, neutrophils can be mobilized at a moment's notice and released in great numbers into the bloodstream (52). Following a gradient of IL-8/C-X-C motif chemokine ligand(CXCL)8, 
neutrophils migrate to the site of insult where they employ a large arsenal of defense mechanisms including phagocytosis, release of inflammatory mediators (including oxygen radicals and cytotoxic chemicals via degranulation), and extrusion of their genetic material to form neutrophil extracellular traps (NETs) (53-55).

The mononuclear phagocyte system (MPS): monocytes, macrophages (M $\varphi$ ), and dendritic cells $(D C)$

(1) Monocytes

Monocytes are the migratory precursors to tissue-resident phagocytes, M $\varphi s$ and DCs. Together, these three cell populations form what is known as the mononuclear phagocyte system (MPS) and bridge innate and adaptive immunity by serving as antigen-presenting cells (APCs) (56). APCs present processed antigen at their cell surface and provide lymphocytes with the stimulatory and co-stimulatory signals necessary to amount an effective immune response (57). Derived from the CMP, monocytes are mobilized from the BM and migrate to areas of active infection and/or inflammation in response to chemokines like CC motif chemokine ligand(CCL) 2 and CCL7 (58). Although once thought of as merely a means to replenish phagocytes in peripheral tissues, monocytes are now known to be distinct immunological effector cells in their own right and not restricted to $\mathrm{DC}$ or $\mathrm{M} \varphi$ differentiation (59). Like neutrophils, monocytes phagocytose and release inflammatory mediators like tumor necrosis factor (TNF) (60). While $\mathrm{M} \varphi s$ and DCs are responsible for the majority of antigen presentation, monocytes can also process and present antigens to lymphocytes via the major histocompatibility complex (MHC) class II molecule (MHC-II) $(61,62)$. 
(2) $\mathrm{M} \varphi \mathrm{s}$

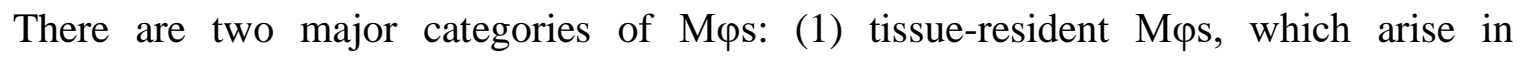
embryogenesis and actively preserve the integrity of the organ, and (2) monocyte-derived M $\varphi s$, which travel from the BM and spleen to sites of injury and mediate pathogen clearance and wound healing $(63,64)$. Tissue-resident phagocytes have a wide range of activities that vary with location. In the lung, alveolar M $\varphi s$ (also known as "dust" cells) actively clear the airways of particulate matter and infectious agents (65). In the liver, Kupffer cells make up the majority of tissue-resident M $\varphi$ s in the body; residing in hepatic sinusoids, they protect hepatocytes from the constant onslaught of microbes and other toxins introduced through the gut (66). In the spleen, marginal metallophilic, marginal zone, and red pulp M $\varphi$ s clear bloodborne pathogens and phagocytose senescent red blood cells (67). In the BM, osteoclasts remodel the extracellular matrix by resorbing bone (68). Microglia of the central nervous system (CNS) are an extremely specialized subset of M $\varphi$ s that maintain neuronal connections and assist in tissue repair (69).

M $\varphi$ s are important APCs that activate antigen-restricted T lymphocytes, particularly in the case of necrotic cell death (70). They are extremely plastic in both phenotype and function, and their actions are context dependent. Depending on the nature of the microenvironment, M $\varphi$ s will modulate their expression of immunoregulatory molecules to adjust the likelihood of successful lymphocyte activation. In response to signals from the surrounding microenvironment, M $\varphi s$ adopt generalized functionalities that favor immunosupportive or suppressive behaviors through a process called M $\varphi$ polarization (71) (Figure 1). The nomenclature used to define $\mathrm{M} \varphi$ polarization is typically based upon expression profile but is continually evolving.

Induced by soluble mediators like cytokines and bacterial products, $\mathrm{M} \varphi$ polarization exists as a continuum between two extremes: M1 (classical activation, immunosupportive), and M2 
(alternative activation, immunosuppressive). Each subset has a unique function, populationdefining protein expression profile, secretome, and signaling milieu that induces polarization (7173). Nonpolarized (i.e., "M0") M 9 s polarize into M1s under the influence of immunosupportive cytokines like IFN- $\gamma$ and lipopolysaccharide (LPS), and are then primed to kill invading microbes. Conversely, M2 M $\varphi$ s restore homeostasis by phagocytosing debris, promoting angiogenesis, and remodeling tissue. A plethora of M2 subtypes (M2a, b, c, etc.) have been classified thus far and new functions are being discovered yearly (71-78). Notably, M1/M2 polarization is much more well-defined in vitro, where the cytokine environment is tightly controlled. However, in vivo, M $\varphi s$ express a wide variety of both M1 and M2 markers. In these cases, it is more appropriate to refer to M $\varphi s$ as adopting either "M1-" or "M2-like" phenotypes, or simply referring to the particular expression profile (79-82). 


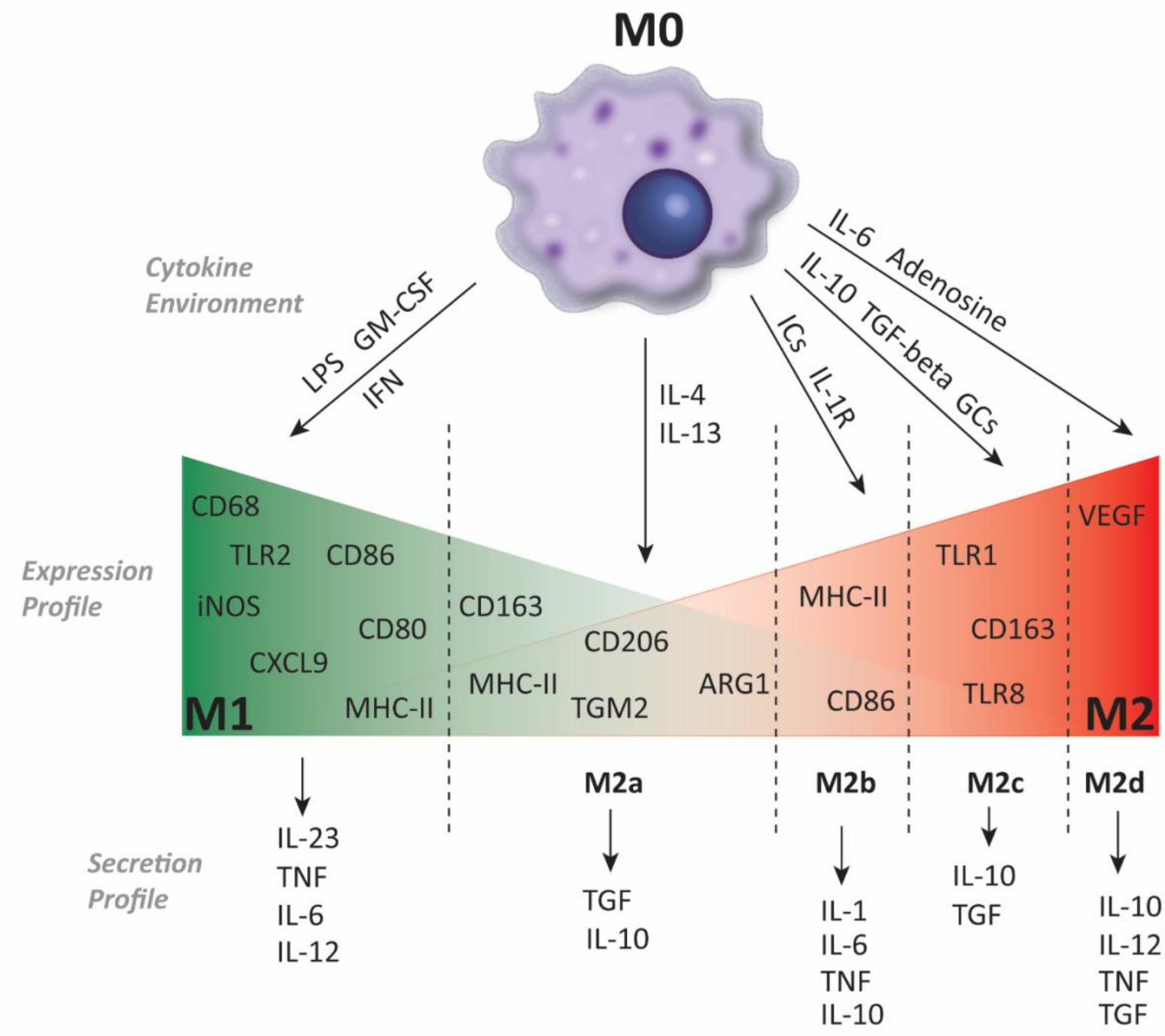

Figure 1. Schematic depicting the differentiation of an unpolarized M0-macrophage towards an M1- or M2-

like phenotype. The cytokine environment to which the macrophage is exposed determines its differentiation pathway, resulting in the upregulation of various intracellular and extracellular protein markers that together define its expression profile. The expression profile can then be used to infer function, one of the major ones being its corresponding secretion profile or "seceretome."

(3) DCs

DCs constitutively express MHC-II and are the most proficient APC. Classically, they carry antigens from a site of injury or infection to draining lymph nodes for presentation to 
lymphocytes. In response to microbial danger signals, DCs undergo maturation which includes phenotypic and functional changes; matured DCs have significantly upregulated antigen processing and presentation capabilities (83). Like M $\varphi s$, DCs differentiate into subsets with specialized functions under the influence of various signals; these subsets are classified based upon function, cell of origin, and expression of certain identifying proteins. When alerted of an infection, DCs undergo a genetic convergence that selects for the proper subtype most suited to address the immunological need (84-86).

The three main types of human DCs are plasmacytoid DCs (pDCs), conventional DCs (cDCs), and monocyte-derived DCs (mDCs) $(86,87)$. pDCs have a short half-life, are not major activators of T cells, and respond to viral genomes by producing large amounts of type 1 IFN. cDCs are the prototypical MHC-IIhigh APC that migrate the lymph nodes to present antigen to lymphocytes; they are further subdivided into type 1 (cDC1) and type 2 (cDC2) depending on transcriptional activity and proficiency in presenting intra- or extracellular pathogens. mDCs are inflammatory in nature, can promote a variety of pro-inflammatory immune responses, and may be functionally dysregulated in neoplastic settings (87-92).

\section{Innate Lymphoid Cells (ILCs) and Natural killer (NK) cells}

Simply put, ILCs are lymphocytes that started down the T/B lymphocyte maturation pathway from the CLP but, under the influence of Id protein 2-suppression, did not develop somatically-recombined antigen-specific receptors (93). Collectively, they serve as the innate immune counterpart to T lymphocytes of adaptive immunity. Like all cells of the innate immune system, they are primed for an immediate response to a perceived threat and react to non-antigenspecific signals such as cytokines and chemokines, microbial products, and inflammatory 
mediators; however, their activities are regulated by mechanisms of both innate and adaptive creation (94). Interestingly, some ILCs express MHC-II and can serve as APCs, thereby linking adaptive and innate immunity together (95).

The most prominent ILC is the NK cell, which can be thought of as the cytotoxic T lymphocyte (CTL) of innate immunity. However, NK cell activation is not restricted to the recognition of specific antigens, and its decision to kill or not kill depends on a delicate balance of activating and inhibitory signals on the extracellular surface of target cells (96). MHC class I polypeptide-related sequence (MIC)A and MICB are two common cellular stress proteins upregulated on infected and transformed cells; NK cells recognize these proteins with immunoreceptor tyrosine-based activating motif(ITAM)-associated receptors, thereby favoring cytotoxic activity $(97,98)$. Conversely, the presentation of self peptide on MHC class I molecules leads to deactivation of NK cell activity via signaling through receptors associated with immunoreceptor tyrosine-based inhibitory motifs (ITIMs) like killer-cell immunoglobulin-like receptors (KIRs) and the type II C-type lectin-like membrane glycoprotein Ly49 (99). Cytokines provide additional information to NK cells and are important for activation, maturation, and proliferation. IL-2 and IL-12 promote while transforming growth factor(TGF)- $\beta$ inhibits NK cell effector functions; cytokine environments become particularly important in the context of a developing malignancy (100-104).

Adaptive immunity: Overview

Adaptive immunity represents increased specificity and the ability to generate memory at the cost of a delayed initial response (typically 4-7 days following exposure). Adaptive CMI is 
mediated by $\mathrm{T}$ lymphocytes, and adaptive humoral immunity is mediated by antibody-secreting plasma B cells. Unlike cells of the innate immune system, T and B lymphocytes require specific peptide antigens presented on MHC molecules to mount responses (105).

Derived from the CLP produced in the BM, T and B lymphocytes mature in the thymus and BM, respectively, and circulate as naïve cells in pursuit of their cognate antigens. Antigen specificity is determined by the BCRs and TCRs, which are generated through a process of somatic recombination. In somatic recombination, $\mathrm{V}, \mathrm{D}$, and $\mathrm{J}$ gene segments are rearranged randomly to produce a wide repertoire of receptors that bind a specific antigen complexed with a specific MHC molecule. Once a naïve B or $\mathrm{T}$ cell finds its cognate pair, it differentiates under the presence of various co-stimulatory signals into effector and memory cells through clonal selection (105-107). Effector lymphocytes are tasked with eradicating the threat, while memory cells patrol the circulation quiescently but proliferate quickly and robustly in response to subsequent antigen exposures; it is the ability of the adaptive immune system to generate memory that forms the basis of vaccine therapy and reduces the chances of reinfection by the same pathogen $(105,108)$.

Adaptive immunity: Prominent Cellular Constituents

Tlymphocytes

T lymphocytes are a subset of immune cells that together make up the adaptive arm of CMI, and are involved in the clearance of microorganisms and transformed cells. Following hematopoiesis in the BM, immature lymphocytes travel to the thymus where they begin their maturation process. Early on in their development, immature "thymocytes" express both CD4 and CD8 co-receptors. The particular T cell subset is defined by the co-receptor expressed, and naïve 
T cells will retain only one upon exiting the thymus. CD4 and CD8 double-positive thymocytes are not normally present in the peripheral circulation but may increase in numbers due to malignancy $(109,110)$.

Each T lymphocyte is equipped with a unique TCR and either a CD4 or CD8 co-receptor that recognizes an MHC molecule:peptide antigen complex; successful ligation of these two receptors provides the primary stimulatory signal for activation. However, for complete activation, a secondary signal must also be sent to the T cell via binding of its CD28 molecule to CD80/86 on the surface of APCs (111). Upon simultaneous receipt of both stimulatory signals, naïve T cells proliferate and differentiate into effector and memory $\mathrm{T}$ cells provided they are in the proper cytokine environment. The translocation of stimulatory signals intracellularly occurs via phosphorylation of the ITAMs associated with CD3, an integral membrane protein present on all T cells regardless of subtype $(105,112-113)$. All of the aforementioned signals are required for an effective response, and removal of any one component can result in a suboptimal response; lymphocytes that receive only the primary signal become anergic and undergo apoptosis (114).

\section{(1) Cytotoxic T lymphocytes (CTLs)}

CTLs are directly involved in the killing of infected or transformed cells that express the MHC class I molecule, which are all nucleated cells. They are distinguished from helper T cells by the presence of the CD8 co-receptor, a defining protein of the CTL class that assists in binding of the TCR to the MHC molecule:peptide complex. Infected and transformed cells constantly digest and present protein antigens on their extracellular surface for sampling by circulating CTLs. When the antigen is endogenous and not a product of malignant transformation (i.e., "self"), no activation signals are sent and the T cell continues searching; however, when the antigen is of viral 
or neoplastic origin (i.e., "non-self"), the T cell becomes activated and, in the presence of the various co-stimulatory signals and cytokines described above, differentiates into both memory and effector $\mathrm{T}$ cells that equip the organism with antigen-specific cytotoxicity $(105,115-116)$. Target cells are killed by the release of perforins and granzymes, which permeate the plasma membrane and activate intracellular apoptosis pathways via caspase cleavage, respectively (117).

\section{(2) Helper T lymphocytes}

Helper T lymphocytes, defined by the presence of a CD4 glycoprotein co-receptor, are not directly involved in the killing of infected or transformed cells but instead help coordinate adaptive immune responses. Unlike CTLs, which find their cognate pair in the form of a peptide:MHC class I molecule complex on the surface of nucleated cells, helper $\mathrm{T}$ cells recognize antigen complexed with MHC-II on professional APCs. Also unlike CTLs, helper $\mathrm{T}$ cells undergo further differentiation that delineates the type of immune response generated against a particular pathogen. The most well-studied of these subsets include helper T cell type 1 (Th1) and 2 (Th2) that are analogous to the M1/M2 general classification scheme discussed previously (118-123).

\section{(3) Regulatory T lymphocytes}

Regulatory $\mathrm{T}$ lymphocytes are a subset of helper $\mathrm{T}$ cells with immunosuppressive functions that have an important role in the prevention of autoimmunity. There are two types of regulatory $\mathrm{T}$ cells, the distinction between the two being related to the timing at which the lymphocyte commits to the regulatory lineage. Natural regulatory $\mathrm{T}$ cells commit to the lineage early on in development within the thymus, and are a major regulator of autoimmunity. Conversely, induced regulatory $\mathrm{T}$ cells are a subpopulation derived from helper $\mathrm{T}$ cells under the influence of TGF- $\beta$ 
(124-126). Regulatory T cells secrete anti-inflammatory cytokines and participate in the clearance of effector T cells that persist following the resolution of an infection (127).

Adaptive immunity: T cell exhaustion (TCE)

T cell exhaustion (TCE) was a phenomenon first described in chronic viral infection, and is defined by a reversible transcriptional reprogramming leading to stepwise loss of effector functions. It is different from senescence, which is an irreversible state of cell cycle arrest induced by telomere shortening (128-131). In addition to chronic infection, TCE can be induced by the administration of exogenous immunostimulatory cytokines like IL-12, and by tumors (128, 132133). Phenotypically, exhausted T cells upregulate expression of programmed cell death protein 1 (PD-1) and T cell immunoglobulin and mucin-containing domain-3 (TIM-3). In the context of a developing malignancy, APCs and tumor cells suppress PD-1+ T cells via expression of its ligand, programmed death ligand 1 (PD-L1); it is also suggested that TIM-3-associated T cell suppression is mediated through galectin-9 (134-137). Increases in PD-1 and TIM-3 expression coincide with functional losses and occur in a stepwise manner; if the process is not reversed, these cells will completely lose their pro-inflammatory functions and eventually undergo apoptosis (128).

\section{Tumor Micro- and Macro-Environment Immunology}

Overview 
Tumor micro- and macro-environment immunology is concerned with characterizing and manipulating the immune response generated against tumor neoantigens both locally (micro) and systemically (macro). The foundation of modern tumor immunology traces back to Coley's toxin, which was a mixture of pyogenic bacterial products injected into tumors that caused regression of disease. Early on, there was controversy over whether tumors cells could be immunogenic, being that the immune system classically recognizes and responds to non-self antigens only. Dr. Coley realized that patients with infected tumors had a higher recovery rate and sought to recreate the same scenario (138-140). It is now widely accepted that the immune system not only recognizes transformed cells, but also participates in each step of the metastatic cascade. $(141,142)$. The field has since matured to a point where the specific antitumor immune responses to various cancers have been elucidated in great detail, leading to extremely effective immunotherapies for a variety of cancers (143-148).

\section{Tumor Immune Microenvironment}

The tumor immune microenvironment (Figure 2) includes the tumor cells themselves, associated stroma, perivascular cells, endothelial cells, and infiltrating leukocytes embedded in extracellular matrix with an emphasis on the immune cells and the influence of the developing tumor on their phenotype and function (149). Importantly, "micro"-environment analyses examine the tumor itself as an organ-like system and consider only the immune cells within the tumor border. The tumor microenvironment harnesses unique structural and spatial distribution of its components that are distinct from normal healthy organs, including aberrant vascular architecture and metabolism driven largely by hypoxia (150-153). Here, the interactions between tumor and immune cells occur across relatively short distances, with greater probability for effects to be 
mediated via cell-cell interactions and autocrine/paracrine feedback loops. Tumors with high nonsynonymous mutation rates (and therefore higher probability of producing neoepitopes) are often associated with a more predominant immune infiltrate (154). While the specific components of each tumor immune microenvironment are patient-dependent, many tumors follow generalizable trends and can be classified into three categories including 1) immune-excluded, 2) infiltrated-excluded, and 3) infiltrated-inflamed (155).

(1) Immune-excluded

Immune-excluded tumor immune microenvironments, sometimes known as immunologically "cold" or "immune desert" tumors, have the lowest level of infiltrating immune cells and represent the extreme lower end of the continuum. A study from 2018 analyzing the tumor immune infiltrates from 177 patients across nine different tumor types showed that ovarian cancer came the closest to being completely immune-excluded (156). Various reasons exist for why some tumors have less immune infiltration than others, and include physical barriers, decreased homing, lack of neoepiptope production by tumors cells, and immune privilege (157).

\section{(2) Infiltrated-excluded}

Infiltrated-excluded tumor immune microenvironments are sometimes lumped in with other "cold" tumors, and may have a paucity of CTLs or CTLs around the outer tumor perimeter only; infiltrated-excluded tumor immune microenvironments are often seen in tumors of epithelial origin such as colorectal carcinoma and pancreatic ductal adenocarcinoma. Patients with tumors of this subtype may have poorer prognoses, and most do not respond well to checkpoint blockade immunotherapies, discussed below (154-158). 


\section{(3) Infiltrated-inflamed}

In contrast to immune-excluded and infiltrated-excluded tumors, infiltrated-inflamed tumor immune microenvironments (otherwise known as "hot" tumors) have significant APC and CTL infiltration that extends deep within the tumor core. Infiltrated-inflamed tumors are also characteristic of cancers of epithelial origin and include melanoma and non-small cell lung cancer $(159,160)$; patients with infiltrated-inflamed tumors show the strongest response to checkpoint blockade immunotherapies (161-163).

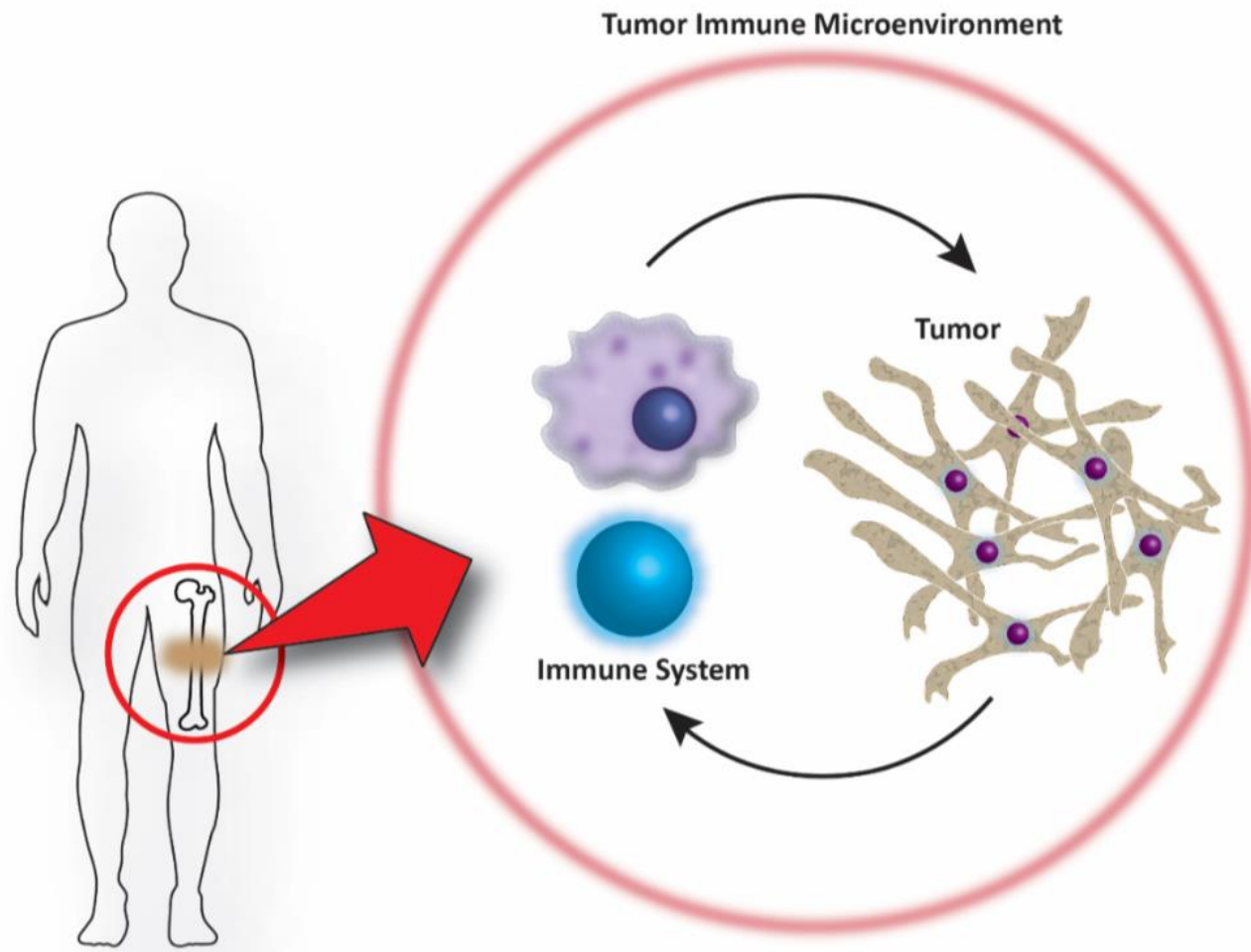

Figure 2. Schematic depicting the relationship between tumor-infiltrating leukocytes and tumor cells within the tumor immune microenvironment. A patient with, in this case a bone tumor, presents at diagnosis with a complex network of interactions between tumor and immune system that shapes the phenotype of both the growing malignancy and the immune cells themselves. The components of this system can be broken down into its cellular constituents and analyzed to yield diagnostic and prognostic implications. 


\section{Tumor Immune Macroenvironment}

The tumor immune macroenvironment is a less well-established idea in the field of tumor immunology and is not easily subdivided into distinctive groups like its microenvironment counterpart. In its most general sense, the idea of a tumor macroenvironment considers the emergent effects of tumor on systemic immunity beyond the immune cells confined within the tumor border (155). The intimate connection between local and systemic antitumor immune responses provides the theoretical framework behind the observed abscopal effect, where treatments directed at primary tumors result in immune responses at distant sites (164).

It is increasingly recognized that the systemic antitumor immune response may be used for prognostic, diagnostic, and therapeutic purposes (165-176). Activation of secondary lymphoid organs outside of the primary tumor is a necessary component of successful tumor immunotherapy (177). Lymphoid and myeloid cell lineages in the blood of cancer patients from virtually all tumor types are significantly different from age-matched healthy controls, with more advanced disease resulting in more dramatic changes (178-187). It is the current understanding that the mechanisms underlying these observed systemic immune effects are mediated in part by secreted molecules from both tumor and immune cells circulating throughout the blood and lymph, leading to significant immunomodulation at distant organ sites (155). Additionally, tumor cells released into circulation and antigen-loaded DCs evoke immune responses from immune cells in secondary lymphoid organs (188). Tumor cells can also secrete signaling molecules that recruit immunosuppressive myeloid cells to primary tumors to promote growth and metastatic tumor sites to prepare organotropic tissues for colonization $(155,189-191)$.

\section{Immunophenotyping}


Immunophenotyping is the process of characterizing immune micro/macro-environments for diagnostic, prognostic, and therapeutic applications (Figure 3). The data used to construct immunophenotypes are often generated using immunohistochemistry (IHC), flow cytometry (FC), or gene expression profiling, and reference expression profiles or matrices are used to extract pertinent cell population information (192-197). Immunophenotyping has utility across a number of various pathologies ranging from postoperative recovery to sepsis and diabetes, and has regained popularity in cancer with the surge of interest in tumor immunotherapies following the success of checkpoint blockers (discussed below) (198-201).

In practice, a solid or liquid tissue sample is harvested and prepared in a manner that preserves (or reconstructs, in the setting of gene set enrichment analysis) the expression profile of individual cell types. In the case of IHC, samples are stained with antibodies recognizing population-defining immune cell markers and analyzed in their anatomical location using microscopy (202). For FC and genomics, reference expression profiles are used to classify each immune cell and assess its relative population distribution. Depending on the choice of components, expression profiles may be redundant, with multiple arrays often appropriately describing a certain population. They are also dependent on the context and nature of the research question being addressed, with further considerations regarding tissue type, species, sex, strain, and age (195, 203-205). From there, additional structure can be imposed in a hierarchical manner using non-population defining markers and/or gene signatures to obtain activation status or whatever else is of interest to the investigator. Currently, the major utilities of immunophenotyping are to make diagnoses, infer prognosis, and determine candidacy for various immunomodulatory drugs $(193,197,202)$. 


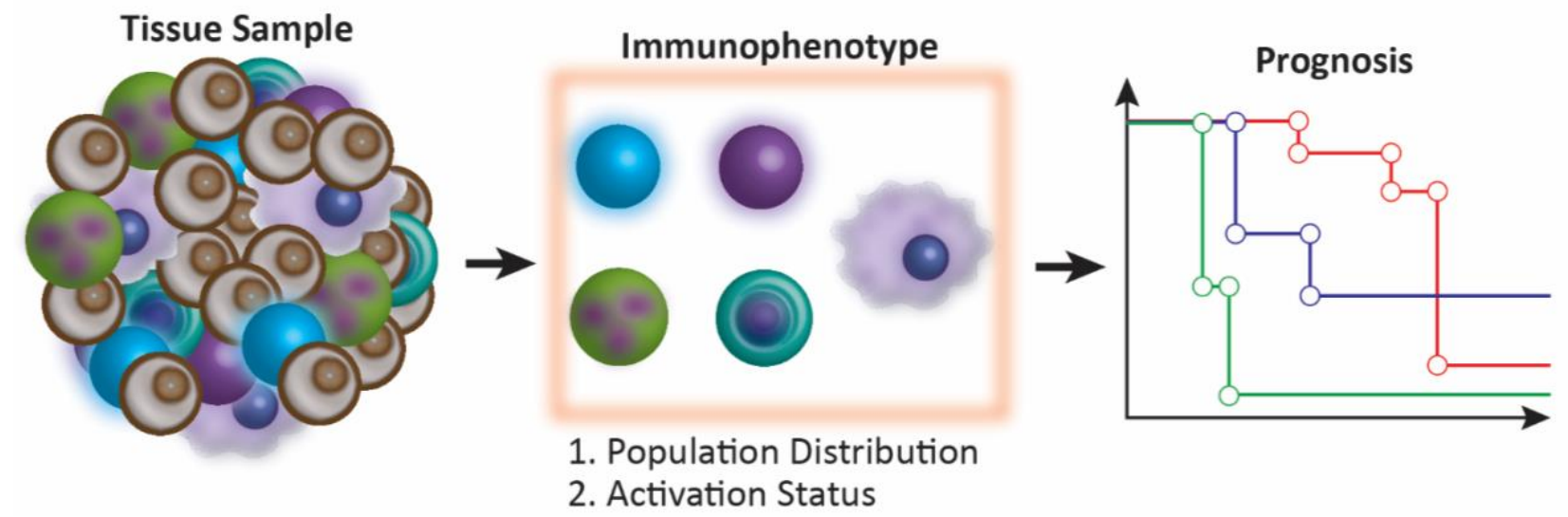

Figure 3. Schematic depicting current utility of creating a tumor immune micro/macro-environment immunophenotype. A tissue sample, often tumor or blood, is dissociated into single cell suspension and immunophenotyped, most often through the use of IHC, flow cytometry, or RNA-seq. The resulting data is analyzed via microscopy (IHC), manual gating or clustering (flow cytometry) or an ever-growing slew of computational approaches (RNA-seq) and constructed into an immunophenotype that may associate with a particular prognosis.

\section{Osteosarcoma Tumor Immunology}

Overview

OS is an orphan disease, and its immunology is not well defined. In contrast to many tumors of epithelial origin, sarcomas harbor a heterogeneous display of immunophenotypes and do not fit neatly into immune-excluded, infiltrated-excluded, or infiltrated-inflamed categories described above. However, there is evidence that the immune system plays an important role in OS tumorigenesis. Indeed, post-operative infections associate with increased survival in OS patients, and early lymphocyte recovery following chemotherapy predicts better prognosis in pediatric patients $(206,207)$. Futhermore, immune cells harvested from OS patients retain their ability to react to IFNs, a family of cytokines with potent antitumor activity (208). A recent study using the 
CIBERSORT algorithm to calculate an immune "score" in 83 OS patients showed that those with a higher immune infiltrate in primary tumors had significantly increased survival (209). Likewise, metastasis suppression has been associated with the upregulation of genes regulating the immune response in primary OS tumors (210).

OS tumors are extremely pleomorphic and unlike carcinomas, have few recurrent clinically-targetable modifications (211). However, the pleomorphism results in a high nonsynonymous mutation rate, with the highest mutational loads seen in metastatic lesions (212). Importantly, OS tumors retain MHC class I and MICA expression, which are recognized by cytotoxic immune cells $(213,214)$. When expressed, nonsynonymous mutations create neoepitopes that can be immunogenic. As such, most of the immune cells found within the OS immune-genomic landscape participate in CMI, and include NK cells, T lymphocytes, monocytes, M $\varphi s$, and immature MLC cells termed myeloid-derived suppressor cells (MDSCs) (215).

Most of the data available on the OS immunophenotype have come from the tumor immune microenvironment, and often present an association between immune infiltrate and prognosis. In general, there is more immune activity in metastatic versus primary lesions, though this increase in activity is coupled to increased tumor immunosuppressive capacity (214, 215). Schematics depicting the main components of lymphoid and myeloid lineage OS tumor immunology are shown in Figures 4 and 5, respectively.

\section{Lymphoid Lineage}

Due to the high levels of neoantigens expressed by OS tumors, they protect themselves by reducing $\mathrm{T}$ cell recruitment, downregulating the expression of neoepitope-generating nonsynonymous mutations, and creating immunosuppressive forces that disrupt the immune 
response $(212,214-216)$. To block lymphocyte recruitment, OS tumors reduce production of CXCL12, a potent $\mathrm{T}$ cell chemoattractant (217). As they metastasize, however, OS tumors develop a significantly higher mutational burden which recruits progressively more $\mathrm{T}$ cells to the tumor (212, 215). Tumor cells suppress infiltrated lymphocytes by activating immune checkpoint receptors like cytotoxic $\mathrm{T}$ lymphocyte-associated protein 4 (CTLA-4) and PD-1, recruiting suppressive myeloid cell populations, and inducing differentiation of regulatory T cells (218-222). NK cells, which are not antigen restricted, are vital in the control of metastatic tumor cells that downregulate antigen expression but are decreased in the blood of pediatric OS patients along with helper and CTLs (223).

Polymorphisms that reduce the capacity of an individual to amount an immunosupportive helper $\mathrm{T}$ cell response have been shown to increase the risk of OS sarcomagensis. IL-12 is a pleiotropic protein cytokine involved in the induction of antitumor helper $\mathrm{T}$ cell responses and activation of NK cell cytotoxicity (100-102, 224). In OS patients, serum levels of IL-12 and related cytokine IL-27 are reduced compared to healthy controls, and replenishment of IL-12 via aerosol gene therapy significantly reduced metastatic rate in preclinical models (225-227); it is hypothesized that NK cell control of metastatic outgrowth is mediated through the targeting of tumor-initiating cells in an NKG2D-NKG2DL manner (228). Likewise, activated NK cells are positively correlated with increased survival in OS patients (229).

Increases in lymphocyte infiltration are coupled with greater immunosuppressive responses by the tumor, such as the induction of regulatory $\mathrm{T}$ cells via the release of exosomes containing TGF- $\beta$, $\alpha$ fetoprotein, and HSPs (230). TGF- $\beta$, a critical regulatory T cell-associated cytokine, is increased in the blood of OS patients with metastases (231). IL-35 is another regulatory T cell-associated cytokine that is increased in the blood of OS patients and contributes to CTL 
dysfunction in the periphery (232). Within the tumor itself, regulatory T cells expressing galectin9 can suppress the activity of TIM-3+ helper and CTLs and interrupt the antigen presentation process $(233,234)$. Blocking regulatory T cells indirectly via TGF- $\beta$ or directly via cell-targeted antibody depletion in vivo restores antitumor immunity and reduces metastasis in preclinical OS mouse models $(235,236)$. Clinically, patients with high CD8+ cytotoxic to Foxp3+ regulatory T cell ratios in their primary tumors have significantly better overall survival (237). Interestingly, antibody blockade of PD-1 has recently been shown to decrease the accumulation of regulatory $\mathrm{T}$ cells within the primary tumor microenvironment (238).

TCE also plays an important role in OS tumor immunology. Helper and cytotoxic T lymphocytes both within the tumor microenvironment and systemically show an exhausted phenotype, which increases in intensity with disease progression and metastasis (239-242). OS tumor cells upregulate PD-L1 expression as disease progresses and suppress PD-1+ T cells (214). Moreover, high PD-L1 expression within the primary tumor is associated with early-onset metastasis (243). Both within the primary tumors and systemically, the exhaustion status of T cells correlates with outcome, and patients with higher percentages of exhausted lymphocytes have worse outcomes $(240,242-244)$. 


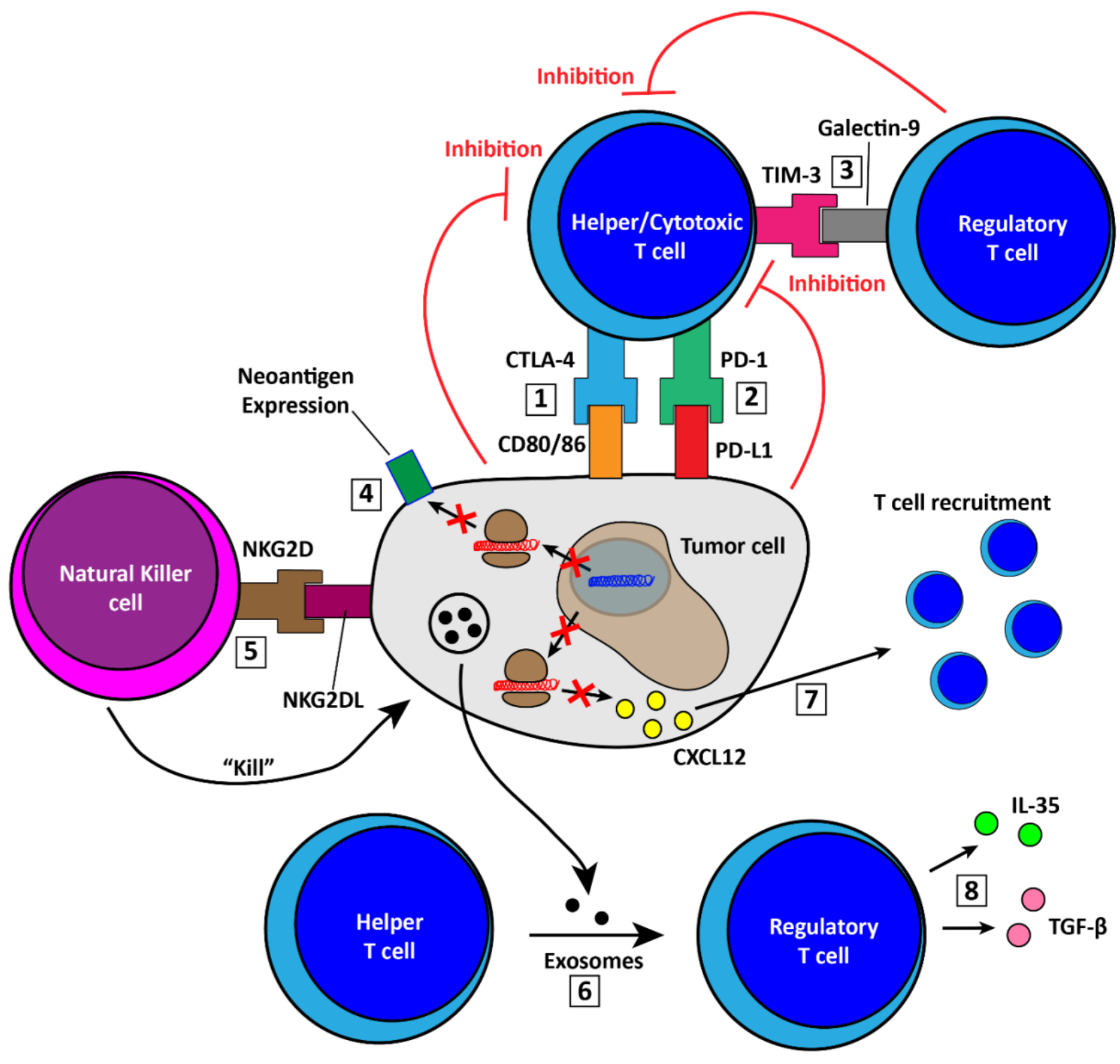

Figure 4. Schematic depicting major lymphoid OS tumor immunology concepts. 1) Tumor cells upregulate CD80 and CD86 that bind to CTLA-4 on T lymphocytes causing inhibition of effector functions 2) Tumor cells upregulate PD-L1 that binds to PD-1 receptors on T lymphocytes causing inhibition of effector functions and exacerbation of T cell exhaustion 3) Galectin-9+ regulatory T cells directly inhibit the effector functions of TIM-3+ helper and cytotoxic T cells. 4) Tumor cells downregulate the expression of nonsynonymous mutations leading to decreased detection by lymphocytes 5) Natural killer cells become activated to kill metastatic NKG2DL+ tumor cells via NKG2D receptor ligation 6) Exosomes secreted by tumor cells induce the differentiation of immunosupportive helper $\mathrm{T}$ cells into regulatory T cells 7) Tumor cells epigenetically downregulate the production of potent T cell chemoattractant CXCL12 
leading to decreased lymphocyte infiltration into the core of the tumor 8) Regulatory T cells secrete cytokines like IL35 and TGF- $\beta$ that suppress the antitumor immune response

\section{Myeloid lineage}

(1) M $\varphi s$ and other APCs

The most well-studied MLC in OS tumor immunology is the M $\varphi$, though there is controversy regarding its role in disease progression. The controversy is mostly attributed to the constantly evolving definition of M1 and M2 polarization in vivo (245). To remove considerable ambiguity from the literature, the markers used to define M1 and M2 will be explicitly stated alongside the bias they describe. With this caveat in place, the majority of data suggests that OS tumors dysregulate polarization of infiltrated $\mathrm{M} \varphi \mathrm{s}$, and that a shift favoring induction of immunosuppressive $\mathrm{M} \varphi$ phenotypes is required for disease progression (246).

One of the first studies showing a relationship between $\mathrm{M} \varphi$ s and outcome in OS patients was published in 2011. In this study, genome-wide gene expression profiling showed that increased primary intratumoral M $\varphi$ density, regardless of M1 (HLA-DR $\alpha_{+}$) or M2 (CD163+) bias, correlated with decreased metastatic burden in patients (247). This finding was contrary to most other cancers, where tumor-associated $\mathrm{M} \varphi$ s are thought to promote metastasis (248); hence, it was thought that polarization status may be the determining factor. Indeed, a subsequent study found that patients with high $\mathrm{M} 1$ (iNOS + ) M $\varphi$ bias in primary tumors developed fewer metastases (249). Still, other studies using similar methods have drawn opposite conclusions; indeed, one study found no association between M2 (CD163+) M $\varphi$ s and prognosis, while another observed that the presence of both tumor-associated M $\varphi$ s and DCs correlated with worse 5-year event-free survival $(250,251)$. The extent of lymphocytic infiltration may provide a reason for these discrepancies, as high levels of M2 (CD163+) M $\varphi$ s have been shown to correlate positively with exhausted TIM- 
$3+\mathrm{PD}-1+\mathrm{T}$ cells; therefore, it is possible that interactions between polarized $\mathrm{M} \varphi \mathrm{s}$ and exhausted $\mathrm{T}$ cells determine outcome (239).

Irrespective of M1/M2 nomenclature, it is well established that OS cells and M $\varphi$ s can engage in immunosuppressive feedback loops that result in disease progression. Exosomes released by tumor cells induce M2(CD206, CD163, and arginase-1)-polarization of M $\varphi s$, and M $\varphi s$ increase OS tumor cell migration via upregulation of cyclooxygenase-2 (COX2) and release exosomes that activate AKT in tumor cells (252-254). Moreover, the density of tumor-associated $\mathrm{M} \varphi \mathrm{s}$ in metastatic nodules is significantly higher than in primary tumors, and they show a strong predilection for M2 (CD206+) versus M1 (CD86+) bias $(253,255)$.

Further support that $\mathrm{M} \varphi$ polarization plays a key role in OS tumor progression has come from the $\mathrm{M} \varphi$ immunomodulatory drug, mifamurtide. Mifamurtide is a synthetic analog of muramyl dipeptide, a component of the cell wall of Mycobacterium species. Currently, it is approved in Europe alongside standard of care for patients with resectable, nonmetastatic OS (256). Similar to LPS, mifamurtide was thought to induce M $\varphi s$ to adopt immunosupportive phenotypes. Recently, however, it was shown that mifamurtide induces an intermediate $\mathrm{M} \varphi$ phenotype, with both immunosupprotive and suppressive functions; these data highlight the shortcomings of M1/M2 nomenclature and help justify the contradictions seen in the literature (257).

\section{(2) Other Myeloid-Lineage Cells (MLCs)}

Outside of M $\mathrm{Ms}$, little work has been done to elucidate the specific roles of other MLCs in OS tumor immunology. There is, however, evidence to suggest potentially important roles for neutrophils, monocytes, and myeloid-derived suppressor cells (MDSCs). High neutrophil- and monocyte-lymphocyte ratios, as well as high overall neutrophil counts in peripheral blood are poor 
prognostic indicators in OS (258-260). Besides blood, neutrophils are also present within tumor samples and have been shown to induce migration of OS tumor cells (261). Additionally, IL8/CXCL8 (also known as neutrophil chemotactic factor) is increased in the serum of OS patients and may play a role in mediation of OS lung tropism $(262,263)$. TIM-3+ monocytes in tumor and sera secrete high levels of immunosuppressive IL-10, preventing the release of IFN- $\gamma$ from galectin-9+ helper T cells (233).

MDCSs are immature myeloid cells that arise in situations of chronic inflammation. In OS tumor-bearing mice, MDSCs accumulate in spleens and primary tumors following an IL-18 and CXCL12 gradient $(264,265)$. They are highly immunosuppressive and have multiple mechanisms of action, including the induction of regulatory $\mathrm{T}$ cells and contact-dependent inhibition of $\mathrm{NK}$ and T cell effector functions (266-269). Another major mechanism by which MDSCs inhibit T cells is via arginase-1-mediated depletion of L-arginine, which is consumed in large amounts by proliferating T cells; L-arginine supplementation in the background of PD-L1 blockade increased survival of OS-tumor-bearing mice $(270,271)$. 


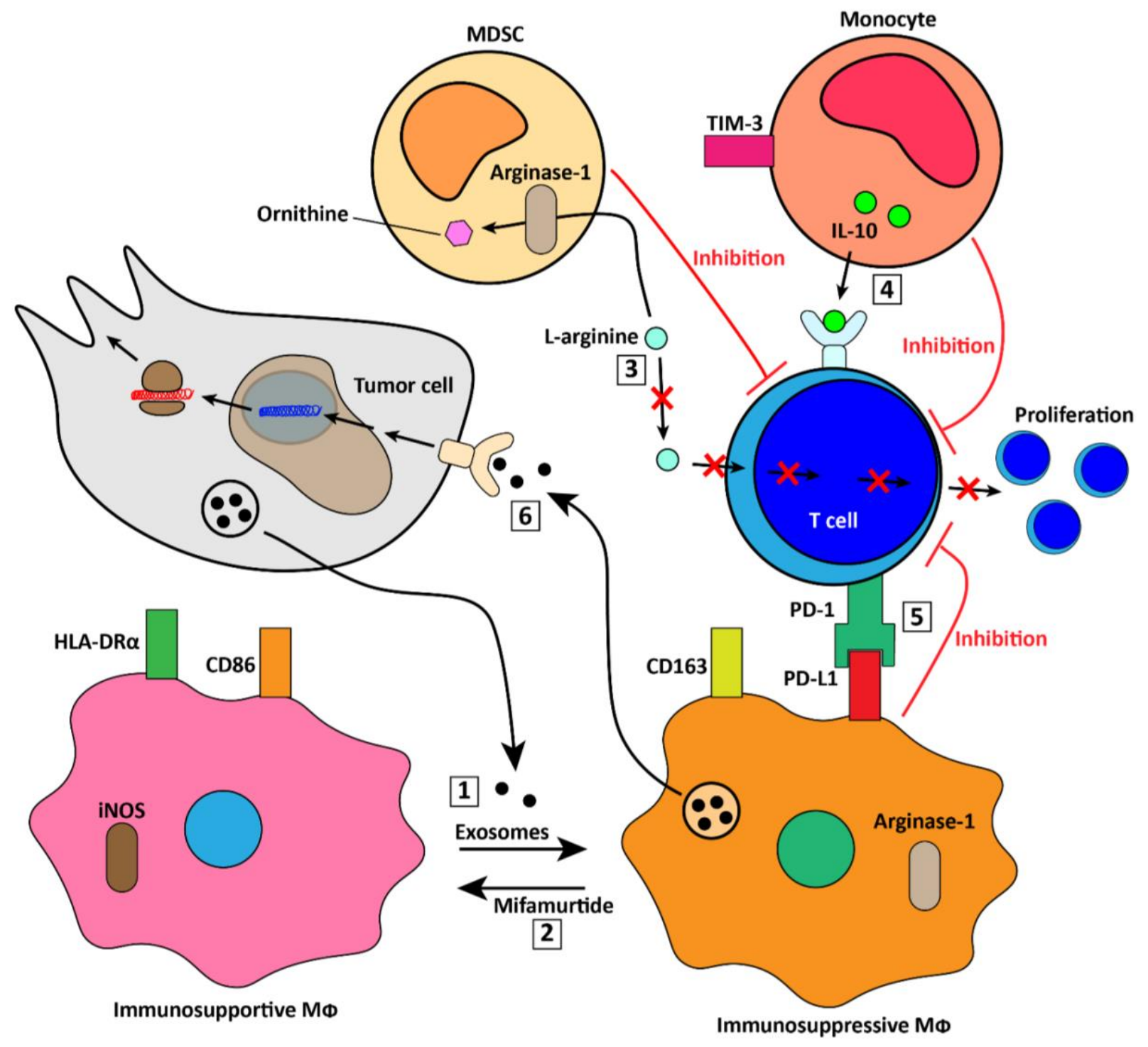

Figure 5. Schematic depicting major myeloid OS tumor immunology concepts. 1) Tumor cells produce exosomes that promote the polarization of $\mathrm{M} \varphi$ s to immunosuppressive phenotypes 2) Mifamurtide promotes the polarization of M $\varphi$ s to immunosupportive phenotypes 3) MDSCs sequester L-arginine from T lymphocytes thus decreasing their ability to proliferate 4) TIM-3+ monocytes produce the immunosuppressive cytokine IL-10 that inhibits T cellmediated antitumor immunity 5) CD163+ immunosuppressive M $\varphi$ s inhibit $\mathrm{T}$ cell-mediated antitumor immunity and exacerbate TCE 6) Exosomes secreted by immunosuppressive M $\varphi$ s increase the invasiveness of OS tumor cells

Experimental Immunotherapeutics: Checkpoint Blockade 
Under normal physiological circumstances, immune cells express a balance of activating and inhibitory ligands and receptors. Various stimuli including infection, trauma, autoimmunity, and neoplasia, tip this balance in one direction or the other and determine the fate of the response. While there are many molecules that contribute to this delicate balance, a select few have thus far proven to be important to OS immunotherapy.

Immunological checkpoints are a family of protein checks and balances that, when expressed, send inhibitory signals to the receiving cell. Checkpoint blockade refers to the use of drugs - mostly monoclonal antibodies — to disrupt these immunosuppressive receptor-ligand axes and promote activation through disinhibition (272-274). OS tumor cells themselves, as well as APCs like M $\varphi$ s and DCs, express the cell surface checkpoint protein PD-L1. Upon binding of PDL1 to PD-1+ leukocytes, the likelihood of an effective antitumor immune response is reduced (214, 275-276). Clinical trials testing antibodies that block the interaction of PD-1 and PD-L1 (e.g., nivolumab and atezolizumab, respectively) have not been successful, and treatment failure has since been attributed to an escape mechanism involving a compensatory increase of CTLA-4 and CD80/86 expression on T lymphocytes and tumor cells, respectively $(218,277)$.

In order to become fully activated, lymphocytes must receive an additional co-stimulatory signal in the form of CD28 binding to CD80 or 86 on the surface of APCs. CTLA-4 is homologous to $\mathrm{CD} 28$ but with greatly increased binding affinity for CD80/86, effectively out competing CD28 for binding sites (278). Ipilibumab is an $\alpha$-CTLA-4 monoclonal antibody that, in combination with $\alpha$-PD-L1, showed enhanced efficacy against pulmonary metastases versus $\alpha$-PD-L1 alone in a preclinical model (218); however, neither ipilibumab as a single agent nor combination checkpoint blockade therapy with nivolumab has shown efficacy in clinical trials (279). 
Figure 6. Schematic depicting dual checkpoint blockade of CTLA-4 and PD-L1. 1) Tumor-expressed CD80/86 binds to CTLA-4 on $\mathrm{T}$ cells causing decreased effector functions 2) Tumorexpressed PD-L1 binds to PD-1 on $\mathrm{T}$ cells causing decreased effector function 3) $\alpha$-CTLA- 4 blocks the ability of T cells to bind to $\mathrm{CD} 80 / 86$ leading to $\mathrm{T}$ cell activation through disinhibition 4) $\alpha$-PDL1 blocks the ability of tumor cells to bind to $\mathrm{T}$ cells leading to $\mathrm{T}$ cell activation through disinhibition 5) With CTLA-4 bound by antibody, CD28 can now bind CD80/86 on APCs and receive the necessary co-stimulatory signals to amount an effective antitumor response
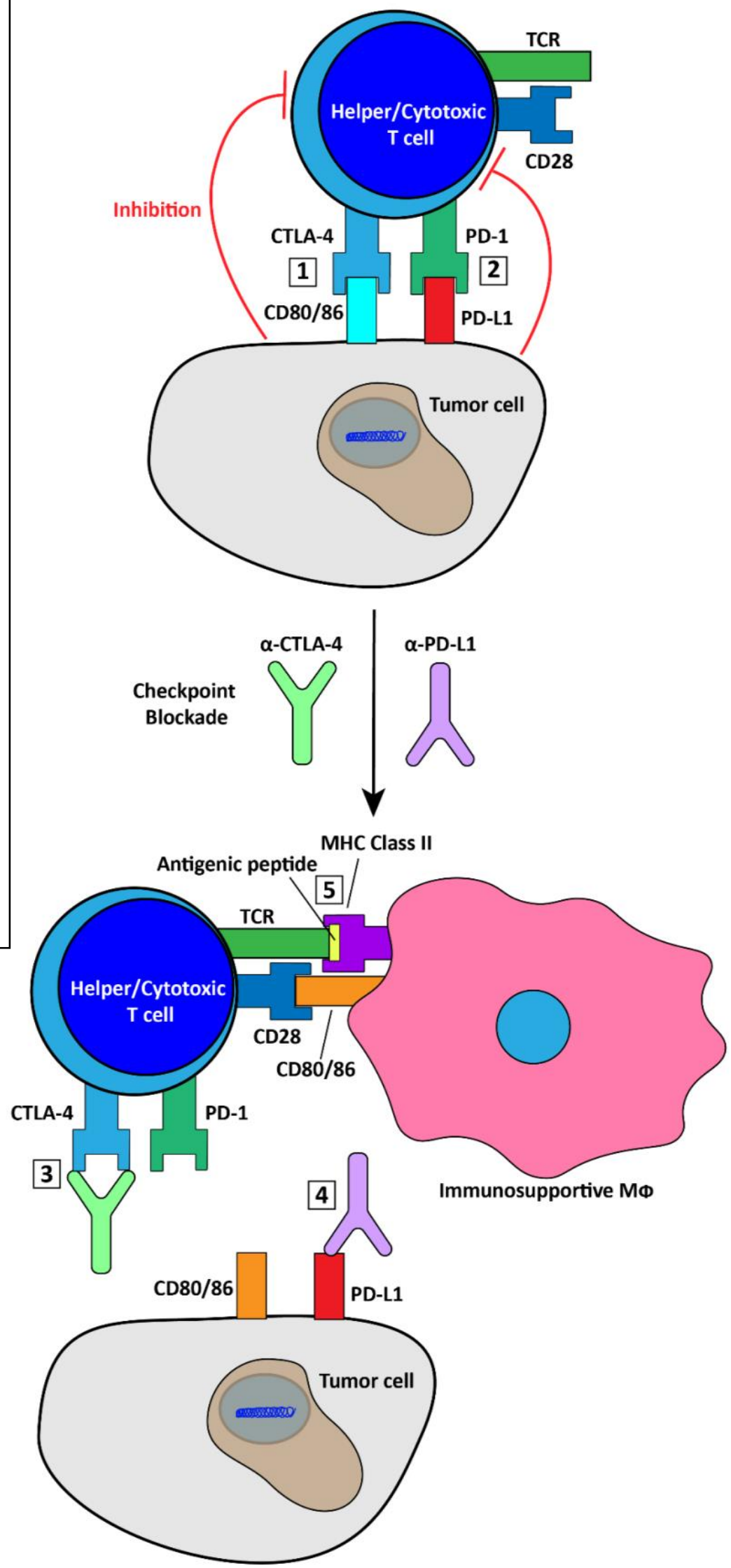


\section{References}

1. Cancer.org. 2020. Key Statistics For Osteosarcoma. [online] Available at: <https://www.cancer.org/cancer/osteosarcoma/about/key-statistics.html> [Accessed 29 March 2020].

2. Hansen, M. F., Seton, M., \& Merchant, A. (2006). Osteosarcoma in Paget's disease of bone. Journal of bone and mineral research, 21(S2), P58-P63.

3. Cancer.org. 2020. Risk Factors For Bone Cancer. [online] Available at: <https://www.cancer.org/cancer/bone-cancer/causes-risks-prevention/risk-factors.html> [Accessed 29 March 2020].

4. Jawad, M. U., Cheung, M. C., Clarke, J., Koniaris, L. G., \& Scully, S. P. (2011). Osteosarcoma: improvement in survival limited to high-grade patients only. Journal of cancer research and clinical oncology, 137(4), 597-607.

5. Lindsey, B. A., Markel, J. E., \& Kleinerman, E. S. (2017). Osteosarcoma overview. Rheumatology and therapy, 4(1), 25-43.

6. Cancer.org. 2020. Survival Rates For Osteosarcoma. [online] Available at: <https://www.cancer.org/cancer/osteosarcoma/detection-diagnosis-staging/survival-rates.html> [Accessed 29 March 2020].

7. Smeland, S., Bielack, S. S., Whelan, J., Bernstein, M., Hogendoorn, P., Krailo, M. D., ... \& Antal, I. (2019). Survival and prognosis with osteosarcoma: outcomes in more than 2000 patients in the EURAMOS-1 (European and American Osteosarcoma Study) cohort. European Journal of Cancer, 109, 36-50.

8. Collier, C. D., Wirtz, E. C., Knafler, G. J., Morris, W. Z., Getty, P. J., \& Greenfield, E. M. (2018). Micrometastatic drug screening platform shows heterogeneous response to MAP chemotherapy in osteosarcoma cell lines. Clinical Orthopaedics and Related Research®, 476(7), 1400-1411.

9. Li, S., Sun, W., Wang, H., Zuo, D., Hua, Y., \& Cai, Z. (2015). Research progress on the multidrug resistance mechanisms of osteosarcoma chemotherapy and reversal. Tumor Biology, 36(3), 1329-1338.

10. Ferrari, S., \& Serra, M. (2015). An update on chemotherapy for osteosarcoma. Expert opinion on pharmacotherapy, 16(18), 2727-2736.

11. D Lamplot, J., Denduluri, S., Qin, J., Li, R., Liu, X., Zhang, H., ... \& Luo, X. (2013). The current and future therapies for human osteosarcoma. Current cancer therapy reviews, 9(1), $55-77$.

12. Selvarajah, S., Yoshimoto, M., Ludkovski, O., Park, P. C., Bayani, J., Thorner, P., ... \& Zielenska, M. (2008). Genomic signatures of chromosomal instability and osteosarcoma progression detected by high resolution array $\mathrm{CGH}$ and interphase FISH. Cytogenetic and genome research, 122(1), 5-15.

13. Chen X, Bahrami A, Pappo A, et al. 2014. Recurrent somatic structural variations contribute to tumorigenesis in pediatric osteosarcoma. Cell Rep 7:104-112.

14. Martin, J. W., Squire, J. A., \& Zielenska, M. (2012). The genetics of osteosarcoma. Sarcoma, 2012.

15. Robl, B., Pauli, C., Botter, S. M., Bode-Lesniewska, B., \& Fuchs, B. (2015). Prognostic value of tumor suppressors in osteosarcoma before and after neoadjuvant chemotherapy. BMC cancer, 15(1), 379. 
16. Li, C., Cong, Y., Liu, X., Zhou, X., Zhou, G., Lu, M., ... \& Wu, S. (2016). The progress of molecular diagnostics of osteosarcoma. lung, 11, 13.

17. Scott, M. C., Sarver, A. L., Tomiyasu, H., Cornax, I., Van Etten, J., Varshney, J., ... \& Modiano, J. F. (2015). Aberrant retinoblastoma (RB)-E2F transcriptional regulation defines molecular phenotypes of osteosarcoma. Journal of Biological Chemistry, 290(47), 28070-28083. 18. Ren, W., \& Gu, G. (2017). Prognostic implications of RB 1 tumour suppressor gene alterations in the clinical outcome of human osteosarcoma: a meta-analysis. European journal of cancer care, 26(1), e12401.

19. Brady, S. W., Ma, X., Bahrami, A., Satas, G., Wu, G., Newman, S., ... \& Edmonson, M. N. (2019). The clonal evolution of metastatic osteosarcoma as shaped by cisplatin treatment. Molecular Cancer Research, 17(4), 895-906.

20. Zhao, E., Xu, H., Wang, L., Kryczek, I., Wu, K., Hu, Y., ... \& Zou, W. (2012). Bone marrow and the control of immunity. Cellular \& molecular immunology, 9(1), 11-19.

21. Kim, C. H. (2010). Homeostatic and pathogenic extramedullary hematopoiesis. Journal of blood medicine, 1, 13.

22. Sarrias, M. R., Farnós, M., Mota, R., Sánchez-Barbero, F., Ibáñez, A., Gimferrer, I., ... \& Lozano, F. (2007). CD6 binds to pathogen-associated molecular patterns and protects from LPSinduced septic shock. Proceedings of the National Academy of Sciences, 104(28), 11724-11729.

23. Akira, S., \& Hemmi, H. (2003). Recognition of pathogen-associated molecular patterns by TLR family. Immunology letters, 85(2), 85-95.

24. Jensen, S., \& Thomsen, A. R. (2012). Sensing of RNA viruses: a review of innate immune receptors involved in recognizing RNA virus invasion. Journal of virology, 86(6), 29002910.

25. Takada, H., \& Uehara, A. (2006). Enhancement of TLR-mediated innate immune responses by peptidoglycans through NOD signaling. Current pharmaceutical design, 12(32), 4163-4172.

26. Ray, A., Cot, M., Puzo, G., Gilleron, M., \& Nigou, J. (2013). Bacterial cell wall macroamphiphiles: pathogen-/microbe-associated molecular patterns detected by mammalian innate immune system. Biochimie, 95(1), 33-42.

27. Vénéreau, E., Ceriotti, C., \& Bianchi, M. E. (2015). DAMPs from cell death to new life. Frontiers in immunology, 6, 422.

28. Graeber, M. B., \& Streit, W. J. (2010). Microglia: biology and pathology. Acta neuropathologica, 119(1), 89-105.

29. Naito, M., Hasegawa, G., Ebe, Y., \& Yamamoto, T. (2004). Differentiation and function of Kupffer cells. Medical Electron Microscopy, 37(1), 16-28.

30. Fels, A. O., \& Cohn, Z. A. (1986). The alveolar macrophage. Journal of applied physiology, 60(2), 353-369.

31. Carleton, H. M. (1934). Studies on epithelial phagocytosis. II.-A method for demonstrating the origin of dust cells. Proceedings of the Royal Society of London. Series B, Containing Papers of a Biological Character, 114(790), 513-523.

32. Roodman, G. D. (1999). Cell biology of the osteoclast. Experimental hematology, 27(8), 1229-1241.

33. Wynn, T. A., \& Vannella, K. M. (2016). Macrophages in tissue repair, regeneration, and fibrosis. Immunity, 44(3), 450-462.

34. Krenkel, O., \& Tacke, F. (2017). Liver macrophages in tissue homeostasis and disease. Nature Reviews Immunology, 17(5), 306. 
35. Amit, I., Winter, D. R., \& Jung, S. (2016). The role of the local environment and epigenetics in shaping macrophage identity and their effect on tissue homeostasis. Nature immunology, 17(1), 18.

36. Henson, P. M., \& Hume, D. A. (2006). Apoptotic cell removal in development and tissue homeostasis. Trends in immunology, 27(5), 244-250.

37. Agrawal, T., Vats, V., Wallace, P. K., Singh, A., Salhan, S., \& Mittal, A. (2009).

Recruitment of myeloid and plasmacytoid dendritic cells in cervical mucosa during Chlamydia trachomatis infection. Clinical microbiology and infection, 15(1), 50-59.

38. Greaves, D. R., \& Schall, T. J. (2000). Chemokines and myeloid cell

recruitment. Microbes and infection, 2(3), 331-336.

39. Shi, C., \& Pamer, E. G. (2011). Monocyte recruitment during infection and inflammation. Nature reviews immunology, 11(11), 762-774.

40. Akashi, K., Traver, D., Miyamoto, T., \& Weissman, I. L. (2000). A clonogenic common myeloid progenitor that gives rise to all myeloid lineages. Nature, 404(6774), 193-197.

41. Kondo, M., Weissman, I. L., \& Akashi, K. (1997). Identification of clonogenic common lymphoid progenitors in mouse bone marrow. Cell, 91(5), 661-672.

42. Prchal, J. T., Throckmorton, D. W., Carroll, A. J., Fuson, E. W., Gams, R. A., \& Prchal, J. F. (1978). A common progenitor for human myeloid and lymphoid cells. Nature, 274(5671), 590-591.

43. Mansson, R., Zandi, S., Welinder, E., Tsapogas, P., Sakaguchi, N., Bryder, D., \& Sigvardsson, M. (2010). Single-cell analysis of the common lymphoid progenitor compartment reveals functional and molecular heterogeneity. Blood, The Journal of the American Society of Hematology, 115(13), 2601-2609.

44. Spits, H., \& Cupedo, T. (2012). Innate lymphoid cells: emerging insights in development, lineage relationships, and function. Annual review of immunology, 30, 647-675.

45. Katsura, Y. (2002). Redefinition of lymphoid progenitors. Nature Reviews Immunology, 2(2), 127-132.

46. Freud, A. G., Yokohama, A., Becknell, B., Lee, M. T., Mao, H. C., Ferketich, A. K., \& Caligiuri, M. A. (2006). Evidence for discrete stages of human natural killer cell differentiation in vivo. Journal of Experimental Medicine, 203(4), 1033-1043.

47. Liu, C. C., Perussia, B., \& Young, J. D. E. (2000). The emerging role of IL-15 in NK-cell development. Immunology today, 21(3), 113-116.

48. Barreda, D. R., Hanington, P. C., \& Belosevic, M. (2004). Regulation of myeloid development and function by colony stimulating factors. Developmental \& Comparative Immunology, 28(5), 509-554.

49. Pether, N. S., Brothwood, J. L., van Berkel, C., Dunwoodie, E. H., Blake, R. L., Price, C. P., ... \& Hall, G. (2017). Comparative diagnostic performance of the granulocyte and neutrophil counts. Practical laboratory medicine, 9, 45-52.

50. Mócsai, A. (2013). Diverse novel functions of neutrophils in immunity, inflammation, and beyond. Journal of Experimental Medicine, 210(7), 1283-1299.

51. Furze, R. C., \& Rankin, S. M. (2008). The role of the bone marrow in neutrophil clearance under homeostatic conditions in the mouse. The FASEB Journal, 22(9), 3111-3119. 52. Kobayashi, S. D., Voyich, J. M., \& DeLeo, F. R. (2003). Regulation of the neutrophilmediated inflammatory response to infection. Microbes and Infection, 5(14), 1337-1344. 
53. Hammond, M. E., Lapointe, G. R., Feucht, P. H., Hilt, S., Gallegos, C. A., Gordon, C. A., ... \& Tekamp-Olson, P. (1995). IL-8 induces neutrophil chemotaxis predominantly via type I IL-8 receptors. The Journal of Immunology, 155(3), 1428-1433.

54. Lee, W. L., Harrison, R. E., \& Grinstein, S. (2003). Phagocytosis by neutrophils. Microbes and infection, 5(14), 1299-1306.

55. Brinkmann, V., Reichard, U., Goosmann, C., Fauler, B., Uhlemann, Y., Weiss, D. S., ... \& Zychlinsky, A. (2004). Neutrophil extracellular traps kill bacteria. science, 303(5663), $1532-$ 1535

56. Tamoutounour, S., Guilliams, M., Sanchis, F. M., Liu, H., Terhorst, D., Malosse, C., ... \& Dalod, M. (2013). Origins and functional specialization of macrophages and of conventional and monocyte-derived dendritic cells in mouse skin. Immunity, 39(5), 925-938.

57. Pieters, J. (1997). MHC class II restricted antigen presentation. Current opinion in immunology, 9(1), 89-96.

58. Tsou, C. L., Peters, W., Si, Y., Slaymaker, S., Aslanian, A. M., Weisberg, S. P., ... \& Charo, I. F. (2007). Critical roles for CCR2 and MCP-3 in monocyte mobilization from bone marrow and recruitment to inflammatory sites. The Journal of clinical investigation, 117(4), 902909.

59. Italiani, P., \& Boraschi, D. (2014). From monocytes to M1/M2 macrophages:

phenotypical vs. functional differentiation. Frontiers in immunology, 5, 514.

60. Chimen, M., Yates, C. M., McGettrick, H. M., Ward, L. S., Harrison, M. J., Apta, B., ... \& Rainger, G. E. (2017). Monocyte subsets coregulate inflammatory responses by integrated signaling through TNF and IL-6 at the endothelial cell interface. The Journal of Immunology, 198(7), 2834-2843.

61. Jakubzick, C. V., Randolph, G. J., \& Henson, P. M. (2017). Monocyte differentiation and antigen-presenting functions. Nature Reviews Immunology, 17(6), 349-362.

62. Lee, J., Tam, H., Adler, L., Ilstad-Minnihan, A., Macaubas, C., \& Mellins, E. D. (2017). The MHC class II antigen presentation pathway in human monocytes differs by subset and is regulated by cytokines. PloS one, 12(8), e0183594.

63. Ginhoux, F., \& Guilliams, M. (2016). Tissue-resident macrophage ontogeny and homeostasis. Immunity, 44(3), 439-449.

64. $\quad$ Sprangers, S., Vries, T. J. D., \& Everts, V. (2016). Monocyte heterogeneity: consequences for monocyte-derived immune cells. Journal of immunology research, 2016.

65. Divangahi, M., King, I. L., \& Pernet, E. (2015). Alveolar macrophages and type I IFN in airway homeostasis and immunity. Trends in immunology, 36(5), 307-314.

66. Bilzer, M., Roggel, F., \& Gerbes, A. L. (2006). Role of Kupffer cells in host defense and liver disease. Liver International, 26(10), 1175-1186.

67. Borges da Silva, H., Fonseca, R., Pereira, R. M., Cassado, A. D. A., Álvarez, J. M., \& D'Império Lima, M. R. (2015). Splenic macrophage subsets and their function during bloodborne infections. Frontiers in immunology, 6, 480.

68. Teitelbaum, S. L. (2000). Bone resorption by osteoclasts. Science, 289(5484), 1504-1508.

69. Colonna, M., \& Butovsky, O. (2017). Microglia function in the central nervous system during health and neurodegeneration. Annual review of immunology, 35, 441-468.

70. Barker, R. N., Erwig, L. P., Hill, K. S. K., Devine, A., Pearce, W. P., \& Rees, A. J. (2002). Antigen presentation by macrophages is enhanced by the uptake of necrotic, but not apoptotic, cells. Clinical \& Experimental Immunology, 127(2), 220-225. 
71. Mantovani, A., Sica, A., \& Locati, M. (2005). Macrophage polarization comes of age. Immunity, 23(4), 344-346.

72. Sica, A., \& Mantovani, A. (2012). Macrophage plasticity and polarization: in vivo veritas. The Journal of clinical investigation, 122(3), 787-795.

73. Liu, H., Wu, X., Gang, N., Wang, S., Deng, W., Zan, L., \& Yu, S. (2015). Macrophage functional phenotype can be consecutively and reversibly shifted to adapt to microenvironmental changes. International journal of clinical and experimental medicine, 8(2), 3044.

74. Müller, E., Christopoulos, P. F., Halder, S., Lunde, A., Beraki, K., Speth, M., ... \& Corthay, A. (2017). Toll-like receptor ligands and interferon- $\gamma$ synergize for induction of antitumor M1 macrophages. Frontiers in immunology, 8, 1383.

75. Ferrante, C. J., \& Leibovich, S. J. (2012). Regulation of macrophage polarization and wound healing. Advances in wound care, 1(1), 10-16.

76. Lu, J., Cao, Q., Zheng, D., Sun, Y., Wang, C., Yu, X., ... \& Wang, X. (2013). Discrete functions of $\mathrm{M} 2 \mathrm{a}$ and $\mathrm{M} 2 \mathrm{c}$ macrophage subsets determine their relative efficacy in treating chronic kidney disease. Kidney international, 84(4), 745-755.

77. Wang, L. X., Zhang, S. X., Wu, H. J., Rong, X. L., \& Guo, J. (2019). M2b macrophage polarization and its roles in diseases. Journal of leukocyte biology, 106(2), 345-358.

78. Wang, Q., Ni, H., Lan, L., Wei, X., Xiang, R., \& Wang, Y. (2010). Fra-1 protooncogene regulates IL-6 expression in macrophages and promotes the generation of $\mathrm{M} 2 \mathrm{~d}$ macrophages. Cell research, 20(6), 701.

79. Vogel, D. Y., Glim, J. E., Stavenuiter, A. W., Breur, M., Heijnen, P., Amor, S., ... \& Beelen, R. H. (2014). Human macrophage polarization in vitro: maturation and activation methods compared. Immunobiology, 219(9), 695-703.

80. Orecchioni, M., Ghosheh, Y., Pramod, A. B., \& Ley, K. (2020). Corrigendum: Macrophage Polarization: Different Gene Signatures in M1 (LPS+) vs. Classically and M2 (LPS-) vs. Alternatively Activated Macrophages. Frontiers in Immunology, 11.

81. Murray, P. J., Allen, J. E., Biswas, S. K., Fisher, E. A., Gilroy, D. W., Goerdt, S., ... \& Locati, M. (2014). Macrophage activation and polarization: nomenclature and experimental guidelines. Immunity, 41(1), 14-20.

82. Martinez, F. O., \& Gordon, S. (2014). The M1 and M2 paradigm of macrophage activation: time for reassessment. F1000prime reports, 6 .

83. Guermonprez, P., Valladeau, J., Zitvogel, L., Théry, C., \& Amigorena, S. (2002). Antigen presentation and T cell stimulation by dendritic cells. Annual review of immunology, 20(1), 621-667.

84. Dalod, M., Chelbi, R., Malissen, B., \& Lawrence, T. (2014). Dendritic cell maturation: functional specialization through signaling specificity and transcriptional programming. The EMBO journal, 33(10), 1104-1116.

85. Boltjes, A., \& Van Wijk, F. (2014). Human dendritic cell functional specialization in steady-state and inflammation. Frontiers in immunology, 5, 131.

86. Manh, T. P. V., Alexandre, Y., Baranek, T., Crozat, K., \& Dalod, M. (2013).

Plasmacytoid, conventional, and monocyte-derived dendritic cells undergo a profound and convergent genetic reprogramming during their maturation. European journal of immunology, 43(7), 1706-1715.

87. Collin, M., \& Bigley, V. (2018). Human dendritic cell subsets: an update. Immunology, 154(1), 3-20. 
88. Colonna, Marco, Giorgio Trinchieri, and Yong-Jun Liu. "Plasmacytoid dendritic cells in immunity." Nature immunology 5.12 (2004): 1219-1226.

89. Schlitzer, A., Sivakamasundari, V., Chen, J., Sumatoh, H. R. B., Schreuder, J., Lum, J., ... \& Renia, L. (2015). Identification of cDC1-and cDC2-committed DC progenitors reveals early lineage priming at the common DC progenitor stage in the bone marrow. Nature immunology, 16(7), 718.

90. León, B., \& Ardavín, C. (2008). Monocyte-derived dendritic cells in innate and adaptive immunity. Immunology and cell biology, 86(4), 320-324.

91. Kiertscher, S. M., Luo, J., Dubinett, S. M., \& Roth, M. D. (2000). Tumors promote altered maturation and early apoptosis of monocyte-derived dendritic cells. The Journal of Immunology, 164(3), 1269-1276.

92. Hasebe, H., Nagayama, H., Sato, K., Enomoto, M., Takeda, Y., Takahashi, T. A., ... \& Eriguchi, M. (2000). Dysfunctional regulation of the development of monocyte-derived dendritic cells in cancer patients. Biomedicine \& pharmacotherapy, 54(6), 291-298.

93. Miyazaki, M., Miyazaki, K., Chen, K., Jin, Y., Turner, J., Moore, A. J., ... \& Lin, Y. C. (2017). The E-Id protein axis specifies adaptive lymphoid cell identity and suppresses thymic innate lymphoid cell development. Immunity, 46(5), 818-834.

94. Klose, C. S., \& Artis, D. (2016). Innate lymphoid cells as regulators of immunity, inflammation and tissue homeostasis. Nature immunology, 17(7), 765.

95. Robinette, M. L., \& Colonna, M. (2016). Innate lymphoid cells and the MHC. Hla, 87(1), 5-11.

96. Pegram, H. J., Andrews, D. M., Smyth, M. J., Darcy, P. K., \& Kershaw, M. H. (2011). Activating and inhibitory receptors of natural killer cells. Immunology and cell biology, 89(2), 216-224.

97. Bauer, S., Groh, V., Wu, J., Steinle, A., Phillips, J. H., Lanier, L. L., \& Spies, T. (1999). Activation of NK cells and T cells by NKG2D, a receptor for stress-inducible

MICA. Science, 285(5428), 727-729.

98. López-Larrea, C., Suárez-Alvarez, B., López-Soto, A., López-Vázquez, A., \& Gonzalez, S. (2008). The NKG2D receptor: sensing stressed cells. Trends in molecular medicine, 14(4), 179-189.

99. Paul, S., \& Lal, G. (2017). The molecular mechanism of natural killer cells function and its importance in cancer immunotherapy. Frontiers in immunology, 8, 1124.

100. Ye, J., Ortaido, J. R., Conlon, K., Winkler-Pickett, R., \& Young, H. A. (1995). Cellular and molecular mechanisms of IFN- $\gamma$ production induced by IL-2 and IL-12 in a human NK cell line. Journal of leukocyte biology, 58(2), 225-233.

101. Ferlazzo, G., Pack, M., Thomas, D., Paludan, C., Schmid, D., Strowig, T., ... \& Münz, C. (2004). Distinct roles of IL-12 and IL-15 in human natural killer cell activation by dendritic cells from secondary lymphoid organs. Proceedings of the National Academy of Sciences, 101(47), 16606-16611.

102. Lehmann, D., Spanholtz, J., Sturtzel, C., Tordoir, M., Schlechta, B., Groenewegen, D., \& Hofer, E. (2014). IL-12 directs further maturation of ex vivo differentiated NK cells with improved therapeutic potential. PLoS One, 9(1).

103. Biron, C. A., Young, H. A., \& Kasaian, M. T. (1990). Interleukin 2-induced proliferation of murine natural killer cells in vivo. The Journal of experimental medicine, 171(1), 173-188. 
104. Wilson, E. B., El-Jawhari, J. J., Neilson, A. L., Hall, G. D., Melcher, A. A., Meade, J. L., \& Cook, G. P. (2011). Human tumour immune evasion via TGF- $\beta$ blocks NK cell activation but not survival allowing therapeutic restoration of anti-tumour activity. PloS one, 6(9).

105. Janeway Jr, C. A., Travers, P., Walport, M., \& Shlomchik, M. J. (2001). Principles of innate and adaptive immunity. In Immunobiology: The Immune System in Health and Disease. 5th edition. Garland Science.

106. Tonegawa, S. (1983). Somatic generation of antibody diversity. Nature, 302(5909), 575581.

107. Burnet, S. F. M. (1959). The clonal selection theory of acquired immunity.

108. Castellino, F., Galli, G., Del Giudice, G., \& Rappuoli, R. (2009). Generating memory with vaccination. European journal of immunology, 39(8), 2100-2105.

109. Shortman, K., Egerton, M., Spangrude, G. J., \& Scollay, R. (1990, January). The generation and fate of thymocytes. In Seminars in immunology (Vol. 2, No. 1, pp. 3-12).

110. Rahemtullah, A., Reichard, K. K., Preffer, F. I., Harris, N. L., \& Hasserjian, R. P. (2006). A double-positive CD4+ CD8+ T-cell population is commonly found in nodular lymphocyte predominant Hodgkin lymphoma. American journal of clinical pathology, 126(5), 805-814.

111. Slavik, J. M., Hutchcroft, J. E., \& Bierer, B. E. (1999). CD28/CTLA-4 and CD80/CD86 families. Immunologic research, 19(1), 1-24.

112. Clevers, H., Alarcon, B., Wileman, T., \& Terhorst, C. (1988). The T cell receptor/CD3 complex: a dynamic protein ensemble. Annual review of immunology, 6(1), 629-662.

113. Cambier, J. C. (1995). Antigen and Fc receptor signaling. The awesome power of the immunoreceptor tyrosine-based activation motif (ITAM). The Journal of Immunology, 155(7), 3281-3285.

114. Schwartz, R. H. (2003). T cell anergy. Annual review of immunology, 21(1), 305-334.

115. Pamer, E., \& Cresswell, P. (1998). Mechanisms of MHC class I-restricted antigen processing. Annual review of immunology, 16(1), 323-358.

116. Hewitt, E. W. (2003). The MHC class I antigen presentation pathway: strategies for viral immune evasion. Immunology, 110(2), 163-169.

117. Trapani, J. A., \& Smyth, M. J. (2002). Functional significance of the perforin/granzyme cell death pathway. Nature Reviews Immunology, 2(10), 735-747.

118. Abbas, A. K., Murphy, K. M., \& Sher, A. (1996). Functional diversity of helper T lymphocytes. Nature, 383(6603), 787-793.

119. Dong, C., \& Flavell, R. A. (2000). Cell fate decision: T-helper 1 and 2 subsets in immune responses. Arthritis Research \& Therapy, 2(3), 179.

120. London, C. A., Abbas, A. K., \& Kelso, A. (1998). Helper T cell subsets: heterogeneity, functions and development. Veterinary immunology and immunopathology, 63(1-2), 37-44.

121. Schmitt, N., \& Ueno, H. (2015). Regulation of human helper T cell subset differentiation by cytokines. Current opinion in immunology, 34, 130-136.

122. Gerloni, M., \& Zanetti, M. (2005, June). CD4 T cells in tumor immunity. In Springer seminars in immunopathology (Vol. 27, No. 1, pp. 37-48). Springer-Verlag.

123. Janeway Jr, C. A., Carding, S., Jones, B., Murray, J., Portoles, P., Rasmussen, R., ... \& Bottomly, K. (1988). CD4+ T cells: specificity and function. Immunological reviews, 101(1), 3980 .

124. Miyara, M., \& Sakaguchi, S. (2007). Natural regulatory T cells: mechanisms of suppression. Trends in molecular medicine, 13(3), 108-116. 
125. von Herrath, M. G., \& Harrison, L. C. (2003). Antigen-induced regulatory T cells in autoimmunity. Nature Reviews Immunology, 3(3), 223-232.

126. Dons, E. M., Raimondi, G., Cooper, D. K., \& Thomson, A. W. (2012). Induced regulatory T cells: mechanisms of conversion and suppressive potential. Human immunology, 73(4), 328-334.

127. Maloy, K. J., \& Powrie, F. (2001). Regulatory T cells in the control of immune pathology. Nature immunology, 2(9), 816-822.

128. Wherry, E. J. (2011). T cell exhaustion. Nature immunology, 12(6), 492-499.

129. Akbar, A. N., \& Henson, S. M. (2011). Are senescence and exhaustion intertwined or unrelated processes that compromise immunity?. Nature Reviews Immunology, 11(4), 289-295.

130. Vallejo, A. N. (2005). CD28 extinction in human T cells: altered functions and the program of T-cell senescence. Immunological reviews, 205(1), 158-169.

131. Effros, R. B. (2003). Replicative senescence: the final stage of memory T cell differentiation?. Current HIV research, 1(2), 153-165.

132. Yang, Z. Z., Grote, D. M., Ziesmer, S. C., Niki, T., Hirashima, M., Novak, A. J., ... \& Ansell, S. M. (2012). IL-12 upregulates TIM-3 expression and induces T cell exhaustion in patients with follicular B cell non-Hodgkin lymphoma. The Journal of clinical investigation, 122(4), 1271-1282.

133. Jiang, Y., Li, Y., \& Zhu, B. (2015). T-cell exhaustion in the tumor microenvironment. Cell death \& disease, 6(6), e1792.

134. Deshpande, A. M., Fontana, R., Zhang, Y., Ruisi, M., Zhang, D., Senger, T., \& Rolfe, P. A. (2018). Galectin-9 drives TIM-3 mediated immune suppression.

135. Li, H., Wu, K., Tao, K., Chen, L., Zheng, Q., Lu, X., ... \& Zou, W. (2012). Tim3/galectin-9 signaling pathway mediates T-cell dysfunction and predicts poor prognosis in patients with hepatitis B virus-associated hepatocellular carcinoma. Hepatology, 56(4), 13421351.

136. Sakuishi, K., Apetoh, L., Sullivan, J. M., Blazar, B. R., Kuchroo, V. K., \& Anderson, A. C. (2010). Targeting Tim-3 and PD-1 pathways to reverse T cell exhaustion and restore antitumor immunity. Journal of Experimental Medicine, 207(10), 2187-2194.

137. Latchman, Y. E., Liang, S. C., Wu, Y., Chernova, T., Sobel, R. A., Klemm, M., ... \& Sharpe, A. H. (2004). PD-L1-deficient mice show that PD-L1 on T cells, antigen-presenting cells, and host tissues negatively regulates T cells. Proceedings of the National Academy of Sciences, 101(29), 10691-10696.

138. Wiemann, B., \& Starnes, C. O. (1994). Coley's toxins, tumor necrosis factor and cancer research: a historical perspective. Pharmacology \& therapeutics, 64(3), 529-564.

139. Starnes, C. O. (1992). Coley's toxins in perspective. Nature, 357(6373), 11-12.

140. Nauts, H. C., Fowler, G. A., \& Bogatko, F. H. (1953). A review of the influence of bacterial infection and of bacterial products (Coley's toxins) on malignant tumors in man; a critical analysis of 30 inoperable cases treated by Coley's mixed toxins, in which diagnosis was confirmed by microscopic examination selected for special study. Acta medica Scandinavica. Supplementum, 276, 1.

141. Gajewski, T. F., Schreiber, H., \& Fu, Y. X. (2013). Innate and adaptive immune cells in the tumor microenvironment. Nature immunology, 14(10), 1014.

142. Kitamura, T., Qian, B. Z., \& Pollard, J. W. (2015). Immune cell promotion of metastasis. Nature Reviews Immunology, 15(2), 73-86. 
143. Emens, L. A., Butterfield, L. H., Hodi, F. S., Marincola, F. M., \& Kaufman, H. L. (2016). Cancer immunotherapy trials: leading a paradigm shift in drug development. Journal for immunotherapy of cancer, 4(1), 42.

144. Reuben, A., Zhang, J., Chiou, S. H., Gittelman, R. M., Li, J., Lee, W. C., ... \& Quek, K. (2020). Comprehensive T cell repertoire characterization of non-small cell lung cancer. Nature communications, 11(1), 1-13.

145. Burugu, S., Asleh-Aburaya, K., \& Nielsen, T. O. (2017). Immune infiltrates in the breast cancer microenvironment: detection, characterization and clinical implication. Breast cancer, 24(1), 3-15.

146. Lieberman, N., Kovar, H., DeGolier, K., Davis, A., Hoglund, V., Stevens, J., ... \& Crane, C. (2018). IMMU-16. CHARACTERIZING TUMOR-IMMUNE INTERACTIONS IN DIFFUSE INTRINSIC PONTINE GLIOMA. Neuro-Oncology, 20(suppl_2), i102-i102. 147. Capietto, A. H., Jhunjhunwala, S., \& Delamarre, L. (2017). Characterizing neoantigens for personalized cancer immunotherapy. Current opinion in immunology, 46, 58-65.

148. Yarchoan, M., Xing, D., Luan, L., Xu, H., Sharma, R. B., Popovic, A., ... \& Taube, J. M. (2017). Characterization of the immune microenvironment in hepatocellular carcinoma. Clinical Cancer Research, 23(23), 7333-7339.

149. Nishida-Aoki, N., \& Gujral, T. S. (2019). Emerging approaches to study cell-cell interactions in tumor microenvironment. Oncotarget, 10(7), 785.

150. Fukumura, D., \& Jain, R. K. (2007). Tumor microenvironment abnormalities: causes, consequences, and strategies to normalize. Journal of cellular biochemistry, 101(4), 937-949.

151. Rankin, E. B., \& Giaccia, A. J. (2016). Hypoxic control of metastasis. Science, 352(6282), 175-180.

152. Finger, E. C., \& Giaccia, A. J. (2010). Hypoxia, inflammation, and the tumor microenvironment in metastatic disease. Cancer and Metastasis Reviews, 29(2), 285-293.

153. Forster, J. C., Harriss-Phillips, W. M., Douglass, M. J., \& Bezak, E. (2017). A review of the development of tumor vasculature and its effects on the tumor microenvironment. Hypoxia, 5, 21.

154. Vareki, S. M. (2018). High and low mutational burden tumors versus immunologically hot and cold tumors and response to immune checkpoint inhibitors. Journal for immunotherapy of cancer, 6(1), 157.

155. Binnewies, M., Roberts, E. W., Kersten, K., Chan, V., Fearon, D. F., Merad, M., ... \& Vonderheide, R. H. (2018). Understanding the tumor immune microenvironment (TIME) for effective therapy. Nature medicine, 24(5), 541-550.

156. Kather, J. N., Suarez-Carmona, M., Charoentong, P., Weis, C. A., Hirsch, D., Bankhead, P., ... \& Schott, S. (2018). Topography of cancer-associated immune cells in human solid tumors. Elife, 7, e36967.

157. Bonaventura, P., Shekarian, T., Alcazer, V., Valladeau-Guilemond, J., ValsesiaWittmann, S., Amigorena, S., ... \& Depil, S. (2019). Cold tumors: a therapeutic challenge for immunotherapy. Frontiers in immunology, 10.

158. Blando, J., Sharma, A., Higa, M. G., Zhao, H., Vence, L., Yadav, S. S., ... \& Wargo, J. (2019). Comparison of immune infiltrates in melanoma and pancreatic cancer highlights VISTA as a potential target in pancreatic cancer. Proceedings of the National Academy of Sciences, 116(5), 1692-1697.

159. Mukherji, B. (2013). Immunology of melanoma. Clinics in dermatology, 31(2), 156-165. 
160. Stankovic, B., Bjørhovde, H. A. K., Skarshaug, R., Aamodt, H., Frafjord, A., Müller, E., ... \& Helland, A. (2019). Immune cell composition in human non-small cell lung cancer. Frontiers in immunology, 9, 3101.

161. Ott, P. A., Hodi, F. S., \& Robert, C. (2013). CTLA-4 and PD-1/PD-L1 blockade: new immunotherapeutic modalities with durable clinical benefit in melanoma patients.

162. Postow, M. A., Callahan, M. K., \& Wolchok, J. D. (2015). Immune checkpoint blockade in cancer therapy. Journal of clinical oncology, 33(17), 1974.

163. Pai-Scherf, L., Blumenthal, G. M., Li, H., Subramaniam, S., Mishra-Kalyani, P. S., He, K., ... \& McKee, A. E. (2017). FDA approval summary: pembrolizumab for treatment of metastatic non-small cell lung cancer: first-line therapy and beyond. The oncologist, 22(11), 1392.

164. Kaminski, J. M., Shinohara, E., Summers, J. B., Niermann, K. J., Morimoto, A., \& Brousal, J. (2005). The controversial abscopal effect. Cancer treatment reviews, 31(3), 159-172. 165. Kobayashi, M., Kubo, T., Komatsu, K., Fujisaki, A., Terauchi, F., Natsui, S., ... \& Morita, T. (2013). Changes in peripheral blood immune cells: their prognostic significance in metastatic renal cell carcinoma patients treated with molecular targeted therapy. Medical Oncology, 30(2), 556.

166. Porrata, L. F., Ristow, K., Colgan, J. P., Habermann, T. M., Witzig, T. E., Inwards, D. J., ... \& Thompson, C. (2012). Peripheral blood lymphocyte/monocyte ratio at diagnosis and survival in classical Hodgkin's lymphoma. Haematologica, 97(2), 262-269.

167. Liotta, F., Gacci, M., Frosali, F., Querci, V., Vittori, G., Lapini, A., ... \& Angeli, R. (2011). Frequency of regulatory T cells in peripheral blood and in tumour-infiltrating lymphocytes correlates with poor prognosis in renal cell carcinoma. BJU international, 107(9), 1500-1506.

168. Aliustaoglu, M., Bilici, A., Ustaalioglu, B. B. O., Konya, V., Gucun, M., Seker, M., \& Gumus, M. (2010). The effect of peripheral blood values on prognosis of patients with locally advanced gastric cancer before treatment. Medical oncology, 27(4), 1060-1065.

169. Twine, N. C., Stover, J. A., Marshall, B., Dukart, G., Hidalgo, M., Stadler, W., ... \& Slonim, D. K. (2003). Disease-associated expression profiles in peripheral blood mononuclear cells from patients with advanced renal cell carcinoma. Cancer research, 63(18), 6069-6075.

170. Baine, M. J., Chakraborty, S., Smith, L. M., Mallya, K., Sasson, A. R., Brand, R. E., \& Batra, S. K. (2011). Transcriptional profiling of peripheral blood mononuclear cells in pancreatic cancer patients identifies novel genes with potential diagnostic utility. PloS one, 6(2).

171. Krijgsman, D., de Vries, N. L., Skovbo, A., Andersen, M. N., Swets, M., Bastiaannet, E., ... \& Kuppen, P. J. (2019). Characterization of circulating T-, NK-, and NKT cell subsets in patients with colorectal cancer: the peripheral blood immune cell profile. Cancer Immunology, Immunotherapy, 68(6), 1011-1024.

172. Pernot, S., Terme, M., Radosevic-Robin, N., Castan, F., Badoual, C., Marcheteau, E., ... \& Ghiringhelli, F. (2020). Infiltrating and peripheral immune cell analysis in advanced gastric cancer according to the Lauren classification and its prognostic significance. Gastric Cancer, 23(1), 73-81.

173. Das, R. K., Vernau, L., Grupp, S. A., \& Barrett, D. M. (2019). Naïve T-cell deficits at diagnosis and after chemotherapy impair cell therapy potential in pediatric cancers. Cancer discovery, 9(4), 492-499.

174. Hogan, S. A., Courtier, A., Cheng, P. F., Jaberg-Bentele, N. F., Goldinger, S. M., Manuel, M., ... \& Raaijmakers, M. I. (2019). Peripheral blood TCR repertoire profiling may 
facilitate patient stratification for immunotherapy against melanoma. Cancer immunology research, 7(1), 77-85.

175. Choi, J., Maeng, H. G., Lee, S. J., Kim, Y. J., Kim, D. W., Lee, H. N., .. \& Park, S. J. (2018). Diagnostic value of peripheral blood immune profiling in colorectal cancer. Annals of surgical treatment and research, 94(6), 312-321.

176. Bose, A., Chakraborty, T., Chakraborty, K., Pal, S., \& Baral, R. (2008). Dysregulation in immune functions is reflected in tumor cell cytotoxicity by peripheral blood mononuclear cells from head and neck squamous cell carcinoma patients. Cancer Immunity Archive, 8(1), 10. 177. Spitzer, M. H., Carmi, Y., Reticker-Flynn, N. E., Kwek, S. S., Madhireddy, D., Martins, M. M., ... \& Fong, L. (2017). Systemic immunity is required for effective cancer immunotherapy. Cell, 168(3), 487-502.

178. Ormandy, L. A., Hillemann, T., Wedemeyer, H., Manns, M. P., Greten, T. F., \& Korangy, F. (2005). Increased populations of regulatory T cells in peripheral blood of patients with hepatocellular carcinoma. Cancer research, 65(6), 2457-2464.

179. Miller, A. M., Lundberg, K., Özenci, V., Banham, A. H., Hellström, M., Egevad, L., \& Pisa, P. (2006). CD4+ CD25 high T cells are enriched in the tumor and peripheral blood of prostate cancer patients. The Journal of Immunology, 177(10), 7398-7405.

180. Wolf, A. M., Wolf, D., Steurer, M., Gastl, G., Gunsilius, E., \& Grubeck-Loebenstein, B. (2003). Increase of regulatory T cells in the peripheral blood of cancer patients. Clinical cancer research, 9(2), 606-612.

181. Kobayashi, M., Kubo, T., Komatsu, K., Fujisaki, A., Terauchi, F., Natsui, S., ... \& Morita, T. (2013). Changes in peripheral blood immune cells: their prognostic significance in metastatic renal cell carcinoma patients treated with molecular targeted therapy. Medical Oncology, 30(2), 556.

182. Ichihara, F., Kono, K., Takahashi, A., Kawaida, H., Sugai, H., \& Fujii, H. (2003). Increased populations of regulatory $\mathrm{T}$ cells in peripheral blood and tumor-infiltrating lymphocytes in patients with gastric and esophageal cancers. Clinical Cancer Research, 9(12), 4404-4408.

183. Wang, L., Simons, D. L., Lu, X., Tu, T. Y., Avalos, C., Chang, A. Y., ... \& Lee, P. P. (2020). Breast cancer induces systemic immune changes on cytokine signaling in peripheral blood monocytes and lymphocytes. EBioMedicine, 52, 102631.

184. Chong, B. F., Wilson, A. J., Gibson, H. M., Hafner, M. S., Luo, Y., Hedgcock, C. J., \& Wong, H. K. (2008). Immune function abnormalities in peripheral blood mononuclear cell cytokine expression differentiates stages of cutaneous T-cell lymphoma/mycosis fungoides. Clinical Cancer Research, 14(3), 646-653.

185. Deng, L., Zhang, H., Luan, Y., Zhang, J., Xing, Q., Dong, S., ... \& Wang, S. (2010). Accumulation of foxp3+ T regulatory cells in draining lymph nodes correlates with disease progression and immune suppression in colorectal cancer patients. Clinical Cancer Research, 16(16), 4105-4112.

186. Showe, M. K., Kossenkov, A. V., \& Showe, L. C. (2012). The peripheral immune response and lung cancer prognosis. Oncoimmunology, 1(8), 1414-1416.

187. Zhang, B., Rong, G., Wei, H., Zhang, M., Bi, J., Ma, L., ... \& Fang, G. (2008). The prevalence of Th17 cells in patients with gastric cancer. Biochemical and biophysical research communications, 374(3), 533-537. 
188. Teijeira, A., Russo, E., \& Halin, C. (2014, March). Taking the lymphatic route: dendritic cell migration to draining lymph nodes. In Seminars in immunopathology (Vol. 36, No. 2, pp. 261-274). Springer Berlin Heidelberg.

189. Wu, C., Ning, H., Liu, M., Lin, J., Luo, S., Zhu, W., ... \& Ren, J. (2018). Spleen mediates a distinct hematopoietic progenitor response supporting tumor-promoting myelopoiesis. The Journal of clinical investigation, 128(8), 3425-3438.

190. Sica, A., Guarneri, V., \& Gennari, A. (2019). Myelopoiesis, metabolism and therapy: a crucial crossroads in cancer progression. Cell stress, 3(9), 284.

191. Wang, Y., Ding, Y., Guo, N., \& Wang, S. (2019). MDSCs: Key criminals of tumor premetastatic niche formation. Frontiers in immunology, 10.

192. Maecker, H. T., McCoy, J. P., \& Nussenblatt, R. (2012). Standardizing immunophenotyping for the human immunology project. Nature Reviews Immunology, 12(3), 191-200.

193. Charoentong, P., Finotello, F., Angelova, M., Mayer, C., Efremova, M., Rieder, D., ... \& Trajanoski, Z. (2017). Pan-cancer immunogenomic analyses reveal genotype-immunophenotype relationships and predictors of response to checkpoint blockade. Cell reports, 18(1), 248-262.

194. Finotello, F., \& Trajanoski, Z. (2018). Quantifying tumor-infiltrating immune cells from transcriptomics data. Cancer Immunology, Immunotherapy, 67(7), 1031-1040.

195. Finak, G., Langweiler, M., Jaimes, M., Malek, M., Taghiyar, J., Korin, Y., ... \& Pontikos, N. (2016). Standardizing flow cytometry immunophenotyping analysis from the Human ImmunoPhenotyping Consortium. Scientific reports, 6(1), 1-11.

196. Borch, W. R., Aguilera, N. S., Brissette, M. D., O'Malley, D. P., \& Auerbach, A. (2019). Practical Applications in Immunohistochemistry: An Immunophenotypic Approach to the Spleen. Archives of pathology \& laboratory medicine, 143(9), 1093-1105.

197. Pages, F., Galon, J., Dieu-Nosjean, M. C., Tartour, E., Sautes-Fridman, C., \& Fridman, W. H. (2010). Immune infiltration in human tumors: a prognostic factor that should not be ignored. Oncogene, 29(8), 1093.

198. Rosenberger, P. H., Ickovics, J. R., Epel, E., Nadler, E., Jokl, P., Fulkerson, J. P., ... \& Dhabhar, F. S. (2009). Surgical stress-induced immune cell redistribution profiles predict shortterm and long-term postsurgical recovery: A prospective study. The Journal of Bone and Joint Surgery. American volume., 91(12), 2783.

199. Oras, A., Peet, A., Giese, T., Tillmann, V., \& Uibo, R. (2019). A study of 51 subtypes of peripheral blood immune cells in newly diagnosed young type 1 diabetes patients. Clinical \& Experimental Immunology, 198(1), 57-70.

200. Schadendorf, D., \& Hauschild, A. (2014). Melanoma-the run of success continues. Nature Reviews Clinical Oncology, 11(2), 75-76.

201. Garon, E. B., Rizvi, N. A., Hui, R., Leighl, N., Balmanoukian, A. S., Eder, J. P., ... \& Carcereny, E. (2015). Pembrolizumab for the treatment of non-small-cell lung cancer. New England Journal of Medicine, 372(21), 2018-2028.

202. De Matos, L. L., Trufelli, D. C., De Matos, M. G. L., \& da Silva Pinhal, M. A. (2010). Immunohistochemistry as an important tool in biomarkers detection and clinical practice. Biomarker insights, 5, BMI-S2185.

203. Walsh, D. A., Da Silva, H. B., Beura, L. K., Peng, C., Hamilton, S. E., Masopust, D., \& Jameson, S. C. (2019). The functional requirement for CD69 in establishment of resident memory CD8+ T cells varies with tissue location. The Journal of Immunology, 203(4), 946-955. 
204. Yudanin, N. A., Schmitz, F., Flamar, A. L., Thome, J. J., Wojno, E. T., Moeller, J. B., ... \& Monticelli, L. A. (2019). Spatial and temporal mapping of human innate lymphoid cells reveals elements of tissue specificity. Immunity, 50(2), 505-519.

205. Ridge, K., Downes, N., \& Finney, B. (2019). Effects of strain, sex and age on immunophenotyping parameters in the rat and mouse. Comparative Clinical Pathology, 28(1), 41-51.

206. Jeys, L. M., Grimer, R. J., Carter, S. R., Tillman, R. M., \& Abudu, A. (2007). Post operative infection and increased survival in osteosarcoma patients: are they associated?. Annals of surgical oncology, 14(10), 2887-2895.

207. Moore, C., Eslin, D., Levy, A., Roberson, J., Giusti, V., \& Sutphin, R. (2010). Prognostic significance of early lymphocyte recovery in pediatric osteosarcoma. Pediatric blood \& cancer, 55(6), 1096-1102.

208. Buddingh, E. P., Ruslan, S. E. N., Berghuis, D., Gelderblom, H., Anninga, J. K., Hogendoorn, P. C., ... \& Lankester, A. C. (2012). Intact interferon signaling in peripheral blood leukocytes of high-grade osteosarcoma patients. Cancer Immunology, Immunotherapy, 61(6), 941-947.

209. Hong, W., Yuan, H., Gu, Y., Liu, M., Ji, Y., Huang, Z., ... \& Ma, L. (2020). Immunerelated prognosis biomarkers associated with osteosarcoma microenvironment. Cancer Cell International, 20(1), 1-12.

210. Li, M., Jin, X., Li, H., Wu, G., Wang, S., Yang, C., \& Deng, S. (2020). Key genes with prognostic values in suppression of osteosarcoma metastasis using comprehensive analysis. $B M C$ cancer, 20(1), 65.

211. Egas-Bejar, D., Anderson, P. M., Agarwal, R., Corrales-Medina, F., Devarajan, E., Huh, W. W., ... \& Subbiah, V. (2014). Theranostic profiling for actionable aberrations in advanced high risk osteosarcoma with aggressive biology reveals high molecular diversity: the human fingerprint hypothesis. Oncoscience, 1(2), 167.

212. Wang, D., Niu, X., Wang, Z., Song, C. L., Huang, Z., Chen, K. N., ... \& Wang, Y. (2019). Multiregion sequencing reveals the genetic heterogeneity and evolutionary history of osteosarcoma and matched pulmonary metastases. Cancer research, 79(1), 7-20.

213. Lu, S. M., Xiao, P., Xue, L., Che, L. H., Yang, P., Li, Y., \& Qiao, H. (2008). Prevalent expression of MHC class I chain-related molecule A in human osteosarcoma. Neoplasma, 55(3), 266-272.

214. Sundara, Y. T., Kostine, M., Cleven, A. H., Bovée, J. V., Schilham, M. W., \& CletonJansen, A. M. (2017). Increased PD-L1 and T-cell infiltration in the presence of HLA class I expression in metastatic high-grade osteosarcoma: a rationale for T-cell-based immunotherapy. Cancer Immunology, Immunotherapy, 66(1), 119-128.

215. Ligon, J. A., Oke, T. F., Choi, W., Fong, M. H., Levin, A., Rhee, D. S., ... \& McConkey, D. J. (2019). The immunosuppressive tumor microenvironment of metastatic osteosarcoma inhibits the cytotoxic effect of tumor-infiltrating lymphocytes.

216. Wu, C. C., Beird, H. C., Livingston, J. A., Advani, S., Mitra, A., Cao, S., ... \& Leung, C. H. (2020). Immuno-genomic landscape of osteosarcoma. Nature Communications, 11(1), 1-11. 217. Li, B., Wang, Z., Wu, H., Xue, M., Lin, P., Wang, S., ... \& Yan, X. (2018). Epigenetic regulation of CXCL12 plays a critical role in mediating tumor progression and the immune response in osteosarcoma. Cancer research, 78(14), 3938-3953.

218. Lussier, D. M., Johnson, J. L., Hingorani, P., \& Blattman, J. N. (2015). Combination immunotherapy with $\alpha$-CTLA-4 and $\alpha$-PD-L1 antibody blockade prevents immune escape and 
leads to complete control of metastatic osteosarcoma. Journal for immunotherapy of cancer, 3(1), 21.

219. Wang, S. D., Li, H. Y., Li, B. H., Xie, T., Zhu, T., Sun, L. L., ... \& Ye, Z. M. (2016). The role of CTLA-4 and PD-1 in anti-tumor immune response and their potential efficacy against osteosarcoma. International immunopharmacology, 38, 81-89.

220. Kelleher, F. C., \& O'Sullivan, H. (2017). Monocytes, macrophages, and osteoclasts in osteosarcoma. Journal of adolescent and young adult oncology, 6(3), 396-405.

221. Uehara, T., Eikawa, S., Nishida, M., Kunisada, Y., Yoshida, A., Fujiwara, T., ... \& Udono, H. (2019). Metformin induces CD11b+-cell-mediated growth inhibition of an osteosarcoma: implications for metabolic reprogramming of myeloid cells and anti-tumor effects. International immunology, 31(4), 187-198.

222. Troyer, R. M., Ruby, C. E., Goodall, C. P., Yang, L., Maier, C. S., Albarqi, H. A., ... \& Bracha, S. (2017). Exosomes from Osteosarcoma and normal osteoblast differ in proteomic cargo and immunomodulatory effects on T cells. Experimental cell research, 358(2), 369-376.

223. Markiewicz, K., Zeman, K., Kozar, A., Gołębiowska-Wawrzyniak, M., \& Woźniak, W. (2012). Evaluation of selected parameters of cellular immunity in children with osteosarcoma at diagnosis. Medycyna wieku rozwojowego, 16(3), 212-221.

224. Knutson, K. L., \& Disis, M. L. (2004). IL-12 enhances the generation of tumour antigenspecific Th1 CD4 T cells during ex vivo expansion. Clinical \& Experimental

Immunology, 135(2), 322-329.

225. Wang, J., Nong, L., Wei, Y., Qin, S., Zhou, Y., \& Tang, Y. (2013). Association of interleukin-12 polymorphisms and serum IL-12p40 levels with osteosarcoma risk. DNA and cell biology, 32(10), 605-610.

226. Tang, Y. J., Wang, J. L., Nong, L. G., Lan, C. G., Zha, Z. G., \& Liao, P. H. (2014). Associations of IL-27 polymorphisms and serum IL-27p28 levels with osteosarcoma risk. Medicine, 93(10).

227. Jia, S. F., Worth, L. L., Densmore, C. L., Xu, B., Duan, X., \& Kleinerman, E. S. (2003). Aerosol gene therapy with PEI: IL-12 eradicates osteosarcoma lung metastases. Clinical cancer research, 9(9), 3462-3468.

228. Fernández, L., Valentin, J., Zalacain, M., Leung, W., Patino-Garcia, A., \& PérezMartínez, A. (2015). Activated and expanded natural killer cells target osteosarcoma tumor initiating cells in an NKG2D-NKG2DL dependent manner. Cancer letters, 368(1), 54-63.

229. Yang, X., Zhang, W., \& Xu, P. (2019). NK cell and macrophages confer prognosis and reflect immune status in osteosarcoma. Journal of cellular biochemistry, 120(5), 8792-8797.

230. Troyer, R. M., Ruby, C. E., Goodall, C. P., Yang, L., Maier, C. S., Albarqi, H. A., ... \& Bracha, S. (2017). Exosomes from Osteosarcoma and normal osteoblast differ in proteomic cargo and immunomodulatory effects on T cells. Experimental cell research, 358(2), 369-376.

231. Xu, S., Yang, S., Sun, G., Huang, W., \& Zhang, Y. (2014). Transforming growth factorbeta polymorphisms and serum level in the development of osteosarcoma. DNA and cell biology, 33(11), 802-806.

232. Liu, M. X., Liu, Q. Y., Liu, Y., Cheng, Z. M., Liu, L., Zhang, L., \& Sun, D. H. (2019). Interleukin-35 suppresses antitumor activity of circulating CD8+ T cells in osteosarcoma patients. Connective tissue research, 60(4), 367-375.

233. Li, X., Chen, Y., Liu, X., Zhang, J., He, X., Teng, G., \& Yu, D. (2017). Tim3/Ga19 interactions between $\mathrm{T}$ cells and monocytes result in an immunosuppressive feedback loop that 
inhibits Th1 responses in osteosarcoma patients. International immunopharmacology, 44, 153159.

234. Haeryfar, S. M., DiPaolo, R. J., Tscharke, D. C., Bennink, J. R., \& Yewdell, J. W. (2005). Regulatory $\mathrm{T}$ cells suppress CD8+ T cell responses induced by direct priming and cross-priming and moderate immunodominance disparities. The Journal of Immunology, 174(6), 3344-3351. 235. Kawano, M., Itonaga, I., Iwasaki, T., Tsuchiya, H., \& Tsumura, H. (2012). Anti-TGF- $\beta$ antibody combined with dendritic cells produce antitumor effects in osteosarcoma. Clinical Orthopaedics and Related Research®, 470(8), 2288-2294.

236. Kozawa, E., Sugiura, H., Wasa, J., Kohyama, K., Yamada, K., Nishioka, A., ... \& Taguchi, O. (2010). Suppression of tumour metastasis in a murine osteosarcoma model with antiCD25 monoclonal antibody treatment. Anticancer research, 30(12), 5019-5022.

237. Fritzsching, B., Fellenberg, J., Moskovszky, L., Sápi, Z., Krenacs, T., Machado, I., ... \& Bernd, L. (2015). CD8+/FOXP3+-ratio in osteosarcoma microenvironment separates survivors from non-survivors: a multicenter validated retrospective study. Oncoimmunology, 4(3), e990800.

238. Yoshida, K., Okamoto, M., Sasaki, J., Kuroda, C., Ishida, H., Ueda, K., .. \& Tanaka, M. (2020). Anti-PD-1 antibody decreases tumour-infiltrating regulatory T cells. BMC cancer, 20(1), $1-10$.

239. Han, Q., Shi, H., \& Liu, F. (2016). CD163+ M2-type tumor-associated macrophage support the suppression of tumor-infiltrating T cells in osteosarcoma. International immunopharmacology, 34, 101-106.

240. Liu, H., Zhi, L., Duan, N., \& Su, P. (2016). Abnormal expression of Tim-3 antigen on peripheral blood $\mathrm{T}$ cells is associated with progressive disease in osteosarcoma patients. FEBS open bio, 6(8), 807-815.

241. Lussier, D. M., O’Neill, L., Nieves, L. M., McAfee, M. S., Holechek, S. A., Collins, A. W., ... \& Blattman, J. N. (2015). Enhanced T-cell immunity to osteosarcoma through antibody blockade of PD-1/PD-L1 interactions. Journal of immunotherapy (Hagerstown, Md.: 1997), 38(3), 96.

242. Zheng, W., Xiao, H., Liu, H., \& Zhou, Y. (2015). Expression of programmed death 1 is correlated with progression of osteosarcoma. Apmis, 123(2), 102-107.

243. Yoshida, K., Okamoto, M., Sasaki, J., Kuroda, C., Ishida, H., Ueda, K., ... \& Takizawa, T. (2019). Clinical outcome of osteosarcoma and its correlation with programmed death-ligand 1 and T cell activation markers. OncoTargets and therapy, 12, 2513.

244. Huang, X., Zhang, W., Zhang, Z., Shi, D., Wu, F., Zhong, B., \& Shao, Z. (2018). Prognostic value of programmed cell death 1 ligand-1 (PD-L1) or PD-1 expression in patients with osteosarcoma: a meta-analysis. Journal of Cancer, 9(14), 2525.

245. Orecchioni, M., Ghosheh, Y., Pramod, A. B., \& Ley, K. (2020). Corrigendum: Macrophage Polarization: Different Gene Signatures in M1 (LPS+) vs. Classically and M2 (LPS-) vs. Alternatively Activated Macrophages. Frontiers in Immunology, 11.

246. Xiao, Q., Zhang, X., Wu, Y., \& Yang, Y. (2014). Inhibition of macrophage polarization prohibits growth of human osteosarcoma. Tumor Biology, 35(8), 7611-7616.

247. Buddingh, E. P., Kuijjer, M. L., Duim, R. A., Bürger, H., Agelopoulos, K., Myklebost, O., ... \& Cleton-Jansen, A. M. (2011). Tumor-infiltrating macrophages are associated with metastasis suppression in high-grade osteosarcoma: a rationale for treatment with macrophage activating agents. Clinical Cancer Research, 17(8), 2110-2119. 
248. Takeya, M., \& Komohara, Y. (2016). Role of tumor-associated macrophages in human malignancies: friend or foe?. Pathology international, 66(9), 491-505.

249. Dumars, C., Ngyuen, J. M., Gaultier, A., Lanel, R., Corradini, N., Gouin, F., ... \& Heymann, M. F. (2016). Dysregulation of macrophage polarization is associated with the metastatic process in osteosarcoma. Oncotarget, 7(48), 78343.

250. Koirala, P., Roth, M. E., Gill, J., Piperdi, S., Chinai, J. M., Geller, D. S., ... \& Gorlick, R. (2016). Immune infiltration and PD-L1 expression in the tumor microenvironment are prognostic in osteosarcoma. Scientific reports, 6(1), 1-10.

251. Palmerini, E., Agostinelli, C., Picci, P., Pileri, S., Marafioti, T., Lollini, P. L., ... \& Ferrari, S. (2017). Tumoral immune-infiltrate (IF), PD-L1 expression and role of CD8/TIA-1 lymphocytes in localized osteosarcoma patients treated within protocol ISG-

OS1. Oncotarget, 8(67), 111836.

252. Cheng, Z., Wang, L., Wu, C., Huang, L., Ruan, Y., \& Xue, W. Tumor-derived exosomes induced M2 macrophage polarization and promoted the metastasis of osteosarcoma cells through Tim-3.

253. Han, Y., Guo, W., Ren, T., Huang, Y., Wang, S., Liu, K., ... \& Liang, X. (2019). Tumorassociated macrophages promote lung metastasis and induce epithelial-mesenchymal transition in osteosarcoma by activating the COX-2/STAT3 axis. Cancer letters, 440, 116-125.

254. Yan, B., Liu, Q., Liu, G., Huang, X., Zhu, G., Gao, L., \& Xu, Y. (2020). Macrophagederived exosomes mediate osteosarcoma cell behavior by activating AKT signaling. RSC Advances, 10(9), 5032-5039.

255. Zhou, Q., Xian, M., Xiang, S., Xiang, D., Shao, X., Wang, J., ... \& He, Q. (2017). Alltrans retinoic acid prevents osteosarcoma metastasis by inhibiting M2 polarization of tumorassociated macrophages. Cancer immunology research, 5(7), 547-559.

256. Ando, K., Mori, K., Corradini, N., Redini, F., \& Heymann, D. (2011). Mifamurtide for the treatment of nonmetastatic osteosarcoma. Expert opinion on pharmacotherapy, 12(2), 285292.

257. Punzo, F., Bellini, G., Tortora, C., Di Pinto, D., Argenziano, M., Pota, E., ... \& Rossi, F. (2020). Mifamurtide and TAM-like macrophages: effect on proliferation, migration and differentiation of osteosarcoma cells. Oncotarget, 11(7), 687.

258. Liu, T., Fang, X. C., Ding, Z., Sun, Z. G., Sun, L. M., \& Wang, Y. L. (2015). Preoperative lymphocyte-to-monocyte ratio as a predictor of overall survival in patients suffering from osteosarcoma. FEBS open bio, 5(1), 682-687.

259. Liu, B., Huang, Y., Sun, Y., Zhang, J., Yao, Y., Shen, Z., ... \& He, A. (2016). Prognostic value of inflammation-based scores in patients with osteosarcoma. Scientific reports, 6, 39862. 260. Wang, B., Tu, J., Yin, J., Zou, C., Wang, J., Huang, G., ... \& Shen, J. (2015).

Development and validation of a pretreatment prognostic index to predict death and lung metastases in extremity osteosarcoma. Oncotarget, 6(35), 38348.

261. Tonglei, Y., Tao, L., Qiong, M., Xiuchun, Q., Yunyan, L., Yanhua, W., \& Baoan, M. Tumor associated neutrophils promote the migration of human osteosarcoma cells SOSP-9607 and its mechanism.

262. Xiao, H., Chen, L., Luo, G., Son, H., Prectoni, J. H., \& Zheng, W. (2014). Effect of the cytokine levels in serum on osteosarcoma. Tumor Biology, 35(2), 1023-1028.

263. Roberts, R. D., Gross, A. C., Bid, H. K., Phelps, D., Wedekind, M. F., \& Houghton, P. J. (2016). Abstract B40: Autocrine and paracrine IL-6 and IL-8 drive osteosarcoma lung tropism and facilitate metastasis. 
264. Guan, Y., Zhang, R., Peng, Z., Dong, D., Wei, G., \& Wang, Y. (2017). Inhibition of IL18-mediated myeloid derived suppressor cell accumulation enhances anti-PD1 efficacy against osteosarcoma cancer. Journal of bone oncology, 9, 59-64.

265. Jiang, K., Li, J., Zhang, J., Wang, L., Zhang, Q., Ge, J., ... \& Hao, D. (2019). SDF1/CXCR4 axis facilitates myeloid-derived suppressor cells accumulation in osteosarcoma microenvironment and blunts the response to anti-PD-1 therapy. International immunopharmacology, 75, 105818.

266. Hoechst, B., Voigtlaender, T., Ormandy, L., Gamrekelashvili, J., Zhao, F., Wedemeyer, H., ... \& Korangy, F. (2009). Myeloid derived suppressor cells inhibit natural killer cells in patients with hepatocellular carcinoma via the NKp30 receptor. Hepatology, 50(3), 799-807. 267. Huang, B., Pan, P. Y., Li, Q., Sato, A. I., Levy, D. E., Bromberg, J., ... \& Chen, S. H. (2006). Gr-1+ CD115+ immature myeloid suppressor cells mediate the development of tumorinduced T regulatory cells and T-cell anergy in tumor-bearing host. Cancer research, 66(2), 1123-1131.

268. Uehara, T., Eikawa, S., Nishida, M., Kunisada, Y., Yoshida, A., Fujiwara, T., ... \& Udono, H. (2019). Metformin induces CD11b+-cell-mediated growth inhibition of an osteosarcoma: implications for metabolic reprogramming of myeloid cells and anti-tumor effects. International immunology, 31(4), 187-198.

269. Pan, P. Y., Ma, G., Weber, K. J., Ozao-Choy, J., Wang, G., Yin, B., ... \& Chen, S. H. (2010). Immune stimulatory receptor CD40 is required for T-cell suppression and T regulatory cell activation mediated by myeloid-derived suppressor cells in cancer. Cancer research, 70(1), 99-108.

270. Raber, P., Ochoa, A. C., \& Rodríguez, P. C. (2012). Metabolism of L-arginine by myeloid-derived suppressor cells in cancer: mechanisms of T cell suppression and therapeutic perspectives. Immunological investigations, 41(6-7), 614-634.

271. He, X., Lin, H., Yuan, L., \& Li, B. (2017). Combination therapy with L-arginine and $\alpha$ PD-L1 antibody boosts immune response against osteosarcoma in immunocompetent mice. Cancer biology \& therapy, 18(2), 94-100.

272. Robainas, M., Otano, R., Bueno, S., \& Ait-Oudhia, S. (2017). Understanding the role of PD-L1/PD1 pathway blockade and autophagy in cancer therapy. OncoTargets and therapy, 10, 1803.

273. Probst, H. C., McCoy, K., Okazaki, T., Honjo, T., \& van den Broek, M. (2005). Resting dendritic cells induce peripheral CD8+ T cell tolerance through PD-1 and CTLA-4. Nature immunology, 6(3), 280-286.

274. Wei, S. C., Duffy, C. R., \& Allison, J. P. (2018). Fundamental mechanisms of immune checkpoint blockade therapy. Cancer discovery, 8(9), 1069-1086.

275. Curiel, T. J., Wei, S., Dong, H., Alvarez, X., Cheng, P., Mottram, P., ... \& David, O. (2003). Blockade of B7-H1 improves myeloid dendritic cell-mediated antitumor immunity. Nature medicine, 9(5), 562-567.

276. Hartley, G. P., Chow, L., Ammons, D. T., Wheat, W. H., \& Dow, S. W. (2018).

Programmed cell death ligand 1 (PD-L1) signaling regulates macrophage proliferation and activation. Cancer immunology research.

277. Burgess, M. A., Crowley, J., Reinke, D. K., Riedel, R. F., George, S., Movva, S., ... \& Attia, S. (2015). SARC 028: A phase II study of the anti-PD1 antibody pembrolizumab (P) in patients (Pts) with advanced sarcomas. 
278. Maszyna, F., Hoff, H., Kunkel, D., Radbruch, A., \& Brunner-Weinzierl, M. C. (2003). Diversity of clonal $\mathrm{T}$ cell proliferation is mediated by differential expression of CD152 (CTLA4) on the cell surface of activated individual T lymphocytes. The Journal of Immunology, 171(7), 3459-3466.

279. Thanindratarn, P., Dean, D. C., Nelson, S. D., Hornicek, F. J., \& Duan, Z. (2019). Advances in immune checkpoint inhibitors for bone sarcoma therapy. Journal of bone oncology, 100221. 


\title{
Chapter 2
}

Using the Spleen as an in vivo Systemic Immune Barometer Alongside Osteosarcoma Disease Progression and Immunotherapy with $\alpha$-PD-L1

\author{
Published in: Sarcoma
}

Sarcoma. 2018 Dec 12. https://doi.org/10.1155/2018/8694397 
Using the Spleen as an in vivo Systemic Immune Barometer Alongside Osteosarcoma Disease Progression and Immunotherapy with $\alpha$-PD-L1

\author{
Justin E. Markel \\ Department of Orthopaedics \\ West Virginia University \\ jemarkel@mix.wvu.edu \\ Jabeen Noore \\ Department of Orthopaedics \\ West Virginia University \\ jabeennoore@gmail.com \\ Eric J. Emery \\ School of Medicine \\ West Virginia University \\ eemery3@mix.wvu.edu \\ Harley J. Bobnar \\ Washington and Jefferson College \\ Harley.01@comcast.net \\ Eugenie S. Kleinerman \\ The University of Texas MD Anderson Cancer Center \\ ekleiner@mdanderson.org \\ Brock A. Lindsey \\ Corresponding Author \\ Department of Orthopaedics \\ West Virginia University \\ PO Box 9196 \\ Morgantown, WV 26506-9196 \\ Phone (304) 293-1317 \\ Fax (304) 293-7042 \\ Email: blindey@hsc.wvu.edu
}




\begin{abstract}
Indications for immunotherapies are still unclear, and there is a great need for real-time patient immune status monitoring. In this study, we confirmed that the local and systemic immune profiles of an orthotopic osteosarcoma model with or without luciferase transfection were statistically equivalent. Next, we used flow cytometry to describe systemic immune cell populations influenced by osteosarcoma disease progression. When compared to vehicle-inoculated sham mice, it was found that tumor-bearing mice had significant immunophenotype disturbances at approximately 11 weeks post-inoculation (at which time $90 \%$ of primary tumor-bearing mice have fulminant pulmonary metastases). Percent populations of Natural Killer cells and T regulatory cells were increased in the spleens of tumor-bearing mice $(\mathrm{p}<0.0083)$ compared to shams. Additionally, $\mathrm{T}$ lymphocytes from spleens of tumor-bearing mice showed increased Tim-3/PD-1 exhaustion status $(\mathrm{p}<0.0083)$. There were also increases in the percent populations of myeloid cells and overall M1/M2 macrophage marker expression on tumor-bearing mice spleens versus controls ( $\mathrm{p}<$ 0.00714). Finally, treatment with $20 \mu \mathrm{g} \alpha$-PD-L1 decreased T cell exhaustion back to sham status, with a corresponding increase in CTLA-4 expression on cytotoxic T cells in the majority of mice tested. Checkpoint inhibition also increased splenic monocyte maturation and returned macrophage M1/M2 marker expression back to sham status. These data suggest that cancer induces systemic immune dysregulation, and that these changes may be elucidated and utilized for treatment purposes by sampling the systemic immune environment via the spleen. In addition, treatment with the checkpoint inhibitor $\alpha$-PD-L1 may neutralize, but not overcome the systemic immunological changes induced by a progressing malignancy.
\end{abstract}

Keywords: immunotherapy; osteosarcoma; tumor microenvironment; PD-L1 


\section{Introduction}

Osteosarcoma is an extremely aggressive tumor of bone, and no new therapeutics have been implemented clinically in over three decades (1). Its extreme pleomorphism and high antigenic load $(2,3)$ make it a seemingly prime candidate for tumor immunotherapies, which can induce sustainable immunity against cancer cell neoantigens thus providing a viable therapeutic strategy for overcoming heterogeneity; however, no immunotherapies are currently implicated as standard of care in the treatment of osteosarcoma. As the malignant process can lead to generalized immune dysfunction and disease progression (4-6), there has been success across multiple tumor types with the introduction of various immunotherapies into systemic circulation including monoclonal antibody checkpoint blockers like those against programmed death-ligand 1 (PD-L1) and its receptor, programmed cell death protein 1 (PD-1) (7). PD-L1 is expressed on tumor and antigen presenting cells (APCs), while its receptor PD-1 is expressed on activated T cells; this interaction suppresses $\mathrm{T}$ cell activity and is associated with $\mathrm{T}$ cell exhaustion (TCE), a state of depressed effector function and anergy brought on by chronic antigen exposure and hypoxia (8).

Osteosarcoma tumors are often positive for PD-L1, and its expression has been shown to upregulate in metastatic versus primary lesions (9). While blockade of this protein interaction has experienced clinical success in some cancers, there is still confusion surrounding its specific indications and only a minority show a response (10); preclinical mouse models of metastatic osteosarcoma have shown resistance to PD-L1/PD-1 blockade (11). Moreover, there is currently no reliable way of monitoring or predicting patient responses to any immunotherapies including checkpoint blockers and the search for relevant biomarkers is ongoing (12). Indeed, data of this nature would potentially allow clinicians to adjust regimens as necessary for maximal efficacy in real-time, and may uncover reasons as to why tumors like osteosarcoma have remained largely 
unresponsive to immunotherapies despite their high neoantigen load. From the few studies currently available on osteosarcoma tumor immunology, data have emerged that support the importance of macrophage M1/M2 polarization and T cell exhaustion and activation in tumor clearance, although the scope has generally been limited to the primary lesions $(11,13-21)$. We suggest that these data warrant further investigation regarding the translation of macrophage-T cell dynamics systemically and how we might use this information clinically to trigger antitumor immunity in patients. In the present study, after confirming immunologic equivalency between two models of osteosarcoma [with or without introduction of a non-viral luciferase reporter vector for in vivo imaging systems (IVIS) visualization], we further defined the immunological consequences of osteosarcoma disease progression over time. We also investigated the systemic effects of monotherapy with checkpoint blockade of PD-L1 using the spleen as a barometer of immune status. The overarching hypothesis is that the spleen can be used as a barometer to assess

clinically relevant changes in the systemic macrophage- $\mathrm{T}$ cell immunophenotype brought on by osteosarcoma disease progression and immunotherapy.

\section{Materials and Methods}

Animals

Female BALB/c mice aged 4-5 weeks and between 20 and 25 grams (mean $=22.5$ grams) in mass were obtained from The Jackson Laboratory (Bar Harbor, ME). Mice were housed individually in ventilated Allentown cages at ambient temperatures within specific pathogen free facilities on corncob bedding with 12 hour light/dark cycles, automatic lixit water, and ad libitum food access. All experiments were approved by the Institutional Animal Care and Use Committee. Growth and preparation of transfected and wild type K7M2 cells 
K7M2 cells were grown and prepared as previously described (22). Briefly, wild-type (WT) and genome-stable luciferase-transfected (TF) K7M2 cells were cultured in Dulbecco's Modified Eagle's Medium (DMEM) containing 10\% fetal bovine serum (FBS) and 100 units/mL penicillin and streptomycin (Thermo Fisher Scientific, Waltham, MA). Passage one cells were used for orthotopic implantation at a density of one million cells in $25 \mu \mathrm{L}$ of phosphate buffered saline (PBS) without calcium or magnesium (Corning Inc., Corning, NY).

Osteosarcoma BALB/c K7M2 syngeneic mouse model

$\mathrm{K} 7 \mathrm{M} 2$ cells or vehicle were surgically inoculated into the tibias of female BALB/c mice as previously described (22). Briefly, $25 \mu \mathrm{L}$ of PBS containing one million TF or WT K7M2 cells were dispensed into a cortical window made through the center of the tibia at the level of the proximal tibial flare.

WT and TF tumor-bearing mice were monitored as previously described (22) in accordance with our institution's tumor burden scoring system. Tumors were monitored daily using digital calipers (greatest width $\mathrm{x}$ greatest length). Tumor volume was calculated using the formula $V=$ (length $x$ width2)/2. The health of each animal was assessed using a tumor score ranging from 0 to $60(0=$ healthy animal; $60=$ requires euthanasia $)$ based on criteria established by our institution regarding overall animal health as previously described (22). Once mice reached a tumor score of 60 (approximately 11 weeks post-inoculation), animals were humanely euthanized using carbon dioxide asphyxiation.

Tumors were harvested as previously described (22). Briefly, animals whose tumors reached an average of $1.7 \mathrm{~cm} 3$ underwent palliative amputation by dissection and hip disarticulation. Skin edges were closed using 4-0 vicryl suture. Tumors were dissociated and prepared for flow cytometry analysis as described below. Once tumor scores reached 60 
(approximately 11 weeks post-inoculation), animals were euthanized and their spleens harvested and prepared for flow cytometry (FC) analysis.

Flow cytometry analysis of tumors and spleens

The excised primary tumor and spleen from each mouse were minced and digested enzymatically using the MACS Tissue Dissociation Kits (Miltenyi Biotec, Auburn, CA), according to the manufacturer's guidelines. After dissociation, each sample was filtered through a $70 \mu \mathrm{M}$ filter (Miltenyi Biotec) and centrifuged at $300 \mathrm{x}$ g for $10 \mathrm{~min}$; the cell pellet was resuspended in RPMI-1640. Each single cell suspension was split into two. One half of the tumor single cell suspension was treated with Red Blood Cell Lysis Solution (Miltenyi Biotec), washed twice, and counted.

Spleen single cell suspensions and the other half of the tumor single cell suspensions were layered over Ficoll-Paque premium (GE Healthcare Life Sciences, Pittsburgh, PA) and centrifuged at $400 \mathrm{x}$ g for $40 \mathrm{~min}$ at $20{ }^{\circ} \mathrm{C}$ to allow density gradient separation of the leukocytes. After Ficoll, the leukocytes (buffy coat) were harvested, washed twice with PBS, and counted.

Single cell suspensions were stained following standard protocols. Briefly, cells were washed using FACs buffer (PBS, 2\% FBS, and 0.02\% sodium azide). Cell pellets were resuspended, blocked using Rat IgG and Mouse IgG (Jackson ImmunoResearch, West Grove, PA), washed, and incubated with the appropriate antibodies described below. After incubation, the cells were washed, fixed in paraformaldehyde, and stored at $4{ }^{\circ} \mathrm{C}$.

Panel 1 antibodies: $\alpha$-CD45-Phycoerithrin Cyanine5 (BD Biosciences, San Jose, CA), $\alpha$-CD4BV510 (BD Bioscience), $\alpha$-Ly6G-V450 (BD Biosciences), $\alpha$-CD8a-Alexa Fluor 488 (BD Biosciences), $\alpha$-NKp46-PE-eFluor 610 (eBioscience, San Diego, CA), $\alpha$-CD11b-Alexa Fluor 700 (eBioscience), $\alpha$-Foxp3-Phycoerithrin (eBioscience) 
Panel 2 antibodies: $\alpha$-CD45-Phycoerithrin Cyanine5 (BD Biosciences), $\alpha$-PD-L1-Phycoerithrin Cyanine7 (eBioscience)

Panel 3 antibodies: $\alpha$-CD45-Phycoerithrin Cyanine5 (BD Bioscience), $\alpha$-CD4-BV510 (BD Bioscience), $\alpha$-CD8a-Alexa Fluor 488 (BD Bioscience), $\alpha$-Tim-3-PerCP/Cy5.5 (BioLegend, San Diego, CA), $\alpha$-PD-1-eFluor 450 (Thermo Fisher Scientific), $\alpha$-CTLA-4-PE/Cy7 (BioLegend), $\alpha-$ Foxp3-Phycoerithrin (eBioscience)

Panel 4 antibodies: $\alpha$-CD45-Phycoerithrin Cyanine5 (BD Biosciences), $\alpha$-CD11b-Alexa Fluor 700 (eBioscience), $\alpha$-Ly6G-V450 (BD Bioscience), $\alpha$-Ly6C-PerCP/Cy5.5 (eBioscience), $\alpha$-CXCL9Phycoerithrin (eBioscience), $\alpha$-NOS2-PE-Cyanine7 (BioLegend), $\alpha$-Tgm2-Alexa488 (Novus Biologicals, Littleton, CO), $\alpha$-Arg1-APC (R\&D Systems, Minneapolis, MN)

All cell populations and/or pertinent markers as they are used in this manuscript are defined below in Table 1.

For Foxp3 staining, after washing cells with FACS buffer, the cells were processed using Foxp3/Transcription Factor Staining Buffer Set (eBioscience) according to the manufacturer's instructions. Briefly, the cell pellet was resuspended and $1.0 \mathrm{~mL}$ of diluted (1:3 ratio) Foxp3 Fixation/Permeabilization buffer was added. Samples were vortexed and incubated at $4{ }^{\circ} \mathrm{C}$ overnight. The next day, the cells were washed twice with 1X Foxp3 buffer and incubated with $\alpha$ Foxp3-Phycoerithrin (eBioscience), washed with 1X Foxp3 buffer, and resuspended in FACS buffer.

Panel 4 included intracellular staining of M1/M2 macrophage markers. For this panel, after washing cells with FACS buffer, the cells were further processed for intracellular antigens using the Intracellular Antigen Staining Buffer Set (BioLegend) according to the manufacturer's instructions. Briefly, cell pellets were resuspended and 100uL of intracellular-fixation buffer was 
added. After a pulse vortex, the cells were incubated at $4{ }^{\circ} \mathrm{C}$ overnight. The next day, the cells were washed twice with 1 X permeabilization buffer, incubated with a master-mix of $\alpha$ - CXCL9Phycoerithrin (eBioscience), $\alpha$-NOS2 -PE-Cyanine7 (BioLegend), $\alpha$-Tgm2- Alexa488 (Novus Biologicals), and $\alpha$-Arg1-APC (R\&D Systems), washed twice with $1 \mathrm{X}$ permeabilization buffer, and resuspended in FACS buffer.

Cells were analyzed and the data were collected on the BD LSRFortessa (BD Biosciences) using BD FACS Diva version 8.0 software located in the Flow Cytometry \& Single Cell Core Facility. Single stained controls were generated using OneComp beads eBeads (eBiosciences). A minimum of 50,000 cells were analyzed for each sample. Data analysis was performed using FCS Express 6 Software (De Novo Software, Glendale, CA).

T cell Exhaustion Status (TCES)

T cells expressing the exhaustion markers PD- 1 and/or T cell immunoglobulin and mucindomain containing-3 (Tim-3) exhibit depressed effector functions and an inability to proliferate (23-25); exhausted Tim-3+ and PD-1+T cells have been found in osteosarcoma tumor tissues (26). In order to properly compare expression of these TCE markers on the T cell repertoire across mice with different cell counts, we normalized for total $\mathrm{T}$ cell percent population to yield an overall exhaustion status we refer to as the TCE status (TCES) calculated by the following equation:

$$
\text { TCES }=\frac{\text { Total } \% P D 1 \text { expression }_{C T L, T h}+\text { Total } \% \text { Tim } 3_{\text {CTL,Th }}}{\text { Total } \% \text { CTL }+ \text { Total } \% \text { Th }}
$$

In words, the TCE status equals the total PD- 1 and Tim- 3 percent expression on both CD4+ T helper cells (Th) and CD8+ cytotoxic T cells (CTLs) divided by the total percent population of all $\mathrm{T}$ cells (both $\mathrm{CD}_{+}$and $\mathrm{CD} 8_{+}$) sampled. This equation yields the ratio of exhaustion-marker expressing T lymphocytes per total lymphocyte percent population and is driven by peer-reviewed data showing that PD- 1 and Tim-3 expression on T cells correlates with the extent of TCE $(23,27-$ 
29). Normalizing for total $\mathrm{T}$ cell percent populations simply allows comparisons to be made across different samples with varying $\mathrm{T}$ cell counts.

$\alpha-P D-L 1$ treatment

Mice were given $20 \mu \mathrm{g}(1 \mathrm{mg} / \mathrm{kg}) \alpha-\mathrm{PD}-\mathrm{L} 1$ (clone MIH5, eBioscience) via intraperitoneal injection every Monday and Thursday starting when tumors were first palpable and continuing until euthanasia (approximately 11 weeks following tumor cell inoculation). The standard dosing used throughout the literature is $200 \mu \mathrm{g}(10 \mathrm{mg} / \mathrm{kg})$; however, $1 \mathrm{mg} / \mathrm{kg}$ is the dosage at which clinical activity in advanced human cancer starts (7), although extended treatment periods with doses as high as $20 \mathrm{mg} / \mathrm{kg}$ have been shown ineffective against metastatic K7M2 tumors [11]. For this manuscript, which focuses purely on the immunological changes associated with PD-L1 blockade, the minimum dose needed to produce a measurable immune response $(1 \mathrm{mg} / \mathrm{kg}) \mathrm{was}$ used as there is no known $\alpha$-PD-L1 dose that produces clinical benefit in osteosarcoma.

\section{Statistical analysis}

Statistical significance between groups was assessed using a Student's T-test with alpha = 0.05 and Bonferroni correction. A minimum of three animals per group were used, which is a widely used starting number and allows for the determination of potential outliers.

\section{Results and Discussion}

The primary FC data for this manuscript can be accessed without restrictions by contacting the primary author.

SECTION 1: The local and systemic immune environments of BALB/c mice orthotopically inoculated with luciferase(+) TF or luciferase(-) WT K7M2 cells are statistically equivalent.

Our laboratory previously developed an orthotopic model of metastatic osteosarcoma using BALB/c syngeneic K7M2 tumor cells transfected with a luciferase reporter for real-time disease 
monitoring using IVIS. This model allows for sensitive visualization and assessment of primary and metastatic lesions without euthanasia in an immunocompetent mouse. From a clinical outcomes perspective, both luciferase(+) TF and luciferase(-) WT models were previously shown to result in statistically equivalent rates of primary lesion formation and pulmonary metastasis (22). However, as luciferase is a foreign intracellular protein under a constitutively active promoter, it is presented on MHC class I molecules (30) and has the potential to elicit atypical immune reactions $(31,32)$. As this manuscript focuses exclusively on characterizing antitumor immune responses, we considered it extremely important to first examine whether the luciferase antigen significantly affected the immune microenvironment. To accomplish this goal, we assessed the local (tumors harvested at approximately $1.7 \mathrm{~cm}_{3}$ in volume) and systemic (splenic) immune microenvironments by flow cytometry. The spleen is a large lymph node-like immune organ with direct access to blood-borne pathogens and antigens, and thus a good representation of systemic immune function.

To determine if the local tumor immune microenvironments were influenced by the presence of the luciferase antigen, we surveyed a variety of key myeloid and lymphoid lineage immune cells (six populations total) with maximal clinical relevance in both WT tumor-bearing (n $=11)$ and TF tumor-bearing $(\mathrm{n}=15)$ mice using Antibody Panel 1. The populations investigated included CD45+CD8+ CTLs, CD45+CD4+ Ths, CD45+CD4+Foxp3+ T regulatory cells (Tregs), CD45+NKp46+ Natural Killer (NK) cells, CD45+CD11b+ myeloid lineage cells (MLCs), and $\mathrm{CD} 45+\mathrm{CD} 11 \mathrm{~b}+\mathrm{Ly} 6 \mathrm{G}+$ granulocytes. A sample gating schema is shown in Supplementary Figures 1-3. Using the Bonferroni correction, individual comparisons were made using $\alpha=0.0083$ and all populations were deemed statistically equivalent $(\mathrm{p}>0.0083$ ) between the two groups (Table 2 ). Additionally, as PD-L1 expression in tumors has been speculated to play an important part in 
osteosarcoma-mediated immune suppression $(33,34)$ we also examined whether PD-L1 expression differed between groups. We looked for expression of PD-L1 on tumor cells using the CD45- gate (which identified all non-leukocyte populations within the tumor) in Antibody Panel 2; a sample gating schema is shown in Supplementary Figure 4. No statistical difference $(\mathrm{p}>0.05)$ was observed in percent PD-L1 expression on tumors generated from both TF tumor-bearing $(\mathrm{n}=$ 15) and WT tumor-bearing $(\mathrm{n}=11)$ mice (Table 3$)$.

To further evaluate the immunological equivalence between TF and WT models, we sought to determine whether the systemic immune microenvironments were influenced by the presence of luciferase. Previous data had shown that approximately eleven weeks following tumor cell inoculation, $90 \%$ of mice in both $\mathrm{TF}$ and WT tumor-bearing groups developed pulmonary metastases (22). At this time, mice were euthanized and splenocytes were immunophenotyped with Antibody Panel 1 to determine if splenic immunomodulation in response to late stage disease was consistent between groups. Using the Bonferroni correction, individual comparisons were made using $\alpha=0.0083$ and, like that for primary tumors, the splenic microenvironments of mice from both groups were almost uniformly statistically equivalent $(\mathrm{p}>0.0083)$, except for the percentage of granulocytes $\left(\mathrm{WT}_{\text {gran }}=15.27 \pm 21.82 \%: \mathrm{n}=10 ; \mathrm{TF}_{\operatorname{gran}}=52.33 \pm 19.02 \%: \mathrm{n}=9 ; \mathrm{p}=0.0011\right)$. The statistics for these analyses are shown in Table 4.

Whenever a foreign reporter protein is introduced into in vivo systems, there is the potential for it to interfere with normal immune processes; however, this aspect of tumor immunology is often overlooked in the literature even though it has the potential to invalidate conclusions. As the manuscript focuses on elucidating the antitumor responses, we wanted evidence to support that introduction of a luciferase reporter did not dramatically alter the antitumor immune response. The white blood cell (WBC) populations examined in Panel 1 presented a broad overview of key 
mediators of tumor immunology including NK cells, CTLs, Ths, Tregs, and myeloid lineage cells (MLCs). Overall, out of the 13 comparisons made between TF and WT tumor-bearing models, only one population (percent $\mathrm{CD} 45+\mathrm{CD} 11 \mathrm{~b}+\mathrm{Ly} 6 \mathrm{G}+$ granulocytes in the spleen) was deemed statistically inequivalent. However, we dismissed it as the probable consequence of a type 1 error at the $95 \%$ confidence interval and concluded that constituent expression of the luciferase reporter did not significantly affect the local (tumor) or systemic (splenic) immune responses. Therefore, we continued our study of the systemic immune response to osteosarcoma using only the TF luciferase(+) model which allows for bioluminescent detection and quantification of disease burden.

SECTION 2: Using the spleen to identify the baseline systemic immune fingerprint associated with disease progression and metastasis in the luciferase(+) K7M2 orthotopic BALB/c mouse model of osteosarcoma.

There is a critical gap in knowledge regarding the effects of malignancy on a patient's systemic immune status, especially in the context of tumor immunotherapy. Many immunotherapies (including monoclonal antibody checkpoint blockers) used in both preclinical and clinical settings are given systemically via the blood and can modulate interactions between $\mathrm{T}$ and antigen presenting cells (APCs) like macrophages (35-39); however, our understanding of what systemic immunomodulations underlie tumor clearance (or resistance) is incomplete. Immunotherapies targeting this axis have shown great antitumor potential and, for the case of Mifamurtide, come the closest to regular clinical use in osteosarcoma (40). Additionally, there is currently no reliable way to monitor immune activation changes over time once primary tumors are removed. Therefore, we sought to establish a baseline systemic immunological profile for the TF tumor-bearing mouse model that can be used by other investigators to examine tumor and/or 
treatment induced immunomodulation. The spleen was chosen as a marker of systemic immune status as it is a central hub for circulating immune cells (including mature macrophages) and is the main filter for blood-borne antigens (including circulating tumor cells and debris). From a feasibility and clinical translational standpoint, splenic biopsies performed with fine needle aspirates are an effective cytological monitoring platform with low complication rates in humans (41).

At 11 weeks post-inoculation (at which time $90 \%$ of tumor-bearing mice have fulminant pulmonary metastases), we used Antibody Panels 3 and 4 to monitor the cellular changes of lymphoid lineage cells (LLCs) and myeloid lineage cells (MLCs), respectively, occurring alongside disease progression in the spleens of TF tumor-bearing mice. Antibody Panel 3 examined the following four LLC subsets of WBC populations: CD45+CD8+CTLs, CD45+CD4+ Ths, CD45+CD4+Foxp3+ Tregs, and CD45+NKp46+ NK cells (example gating schema seen in Supplementary Figure 5). We also investigated the exhaustion status of the CD4+ and CD8+ Tlymphocyte populations using the prototypical cell-surface markers PD-1 and Tim-3. T cell exhaustion (TCE) is a phenomenon characterized by decreased effector cell function first described in the instance of chronic viral infection that correlates with increases in the expression levels of PD-1 and Tim-3 (23, 27-29). Indeed, it has been shown that, like viral infections, cancers can cause similar immunological disruptions in lymphocyte function (42). An additional $\mathrm{T}$ cell surface molecule Cytotoxic T Lymphocyte Associated Protein 4 (CTLA-4) which competes with CD28 for binding to the $\mathrm{B} 7$ molecules CD80 and 86 on APCs inhibiting CTL function can also play a role in the immunological response to cancer (43). An example gating schema for PD-1, Tim-3, and CTLA-4 expression on T cells can be seen in Supplementary Figure 6. The expression profile data for Antibody Panel 3 from the spleens of five mice are shown in the left column of 
Table 5. In summary, NK cell percent population averaged $10.78 \pm 4.5 \%$; percent populations of CTLs, Ths, and Tregs averaged $2.73 \pm 1.22 \%, 6.45 \pm 3.08 \%$, and $21.09 \pm 5.12 \%$, respectively. CTLA-4 expression on CTLs and TCES averaged $18.35 \pm 10.12 \%$ and $18.23 \pm 4.58 \%$, respectively.

Antibody Panel 4 investigated the MLC component of the splenic immunophenotype. The three cell populations examined included CD45+CD11b+ MLCs, CD45+CD11b+Ly6G-Ly6C+ monocytes/macrophages, and CD45+CD11b+Ly6C-Ly6G+ granulocytes (example gating schema in Supplementary Figure 7). Additionally, the expression of the following four macrophage polarization markers transglutaminase 2 (Tgm2), arginase 1 (Arg1), chemokine (C-X-C motif) ligand 9 (Cxc19), and nitric oxide synthase 2 (Nos2) was also assessed (example gating schema in Supplementary Figure 8). Tgm2/Arg1 and Cxc19/Nos2 are classical M2- and M1-like macrophage markers typically associated with pro-tumor anti-inflammatory or antitumor pro-inflammatory properties, respectively. CD45+CD11b+ MLCs were separated into Ly6C+Ly6G- monocytes and Ly6G+Ly6C-granulocytes. From there, the total percent expression of the following four M1/M2 macrophage markers on Ly6C+Ly6G- monocytes was determined and the expression profile data for these markers from the spleens of five mice is shown in the left column of Table 6. In summary, MLC, monocyte/macrophage, and granulocyte percent populations averaged $5.27 \pm 1.11 \%, 4.92 \pm$ $1.29 \%$, and $57.49 \pm 9.62 \%$, respectively. Percent expression of individual M1/M2 markers Tgm2, Arg1, Cxc19, and Nos2 on macrophages was $18.58 \pm 5.69 \%, 0.3 \pm 0.56 \%, 18.28 \pm 5.38 \%$, and $97.99 \pm 1.62 \%$, respectively

Here, we have developed two in-depth antibody panels (Panels 3 and 4) that can be used to assess the splenocyte immune fingerprint. The data gathered can be used as a baseline by which to assess immunomodulation in response to therapeutic intervention. To date, the splenic lymphoid 
and myeloid immune fingerprints of osteosarcoma tumor-bearing mice at time of pulmonary metastasis have never been investigated. Note that in this study, macrophages were not classified overtly as M1 or M2; rather, we report only the percent expression of each individual marker expressed by the entire macrophage population. As opposed to studies that choose the presence or absence of a single marker to identify $\mathrm{M} 1 / \mathrm{M} 2$ polarization, our paradigm provides a more objective view of macrophage phenotypes as complex disease processes like cancer influence macrophages to take on intermediate phenotypes and co-express both M1 and M2 markers (44, 45). Analyzing the percent expression of each individual marker on the macrophage population allows for the full spectrum of macrophage phenotypes to be appreciated, compared with using single markers to incorrectly generalize and simplify a complex population.

SECTION 3: Osteosarcoma disease progression and metastasis induce systemic immunomodulation as visualized by splenocyte immunophenotyping.

Evidence suggests that both lymphoid and myeloid lineage activation status in both the local and systemic immune environments may carry clinically useful information regarding osteosarcoma disease progression, metastasis, and survival $(16,17,46)$. However, once the primary tumor is removed, it can no longer provide up-to-date information on the evolving antitumor immune response. Peripheral blood has been used to determine immune status outside of the primary tumor with promising results in other cancers (47-51); however, the story is incomplete as mature macrophage data cannot be obtained in this fashion. Therefore, we sought to determine if the spleen can be used to detect tumor-induced immunomodulation by comparing the spleens of TF tumor-bearing mice to those undergoing sham $(\mathrm{SH})$ surgery procedures to examine the extent of systemic splenic immunomodulation induced by malignancy. SH mice underwent the same surgical procedures and Antibody Panels (3 and 4) as tumor-bearing mice; 
however, they were inoculated with vehicle as opposed to tumor cells. Using the Bonferroni correction, individual comparisons were made between TF tumor-bearing $(\mathrm{n}=5)$ and $\mathrm{SH}(\mathrm{n}=4)$ mice via the Student's T-test using $\alpha=0.0083$ and $\alpha=0.00714$ for Antibody Panels 3 and 4 , respectively, to assess significance at the $95 \%$ confidence interval. Between the two panels, numerous statistically significant differences between TF tumor-bearing and SH mice were discovered. For Panel 3, NK cell $\left(\mathrm{TFNK}_{\mathrm{N}}=10.78 \pm 4.5 \%\right.$; $\mathrm{SHNK}=1.85 \pm 0.4 \% ; \mathrm{p}=0.006$, Figure $1 \mathrm{~A})$ and Treg $\left(\mathrm{TFTreg}_{\mathrm{T}}=21.09 \pm 5.12 \% ; \mathrm{SH}\right.$ reg $=7.25 \pm 0.23 \% ; \mathrm{p}=0.0011$, Figure 1B $)$ percentages were significantly increased in the spleens of TF tumor-bearing versus SH mice. Also, the exhaustion status was significantly elevated in the spleens of tumor-bearing compared to SH mice $($ TFtCES $=18.23 \pm 4.58 ;$ SHTCES $=0.16 \pm 0.054, p=0.0001$, Figure 1C). Interestingly, there was also an increase in the percentage of CTLA- $4+$ CTLs in tumor-bearing mice that was trending toward statistical significance $(\mathrm{p}=0.014)$. Importantly, the overall population percentages of CTLs and Ths were statistically equivalent ( $p>0.0083$ ). For Panel 4, the overall percentage of MLCs within the tumor-bearing mouse spleens was significantly elevated compared to SH (TFMLC $=5.27$ $\pm 1.11 \%$; SHмLC $=1.39 \pm 0.4 \%, p=0.00031$, Figure $2 \mathrm{~A})$. However, the percent populations of both monocytes/macrophages and granulocytes from tumor-bearing mouse spleens versus $\mathrm{SH}$ were decreased and trending toward statistical significance $(p=0.036$ and 0.014 , respectively), potentially suggesting a percent increase of immature MLCs exhibiting intermediate phenotypes. Strikingly, the M1 markers Cxc19 and Nos2 and M2 marker Tgm2 were significantly upregulated in the macrophage populations of tumor-bearing mice $(\mathrm{p}=0.00052$ [Figure 2B], $4.2 \mathrm{E}-07$ [Figure $2 \mathrm{C}$ ], and 0.0025 [Figure 2D] respectively), displaying no clear transition in terms of polarization. The M2 marker Arg1 did not stain well in any of the populations examined. The statistics for each panel are summarized in Tables 5 and 6 for Panels 3 and 4, respectively. 
Out of the thirteen cell populations compared between TF tumor-bearing and SH groups, seven were found to be statistically significant. On the lymphoid side, we saw significant increases in both NK cells and Tregs; this increase in splenic Tregs has been observed across multiple tumor types in preclinical models, correlates with tumor size, and is generally associated with an overall state of immune suppression $(52,53)$. The splenic myeloid compartment of tumor-bearing mice was altered both in terms of myeloid lineage cell pool and macrophage maturation, which reflects a situation of increased inflammatory monocyte recruitment that may act as a reservoir for fast deployment to peripheral tissues during disease processes (54). Additionally, it may also represent an accumulation of immature myeloid cells with immunosuppressive phenotypes, as suggested for other tumor models (55). The status of macrophage activation was also significantly altered in tumor-bearing mice. Interestingly, the expression of macrophage M1 markers Nos2/Cxc19 and M2 marker Tgm2 all increased significantly, with no clear M1/M2 bias. These data support the evolving hypothesis that macrophages with intermediate phenotypes predominate in microenvironments associated with complex tumorigenic processes, and that simple M1/M2 classification may lead to gross oversimplifications that lead to incorrect conclusions. Overall, these data suggest that there is a systemic dysregulation of lymphocyte and myeloid populations created by malignancy, which can be monitored using these panels we described. The next step in this process is to more clearly define the specific functions that are associated with the observed phenotypes and what clinical outcomes they associate with, which may be used to help determine extent of disease progression and inform prognostics.

SECTION 4: The spleen can be used as a barometer of the systemic immune response to treatment with monoclonal antibody and immunological checkpoint blocker $\alpha-P D-L 1$. 
In Section 3, we determined that our antibody panels could accurately characterize malignancy-induced immunomodulations occurring alongside disease progression. As such, if we are able to assess how the immune system is changing in response to immunotherapeutics in realtime, we may be able to adapt treatment regimens so that the appropriate immune responses are elicited in the right way at the right time on a patient-by-patient basis. Therefore, if patients' systemic immune systems are sampled regularly, important tumor immune escape mechanisms such as these may be exposed and properly accounted for therapeutically. Therefore, we sought to determine whether the spleen could be used as a systemic immunologic barometer to assess immunotherapy-induced microenvironment changes. The immunotherapy chosen for this portion of the study was $\alpha$-PD-L1, which is used in different cancers with varying success rates (6). As $\alpha$ PD-L1 blockade activity is already well-established in the literature $(56,57)$ and overall treatment efficacy was not the desired outcome of study, no isotype controls were deemed necessary.

For these studies, transfected tumor-bearing mice $(n=3)$ were treated intraperitoneally with $20 \mu \mathrm{g}$ of $\alpha$-PD-L1 antibody in PBS twice a week starting at the first palpable sign of primary lesion. Primary tumors were removed at approximately $1.7 \mathrm{~cm} 3$ and mice were euthanized at approximately 11 weeks post-inoculation. Harvested spleens were subjected to a modified (mod) Panel 3 (lacking the NKp46 NK cell identifier) and Panel 4. Using the Bonferroni correction, individual comparisons to assess significance between $\alpha$-PD-L1 treated $(n=3)$ and untreated $(n=$ 5) TF tumor-bearing mice were made via Student's T-test using $\alpha=0.01$ and $\alpha=0.00714$ for Antibody Panels 3 (mod) and 4 at the $95 \%$ confidence interval, respectively. Of those populations investigated in Panel 3 (mod), $\alpha-P D-L 1$ treatment was shown to significantly reduce splenic TCES $\left(\mathrm{TF}_{(-\alpha \mathrm{PD}-\mathrm{L} 1)}=18.23 \pm 4.58 ; \mathrm{TF}_{(+\alpha \mathrm{PD}-\mathrm{L} 1)}=0.79 \pm 0.71, \mathrm{p}=0.00072\right)$ as shown in Figure 3A. Interestingly, however, two out of the three mice that showed decreased TCES in response to $\alpha$ - 
PD-L1 therapy also showed a large increase in the percentage of CTLA-4+ expressed on CTLs (Figure 3B), perhaps suggestive of a potential escape mechanism by which tumors can depress $\mathrm{T}$ cell activity. $\alpha$-PD-L1 treatment also greatly increased the percent population of monocytes/macrophages (Figure 3C: $\mathrm{TF}_{(-\alpha \mathrm{PD}-\mathrm{L} 1)}=4.92 \pm 1.29 \% ; \mathrm{TF}(+\alpha \mathrm{PD}-\mathrm{L} 1)=12.8 \pm 2.92 \%, \mathrm{p}=$ 0.0016) and decreased the percentage of macrophages expressing Tgm $2($ Figure $3 \mathrm{D}: \mathrm{TF}(-\alpha \mathrm{PD}-\mathrm{L} 1)=$ $18.58 \pm 5.69 \% ; \mathrm{TF}(+\alpha \mathrm{PD}-\mathrm{L} 1)=2.1 \pm 2.17 \%, \mathrm{p}=0.0034), \mathrm{Cxcl}$ (Figure $3 \mathrm{E}: \mathrm{TF}(-\alpha \mathrm{PD}-\mathrm{L} 1)=18.28 \pm$ $\left.5.38 \% ; \mathrm{TF}_{(+\alpha \mathrm{PD}-\mathrm{L} 1)}=1.52 \pm 1.24 \%, \mathrm{p}=0.002\right)$, and Nos2 $($ Figure $3 \mathrm{~F}: \mathrm{TF}(-\alpha \mathrm{PD}-\mathrm{L} 1)=97.99 \pm 1.62 \%$; $\left.\mathrm{TF}_{(+\alpha \mathrm{PD}-\mathrm{L} 1)}=50.89 \pm 18.07 \%, \mathrm{p}=0.00086\right)$, essentially normalizing the M1/M2 macrophage polarization distribution back to SH status. Complete data summaries can be found in Tables 7 and 8 for Panels 3 (mod) and 4, respectively.

Immunotherapies that include PD-1/PD-L1 blockade have shown prolonged clinical activity against various human malignancies (58-60) excluding osteosarcoma $(10,61)$, despite the fact that there is evidence that PD-L1 contributes to osteosarcoma disease progression $(33,34)$. Therefore, $\alpha$-PD-L1 was chosen as our quintessential immunotherapeutic for the dual purposes of testing the ability of our antibody panels to detect therapy-induced changes in splenic microenvironment and to potentially shed some light on the immunologic mechanisms behind its therapeutic shortcomings. Interestingly, we found that while both $\alpha$-PD-L1-treated and non-treated mice eventually succumbed to disease, $\alpha$-PD-L1 monotherapy alone was sufficient to substantially reverse many immunosuppressive phenomena observed in non-treated tumor-bearing mice. However, while individual M1 and M2 markers between treated and untreated groups did show significant changes, the M1:M2 marker ratios were statistically equivalent $(\mathrm{p}=0.21)$. The data suggest that $\alpha$-PD-L1 monotherapy alone can stabilize but not induce generalized M1-biased macrophage polarization that has been associated with decreased metastasis (62). Still, these data 
offer a possible explanation for why combination therapies including $\alpha$-PD-L1 therapy are more effective (61), as it appears to neutralize many of the immunological effects induced by malignancy. Interestingly, two out of three mice treated with $\alpha$-PD-L1 therapy showed drastically increased expression of CTLA-4 on CTLs, revealing perhaps a compensatory mechanism by which tumors can circumnavigate $\alpha$-PD-L1 monotherapeutic intervention which supports previous findings (61).

Taken together, these data argue for the use of immunotherapeutic "cocktails" that target multiple pathways similar to the approach taken for human immunodeficiency virus/acquired immune deficiency syndrome (HIV/AIDS) therapy. Furthermore, we have provided a reasonable explanation for why $\alpha$-PD-L1 monotherapy for osteosarcoma has been less successful in the past. As such, we propose that the spleen may serve as a barometer for real-time monitoring of disease progression and patient response to immunotherapy and deserves further investigation.

\section{Conclusions}

Introduction of a non-viral luciferase reporter vector into K7M2 osteosarcoma tumor cells had no significant effect on the local or systemic immune responses to disease progression and metastasis in an orthotopic murine model of osteosarcoma, confirming its utility for accurately modeling complex immune microenvironment dynamics despite the luciferase reporter transfection. Our model can be used to accurately assess both disease burden (via IVIS) and immune dynamics with confidence that the reporter protein will not cause significant immunological disturbances. Additionally, it was shown that osteosarcoma disease progression and metastasis induce systemic dysregulation of immune cell populations including macrophage maturation and M1/M2 marker expression, TCE, and NK cell percent populations when compared to disease-free sham controls. These data suggest that osteosarcoma-induced immunomodulation 
is observable in the spleen with the antibody panels described and offer new insight into the complex role that macrophages play in osteosarcoma disease progression. Indeed, both the prevalence of tumor-associated macrophages and expression of single macrophage M1/M2 polarization markers have been previously associated with disease events that when taken together seem contradictory $(13,26,62,63)$.

In this study, we showed that osteosarcoma disease progression induces clear dysregulation of macrophage activation with increased expression of both M1 and M2 markers, offering further proof as to why single marker M1/M2 macrophage identifiers do not accurately assess polarization status in complex malignant settings. Our studies also showed that low-dose $\alpha$-PD-L1 therapy alone normalized osteosarcoma-induced systemic immunodysregulation back to that of sham status without changing clinical outcome; however, a compensatory increase in the immunosuppressive marker CTLA-4 on CTLs was observed following PD-L1 blockade indicating a potential tumor escape mechanism. These data suggest that immune escape mechanisms like PDL1/CTLA-4 counter-regulation may be uncovered by immunophenotyping systemic immune organs like the spleen, which could be extremely useful clinically by adapting therapies to the immunophenotype that is occurring at a specific time point. 


\section{Conflicts of Interest}

BAL declares paid consultancy for Highmark and Zimmer Biomet; research support from Stryker and Globus Life Technologies; and he is a paid speaker for Hangar Prosthetics. The other authors report no conflicts of interest.

\section{Funding Statement}

This paper is generated from work supported by the WVU CTSI IDeA CTR Support under NIH/NIGMS Award Number U54GM104942 and the WVU Flow Cytometry \& Single Cell Core under grant numbers P30GM103488 and S10OD016165. 


\section{References}

1. Bielack, S. S., Hecker-Nolting, S., Blattmann, C., \& Kager, L. (2016). Advances in the management of osteosarcoma. F1000Research, 5.

2. Chen, X., Bahrami, A., Pappo, A., Easton, J., Dalton, J., Hedlund, E., ... \& Parker, M. (2014). Recurrent somatic structural variations contribute to tumorigenesis in pediatric osteosarcoma. Cell reports, 7(1), 104-112.

3. Wang, D., Wang, J., Niu, X., Huang, Z., Wang, Z., Zhang, Q., ... \& Hao, L. (2017). Clone evolution and genomic alteration analysis of osteosarcoma and matched lung metastasis.

4. Von Bernstorff, W., Voss, M., Freichel, S., Schmid, A., Vogel, I., Jöhnk, C., ... \& Kalthoff, $\mathrm{H}$.

(2001). Systemic and local immunosuppression in pancreatic cancer patients. Clinical Cancer Research, 7(3), 925s-932s.

5. Lippitz, B. E. (2013). Cytokine patterns in patients with cancer: a systematic review. The lancet oncology, 14(6), e218-e228.

6. Gabrilovich, D. I., \& Nagaraj, S. (2009). Myeloid-derived suppressor cells as regulators of the immune system. Nature reviews immunology, 9(3), 162.

7. Brahmer, J. R., Tykodi, S. S., Chow, L. Q., Hwu, W. J., Topalian, S. L., Hwu, P., ... \& Pitot, H. C. (2012). Safety and activity of anti-PD-L1 antibody in patients with advanced cancer. New England Journal of Medicine, 366(26), 2455-2465.

8. Zhang, Y., \& Ertl, H. C. (2016). Starved and asphyxiated: How can CD8+ T cells within a tumor microenvironment prevent tumor progression. Frontiers in immunology, 7, 32 9. Sundara, Y. T., Kostine, M., Cleven, A. H., Bovée, J. V., Schilham, M. W., \& CletonJansen, A. M. (2017). Increased PD-L1 and T-cell infiltration in the presence of HLA class I expression in metastatic high-grade osteosarcoma: a rationale for T-cell-based immunotherapy. Cancer Immunology, Immunotherapy, 66(1), 119-128.

10. Ma, W., Gilligan, B. M., Yuan, J., \& Li, T. (2016). Current status and perspectives in translational biomarker research for PD-1/PD-L1 immune checkpoint blockade therapy. Journal of hematology \& oncology, 9(1), 47.

11. Lussier, D. M., O’Neill, L., Nieves, L. M., McAfee, M. S., Holechek, S. A., Collins, A. W., ... \& Blattman, J. N. (2015). Enhanced T-cell immunity to osteosarcoma through antibody blockade of PD-1/PD-L1 interactions. Journal of Immunotherapy, 38(3), 96-106.

12. Topalian, S. L., Taube, J. M., Anders, R. A., \& Pardoll, D. M. (2016). Mechanism-driven biomarkers to guide immune checkpoint blockade in cancer therapy. Nature Reviews Cancer, 16(5), 275.

13. Buddingh, E. P., Kuijjer, M. L., Duim, R. A., Bürger, H., Agelopoulos, K., Myklebost, O., ... \& Cleton-Jansen, A. M. (2011). Tumor-infiltrating macrophages are associated with metastasis suppression in high-grade osteosarcoma: a rationale for treatment with macrophageactivating agents. Clinical Cancer Research, clincanres-2047.

14. Pahl, J. H., Kwappenberg, K. M., Varypataki, E. M., Santos, S. J., Kuijjer, M. L., Mohamed, S., ... \& Jiskoot, W. (2014). Macrophages inhibit human osteosarcoma cell growth after activation with the bacterial cell wall derivative liposomal muramyl tripeptide in combination with interferon- $\gamma$. Journal of Experimental \& Clinical Cancer Research, 33(1), 27. 15. Xiao, Q., Zhang, X., Wu, Y., \& Yang, Y. (2014). Inhibition of macrophage polarization prohibits growth of human osteosarcoma. Tumor Biology, 35(8), 7611-7616. 
16. Li, X., Chen, Y., Liu, X., Zhang, J., He, X., Teng, G., \& Yu, D. (2017). Tim3/Gal9 interactions between $T$ cells and monocytes result in an immunosuppressive feedback loop that inhibits Th1 responses in osteosarcoma patients. International immunopharmacology, 44, 153159.

17. Liu, H., Zhi, L., Duan, N., \& Su, P. (2016). Abnormal expression of Tim-3 antigen on peripheral blood T cells is associated with progressive disease in osteosarcoma patients. FEBS open bio, 6(8), 807-815.

18. Heymann, M. F., Lézot, F., \& Heymann, D. (2017). The contribution of immune infiltrates and the local microenvironment in the pathogenesis of osteosarcoma. Cellular immunology.

19. Nardin, A., Lefebvre, M. L., Labroquere, K., Faure, O., \& Abastado, J. P. (2006). Liposomal muramyl tripeptide phosphatidylethanolamine: Targeting and activating macrophages for adjuvant treatment of osteosarcoma. Current cancer drug targets, 6(2), 123-133.

20. Endo-Munoz, L., Evdokiou, A., \& Saunders, N. A. (2012). The role of osteoclasts and tumour-associated macrophages in osteosarcoma metastasis. Biochimica et Biophysica Acta (BBA)-Reviews on Cancer, 1826(2), 434-442.

21. Jia, S. F., Worth, L. L., Densmore, C. L., Xu, B., Duan, X., \& Kleinerman, E. S. (2003). Aerosol gene therapy with PEI: IL-12 eradicates osteosarcoma lung metastases. Clinical cancer research, 9(9), 3462-3468.

22. Grisez, B. T., Ray, J. J., Bostian, P. A., Markel, J. E., \& Lindsey, B. A. (2018). Highly metastatic K7M2 cell line: A novel murine model capable of in vivo imaging via luciferase vector transfection. Journal of Orthopaedic Research ${ }^{\circledR}$.

23. Sakuishi, K., Apetoh, L., Sullivan, J. M., Blazar, B. R., Kuchroo, V. K., \& Anderson, A. C. (2010). Targeting Tim-3 and PD-1 pathways to reverse T cell exhaustion and restore antitumor immunity. Journal of Experimental Medicine, 207(10), 2187-2194.

24. Ahmadzadeh, M., Johnson, L. A., Heemskerk, B., Wunderlich, J. R., Dudley, M. E., White, D. E., \& Rosenberg, S. A. (2009). Tumor antigen-specific CD8 T cells infiltrating the tumor express high levels of PD-1 and are functionally impaired. Blood, 114(8), 1537-1544.

25. Anderson, A. C. (2012). Tim-3, a negative regulator of anti-tumor immunity. Current opinion in immunology, 24(2), 213-216.

26. Han, Q., Shi, H., \& Liu, F. (2016). CD163+ M2-type tumor-associated macrophage support the suppression of tumor-infiltrating $\mathrm{T}$ cells in osteosarcoma. International immunopharmacology, 34, 101-106.

27. Fourcade, J., Sun, Z., Benallaoua, M., Guillaume, P., Luescher, I. F., Sander, C., ... \& Zarour, H. M. (2010). Upregulation of Tim-3 and PD-1 expression is associated with tumor antigen-specific CD8+ T cell dysfunction in melanoma patients. Journal of Experimental Medicine, 207(10), 2175-2186.

28. Baitsch, L., Fuertes-Marraco, S. A., Legat, A., Meyer, C., \& Speiser, D. E. (2012). The three main stumbling blocks for anticancer T cells. Trends in immunology, 33(7), 364-372.

29. Wherry, E. J., \& Kurachi, M. (2015). Molecular and cellular insights into T cell exhaustion. Nature Reviews Immunology, 15(8), 486. England Journal of Medicine, 366(26), 2455-2465.

30. Monaco, J. J. (1992). A molecular model of MHC class-I-restricted antigen processing. Immunology today, 13(5), 173-179. 
31. Ansari, A. M., Ahmed, A. K., Matsangos, A. E., Lay, F., Born, L. J., Marti, G., ... \& Sun, Z. (2016). Cellular GFP toxicity and immunogenicity: potential confounders in in vivo cell tracking experiments. Stem Cell Reviews and Reports, 12(5), 553-559.

32. Baklaushev, V. P., Kilpeläinen, A., Petkov, S., Abakumov, M. A., Grinenko, N. F., Yusubalieva, G. M., ... \& Abakumova, T. O. (2017). Luciferase expression allows

bioluminescence imaging but imposes limitations on the Orthotopic mouse (4T1) model of breast Cancer. Scientific reports, 7(1), 7715.

33. Zheng, W., Xiao, H., Liu, H., \& Zhou, Y. (2015). Expression of programmed death 1 is correlated with progression of osteosarcoma. Apmis, 123(2), 102-107.

34. Koirala, P., Roth, M. E., Gill, J., Piperdi, S., Chinai, J. M., Geller, D. S., ... \& Gorlick, R. (2016). Immune infiltration and PD-L1 expression in the tumor microenvironment are prognostic in osteosarcoma. Scientific reports, 6, 30093.

35. Wolchok, J. D., \& Saenger, Y. (2008). The mechanism of anti-CTLA-4 activity and the negative regulation of T-cell activation. The Oncologist, 13(Supplement 4), 2-9.

36. Latchman, Y. E., Liang, S. C., Wu, Y., Chernova, T., Sobel, R. A., Klemm, M., ... \& Sharpe, A. H. (2004). PD-L1-deficient mice show that PD-L1 on T cells, antigen-presenting cells, and host tissue negatively regulates T cells. Proceedings of the National Academy of Sciences of the United States of America, 101(29), 10691-10696.

37. Zhang, Y., Zhou, Y., Lou, J., Li, J., Bo, L., Zhu, K., ... \& Cai, Z. (2010). PD-L1 blockade improves survival in experimental sepsis by inhibiting lymphocyte apoptosis and reversing monocyte dysfunction. Critical care, 14(6), R220.

38. Lau, J., Cheung, J., Navarro, A., Lianoglou, S., Haley, B., Totpal, K., ... \& Chiu, H. (2017). Tumour and host cell PD-L1 is required to mediate suppression of anti-tumour immunity in mice. Nature Communications, 8, 14572.

39. Lafleur, E. A., Jia, S. F., Worth, L. L., Zhou, Z., Owen-Schaub, L. B., \& Kleinerman, E. S. (2001). Interleukin (IL)-12 and IL-12 gene transfer up-regulate Fas expression in human osteosarcoma and breast cancer cells. Cancer research, 61(10), 4066-4071.

40. Jimmy, R., Stern, C., Lisy, K., \& White, S. (2017). Effectiveness of mifamurtide in addition to standard chemotherapy for high-grade osteosarcoma: a systematic review. JBI database of systematic reviews and implementation reports, 15(8), 2113-2152.

41. Gochhait, D., Dey, P., Rajwanshi, A., Nijhawan, R., Gupta, N., Radhika, S., \& Lal, A. (2015). Role of fine needle aspiration cytology of spleen. APMIS, 123(3), 190-193.

42. Blank, C., \& Mackensen, A. (2007). Contribution of the PD-L1/PD-1 pathway to T-cell exhaustion: an update on implications for chronic infections and tumor evasion. Cancer immunology, immunotherapy, 56(5), 739-745.

43. Vandenborre, K., Van Gool, S. W., Kasran, A., Ceuppens, J. L., Boogaerts, M. A., \& Vandenberghe, P. (1999). Interaction of CTLA-4 (CD152) with CD80 or CD86 inhibits human T-cell activation. Immunology, 98(3), 413-421.

44. Martinez, F. O., \& Gordon, S. (2014). The M1 and M2 paradigm of macrophage activation: time for reassessment. F1000prime reports, 6 .

45. Murray, P. J., Allen, J. E., Biswas, S. K., Fisher, E. A., Gilroy, D. W., Goerdt, S., ... \& Locati, M. (2014). Macrophage activation and polarization: nomenclature and experimental guidelines. Immunity, 41(1), 14-20.

46. Fritzsching, B., Fellenberg, J., Moskovszky, L., Sápi, Z., Krenacs, T., Machado, I., ... \& Bernd, L. (2015). CD8+/FOXP3+-ratio in osteosarcoma microenvironment separates survivors 
from non-survivors: a multicenter validated retrospective study. Oncoimmunology, 4(3), e990800.

47. Wolf, A. M., Wolf, D., Steurer, M., Gastl, G., Gunsilius, E., \& Grubeck-Loebenstein, B. (2003). Increase of regulatory T cells in the peripheral blood of cancer patients. Clinical cancer research, 9(2), 606-612.

48. Giraldo, N. A., Becht, E., Vano, Y., Petitprez, F., Lacroix, L., Validire, P., ... \& Buttard, B. (2017). Tumor-infiltrating and peripheral blood T cell immunophenotypes predict early relapse in localized clear cell renal cell carcinoma. Clinical Cancer Research, clincanres-2848.

49. Mego, M., Gao, H., Cohen, E. N., Anfossi, S., Giordano, A., Tin, S., ... \& Alvarez, R. H. (2017). Circulating tumor cells (CTCs) are associated with abnormalities in peripheral blood dendritic cells in patients with inflammatory breast cancer. Oncotarget, 8(22), 35656.

50. Grimm, M., Feyen, O., Hofmann, H., Teriete, P., Biegner, T., Munz, A., \& Reinert, S. (2016). Immunophenotyping of patients with oral squamous cell carcinoma in peripheral blood and associated tumor tissue. Tumor Biology, 37(3), 3807-3816.

51. Marini, O., Spina, C., Mimiola, E., Cassaro, A., Malerba, G., Todeschini, G., ... \& Cassatella, M. (2016). Identification of granulocytic myeloid-derived suppressor cells (GMDSCs) in the peripheral blood of Hodgkin and non-Hodgkin lymphoma patients. Oncotarget, 7(19), 27676.

52. Du, Y., Chen, X., Huang, Z. M., Ye, X. H., \& Niu, Q. (2012). Increased frequency of Foxp3+ regulatory T cells in mice with hepatocellular carcinoma. Asian Pacific Journal of Cancer Prevention, 13(8), 3815-3819.

53. Liu, J. Y., Zhang, X. S., Ding, Y., Peng, R. Q., Cheng, X., Zhang, N. H., .. \& Zeng, Y. $\mathrm{X}$. (2005). The changes of CD4+ CD25+/CD4+ proportion in spleen of tumor-bearing BALB/c mice. Journal of translational medicine, 3(1), 5.

54. Swirski, F. K., Nahrendorf, M., Etzrodt, M., Wildgruber, M., Cortez-Retamozo, V., Panizzi, P., ... \& Aikawa, E. (2009). Identification of splenic reservoir monocytes and their deployment to inflammatory sites. Science, 325(5940), 612-616.

55. Gabrilovich, D. I., Ostrand-Rosenberg, S., \& Bronte, V. (2012). Coordinated regulation of myeloid cells by tumours. Nature Reviews Immunology, 12(4), 253.

56. Hirano, F., Kaneko, K., Tamura, H., Dong, H., Wang, S., Ichikawa, M., ... \& Tamada, K. (2005). Blockade of B7-H1 and PD-1 by monoclonal antibodies potentiates cancer therapeutic immunity. Cancer research, 65(3), 1089-1096.

57. Hamanishi, J., Mandai, M., Matsumura, N., Abiko, K., Baba, T., \& Konishi, I. (2016). PD-1/PD-L1 blockade in cancer treatment: perspectives and issues. International journal of clinical oncology, 21(3), 462-473.

58. Powles, T., Eder, J. P., Fine, G. D., Braiteh, F. S., Loriot, Y., Cruz, C., ... \& Shen, X. (2014). MPDL3280A (anti-PD-L1) treatment leads to clinical activity in metastatic bladder cancer. Nature, 515(7528), 558.

59. Hamid, O., Sosman, J. A., Lawrence, D. P., Sullivan, R. J., Ibrahim, N., Kluger, H. M., ... \& Mokatrin, A. (2013). Clinical activity, safety, and biomarkers of MPDL3280A, an engineered PD-L1 antibody in patients with locally advanced or metastatic melanoma (mM).

60. Reck, M., Rodríguez-Abreu, D., Robinson, A. G., Hui, R., Csőszi, T., Fülöp, A., ... \& O'Brien, M. (2016). Pembrolizumab versus chemotherapy for PD-L1-positive non-small-cell lung cancer. New England Journal of Medicine, 375(19), 1823-1833.

61. Lussier, D. M., Johnson, J. L., Hingorani, P., \& Blattman, J. N. (2015). Combination immunotherapy with $\alpha$-CTLA-4 and $\alpha$-PD-L1 antibody blockade prevents immune escape and 
leads to complete control of metastatic osteosarcoma. Journal for immunotherapy of cancer, 3(1), 21.

62. Dumars, C., Ngyuen, J. M., Gaultier, A., Lanel, R., Corradini, N., Gouin, F., ... \& Heymann, M. F. (2016). Dysregulation of macrophage polarization is associated with the metastatic process in osteosarcoma. Oncotarget, 7(48), 78343.

63. Meyers, P. A., Schwartz, C. L., Krailo, M. D., Healey, J. H., Bernstein, M. L., Betcher, D., ... \& Kleinerman, E. (2008). Osteosarcoma: the addition of muramyl tripeptide to chemotherapy improves overall survival-a report from the Children's Oncology Group. Journal of Clinical Oncology, 26(4), 633-638. 


\section{Figure Legends}

Figure 1. Osteosarcoma induces systemic immunomodulation in splenic LLC populations at 11 weeks. The spleens of TF tumor-bearing $(n=5)$ and $\mathrm{SH}(\mathrm{n}=4)$ mice were harvested at time of metastatic disease (approximately 11 weeks) post-inoculation and analyzed via flow cytometry for LLC immunophenotyping. The data spread of the following percent populations (A) CD45+NKp46+ NK cells and (B) CD45+CD4+Foxp3+ Tregs between groups are displayed. (C) Data spread of TCES (total PD-1 and Tim-3 expression normalized to total percent T-lymphocyte population) for each group. An asterisk denotes statistical significance $(\mathrm{p}<0.0083)$ between groups as determined by a two-tailed Student's T-test with Bonferroni correction and 95\% confidence. $\mathrm{LLC}=$ lymphoid lineage cell; $\mathrm{TF}=$ transfected; $\mathrm{SH}=$ sham; $\mathrm{NK}=$ natural killer; Treg $=\mathrm{T}$ regulatory cell; $\mathrm{TCES}=\mathrm{T}$ cell exhaustion status $; \mathrm{PD}-1=$ programmed cell death protein 1 ; Tim-3 = T cell immunoglobulin and mucin-domain containing-3

Figure 2. Osteosarcoma induces systemic immunomodulation in splenic MLC populations at 11 weeks. The spleens of TF tumor-bearing $(n=5)$ and $\mathrm{SH}(\mathrm{n}=4)$ mice were harvested at time of metastatic disease (approximately 11 weeks) post-inoculation and analyzed via flow cytometry for MLC immunophenotyping. The data spread of the percent parent populations (A) CD45+CD11b+ MLCs is shown. Also, the spreads of an M2 macrophage polarization marker (B) Tgm2 and two M1 macrophage polarization markers (C) Cxc19 and (D) Nos2 on CD45+CD11b+Ly6C+Ly6Gmonocytes/macrophages are shown. An asterisk denotes statistical signifance with p-values < 0.00714 from a two-tailed Student's T-test with Bonferroni correction and 95\% confidence. MLC 
$=$ myleoid lineage cell $; \mathrm{TF}=$ transfected $; \mathrm{SH}=$ sham; $\mathrm{Tgm} 2=$ transglutaminase $2 ; \mathrm{Cxc1} 9=$ chemokine (C-X-C motif) ligand 9; Nos2 = nitric oxide synthase 2

\section{Figure 3. Treatment with $\alpha$-PD-L1 significantly reverses osteosacoma-induced immunomodulation in LLC and MLC populations in the spleens of tumor-bearing mice.}

Spleens were harvested at time of metastatic disease (approximately 11 weeks) post-inoculation and analyzed via flow cytometry for LLC and MLC immunophenotyping from $n=5(-) \alpha$-PD-L1 and $\mathrm{n}=3(+) \alpha$-PD-L1 TF tumor-bearing mice using Antibody Panels 3 (mod) and 4, respectively. For treated mice, $20 \mu \mathrm{g}$ of $\alpha$-PD-L1 was given in phosphate buffered saline via intraperitoneal injection twice a week (Mondays and Thursdays) starting when tumors were first palpable. The data spreads of (A) TCES and (B) CTLA-4+ CTLs from Antibody Panel 3 (mod) are shown with significance being assigned to those populations presenting $\mathrm{p}$-values $<0.01$ from a two-tailed Student's T-test with Bonferroni correction and 95\% confidence as denoted by an asterisk. The data spreads of (C) monocytes/macrophages, (D)Tgm2+ macrophages, (E) Cxc19+ macrophages, and (F) Nos2+ macrophages from Antibody Panel 4 are shown with significance being assigned to those populations presenting p-values $<0.00714$ from a two-tailed Student's T-test with Bonferroni correction and $95 \%$ confidence as denoted by an asterisk. PD-L1 = programmed deathligand $;$ LLC $=$ lymhpoid lineage cell $;$ MLC $=$ myleoid lineage cell; $\mathrm{TF}=$ transfected $; \mathrm{TCES}=\mathrm{T}$ cell exhaustion status; $\mathrm{CTLA}-4=$ cytotoxic $\mathrm{T}$ lymphocyte associated protein $4 ; \mathrm{CTL}=$ cytotoxic T lymphocyte; $\operatorname{Tgm} 2=$ transglutaminase $2 ; \mathrm{Cxc19}=$ chemokine $(\mathrm{C}-\mathrm{X}-\mathrm{C}$ motif $)$ ligand 9; Nos2 = nitric oxide synthase 2 
Supplementary Figure 1. Example gating schema for myeloid lineage cells, monocytes/macrophages, and granulocytes from Antibody Panel 1. Cells were gated into CD45+ and CD45- groups; from there, CD4-CD8-CD11b+cells myeloid lineage cells were gated into Ly6C+Ly6G- macrophages/monocytes and Ly6G+Ly6C- granulocytes.

Supplementary Figure 2. Example gating schema for $T$ helper cells, cytotoxic $T$ cells, and $T$ regulatory cells from Antibody Panel 1. Cells were gated into CD45+ and CD45-groups; from there, cells were gated into $\mathrm{CD} 4+\mathrm{T}$ helper cells and CD8+ cytotoxic $\mathrm{T}$ cells. $\mathrm{CD} 4+$ cells were further gated using Foxp3+ as a defining marker of T regulatory cells.

Supplementary Figure 3. Example gating schema for Natural Killer cells from Antibody Panel 1. Cells were gated into CD45+ and CD45-groups; from there, CD45+ cells were gated into NKp46+ Natural Killer cells.

Supplementary Figure 4. Example gating schema for PD-L1 positive non-white blood cell tumor cells from Antibody Panel 2. Tumor cells were gated into CD45+ and CD45- groups; from there, CD45-non-white blood cell tumor cells were assessed for programmed death-ligand 1 (PDL1) positivity. Note that, although the figure displays staining for CD95 positivity, this marker was not included in the analysis for PD-L1 expression in the manuscript.

Supplementary Figure 5. Example gating schema for cytotoxic $T$ cells, $T$ helper cells, Natural Killer cells, and T regulatory cells from Antibody Panel 3. Cells were gated into CD45+ and CD45- groups; from there, CD45+ cells were gated into NKp46+ Natural Killer cells, CD45+CD8+ 
cytotoxic $\mathrm{T}$ cells, and CD45+CD4+ $\mathrm{T}$ helper cells. CD45+CD4+ $\mathrm{T}$ cells were further gated for Foxp3 positivity to define CD45+CD4+Foxp3+ T regulatory cells.

\section{Supplementary Figure 6. Example gating schema for $\mathrm{T}$ cell exhaustion and functional status}

from Antibody Panel 3. CD4+ T helper cells and CD8+ cytotoxic $\mathrm{T}$ cells were assessed for expression of the $\mathrm{T}$ cell exhaustion markers programmed cell death protein 1 (PD-1) and $\mathrm{T}$ cell immunoglobulin and mucin-domain containing-3 (Tim-3). CD8+ T cells were also separately assessed for expression of inhibitory molecule cytotoxic $\mathrm{T}$ lymphocyte-associated protein 4 (CTLA-4).

Supplementary Figure 7. Example gating schema for myeloid lineage cells, monocytes/macrophages, and granulocytes from Antibody Panel 4. Cells were gated into CD45+CD11b+ myeloid lineage cells; from the CD45+CD11b+quadrant, cells were further gated into Ly6C+Ly6G- macrophages/monocytes and Ly6G+Ly6C- granulocytes.

Supplementary Figure 8. Example gating scheme for markers of macrophage M1/M2 polarization from Antibody Panel 4. CD11b+Ly6C+monocytes/macrophages were assessed for expression of M1 activation markers chemokine (C-X-C motif) ligand 9 (Cxc19), and nitric oxide synthase 2 (Nos2) and M2 activation markers transglutaminase 2 (Tgm2) and arginase 1 (Arg1). 
Figure 1

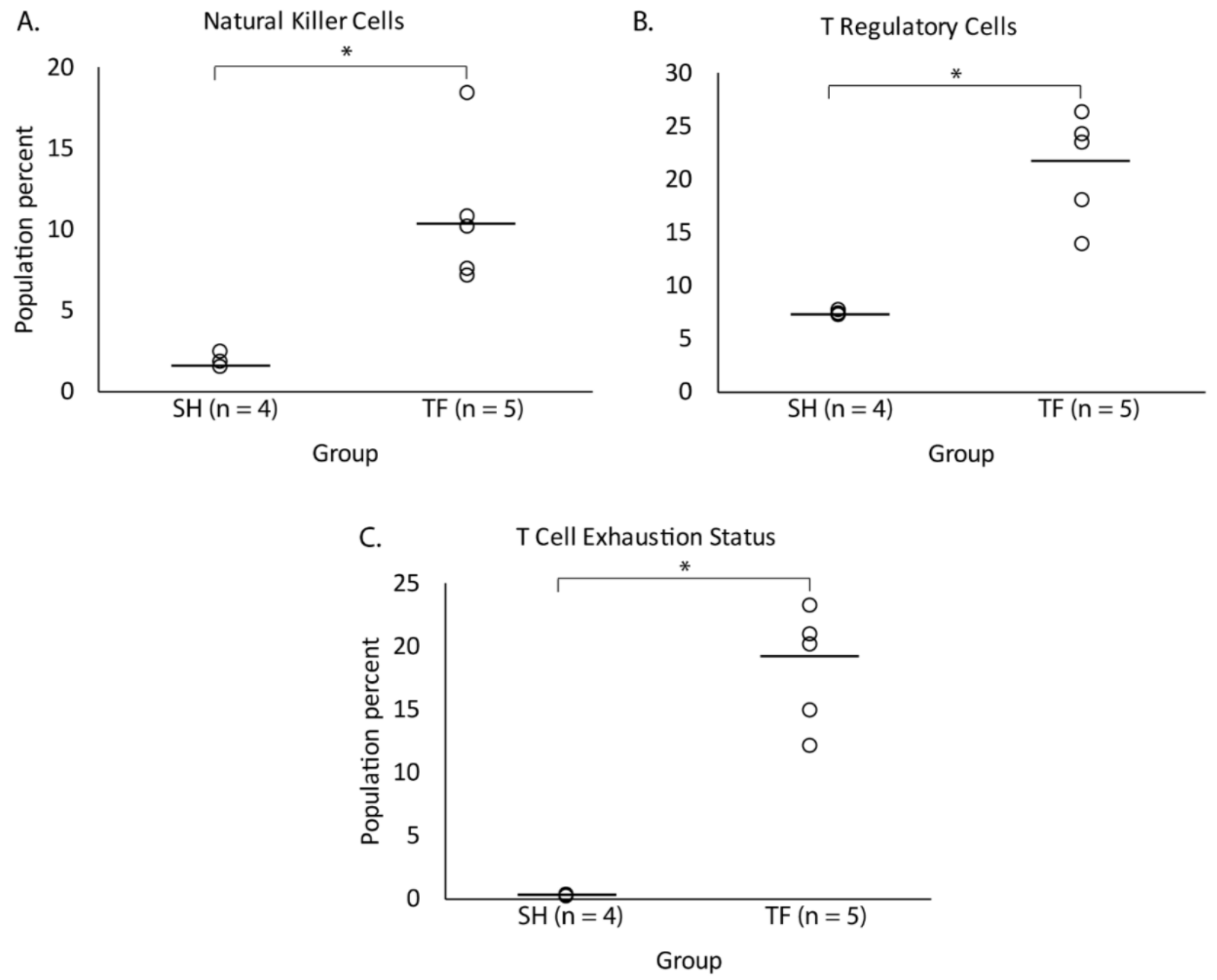




\section{Figure 2}

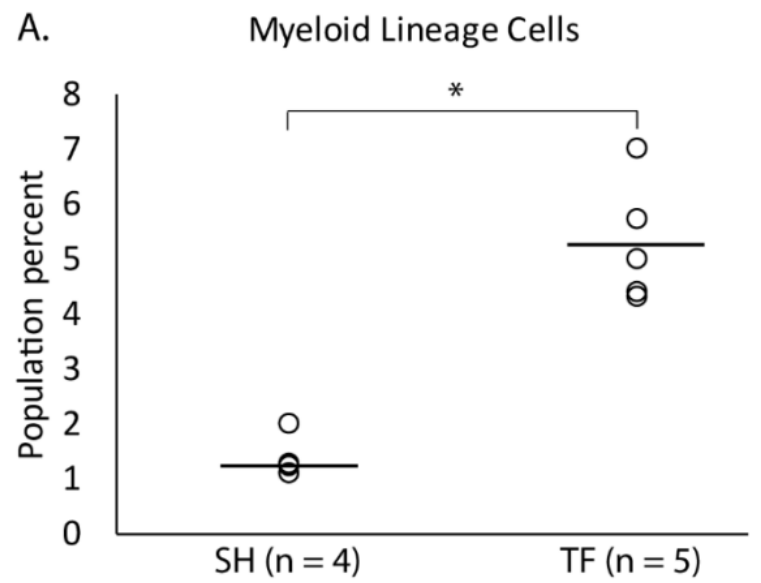

B.

$\mathrm{Tgm2}^{+}$Macrophages

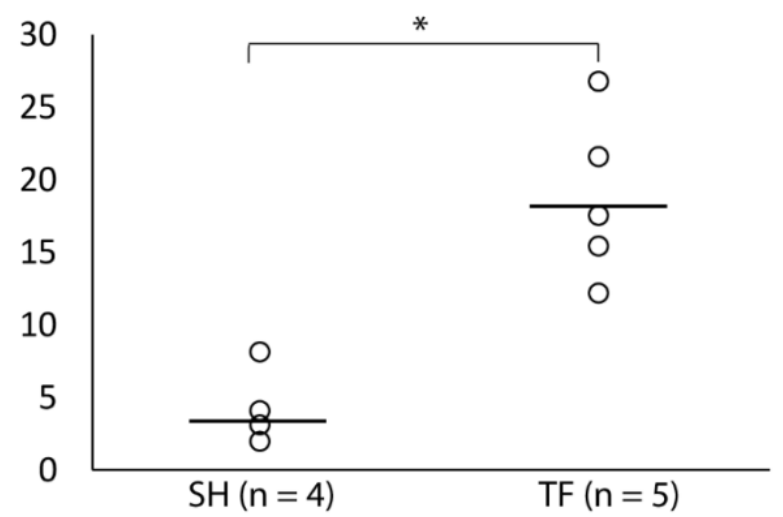

C.

Cxcl9+ Macrophages

D.
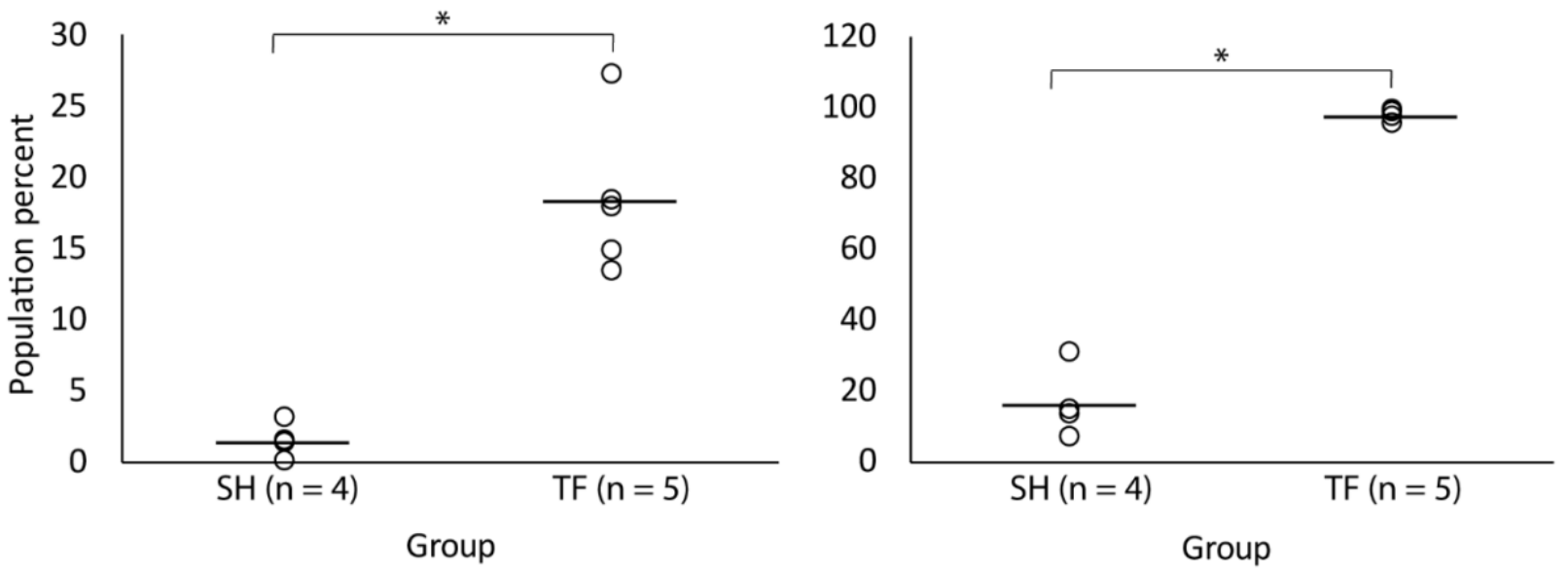


\section{Figure 3}

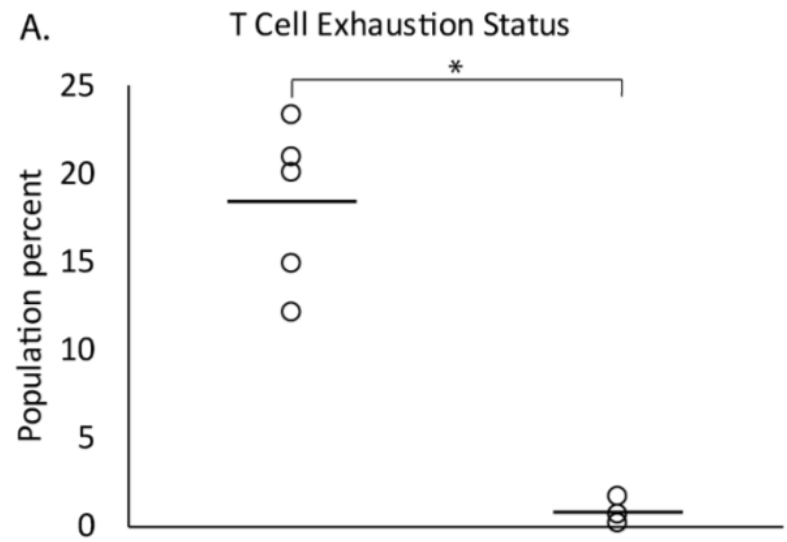

C.

Monocytes and Macrophages

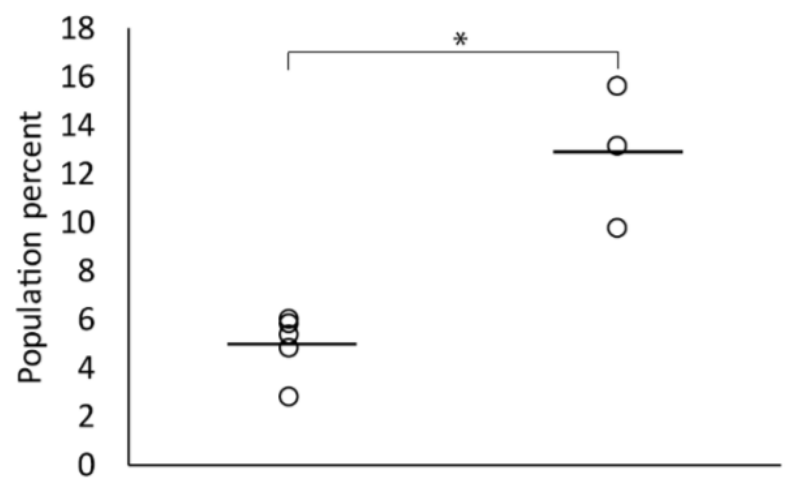

E.

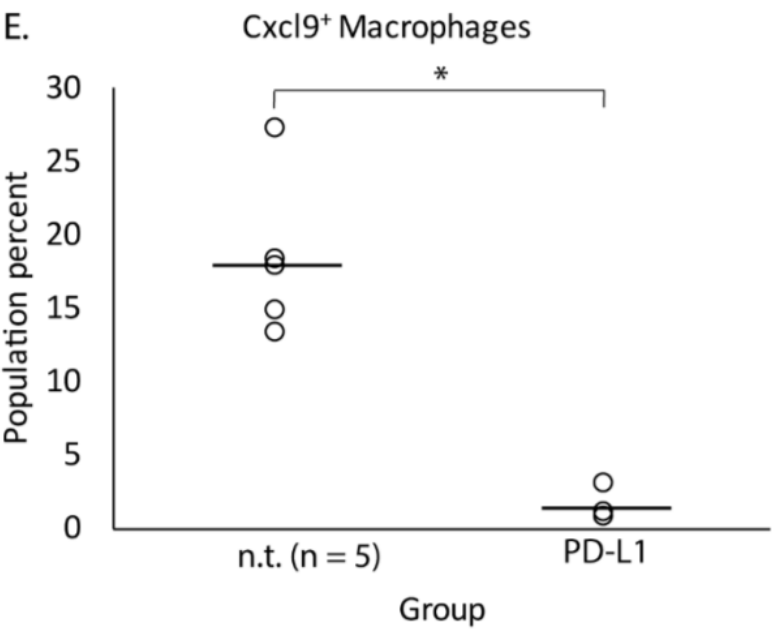

B. $\quad$ CTLA $-4^{+}$Cytotoxic T Cells

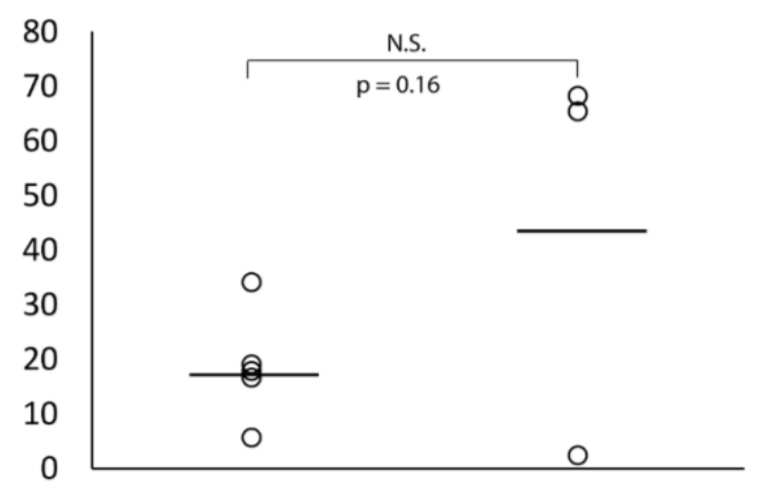

D.

$\operatorname{Tgm2}^{+}$Macrophages

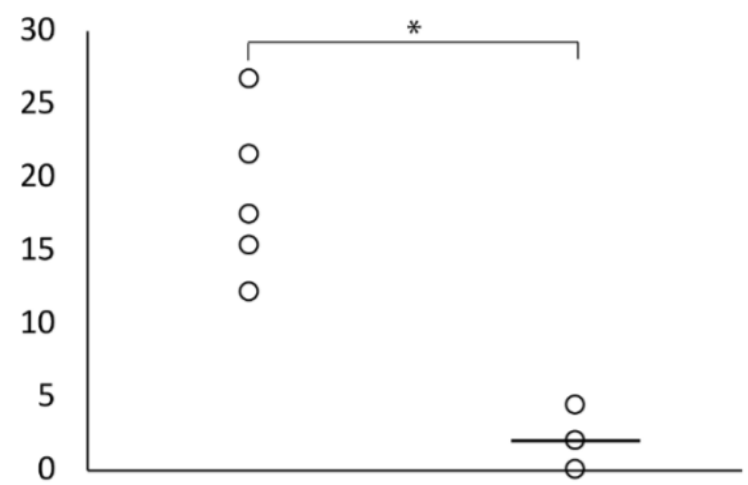

F.

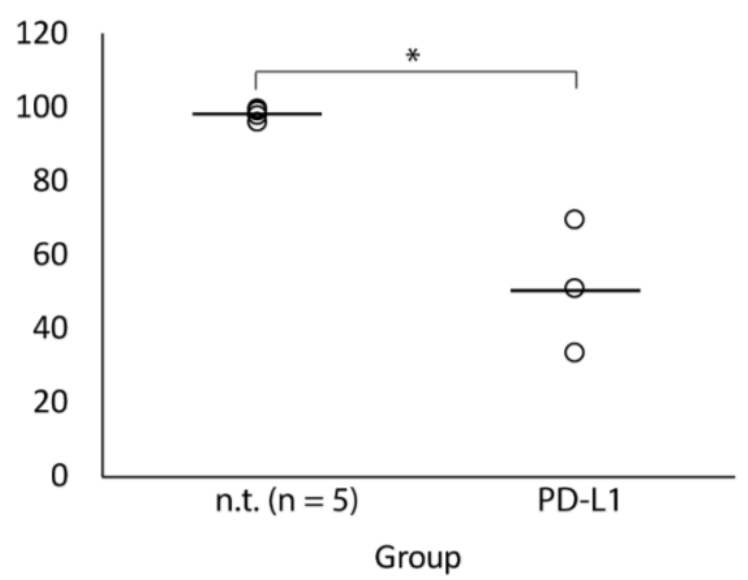




\section{Supplementary Figure 1}
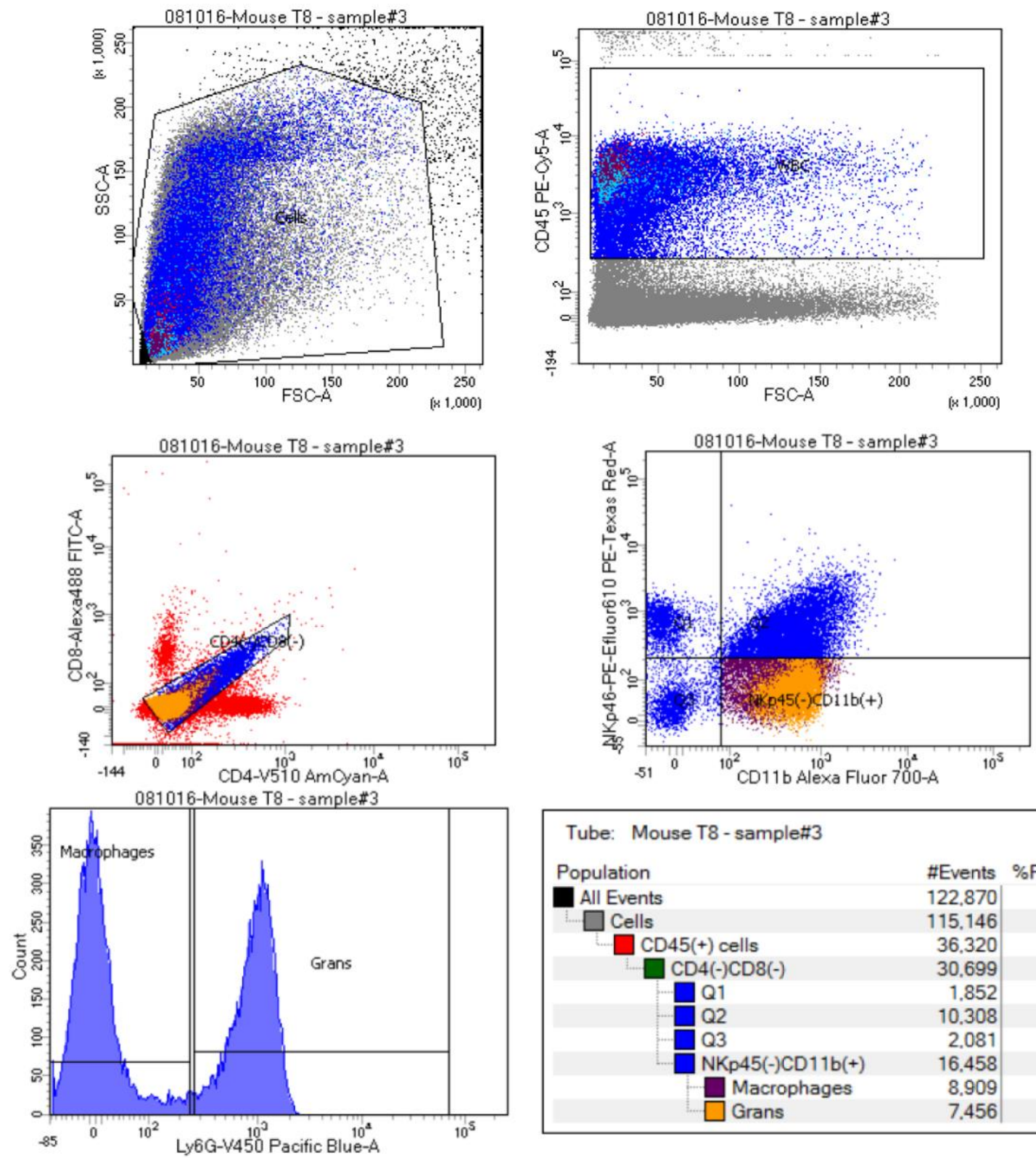

\begin{tabular}{|c|c|c|c|}
\hline Population & \#Events & \%Parent & \%Total \\
\hline All Events & 122.870 & \#\#\#\# & 100.0 \\
\hline$\square$ Cells & 115,146 & 93.7 & 93.7 \\
\hline$\square$ CD45(+) cells & 36.320 & 31.5 & 29.6 \\
\hline$\square \operatorname{CD} 4(-) \operatorname{CD} 8(-)$ & 30.699 & 84.5 & 25.0 \\
\hline Q1 & 1.852 & 6.0 & 1.5 \\
\hline Q2 & 10.308 & 33.6 & 8.4 \\
\hline Q3 & 2.081 & 6.8 & 1.7 \\
\hline NKp45(-)CD11b(+) & 16.458 & 53.6 & 13.4 \\
\hline Macrophages & 8.909 & 54.1 & 7.3 \\
\hline Grans & 7.456 & 45.3 & 6.1 \\
\hline
\end{tabular}




\section{Supplementary Figure 2}
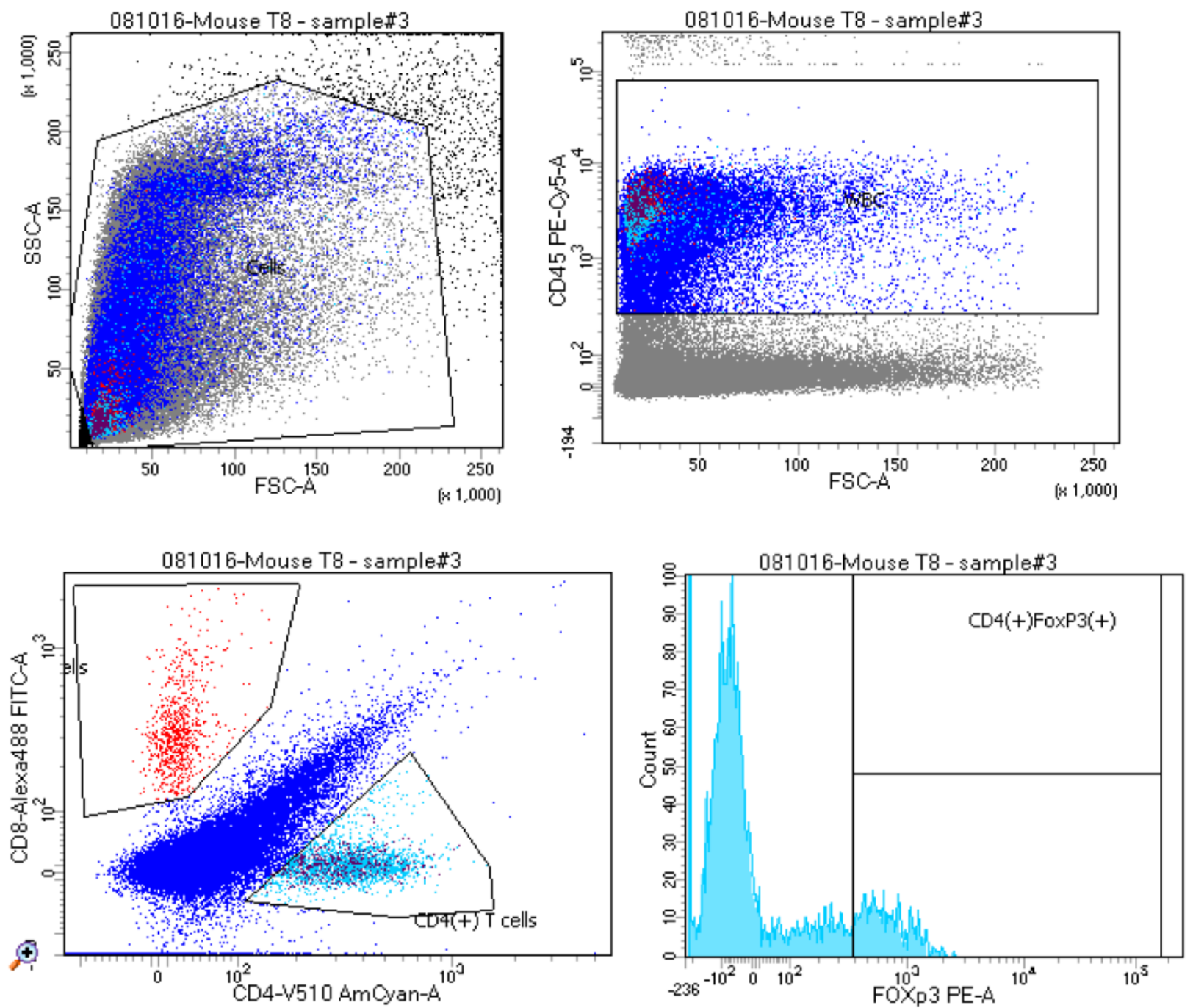

\begin{tabular}{|lr|r|r|}
\hline Tube: Mouse T8 - sample\#3 & & & \\
Population & \#Events & \%Parent & \% Total \\
All Events & 122,870 & \#\#\# & 100.0 \\
$\square$ Cells & 114.596 & 93.3 & 93.3 \\
$\square$ WBC & 37.984 & 33.1 & 30.9 \\
$\square$ CD8(+) Tcells & 816 & 2.1 & 0.7 \\
$\square$ CD4(+) T cells & 2.570 & 6.8 & 2.1 \\
$\square$ CD4(+)FoxP3(+) & 404 & 15.7 & 0.3 \\
\hline
\end{tabular}




\section{Supplementary Figure 3}

081016-Mouse T8 - sample\#3

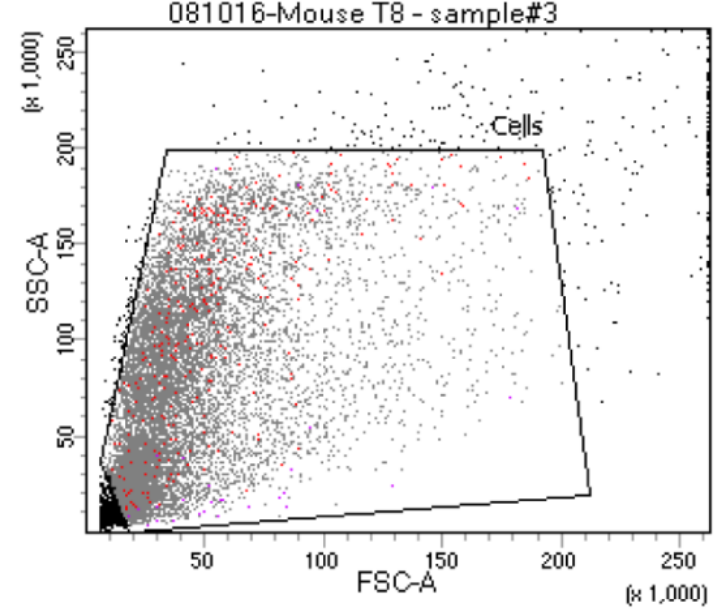

081016-Mouse T8 - sample\#3

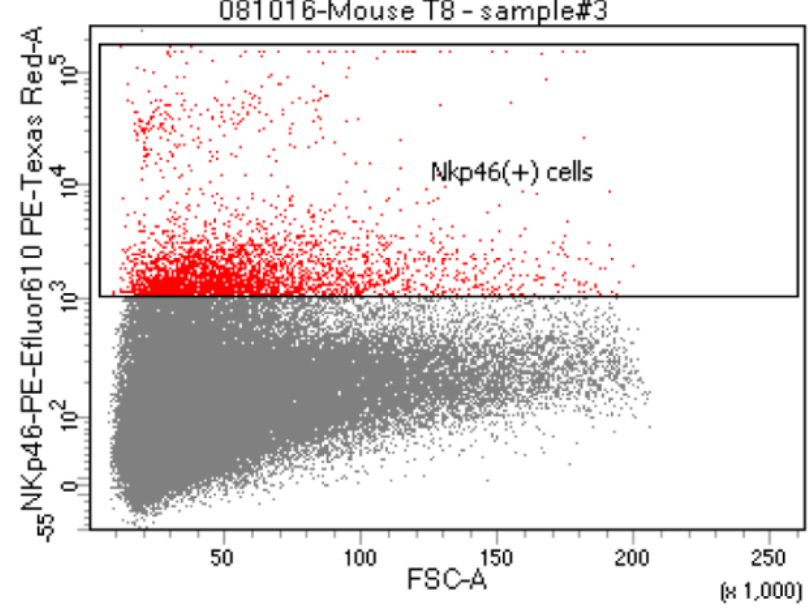

\begin{tabular}{lr|r|r|}
\hline Tube: Mouse T8 - sample\#3 & & & \\
& & & \\
Population & \#Events & $\%$ Parent & $\%$ Total \\
All Events & 122,870 & \#\#\# & 100.0 \\
$\square$ Cells & 108,096 & 88.0 & 88.0 \\
\hline$\square$ Nkp46(+) cells & 3,355 & 3.1 & 2.7 \\
\hline
\end{tabular}




\section{Supplementary Figure 4}
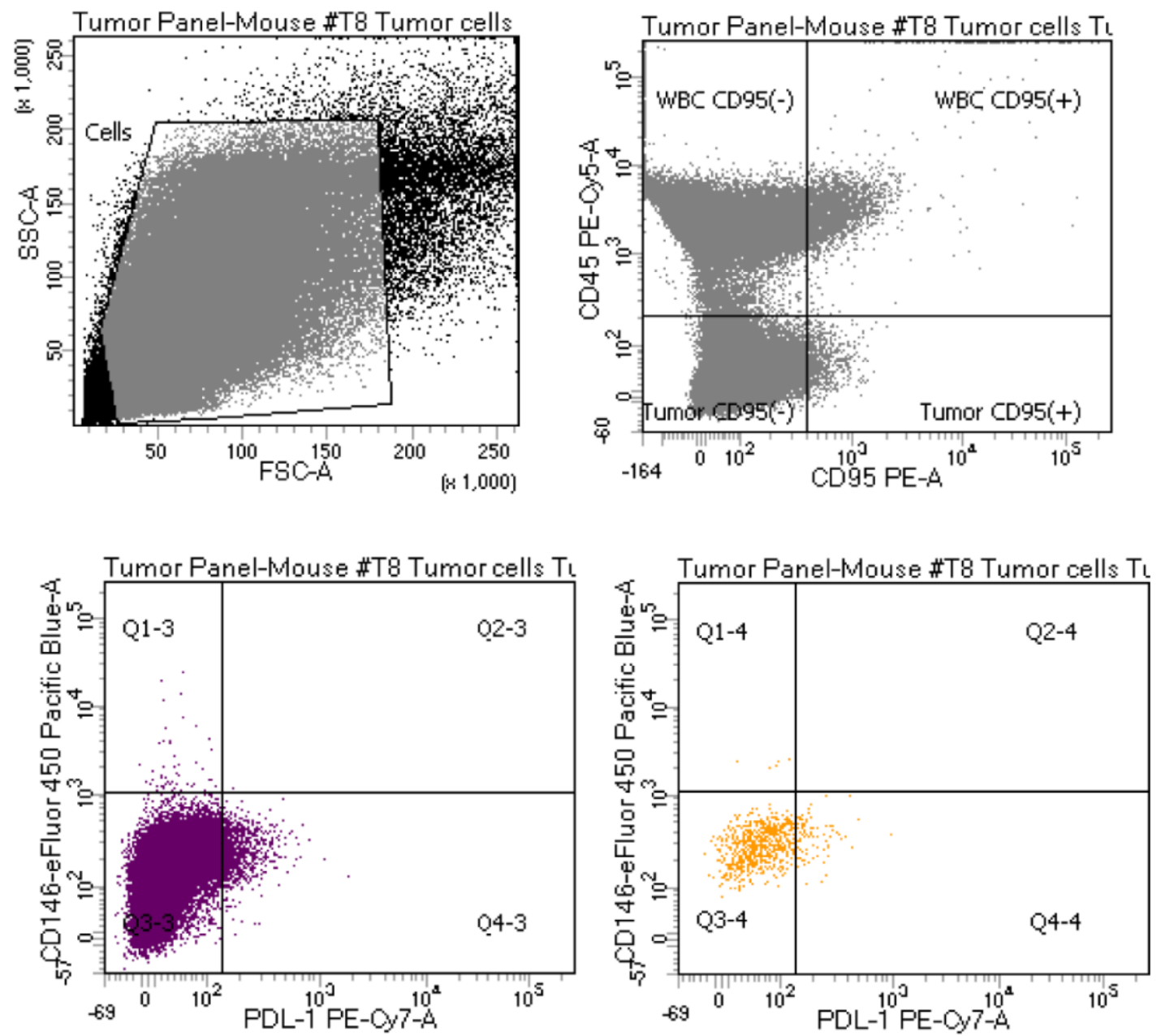

\begin{tabular}{|c|c|c|c|}
\hline Population & \#Events & \%Parent & \%Total \\
\hline All Events & 126.420 & \#\#\# & 100.0 \\
\hline$\square$ Cells & 96,031 & 76.0 & 76.0 \\
\hline$\square$ Tumor CD95(-) & 56.962 & 59.3 & 45.1 \\
\hline$\square$ Q1-3 & 63 & 0.1 & 0.0 \\
\hline Q2-3 & 1 & 0.0 & 0.0 \\
\hline Q3-3 & 56,015 & 98.3 & 44.3 \\
\hline$\square$ Q4-3 & 883 & 1.6 & 0.7 \\
\hline$\square$ Tumor CD95(+) & 732 & 0.8 & 0.6 \\
\hline$\square$ Q1-4 & 5 & 0.7 & 0.0 \\
\hline$\square$ Q2-4 & 0 & 0.0 & 0.0 \\
\hline$\square$ Q3-4 & 647 & 88.4 & 0.5 \\
\hline$\square$ Q4-4 & 80 & 10.9 & 0.1 \\
\hline
\end{tabular}




\section{Supplementary Figure 5}

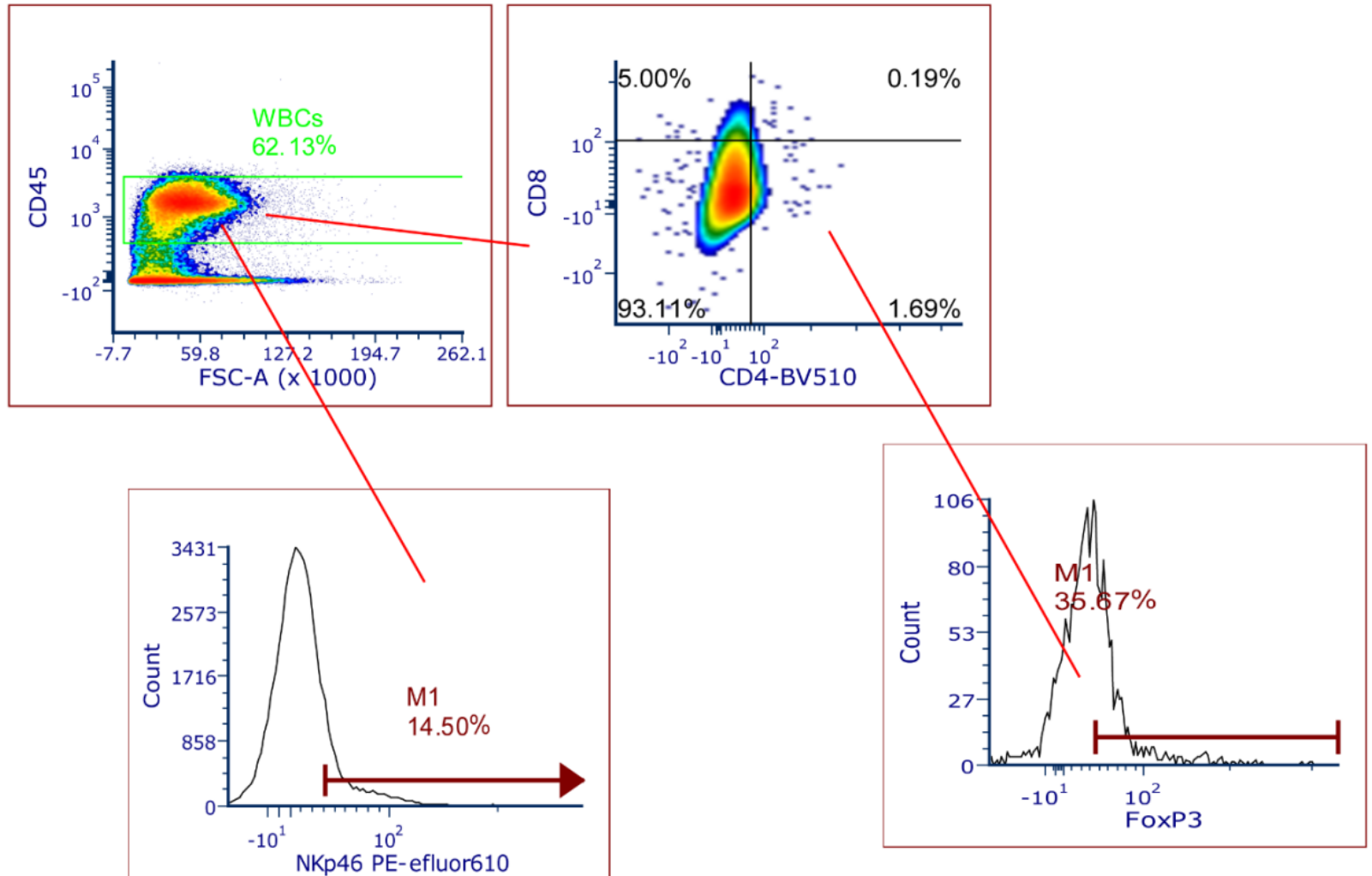




\section{Supplementary Figure 6}

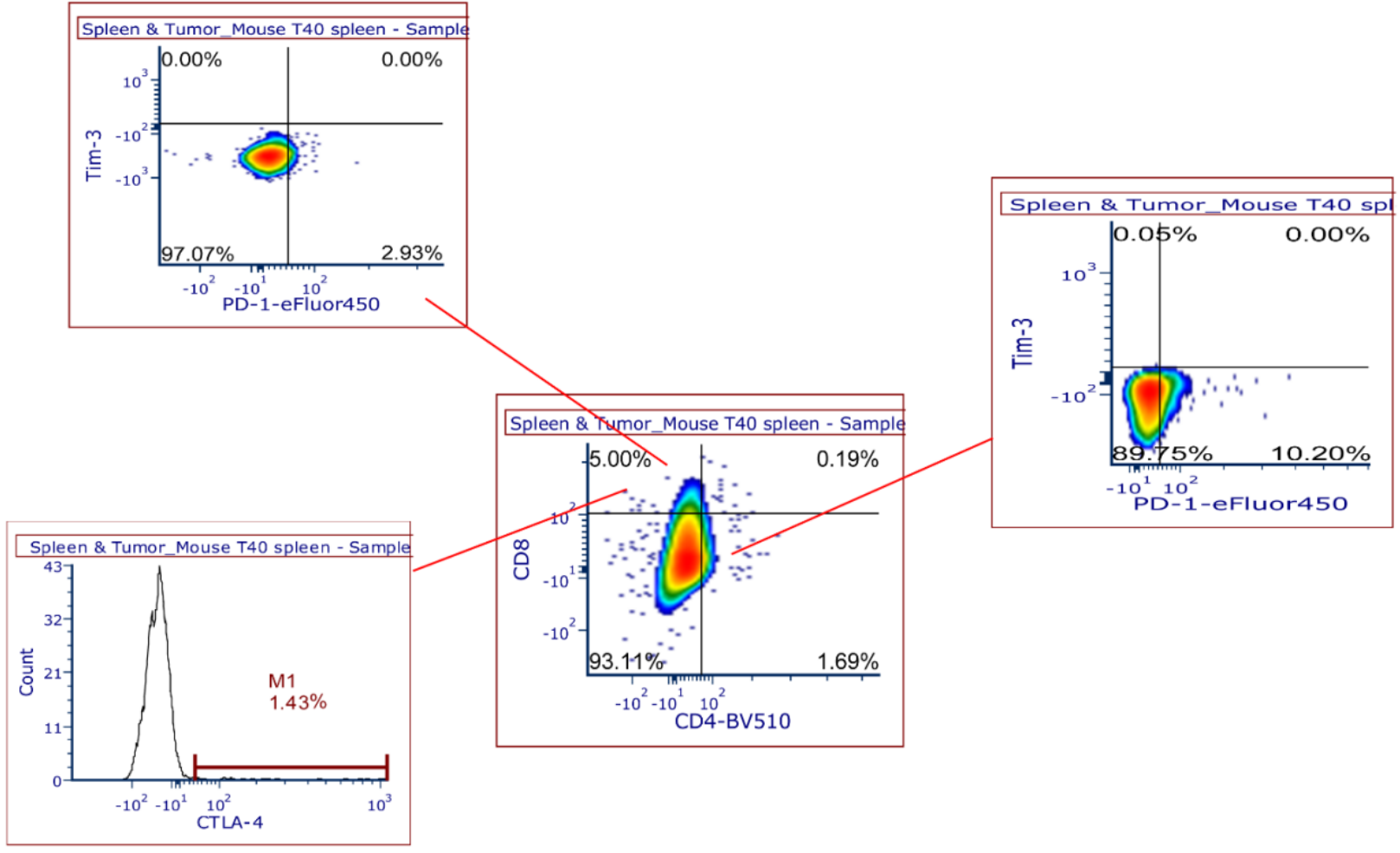




\section{Supplementary Figure 7}

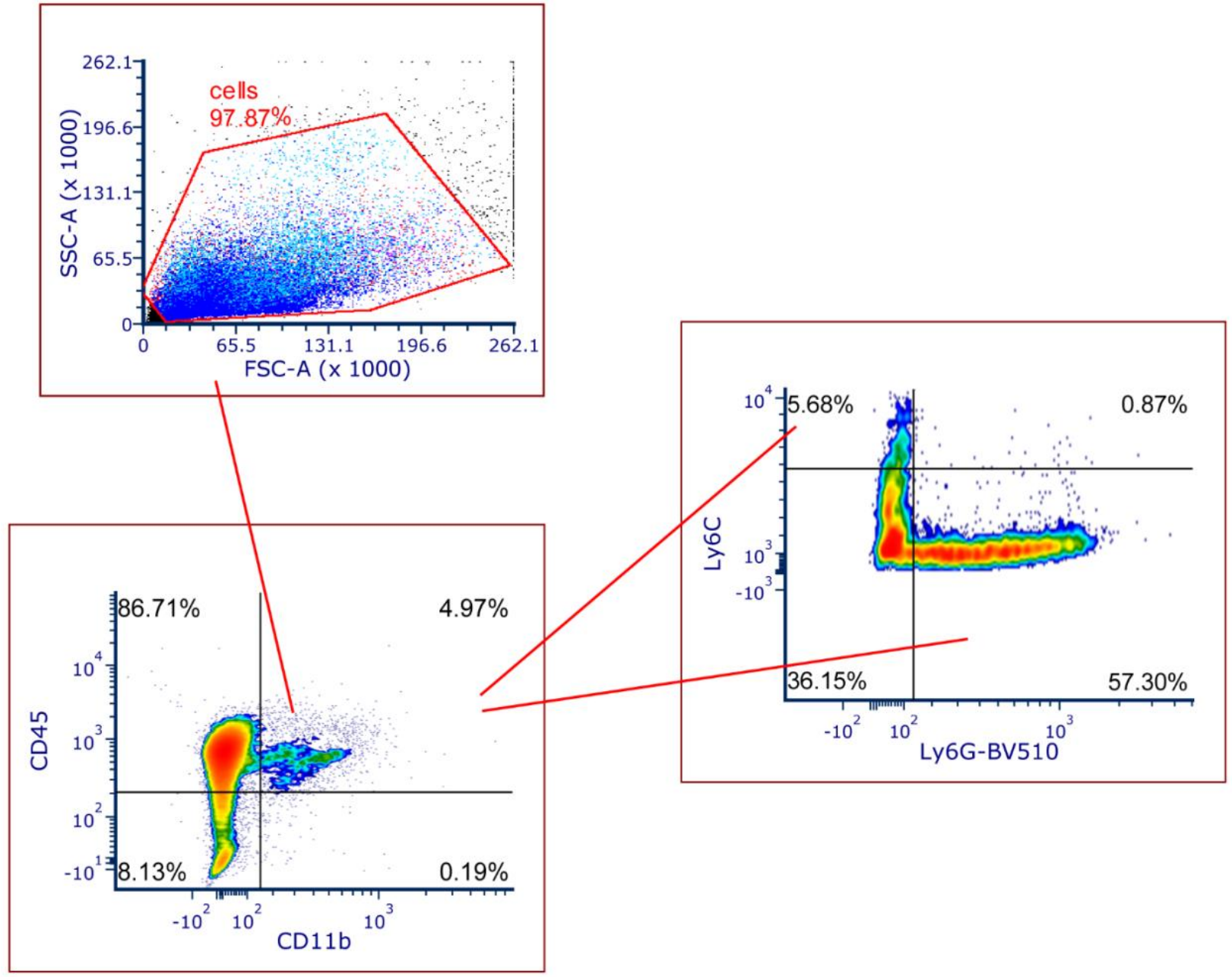




\section{Supplementary Figure 8}

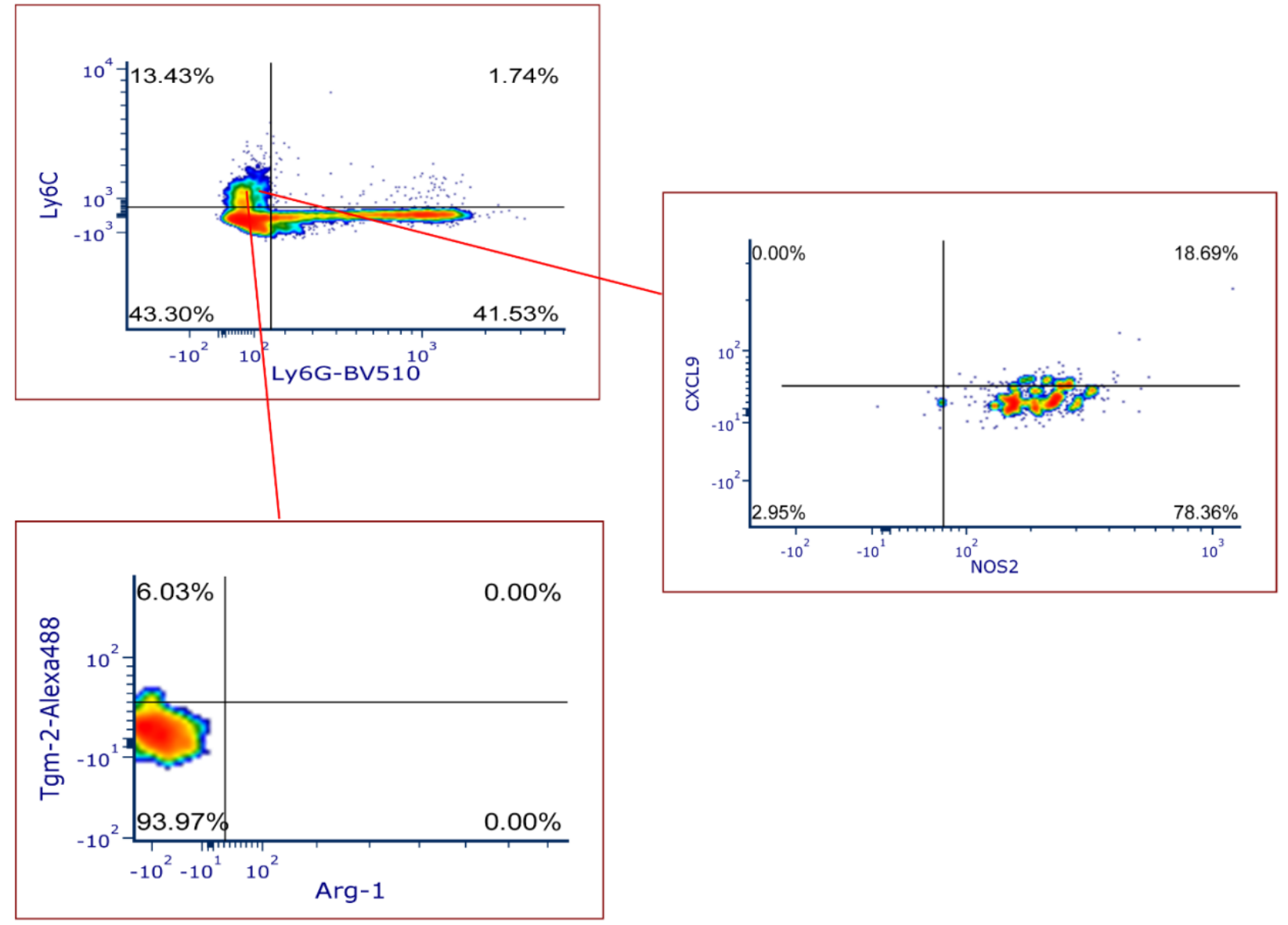


Table 1. Specific cell populations discussed and their corresponding defining markers.

\begin{tabular}{|l|l|}
\hline Cell Population & Defining Markers \\
\hline Tumor cells and stroma & CD45-PD-L1 \pm \\
\hline Cytotoxic T-lymphocyte & CD45+CD8+ \\
\hline T helper lymphocyte & CD45+CD4+ \\
\hline Myeloid lineage cell & CD45+CD11b+ \\
\hline Granulocyte & CD45+CD11b+Ly6G+Ly6C- \\
\hline Monocyte/macrophage & CD45+CD11b+Ly6C+Ly6G- \\
\hline Exhausted cytotoxic T-lymphocyte & CD45+CD8+PD- $1_{ \pm}$Tim-3 $3_{ \pm}$ \\
\hline Exhausted Thelper lymphocyte & CD45+CD4+PD-1 $1_{ \pm}$Tim-3 $3_{ \pm}$ \\
\hline CTLA-4-expressing cytotoxic T-lymphocyte & CD45+CD8+CTLA-4+ \\
\hline M1-like macrophage & Cxcl9 ${ }_{ \pm}$Nos2 $2_{ \pm}$ \\
\hline M2-like macrophage & Arg1 $1_{ \pm}$Tgm2 ${ }_{ \pm}$ \\
\hline
\end{tabular}

PD-L1 = programmed death-ligand 1; PD-1 = programmed cell death protein 1; Tim-3 = T cell immunoglobulin and mucin-domain containing-3; CTLA-4+ $=$ cytotoxic T-lymphocyte-associated protein 4; $\mathrm{Cxcl} 9=$ chemokine $(\mathrm{C}-\mathrm{X}-\mathrm{C})$ motif ligand 9; Nos2 = nitric oxide synthase $2 ; \operatorname{Arg} 1=$ arginase $1 ; \operatorname{Tgm} 2=$ transglutaminase 2 
Table 2. Population distribution comparisons of WBCs isolated from primary tumors of BALB/c mice inoculated with TF $(n=15)$ or WT $(n=11)$ K7M2 cells. The statistics for each group are summarized accordingly with significance being assigned to those populations presenting $\mathrm{p}$-values $<0.0083$ from a two-tailed Student's T-test with Bonferroni correction and $95 \%$ confidence as denoted by an asterisk.

\begin{tabular}{|l|l|l|l|}
\hline Population & WT \% Expression & TF \% Expression & p \\
\hline NK cells & $19.76 \pm 4.76 \%$ & $20.94 \pm 12.67 \%$ & 0.77 \\
\hline CTLs & $5.45 \pm 3.09 \%$ & $6.07 \pm 7.55 \%$ & 0.8 \\
\hline Ths & $6.96 \pm 1.69 \%$ & $5.42 \pm 3.39 \%$ & 0.18 \\
\hline Tregs & $16.95 \pm 10.44 \%$ & $24.02 \pm 15.37 \%$ & 0.2 \\
\hline MLCs & $36.5 \pm 23.12 \%$ & $34.74 \pm 17.53 \%$ & 0.95 \\
\hline Granulocytes & $35.8 \pm 18.32 \%$ & $36.59 \pm 16.38 \%$ & 0.91 \\
\hline
\end{tabular}

$\mathrm{WBC}=$ white blood cell; $\mathrm{TF}=$ transfected; $\mathrm{WT}=$ wild type; $\mathrm{NK}=$ natural killer; $\mathrm{CTL}=$ cytotoxic T-lymphocyte; $\mathrm{Th}=\mathrm{T}$ helper cell; Treg $=\mathrm{T}$ regulatory cell $; \mathrm{MLC}=$ myeloid lineage cell 
Table 3. Non-WBC cell PD-L1 expression of primary tumors isolated from BALB/c mice inoculated with TF $(n=15)$ or WT $(n=11)$ K7M2 cells. Statistical significance was assessed using a two-tailed Student's T-test and $95 \%$ confidence.

\begin{tabular}{|l|l|l|l|}
\hline Marker & WT \% Expression & TF \% Expression & p \\
\hline PD-L1 & $1.96 \pm 0.77 \%$ & $1.91 \pm 1.28 \%$ & 0.9 \\
\hline
\end{tabular}

$\mathrm{WBC}=$ white blood cell; $\mathrm{PD}-\mathrm{L} 1=$ programmed death-ligand $1 ; \mathrm{TF}=$ transfected; $\mathrm{WT}=$ wild type 
Table 4. Population distribution comparison of WBCs isolated from the spleens of BALB/c mice inoculated with TF $(n=9)$ or WT $(n=10)$ K7M2 cells. The statistics for each group are summarized accordingly with significance being assigned to those populations presenting $\mathrm{p}$-values $<0.0083$ from a two-tailed Student's T-test with Bonferroni correction and $95 \%$ confidence as denoted by an asterisk.

\begin{tabular}{|l|l|l|l|}
\hline Population & WT \% Expression & TF \% Expression & p \\
\hline NK cells & $5.35 \pm 4.58 \%$ & $7.55 \pm 5 \%$ & 0.33 \\
\hline CTLs & $7.23 \pm 2.41 \%$ & $5.03 \pm 2.64 \%$ & 0.08 \\
\hline Ths & $18 \pm 13.61 \%$ & $11.71 \pm 12.95 \%$ & 0.32 \\
\hline Tregs & $12.68 \pm 6.01 \%$ & $16.19 \pm 8.43 \%$ & 0.31 \\
\hline MLCs & $5.2 \pm 11.41 \%$ & $9.76 \pm 10.76 \%$ & 0.38 \\
\hline Granulocytes & $15.27 \pm 21.82 \%$ & $52.33 \pm 19.02 \%$ & $0.0011 *$ \\
\hline
\end{tabular}

$\mathrm{WBC}=$ white blood cell; $\mathrm{TF}=$ transfected; $\mathrm{WT}=$ wild type; $\mathrm{NK}=$ natural killer; $\mathrm{CTL}=$ cytotoxic

T-lymphocyte; $\mathrm{Th}=\mathrm{T}$ helper cell; Treg $=\mathrm{T}$ regulatory cell; $\mathrm{MLC}=$ myeloid lineage cell 
Table 5. Population distribution comparison of LLCs isolated from the spleens of mice from TF tumor-bearing $(\mathrm{n}=5)$ and $\mathrm{SH}(\mathrm{n}=4)$ groups. The statistics for each group are summarized accordingly with significance being assigned to those populations presenting p-values $<0.0083$ from a two-tailed Student's T-test with Bonferroni correction and 95\% confidence as denoted by an asterisk.

\begin{tabular}{|l|l|l|l|}
\hline Population & TF \% Expression/TCES & SH \% Expression/TCES & p \\
\hline NK cells & $10.78 \pm 4.5 \%$ & $1.85 \pm 0.4 \%$ & $0.006^{*}$ \\
\hline CTLs & $2.73 \pm 1.22 \%$ & $3.72 \pm 0.86 \%$ & 0.21 \\
\hline Ths & $6.45 \pm 3.08 \%$ & $7.72 \pm 1.1 \%$ & 0.46 \\
\hline Tregs & $21.09 \pm 5.12 \%$ & $7.25 \pm 0.23 \%$ & $0.0011^{*}$ \\
\hline CTLA-4+CTLs & $18.35 \pm 10.12 \%$ & $1.72 \pm 0.42 \%$ & 0.014 \\
\hline TCES & $18.23 \pm 4.58$ & $0.16 \pm 0.054$ & $0.0001^{*}$ \\
\hline
\end{tabular}

LLC = lymphoid lineage cell; $\mathrm{TF}=$ transfected; $\mathrm{NK}=$ natural killer; $\mathrm{CTL}=$ cytotoxic $\mathrm{T}$ lymphocyte; $\mathrm{Th}=\mathrm{T}$ helper cell; $\mathrm{Treg}=\mathrm{T}$ regulatory cell; CTLA- $4+\mathrm{CTL}=$ cytotoxic $\mathrm{T}-$ lymphocyte-associated protein 4 positive cytotoxic $\mathrm{T}$-lymphocyte; TCES $=\mathrm{T}$ cell exhaustion status 
Table 6. Population distribution comparison of MLCs isolated from the spleens of mice from TF tumor-bearing $(\mathrm{n}=5)$ and $\mathrm{SH}(\mathrm{n}=4)$ groups. The statistics for each group are summarized accordingly with significance being assigned to those populations presenting $\mathrm{p}$-values $<0.00714$ from a two-tailed Student's T-test with Bonferroni correction and 95\% confidence as denoted by an asterisk.

\begin{tabular}{|l|l|l|l|}
\hline Population & TF \% Expression & SH \% Expression & p \\
\hline MLCs & $5.27 \pm 1.11 \%$ & $1.39 \pm 0.4 \%$ & $0.00031^{*}$ \\
\hline Monocytes/Macrophages & $4.92 \pm 1.29 \%$ & $7.49 \pm 1.7 \%$ & 0.036 \\
\hline Granulocytes & $57.49 \pm 9.62 \%$ & $74.69 \pm 4.55$ & 0.014 \\
\hline Tgm2+ macrophages & $18.58 \pm 5.69 \%$ & $4.22 \pm 2.66 \%$ & $0.0025^{*}$ \\
\hline Arg1+macrophages & $0.3 \pm 0.56 \%$ & $0 \pm 0 \%$ & 0.32 \\
\hline Cxc19+ macrophages & $18.28 \pm 5.38 \%$ & $1.44 \pm 1.23 \%$ & $0.00052^{*}$ \\
\hline Nos2+ macrophages & $97.99 \pm 1.62 \%$ & $16.56 \pm 10.19 \%$ & $4.2 \mathrm{E}-07^{*}$ \\
\hline
\end{tabular}

MLC = myeloid lineage cell; $\mathrm{TF}=$ transfected; Tgm2 = transglutaminase 2; Arg1 = arginase 1; Cxc19 = chemokine $(\mathrm{C}-\mathrm{X}-\mathrm{C})$ motif ligand 9; Nos2 = nitric oxide synthase 2 
Table 7. Treatment with $\alpha$-PD-L1 significantly reduces TCES but does not affect parent populations of LLCs in the spleens of TF tumor-bearing mice. Spleens were harvested at approximately 11 weeks post-inoculation and the tissue was subjected to Panel 3 (mod) for immune cell population analysis from $n=5(-) \alpha-P D-L 1$ and $n=3(+) \alpha-P D-L 1$ tf tumor-bearing mice. For treated mice, $20 \mu \mathrm{g}$ of $\alpha$-PD-L1 was given in PBS via intraperitoneal injection twice a week starting when tumors were first palpable. The statistics for each group are summarized accordingly with signifiance being assigned to those populations presenting $\mathrm{p}$-values $<0.01$ from a two-tailed Student's T-test with Bonferroni correction and 95\% confidence as denoted by an asterisk.

\begin{tabular}{|l|l|l|l|}
\hline Population & TF $(-) \boldsymbol{\alpha}$-PD-L1 \% Expression/TCES & TF $(+) \boldsymbol{\alpha}$-PD-L1 \% Expression/TCES & p \\
\hline CTLs & $2.73 \pm 1.22 \%$ & $2.85 \pm 1.13 \%$ & 0.9 \\
\hline Ths & $6.45 \pm 3.08 \%$ & $5.14 \pm 3.74 \%$ & 0.61 \\
\hline Tregs & $21.09 \pm 5.12 \%$ & $26.63 \pm 7.02 \%$ & 0.24 \\
\hline CTLA-4+ CTLs & $18.35 \pm 10.12 \%$ & $44.95 \pm 37.19 \%$ & 0.16 \\
\hline TCES & $18.23 \pm 4.58$ & $0.79 \pm 0.71$ & $0.00072^{*}$ \\
\hline
\end{tabular}

PD-L1 = Programmed death-ligand $1 ; \mathrm{TF}=$ transfected; LLC: lymphoid lineage cell; $\mathrm{CTL}=$ cytotoxic T-lymphocyte; $\mathrm{Th}=\mathrm{T}$ helper cell; $\mathrm{Treg}=\mathrm{T}$ regulatory cell; CTLA- $4+\mathrm{CTL}=$ cytotoxic T-lymphocyte-associated protein 4 positive cytotoxic T-lymphocyte; TCES $=\mathrm{T}$ cell exhaustion status 
Table 8. Treatment with $\alpha$-PD-L1 dramatically affects macrophage maturation and M1/M2 polarization in the spleens of TF tumor-bearing mice. Spleens were harvested at approximately 11 weeks post-inoculation and the tissue was subjected to Panel 4 for immune cell population analysis from $\mathrm{n}=5(-) \alpha$-PD-L1 and $\mathrm{n}=3(+) \alpha$-PD-L1 tf tumor-bearing mice. For treated mice, $20 \mathrm{ug}$ of $\alpha$-PD-L1 was given in PBS via intraperitoneal injection twice a week starting when tumors were first palpable. The statistics for each group are summarized accordingly with significance being assigned to those populations presenting p-values $<0.00714$ from a two-tailed Student's T-test with Bonferroni correction and 95\% confidence as denoted by an asterisk.

\begin{tabular}{|l|l|l|l|}
\hline Population & TF (-) a-PD-L1 \% Expression & TF (+) $\boldsymbol{\alpha}$-PD-L1 \% Expression & p \\
\hline MLCs & $5.27 \pm 1.11 \%$ & $5.73 \pm 1.68 \%$ & 0.65 \\
\hline Monocytes/Macrophages & $4.92 \pm 1.29 \%$ & $12.8 \pm 2.92 \%$ & $0.0016^{*}$ \\
\hline Granulocytes & $57.49 \pm 9.62 \%$ & $51.37 \pm 9.11 \%$ & 0.41 \\
\hline Tgm2+ macrophages & $18.58 \pm 5.69 \%$ & $2.1 \pm 2.17 \%$ & $0.0034^{*}$ \\
\hline Arg1+ macrophages & $0.3 \pm 0.56 \%$ & $0.23 \pm 0.17 \%$ & 0.84 \\
\hline Cxc19+ macrophages & $18.28 \pm 5.38 \%$ & $1.52 \pm 1.24 \%$ & $0.002^{*}$ \\
\hline Nos2+ macrophages & $97.99 \pm 1.62 \%$ & $50.89 \pm 18.07 \%$ & $0.00086^{*}$ \\
\hline
\end{tabular}

PD-L1 = Programmed death-ligand $1 ; \mathrm{TF}=$ transfected $; \mathrm{MLC}=$ myeloid lineage cell; $\mathrm{Tgm} 2=$ transglutaminase $2 ; \operatorname{Arg} 1=$ arginase $1 ; \mathrm{Cxcl} 9=$ chemokine $(\mathrm{C}-\mathrm{X}-\mathrm{C})$ motif ligand 9; Nos2 = nitric oxide synthase 2 


\section{Chapter 3}

\section{Systems-Wide Immunophenotyping Defines Distinct Malignancy-Induced Immunological}

Changes in an Immunocompetent K7M2 Orthotopic Murine Model of Osteosarcoma

Rejected from Cancer Immunology Research and Journal of Immunology

Soon to be submitted to: Cancer Immunology, Immunotherapy 


\title{
Systems-Wide Immunophenotyping Defines Distinct Malignancy-Induced Immunological Changes in an Immunocompetent K7M2 Orthotopic Murine Model of Osteosarcoma
}

Justin E. Markel1, Ryan A. Lacinski*, Amanda B. Stewart*, Justin Vaida*, Hillary G. Pratt*, Ryan

M. Reinbeau*, Alan D. Mizener*2, Brock A. Lindsey*3

\begin{abstract}
Osteosarcoma (OS) is the most common primary tumor of bone and largely affects children and young adults. When metastatic, survival rates are extremely poor. While the primary tumor immune microenvironment has been extensively studied, immunotherapies (IMTs) have not yet been effective for patients with systemic disease. Metastatic OS tumors harbor high mutation rates and contain infiltrated leukocytes; however, little is known about the immune response beyond the tumor border. Real-time tracking of the systemic antitumor immune response may allow for the creation of personalized IMT regimens that regress metastatic lesions. Here, we report systems-wide immunological disturbances that occur alongside disease progression in an orthotopic model of metastatic OS. We have identified three populations of tumor-sensitive immune cells, including Natural Killer (NK) cells, T helper ( with systemic NK cell depletion being specific to advancing disease. Additionally, an innovative metric of OS disease burden was generated using co-expression percentages of programmed deathligand 1 (PD-L1) and major histocompatibility complex II (MHC-II) on monocytic-myeloidderived suppressor cells (M-MDSCs) to produce the PD-L1+/MHC-II+ (P[1]/M[II]) ratio. In blood, this ratio showed a significant linear association with tumor burden, increased in magnitude with advancing disease, and provided insight into the immunophenotype of the lung. The ratio was also
\end{abstract}

\footnotetext{
1 Department of Orthopaedics, West Virginia University, Morgantown, WV, USA

2 University of Pittsburgh, Pittsburgh, PA, USA

${ }_{3}$ Corresponding Author: Brock A. Lindsey, 26508 Morgantown, West Virginia University, United States of America. Phone: 304-293-1054. Fax: 304-293-7042 E-mail: blindsey@hsc.wvu.edu.
} 
increased in the lungs and bone marrow (BM) of mice with advanced disease compared to sham controls. Moreover, all malignancy-induced immunological disturbances appeared to normalize following disease clearance. Thus, we present the first ever placement of systemic OS-induced immune disturbances within the context of disease progression which may ultimately lead to successful IMT for metastatic OS.

Keywords: osteosarcoma, immunophenotype, myeloid-derived suppressor cell, PD-L1, MHC-II

\section{Declarations}

Funding: This work was supported by the WVU Flow Cytometry \& Single Cell Core under TME CoBRE grant: GM121322 and Fortessa S10 grants: OD016165 and WV-INBRE 103434.

Conflicts of Interest/Competing Interests: BAL declares paid consultancy for Highmark and Zimmer Biomet and research support from Stryker and Globus Life Technologies, and he is a paid speaker for Hangar Prosthetics. The other authors report no conflicts of interest.

Ethics approval: All animal experiments were approved by the Institutional Animal Care and Use Committee (IACUC).

Consent to Participate: Not applicable.

Consent for publication: Not applicable.

Availability of data and material: All data is available upon request by contacting Brock A. Lindsey; blindsey@hsc.wvu.edu.

Code availability: Not applicable.

Authors' contributions: All authors contributed to the study conception and design. Material preparation, data collection and analysis were performed by Justin E. Markel, Ryan A. Lacinski, 
Amanda B. Steward, Justin Vaida, Hillary G. Pratt, Ryan M. Reinbeau, Alan D. Mizener, and Brock A. Lindsey. The first draft of the manuscript was written by Justin E. Markel and all authors commented on previous versions of the manuscript. All authors have read and approved the final manuscript.

\section{Introduction}

Despite the presence of nonsynonymous mutations and immune infiltrates, metastatic OS tumors have not responded to immunotherapies (IMTs) [1, 2]. The IMTs tested thus far have been generated largely in response to data from primary tumors; however, primary and metastatic tumor microenvironments are immunophenotypically distinct [3-8]. There is also no meaningful way to assess immune status after the primary tumor is removed, and clinically actionable malignancyinduced immune deficits may be missed.

Unlike the primary tumor immune microenvironment, the systemic immune system can be sampled continuously. Our group has shown previously that the systemic immune system can serve as a surrogate marker of OS disease progression; however, only one tissue (spleen) and time point (end-stage disease) were assessed [8]. Therefore, the objective of this study was to extend our analysis of the systemic anti-OS immune response to include other tissues across multiple time points. As such, herein we describe the utility of systems-wide immunophenotyping for placing malignancy-induced immunomodulation within the context of OS disease progression.

Using an orthotopic tumor model, we have quantified disease burden over time using luciferasetransfected K7M2 (luc-K7M2) tumor cells and In Vivo Imaging Systems (IVIS). At intervals described in Fig. 1, we conducted systems-wide flow cytometry (FC) surveys on blood, spleen, lung, and bone marrow (BM). The survey included pertinent components of the OS immuno- 
genomic landscape that may be targeted therapeutically, including the mononuclear phagocyte system (monocytes, myeloid-derived suppressor cells [MDSCs], and macrophages), T cells, and Natural Killer (NK) cells [9-16].

FC antibody panels (one focusing on lymphoid- [panel L] and one myeloid- [panel M] lineage cell populations) used in this study were optimized using the markers shown in supplementary Table S1 but are focused on CD45+NKp46+ NK cells, CD45+CD4+ T helper $(\mathrm{TH})$ cells, CD45+CD8+ cytotoxic $\quad \mathrm{T} \quad(\mathrm{Tc}) \quad$ cells, $\mathrm{CD} 45+\mathrm{CD} 11 \mathrm{~b}+\mathrm{Ly} 6 \mathrm{C}+\mathrm{Ly} 6 \mathrm{G}-\quad$ monocytic(M)-MDSCs, and CD45+CD11b+Ly6Clo/Ly6G+ polymorphonuclear(PMN)-MDSCs, which showed sensitivity to tumor burden. Hence, herein we describe a distinct combination of OS-induced immune events within the context of disease progression; these events occur consistently across multiple tissue types and may be followed in real-time for clinical benefit.

\section{Materials and Methods}

\section{Mice}

Male and female BALB/c mice aged 4-5 weeks (Stock Number: 000651) were obtained from The Jackson Laboratory and housed individually in ventilated Allentown cages within specific pathogen-free facilities on corncob bedding with 12 hour light/dark cycles, automatic lixit water, and ad libitum food access. All experiments were approved by the Institutional Animal Care and Use Committee (IACUC).

\section{OS BALB/c K7M2 Syngeneic Mouse Model with Time Point Blood and Spleen Collection Inoculation of luc-K7M2 cells or vehicle into the proximal tibia (INOC, week 0) (Fig. 1A)}


K7M2 murine OS tumor cells (ATCC CRL-2836, ATCC) were kindly donated by Dr. Kurt Weiss, MD (University of Pittsburgh Medical Center, Pittsburgh, PA) in April 2014 and transfected with the $l u c 2$ reporter as previously described [17]. Luc-K7M2 cells were Mycoplasma free and their identity confirmed in 2018 via IDEXX BioResearch Case \#6926-2018 (ID 3). For inoculations, luc-K7M2 cells $(1 \times 106)$ suspended in DMEM or vehicle (media alone) were administered intratibially as previously described [17]. An initial cohort of $n=10$ (five male and five female) $\mathrm{BALB} / \mathrm{c}$ mice were randomized into tumor-bearing (TUMOR) and age- and sex-matched sham (SHAM) groups prior to inoculation with tumor cells or media only, respectively. Due to high mortality rate, additional mice were added to achieve an $n$ of 5 for each group per time point. To further correct for fluctuation in tumor growth rates, each TUMOR mouse was matched with a same-sex SHAM that underwent surgical procedures at the same intervals. As a result, the analyses herein were conducted using $n=14$ TUMOR (six male and eight female) and $n=11$ SHAM (four male and seven female) BALB/c mice Primary tumor amputation (AMP, weeks 4-7) (Fig. 1B) TUMOR mice were monitored as previously described [17] in accordance with our institution's tumor burden scoring system (supplementary Table S2). Primary tumor size was measured on both longitudinal (length) and transverse (width) axes. Between four and seven weeks when tumor volumes measured $>1.5 \mathrm{~cm} 3\left(\mathrm{~V}_{\text {tumor }}=\right.$ length $\mathrm{x}$ width $\left.) 2 / 2\right)$, TUMOR mice were imaged via IVIS before undergoing cheek bleed, leg amputation, and spleen biopsy procedures within 24 hours. Cheek bleeds were performed by piercing the facial vein plexus with a 4mm Goldenrod Animal Lancet (Braintree Scientific) to collect $100 \mu \mathrm{L}$ whole blood; the tumor-bearing limb was removed as previously described [17]. To obtain the spleen biopsy, a small $(5 \mathrm{~mm})$ incision was made in the skin and peritoneum over the left upper abdominal quadrant. The specimen $(23.3 \pm 10.4 \mathrm{mg}$ 
tissue) was excised from the anterior extremity of the diaphragmatic surface parallel to the segmental vessels $[18,19]$. Care was taken to ensure that the splenic artery was not severed to preserve tissue viability [20-25]. SHAM mice underwent identical blood draw and surgical procedures as their tumor-bearing mouse counterparts. Despite our efforts, the surgical insult of repeated splenic biopsy caused tissue death and cell loss that disrupted its natural immune response; for clarity, these data are not included in this manuscript but can be made available upon request.

Post-Amputation Time Points 1 (T1) and 2 (T2) (Fig. 1C, D)

Four (T1) and six (T2) weeks following AMP, TUMOR mice were imaged via IVIS. The next day, TUMOR and SHAM mice underwent a second (T1) and third (T2) cheek bleed and spleen biopsy procedure.

Euthanasia (EUTH) (Fig. 1E)

Eight weeks following AMP, TUMOR mice were imaged via IVIS. The next day, both TUMOR and SHAM mice underwent a third and final cheek bleed before euthanasia and tissue (BM, spleen, and lung) harvest. In this manuscript, local recurrence was defined as any extra-pulmonary disease burden but was mainly confined to the peritoneum within the ipsilateral abdominal quadrant from which the affected limb was removed; metastasis was defined by pulmonary nodules via IVIS or gross inspection.

There were two special scenarios that arose defined as early euthanasia (EE) and unplanned death (UD). In the case of EE, animals with advanced (post-AMP) disease that met mandatory euthanasia criteria (tumor burden score > 30 [supplementary Table S2]) must be prematurely euthanized; in this instance, an immediate EUTH time point surgery was scheduled so that all tissues could be 
harvested. Accordingly, all EE blood, spleen, lung, and BM tissue data for said mouse were then moved to the EUTH time point for group average purposes. An UD resulted when a mouse died from tumor burden before its scheduled time point was reached (early or otherwise) and no viable tissue was collected; in this instance, data collected at the previous time point were moved to EUTH for group average purposes. These adjustments were made to account for differences in tumor burden between animals over time and ensure that EUTH data points were assembled from time points when each mouse was at its most advanced stage of disease progression. Of the nonUD mice surviving to a terminal time point, $87.5 \%$ were macrometastatic disease positive (via IVIS imaging and/or gross inspection).

\section{In vivo imaging system (IVIS) imaging of tumor burden}

Animals were IVIS-imaged 24 hours prior to each time point (AMP, T1, T2, and EUTH) using the IVIS Spectrum CT imaging system (PerkinElmer Life Sciences) with Living Image version 4.5 Software to monitor disease burden. To visualize disease burden, mice received $150 \mathrm{mg} / \mathrm{kg}$ intraperitoneal (i.p.) D-luciferin (Caliper Life Sciences); to visualize metastatic disease in the lung (time points T1-EUTH), mice received an additional $15 \mathrm{mg} / \mathrm{kg}$ of intranasal (i.n.) D-luciferin (approximately $30 \mu \mathrm{L}$ ). Images were captured using auto-exposure within the predetermined interval of maximum bioluminescence.

\section{Solid tissue single-cell preparation}

Solid tissues were disassociated using a gentleMACS Dissociator and mouse Tumor Disassociation Kit (Miltenyi Biotec, Auburn, CA) or Lung Dissociation Kit (Miltenyi Biotec), respectively. Cell suspensions from lung tissue were enriched for CD45+ leukocytes using CD45 
MicroBeads and autoMACS Pro Separator per manufacturer's recommendations (Miltenyi Biotec). Red blood cells were lysed with Red Blood Cell (RBC) Lysis Solution (Miltenyi Biotec).

\section{Flow cytometry (FC)}

Single cell suspensions were split and incubated with either the lymphoid (L) or myeloid (M) panel antibody panels (supplementary Table S1), according to the manufacturer's instructions; a minimum of $1 \times 10_{4}$ events were analyzed per sample. Sample gating strategies can be found in supplementary Figs. S1 and S2. The average CD45+ percentage of cells harvested from all tumorbearing and sham animal tissues was statistically equivalent across all time points measured (supplementary Fig. S3).

\section{Statistical Analysis}

For all parameters measured, combined male/female group (TUMOR versus SHAM) averages were calculated for each tissue and time point and the standard deviations (SD) calculated. Power analyses based upon a range of markers that vary significantly in expression between TUMOR and SHAM mice determined that the minimum required sample size to achieve at least $90 \%$ power was $n=8$ per time point (supplementary Table S3). Statistical significance between TUMOR and SHAM group immunophenotypes was determined with Student unpaired two-tailed $t$ test and the strength of associations was evaluated using Pearson's correlation test. Although there was a high drop in $n$ over time due to surgical insult and/or tumor burden, all statistics were performed with $n \geq 5$ mice per group. For comparisons across multiple time points, a preliminary Fisher's least significant differences determined that test variables "TUMOR versus SHAM" and "time" differed significantly $(\mathrm{p}<0.05)$. 


\section{Results}

\section{OS disease progression induces accelerated lymphocyte depletion in blood}

NKp46+ NK, CD4+CD8- TH, and CD8+CD4- Tc cells exhibited distinct downward trajectories in TUMOR mice as disease progressed (Fig. 2A-B and 2D-F, respectively). Across these three cell populations, a pattern of statistical significance was achieved where differences between TUMOR and SHAM mice were greatest at times of highest mean tumor bioluminescence (AMP, Supplementary Fig. S4G) and point of furthest disease progression/highest tumor burden score (EUTH, supplementary Table S2). NK cell percentages in TUMOR mice were also significantly lower than those of SHAM at T1 (Fig. 2B). From a longitudinal perspective, NK cells were the only lymphocyte population that changed significantly in TUMOR but not SHAM mice across AMP and EUTH time points (Fig. 2C), suggesting that NK cell depletion in blood was specific to OS disease progression. The overall change in TH cells across AMP and EUTH time points was significant for both TUMOR and SHAM animals (Fig. 2G), while the change in TC was not significant in either group (Fig. 2H).

The differences in circulating lymphocyte populations between TUMOR and SHAM mice over time became magnified when comparing individual time- and sex-matched pairs. IVIS-reported disease burden of TUMOR mice for three exemplary matched pairs (supplementary Fig. S5A, $\mathrm{S} 5 \mathrm{H}$, and $\mathrm{S} 5 \mathrm{O}$ ) is shown alongside blood $\mathrm{NK}, \mathrm{T}_{\mathrm{H}}$, and $\mathrm{T}_{\mathrm{C}}$ cell trajectories in supplementary Fig. S5B-D, S5I-K, and S5P-R, respectively. Note that Male Tumor 1 mouse (supplementary Fig. S5AG) had local recurrence and pulmonary metastasis on necropsy prior to tissue processing while 
Male Tumor 2 mouse (supplementary Fig. S5H-N) had local recurrence only. Importantly, Female Tumor 1 mouse (supplementary Fig. S5O-U) was disease-free following the removal of its primary lesion at AMP; strikingly, its lymphocyte populations returned roughly to that of the matched-pair sham for subsequent time points.

\section{OS disease progression induces changes in PMN- and M-MDSC populations in blood}

While lymphocyte percentages of TUMOR mice were significantly decreased compared to SHAM at AMP and EUTH time points, immunosuppressive CD11b+Ly6Clo/-Ly6G+ PMN-MDSCs were significantly increased (Fig. 2I, J). Unlike the progressive depletion of circulating lymphocytes, PMN-MDSCs remained consistently elevated across time points with no clear trend (Fig. 2J). Conversely, TUMOR mice M-MDSCs were significantly elevated at AMP only and decreased over time (Fig. 2I, K). Neither PMN- nor M-MDSC populations changed significantly across AMP and EUTH time points in TUMOR or SHAM mice groups (Fig. $2 \mathrm{~L}$ and $2 \mathrm{M}$, respectively). The IVIS-reported disease burden of TUMOR mice for three exemplary matched pairs (supplementary Fig. S5A, S5H, and S5O) is shown alongside blood PMN-MDSC and M-MDSC trajectories in supplementary Fig. S5E and S5F, S5L and S5M, and S5S and S5T, respectively.

\section{The PD-L1/MHC-II ratio on blood M-MDSCs correlates with increasing tumor burden and is a specific marker of OS disease progression}

Expression of Human Leukocyte Antigen DR isotype (HLA-DR), an immunostimulatory molecule and member of the major histocompatibility complex II (MHC-II) family that presents foreign peptides to $\mathrm{TH}$ cells [26], is lost on monocytes in pediatric patients with aggressive sarcomas and postulated to be one of the first steps in the transition of monocytes to M-MDSCs 
[27, 28]. As decreased MHC-II expression is linked to increased PD-L1 expression and immunosuppression $[29,30]$, we investigated their co-expression levels on CD11b+Ly6C+Ly6GM-MDSCs over time. Indeed, as disease progressed, M-MDSCs in TUMOR mice gained expression of PD-L1 and lost expression of MHC-II as shown by the representative histograms in Fig. 3A and B, respectively. We then considered whether increasing tumor burden was associated with increasing \%PD-L1 to \%MHC-II (P[1]/M[II]) ratio on M-MDSCs. Note that luciferinmediated tumor bioluminescence can paradoxically decrease with increasing tumor size in cases where rapid growth prompts central necrosis [31, 32]. Supplementary Fig. S4A shows a representative TUMOR mouse with central necrosis of a local recurrence between time points T2 and EUTH, as indicated by the loss of bioluminescent signal coupled with increasing tumor diameter (Fig. S4B and S4C, respectively). In contrast, the animal in Supplementary Fig. S4D displayed concurrent increases in both tumor bioluminescence and diameter (Fig. S4E and S4F, respectively). To account for this imaging limitation, associations were assessed using data points from mice with corresponding increases in both tumor bioluminescence and radius only. In doing so, it was found that the ratio of \%PD-L1 to \%MHC-II (P[1]/M[II]) expression on blood MMDSCs correlated strongly and positively with tumor bioluminescence over time (Fig. 3C: $\mathrm{R}=$ 0.96; $\mathrm{p}=0.0006$ ), while \%PD-L1 and \%MHC-II alone (Fig. 3D and 3E, respectively) did not.

When the average $\mathrm{P}[1] / \mathrm{M}[\mathrm{II}]$ ratios were plotted over time, they increased in TUMOR mice only and showed greatest divergence from SHAM mice at EUTH (Fig. 3F), the time point at which disease was most advanced. Longitudinally, the M-MDSC P[1]/M[II] ratio was significantly increased across AMP and EUTH time points in TUMOR but not SHAM mice (Fig. 3G), suggesting that its change was specific to disease progression. Following amputation of its tumor- 
bearing limb, Female Tumor 1 mouse remained disease-free throughout the remainder of the study and its ratio returned to that of the matched-pair sham by T2 (supplementary Fig. S5U). Conversely, the ratios of Male Tumor 1 and 2 mice with relapses increased rapidly (supplementary Fig. S5G and S5N, respectively).

\section{Decreased lymphocytic infiltration and abnormal myeloid activation in lung and BM reflect disease status}

At EUTH, lungs and BM were collected (in addition to blood and spleen) and analyzed for diseasespecific changes in immune composition. NKp46+ NK and CD8+ Tc cells were significantly decreased in the lungs and BM of TUMOR versus SHAM mice (Fig. 4A-B and 4C-D), respectively. Conversely, the M-MDSC P[1]/M[II] ratio was increased in the lungs and BM of TUMOR versus SHAM mice (Fig. 4G-H), which was significant in lung. Interestingly, while there were no statistical differences in percent populations of CD4+ TH cells or CD11b+Ly6Clo-Ly6G+ PMN-MDSCs in lung and BM (Fig. 4C-D and 4E-F respectively), CD11b+Ly6C+Ly6G- MMDSCs were increased in lungs and significantly decreased in the BM of TUMOR versus SHAM mice (Fig. 4E-F). For reference, the average immunophenotype of the primary tumors is shown in supplementary Fig. S6.

\section{Blood and BM M-MDSC P[1]/M[II] ratios correlate with lung M-MDSC P[1]/M[II] ratios in TUMOR mice only}

Lung metastasis is the leading cause of death in OS patients [33], and as M-MDSCs are a vital component of the metastatic niche [34], we investigated the utility of the M-MDSC P[1]/M[II] ratio as a diagnostic and/or prognostic tool. In primary tumors, the $\mathrm{P}[1] / \mathrm{M}[\mathrm{II}]$ ratio at AMP did 
not correlate with the M-MDSC $\mathrm{P}[1] / \mathrm{M}[\mathrm{II}]$ ratio in lung (Fig. $5 \mathrm{~A}: \mathrm{R}=0.15 ; \mathrm{p}=0.81$ ). However, there was a strong positive correlation between the M-MDSC P[1]/M[II] ratios of blood at EUTH and lung in TUMOR mice (Fig. 5B: $\mathrm{R}=0.972 ; \mathrm{p}=0.0056$ ). The correlation between $\mathrm{BM}$ and lung ratios, while not significant, showed a similar effect in TUMOR mice (Fig. 5B: $\mathrm{R}=0.849 ; \mathrm{p}=$ 0.069). Importantly, these correlations were nonexistent in the blood (Fig. $5 B: R=0.255 ; p=0.58$ ) and BM (Fig. 5B: $\mathrm{R}=0.267 ; \mathrm{p}=0.56$ ) of SHAM mice, suggesting disease specificity.

\section{Discussion}

Primary tumor data is often used to direct immunotherapeutic approaches despite the fact that they are not representative of systemic immune status [3-8]. Additionally, the primary tumor provides no further information once it is removed. While some studies have investigated OS serum biomarkers, most have focused on highly variable, non-cellular entities [35]. Herein, we described the systemic immune response to disease burden and evaluated the potential of its cellular components as real-time immune biomarkers. In doing so, we have identified several diseaseresponsive lymphoid and myeloid immune cell populations that warrant further investigation.

It has been shown that pediatric OS patients have decreased numbers of circulating lymphocytes versus healthy age-matched controls [36]. Our data support this conclusion, as all three lymphocyte populations studied (NK, $\mathrm{TH}_{\mathrm{H}}$, and Tc cells) were significantly decreased in TUMOR versus SHAM mice blood at AMP (i.e., the time point analogous to when patients would present in clinic). Our data suggest that circulating lymphocyte percentages continue to decline at an accelerated rate as disease progresses. From a longitudinal perspective, the depletion of circulating NK cells was 
significant and specific to advancing disease (Fig. 2A-C). Not surprisingly, NK cells were also significantly decreased in the lungs of TUMOR versus SHAM mice (Fig. 4A-B), of which $87.5 \%$ had macrometastatic pulmonary disease. Clinically, it has been shown that increased circulating percentages of NK cells in pediatric OS patients correlate with better outcomes [37]. Our data suggest that NK cell percentages will continue to fluctuate in direct response to tumor burden and therefore may serve as a useful biomarker of disease progression. Average $\mathrm{TH}_{\mathrm{H}}$ and $\mathrm{TC}_{\mathrm{c}}$ cell percentages in the lungs of TUMOR mice, while decreased versus SHAM, were more variable and did not achieve statistical significance; it is possible that suppressive myeloid cells within the lung parenchyma sufficiently inhibit $\mathrm{T}$ lymphocytes responses regardless of their tumor-infiltrating capacity [16].

Very little is known about the myeloid component of systemic immune responses to OS. Here, we have shown that circulating PMN- and M-MDSC percent populations are significantly elevated in TUMOR mice at AMP and variable throughout the remaining course of disease (Fig. 2I-K), with neither population showing disease-specific outcomes (Fig. 2L-M). Interestingly, PMN-MDSC percentages were significantly increased in blood (Fig. 2I-J) but not lungs of TUMOR versus SHAM mice (Fig. 4E-F). Conversely, M-MDSC percentages were decreased in BM and increased in lungs of TUMOR versus SHAM mice (Fig. 4E-F); together, these data suggest that M-MDSCs may play a more direct role in supporting OS metastases than PMN-MDSCs.

The $\mathrm{P}[1] / \mathrm{M}[\mathrm{II}]$ ratio, a novel immune biomarker of OS disease progression, not only allowed for magnification of effect size for each observation, but also correlated significantly with increasing tumor burden (Fig. 3E) and progressively increased in magnitude over time (Fig. 3F) in a disease- 
specific manner (Fig. 3G). It is possible that this ratio could be partially describing the monocyteto-M-MDSC dedifferentiation phenomenon seen in pediatric patients with aggressive sarcomas, where monocytes lose MHC-II and gain PD-L1 expression [27-30]. In contrast to SHAM, the $\mathrm{P}[1] / \mathrm{M}[\mathrm{II}]$ ratio of blood and BM from TUMOR mice showed strong correlations with that of lung (Fig. 5B), suggesting that 1 ) increases in ratio magnitude are specific to disease process, and 2) systemic tissue sampling (i.e., via a blood draw) may provide insight into the immunophenotype of the lung. The ability to infer immunological information about lung from blood is clinically relevant, as it would otherwise require biopsy. Primary tumors had no predictive value regarding the $\mathrm{P}[1] / \mathrm{M}[\mathrm{II}]$ status in the lung at EUTH. Also, the $12.5 \%$ of non-UD mice with local recurrences but without confirmed macrometastases had similar changes in their systemic immunophenotypes, suggesting that the presence of tumor produces similar immunologic disturbances regardless of location.

In conclusion, primary tumors are not always good indicators of systemic disease burden as they provide only a snapshot of disease and exclude the systemic impact of the progressing malignancy. Our data suggest that there is clinically useful information that can be extrapolated from blood, which unlike primary and metastatic tumors, can be sampled continually and non-invasively. Therefore, we suggest that, aside from its diagnostic and prognostic application potential, systemswide immunophenotyping may greatly increase the success rate of all IMTs currently in use and allow for the creation of individualized immunotherapeutic regimens tailored to the specific needs of each patient. Also, these data suggest that IMTs specifically targeting monocyte-to-M-MDSC dedifferentiation may be effective against metastatic OS. 


\section{References}

1. Wang, D., Niu, X., Wang, Z., Song, C. L., Huang, Z., Chen, K. N., ... \& Wang, Y. (2019). Multiregion sequencing reveals the genetic heterogeneity and evolutionary history of osteosarcoma and matched pulmonary metastases. Cancer research, 79(1), 7-20.

2. Thanindratarn, P., Dean, D. C., Nelson, S. D., Hornicek, F. J., \& Duan, Z. (2019). Advances in immune checkpoint inhibitors for bone sarcoma therapy. Journal of bone oncology, 100221.

3. Buddingh, E. P., Kuijjer, M. L., Duim, R. A., Bürger, H., Agelopoulos, K., Myklebost, O., ... \& Cleton-Jansen, A. M. (2011). Tumor-infiltrating macrophages are associated with metastasis suppression in high-grade osteosarcoma: a rationale for treatment with macrophage activating agents. Clinical Cancer Research, 17(8), 2110-2119.

4. Gomez-Brouchet, A., Illac, C., Gilhodes, J., Bouvier, C., Aubert, S., Guinebretière, J. M., ... \& Filleron, T. (2017). CD163-positive tumor-associated macrophages and CD8-positive cytotoxic lymphocytes are powerful diagnostic markers for the therapeutic stratification of osteosarcoma patients: an immunohistochemical analysis of the biopsies fromthe French OS2006 phase 3 trial. Oncoimmunology, 6(9), e1331193.

5. Koirala, P., Roth, M. E., Gill, J., Piperdi, S., Chinai, J. M., Geller, D. S., ... \& Gorlick, R. (2016). Immune infiltration and PD-L1 expression in the tumor microenvironment are prognostic in osteosarcoma. Scientific reports, 6(1), 1-10.

6. Sundara, Y. T., Kostine, M., Cleven, A. H., Bovée, J. V., Schilham, M. W., \& CletonJansen, A. M. (2017). Increased PD-L1 and T-cell infiltration in the presence of HLA class I expression in metastatic high-grade osteosarcoma: a rationale for T-cell-based immunotherapy. Cancer Immunology, Immunotherapy, 66(1), 119-128.

7. Xiao, H., Luo, G., Son, H., Zhou, Y., \& Zheng, W. (2014). Upregulation of peripheral CD4+ CXCR5+ T cells in osteosarcoma. Tumor Biology, 35(6), 5273-5279.

8. Markel, J. E., Noore, J., Emery, E. J., Bobnar, H. J., Kleinerman, E. S., \& Lindsey, B. A. (2018). Using the Spleen as an In Vivo Systemic Immune Barometer Alongside Osteosarcoma Disease Progression and Immunotherapy with $\alpha$-PD-L1. Sarcoma, 2018.

8. $\quad$ Spitzer, M. H., Carmi, Y., Reticker-Flynn, N. E., Kwek, S. S., Madhireddy, D., Martins, M. M., ... \& Fong, L. (2017). Systemic immunity is required for effective cancer immunotherapy. Cell, 168(3), 487-502.

9. Uehara, T., Eikawa, S., Nishida, M., Kunisada, Y., Yoshida, A., Fujiwara, T., ... \& Udono, H. (2019). Metformin induces CD11b+-cell-mediated growth inhibition of an osteosarcoma: implications for metabolic reprogramming of myeloid cells and anti-tumor effects. International immunology, 31(4), 187-198.

10. Lussier, D. M., Johnson, J. L., Hingorani, P., \& Blattman, J. N. (2015). Combination immunotherapy with $\alpha$-CTLA-4 and $\alpha$-PD-L1 antibody blockade prevents immune escape and leads to complete control of metastatic osteosarcoma. Journal for immunotherapy of cancer, 3(1), 21.

11. Guma, S. R., Lee, D. A., Yu, L., Gordon, N., Hughes, D., Stewart, J., ... \& Kleinerman, E. S. (2014). Natural killer cell therapy and aerosol interleukin-2 for the treatment of osteosarcoma lung metastasis. Pediatric blood \& cancer, 61(4), 618-626.

12. Dumars, C., Ngyuen, J. M., Gaultier, A., Lanel, R., Corradini, N., Gouin, F., ... \& Heymann, M. F. (2016). Dysregulation of macrophage polarization is associated with the metastatic process in osteosarcoma. Oncotarget, 7(48), 78343. 
13. Jimmy, R., Stern, C., Lisy, K., \& White, S. (2017). Effectiveness of mifamurtide in addition to standard chemotherapy for high-grade osteosarcoma: a systematic review. JBI database of systematic reviews and implementation reports, 15(8), 2113-2152.

14. Liu, T., Fang, X. C., Ding, Z., Sun, Z. G., Sun, L. M., \& Wang, Y. L. (2015). Preoperative lymphocyte-to-monocyte ratio as a predictor of overall survival in patients suffering from osteosarcoma. FEBS open bio, 5(1), 682-687.

15. Li, X., Chen, Y., Liu, X., Zhang, J., He, X., Teng, G., \& Yu, D. (2017). Tim3/Gal9 interactions between $\mathrm{T}$ cells and monocytes result in an immunosuppressive feedback loop that inhibits Th1 responses in osteosarcoma patients. International immunopharmacology, 44, 153159.

16. Wu, C. C., Beird, H. C., Livingston, J. A., Advani, S., Mitra, A., Cao, S., ... \& Leung, C. H. (2020). Immuno-genomic landscape of osteosarcoma. Nature communications, 11(1), 1-11. 17. Grisez, B. T., Ray, J. J., Bostian, P. A., Markel, J. E., \& Lindsey, B. A. (2018). Highly metastatic K7M2 cell line: a novel murine model capable of in vivo imaging via luciferase vector transfection. Journal of Orthopaedic Research®, 36(8), 2296-2304.

18. Liu, D. L., Xia, S., Xu, W., Ye, Q., Gao, Y., \& Qian, J. (1996). Anatomy of vasculature of 850 spleen specimens and its application in partial splenectomy. surgery, 119(1), 27-33.

19. Upadhyaya, P., Nayak, N. C., \& Moitra, S. (1971). Experimental study of splenic trauma in monkeys. Journal of pediatric surgery, 6(6), 767-773.

20. Malangoni, M. A., Dawes, L. G., Droege, E. A., Rao, S. A., Collier, B. D., \& Almagro, U. A. (1985). Splenic phagocytic function after partial splenectomy and splenic autotransplantation. Archives of Surgery, 120(3), 275-278.

21. Okinaga, K., Giebink, G. S., Rich, R. H., Baesl, T. J., Krishnanaik, D., \& Leonard, A. S. (1981). The effect of partial splenectomy on experimental pneumonoccal bacteremia in an animal model. Journal of pediatric surgery, 16(5), 717-724.

22. Jahn, S., Bauer, B., Schwab, J., Kirchmair, F., Neuhaus, K., Kiessig, S. T., .. \& Specht, U. (1993). Immune restoration in children after partial splenectomy. Immunobiology, 188(4-5), 370-378.

23. de Buys Roessingh, A. S., De Lagausie, P., Rohrlich, P., Berrebi, D., \& Aigrain, Y. (2002). Follow-up of partial splenectomy in children with hereditary spherocytosis. Journal of pediatric surgery, 37(10), 1459-1463.

24. Tracy, E. T., Haas, K. M., Gentry, T., Danko, M., Roberts, J. L., Kurtzberg, J., \& Rice, H. E. (2011). Partial splenectomy but not total splenectomy preserves immunoglobulin M memory B cells in mice. Journal of pediatric surgery, 46(9), 1706-1710.

25. Diesen, D. L., Zimmerman, S. A., Thornburg, C. D., Ware, R. E., Skinner, M., Oldham, K. T., \& Rice, H. E. (2008). Partial splenectomy for children with congenital hemolytic anemia and massive splenomegaly. Journal of pediatric surgery, 43(3), 466-472.

26. Neefjes, J., Jongsma, M. L., Paul, P., \& Bakke, O. (2011). Towards a systems understanding of MHC class I and MHC class II antigen presentation. Nature Reviews Immunology, 11(12), 823.

27. Hingorani, P., Maas, M. L., Gustafson, M. P., Dickman, P., Adams, R. H., Watanabe, M., ... \& Dietz, A. B. (2015). Increased CTLA-4+ T cells and an increased ratio of monocytes with loss of class II (CD14+ HLA-DR lo/neg) found in aggressive pediatric sarcoma patients. Journal for immunotherapy of cancer, 3(1), 35 . 
28. Bergenfelz, C., Larsson, A. M., von Stedingk, K., Gruvberger-Saal, S., Aaltonen, K., Jansson, S., ... \& Rydén, L. (2015). Systemic monocytic-MDSCs are generated from monocytes and correlate with disease progression in breast cancer patients. PloS one, 10(5).

29. Huang, H., Zhang, G., Li, G., Ma, H., \& Zhang, X. (2015). Circulating CD14+ HLADR-/low myeloid-derived suppressor cell is an indicator of poor prognosis in patients with ESCC. Tumor Biology, 36(10), 7987-7996.

30. Huang, A., Zhang, B., Wang, B., Zhang, F., Fan, K. X., \& Guo, Y. J. (2013). Increased CD14+ HLA-DR-/low myeloid-derived suppressor cells correlate with extrathoracic metastasis and poor response to chemotherapy in non-small cell lung cancer patients. Cancer Immunology, Immunotherapy, 62(9), 1439-1451.

31. Lee, S. Y., Ju, M. K., Jeon, H. M., Jeong, E. K., Lee, Y. J., Kim, C. H., ... \& Kang, H. S. (2018). Regulation of tumor progression by programmed necrosis. Oxidative medicine and cellular longevity, 2018.

32. Lim, E., Modi, K. D., \& Kim, J. (2009). In vivo bioluminescent imaging of mammary tumors using IVIS spectrum. JoVE (Journal of Visualized Experiments), (26), e1210.

33. Ottaviani, G., \& Jaffe, N. (2009). The epidemiology of osteosarcoma. In Pediatric and adolescent osteosarcoma (pp. 3-13). Springer, Boston, MA.

34. Shi, H., Zhang, J., Han, X., Li, H., Xie, M., Sun, Y., ... \& Zeng, X. (2017). Recruited monocytic myeloid-derived suppressor cells promote the arrest of tumor cells in the premetastatic niche through an IL-1 $\beta$-mediated increase in E-selectin expression. International journal of cancer, 140(6), 1370-1383.

35. Savitskaya, Y. A., Rico-Martínez, G., Linares-González, L. M., Delgado-Cedillo, E. A., Téllez-Gastelum, R., Alfaro-Rodríguez, A. B., ... \& Ibarra-Ponce de León, J. C. (2012). Serum tumor markers in pediatric osteosarcoma: a summary review. Clin. Sarcoma Res, 2(9).

36. Markiewicz, K., Zeman, K., Kozar, A., Gołębiowska-Wawrzyniak, M., \& Woźniak, W. (2012). Evaluation of selected parameters of cellular immunity in children with osteosarcoma at diagnosis. Medycyna wieku rozwojowego, 16(3), 212-221.

37. Luksch, R., Perotti, D., Cefalo, G., Passerini, C. G., Massimino, M., Spreafico, F., ... \& Gambirasio, F. (2003). Immunomodulation in a treatment program including pre-and postoperative interleukin-2 and chemotherapy for childhood osteosarcoma. Tumori Journal, 89(3), 263-268. 


\section{Figure Legends}

Fig. 1 Experimental design for systems-wide immunophenotyping in an orthotopic murine model of metastatic OS. (A) Inoculation: at week 0, male and female BALB/c mice received inoculums in the proximal tibia comprised of either 1 x 106 luc-K7M2 tumor cells in media (TUMOR) or media only (SHAM). (B) Amputation/AMP: primary tumors reached an average volume $>1.5 \mathrm{~cm} 3$ within 4-7 weeks. The day preceding amputation, TUMOR mice were given 150 $\mathrm{mg} / \mathrm{kg}$ D-luciferin intraperitoneal (i.p.) and IVIS-imaged for bioluminescence intensity. The following day, TUMOR and SHAM mice were cheek bled for $100 \mu \mathrm{L}$ whole blood followed by leg amputation and splenic biopsy. Blood, spleen, and tumor samples were stained with lymphoid and myeloid antibody panels for immunophenotyping via flow cytometry (FC). Four (C) and six (D) weeks following limb amputation (time points 1 [T1] and 2 [T2], respectively), TUMOR and SHAM mice underwent additional cheek bleeds and spleen biopsies; samples were stained and immunophenotyped. The day preceding each time point, TUMOR mice were given $150 \mathrm{mg} / \mathrm{kg} \mathrm{D}$ luciferin i.p. plus an additional $15 \mathrm{mg} / \mathrm{kg}$ intranasal (i.n.) and IVIS-imaged for bioluminescence intensity. (E) Eight weeks following amputation, TUMOR and SHAM mice were cheek bled one final time before euthanasia (EUTH) and tissue harvest; all tissues (blood, spleen, lung, and bone marrow $[\mathrm{BM}])$ were stained and immunophenotyped. The day preceding the EUTH time point, TUMOR mice were given $150 \mathrm{mg} / \mathrm{kg}$ D-luciferin i.p. plus an additional $15 \mathrm{mg} / \mathrm{kg}$ i.n. and IVISimaged for bioluminescence intensity.

Fig. 2 OS disease progression induces changes in percentages of circulating lymphocytes, PMN-MDSCs, and M-MDSCs. Mice were inoculated intratibially with luc-K7M2 tumor cells 
(TUMOR) or vehicle (SHAM) and followed over 12 weeks with time point tissue sampling. Representative flow cytometry (FC) plots of select peripheral blood cell populations for TUMOR and SHAM animals at each time point are shown for (A) NKp46+ NK cells, (D) CD4+ TH and CD8+ Tc cells, and (I) CD11b+Ly6Clo/-Ly6G+ PMN-MDSCs and CD11b+Ly6C+Ly6G- MMDSCs. Average (mean \pm SD) percent of peripheral blood CD45+ leukocytes in TUMOR versus SHAM mice are shown for NKp46+ NK cells $(\mathbf{B})$ along the entire course of disease and $(\mathbf{C})$ across AMP and EUTH time points only, CD4+ TH cells $(\mathbf{E})$ along the entire course of disease and $(\mathbf{G})$ across AMP and EUTH time points only, CD8+ Tc cells $(\mathbf{F})$ along the entire course of disease and (H) across AMP and EUTH time points only, CD11b+Ly6Clo/-Ly6G+ PMN-MDSCs (J) along the entire course of disease and (L) across AMP and EUTH time points only, and CD11b+Ly6C+Ly6GM-MDSCs (K) along the entire course of disease and (M) across AMP and EUTH time points only. Trendlines represent linear curves of best fit for tumor-bearing mice. *, p < 0.05; **, p < 0.01 ; ***, $\mathrm{p}<0.001$ by an unpaired two-tailed $t$ test $($ TUMOR versus SHAM). N.S. $=$ not significant. Data are representative of $n \geq 5$ mice (male/female combined) per time point.

Fig. 3 The ratio of PD-L1/MHC-II (P[1]/M[II) expression on the surface of systemically circulating M-MDSCs correlates with increasing tumor burden and is specific to disease progression. Mice were inoculated intratibially with luc-K7M2 tumor cells (TUMOR) or vehicle (SHAM) and followed over 12 weeks with time point tissue sampling. (A) Representative flow cytometry (FC) plot showing the percent expression of PD-L1 on the surface of peripheral blood M-MDSCs in TUMOR (red) and SHAM (black) mice. (B) Representative FC plot showing the percent expression of MHC-II on the surface of blood M-MDSCs in TUMOR (red) and SHAM (black) mice. (C-E) IVIS-reported increases in tumor bioluminescence were used to test for 
associations with $(\mathbf{C})$ peripheral blood M-MDSC surface PD-L1 expression alone $(n=9),(\mathbf{D})$ peripheral blood M-MDSC surface MHC-II expression alone $(n=9)$, and $(\mathbf{E})$ peripheral blood MMDSC P[1]/M[II] ratio $(n=9)$ using Pearson's correlation test. Trendlines were centered at the origin and translated to begin at $x=0$ using the means of each group. (F) Average (mean \pm SD) magnitude of the M-MDSC P[1]/M[II] ratio in TUMOR versus SHAM mice along the entire course of disease and (G) across AMP and EUTH time points only. The trendline represents the linear curve of best fit for TUMOR mice. *, $\mathrm{p}<0.05 ;{ }^{*}, \mathrm{p}<0.01$; ***, $\mathrm{p}<0.001$ by an unpaired two-tailed $t$ test (TUMOR versus SHAM). N.S. = not significant. In $(\mathbf{F})$ and $(\mathbf{G})$, the data are representative of $n \geq 5$ mice (male/female combined) per time point.

Fig. 4 Decreased lymphocytic infiltration and abnormal myeloid activation in lung and bone marrow reflect disease status and mirror systemic changes in blood. Mice were inoculated intratibially with luc-K7M2 tumor cells (TUMOR) or vehicle (SHAM) and followed over 12 weeks with time point tissue sampling. Representative flow cytometry (FC) plots of select lung and bone marrow (BM) cell populations for TUMOR and SHAM animals at euthanasia (EUTH) are shown for $(\mathbf{A})$ NKp46+ NK cells, $(\mathbf{C})$ CD4+ TH and CD8+ Tc cells, and (E) CD11b+Ly6Clo/Ly6G+ PMN-MDSCs and CD11b+Ly6C+Ly6G- M-MDSCs. Boxplots of (B) NKp46+ NK cells, (D) $\mathrm{CD}_{+}+\mathrm{TH}_{\mathrm{H}}$ and CD8+ $\mathrm{Tc}$ cells, and (F) CD11b+Ly6Clo/-Ly6G+ PMN-MDSCs and CD11b+Ly6C+Ly6G- M-MDSCs are shown as a percent of total CD45+ leukocytes in lungs and BM of TUMOR versus SHAM mice. (G) Exemplary FC plot showing the percent expression of PD-L1 and MHC-II on the surface of lung and BM M-MDSCs in TUMOR (red) and SHAM (black) mice. (H) Boxplots of the M-MDSC P[1]/M[II] ratio are shown for lungs and BM of TUMOR versus SHAM mice. *, $\mathrm{p}<0.05$ * $^{* *}, \mathrm{p}<0.01$ * $^{* *}, \mathrm{p}<0.001$ by an unpaired two-tailed 
$t$ test (TUMOR versus SHAM). Data are representative of $n \geq 5$ mice (male/female combined) per time point.

Fig. 5 The M-MDSC P[1]/M[II] ratio is a specific and systemic marker of OS disease. Mice were inoculated intratibially with luc-K7M2 tumor cells (TUMOR) or vehicle (SHAM) and followed over 12 weeks with time point tissue sampling. (A) Pearson correlation in TUMOR mice between the M-MDSC P[1]/M[II] ratios of primary tumors harvested at amputation (AMP) versus the M-MDSC P[1]/M[II] ratios of lung tissue collected at euthanasia (EUTH) $(n=5)$. (B) Pearson correlation in TUMOR (triangles) and SHAM (squares) mice between the M-MDSC P[1]/M[II] ratios of blood (left: TUMOR $n=5$; SHAM $n=7$ ) and BM (right: TUMOR $n=5$, SHAM $n=7$ ) at EUTH and the M-MDSC P[1]/M[II] ratio of lung.

Supplementary Fig. S1 Sample gating strategy for NK cells, $\mathrm{T}_{\text {н }}$ cells, and $\mathrm{T}_{\mathrm{c}}$ cells from antibody panel L. Intact cells were separated from debris using SSC-A versus FSC-A (A), which were then gated to exclude doublets using FSC-A versus FSC-W (B). CD45+LIVE/DEAD- live white blood cells $(\mathbf{C})$ were then gated into (D) NKp46+ NK cells and (E) CD4+ TH and CD8+ Tc cells using a univariate and bivariate plots, respectively.

\section{Supplementary Fig. S2 Sample gating strategy for PMN- and M-MDSCs from antibody} panel M. Intact cells were separated from debris using SSC-A versus FSC-A (A), which were then gated to include only CD45+ white blood cells $(\mathbf{B})$. CD45+ white blood cells were further gated into CD11b+ myeloid lineage cells (C), which were separated into (D) Ly6C+Ly6G- M-MDSCs 
and Ly6Clo/-Ly6G+PMN-MDSCs with a bivariate plot. Percent expression of PD-L1 and MHC-II on M-MDSCs was then determined using univariate histograms (E).

\section{Supplementary Fig. S3 CD45+ percent of total cells harvested from tumor, lung, BM, and}

blood tissues. Mice were inoculated intratibially with luc-K7M2 tumor cells (TUMOR) or vehicle (SHAM) and followed over 12 weeks with time point tissue sampling. Blood and spleen samples were collected from TUMOR and SHAM mice at amputation (AMP) and again at four (T1), six (T2), and eight (EUTH) weeks post-AMP. Lung and BM were harvested in addition to blood and spleen at the EUTH time point and all tissues were stained with lymphoid- and myeloid-specific antibody panels and immunophenotyped via flow cytometry (FC). (A) CD45+ percent of total cells harvested from primary tumors at AMP from TUMOR mice and lung and BM harvested at EUTH from TUMOR and SHAM mice. (B) CD45+ percent of total cells harvested from blood of TUMOR and SHAM mice at AMP, T1, T2, and EUTH time points. Data are representative of $n \geq 5$ mice (male/female combined) for each time point and/or tissue.

\section{Supplementary Fig. S4 Luciferin-mediated tumor bioluminescence can paradoxically} decrease with increasing tumor size in cases where rapid growth promotes central necrosis. Mice were inoculated intratibially with luc-K7M2 tumor cells (TUMOR) or vehicle (SHAM) and followed over 12 weeks with time point tissue sampling. The day preceding each time point, TUMOR mice were IVIS-imaged for bioluminescence intensity. IVIS images of representative TUMOR mice with (A) and without (D) central tumor necrosis are shown across four time points. IVIS-reported tumor bioluminescence in average radians $([\mathrm{p} / \mathrm{s}] /[\mathrm{cm} 2 / \mathrm{sr}])$ is shown at each time point for representative TUMOR mice with $(\mathbf{B})$ and without $(\mathbf{E})$ central tumor necrosis. Magnified 
IVIS images of select time points showing representative TUMOR mice with $(\mathbf{C})$ and without $(\mathbf{F})$ central tumor necrosis highlighting the relationship between tumor radius (red dotted circle and arrows) and signal intensity. (G) Average (mean \pm SD) tumor burden bioluminescence in average radians $\left([\mathrm{p} / \mathrm{s}] /\left[\mathrm{cm}_{2} / \mathrm{sr}\right]\right)$ across each time point as measured by IVIS imaging.

\section{Supplementary Fig. S5 Individual matched-pair comparisons highlight the temporal} relationship cellular immune biomarkers in blood and OS disease burden. Mice were inoculated intratibially with luc-K7M2 tumor cells (TUMOR) or vehicle (SHAM) and followed over 12 weeks with time point tissue sampling. Blood samples were collected from TUMOR and SHAM mice at amputation (AMP) and again at four (T1), six (T2), and eight (EUTH) weeks postAMP and stained with both lymphoid- and myeloid-specific antibody panels for immunophenotyping via flow cytometry (FC). The day preceding each time point, TUMOR mice were IVIS-imaged for bioluminescence intensity. (A) Top panel: Male Tumor 1 mouse with a primary lesion of the left lower extremity leading to local recurrence and peritoneal infiltration at T2 (Day 70) and widespread disease with pulmonary metastasis (upon visual inspection of lung) at EUTH (Day 84). Bottom panel: Male Tumor 1 mouse IVIS-reported tumor burden in average radians $\left([\mathrm{p} / \mathrm{s}] /\left[\mathrm{cm}_{2} / \mathrm{sr}\right]\right)$ at each time point. From the lymphoid antibody panel, populations of $(\mathbf{B})$ NKp46+NK, (C) CD4+ TH, and (D) CD8+ Tc cells as a percent of total CD45+ leukocytes in the blood of Male Tumor 1 mouse (triangle) versus a matched-pair sham (square). From the myeloid antibody panel, populations of (E) CD11b+Ly6Clo/-Ly6G+ PMN-MDSCs and (F) CD11b+Ly6C+Ly6G- M-MDSCs as a percent of total CD45+ leukocytes blood of Male Tumor 1 mouse versus a matched-pair sham. (G) Male Tumor 1 mouse blood M-MDSC P[1]/M[II] ratio versus matched-pair sham. (H) Top panel: Male Tumor 2 mouse with a primary lesion of the right 
lower extremity leading to local recurrence and peritoneal infiltration at T1 (Day 70) and widespread disease with pulmonary metastasis at T2 (Day 84). As this mouse reached euthanasia criteria early (early euthanasia $=\mathrm{EE})$, it was euthanized at its T2 time point. Bottom panel: Male Tumor 2 mouse IVIS-reported tumor burden in average radians at each time point. From the lymphoid antibody panel, populations of (I) NKp46+ NK, (J) CD4+ TH, and (K) CD8+ Tc cells as a percent of total CD45+ leukocytes in the blood of Male Tumor 2 mouse versus a matched-pair sham. From the myeloid antibody panel, populations of $(\mathbf{L}) \mathrm{CD} 11 \mathrm{~b}+\mathrm{Ly} 6 \mathrm{Clo} / \mathrm{Ly} 6 \mathrm{G}+\mathrm{PMN}-\mathrm{MDSCs}$ and (M) CD11b+Ly6C+Ly6G- M-MDSCs as a percent of total CD45+ leukocytes in blood of Male Tumor 1 mouse versus a matched-pair sham. (N) Male Tumor 2 mouse blood M-MDSC P[1]/M[II] versus matched-pair sham. (O) Top panel: Female Tumor 1 mouse with a primary lesion of the right lower extremity which was cured at AMP (Day 77) and endured no further disease burden all the way to its EUTH time point (Day 105). Bottom panel: Female Tumor 1 mouse IVISreported tumor burden in average radians at each time point. From the lymphoid antibody panel, populations of $(\mathbf{P})$ NKp46+ NK, (Q) CD4+ TH, and $(\mathbf{R}) \mathrm{TC}_{\mathrm{C}} \mathrm{CD} 8+$ cells as a percent of total CD45+ leukocytes in the blood of Female Tumor 1 mouse versus matched-pair sham. From the myeloid antibody panel, populations of (S) CD11b+Ly6Clo/-Ly6G+ PMN-MDSCs and (T) CD11b+Ly6C+Ly6G- M-MDSCs as a percent of total CD45+ leukocytes in the blood of Female Tumor 1 mouse versus matched-pair sham. (U) Female Tumor 1 mouse M-MDSC P[1]/M[II] ratio versus matched-pair sham.

\section{Supplementary Fig. S6 Immunophenotype of primary tumors removed at time point AMP.}

Mice were inoculated intratibially with luc-K7M2 tumor cells (TUMOR) or vehicle (SHAM) and followed over 12 weeks with time point tissue sampling. Lymphoid (A) and myeloid (B) lineage 
immune cell populations as a percent of total CD45+ leukocytes in primary tumors harvested from TUMOR mice at time point AMP. (C) The M-MDSC P[1]/M[II] ratio of primary tumors harvested from tumor-bearing mice at time point AMP. Data (mean $+\mathrm{SD})$ are representative of $n \geq 5$ mice (male/female combined). 
Figure 1

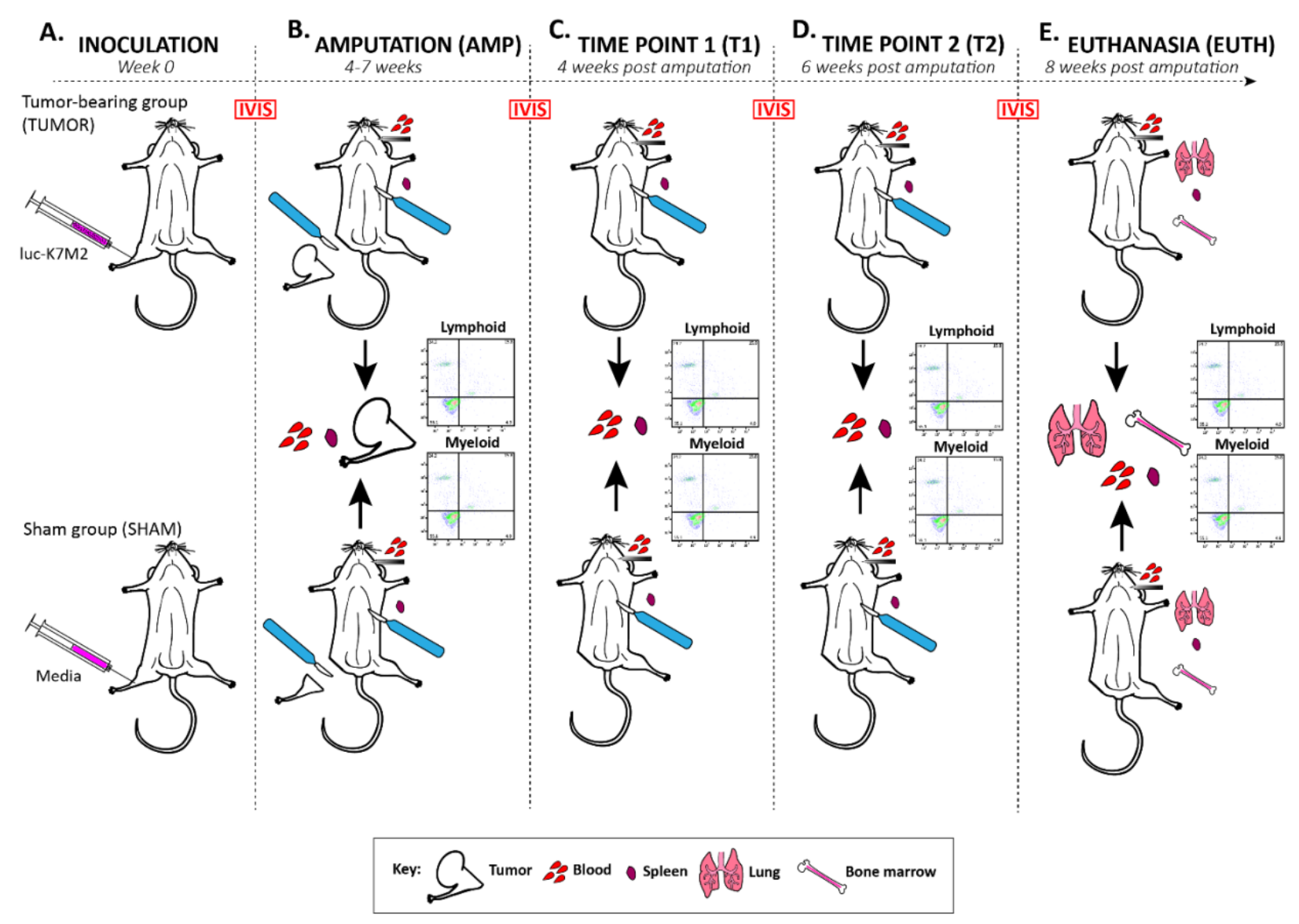


Figure 2
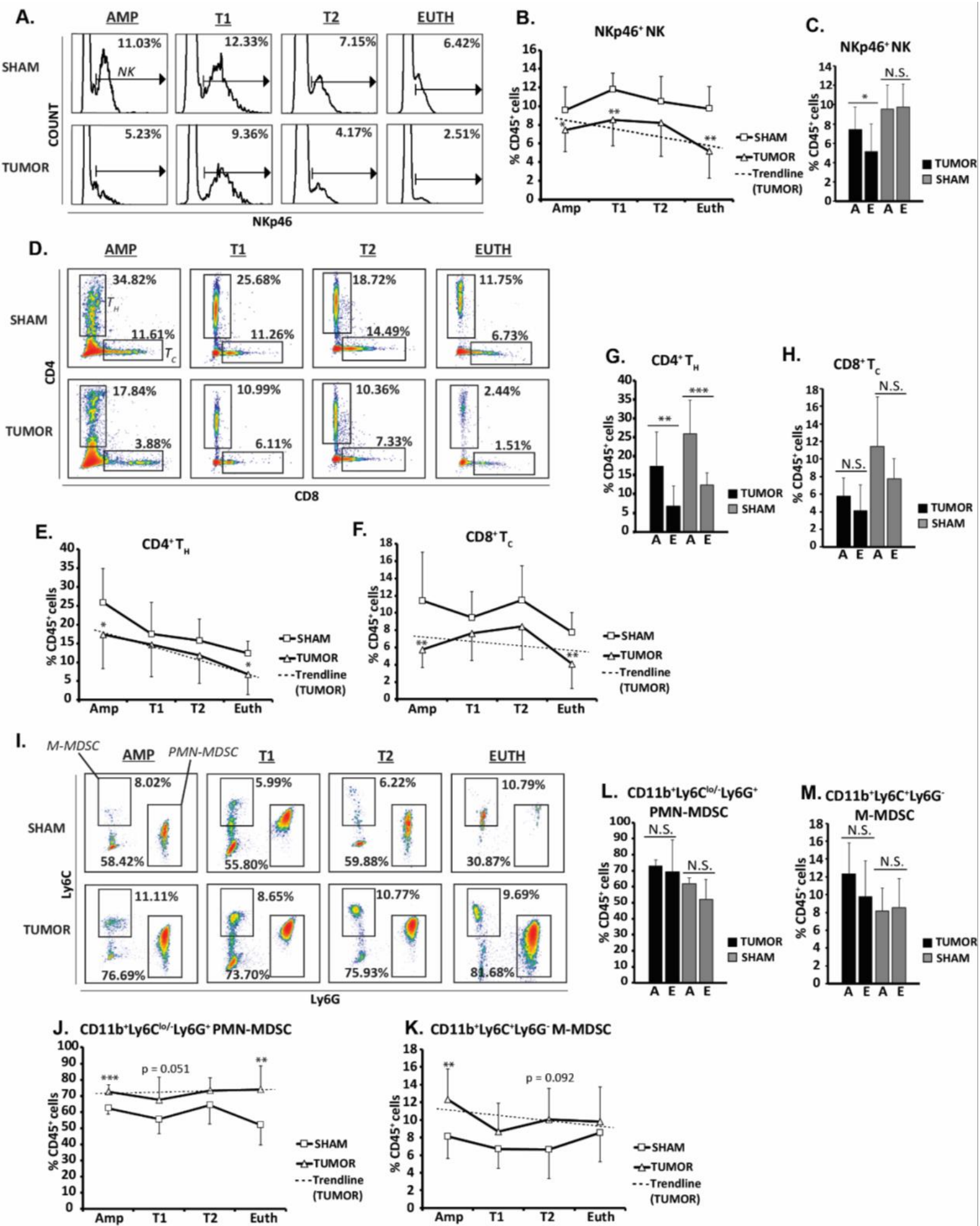
Figure 3

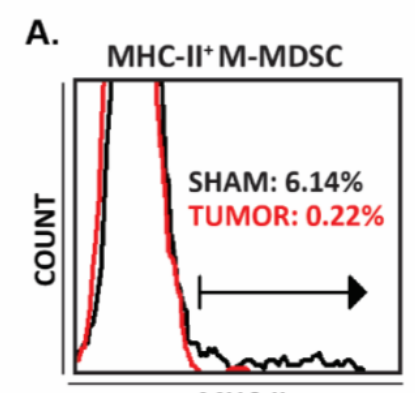

MHC-II

B.

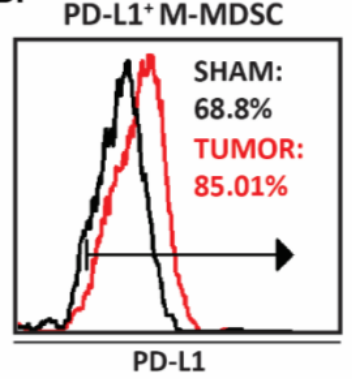

c.

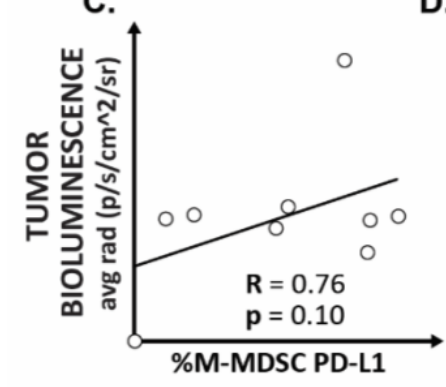

D.

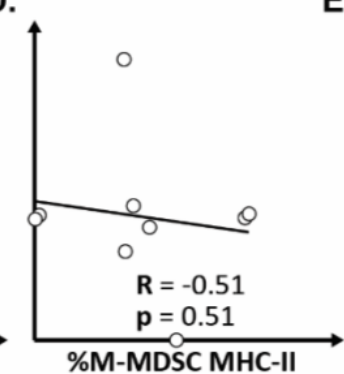

E.

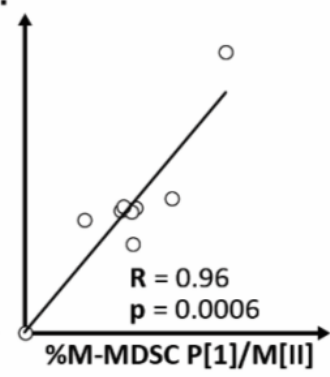

F.
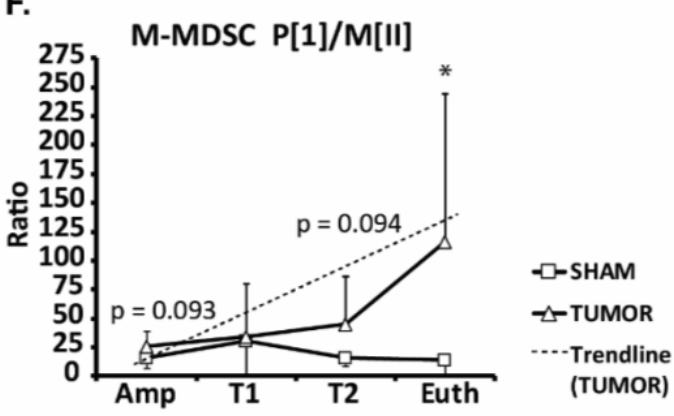

G. M-MDSC

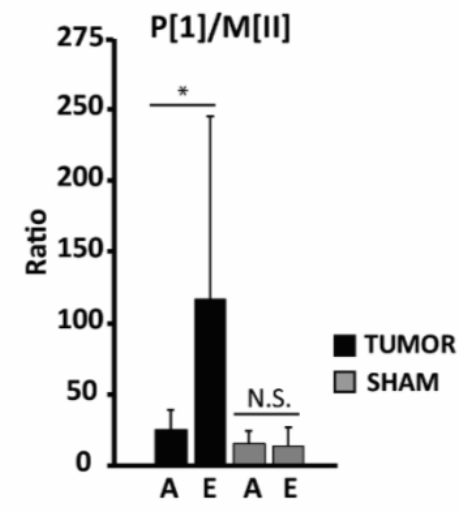




\section{Figure 4}

A. $\mathrm{NKp} 46^{+} \mathrm{NK}$

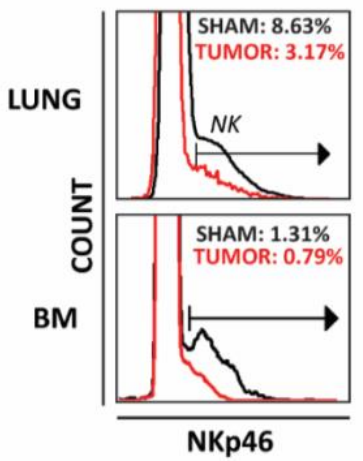

C. $\mathrm{CD}^{+} \mathrm{T}_{\mathrm{H}}$

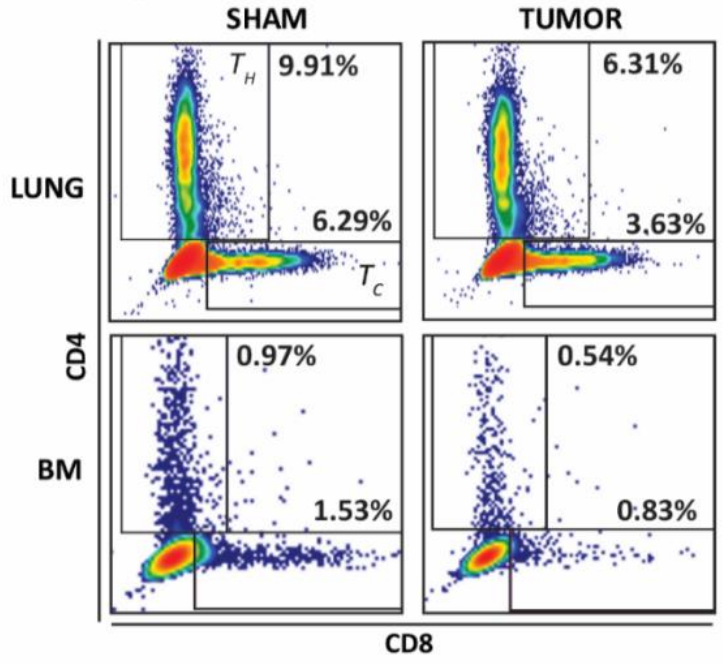

D.

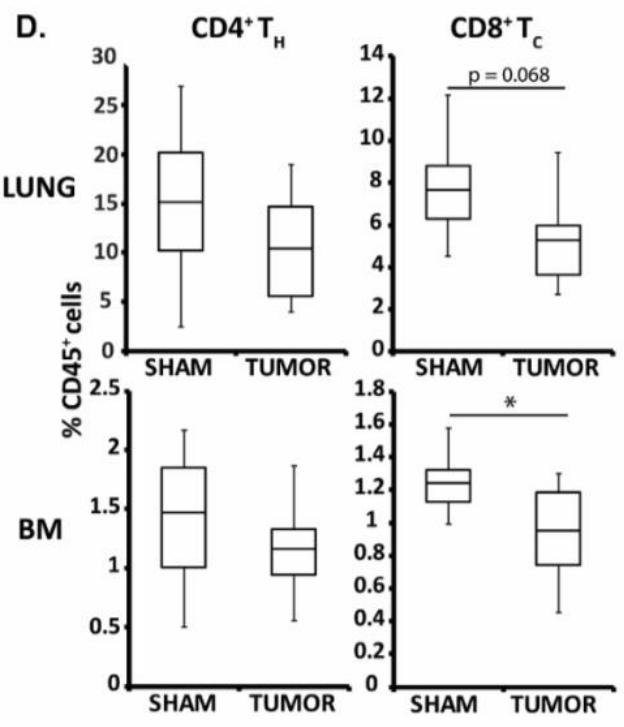

B. NKp46 ${ }^{+} \mathrm{NK}$

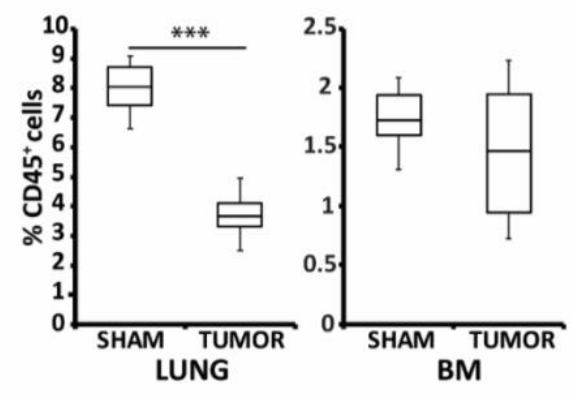

E. CD11b ${ }^{+}$Ly6C $^{10 /} /$ Ly $6 \mathrm{G}^{+}$PMN-MDSC

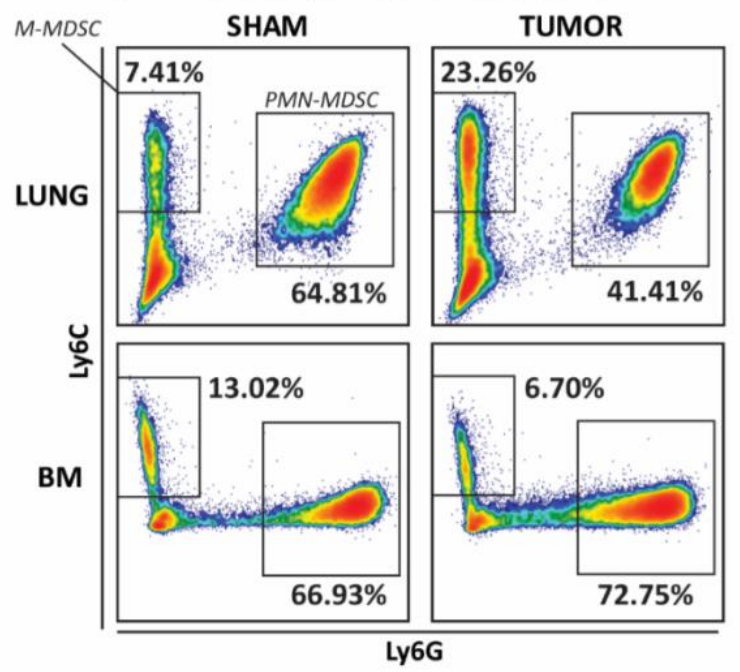

F. CD11b'Ly6C $\mathrm{C}^{10 / \text { Ly6G }^{+}}$CD11b+Ly6C Ly6G

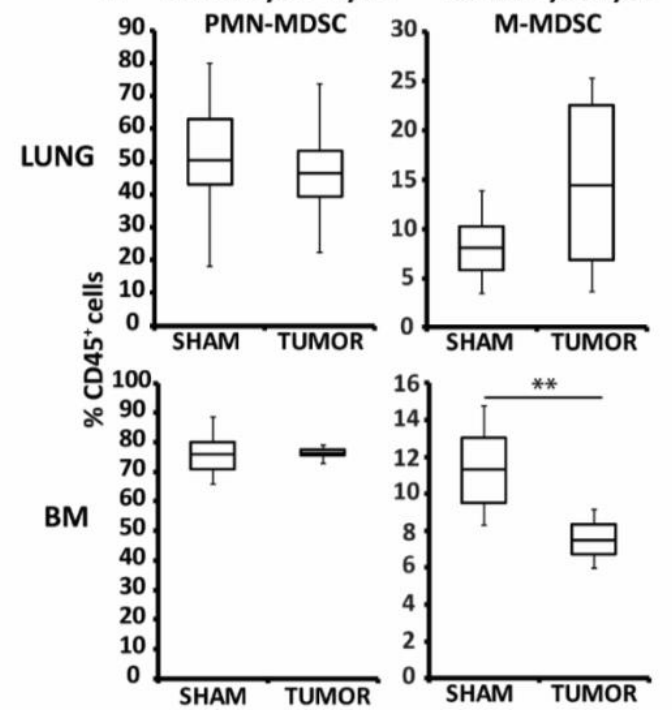


G. $\mathrm{M}-\mathrm{MDSC} \mathrm{P}[1] / \mathrm{M}[\mathrm{II}]$

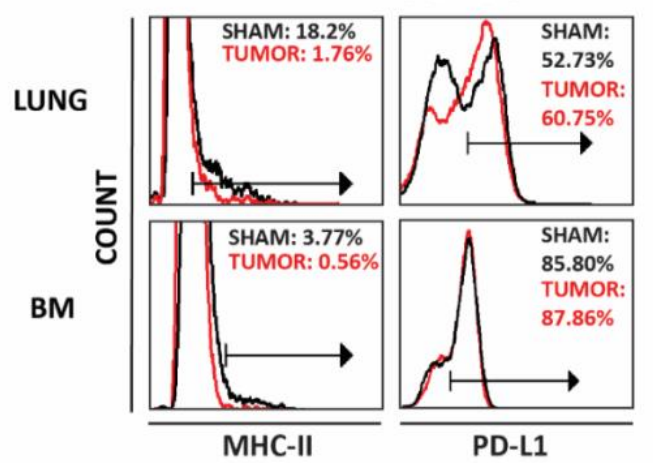

H. M-MDSC P[1]/M[II]

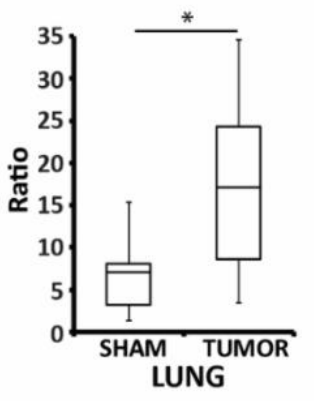


Figure 5

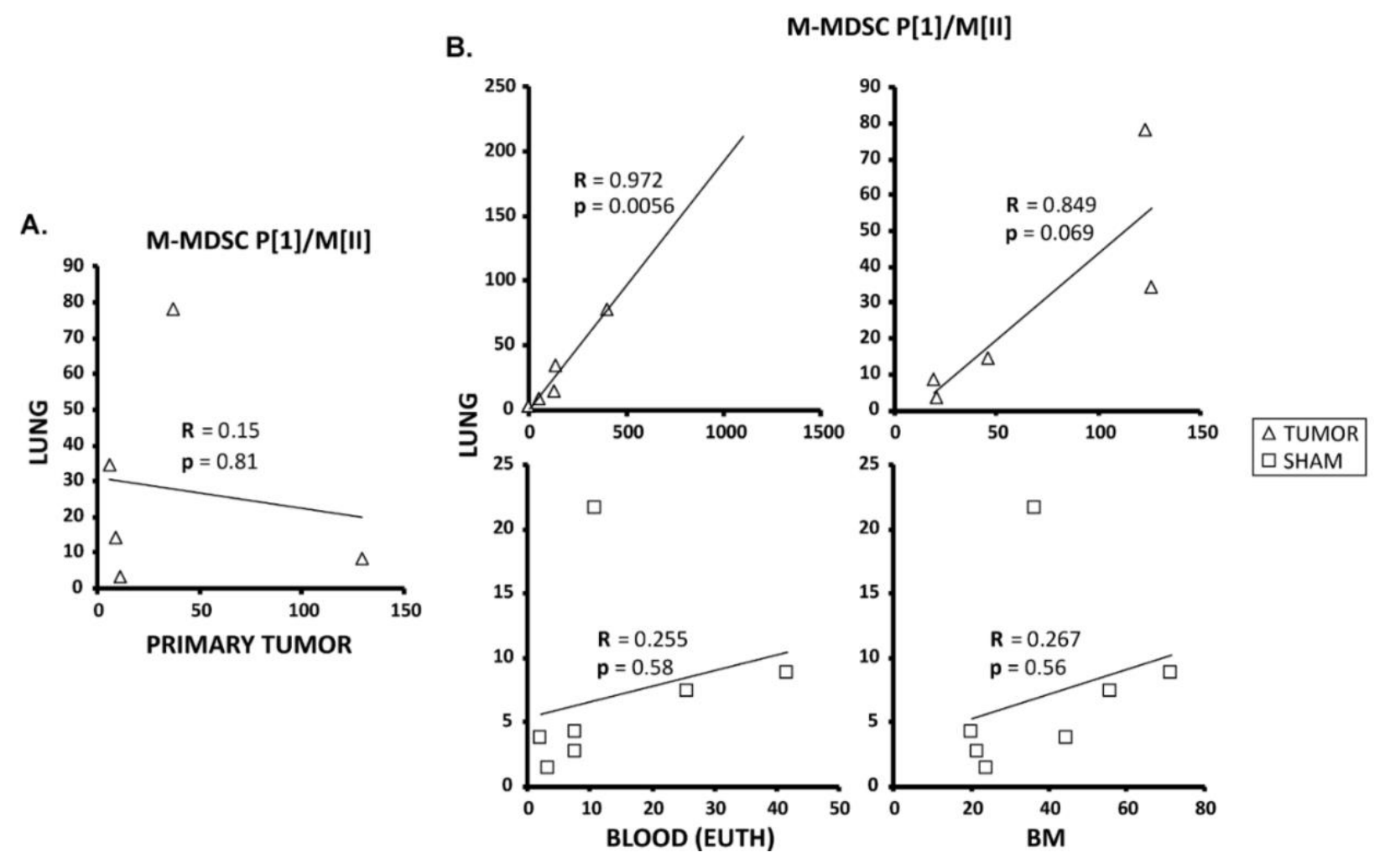




\section{Supplementary Figure S1}

A.

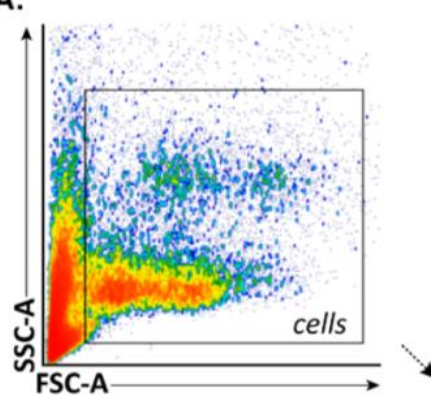

B.

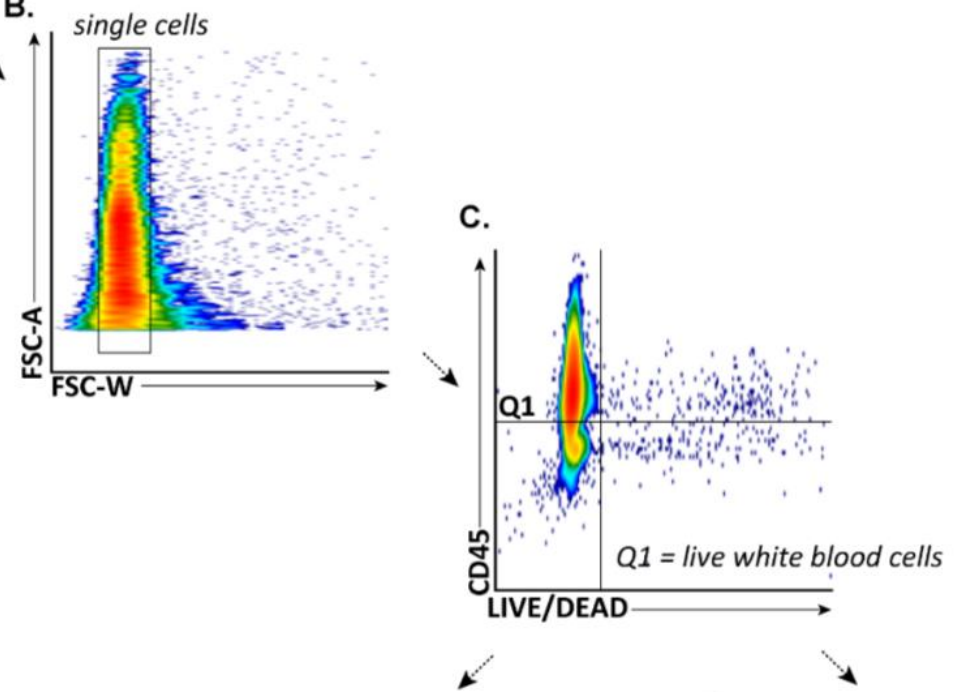

D.
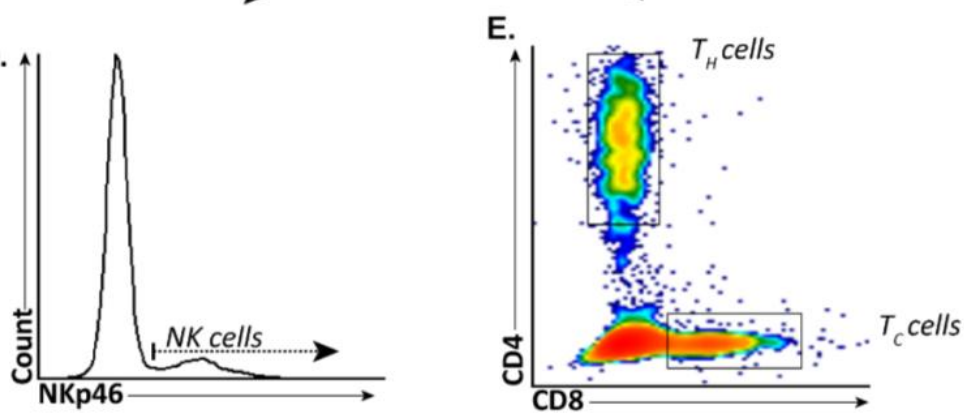


\section{Supplementary Figure S2}

A.
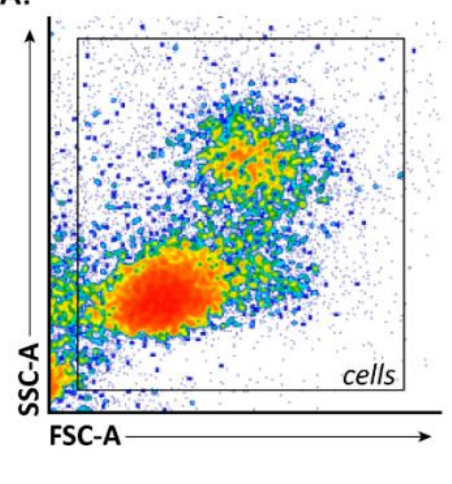

B.
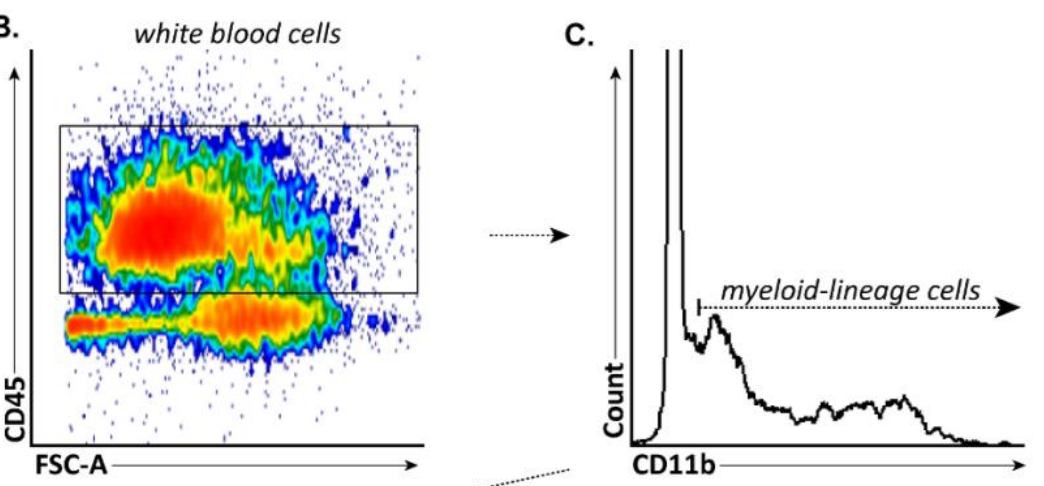
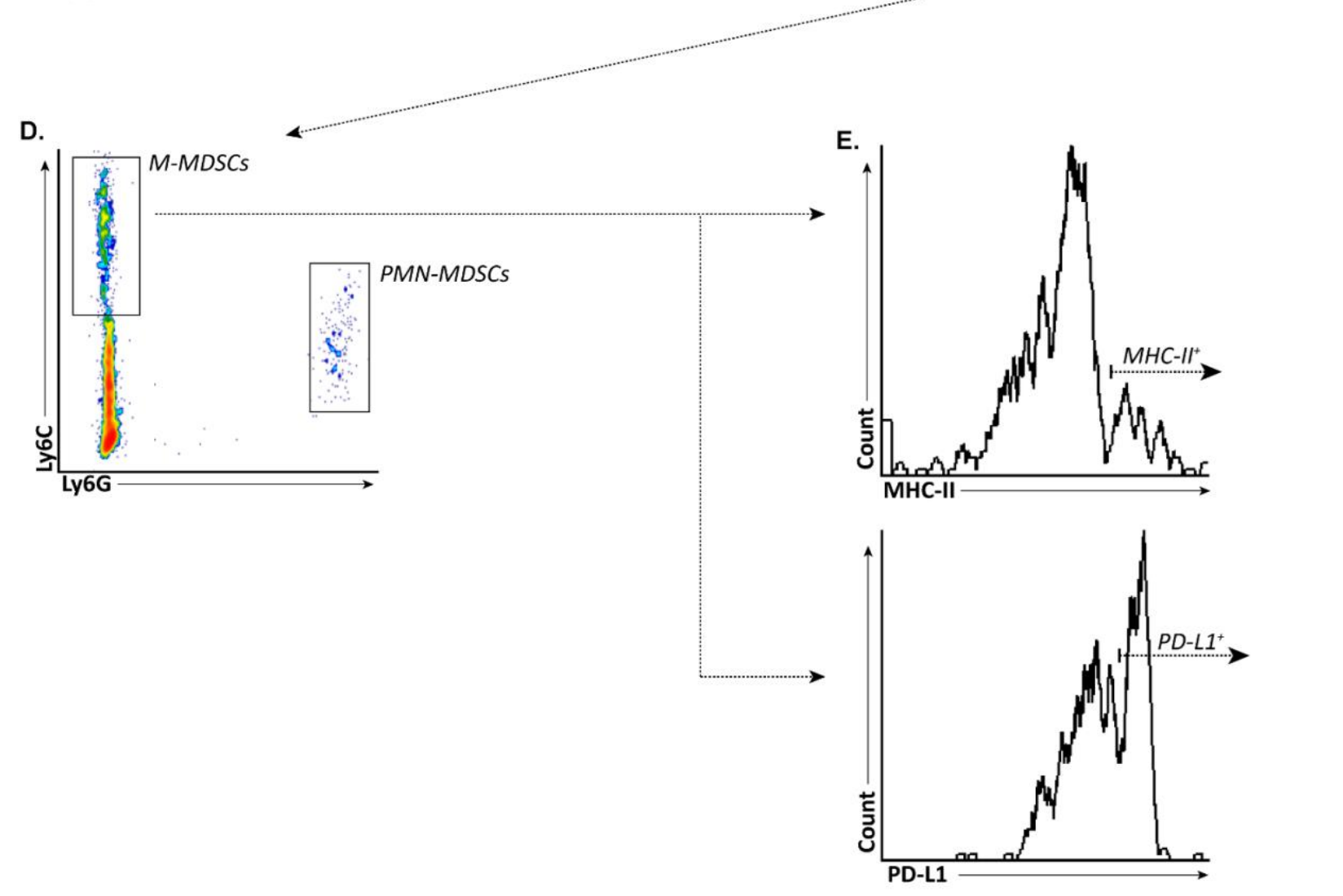


\section{Supplementary Figure S3}

A. Single Sample Tissues

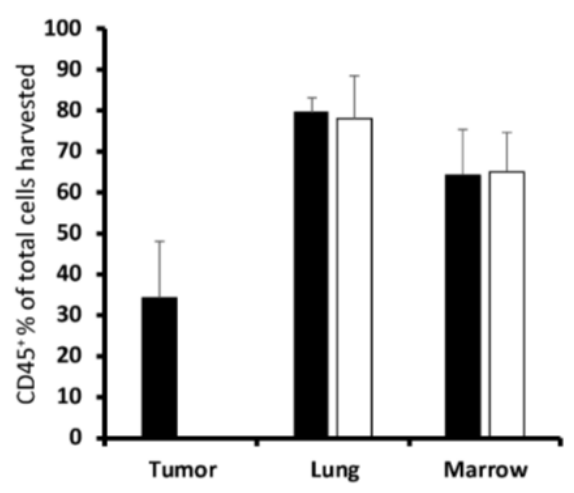

B.

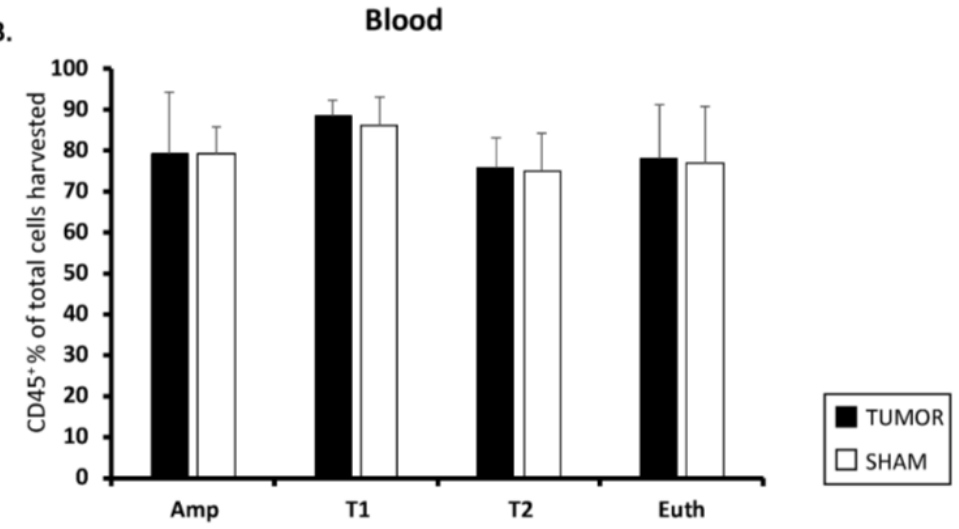




\section{Supplementary Figure S4}
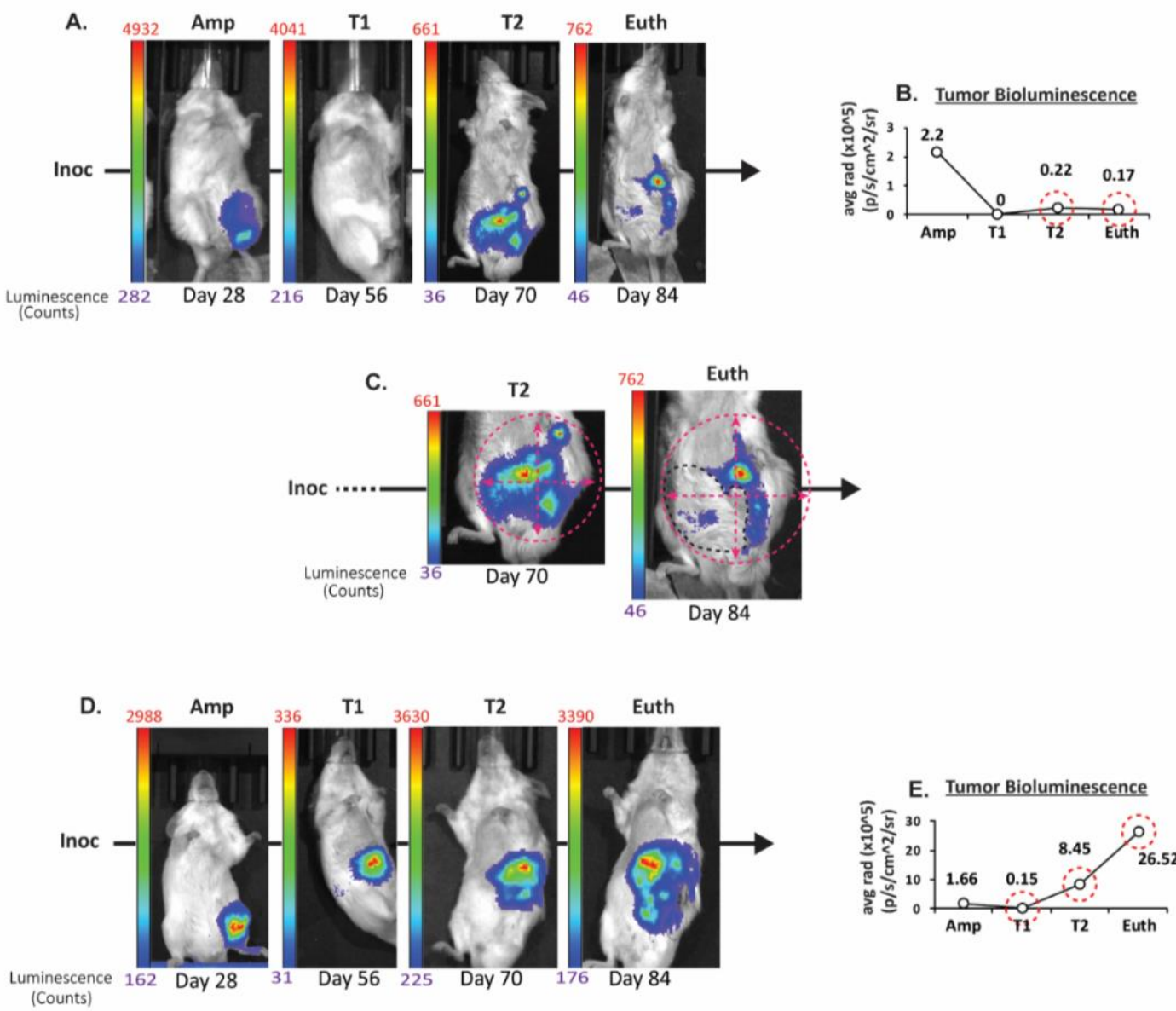

(Counts)
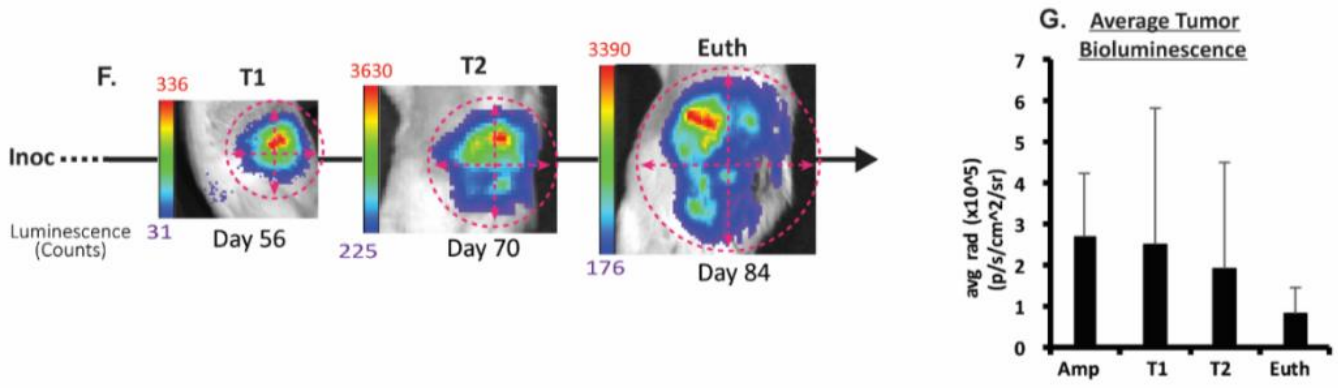


\section{Supplementary Figure S5}

A.

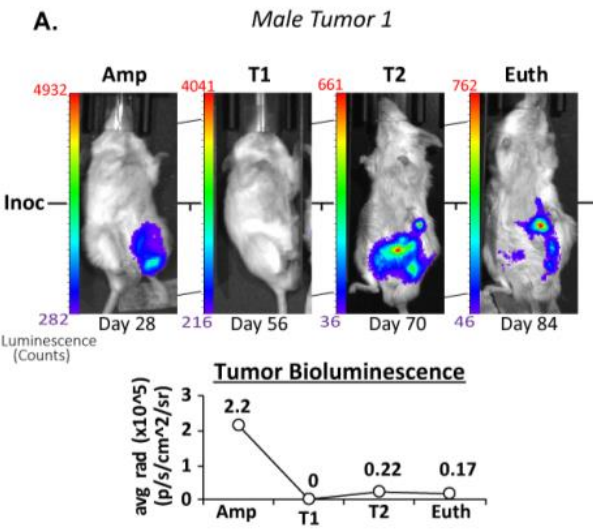

H. Male Tumor 2
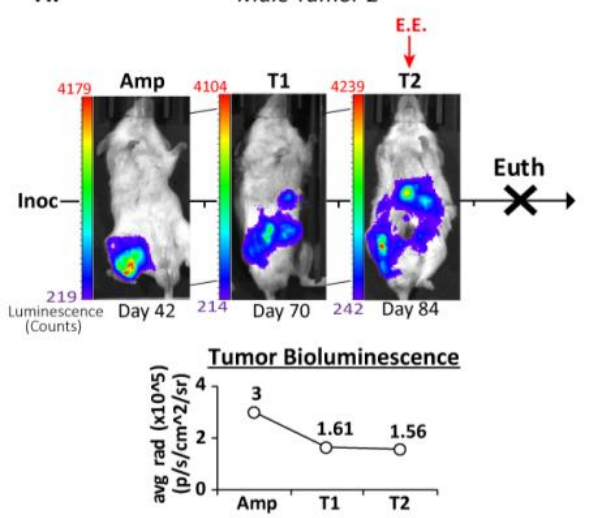

O.
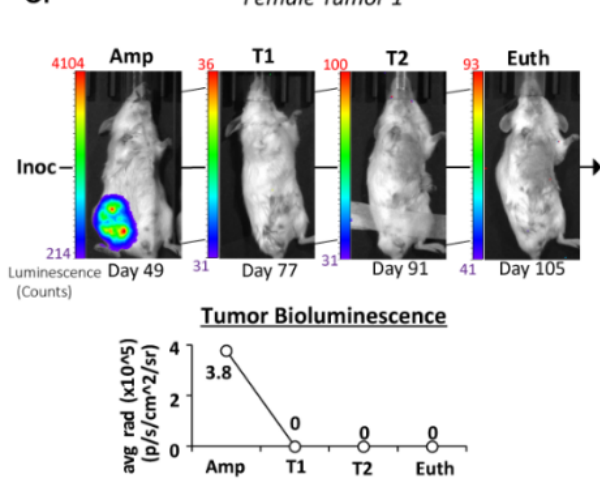
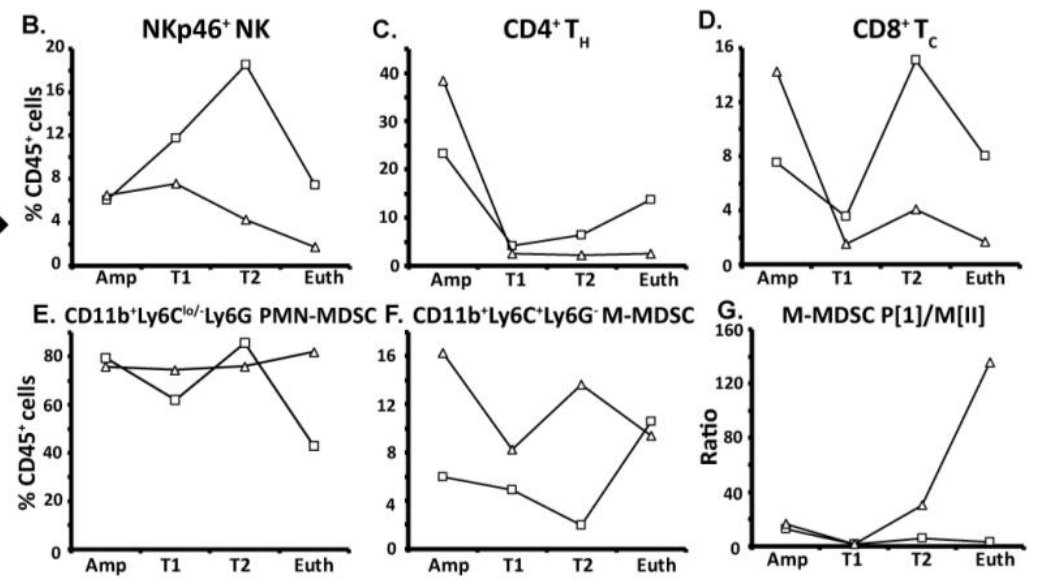
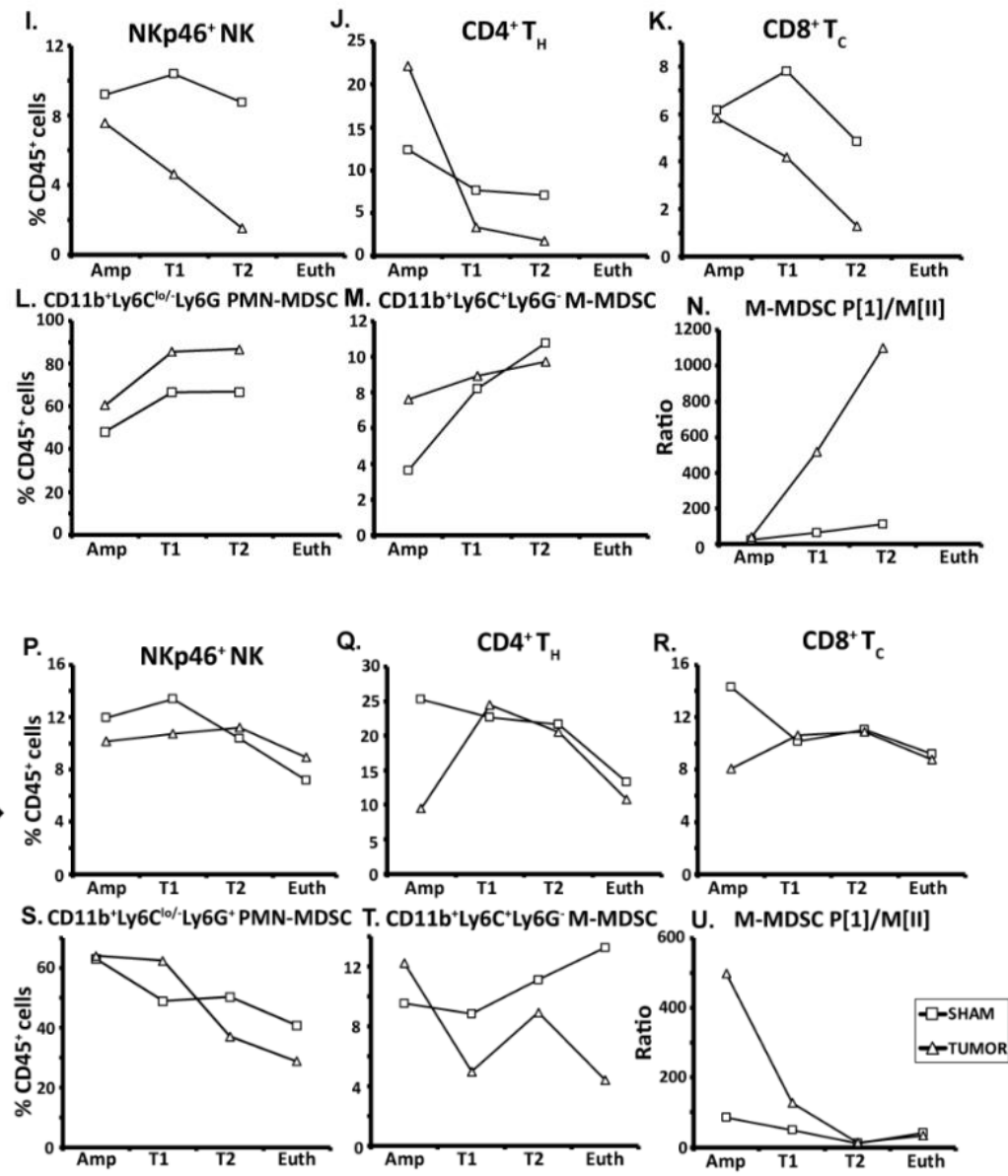
Supplementary Figure S6

TUMOR

A.

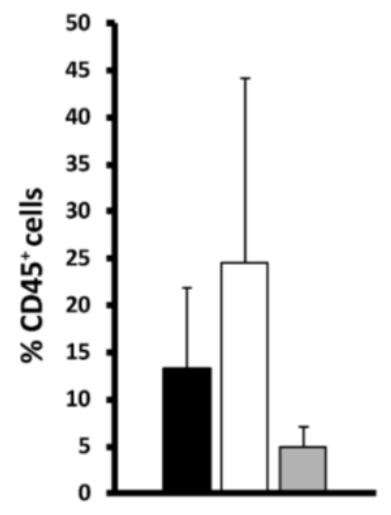

B.

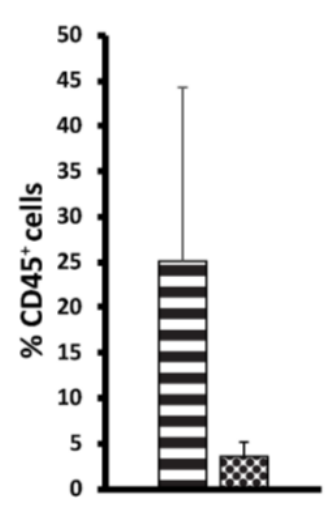

C.

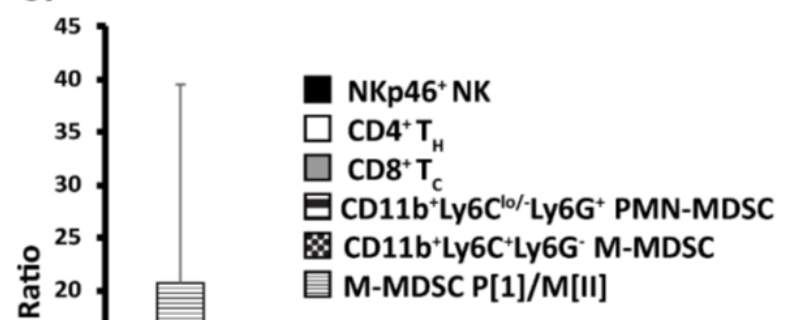


Supplementary Table S1: Markers used to describe the Lymphoid (L) and Myeloid (M)

\section{Antibody Panels}

\begin{tabular}{|c|c|c|c|c|}
\hline ANTIGEN/DYE & PANEL & CLONE & FLUOROCHROME & MANUFACTURER \\
\hline LIVE/DEAD & Lymphoid & & Far red & $\begin{array}{l}\text { Life Technologies, Fisher } \\
\text { Scientific }\end{array}$ \\
\hline CD45 & Lymphoid & MEM-45 & AF405 & Novus \\
\hline CD45 & Myeloid & $30-11$ & BV605 & BD Biosciences \\
\hline CD4 & Lymphoid & RM4-5 & PE-Cy5 & BD Biosciences \\
\hline CD8 & Lymphoid & $53-6.7$ & APC-Сy5.5 & Southern Biotech \\
\hline CD335 (NKP46) & Lymphoid & 29A 1.4 & APC-eFLuor 780 & Invitrogen \\
\hline CD152 (CTLA-4) & Lymphoid & 1B8 & DyLight 488 & Novus \\
\hline CD279 (PD-1) & Lymphoid & $\mathrm{J} 43$ & PE-CF594 & BD Biosciences \\
\hline FOXP3 & Lymphoid & MF23 & $\mathrm{PE}$ & BD Biosciences \\
\hline GALECTIN-9 & Lymphoid & 108A2 & AF 594 & BioLegend \\
\hline KI67 & Lymphoid & REA183 & PE-Vio 770 & Miltenyi Biotec \\
\hline CD69 & Lymphoid & $\mathrm{H} 1.2 \mathrm{~F} 3$ & Brilliant Violet 510 & BioLegend \\
\hline CD366 (TIM-3) & Lymphoid & 5D12 & BB700 & BD Biosciences \\
\hline CD11B & Myeloid & M1/70 & PE-CF594 & BD Biosciences \\
\hline LY6C & Myeloid & HK1.4 & AF700 & BioLegend \\
\hline LY6G & Myeloid & RB6-8C5 & PE-Cy5 & Invitrogen \\
\hline CD124 (IL4R-A) & Myeloid & $\begin{array}{l}\text { Polyclonal goat } \\
\text { IgG }\end{array}$ & Fluoroscein (FAM) & RnD Systems \\
\hline $\begin{array}{l}\text { CD206 } \\
\text { (MANNOSE } \\
\text { RECEPTOR) }\end{array}$ & Myeloid & MR5D3 & AF647 & Bio-Rad \\
\hline CD119 (IFN-ГR1) & Myeloid & RM0093-7K23 & DyLight 550 & Novus \\
\hline TLR2 & Myeloid & 203325 & AF594 & RnD Systems \\
\hline MHC CLASS II & Myeloid & 114.15 .2 & APC-eFluor 780 & Invitrogen \\
\hline CD274 (PD-L1) & Myeloid & 10F9G2 & PE-Cy7 & BioLegend \\
\hline CD38 & Myeloid & 09.4 & VioBlue & Miltenyi Biotec \\
\hline F4/80 & Myeloid & BM8 & PerCP-Cy5.5 & BioLegend \\
\hline
\end{tabular}




\section{Supplementary Table S2: OLAR Tumor Burden Scoring System}

\begin{tabular}{|c|c|c|}
\hline PARAMETER & OBSERVATION & SCORE \\
\hline \multirow[t]{4}{*}{$\begin{array}{l}\text { GENERAL } \\
\text { APPEARANCE }\end{array}$} & $\begin{array}{l}\text { Normal } \\
\text { (e.g. appropriate body condition; healthy appearing fur; pink } \\
\text { mucus membranes, bright, alert, responsive) }\end{array}$ & $\mathbf{0}$ \\
\hline & $\begin{array}{l}\text { Mild abnormal } \\
\text { (e.g. rough/scruffy fur, slightly decrease activity and grooming, } \\
\text { pale mucus membranes) }\end{array}$ & 10 \\
\hline & $\begin{array}{l}\text { Moderately abnormal } \\
\text { (e.g. hunched posture, squinted eyes, reluctant to move, cachectic } \\
\text { body condition, white mucus membranes) }\end{array}$ & 20 \\
\hline & $\begin{array}{l}\text { Severely compromised } \\
\text { (e.g. minimally to non-responsive, closed eyes) }\end{array}$ & 30 \\
\hline \multirow[t]{3}{*}{ BODY CONDITION } & Normal to Overweight body condition & $\mathbf{0}$ \\
\hline & Thin - obvious dorsal vertebrae & \\
\hline & Severe Cachexia - prominent dorsal vertebrae and skeleton & \\
\hline \multirow[t]{3}{*}{$\begin{array}{l}\text { TUMOR } \\
\text { APPEARANCE }\end{array}$} & $\begin{array}{l}\text { Non-ulcerated } \\
\text { Not limiting normal mobility } \\
\text { Not limiting ability to eat or breathe }\end{array}$ & $\mathbf{0}$ \\
\hline & $\begin{array}{l}\text { [One or more listed below] } \\
\text { Non-ulcerated wound associated with tumor-intact healing or } \\
\text { scab present } \\
\text { Limiting normal mobility } \\
\text { Limiting ability to reach food and/or water }\end{array}$ & 20 \\
\hline & $\begin{array}{l}\text { [One or more listed below] } \\
\text { Ulcerated or actively bleeding } \\
\text { Preventing mobility, or so cannot eat or drink } \\
\text { Limiting ability to breathe, or any combination thereof }\end{array}$ & 30 \\
\hline \multirow[t]{3}{*}{ RESPIRATION } & Normal rate and effort for species/strain & $\mathbf{0}$ \\
\hline & Increased rate and/or effort for species/strain & 30 \\
\hline & $\begin{array}{l}\text { Severe respiratory distress or gasping (agonal) breathing } \\
\text { pattern }\end{array}$ & 60 \\
\hline
\end{tabular}




\section{Supplementary Table S3}

\begin{tabular}{l|ccccc}
\multicolumn{1}{l}{ POPULATION } & $\boldsymbol{\alpha}$ & $\boldsymbol{\sigma}$ & $\boldsymbol{\delta}$ & NUMBER & POWER \\
\hline NK CELLS & 0.05 & 2.5 & 9 & 8 & 1.0000 \\
T REGULATORY CELLS & 0.05 & 2.6 & 14 & 8 & 1.0000 \\
T CELL EXHAUSTION SCORE & 0.05 & 2.5 & 18 & 8 & 1.0000 \\
MYELOID LINEAGE CELLS & 0.05 & 0.75 & 3.88 & 8 & 0.9786 \\
GRANULOCYTES & 0.05 & 1.5 & 2.57 & 8 & 0.9994 \\
TGM2+ MACROPHAGES & 0.05 & 4 & 14 & 8 & 1.0000 \\
CXCL9+ MACROPHAGES & 0.05 & 3.5 & 15 & 8 & 1.0000 \\
NOS2+ MACROPHAGES & 0.05 & 6 & 60 & 8 & 1.0000
\end{tabular}

TGM2 = transglutaminase 2; CXCL9 = C-X-C motif chemokine ligand 9; NOS2 $=$ nitric oxide synthase 2 


\title{
Chapter 4
}

IL-12-loaded Poly(lactic-co-glycolic) acid (PLGA) Nanospheres Reduce Metastasis and Increase Cure Rate in a NK cell-dependent Fashion in an Immunocompetent K7M2 Orthotopic Murine Model of Osteosarcoma

\author{
Unpublished data
}




\section{Abstract}

Interleukin(IL)-12 is a protein cytokine that greatly increases the ability of Natural Killer (NK) cells to attack tumors, and IL-12-based immunotherapies have shown activity against osteosarcoma (OS) in preclinical models. The antitumor effects of recombinant IL-12 are greatest when administered systemically; however, intravenous delivery of large bolus doses of IL-12 is associated with severe side effects. Encapsulating IL-12 within biodegradable nanoscale poly(lactic-co-glycolic) acid (PLGA) vectors (nIL-12) may allow for enhanced biodistribution while minimizing side effects. In this study, we first established that high doses of intravenously administered recombinant mouse(rm)IL-12 produced immunologic consequences similar to that seen in humans, including NK cell depletion, T cell exhaustion (TCE), and polymorphonuclearmyeloid-derived suppressor cell (PMN-MDSC) myelocytosis in healthy BALB/c mice. Next, we evaluated the survival benefits of nIL-12 on the luciferase-transfected K7M2 (luc-K7M2) mouse model of metastatic OS while monitoring the systemic immunophenotype. It was observed that luc-K7M2 tumor-bearing mice treated with nIL-12 had a $35.3 \%$ reduction in pulmonary metastases $(\mathrm{p}=0.007)$, as well as a $27.1 \%$ increase in disease-free rate $(\mathrm{p}=0.008)$ at 12 weeks compared to 34 untreated mice from previous experiments utilizing the same model. The immunophenotypes of nIL-12-treated tumor-bearing mice on a per-dose group basis followed the same trajectories across all seven time points assessed, suggesting that dose-dependent nIL-12-mediated IL-12 immunotoxicity was unlikely. Importantly, the nIL-12-treated mice with residual disease at 12 weeks had lower NK cell percentages in their peripheral blood at primary tumor amputation versus disease-free mice; upon stratifying nIL-12-treated mice around the median blood NK cell percentage at primary tumor amputation, it was found that mice with NK cell percentages above the median $(8.21 \%)$ value developed significantly fewer metastases than those below $(\mathrm{p}=0.038)$. 
While more controls are needed to flesh out the details of nIL-12 therapy for OS, these data support the fact that immunophenotyping may be used alongside immunotherapy to increase efficacy and assess immunotoxicity and thus warrant further investigation.

\section{Introduction}

While rare, osteosarcoma (OS) is the most common primary malignancy of bone and is most prevalent in children and young adults (1). When metastatic, patients have extremely low survival rates and no new therapies have been introduced since MAP (methotrexate, doxorubicin, and cisplatin) combination chemotherapy several decades ago $(1,2)$. OS tumors are extremely heterogeneous; the spectrum of mutations comprising individual tumors has been likened to that of "fingerprints," where each patient presents with a unique constellation of mutations driving sarcomagenesis $(3,4)$. As such, there are few genomic alterations of which direct targeting has yielded clinical benefits, and a new treatment perspective is warranted.

High genomic instability in OS tumors leads to extensive nonsynonymous mutation rates, with the highest density of neoantigens expressed in metastatic lesions. Increased neoantigen production is associated with increased tumor-infiltrating leukocytes and tumor immunosuppressive forces (5). Although the immunogenicity of metastases is greater than that of primary tumors, advancing disease brings systemic malignancy-induced immunosuppression in the form of stepwise depletion of circulating lymphyoctes and the accumulation of suppressive myeloid cells (5-7).

Natural killer (NK) cells are cytotoxic lymphocytes of the innate immune system that are depleted in the peripheral circulation of OS patients at diagnosis and continue to decrease as 
disease progresses $(6,8)$. They are a subset of innate lymphoid cells (ILC) that kill infected and/or malignant-transformed cells by recognizing nonspecific cellular stress proteins. NK cells are an important player in tumor immunosurveillance as they prevent metastatic outgrowth by disseminated tumor cells $(9,10)$. Various preclinical studies have confirmed the importance of NK cells in the eradication of metastatic tumors, and they have been shown to kill OS tumor-iniating cells (11-13).

Interleukin(IL)-12 is a heterodimeric protein cytokine that promotes cell-mediated immune responses against microorganisms and cancers (15). It is mainly released by activated antigen presenting cells (APCs) to assist in the priming of naïve helper T cells (16). In an IL-12-rich environment, helper T cells polarize into type 1 ( $\left.\mathrm{T}_{\mathrm{H}} 1\right)$ subsets and NK cells become activated and proliferate. The highly antitumorigenic cytokine interferon(IFN)- $\gamma$ is released by activated T and NK cells and is an important mediator of IL-12 immunostimulation (17-20). Exogeneous administration of recombinant IL-12 has shown efficacy against a variety of murine tumors and infections, though the development of standardized dosing regimens has been complicated by extensive side effect profiles in human patients (21-25).

Recombinant IL-12 induces its most powerful immune responses when given systemically, allowing for more direct activation of immune cells in secondary lymphoid organs $(26,27)$. However, systemic administration of recombinant protein requires high loading doses to achieve adequate biodistribution and may lead to systemic toxicities associated with $\mathrm{T}$ cell exhaustion (TCE), depletion of circulating lymphocytes, and neutrophilia (24-29). To overcome these limitations, IL-12 can be encapsulated within poly(lactic-co-glyolic) acid (PLGA) nanospheres (nIL-12). Nanoencapsulation results in entrapment of the encapsulated protein within a PLGA polymer web, which may allow for therapeutic doses of IL-12 to reach tissues while mitigating 
side effects. Indeed, in contrast to bolus administration of free cytokine, nanosphere encapsulation decreases the amount of bioavailable IL-12 per unit time as it is released more slowly pending polymer hydrolysis (30-32).

Previous studies from our group have shown that systemic NK cell depletion is a specific malignancy-induced immune deficit that worsens with increasing disease burden; as IL-12 has both mitogenic and activating influences on NK cells, we tested the utility of systemic nIL-12 therapy alongside systemic immunophenotyping to correct malignancy-induced immune deficits and inhibit metastasis formation in OS tumor-bearing mice $(9,18)$.

\section{Materials and Methods}

\section{Mice}

Male and female BALB/c mice aged 4-5 weeks (Stock Number: 000651) were obtained from The Jackson Laboratory and housed individually in ventilated Allentown cages within specific pathogen-free facilities on corncob bedding with 12 hour light/dark cycles, automatic lixit water, and ad libitum food access. All experiments were approved by the Institutional Animal Care and Use Committee (IACUC).

\section{In vivo induction of free IL-12 toxicity in healthy mice}

Two groups of four BALB/c mice (two female and two male per group) were cheek bled by piercing the facial venous plexus with a sterile $4 \mathrm{~mm}$ Goldenrod Animal Lancet (Braintree Scientific, Braintree, MA) to gather $100 \mu \mathrm{L}$ whole blood for baseline immunophenotyping. Immediately following cheek bleeds, each group was given a retro-orbital (r.o.) injection of either 
a low (100 ng) or high $(100 \mu \mathrm{g})$ dose of free recombinant mouse (rm)IL-12 (eBiolegend, San Diego, CA) with $0.1 \%$ mouse serum albumin (MSA, Sigma, St. Louis, MO) in $150 \mu$ L DPBS. At 24 and 48 hours following r.o. injections, mice underwent additional cheek bleeds for immunophenotyping.

\section{nIL-12 synthesis}

nIL-12 nanospheres were prepared using the double emulsion solvent evaporation technique (33). Briefly, $150 \mu \mathrm{L}$ of $83.3 \mathrm{mg} / \mathrm{mL}$ recombinant mouse IL-12 (rmIL-12, (eBiolegend, San Diego, CA) with 10\% mouse serum albumin (MSA, Sigma, St. Louis, MO) in DPBS was added to $250 \mathrm{mg}$ PLGA resomer RG 503H (Sigma) dissolved in $1.51 \mathrm{~mL}$ dicholoromethane (Sigma) with 14\% w/w Span 60 (Sigma); the resulting emulsion was sonicated at 50W for 10 seconds on ice before being added to $5 \mathrm{~mL}$ of $1 \% \mathrm{w} / \mathrm{v}$ polyvinyl alcohol (PVA, Sigma) with $4 \%$ w/v Tween 80 (Sigma) and sonicated again on ice at the same parameters. The resulting mixture was stirred for three hours at room temperature and washed four times; particles were stored at $80{ }^{\circ} \mathrm{C}$ until usage. Representative rmIL-12 drug elution profiles and scanning electron microscopy (SEM) nIL-12 images are displayed in supplementary Figure S1A and S1B, respectively.

\section{K7M2 syngeneic orthotopic BALB/c mouse model of metastatic OS}

K7M2 murine OS tumor cells (ATCC CRL-2836, ATCC) were kindly donated by Dr. Kurt Weiss, MD (University of Pittsburgh Medical Center, Pittsburgh, PA) in April 2014 and transfected non-virally with the Promega luc2 reporter vector (luc-K7M2) as previously described (34). Luc-K7M2 cells were Mycoplasma free and their identity confirmed in 2018 via IDEXX BioResearch Case \#6926-2018 (ID 3). For the orthotopic inoculation, luc-K7M2 cells (1 x 106) 
suspended in DMEM media were injected intratibially into 30 mice as previously described (34). Four weeks later, 12 mice with the smallest and most similarly-sized IVIS-confirmed primary tumors were randomized into three log-scale dose treatment groups 1$)$ low dose $(0.1 \mathrm{mg} \mathrm{nIL}-12)$, 2) medium dose (1 mg nIL-12), and 3) high dose (10 mg nIL-12). One week following IVISconfirmation of primary tumors, mice underwent amputation of their tumor-bearing limbs as previously described (34). At 12 weeks post-inoculation, mice were euthanized. A schematic of the full experimental design can be seen in Figure 1. The dose group and disease status for each nIL-12 treated mouse are shown in supplementary Table S1.

\section{Time point cheek bleeds in OS tumor-bearing mice}

Prior to inoculation with tumor cells (week 0), all 30 male BALB/c mice were cheek bled to collect $100 \mu \mathrm{L}$ whole blood for baseline immunophenotyping. Cheek bleeds were repeated at first sign of palpable primary tumor (week 4), prior to amputation of the affected limb (week five), and again at weeks 6 (T1), 8 (T2), 10 (T3), and 12 (euthanasia/EUTH), as shown in Figure 1.

\section{nIL-12 treatment of OS tumor-bearing mice}

Twelve tumor-bearing mice were randomized into three $n=4$ dose groups; 0.1 (low), 1 (medium), or $10 \mathrm{mg}$ (high) of nIL-12 were suspended in 0.1\% MSA in sterile DPBS. Mice received their first dose of nIL-12 following IVIS confirmation of palpable primary tumors, and then at weekly time points thereafter for a total of eight doses, as shown in Figure 1. Each nIL-12 dose was administered intraperitoneal (i.p.) in a total volume of $500 \mu \mathrm{L}$.

\section{In vivo imaging system (IVIS) imaging of OS tumor-bearing mice}


Animals were IVIS-imaged from the first sign of palpable primary tumor and at weekly intervals thereafter using the IVIS Spectrum CT imaging system (PerkinElmer Life Sciences, Waltham, MA) with Living Image version 4.5 Software to monitor disease burden. At each session, mice received $150 \mathrm{mg} / \mathrm{kg}$ i.p. D-luciferin (Caliper Life Sciences, Hopkinton, MA); to visualize metastatic disease in the lung, mice received an additional $15 \mathrm{mg} / \mathrm{kg}$ of intranasal Dluciferin (approximately $30 \mu \mathrm{L}$ ). Images were captured using auto-exposure within the predetermined interval of maximum bioluminescence.

\section{Flow cytometry (FC)}

Red blood cells were lysed with Red Blood Cell (RBC) Lysis Solution (Miltenyi Biotec, Auburn, CA). Single cell suspensions were split and incubated with optimized lymphoid (L) and myeloid (M) antibody panels (supplementary Table S2) according to the manufacturer's instructions; a minimum of $1 \times 104$ events were analyzed for each sample.

\section{Histology}

Histology was performed on the lungs of all nIL-12-treated mice that did not have IVISpositive pulmonary metastases at euthanasia; the metastatic rate of the untreated control group was assessed via a combination of histology and IVIS positivity. Lungs of nIL-12-treated mice were immediately harvested en bloc following euthanasia, placed in neutral buffered formalin, and mounted on a microtome; $50 \mu \mathrm{m}$ sections were obtained every $250 \mu \mathrm{m}$ and stained with hematoxylin and eosin, and specimens were analyzed for the presence or absence of metastases by a board-certified pathologist. 


\section{Statistical Analysis}

To determine significance between categorical clinical data (metastatic and disease-free rates), two-sided chi-squared tests were used. To compare the NK percent populations in peripheral blood between healthy and diseased nIL-12-treated mice, average values for both groups were determined at each time point and the standard deviations (SD) calculated; statistical significance was determined via unpaired two-tailed $t$ tests.

\section{Results}

Both low and high doses of free rmIL-12 induce TCE and PMN-MDSC myelocytosis while high doses induce additional NK cell depletion in peripheral blood of healthy BALB/c mice at 48 hours

In mice, the specific toxicities associated with recombinant IL-12 therapy are strain dependent (35). Therefore, we first tested the ability of rmIL-12 to induce immunotoxic events in $\mathrm{BALB} / \mathrm{c}$ mice to provide a reference for monitoring nIL-12-associated toxicities. It was observed that both low $(100 \mathrm{ng})$ and high $(100 \mu \mathrm{g})$ doses induced significant increases in TCE at 48 hours in all three $\mathrm{T}$ cell populations surveyed, including CD45+CD8-CD4+ helper T cells, CD45+CD4CD8+ cytotoxic $\mathrm{T}$ cells, and CD45+CD8-CD4+Foxp3+CD25+/- regulatory $\mathrm{T}$ cells (Figure 2). Furthermore, both doses induced strong PMN-MDSC myelocytosis at 48 hours, with low and high doses producing an average increase of $43.6 \%$ and $62.54 \%$, respectively (Figure 3); however, only high dose rmIL-12 induced significant NK cell depletion (Figure 4). Helper and cytotoxic T cells were also decreased in response to both low and high dosages; however, smaller effect sizes and 
substantial variability decreased the statistical power, and a larger $n$ will be needed to determine significance (data not shown).

\section{nIL-12 treatment reduces metastatic disease and increases disease-free rate in OS tumor- bearing mice at 12 weeks}

Between all three nIL-12 dose groups, the immunophenotypes assessed across all seven time points were surprisingly uniform (supplementary Figure S1A-H). Therefore, we conducted further analyses on combined $(n=12)$ data to assess the nIL-12 response irrespective of dose. As such, we compared the metastatic and cure rates of the 12 nIL-12-treated OS tumor-bearing mice to 34 untreated tumor-bearing mice from previous experiments. It was observed that treated mice had a combined $35.3 \%$ reduction in metastatic disease at 12 weeks, which was significant $(\mathrm{p}=$ 0.007). Further, the percentage of disease-free "healthy" (i.e., metastasis and local-recurrence negative) mice in the nIL-12 treated group was 33\% versus $5.9 \%$ in the untreated group, which was also significant $(\mathrm{p}=0.008)$; these data are displayed in Table 1 .

\section{Peripheral blood NK cell percentages above $8.21 \%$ at primary tumor amputation separate non-metastatic from metastatic mice at 12 weeks}

Of the 12 tumor-bearing mice that received nIL-12, it was observed that four were completely healthy and eight had residual disease at 12 weeks. As there can be considerable NK cell heterogeneity within mice of the same strain (36), we investigated whether differences in the NK cell immunophenotype could provide insight into whether nIL-12-treated mice achieve complete (i.e., "healthy" mice) or incomplete/no responses (i.e., "diseased" mice). Indeed, significant differences in NK cell percentages were found between healthy and diseased mice at 
both amputation and T3 time points (Figure 5A). Following the first dose of nIL-12 at palpable primary tumor (PT), healthy mice had steady-state NK cell percentages in blood for the duration of the study, whereas the NK cell percentages of diseased mice dropped significantly at time point T3 and remained low at euthanasia. Importantly, diseased mice showed a significant decrease in NK cell percentages at amputation (AMP), a time point that proceeds the development of metastases in the majority of OS patients (2). We also investigated whether there existed an NK cell "threshold" at amputation, below which nIL-12-treated mice have worse outcomes. To do this, the median NK cell percentage in peripheral blood was determined for nIL-12-treated mice at amputation. Next, mice were stratified into two groups based upon whether their NK cell percentages fell above or below the median; remarkably, mice with NK cell percentages above the median had a significantly lower metastatic rate than those with NK cell percentages below the median $(16.67 \%$ versus $66.67 \%$, respectively; $p=0.038$, Figure 5B).

\section{Discussion}

While immunotherapies are currently being used to treat a variety of different tumors, there is extreme heterogeneity amongst patient responses (37). Even within inbred laboratory mouse strains, immunological variables are pervasive; indeed, immune-related gene polymorphisms, Tcell repertoire diversity, gut microbiome diversity, variations in interactions with investigators (e.g., surgeries), and unique allelelic variations contribute to immune heterogeneity (38-42). Obviously, immune variability amongst humans is astronomically greater; therefore, it is essential to perform longitudinal real-time asessments of immune status to continually match the most effective therapeutic to the patient. 
The current method for monitoring immunotherapy efficacy is limited to evaluating disease burden, which is restricted by instrument detection limits and often misleading (e.g., pseudoprogression and hyperprogression) (43-45). Previous studies from our group have highlighted both the feasibility and clinical utility of using real-time systemic immunophenotyping to uncover tumor immune escape mechanisms and identify targetable malignancy-induced immune deficits (8). As such, this manuscript describes our preliminary in vivo investigation of the efficacy of nIL-12 therapy against metastatic OS while simultaneously conducting real-time immunophenotyping to assess systemic immune responses.

It was observed that nIL-12-treated mice, irrespective of dose, showed significantly better outcomes than untreated mice. Further, the decrease in metastatic rates was linked to increased NK cell percentages in peripheral blood at amputation. The observation that a particular component of the blood immunophenotype (NK cell percentage > $8.21 \%$ ) could potentially distinguish between complete and non-responders at such an early clinical time point (amputation) has obvious clinical implications. It is possible that the observed treatment effects are mediated by IL-12-dependent NK cell activation and repletion, although more data will be needed to test this hypothesis $(1,15)$. However, as NK cells play an integral role in tumor immunosurveillance, it is reasonable to assume that immediate correction of $\mathrm{NK}$ cell deficits and/or increased activation may lead to regression of metastatic disease in OS patients $(6,8,11-13,46)$.

Although the current study does not offer conclusive evidence regarding immunemonitoring of drug-related toxicites, we have provided a solid foundation for well-informed hypothesis generation. Indeed, the free rmIL-12 toxicity study highlighted IL-12-induced toxicities specific to $B A L B / c$ mice that could be surveyed alongside nIL-12 treatment, namely TCE status, 
PMN-MDSC myelocytosis, and NK cell depletion; these changes are similar to those seen in IL12-treated humans and nonhuman primates (24-28).

Interestingly, the TCE status of nIL-12-treated tumor-bearing mice progressed similarly across the three dose goups for all three T cell popululations assessed. Further, PMN-MDSC myelocytosis and NK cell percentages were similar across all dose groups and time points; these data may indicate that the study is underpowered to detect dose-dependent differences in immunophenotype. However, it is also possible that, due to immune heterogeneity, each response must be assessed and treated as a separate entity (i.e., using pre-treatment immunophenotype data as baseline and making subsequent assessments relative). It is also possible that the proposed antibody panels were not sensitive enough to determine dose-related differences in immunomodulation, and other immune populations or activation/deactivation markers may need to be considered. Overall, more data will be needed to make robust conclusions about using systemic immunophenotyping to assess for nIL-12-related immunotoxicity and ultimately define the upper threshold of the therapeutic window (i.e., immunotoxic threshold); no direct comparisons can be made between the free and nanoencapsulated IL-12 studies included in this manuscript, as healthy and tumor-bearing mice have significantly different background immunophenotypes $(8$, 47).

While the study described herein is preliminary, we have clearly established that free rmIL12 induces distinguishable changes in the BALB/c systemic imunopheneotype. Further, we have observed that nIL-12 therapy is efficacious against metastatic OS tumors and decreases the chances of disease relapse. Additionally, we have found that monitoring the NK cell percentages in peripheral blood may provide important information about response to nIL-12 and outcome. However, more controls will be needed to determine the therapeutic contributions of IL-12 and 
PLGA individually, and the exact amounts of IL-12 delivered per mg nIL-12 will need to be calculated. Further, nIL-12 will need to be tested in female OS tumor-bearing mice to evaluate for sex-induced differences in response rates. Regardless, we conclude that systemic immunophenotyping may be used alongside immunotherapy for clinical benefit, and its utility for monitoring drug-related immunotoxicities warrants further investigation; these data support the fact that systemic immunophenotyping and experimntal immunotherapies may be used hand-inhand to generate efficacious immunotherapeutic regimens for metastatic OS. 


\section{References}

1. Ottaviani, G., \& Jaffe, N. (2009). The epidemiology of osteosarcoma. In Pediatric and adolescent osteosarcoma (pp. 3-13). Springer, Boston, MA.

2. Lindsey, B. A., Markel, J. E., \& Kleinerman, E. S. (2017). Osteosarcoma overview. Rheumatology and therapy, 4(1), 25-43.

3. Egas-Bejar, D., Anderson, P. M., Agarwal, R., Corrales-Medina, F., Devarajan, E., Huh, W. W., ... \& Subbiah, V. (2014). Theranostic profiling for actionable aberrations in advanced high risk osteosarcoma with aggressive biology reveals high molecular diversity: the human fingerprint

4. Martin, J. W., Squire, J. A., \& Zielenska, M. (2012). The genetics of osteosarcoma. Sarcoma, 2012.

5. Wang, D., Niu, X., Wang, Z., Song, C. L., Huang, Z., Chen, K. N., ... \& Wang, Y. (2019). Multiregion sequencing reveals the genetic heterogeneity and evolutionary history of osteosarcoma and matched pulmonary metastases. Cancer research, 79(1), 7-20.

6. Markiewicz, K., Zeman, K., Kozar, A., Gołębiowska-Wawrzyniak, M., \& Woźniak, W. (2012). Evaluation of selected parameters of cellular immunity in children with osteosarcoma at diagnosis. Medycyna wieku rozwojowego, 16(3), 212-221.

7. Uehara, T., Eikawa, S., Nishida, M., Kunisada, Y., Yoshida, A., Fujiwara, T., ... \& Udono, H. (2019). Metformin induces CD11b+-cell-mediated growth inhibition of an osteosarcoma: implications for metabolic reprogramming of myeloid cells and anti-tumor effects. International immunology, 31(4), 187-198.

8. Markel, J. E., Lacinski, R. A., Stewart, A. B., Vaida, J., Pratt, H. G., Reinbeau, R. M., Mizener, A. D., Lindsey, B. A. (2020). Systems-Wide Immunophenotyping Defines MalignancyInduced Immunological Changes in an Immunocompetent K7M2 Orthotopic Murine Model of Osteosarcoma. Unpublished manuscript.

9. Gonzalez, H., Robles, I., \& Werb, Z. (2018). Innate and acquired immune surveillance in the postdissemination phase of metastasis. The FEBS journal, 285(4), 654-664.

10. Smyth, M. J., Swann, J., \& Hayakawa, Y. (2007). Innate tumor immune surveillance. In Crossroads between Innate and Adaptive Immunity (pp. 103-111). Springer, Boston, MA.

11. Guma, S. R., Lee, D. A., Yu, L., Gordon, N., Hughes, D., Stewart, J., ... \& Kleinerman, E. S. (2014). Natural killer cell therapy and aerosol interleukin-2 for the treatment of osteosarcoma lung metastasis. Pediatric blood \& cancer, 61(4), 618-626.

12. Guma, S. R., Lee, D. A., Ling, Y., Gordon, N., \& Kleinerman, E. S. (2014). Aerosol interleukin-2 induces natural killer cell proliferation in the lung and combination therapy improves the survival of mice with osteosarcoma lung metastasis. Pediatric blood \& cancer, 61(8), 1362-1368.

13. Fernández, L., Valentin, J., Zalacain, M., Leung, W., Patino-Garcia, A., \& PérezMartínez, A. (2015). Activated and expanded natural killer cells target osteosarcoma tumor initiating cells in an NKG2D-NKG2DL dependent manner. Cancer letters, 368(1), 54-63.

14. Gee, K., Guzzo, C., Mat, C., Nor, F., Ma, W., \& Kumar, A. (2009). The IL-12 family of cytokines in infection, inflammation and autoimmune disorders. Inflammation \& Allergy-Drug Targets (Formerly Current Drug Targets-Inflammation \& Allergy), 8(1), 40-52.

15. Lu, X. (2017). Impact of IL-12 in Cancer. Current cancer drug targets, 17(8), 682-697. 16. Lasek, W., Zagożdżon, R., \& Jakobisiak, M. (2014). Interleukin 12: still a promising candidate for tumor immunotherapy?. Cancer Immunology, Immunotherapy, 63(5), 419-435. 
17. Hsieh, C. S., Macatonia, S. E., Tripp, C. S., Wolf, S. F., O'Garra, A., \& Murphy, K. M. (1993). Development of TH1 CD4+ T cells through IL-12 produced by Listeria-induced macrophages. Science, 260(5107), 547-549.

18. Perussia, B. I. C. E., Chan, S. H., D'Andrea, A. N. N. A. L. I. S. A., Tsuji, K. O. J. I. R. O., Santoli, D. A. N. I. E. L. A., Pospisil, M. I. L. A. N., ... \& Trinchieri, G. (1992). Natural killer (NK) cell stimulatory factor or IL-12 has differential effects on the proliferation of TCR-alpha beta+, TCR-gamma delta+ T lymphocytes, and NK cells. The Journal of Immunology, 149(11), 3495-3502.

19. Tripp, C. S., Wolf, S. F., \& Unanue, E. R. (1993). Interleukin 12 and tumor necrosis factor alpha are costimulators of interferon gamma production by natural killer cells in severe combined immunodeficiency mice with listeriosis, and interleukin 10 is a physiologic antagonist. Proceedings of the National Academy of Sciences, 90(8), 3725-3729.

20. Nastala, C. L., Edington, H. D., McKinney, T. G., Tahara, H., Nalesnik, M. A., Brunda, M. J., ... \& Storkus, W. J. (1994). Recombinant IL-12 administration induces tumor regression in association with IFN-gamma production. The Journal of Immunology, 153(4), 1697-1706.

21. Uemura, A., Takehara, T., Miyagi, T., Suzuki, T., Tatsumi, T., Ohkawa, K., ... \& Hayashi, N. (2010). Natural killer cell is a major producer of interferon $\gamma$ that is critical for the IL-12-induced anti-tumor effect in mice. Cancer immunology, immunotherapy, 59(3), 453.

22. Hashimoto, W., Osaki, T., Okamura, H., Robbins, P. D., Kurimoto, M., Nagata, S., ... \& Tahara, H. (1999). Differential antitumor effects of administration of recombinant IL-18 or recombinant IL-12 are mediated primarily by Fas-Fas ligand-and perforin-induced tumor apoptosis, respectively. The Journal of Immunology, 163(2), 583-589.

23. Brunda, M. J., Luistro, L., Warrier, R. R., Wright, R. B., Hubbard, B. R., Murphy, M., ... \& Gately, M. K. (1993). Antitumor and antimetastatic activity of interleukin 12 against murine tumors. The Journal of experimental medicine, 178(4), 1223-1230.

24. Atkins, M. B., Robertson, M., Gordon, M. S., Lotze, M. T., DuBois, J., Ritz, J., ... \& Sherman, M. L. (1996). Phase I evaluation of intravenous recombinant human interleukin 12 (rhIL-12) in patients with advanced malignancies. In Proc Am Soc Clin Oncol (Vol. 15, p. 270). 25. Cohen, J. (1995). IL-12 deaths: explanation and a puzzle. Science, 270(5238), 908-909. 26. Cavallo, F., Di Carlo, E., Butera, M., Verrua, R., Colombo, M. P., Musiani, P., \& Forni, G. (1999). Immune events associated with the cure of established tumors and spontaneous metastases by local and systemic interleukin 12. Cancer research, 59(2), 414-421.

27. Spitzer, M. H., Carmi, Y., Reticker-Flynn, N. E., Kwek, S. S., Madhireddy, D., Martins, M. M., ... \& Fong, L. (2017). Systemic immunity is required for effective cancer immunotherapy. Cell, 168(3), 487-502.

28. Yang, Z. Z., Grote, D. M., Ziesmer, S. C., Niki, T., Hirashima, M., Novak, A. J., ... \& Ansell, S. M. (2012). IL-12 upregulates TIM-3 expression and induces T cell exhaustion in patients with follicular B cell non-Hodgkin lymphoma. The Journal of clinical investigation, 122(4), 1271-1282.

29. Heim, C. E., Vidlak, D., Scherr, T. D., Hartman, C. W., Garvin, K. L., \& Kielian, T. (2015). IL-12 promotes myeloid-derived suppressor cell recruitment and bacterial persistence during Staphylococcus aureus orthopedic implant infection. The Journal of Immunology, 194(8), 3861-3872. (4-7).

30. Semete, B., Booysen, L., Lemmer, Y., Kalombo, L., Katata, L., Verschoor, J., \& Swai, H. S. (2010). In vivo evaluation of the biodistribution and safety of PLGA nanoparticles as drug delivery systems. Nanomedicine: Nanotechnology, Biology and Medicine, 6(5), 662-671. 
31. Snehalatha, M., Venugopal, K., Saha, R. N., Babbar, A. K., \& Sharma, R. K. (2008). Etoposide loaded PLGA and PCL nanoparticles II: biodistribution and pharmacokinetics after radiolabeling with Tc-99m. Drug delivery, 15(5), 277-287.

32. Makadia, H. K., \& Siegel, S. J. (2011). Poly lactic-co-glycolic acid (PLGA) as biodegradable controlled drug delivery carrier. Polymers, 3(3), 1377-1397.

33. Iqbal, M., Zafar, N., Fessi, H., \& Elaissari, A. (2015). Double emulsion solvent evaporation techniques used for drug encapsulation. International journal of pharmaceutics, 496(2), 173-190.

34. Grisez, B. T., Ray, J. J., Bostian, P. A., Markel, J. E., \& Lindsey, B. A. (2018). Highly metastatic K7M2 cell line: a novel murine model capable of in vivo imaging via luciferase vector transfection. Journal of Orthopaedic Research®, 36(8), 2296-2304.

35. Car, B. D., Eng, V. M., Lipman, J. M., \& Anderson, T. D. (1999). The toxicology of interleukin-12: a review. Toxicologic pathology, 27(1), 58-63.

36. Morelli, L., Lusignan, Y., \& Lemieux, S. (1992). Heterogeneity of natural killer cell subsets in NK-1.1+ and NK-1.1- inbred mouse strains and their progeny. Cellular immunology, 141(1), 148-160.

37. Caswell, D. R., \& Swanton, C. (2017). The role of tumour heterogeneity and clonal cooperativity in metastasis, immune evasion and clinical outcome. BMC medicine, 15(1), 133.

38. Nikolich-Žugich, J., Slifka, M. K., \& Messaoudi, I. (2004). The many important facets of T-cell repertoire diversity. Nature Reviews Immunology, 4(2), 123-132.

39. Casellas, J. (2011). Inbred mouse strains and genetic stability: a review. Animal, 5(1), 17.

40. Brodin, P., Jojic, V., Gao, T., Bhattacharya, S., Angel, C. J. L., Furman, D., ... \& Maecker, H. T. (2015). Variation in the human immune system is largely driven by non-heritable influences. Cell, 160(1-2), 37-47.

41. Friswell, M. K., Gika, H., Stratford, I. J., Theodoridis, G., Telfer, B., Wilson, I. D., \& McBain, A. J. (2010). Site and strain-specific variation in gut microbiota profiles and metabolism in experimental mice. PloS one, 5(1).

42. Campbell, J. H., Foster, C. M., Vishnivetskaya, T., Campbell, A. G., Yang, Z. K., Wymore, A., ... \& Podar, M. (2012). Host genetic and environmental effects on mouse intestinal microbiota. The ISME journal, 6(11), 2033-2044.

43. Chiou, V. L., \& Burotto, M. (2015). Pseudoprogression and immune-related response in solid tumors. Journal of Clinical Oncology, 33(31), 3541.

44. Duchnowska, R. (2017). Pseudoprogression during immunotherapy of cancers. Oncology in Clinical Practice, 13(2), 57-60.

45. Denis, M., Duruisseaux, M., Brevet, M., \& Dumontet, C. (2020). How Can Immune Checkpoint Inhibitors Cause Hyperprogression in Solid Tumors?. Frontiers in Immunology, 11. 46. Waldhauer, I., \& Steinle, A. (2008). NK cells and cancer immunosurveillance. Oncogene, 27(45), 5932-5943.

47. Markel, J. E., Noore, J., Emery, E. J., Bobnar, H. J., Kleinerman, E. S., \& Lindsey, B. A. (2018). Using the Spleen as an in vivo systemic immune barometer alongside osteosarcoma disease progression and Immunotherapy with $\alpha$-PD-L1. Sarcoma, 2018. 


\section{Figure Legends}

Figure 1 Schematic depicting experimental design. Thirty male BALB/c mice were cheek bled (red arrows) for $100 \mu \mathrm{L}$ whole blood before being inoculated intratibially with 1 x 106 luc-K7M2 OS tumor cells. The first 12 mice to establish primary tumors were cheek bled again and subsequently randomized into three $n=4 \mathrm{nIL}-12$ dosing groups (low dose: $0.1 \mathrm{mg}$, medium dose: $1 \mathrm{mg}$, or high dose: $10 \mathrm{mg}$ ). The first dose of nIL-12 (blue arrows) was given via intraperitoneal (i.p.) injection following IVIS-confirmation of primary tumors. Mice were imaged via IVIS and dosed at weekly intervals thereafter, and successive cheek bleeds were performed at weeks 5, 6, 8, 10, and 12. Immediately following the week 12 cheek bleed, mice were euthanized. At week 5 , the tumor-bearing limbs were amputated.

Figure 2 rmIL-12-induced $T$ cell exhaustion of helper, cytotoxic, and regulatory $T$ lymphocyte subsets in peripheral blood. Twelve BALB/c mice were cheek bled for $100 \mu \mathrm{L}$ whole blood (baseline) before being dosed with either $100 \mathrm{ng}$ (L: low dose) or $100 \mu \mathrm{g}$ (H: high dose) of recombinant mouse(rm)IL-12 via retro-orbital (r.o.) injection. Mice were cheek bled again at 24 and 48 hours (48h), and blood samples were immunophenotyped via flow cytometry. CD8CD4+ helper $\mathrm{T}$ cells (left, white), CD4-CD8+ cytotoxic T cells (middle, gray), and CD8CD4+FOXP3+CD25+/- regulatory T cells (right, black) were assessed for the extent of PD1+TIM$3+$ positivity, which is indicative of exhaustion. Shown here are T cell data from baseline and $48 \mathrm{~h}$ time points. Individual bars (top half) represent group averages $+\mathrm{SD}$. The table (bottom half) shows the group averages \pm SD for each treatment group and T cell subset. Baseline blood samples 
( $n=4 ; 2$ male and 2 female per group) were compared to $48 \mathrm{~h}$ samples using unpaired two-tailed $t$ tests. ${ }^{*} \mathrm{p}<0.05, * * \mathrm{p}<0.01, * * * \mathrm{p}<0.001$.

Figure 3 rmIL-12-induced polymorphonuclear-myeloid-derived suppressor cell (PMNMDSC) myelocytosis in peripheral blood. Twelve BALB/c mice were cheek bled for $100 \mu \mathrm{L}$ whole blood (baseline) before being dosed with either $100 \mathrm{ng}$ (L: low dose) or $100 \mu \mathrm{g}$ (H: high dose) of recombinant mouse(rm)IL-12 via retro-orbital (r.o.) injection. Mice were cheek bled again at 24 and 48 hours (48h), and blood samples were immunophenotyped via flow cytometry. Shown here are PMN-MDSC data from baseline and 48h time points. Individual bars (top half) represent group averages $+\mathrm{SD}$. The table (bottom half) shows the group averages \pm SD for each treatment group. Baseline blood samples ( $n=4 ; 2$ male and 2 female per group) were compared to $48 \mathrm{~h}$ samples using unpaired two-tailed $t$ tests. $* \mathrm{p}<0.05, * * \mathrm{p}<0.01, * * * \mathrm{p}<0.001$.

Figure 4 IL-12-induced decrease of circulating Natural Killer (NK) cells. Twelve BALB/c mice were cheek bled for $100 \mu \mathrm{L}$ whole blood (baseline) before being dosed with either $100 \mathrm{ng}$ (L: low dose) or $100 \mu \mathrm{g}$ (H: high dose) of recombinant mouse(rm)IL-12 via retro-orbital (r.o.) injection. Mice were cheek bled again at 24 and 48 hours (48h), and blood samples were immunophenotyped via flow cytometry. Invidual bars (top half) represent group averages + S.D. The table (bottom half) shows the group averages \pm SD for each treatment group. Baseline blood samples ( $\mathrm{n}=4 ; 2$ male and 2 female per group) were compared to $48 \mathrm{~h}$ samples using unpaired two-tailed $t$ tests. $* \mathrm{p}<0.05, * * \mathrm{p}<0.01, * * * \mathrm{p}<0.001$ 
Figure 5 Blood NK cell percentages above $8.21 \%$ at amputation separate non-metastatic from metastatic mice at 12 weeks. nIL-12-treated OS tumor-bearing mice were separated into two groups based upon disease status at 12 weeks including $n=8$ diseased (metastasis and/or local recurrence positive) and $n=4$ healthy (both metastasis and local recurrence negative) mice. (A) Average $( \pm$ SD) percentage of NKp46+ Natural Killer (NK) cells in the peripheral blood of healthy (tumor burden negative at 12 weeks, green circles) versus diseased (tumor burden positive at 12 weeks, red triangles) compared across seven time points for 12 weeks. *, p < 0.05; **, p < 0.01; ***, $\mathrm{p}<0.001$ using unpaired two-tailed $t$ tests. (B) Mice were stratified based upon their NK cell percentages in peripheral blood at amputation. Each bar represents the extent to which that individual mouse's NK cell percentage deviated from the median value $(8.21 \%)$. The metastatic rate of mice above the median was compared to that of those below using a one-sided chi-squared test; $p$ met $=p$ value for the metastatic rate between groups with NK cell percentages above and below the median at amputation.

\section{Supplementary Figure S1 rmIL-12 elution profile and morphology of nIL-12. (A)} Representative in vitro nIL-12 elution profile over the course of 15 days. 100 billion particles suspended in $0.5 \mathrm{~mL}$ of protein release buffer (10\% heat-inactivated fetal bovine serum in DPBS) were incubated at $37^{\circ} \mathrm{C}$ with vigorous agitation over 15 days. Every 24 hours, the supernatant was sampled by pelleting, aspiration, and buffer replacement; protein concentrations were determined via ELISA. (B) Representative scanning electron micrograph of nIL-12 particles showing the characteristic spherical morphology and submicron diameter. 


\section{Supplementary Figure S2 Pertinent lymphoid and myeloid lineage immunophenotypes for}

nIL-12-treated OS tumor-bearing mice by dose group. At the first sign of palpable primary tumors, 12 OS tumor-bearing male BALB/c mice were randomized into three groups of $n=$ four and treated with i.p. nIL-12 at weekly intervals. Each group received the same dose $(0.1 \mathrm{mg}$ [low/orange square], $1 \mathrm{mg}$ [medium/purple triangle], or $10 \mathrm{mg}$ [high/blue circle]) for a total of eight doses across 12 weeks. Mice were cheek bled for $100 \mu \mathrm{L}$ whole blood at seven time points throughout the study for immunophenotyping purposes. Shown here are eight pertinent components of the systemic (peripheral blood) immunophenotype for each dose group, including (A) NKp46 NK cells, (B) CD8-CD4+ helper T lymphocytes, (C) CD4-CD8+ cytotoxic T lymphocytes, (D) CD8-CD4+Foxp3+ regulatory T lymphocytes, (E) PD-1+TIM-3+ helper T lymphocytes, (F) PD-1+TIM-3+ cytotoxic T lymphocytes, (G) PD-1+TIM-3+ regulatory T lymphocytes, and (H) CD11b+Ly6Clo/Ly6G+ PMN-MDSCs. Individual data points represent the group average $\pm \mathrm{SD}$ across all seven time points for each immune constituent. INOC $=$ inoculation (week 0), PT = IVIS-confirmed primary tumor $($ week 4), AMP = amputation of tumor-bearing limb (week 5), T1 = post-amputation time point $1($ week 6$), \mathrm{T} 2=$ post-amputation time point 2 (week 8$),$ T3 = post-amputation time point 3 (week 10), EUTH = euthanasia (week 12). 
Figure 1

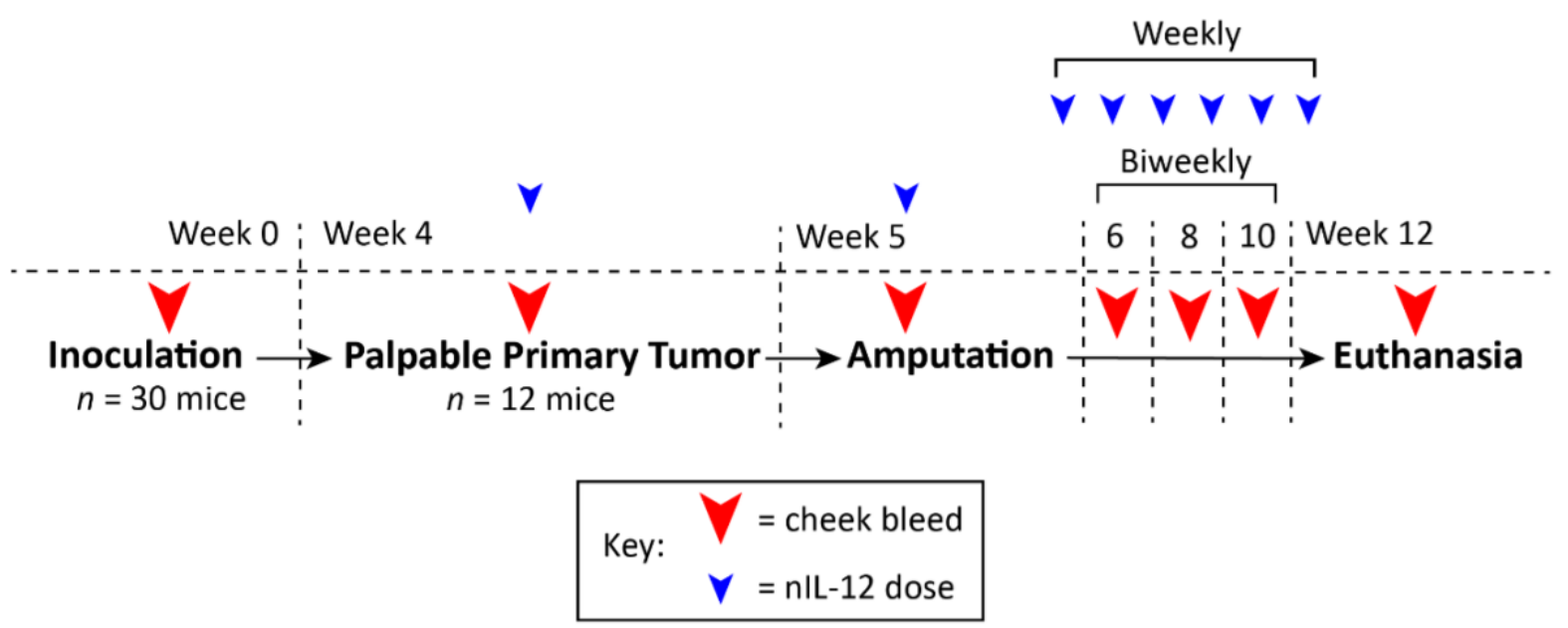


Figure 2

\section{$\underline{\text { IL-12-Induced T cell Exhaustion }}$}

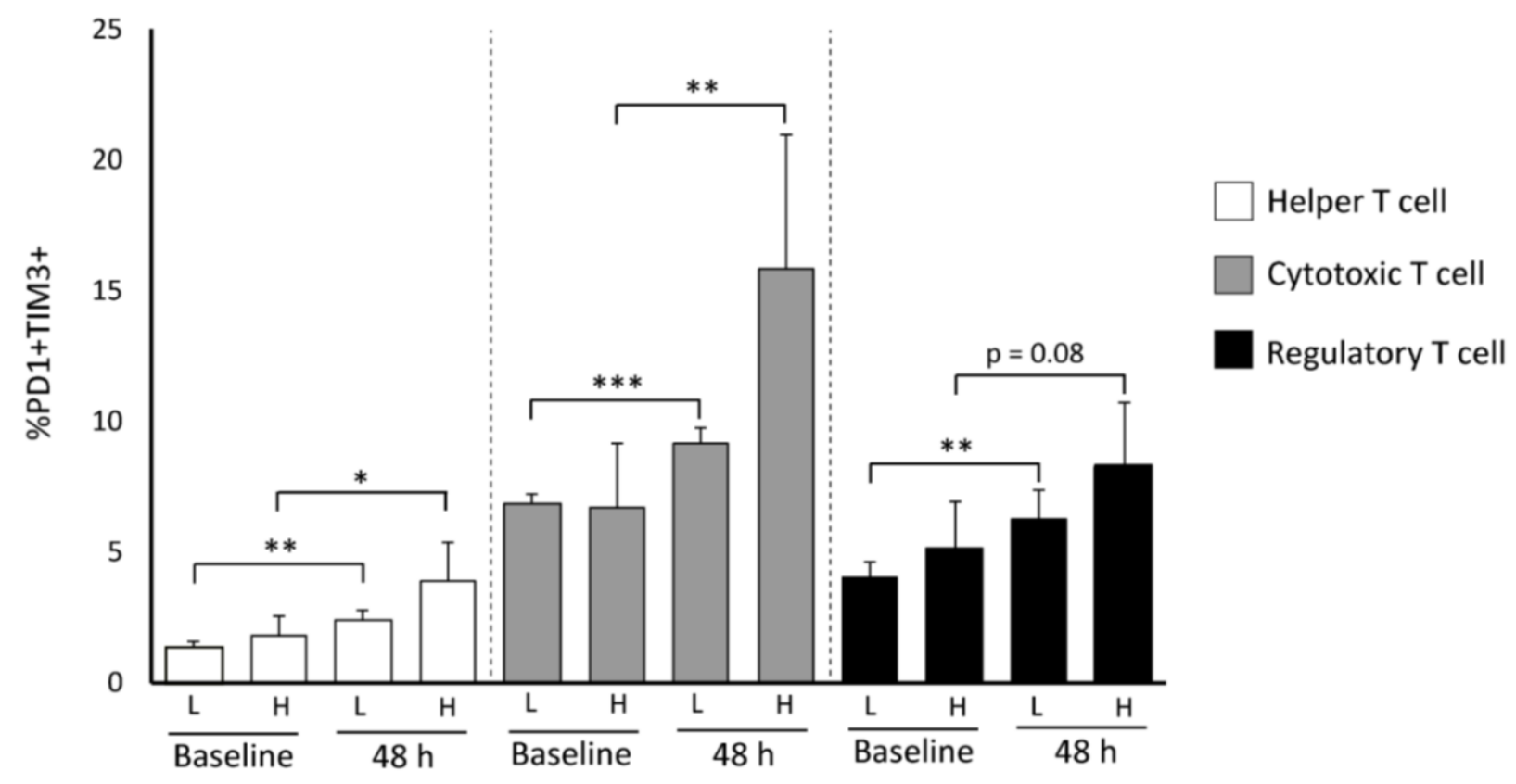

\begin{tabular}{|l|c|c|c|}
\hline T cell Subset & Dose & $\begin{array}{c}\text { Baseline } \\
\text { (\% Tim3+PD1+) }\end{array}$ & $\begin{array}{c}48 \mathrm{~h} \\
\text { (\% Tim3+PD1+) }\end{array}$ \\
\hline \multirow{2}{*}{ Helper T cell } & $\mathrm{L}$ & $1.36 \pm 0.23$ & $2.39 \pm 0.38$ \\
\cline { 2 - 4 } & $\mathrm{H}$ & $1.80 \pm 0.79$ & $3.87 \pm 1.50$ \\
\hline Cytotoxic T cell & $\mathrm{L}$ & $6.84 \pm 0.39$ & $9.15 \pm 0.61$ \\
\cline { 2 - 4 } & $\mathrm{H}$ & $6.70 \pm 2.50$ & $15.87 \pm 5.12$ \\
\hline Regulatory T cell & $\mathrm{L}$ & $4.05 \pm 0.59$ & $6.25 \pm 1.13$ \\
\cline { 2 - 4 } & $\mathrm{H}$ & $5.18 \pm 1.74$ & $8.33 \pm 2.43$ \\
\hline
\end{tabular}


Figure 3

\section{$\underline{\text { IL-12-Induced PMN-MDSC Increase }}$}

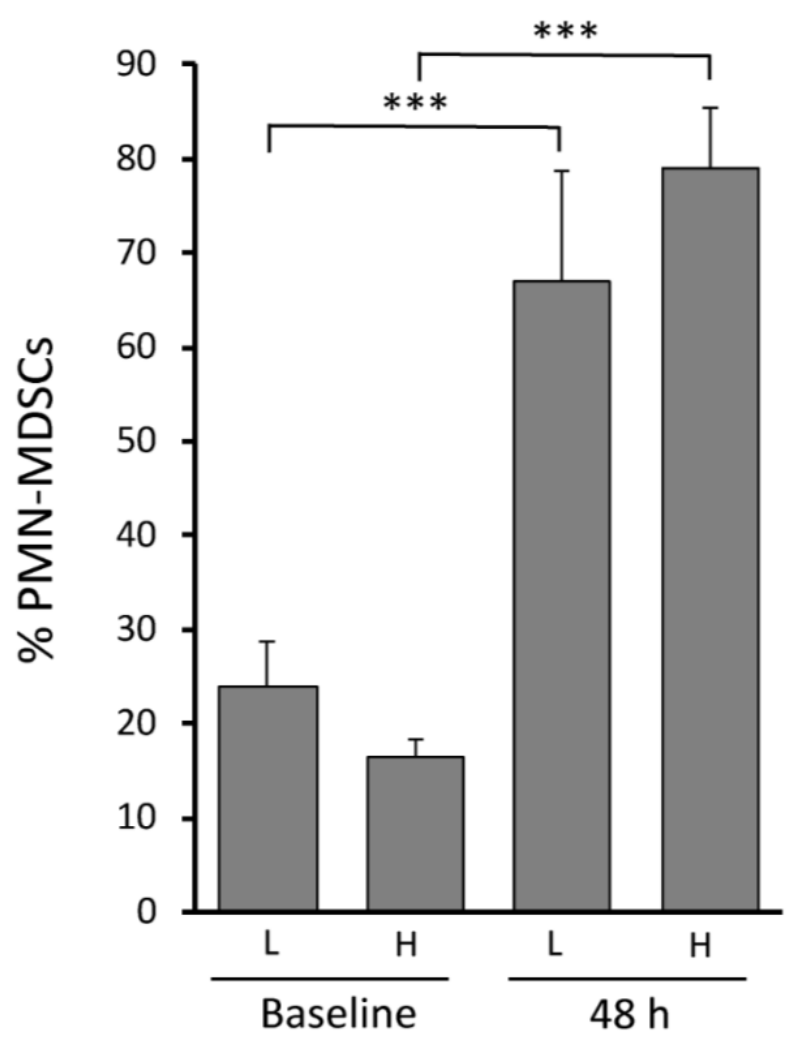

\begin{tabular}{|l|c|c|}
\hline Dose & $\begin{array}{c}\text { Baseline } \\
\text { (\% PMN-MDSCs) }\end{array}$ & $\begin{array}{c}48 \mathrm{~h} \\
\text { (\% PMN-MDSCs) }\end{array}$ \\
\hline L & $23.85 \pm 4.95$ & $67.10 \pm 11.74$ \\
\hline H & $16.39 \pm 2.00$ & $78.93 \pm 6.49$ \\
\hline
\end{tabular}


Figure 4

\section{IL-12-Induced NK cell Decrease}

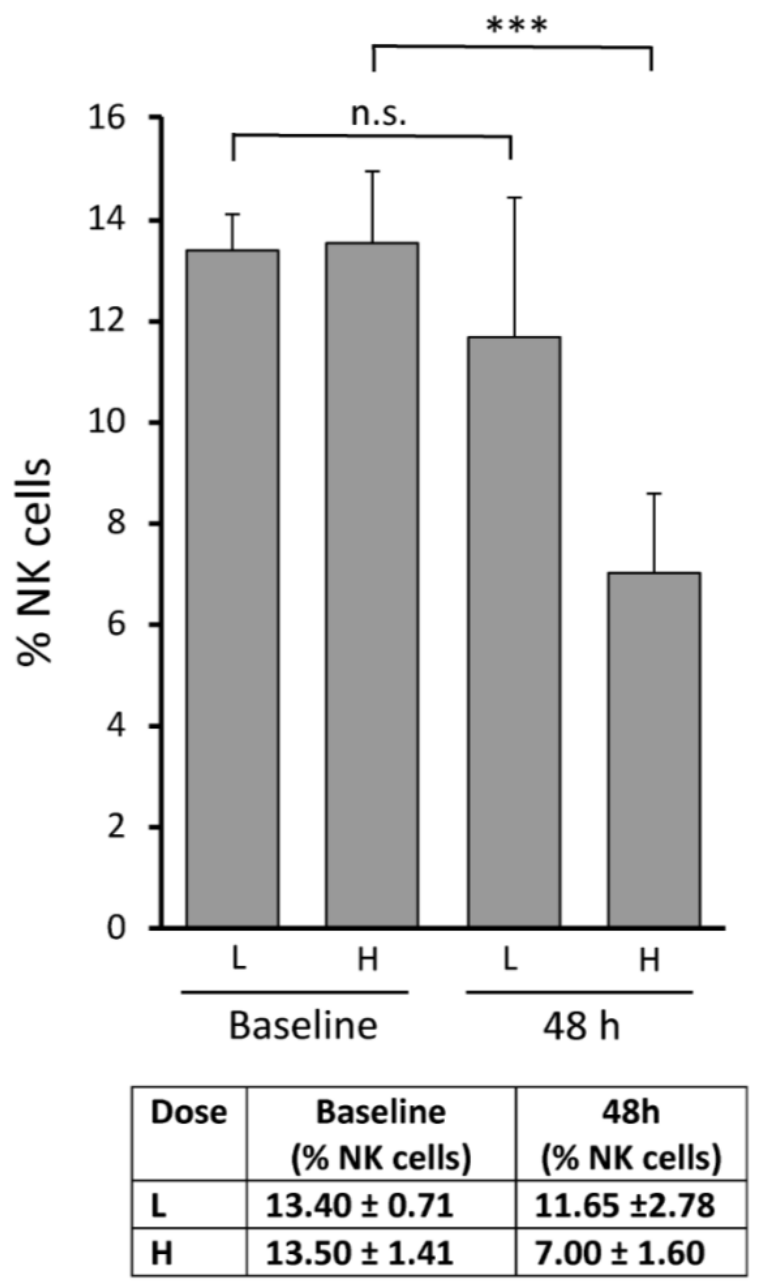


Figure 5

A.

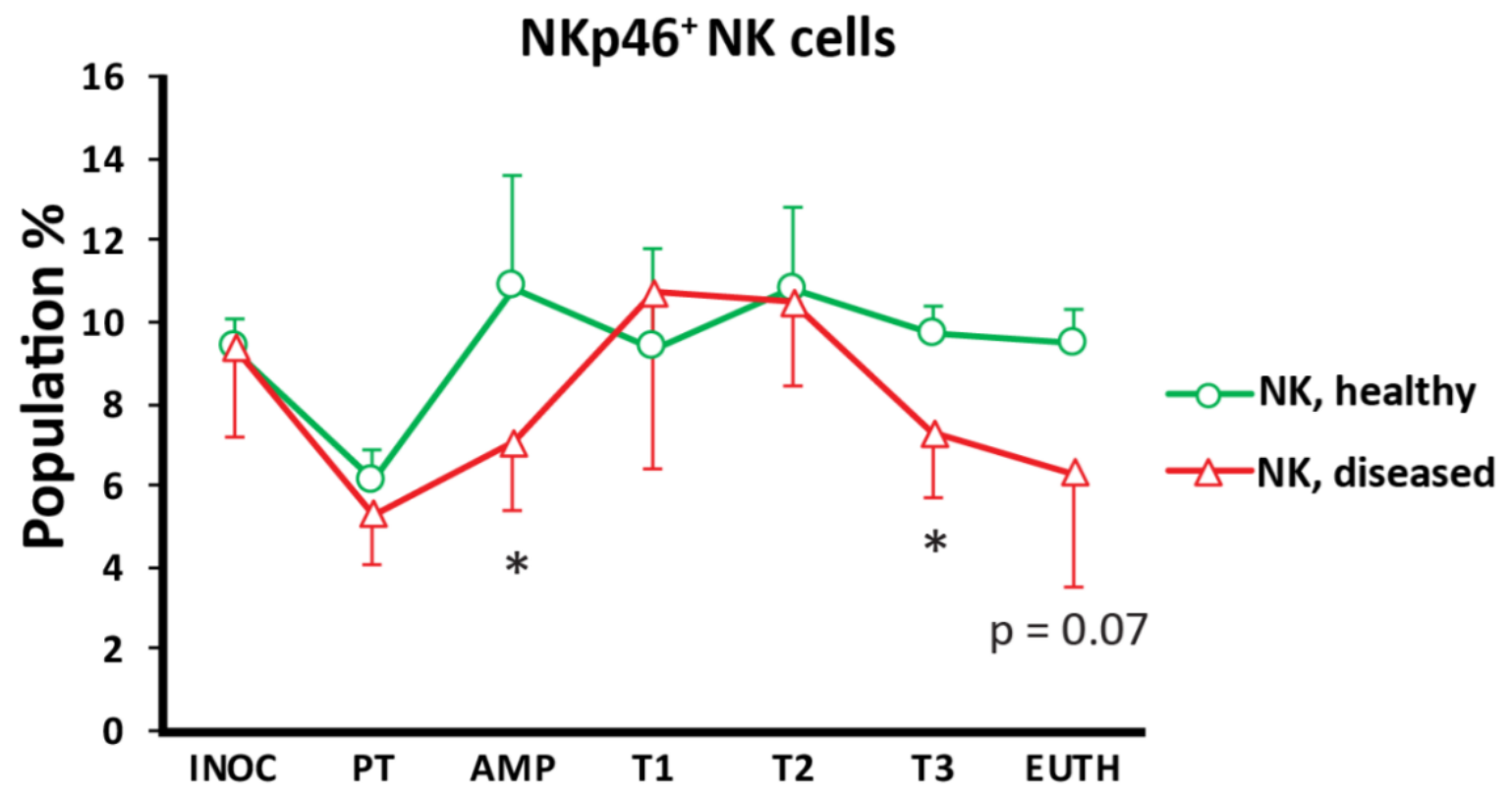

B.

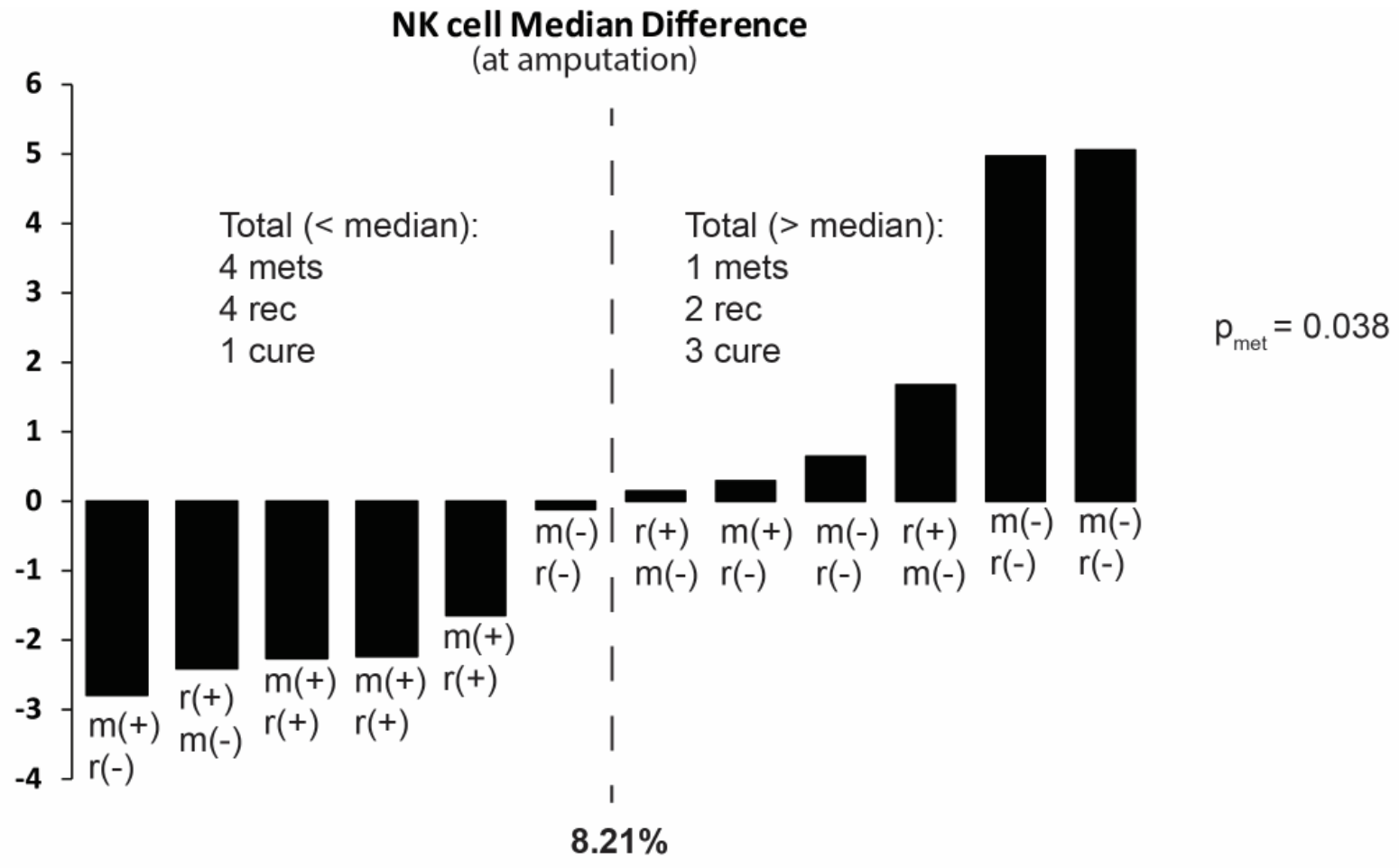




\section{Supplementary Figure S1}

A.

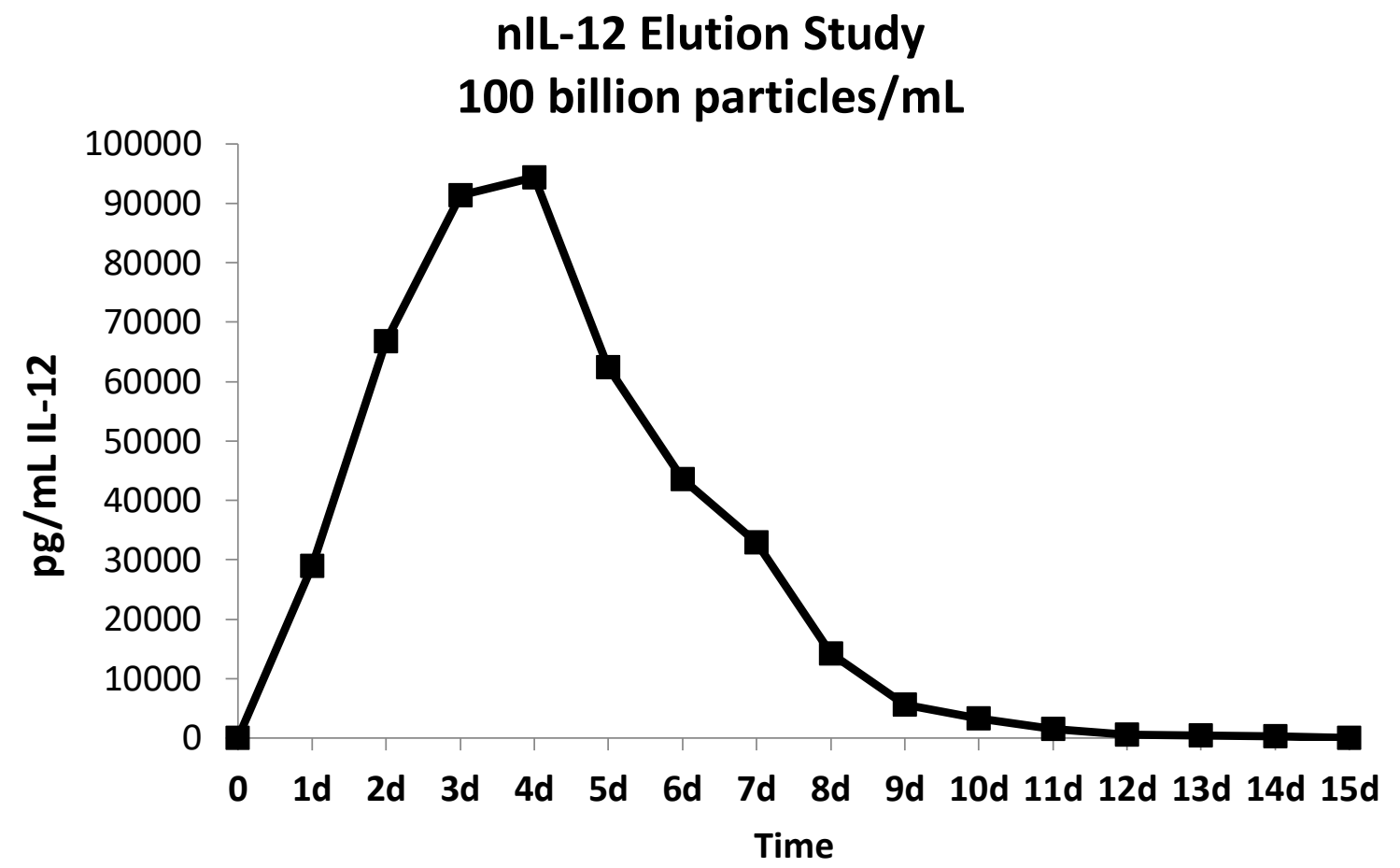

B.

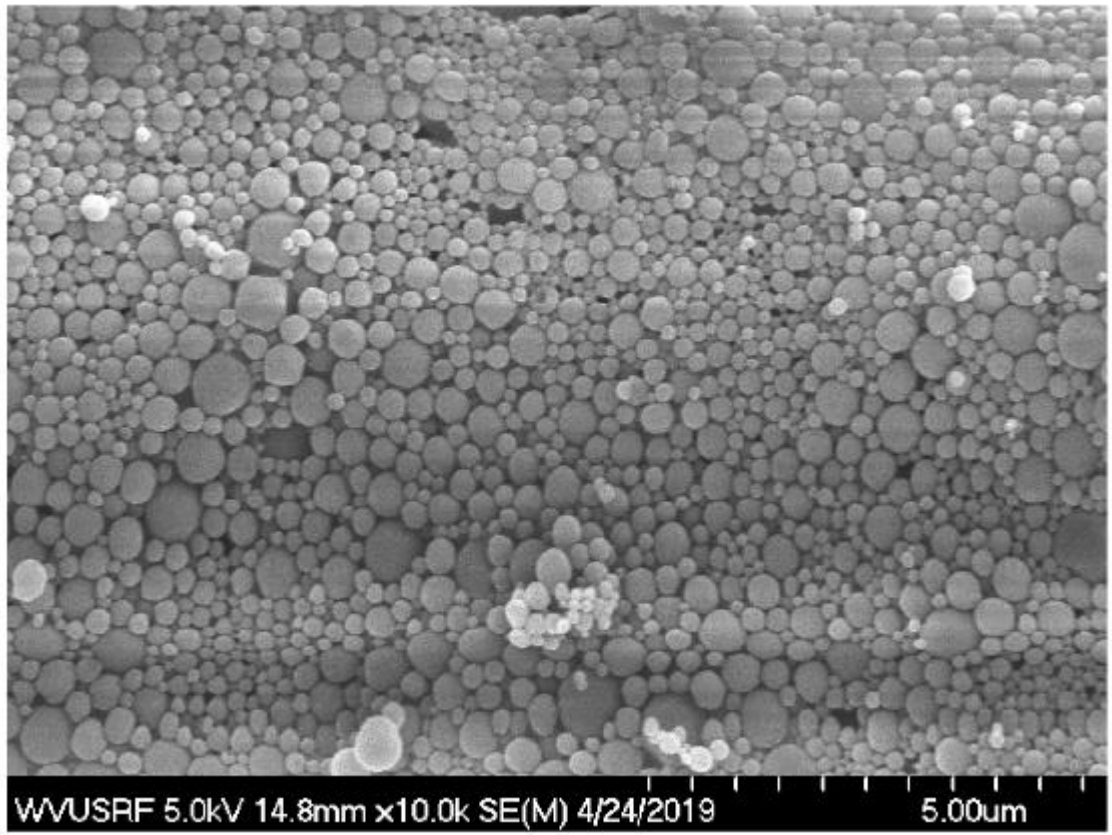




\section{Supplementary Figure S2}

A.

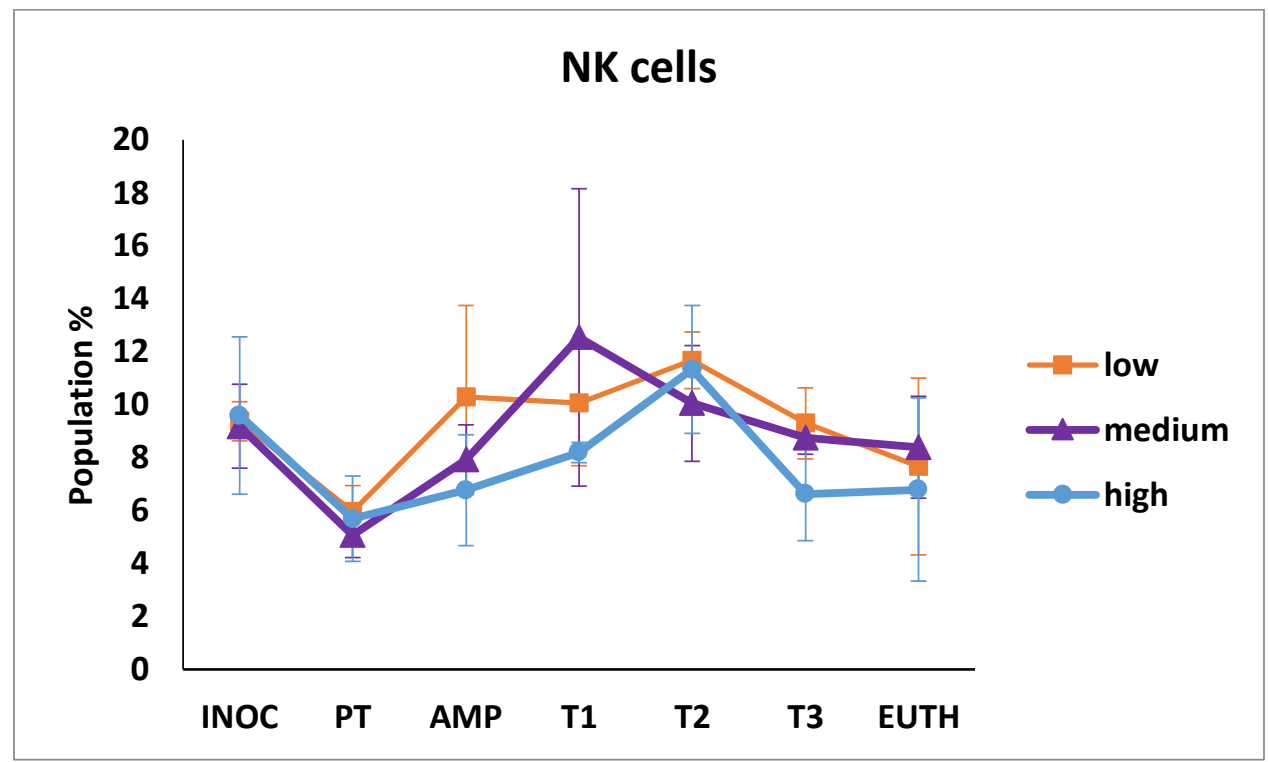

B.

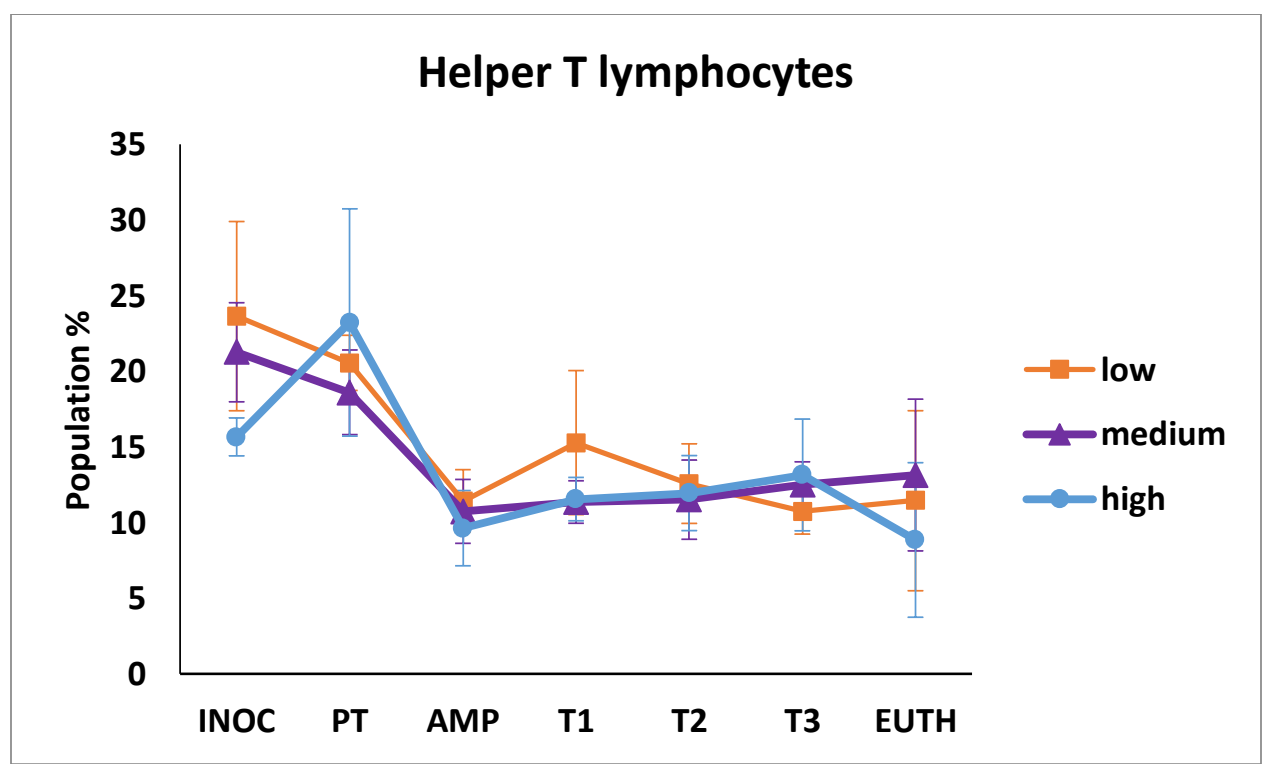


c.

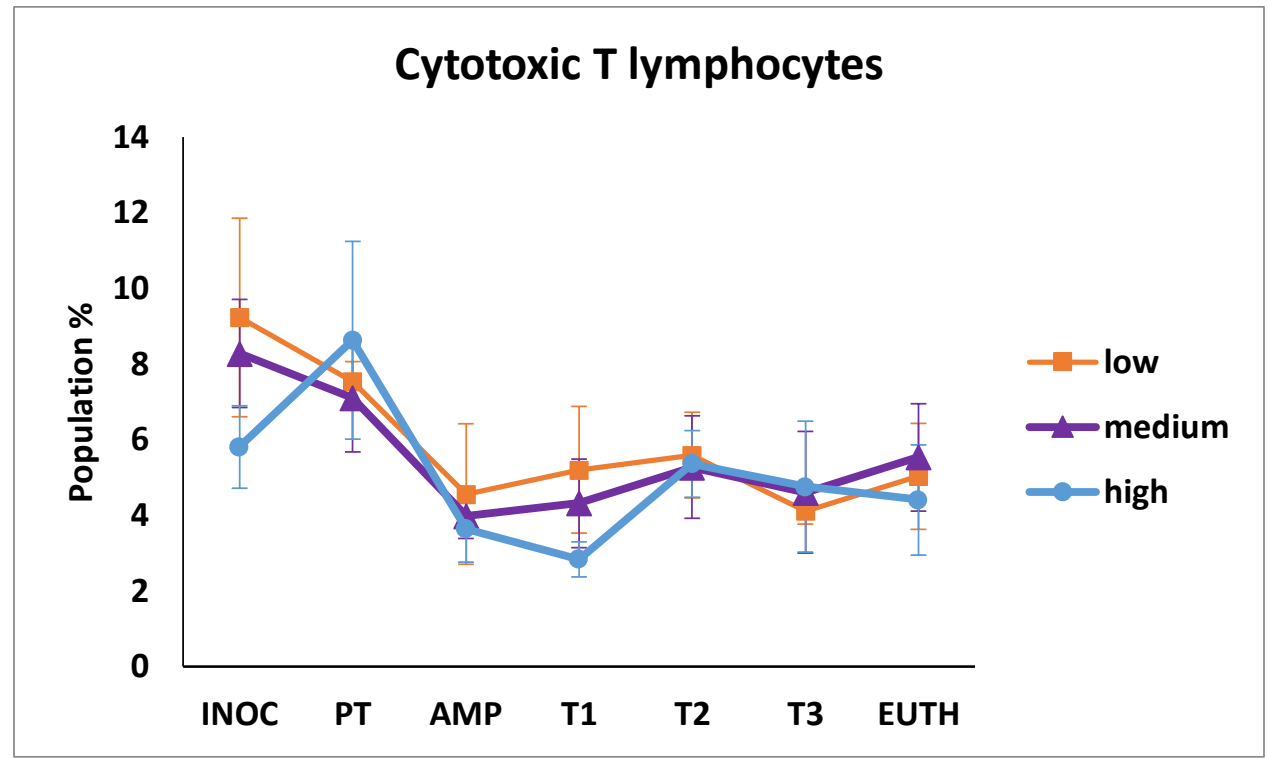

D.

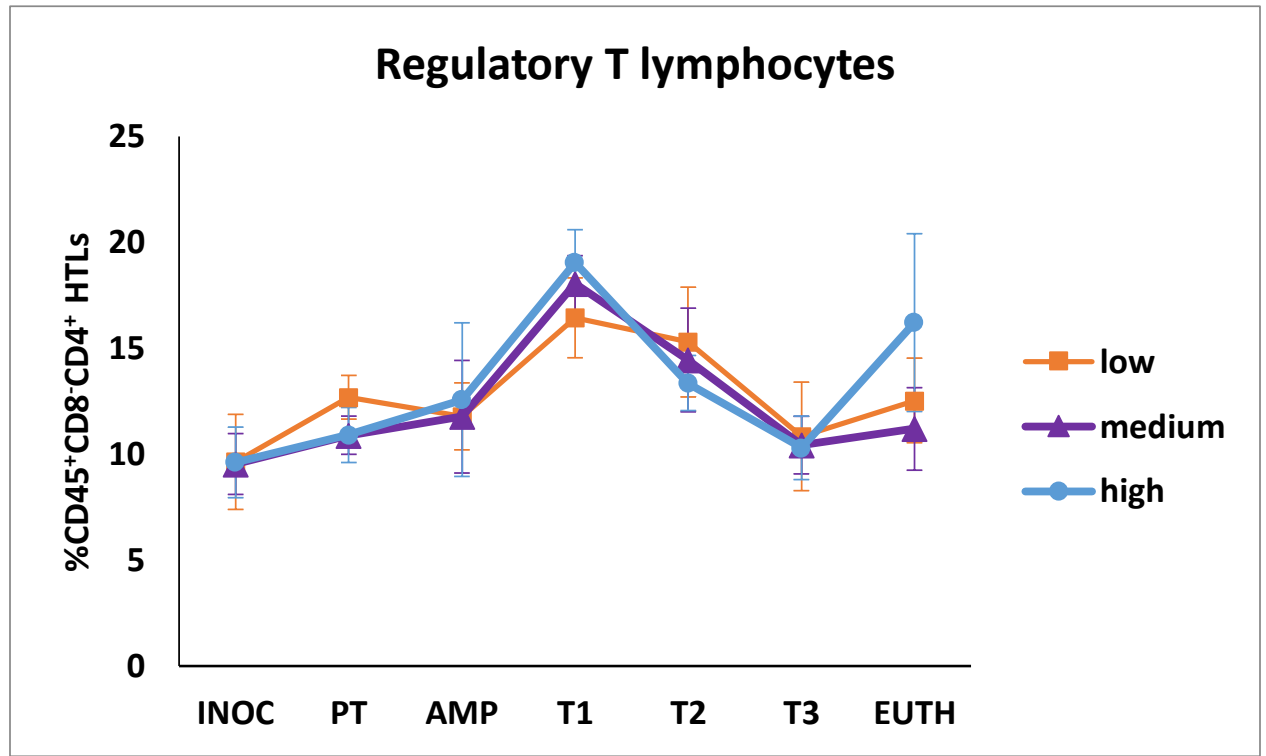


E.

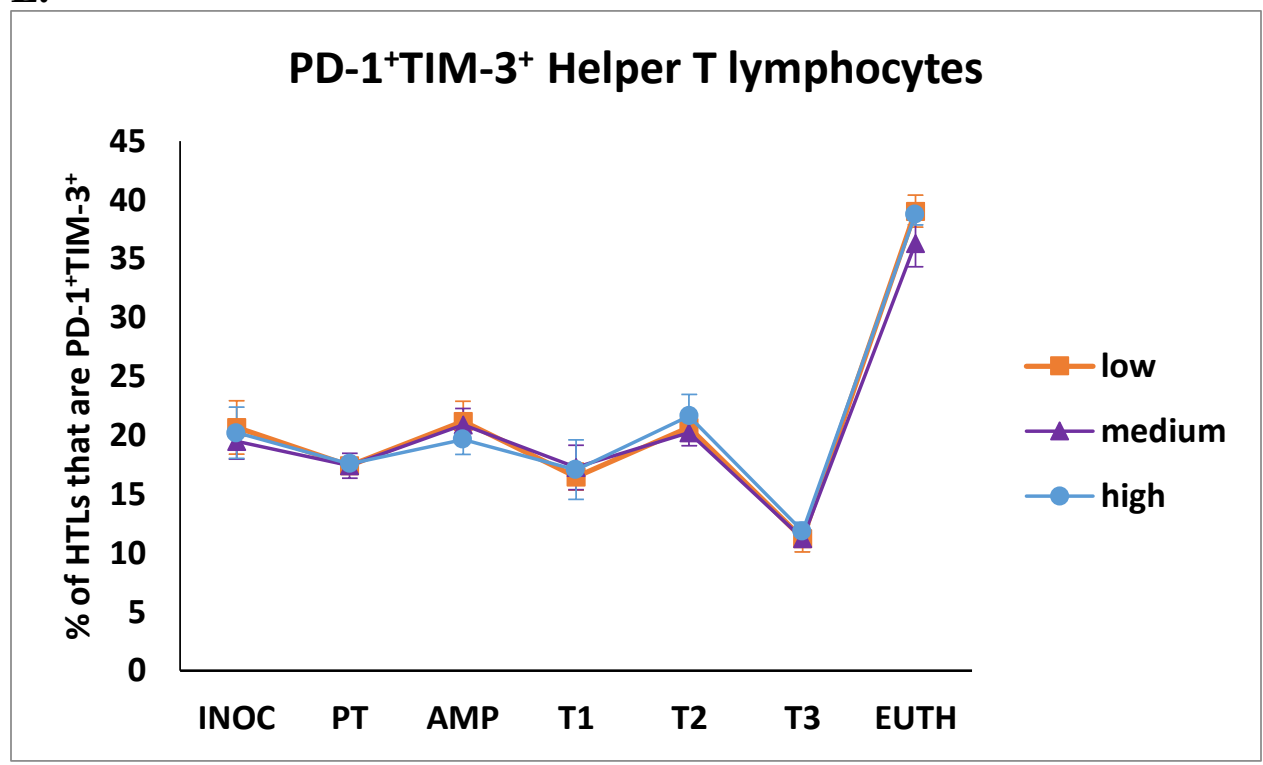

F.

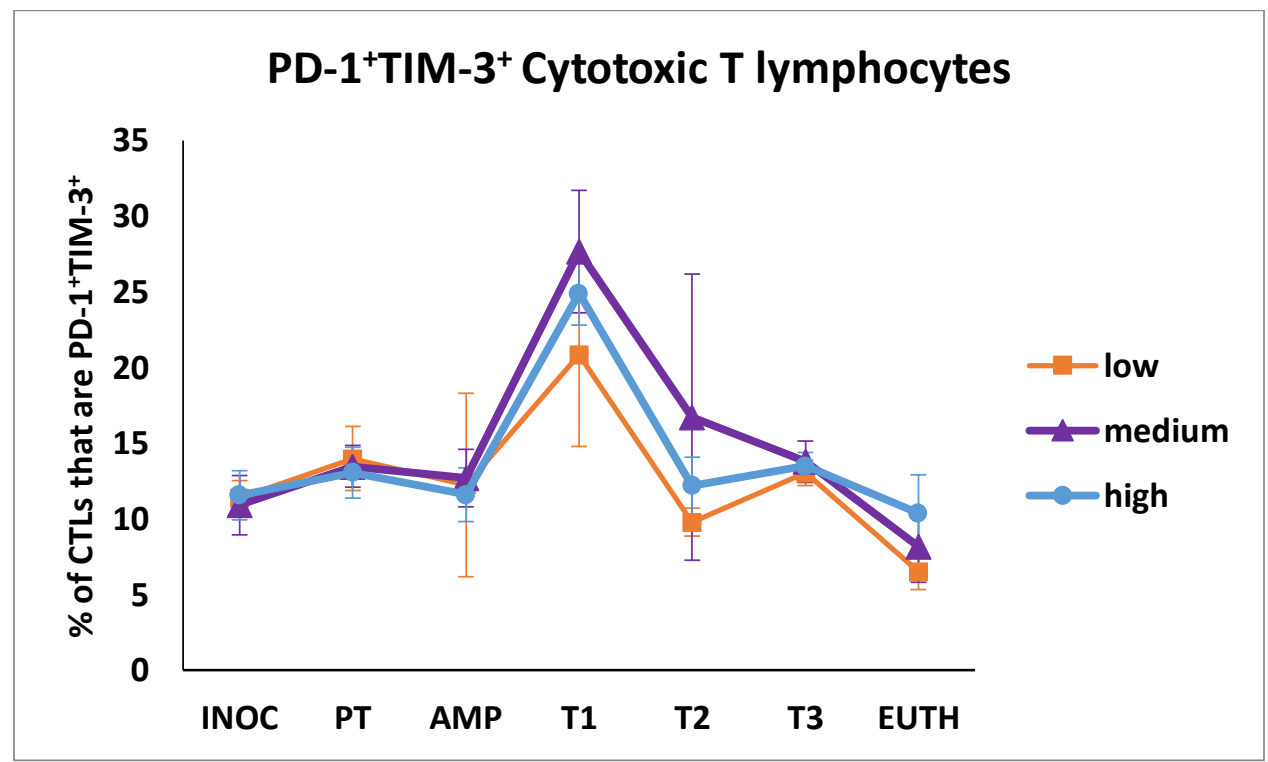


G.

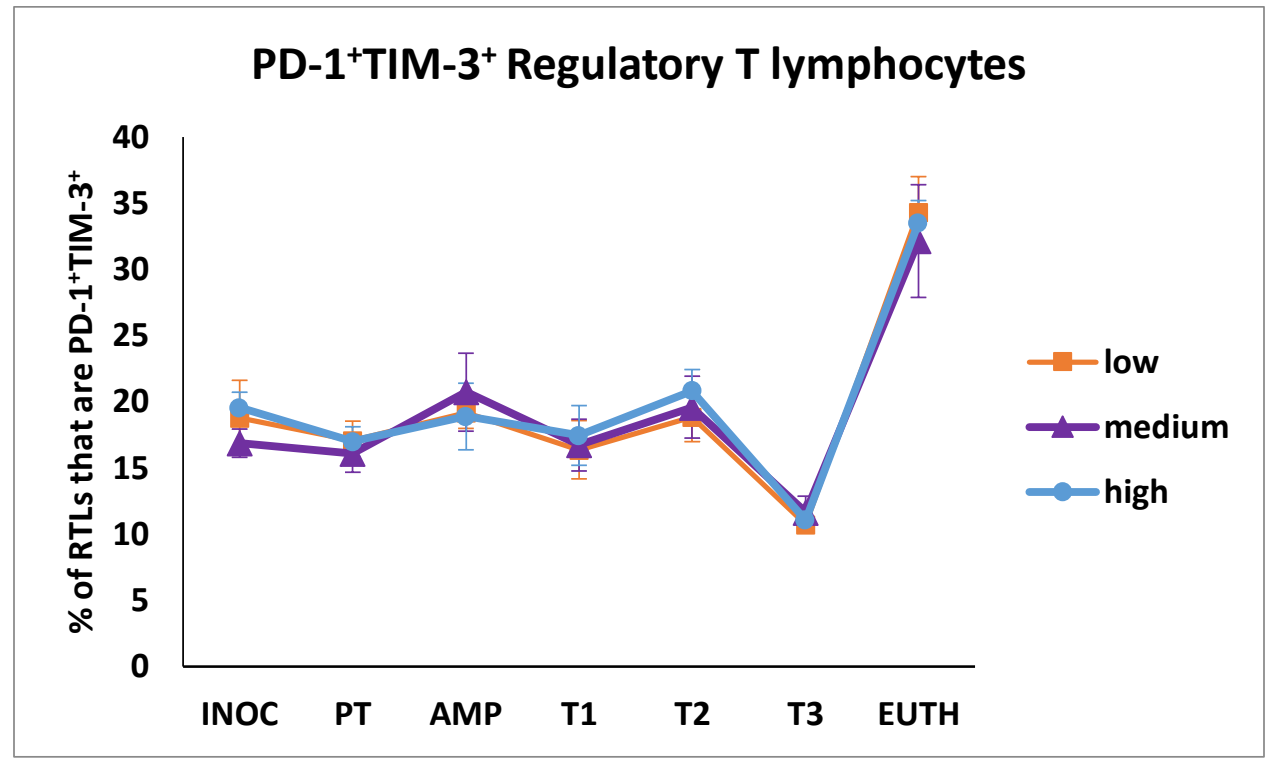

H.

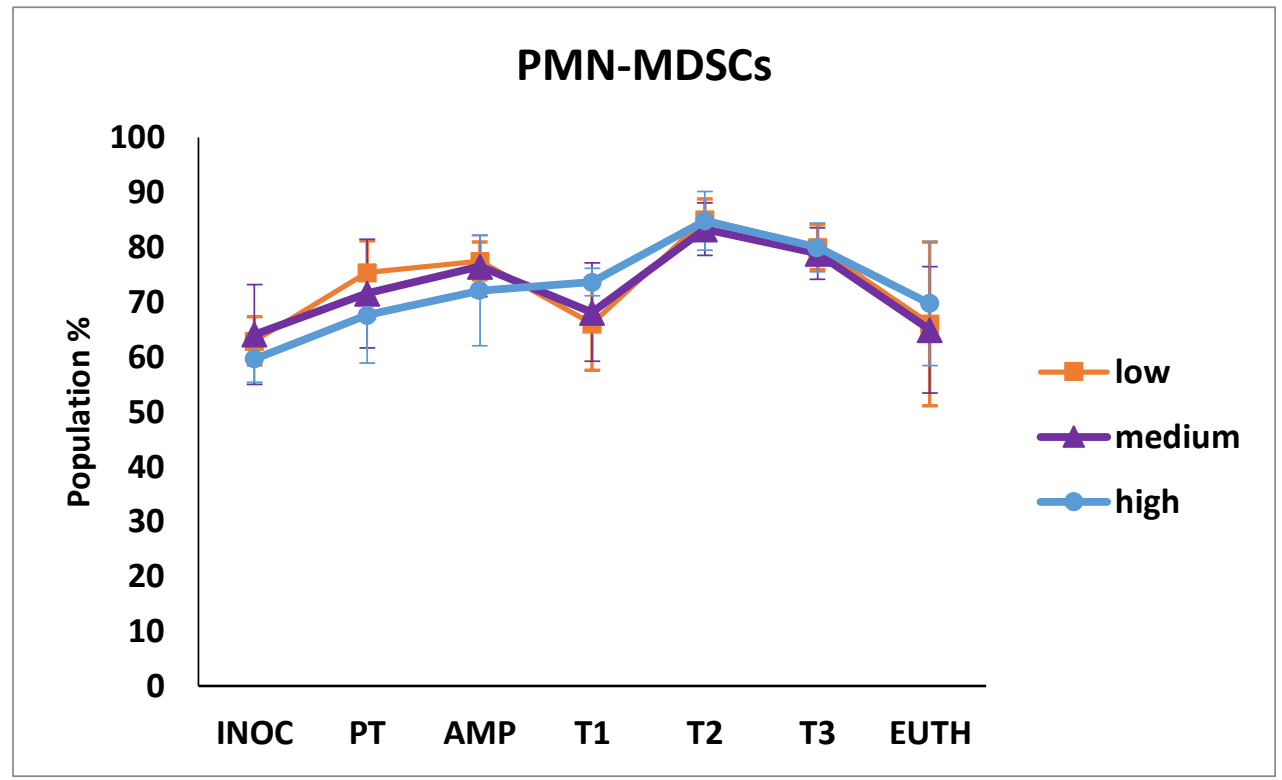


Table 1: nIL-12 treatment reduces metastatic disease and increases cure rates in OS tumor-bearing mice at 12 weeks.

\begin{tabular}{|l|c|c|}
\hline Group & Proportion Metastatic & Proportion Disease-free \\
\hline Untreated (n = 34) & 0.853 & 0.059 \\
\hline nIL-12 (n = 12) & 0.5 & 0.33 \\
\hline p & 0.007 & 0.008 \\
\hline
\end{tabular}


Supplementary Table S1: Disease (local and systemic) status of nIL-12 treated mice by dose at 12 weeks.

\begin{tabular}{|l|c|c|c|}
\hline Mouse ID number & Dose & Pulmonary MT & Local RC \\
\hline $\mathbf{2}$ & Medium $(1 \mathrm{mg})$ & Positive & Negative \\
\hline $\mathbf{3}$ & High $(10 \mathrm{mg})$ & Negative & Positive \\
\hline $\mathbf{4}$ & High $(10 \mathrm{mg})$ & Negative & Positive \\
\hline $\mathbf{5}$ & Medium $(1 \mathrm{mg})$ & Negative & Negative \\
\hline $\mathbf{6}$ & Low $(0.1 \mathrm{mg})$ & Negative & Negative \\
\hline $\mathbf{1 1}$ & Medium $(1 \mathrm{mg})$ & Positive & Positive \\
\hline $\mathbf{1 5}$ & High $(10 \mathrm{mg})$ & Positive & Positive \\
\hline $\mathbf{1 8}$ & Low $(0.1 \mathrm{mg})$ & Positive & Positive \\
\hline $\mathbf{1 9}$ & High $(10 \mathrm{mg})$ & Positive & Negative \\
\hline $\mathbf{2 2}$ & Low $(0.1 \mathrm{mg})$ & Negative & Negative \\
\hline $\mathbf{2 5}$ & Low $(0.1 \mathrm{mg})$ & Negative & Negative \\
\hline $\mathbf{2 8}$ & Medium $(1 \mathrm{mg})$ & Positive & Positive \\
\hline
\end{tabular}

MT $=$ metastasis $; \mathrm{RC}=$ recurrence 
Supplementary Table S2: Lymphoid (L) and myeloid (M) lineage antibody panels

\begin{tabular}{|c|c|c|c|c|}
\hline ANTIGEN/DYE & PANEL & CLONE & FLUOROCHROME & MANUFACTURER \\
\hline LIVE/DEAD & $\begin{array}{l}\text { Lymphoid } \\
\text { Myeloid }\end{array}$ & & Far red & $\begin{array}{l}\text { Life Technologies, Fisher } \\
\text { Scientific }\end{array}$ \\
\hline CD4 & Lymphoid & RM4-5 & PE-Сy5 & BD Biosciences \\
\hline CD8 & Lymphoid & $53-6.7$ & APC-Сy5.5 & Southern Biotech \\
\hline CD335 (NKP46) & Lymphoid & 29A1.4 & APC-eFLuor 780 & Invitrogen \\
\hline CD279 (PD-1) & Lymphoid & $\mathrm{J} 43$ & PE-CF594 & BD Biosciences \\
\hline FOXP3 & Lymphoid & MF23 & $\mathrm{PE}$ & BD Biosciences \\
\hline CD366 (TIM-3) & Lymphoid & $5 \mathrm{D} 12$ & BB700 & BD Biosciences \\
\hline CD25 & Lymphoid & $3 \mathrm{C} 7$ & BV510 & BD Biosciences \\
\hline CD11B & Myeloid & M1/70 & PE-CF594 & BD Biosciences \\
\hline LY6C & Myeloid & HK1.4 & AF700 & BioLegend \\
\hline LY6G & Myeloid & RB6-8C5 & PE-Cy5 & Invitrogen \\
\hline MHC CLASS II & Myeloid & 114.15 .2 & APC-eFluor 780 & Invitrogen \\
\hline CD274 (PD-L1) & Myeloid & 10F9G2 & PE-Cy7 & BioLegend \\
\hline
\end{tabular}




\section{Chapter 5}

\section{Overall Conclusions and Future Directions}




\section{Overall Conclusions}

The immune microenvironment of primary OS tumors has been extensively related to prognosis in multiple contexts. Additionally, it has been documented that certain systemic immune deficits can be associated with the development of OS tumors (1-9). However, these data have not led to increases in survival for metastatic OS patients $(10,11)$. Our work has built upon these foundations by developing protocols for measuring the evolving tumor immune macroenvironment of metastatic individuals in real-time. We have identified that OS immune deficits are propagated throughout the course of disease progression. Furthermore, by determining that the immune system changes in direct response to increasing tumor burden, we have identified therapeutic targets that may present opportunities for therapeutic intervention.

We have developed an immunocompetent mouse model that closely resembles the clinical course of disease in patients. With the introduction of luciferase into the tumor cells, this model allows for real-time tracking and quantification of pulmonary metastatic disease burden to a high degree of sensitivity while preserving the integrity of the antitumor immune response. Investigators can now test experimental immunotherapeutics in vivo while monitoring and adjusting therapy to meet disease burden in real-time.

We have also developed antibody panels that allow for monitoring of the tumor immune microenvironment across multiple tissues along the entire course of disease progression. It was found that the outstretched arm of malignant immunomodulation extends far beyond the primary tumor and throughout the entire organism. Also, similar to that described for primary tumors, data from the spleens of tumor-bearing mice provide evidence that the M1/M2 convention alone is not appropriate to describe systemic $\mathrm{M} \varphi$ polarization in OS. Furthermore, we have determined that immunotherapy can be used to directly affect malignancy-induced immunomodulation, which can 
be measured using specific antibody panels. Lastly, we present exciting data from blood showing that NK cell percent population and the M-MDSC $\mathrm{P}[1] / \mathrm{M}[\mathrm{II}]$ ratio change in direct response to disease burden and can be easily measured from a peripheral venous blood draw. It is possible that these parameters may have clinical utility in OS for diagnostic, prognostic, and therapeutic purposes.

\section{Future Directions}

The next steps to be taken are two-fold. First, it is obvious that the panels described in this manuscript must be translated to humans. Very few immunophenotypes translate directly between humans and mice on a one-to-one basis (12). Therefore, it is imperative that these findings be validated in human patients with the proper panels. Moreover, it will likely be essential to develop and maintain large data sets, as many patients, time points, and tissues will be included in the analyses. Such data sets may be filed on a per-patient basis and include demographics, diagnosis, treatment, co-morbidities, as well as any clinical components that change along the course of disease. We hope to use these large datasets to discover trends that describe the real-time capacity of the antitumor immune response to control disease progression. Not only would this data have prognostic and diagnostic utility, it may act as a guide to identify what specific immune deficits could be corrected with therapy. Therefore, the second step is to evaluate and test the malignancy-

defining immunophenotypes described herein for their ability to respond dynamically to immunotherapy. One such preliminary experiment is described in Chapter 4, where NK cells are used to define a treatment threshold for nanoscale IL-12 therapy.

The long-term goal of this project is described in Figure 7, which combines endogenous (tumorigenic and exogenous (immunotherapeutics) immunomodulation in a single hypothetical 
clinical scenario. Here, a patient presents to the clinic with a primary tumor and is diagnosed with OS. An initial blood immunophenotype is taken, and an individualized immunotherapeutic treatment plan is constructed. The initial immunophenotype analysis identifies a deficit in a certain immune parameter "X," and immunotherapy is started (box 1, red arrow) to correct it. At a followup appointment (box 2), a second immunophenotype is taken and parameter " $\mathrm{X}$ " is shown to have increased to within the therapeutic window (previously determined to be the range in which most patients develop an effective antitumor immune response). At the third (box 3) follow-up appointment, immunophenotyping shows that the parameter has dropped to the lower threshold of the therapeutic window, and the patient is given a second dose of the immunotherapeutic (red arrow). However, at the next appointment, immune parameter " $\mathrm{X}$ " is shown to be approaching the upper limit of the therapeutic window, above which has associations with higher morbidity due to increased risk of TCE. No immunotherapy is given at this time point. Finally, the patient presents at a follow-up appointment with the parameter at the center of the therapeutic window and no additional dose is delivered.

Over 100 years ago, Dr. Coley recognized the importance of the antitumor immune response and used a crude mixture of microbial products to promote inflammation (13). Now, a multitude of technologies exist for the creation of detailed immunophenotypes from any tissue in the body (14-18). As demonstrated throughout this manuscript, once the immune components that vary predictably and in direct response to relevant immunomodulatory forces are determined, systemic immunophenotypes may be created that allow clinicians to monitor disease progression and adjust therapeutic regimens in real-time. From the data presented in this manuscript, NK cells and the $[\mathrm{P} 1] /[\mathrm{MII}] \mathrm{M}-\mathrm{MDSC}$ ratio appear to be the most promising candidates to include when immunophenotyping OS patients; however, as both of these cell types play important roles in many 
cancers, it is possible that these concepts translate to other malignancies (19-24). Indeed, while each specific tumor type and patient come with their own unique sets of immunological variables, the prognostic significance of strong cell-mediated antitumor immune responses and immunosupportive $\mathrm{M} \varphi$ polarizations are common themes throughout all immunogenic tumor types; therefore, we hypothesize that once systemic immunophenotyping comes of age, it may be used across multiple cancer types to assess the extent of disease progression, identify targetable immune deficits allowing for treatment adjustments in real-time, and even determine the therapeutic windows for strong immunostimulatory agents like IL-12 whose benefits have thus far been offset by toxic side effect profiles. 


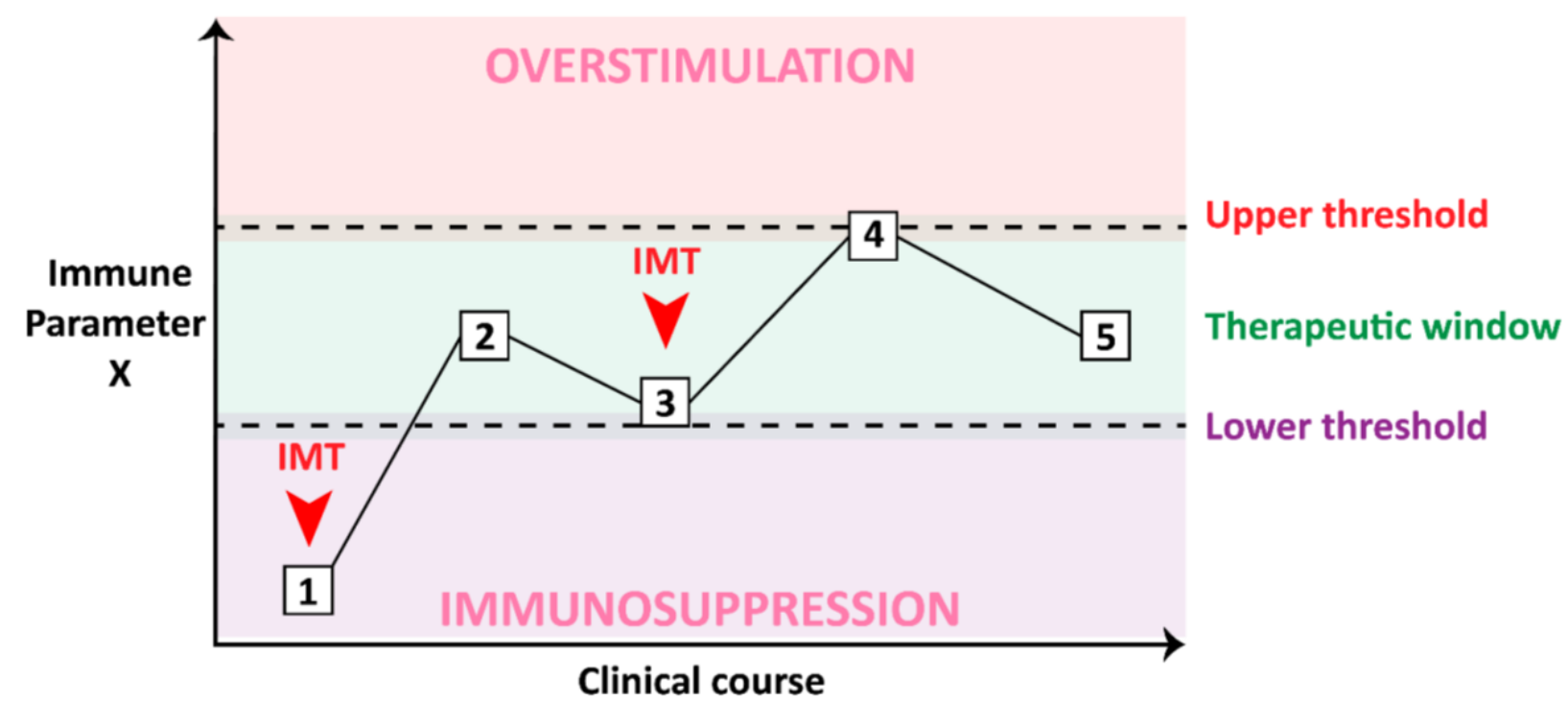

Figure 7. Schematic depicting a hypothetical clinical scenario describing the long-term goal of the project. 1)

Patient presents to clinic with tumor and is diagnosed with OS; preliminary immunophenotyping identifies a potentially correctable immune deficit in some immune parameter " $\mathrm{X}$ " and the appropriate immunotherapy is given (red arrow) 2) At first follow-up, parameter " $X$ " has responded to therapy and is now within the therapeutic window; patient is on track for a good overall prognosis 3) At second follow-up, parameter " $\mathrm{X}$ " has dropped to the lower threshold of the therapeutic window and is in danger of tumor immune escape; patient is given a second dose of appropriate immunotherapeutic 4) At third follow-up, parameter "X" has rebounded and is now at the upper level of the therapeutic window; patient is in danger of immune stimulation and is not given a dose at this time 5) At fourth follow-up, parameter " $\mathrm{X}$ " has returned to a safe value within the therapeutic window and is not given a dose at this time. 


\section{References}

1. Hong, W., Yuan, H., Gu, Y., Liu, M., Ji, Y., Huang, Z., ... \& Ma, L. (2020). Immunerelated prognosis biomarkers associated with osteosarcoma microenvironment. Cancer Cell International, 20(1), 1-12.

2. Li, M., Jin, X., Li, H., Wu, G., Wang, S., Yang, C., \& Deng, S. (2020). Key genes with prognostic values in suppression of osteosarcoma metastasis using comprehensive analysis. BMC cancer, 20(1), 65.

3. Sundara, Y. T., Kostine, M., Cleven, A. H., Bovée, J. V., Schilham, M. W., \& CletonJansen, A. M. (2017). Increased PD-L1 and T-cell infiltration in the presence of HLA class I expression in metastatic high-grade osteosarcoma: a rationale for T-cell-based immunotherapy. Cancer Immunology, Immunotherapy, 66(1), 119-128.

4. Liu, H., Zhi, L., Duan, N., \& Su, P. (2016). Abnormal expression of Tim-3 antigen on peripheral blood $\mathrm{T}$ cells is associated with progressive disease in osteosarcoma patients. FEBS open bio, 6(8), 807-815.

5. Zheng, W., Xiao, H., Liu, H., \& Zhou, Y. (2015). Expression of programmed death 1 is correlated with progression of osteosarcoma. Apmis, 123(2), 102-107.

6. Huang, X., Zhang, W., Zhang, Z., Shi, D., Wu, F., Zhong, B., \& Shao, Z. (2018).

Prognostic value of programmed cell death 1 ligand-1 (PD-L1) or PD-1 expression in patients with osteosarcoma: a meta-analysis. Journal of Cancer, 9(14), 2525.

7. Buddingh, E. P., Kuijjer, M. L., Duim, R. A., Bürger, H., Agelopoulos, K., Myklebost, O., ... \& Cleton-Jansen, A. M. (2011). Tumor-infiltrating macrophages are associated with metastasis suppression in high-grade osteosarcoma: a rationale for treatment with macrophage activating agents. Clinical Cancer Research, 17(8), 2110-2119.

8. $\quad$ Koirala, P., Roth, M. E., Gill, J., Piperdi, S., Chinai, J. M., Geller, D. S., ... \& Gorlick, R. (2016). Immune infiltration and PD-L1 expression in the tumor microenvironment are prognostic in osteosarcoma. Scientific reports, 6(1), 1-10.

9. Markiewicz, K., Zeman, K., Kozar, A., Gołębiowska-Wawrzyniak, M., \& Woźniak, W. (2012). Evaluation of selected parameters of cellular immunity in children with osteosarcoma at diagnosis. Medycyna wieku rozwojowego, 16(3), 212-221.

10. Thanindratarn, P., Dean, D. C., Nelson, S. D., Hornicek, F. J., \& Duan, Z. (2019). Advances in immune checkpoint inhibitors for bone sarcoma therapy. Journal of bone oncology, 100221.

11. Heymann, M. F., Schiavone, K., \& Heymann, D. (2020). Bone sarcomas in the immunotherapy era. British Journal of Pharmacology.

12. Bjornson-Hooper, Z. B., Fragiadakis, G. K., Spitzer, M. H., Madhireddy, D., McIlwain, D., \& Nolan, G. P. (2019). A comprehensive atlas of immunological differences between humans, mice and non-human primates. bioRxiv, 574160.

13. McCarthy, E. F. (2006). The toxins of William B. Coley and the treatment of bone and soft-tissue sarcomas. The Iowa orthopaedic journal, 26, 154.

14. Charoentong, P., Finotello, F., Angelova, M., Mayer, C., Efremova, M., Rieder, D., ... \& Trajanoski, Z. (2017). Pan-cancer immunogenomic analyses reveal genotype-immunophenotype relationships and predictors of response to checkpoint blockade. Cell reports, 18(1), 248-262. 15. Lanasa, M. C., Allgood, S. D., Slager, S. L., Dave, S. S., Love, C., Marti, G. E., ... \& Goldin, L. R. (2011). Immunophenotypic and gene expression analysis of monoclonal B-cell 
lymphocytosis shows biologic characteristics associated with good prognosis CLL. Leukemia, 25(9), 1459-1466.

16. Ornatsky, O., Bandura, D., Baranov, V., Nitz, M., Winnik, M. A., \& Tanner, S. (2010). Highly multiparametric analysis by mass cytometry. Journal of immunological methods, 361(12), 1-20.

17. Bjornson, Z. B., Nolan, G. P., \& Fantl, W. J. (2013). Single-cell mass cytometry for analysis of immune system functional states. Current opinion in immunology, 25(4), 484-494.

18. Papay, J., Krenacs, T., Moldvay, J., Stelkovics, E., Furak, J., Molnar, B., \& Kopper, L. (2007). Immunophenotypic profiling of nonsmall cell lung cancer progression using the tissue microarray approach. Applied immunohistochemistry \& molecular morphology, 15(1), 19-30.

19. Souza-Fonseca-Guimaraes, F., Cursons, J., \& Huntington, N. D. (2019). The emergence of natural killer cells as a major target in cancer immunotherapy. Trends in immunology, 40(2), $142-158$.

20. Di Vito, C., Mikulak, J., Zaghi, E., Pesce, S., Marcenaro, E., \& Mavilio, D. (2019, May). NK cells to cure cancer. In Seminars in Immunology. Academic Press.

21. De Sanctis, F., Solito, S., Ugel, S., Molon, B., Bronte, V., \& Marigo, I. (2016). MDSCs in cancer: conceiving new prognostic and therapeutic targets. Biochimica et Biophysica Acta (BBA)-Reviews on Cancer, 1865(1), 35-48.

22. Bergenfelz, C., Larsson, A. M., von Stedingk, K., Gruvberger-Saal, S., Aaltonen, K., Jansson, S., ... \& Rydén, L. (2015). Systemic monocytic-MDSCs are generated from monocytes and correlate with disease progression in breast cancer patients. PloS one, 10(5).

23. Chi, N., Tan, Z., Ma, K., Bao, L., \& Yun, Z. (2014). Increased circulating myeloidderived suppressor cells correlate with cancer stages, interleukin- 8 and- 6 in prostate cancer. International journal of clinical and experimental medicine, 7(10), 3181.

24. Fujimura, T., Mahnke, K., \& Enk, A. H. (2010). Myeloid derived suppressor cells and their role in tolerance induction in cancer. Journal of dermatological science, 59(1), 1-6. 


\section{Justin E. Markel}

3103 Apple Lane, Morgantown WV 26505

PHONE: (412) 512-4805

EMAIL: jemarkel@mix.wvu.edu

\section{EDUCATION}

Ph.D. M.D.-Ph.D. Dual degree scholar: 07/2014-05/2022

Anticipated degree date: Ph.D.: 05/2022
M.D. M.D.-Ph.D. Dual degree scholar: 07/2014-05/2022

Anticipated degree date: M.D.: 05/2022

B.A. Biochemistry (major): (Summa 08/2009-05/2013 Cum Laude)

B.A. Mathematics (minor): (Summa 08/2009-05/2013 Cum Laude)
West Virginia University, Morgantown, WV

Department of Orthopaedics, Program in Cancer Cell Biology, School of Medicine Dissertation Title: Monitoring the Systemic Immune System to Understand and Improve the Efficacy of Immunotherapy for Metastatic Osteosarcoma Dissertation Advisors: Brock A. Lindsey M.D. and Timothy D. Eubank Ph.D.

Dissertation Defense Date: 04/24/2020

West Virginia University, Morgantown, WV

School of Medicine

Washington \& Jefferson College, Washington, PA

Department of Biochemistry

Advisor: Michael S. Leonard Ph.D.

Washington \& Jefferson College, Washington, PA

Department of Mathematics

Advisor: Roman Wong Ph.D.

\section{Research Experience}

MD/PhD Trainee, Dept. of Orthopaedics, WVU Advisors: Brock A. Lindsey M.D. and Timothy D. Eubank Ph.D. Dissertation Title: "Monitoring the System Immune System to Understand and Improve the Efficacy of Immunotherapy for Metastatic Osteosarcoma” 2016-2020

Student Research Fellowship - University of Pittsburgh Department of Surgery

Summer 2015

Research Specialist - University of Pittsburgh Department of Surgery

January-July 2014

Biophysical Chemistry Research, Washington \& Jefferson College Advisor: Damian Carrieri Ph.D. " $A$ comparative study of the binding constants for caffeine in bovine, equine, and human serum albumin using a competitive spectrofluorometric technique"

Spring 2013

Physical Science Technician - Centers for Disease Control, South Park, PA

Summer 2012

Differential Equations Research, Washington \& Jefferson College Advisor: Roman Wong Ph. D. "Modeling the mechanisms of a hemodialyzer using differential equations"

Spring 2010 


\section{Publications}

Markel, J. E., Noore, J., Emery, E. J., Bobnar, H. J., Kleinerman, E. S., \& Lindsey, B. A. (2018). Using the Spleen as an in vivo systemic immune barometer alongside osteosarcoma disease progression and Immunotherapy with $\alpha$ PD-L1. Sarcoma, 2018.

Grisez, B. T., Ray, J. J., Bostian, P. A., Markel,J. E., \& Lindsey, B. A. (2018). Highly metastatic K7M2 cell line: a novel murine model capable of in vivo imaging via luciferase vector transfection. Journal of Orthopaedic Research ${ }^{\circledR}, 36(8), 2296-2304$.

Lindsey, B. A., Markel, J. E., \& Kleinerman, E. S. (2017). Osteosarcoma overview. Rheumatology and therapy, 4(1), 25-43.

Vogel, S., Bodenstein, R., Chen, Q., Feil, S., Feil, R., Rheinlaender, Schäffer, T. E., Bohn, E., Frick, J. S., Borst, O., Münzer, P., Walker, B., Markel, J.E., Csanyi, G., Pagano, P. J., Loughran, P., Jessup, M. E., Watkins, S. C, Bullock, G. C., Sperry, J. L., Zuckerbraun, B. S., Billiar, T. R., Lotze, M. T., Gawaz, M., \& Neal, M.D. (2015). Platelet-derived HMGB1 is a critical mediator of thrombosis. The Journal of clinical investigation, 125(12), 4638-4654.

\section{Unpublished Manuscript(s)}

Markel, J. E., Lacinski, R. A., Stewart, A. B., Vaida, J., Pratt, H. G., Reinbeau, R. M., Mizener, A. D., Lindsey, B. A. (2020). Systems-Wide Immunophenotyping Defines Malignancy-Induced Immunological Changes in an Immunocompetent K7M2 Orthotopic Murine Model of Osteosarcoma. Unpublished manuscript

\section{Abstracts}

Neal MD, J. Markel, B. S. Zuckerbraun, J. L. Sperry, P. Loughran, T. R. Billiar. HMGB1 Released by Platelets Leads to Microvascular Thrombosis Following Trauma and Hemorrhage. Academic Surgical Congress. Las Vegas, NV. February 4, 2015.

Chen Q, Markel J, Gutierrez A, Hassoune A, Vogel A, Timothy B, Neal MD. HMGB1 Enriched Microparticles Increase after Trauma and Contribute to Thrombosis. 13th Annual Academic Surgical Congress. January 30, 2016

\section{Techniques}

Cell Biology, Mouse Surgery, Organic Nanoencapusulation of Proteins: Flow cytometry-based assays and manual gating, tissue cell culture with sterile technique, PCR Primer Design, RNA isolation, immunoblotting (western blot), qRT-PCR, in vitro, ex vivo, and in vivo luciferase reporter assays, synthesis of poly(lactic-co-glycolic) acid nanospheres via homogenization with ultracentrifugation and sonication, ELISA, nanosphere protein elution, nanosphere sample prep for scanning electron microscopy, nanosphere zeta potential assay (Zetasizer $\left.{ }^{\circledR}\right)$, nanosphere particle count assay (Nanosight ( )), magnetic bead cell separation chromatography, BCA protein quantification, murine intratibial tumor cell inoculation survival surgery, murine lower limb disarticulation and amputation survival surgery, murine splenic biopsy survival surgery, murine inferior vena cava ligation (deep vein thrombosis model) survival surgery, working with murine models of osteosarcoma

\section{HONORS \& AWARDS}

Class Honors (Top 15\%), West Virginia University School of Medicine

2014-2015

- Awarded in Neurobiology (Summer 2015), Behavioral Science/

Psychopathology, Immunity/Infection and Disease, Medical Pharmacology (Fall 2015)

2015 Alpha Omega Alpha Carolyn L. Kuckein Student Research Fellowship (\$5000) 


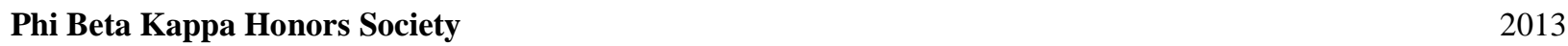

$\begin{array}{ll}\text { Gamma Sigma Epsilon Chemistry Honors Society } & 2013\end{array}$

Pi Mu Epsilon Mathematics Honors Society 2013

Dr. Mariano Garcia '39 Award in Mathematics 2011

Alpha Lambda Delta Honors Society 2009

Presentations

Markel JE, Lindsey BA. Monitoring the Systemic Immune System to Understand and Improve the Efficacy of Immunotherapy for Metastatic Osteosarcoma. Department of Orthopaedics - Cancer Cell Biology Dissertation Defense. April 24th, 2020

Markel JE, Lindsey BA. Using the Spleen as an in vivo systemic immune barometer alongside osteosarcoma disease progression and Immunotherapy with $\alpha$-PD-L1 - Translational Research Cancer Centers Consortium (TRCCC). February 13th, 2019

Zettel K, Raval JS, Hart K, Markel JE, Billiar TR, Neal MD. Human Stored Red Blood Cell Supernatant Leads to Suppression of Pro-Inflammatory Cytokines and Inhibits Leukocyte Phagocytosis after Trauma in a Murine Model. 37th Annual Conference on Shock. June 10th, 2014

Neal MD, Hoffman R, Chen Q, Markel JE, Loughran P, Billiar TR. Platelet Derived HMGB1 Regulates Platelet Aggregation and Mediates Organ Injury Following Trauma and Hemorrhagic Shock. 37th Annual Shock Society Meeting. June 10th, 2014

\section{TEACHING EXPERIENCE}

\section{WORK EXPERIENCE}

Lindsey Laboratory Graduate Researcher

- West Virginia University - Department of Orthopaedics

Research Specialist - University of Pittsburgh Department of Surgery

\section{VOLUNTEER ACTIVITIES}

\section{Community Service}

Problem-Based Learning Facilitator for Pipeline students

- Facilitated a problem-based learning case presentation for Pipeline students interested in medicine 
Whitewater kayaking and rafting, music, general relativity and tensor calculus, foreign languages 


\section{Appendix AI}

Lindsey, B. A., Markel, J. E., \& Kleinerman, E. S. (2017). Osteosarcoma overview. Rheumatology and therapy, 4(1), 25-43. 


\title{
Osteosarcoma Overview
}

\author{
Justin E. Markel, BA \\ Department of Orthopaedics \\ West Virginia University \\ Morgantown, WV US \\ Brock A. Lindsey, MD \\ Department of Orthopaedics \\ West Virginia University \\ Morgantown, WV US \\ Eugenie S. Kleinerman, MD \\ MD Anderson Cancer Center \\ Division of Pediatrics \\ Houston, TX US
}

\author{
Corresponding Author: \\ Brock A. Lindsey, MD \\ Department of Orthopaedics \\ West Virginia University \\ PO Box 9196 \\ Morgantown, WV 26506-9196 \\ Phone (304) 293-1317 \\ Fax (304) 293-7042 \\ Email: blindsey@hsc.wvu.edu
}

\begin{abstract}
Osteosarcoma (OS) is the most common primary malignancy of bone and patients with metastatic disease or recurrences continue to have very poor outcomes. Unfortunately, little prognostic improvement has been generated from the last 20 years of research and a new perspective is warranted. OS is extremely heterogeneous in both its origins and manifestations. Although multiple associations have been made between the development of osteosarcoma and race, gender, age, various genomic alterations, and exposure situations among others, the etiology remains unclear and controversial. Noninvasive diagnostic methods include serum markers like alkaline phosphatase and a growing variety of imaging techniques
\end{abstract}


including X-ray, computed tomography, magnetic resonance imaging, and positron emission as well as combinations thereof. Still, biopsy and microscopic examination are required to confirm the diagnosis and carry additional prognostic implications such as subtype classification and histological response to neoadjuvant chemotherapy. The current standard of care combines surgical and chemotherapeutic techniques, with a multitude of experimental biologics and small molecules currently in development and some in clinical trial phases. In this review, in addition to summarizing the current understanding of OS etiology, diagnostic methods, and the current standard of care, our group describes various experimental therapeutics and provides evidence to encourage a potential paradigm shift toward the introduction of immunomodulation, which may offer a more comprehensive approach to battling cancer pleomorphism.

Key Words: osteosarcoma; metastatic osteosarcoma; bone sarcoma; treatment for sarcoma; bone cancer

Compliance with Ethics Guidelines: This article is based on previously conducted studies and does not involve any new studies of human or animal subjects performed by any of the authors. 


\section{INTRODUCTION}

Osteosarcoma (OS) is an osteoid-producing malignancy of mesenchymal origins. This high-grade tumor is the most common primary malignancy of bone and is often fatal in both children and adults. While primary bone cancers represent less than $0.2 \%$ of all cancers (1), according to the National Cancer Institute SEER (Surveillance, Epidemiology, and End Results) program, their frequency has been increasing by $0.3 \%$ per year over the last decade (2). While OS occurs most frequently in patients between five years of age and early adulthood, incidence peaks again in the older $(>65)$ populations and has been associated with pre-existing Paget's disease and prior radiation therapy (3-7). Collectively, the metaphysis of the lower long bones, specifically the distal femur and proximal tibia, are the most commonly involved primary sites, with patients over 25 displaying a greater variety of bony locations (3).

Metastatic disease is classified by location as either pulmonary or extrapulmonary and is the major cause of OS-related death (8-10). While bony metastases are associated with poorer prognoses (with reports of $13 \%$ survival at five years [11]), the lung is involved in approximately $80 \%$ of cases (12) and subsequent respiratory compromise is responsible for most of the death toll (13). Compared with a potential cure rate of over $60 \%$ in patients presenting with nonmetastatic disease $(12,14)$, those with detectable metastases at the time of diagnosis (approximately 15-20\% [15,16]) have the poorest overall prognoses (17-22), with reports of five-year survival rates as low as $19 \%(17,23)$. Moreover, even in the subset of patients free of primary metastases, $40 \%$ will go on to eventually develop a secondary metastasis (12); in one study, survival rates of patients with nonmetastatic high-grade OS with subsequent metastases were $23 \%$ at five years and $0 \%$ at four years for pulmonary and bony metastases, respectively (24).

In contrast to distant pulmonary and extrapulmonary metastases, skip lesions (also known as skip metastases or synchronous regional bone metastases) are local and yet potentially more serious metastatic complications. These small, anatomically isolated cancerous foci are distinct from the primary tumor and 
located either within the same bone or transarticular to it $(25,26)$. Classically, they are associated with extensive metastatic dissemination, robust therapeutic resistance, and particularly poor prognoses (27-29).

Similar to those with metastatic OS, patients with recurrent disease have comparably dismal five-year post-relapse-overall-survival (PROS) rates (18). In addition, features such as axial tumor site $(30,31)$, male sex (30), and advancing age (31) have all shown correlation with inferior patient outcomes. Notably, the pre-operative histologic response to neoadjuvant chemotherapy, as judged by the extent of tumor necrosis, offers some of the most important predictive value regarding overall patient survival $(30,32$ $34)$.

\section{ETIOLOGY AND GENOMICS}

OS etiology is complex and not well understood. The molecular pathogeneses and genetics of OS are vast and extremely heterogeneous (35), with discrepancies in the literature regarding its demographic and environmental influences further complicating the story. Most cases are sporadic; however, increased risk has been associated with multiple germline mutation disorders including hereditary retinoblastoma (36, 37), Rothmund-Thomson syndrome (38, 39), Li Fraumeni syndrome (40), and Bloom syndrome (41) among others.

Classically, alterations in the Retinoblastoma $(\mathrm{Rb})$ gene have been strongly associated with a predisposition for developing OS (43-44) and loss of heterozygosity has been reported to occur in up in 40-70\% of cases (45-49). Additionally, altered p53 loci, which have been reported to occur in approximately $10-39 \%$ of cases (49-59), display synergistic tumorigenic properties (50,60-62). Whole genome sequencing studies have attempted to elucidate recurrent chromosomal structural patterns, most recently with loci at $6 \mathrm{p} 21.3$ and 2p25.2 displaying potentially genome wide significance $(63,64)$. However, despite these and countless other genetic similarities discovered across cell lines, OS continues 
rapidly modifying its genotype thus making potential targeted molecular therapeutics increasingly impractical. To date, no single genetic target has proven therapeutically successful and this wealth of information has yet to lead to a significant decrease in mortality (35).

As per the central dogma, this genotypic heterogeneity has translated into a wide variety of macromolecular biomarker expression profiles with potentially useful clinical implications. Indeed, phenotypic trends have been found and characterized across various OS cell lines. Multiple studies have identified characteristic protein and mRNA expression profiles showcasing anomalous levels of ErbB-2 (65,66), cathepsin D (67), FBXW7 (68), miR-421 (69), and HMGB1 (70) among others. It has also been suggested that matrix-Gla protein expression may play a role in facilitating tumor spread to the lungs (71). However, as of yet, the true diagnostic, etiologic, and clinical significance of these biomarkers is ongoing and controversial.

Apart from genetic mutations and expression profiles, studies have also discovered correlations between poor prognosis (or increased incidence) and various demographics such as male gender (72), old age $(73,74)$, height (75), and African American descent (76). Others have suggested an association between bone growth and incidence rates (77-81), but the relative strength and importance of this relationship have been challenged (82). Environmental factors that have been associated with increased risk of future OS development include exposure to radiation $(6,7)$, teriparatide usage (83), and consumption of fluorinated drinking water in childhood $(84,85)$; however, more recent studies have disputed the latter two relationships (86-89).

\section{DIAGNOSIS AND PATHOLOGY}

Due to the complex nature of primary bone malignancies, diagnosis of OS is best accomplished via a comprehensive multidisciplinary approach (90). Often, the first sign of potential bone malignancy is 
intermittent pain around the affected area with or without a palpable mass (91). When involving areas around the knee joint, pain exacerbated by weight bearing may manifest as a limp; occasionally, patients will present with a recent bout of bone trauma (92). As previously mentioned, any older adult with a history of Paget's disease has an increased risk of developing OS, most notably of the pelvis (93), and this transformation is associated with a poor prognosis (94).

Various serum markers have been investigated for their utility in diagnosing and tracking progression and recurrence. Alkaline phosphatase (ALP) and lactose dehydrogenase (LDH) are useful serum biomarkers, with ALP carrying the most diagnostic value in OS $(95,96)$. ALP has also been shown to correlate positively with tumor volume, which carries additional useful prognostic implications (95-97). LDH has also been shown to correlate with tumor volume; however, the correlation is weaker than for ALP and is mainly a result of nonspecific metabolic demand (96). If disease is suspected, the first step is to gather plain radiographs of the involved bone and adjacent joint. Any abnormal films then warrant further radiological investigation of soft tissue involvement and possible primary lung metastasis via magnetic resonance imaging (MRI) and computed tomography (CT), respectively (98). Bone scintigraphy (BS) is often used in conjunction with CT to identify metastases and the presence or absence of metastatic disease remains one of the most important predictors of patient outcome $(98,99)$. For a more detailed, flow-chart style approach to patient work-up, please refer to the guidelines put forth by the National Comprehensive Cancer Network (NCCN) for bone cancer, Version 2.2017 (100).

Although they have not yet taken the place of BS in OS diagnosis, various experimental test procedures such as positron emission tomography (PET) scans are currently being investigated for their potentially superior ability to diagnose and track the progression of bone cancers. In 2009, Costelloe et al. demonstrated that combined PET/CT could be used to reliably predict the progression-free survival (PFS), overall survival, and extent of tumor necrosis in OS (101). Studies have also suggested enhanced sensitivity with the use of fludeoxyglucose- (18F-FDG)-PET-CT over BS for detecting metastases (102). 
Hyung-Jun Im et al. (2016) used a similar technique to show that initial baseline metabolic tumor volume and total lesion glycolysis have independent prognostic value in determining survival in pediatric osteosarcoma populations (103). Importantly, combined PET/CT scanning has been shown to accurately determine the extent of histological osteonecrosis and may offer a less invasive alternative to the typical biopsy-requiring Huvos grading system (104), as previously described (105). Please refer to Table 1 for a list of test properties, including sensitivity, specificity, and a brief note on diagnostic application (as indicated by the study from which those numbers were derived). Note, however, that despite the promising aspects of these non-invasive techniques, a biopsy is always required to confirm the diagnosis (106). As such, proper disease management mandates tissue collection with the correct identification of the specific histologic subtype (107). While many subtypes exist and correlate specifically to corresponding chemotherapeutic responses (108), the unifying histological feature is the presence of osteoid-producing malignant cells (106).

Incisional or core needle biopsy is the final step in the diagnostic process (98) and the tumor is staged using the Musculoskeletal Tumor Society staging scheme (109) or the American Joint Commission on Cancer (AJCC) system. In the AJCC system, the tumor is described based on four important factors represented by letters (110): T (primary tumor size), N (spread to regional lymph nodes), M (metastasis), and G (grade). Each letter is then assigned a particular number (e.g., T1) that describes the tumor's pathologic extent (T, N, and M) and histological appearance $(\mathrm{G})$. Once assigned its corresponding TxNxMxGx code, the tumor can then be grouped into its corresponding stage, with Stage 1A being the most localized and Stage IVB the most invasive. This information can be used to determine prognosis, assess response to therapy, and monitor disease progression (111).

Osteosarcoma subtypes include osteoblastic, chondroblastic, fibroblastic, small cell, telangietactic, highgrade surface, extra-skeletal, and other lower grade forms including periosteal and parosteal (112). Based upon their histological appearances, OS subtypes can be grouped into three categories: high-grade, 
intermediate-grade, and low-grade. Parosteal OS is a low-grade subtype that is fibroblastic in appearance and limited to the bone surface; however, with time, it may progress to involve deeper structures. For this and other low-grade subtypes, treatment involves surgery alone and carries a favorable prognosis.

Periosteal OS is chondroblastic on histology and is the only subtype that falls into the intermediate-grade category. Depending on extent of invasion, treatment for intermediate-grade OS often includes systemic chemotherapy. High-grade OS, which includes the classic osteoblastic subtype, is the fastest growing and most aggressive group. The majority of OS subtypes fall under this category and include osteoblastic, chondroblastic, fibroblastic, small cell, telangiectatic, high-grade surface, and extra-skeletal.

Telangiectatic OS is notable for its profuse vascularity and scant osteoid production, which often complicates tissue biopsy and radiographic identification, respectively (113). Telangiectatic OS also carries with it an anatomical predilection to the epiphyseal region of the bone. All high-grade OS should be considered micrometastatic at diagnosis and treated with surgery and systemic chemotherapy.

\section{TREATMENT}

The current standard for osteosarcoma treatment employs neoadjuvant chemotherapy, surgery, and then post-operative adjuvant chemotherapy. Multiple different chemotherapy regimens containing anywhere from two to seven drugs have been used $(98,114,115)$. The four drugs that have shown consistent activity are cisplatin, doxorubicin, high-dose methotrexate with leucovorin rescue, and isofosfamide with or without etoposide. A recent meta-analysis showed that patients who were treated with three drugs had a superior outcome to those that received two drugs. However, there was no benefit in using four drugs compared to three drugs (116). Indeed, adding standard or high-dose ifosfamide significantly increased toxicity while having negligible effects on outcome $(117,118)$ which was recently confirmed by an international trial which showed that there was no benefit in adding high-dose ifosphamide plus etoposide to the cisplatin, doxorubicin, methotrexate combination (MAP) (119). Therefore, MAP multi-agent chemotherapy is the first-line treatment and the standard of care at the present time. For a more thorough 
investigation of OS chemotherapeutics, please refer to the meta-analysis conducted in 2011 by Anninga et al. (116).

Neoadjuvant (pre-operative) chemotherapy greatly increases relapse-free survival (RFS) of patients with non-metastatic disease $(120,121)$. Furthermore, it allows for tumor categorization into histological response subgroups, which has significant prognostic and clinical value and provides the opportunity to alter the post-operative treatment strategies (122). The goal for a positive treatment with neoadjuvant chemotherapy is to achieve at least $90 \%$ necrosis on the surgically resected tumor (122). If the percent necrosis is below $90 \%$ at resection after neoadjuvant treatment then the post-resection adjuvant chemotherapy regimen can be altered. However, changing the chemotherapy regimen post-operatively based on response has not been shown to have a positive impact on patient outcome (119); indeed, another finding from the EURAMOS-1 randomized control trial series has reported increased toxicity and secondary malignancies from intensifying chemotherapy regimens (adding both ifosfamide and etoposide to MAP therapy, i.e., MAPIE) for poor responders (123). For full treatment guidelines with detailed workup flow charts, please refer to the NCCN guidelines for bone cancer, Version 2.2017 (100).

\section{SURGERY AND RADIATION THERAPY}

Surgical excision usually involves tumor resection with negative margins, as multiple studies have linked positive margins with an increased risk of local recurrence (LR) and inferior survival (124). Classically, Enneking et al. described four different types of surgical margins: intralesional, marginal, wide, and radical (125). An intralesional margin, as the name suggests, is obtained when the specimen is taken from within the tumor itself. Although these margins have little therapeutic utility, they are often used for biopsies and have diagnostic purposes. Marginal and wide margins remove the lesion en bloc, with wide leaving a substantial border of normal tissue. This border of normal tissue surrounding the tumor is absolutely paramount in the treatment of OS. Despite these definitions, however, the proper margin to 
obtain on a case-by-case basis has been continually met with controversy; to date, no general consensus has been born out of the literature. In 2012, a retrospective cohort of 47 patients found no significant difference in LR between groups with close (tumor $<5 \mathrm{~mm}$ from resection margin) and wide (tumor $>$ $5 \mathrm{~mm}$ from resection margin) margins (126). The following year, Jeon et al. suggested that negative tumor margins correlate with significantly lower risks of LR in bone and perineurovascular resection planes while having little influence on LR in soft tissue (127). Furthermore, there is also evidence to support that the risk of LR is higher in patients treated with closer margins (113). In either case, when adequate margins cannot be achieved, amputation should be considered (128). Naturally, limb-salvage is greatly preferred over amputation; over $85 \%$ of patients are candidates for this type of procedure (129-132).

In patients with disseminated disease, the complete resection of pulmonary metastases is vital when possible $(133,134)$, as lung metastectomy has been shown to significantly prolong survival in this population $(135,136)$. Patients with recurrent unresectable metastases usually have poorer prognoses, even when treated aggressively with pre-operative chemotherapy (137). For recurrent or refractory disease, some studies have suggested that incorporating etoposide into the chemotherapy treatment regimen may be beneficial (138); however, these data are controversial and associated with severe toxicities (139). Whenever possible, surgical resection of recurrent disease is first-line over systemic therapy, which is less effective and reserved for unresectable cases (20). Radiation therapy may also be used to help clear microscopic or minimal residual disease when substantial surgical resection is not possible (140); however, for a majority of cases, radiation is not used.

\section{NEW THERAPEUTIC APPROACHES}

Effective new OS therapies have plateaued over the last several decades; this lack of new treatment strategies is reflected by unchanging survival rates $(3,141)$. As OS cells tend to exhibit extreme genetic pleomorphism, therapeutic attempts to target specific cell receptors and intracellular signaling molecules 
have not significantly increased survival. In addition, and likely as a result of its pleomorphism, OS cells exhibit strong chemotherapy resistance, most notably in the $15-20 \%$ of patients initially presenting with detectable metastases at the time of diagnosis (15), and who consequently have the poorest prognoses (1722). Therefore, MAP adjuvant treatment strategies have had minimal beneficial effects on this subset of patients (142); as such, a number of alternative therapeutic modalities have been investigated.

Various biologics and small molecules have been used to target cell surface receptors and downstream signaling pathways involved in OS pathogenesis. For example, as HER2 is often expressed in a subset of OS cell lines and has been associated with poorer prognoses (143), a phase II trial was conducted using trastuzumab to target HER2-positive OS; however, despite minimal drug reactions and additional toxicity, no significant difference in groups was observed (144). Pappo et al. (2014) conducted a similar phase II trial targeting another receptor commonly expressed by malignant cells, IGF-1R (145); again, clinical responses were underwhelming (146). The PI3K/mTOR pathway $(147,148)$ and mitogenactivated protein kinases $(148,149)$ have also recently been recognized as potential targets and their therapeutic significance is currently under investigation.

Over the past few decades, increasing evidence has suggested that platelets and other mesenchymal cells, notably the PDGFR-alpha-R for OS (150), can assist tumor cell pathogenesis (151). Indeed, Labelle et al. (2011) showed that platelet-tumor cell interactions, mediated by activation of transforming growth factor (TGF)-beta/SMAD (small mothers against decapentaplegic) and nuclear factor (NF)-kappa-B, could promote metastasis by inducing an epithelial-mesenchymal-like transition (152). As such, Takagi et al. (2014) was able to significantly inhibit platelet-induced OS cell proliferation by blocking Akt-mediated downstream signaling using sunitinib (153). Likewise, sunitinib has since been shown to reduce tumor burden and lung metastasis in mice (154); however, the clinical significance of these initial data is yet to be determined. 
Elucidating the mechanisms of OS's robust chemoresistance has yielded other potentially promising therapeutic targets. Recently, it has been shown that HMGB1-induced autophagy contributes to OS chemotherapy resistance (155); hence, this and other chemoresistance-promoting pathways provide the means for new therapeutic approaches and their inhibitors deserve further investigation. Other potential therapeutics currently under investigation include zoledronic acid (156) and even the natural phenolic compound, curcumin (157).

Due to the vast heterogeneity of OS molecular profiles $(35-40,63,64)$, the future of OS treatment may be moving away from targeted anti-oncogenic paradigms and toward more generalized immunomodulatory/immunoeditory approaches (158). Cancer immunotherapy, although still in its infancy, attempts to enhance tumor immunogenicity and stimulate tumorocidal activity, thereby reallocating the burden of disease clearance back to the patient's own body. Nonspecific immunogens, cytokines, adoptive T-cells, vaccines, oncolytic virotherapies, and checkpoint blockades have all shown potential therapeutic promise (159). If deemed clinically advantageous, these new immunotherapeutics will likely be administered as adjuvants and integrated into the current standard of care.

Muramyl tripetide (MTP), which has been shown to activate NF-kB (160) and increase circulating levels of TNF alpha and interleukin-6 (IL-6) in patients with OS (161), can be packaged within liposomes (162) and injected $(163,164)$. This liposomal product (Mifamurtide) allows for particle ingestion by monocytes and macrophages and the subsequent activation of their cytotoxic function against tumor cells (165). Tumor-associated macrophages, although mostly thought of as being pro-tumorigenic (166), have been found to play a potentially significant role in preventing metastasis in high-grade OS (167). In patients with no clinically detectable metastases and in those with resectable disease, the addition of Mifamurtide to multi-agent chemotherapy has been shown to significantly increase event-free survival (EFS) and overall survival with a $29 \%$ reduction in the mortality rate at eight years (168). The addition of Mifamurtide also improved the outcomes of patients who presented with metastases at the time of 
diagnosis (169). A therapeutic synergism of MTP with zoledronic acid on primary tumor progression has also been suggested (170).

There have also been attempts to target immunotherapy directly to the lungs via the use of aerosols, which have the potential benefit of lowering systemic toxicity by being delivered directly to the site of action (171). OS relapse most commonly occurs as pulmonary metastasis (106), with patient survival often being below $30 \%(172,173)$ and as low as $14 \%(20)$ in these cases. Granulocyte-macrophage colony stimulating factor (GM-CSF), a molecule with multiple roles in immune regulation and phagocyte maturation (174), was recently used in a phase II trial of post-relapse OS patients to investigate its effect on disease-free survival (DFS). However, despite the ability to reach adequately high doses with minimal side effects, no significant improvement in survival was seen (175). While these results are disappointing, they may indicate that the immunomodulatory effects of GM-CSF alone were not enough to influence a tumorocidal environment. Unfortunately there was no investigation of whether GM-CSF treatment resulted in any biologic effect on the lung tumor nodules. Therefore, it is hard to assess whether this therapy resulted in the desired effect in terms of activating an immune response in the lung. Thus, the possibility remains that the lack of therapeutic effect was secondary to the inability of GM-CSF to stimulate an immune response in the lung. Indeed, there is growing evidence to support that the chemical profile of tumor microenvironments comprises an astonishingly complex constellation of signaling molecules in various distributions $(176,177)$ and it is highly possible that further manipulation may be necessary to achieve an effective tumorocidal environment (178). As such, Zeidner et al. (2014) and Wang et al. (2001) have showed that combinations of GM-CSF with interferon (IFN) and IL-12 therapy improved outcomes for chronic myeloid leukemia patients (179) and increased antitumor effects against murine hepatocellular carcinoma (180), respectively. Another more recent immunotherapeutic attempt at combatting OS pulmonary metastasis with combination immunotherapy included aerosol IL-2 with adjuvant natural killer (NK) cell infusions, which has shown enhanced efficacy compared to IL-2 or NK cell infusions alone (181). Other cytokines, including IL-15 and IL-12, have been shown to increase 
natural killer cell-mediated lysis of chemotherapy-resistant OS cells (182) and suppress pulmonary metastasis formation (183), respectively.

IFN immunotherapy has also shown promise in the treatment of OS. IFN- $\alpha$, while initially recognized for its ability to inhibit viral replication, is now used in the treatment of a variety of different solid and hematological cancers (184). In OS, it has been shown to suppress tumor invasion as well as enhance the cytotoxic effects of cisplatin (185). In 2015, Bielack et al. conducted an international randomized, controlled trial comparing the efficacy of MAP therapy alone versus MAP plus pegylated interferon alpha- $2 \mathrm{~b}$ in 2260 registered patients; however, the results were complicated by insufficient patient adherence and no statistical difference in outcome was found (186). In 2015, Gao et al. revealed that IFNlambda1, a relatively new member of the interferon family (187), inhibits the invasive properties of MG63 human osteosarcoma cell lines in vitro (188). Another in vitro study showed that INF-gamma can enhance the ability of $\gamma \delta$ T cells to target and kill HOS and U2OS OS cell lines (189).

As it is now widely accepted that tumors often promote suppression of the immune system in order to facilitate their pathogenesis (190), many immunotherapies have shown promise by targeting immunoregulatory cell surface markers. Programmed death ligand 1 (PD-L1) and cytotoxic Tlymphocyte-associated protein 4 (CTLA-4) are surface receptors involved in down-regulating the cytotoxic T cell response (191-193) and their blockade has been implicated in the treatment of a variety of cancers (194). Interestingly, combined blockade of PD-L1 and CTLA-4 has been shown not only to completely eliminate metastatic osteosarcoma in murine models, but also to induce immunity to further inoculation $(195,196)$.

\section{CONCLUSIONS}


Over the past 25 years, altering or intensifying the chemotherapy regimens for newly diagnosed osteosarcoma patients has failed to improve the $65-70 \%$ long-term survival. The only success in improving patient outcomes was the addition of Mifamurtide to the three-drug or four-drug regimen. Combining Mifamurtide with chemotherapy increased long-term survival from 70-78\% at eight years (168) and improved the outcome of patients who presented with metastases at diagnosis (169). This improvement shows that immunotherapy is effective against this cancer. As OS therapies move forward over the next five years, it is likely that both immunostimulation and suppression blockade immunotherapies will play emerging roles. The genetic heterogeneity and morphological adaptability of OS necessitates a more comprehensive treatment approach, as the disease's molecular repertoire is too vast to be treated successfully by targeted therapies alone. Successful treatment will almost certainly require a combination of these different techniques to best achieve an effective tumorocidal environment; the key will lie in recognizing what specific role each immune cell plays and how best to assist its function. 


\section{Acknowledgements:}

This article is based on previously conducted studies and does not involve any new studies of human or animal subjects performed by any of the authors. No funding or sponsorship was received for this study or publication of this article. All named authors meet the International Committee of Medical Journal Editors (ICMJE) criteria for authorship for this manuscript, take responsibility for the integrity of the work as a whole, and have given final approval for the version to be published. The authors have no conflicts of interest to disclose that are relevant to the review topic. 
Table 1: OS serological and radiological test properties with diagnostic applications.

\begin{tabular}{|c|c|c|c|}
\hline Test & Sensitivity & Specificity & Application \\
\hline Serum ALP (96) & 0.78 & 0.94 & $\begin{array}{l}\text {-Correlation }(\mathrm{r}=0.5) \text { with tumor } \\
\text { volume } \\
\text {-Most descriptive for osteoblastic } \\
\text { subtype }\end{array}$ \\
\hline Serum LDH (96) & 0.82 & 0.97 & $\begin{array}{l}\text {-Correlation }(\mathrm{r}=0.4) \text { with tumor } \\
\text { volume } \\
\text {-Describes tumor metabolic } \\
\text { demand }\end{array}$ \\
\hline Spiral CT (197) & 0.75 & 1.00 & Pulmonary metastases \\
\hline FDG-PET (197) & 0.5 & 0.98 & $\begin{array}{l}\text { Pulmonary metastases; } \\
\text { confirmation of CT abnormality }\end{array}$ \\
\hline PET/CT (198) & 0.95 & 0.98 & $\begin{array}{l}\text { Bony metastases (examination- } \\
\text { based analysis) }\end{array}$ \\
\hline BS (198) & 0.76 & 0.97 & $\begin{array}{l}\text { Bony metastases (examination- } \\
\text { based analysis) }\end{array}$ \\
\hline $\begin{array}{l}\mathrm{PET} / \mathrm{CT}+\mathrm{BS} \\
(198)\end{array}$ & 1.00 & 0.96 & $\begin{array}{l}\text { Bony metastases (examination- } \\
\text { based analysis) }\end{array}$ \\
\hline $\begin{array}{l}\text { FDG-PET/CT } \\
(199)\end{array}$ & 0.947 & Not reported & $\begin{array}{l}\text { Initial staging or assessment of } \\
\text { recurrent disease }\end{array}$ \\
\hline
\end{tabular}




\section{REFERENCES:}

1. Biermann, J. S., Adkins, D. R., Agulnik, M., Benjamin, R. S., Brigman, B., Butrynski, J. E., ... \& Frassica, F. J. (2013). Bone cancer. Journal of the National Comprehensive Cancer Network, 11(6), 688723.

2. SEER Stat Fact Sheets: Bone and Joint Cancer

3. Mirabello, L., Troisi, R. J. and Savage, S. A. (2009), Osteosarcoma incidence and survival rates from 1973 to 2004. Cancer, 115: 1531-1543. doi: 10.1002/cncr.24121

4. Dorfman, H. D. and Czerniak, B. (1995), Bone cancers. Cancer, 75: 203-210. doi: 10.1002/10970142(19950101)75:1+<203::AID-CNCR2820751308>3.0.CO;2-V

5. Ottaviani, G., \& Jaffe, N. (2009). The epidemiology of osteosarcoma. In Pediatric and adolescent osteosarcoma (pp. 3-13). Springer US.

6. Le Vu, B., De Vathaire, F., Shamsaldin, A., Hawkins, M. M., Grimaud, E., Hardiman, C., ... \& Panis, X. (1998). Radiation dose, chemotherapy and risk of osteosarcoma after solid tumours during childhood. International journal of cancer, 77(3), 370-377.

7. Arlen, M., Higinbotham, N. L., Huvos, A. G., Marcove, R. C., Miller, T., \& Shah, I. C. (1971). Radiation-induced sarcoma of bone. Cancer, 28(5), 1087-1099.

8. Eccles, S. A., \& Welch, D. R. (2007). Metastasis: recent discoveries and novel treatment strategies. The Lancet, 369(9574), 1742-1757.

9. Pradelli, E., Karimdjee-Soilihi, B., Michiels, J. F., Ricci, J. E., Millet, M. A., Vandenbos, F., ... \& Kleinerman, E. S. (2009). Antagonism of chemokine receptor CXCR3 inhibits osteosarcoma metastasis to lungs. International journal of cancer, 125(11), 2586-2594.

10. Hughes, D. P. (2009). Strategies for the targeted delivery of therapeutics for osteosarcoma. Expert opinion on drug delivery, 6(12), 1311-1321.

11. Bacci, G., Longhi, A., Bertoni, F., Briccoli, A., Versari, M., Pignotti, E., \& Picci, P. (2006). Bone metastases in osteosarcoma patients treated with neoadjuvant or adjuvant chemotherapy The Rizzoli experience in 52 patients. Acta orthopaedica, 77(6), 938-943.

12. PosthumaDeBoer, J., Witlox, M. A., Kaspers, G. J. L., \& Van Royen, B. J. (2011). Molecular alterations as target for therapy in metastatic osteosarcoma: a review of literature. Clinical \& experimental metastasis, 28(5), 493-503.

13. Snyder, C. L., Saltzman, D. A., Ferrell, K. L., THOMPSON, R. C., \& Leonard, A. S. (1991). A new approach to the resection of pulmonary osteosarcoma metastases: results of aggressive metastasectomy. Clinical orthopaedics and related research, 270, 247-253.

14. Bacci, G., Ferrari, S., Bertoni, F., Ruggieri, P., Picci, P., Longhi, A., ... \& Campanacci, M. (2000). Long-term outcome for patients with nonmetastatic osteosarcoma of the extremity treated at the istituto ortopedico rizzoli according to the istituto ortopedico rizzoli/osteosarcoma-2 protocol: an updated report. Journal of Clinical Oncology, 18(24), 4016-4027. 
15. Meyers, P. A., Heller, G., Healey, J. H., Huvos, A., Applewhite, A., Sun, M. I. N. G., \& LaQuaglia, M. (1993). Osteogenic sarcoma with clinically detectable metastasis at initial presentation. Journal of Clinical Oncology, 11(3), 449-453.

16. Kaste, S. C., Pratt, C. B., Cain, A. M., Jones-Wallace, D. J., \& Rao, B. N. (1999). Metastases detected at the time of diagnosis of primary pediatric extremity osteosarcoma at diagnosis. Cancer, 86(8), 16021608.

17. Kager, L., Zoubek, A., Pötschger, U., Kastner, U., Flege, S., Kempf-Bielack, B., ... \& Jundt, G. (2003). Primary metastatic osteosarcoma: presentation and outcome of patients treated on neoadjuvant Cooperative Osteosarcoma Study Group protocols. Journal of Clinical Oncology, 21(10), 2011-2018.

18. Bacci, G., Briccoli, A., Longhi, A., Ferrari, S., Mercuri, M., Faggioli, F., ... \& Picci, P. (2005). Treatment and outcome of recurrent osteosarcoma: experience at Rizzoli in 235 patients initially treated with neoadjuvant chemotherapy. Acta Oncologica, 44(7), 748-755.

19. Hawkins, D. S., \& Arndt, C. A. (2003). Pattern of disease recurrence and prognostic factors in patients with osteosarcoma treated with contemporary chemotherapy. Cancer, 98(11), 2447-2456.

20. Ferrari, S., Briccoli, A., Mercuri, M., Bertoni, F., Picci, P., Tienghi, A., ... \& Bacci, G. (2003). Postrelapse survival in osteosarcoma of the extremities: prognostic factors for long-term survival. Journal of Clinical Oncology, 21(4), 710-715.

21. Schaller, R. T., Haas, J., Schaller, J., Morgan, A., \& Bleyer, A. (1982). Improved survival in children with osteosarcoma following resection of pulmonary metastases. Journal of pediatric surgery, 17(5), 546550 .

22. Walters, D. K., Steinmann, P., Langsam, B., Schmutz, S., Born, W., \& Fuchs, B. (2008).

Identification of potential chemoresistance genes in osteosarcoma. Anticancer research, 28(2A), 673-679.

23. Mialou, V., Philip, T., Kalifa, C., Perol, D., Gentet, J. C., Marec-Berard, P., ... \& Hartmann, O.

(2005). Metastatic osteosarcoma at diagnosis. Cancer, 104(5), 1100-1109.

24. Ward, W. G., Mikaelian, K., Dorey, F., Mirra, J. M., Sassoon, A., Holmes, E. C., ... \& Eckardt, J. J. (1994). Pulmonary metastases of stage IIB extremity osteosarcoma and subsequent pulmonary metastases. Journal of clinical oncology, 12(9), 1849-1858.

25. Enneking, W. F., \& Kagan, A. (1975). "Skip" metastases in osteosarcoma. Cancer, 36(6), 2192-2205.

26. Kager, L., Zoubek, A., Kastner, U., Kempf-Bielack, B., Potratz, J., Kotz, R., ... \& Jürgens, H. (2006). Skip metastases in osteosarcoma: experience of the Cooperative Osteosarcoma Study Group. Journal of clinical oncology, 24(10), 1535-1541.

27. Leavey, P. J., Day, M. D., Booth, T., \& Maale, G. (2003). Skip metastasis in osteosarcoma. Journal of pediatric hematology/oncology, 25(10), 806-808.

28. Sajadi, K. R., Heck, R. K., Neel, M. D., Rao, B. N., Daw, N., Rodriguez-Galindo, C., ... \& Simon, M. A. (2004). The incidence and prognosis of osteosarcoma skip metastases. Clinical orthopaedics and related research, 426, 92-96. 
29. Malawer, M. M., \& Dunham, W. K. (1983). Skip metastases in osteosarcoma: recent experience. Journal of surgical oncology, 22(4), 236-245.

30. Bielack, S. S., Kempf-Bielack, B., Delling, G., Exner, G. U., Flege, S., Helmke, K., ... \& Zoubek, A. (2002). Prognostic factors in high-grade osteosarcoma of the extremities or trunk: an analysis of 1,702 patients treated on neoadjuvant cooperative osteosarcoma study group protocols. Journal of Clinical Oncology, 20(3), 776-790.

31. Bentzen, S. M., Poulsen, H. S., Kaae, S., Myhre Jensen, O., Johansen, H., Mouridsen, H. T., ... \& Arnoldl, C. (1988). Prognostic factors in osteosarcomas. A regression analysis. Cancer, 62(1), 194-202.

32. Raymond, A. K., Chawla, S. P., Carrasco, C. H., Ayala, A. G., Fanning, C. V., Grice, B., ... \& Edeiken, J. (1987, August). Osteosarcoma chemotherapy effect: a prognostic factor. In Seminars in diagnostic pathology (Vol. 4, No. 3, pp. 212-236).

33. Davis, A. M., Bell, R. S., \& Goodwin, P. J. (1994). Prognostic factors in osteosarcoma: a critical review. Journal of Clinical Oncology, 12(2), 423-431.

34. Hudson, M., Jaffe, M. R., Jaffe, N., Ayala, A., Raymond, A. K., Carrasco, H., ... \& Robertson, R. (1990). Pediatric osteosarcoma: therapeutic strategies, results, and prognostic factors derived from a 10year experience. Journal of Clinical Oncology, 8(12), 1988-1997.

35. Martin, J. W., Squire, J. A., \& Zielenska, M. (2012). The genetics of osteosarcoma. Sarcoma, 2012.

36. Matsunaga, E. (1980). Hereditary retinoblastoma: host resistance and second primary tumors. Journal of the National Cancer Institute, 65(1), 43-46.

37. Draper, G. J., Sanders, B. M., \& Kingston, J. E. (1986). Second primary neoplasms in patients with retinoblastoma. British journal of cancer, 53(5), 661.

38. Wang, L. L., Gannavarapu, A., Kozinetz, C. A., Levy, M. L., Lewis, R. A., Chintagumpala, M. M., ... \& Lev, D. (2003). Association between osteosarcoma and deleterious mutations in the RECQL4 gene in Rothmund-Thomson syndrome. Journal of the National Cancer Institute, 95(9), 669-674.

39. Hicks, M. J., Roth, J. R., Kozinetz, C. A., \& Wang, L. L. (2007). Clinicopathologic features of osteosarcoma in patients with Rothmund-Thomson syndrome. Journal of clinical oncology, 25(4), 370375.

40. Porter, D. E., Holden, S. T., Steel, C. M., Cohen, B. B., Wallace, M. R., \& Reid, R. (1992). A significant proportion of patients with osteosarcoma may belong to Li-Fraumeni cancer families. Bone \& Joint Journal, 74(6), 883-886.

41. BLOOM, D. (1954). Congenital telangiectatic erythema resembling lupus erythematosus in dwarfs: probably a syndrome entity. AMA American journal of diseases of children, 88(6), 754-758.

42. ARAKI, N., UCHIDA, A., KIMURA, T., YOSHIKAWA, H., AOKI, Y., UEDA, T., ... \& ONO, K. (1991). Involvement of the retinoblastoma gene in primary osteosarcomas and other bone and soft-tissue tumors. Clinical orthopaedics and related research, 270, 271-277. 
43. Belchis, D. A., Meece, C. A., Benko, F. A., Rogan, P. K., Williams, R. A., \& Gocke, C. D. (1996). Loss of heterozygosity and microsatellite instability at the retinoblastoma locus in osteosarcomas. Diagnostic Molecular Pathology, 5(3), 214-219.

44. Deshpande, A., \& Hinds, P. W. (2006). The retinoblastoma protein in osteoblast differentiation and osteosarcoma. Current molecular medicine, 6(7), 809-817.

45. Wadayama, B. I., Toguchida, J., Shimizu, T., Ishizaki, K., Sasaki, M. S., Kotoura, Y., \& Yamamuro, T. (1994). Mutation spectrum of the retinoblastoma gene in osteosarcomas. Cancer research, 54(11), 3042-3048.

46. Toguchida, J., Ishizaki, K., Sasaki, M. S., Ikenaga, M., Sugimoto, M., Kotoura, Y., \& Yamamuro, T. (1988). Chromosomal reorganization for the expression of recessive mutation of retinoblastoma susceptibility gene in the development of osteosarcoma. Cancer research, 48(14), 3939-3943.

47. Feugeas, O., Guriec, N., Babin-Boilletot, A., Marcellin, L., Simon, P., Babin, S., ... \& BrunatMentigny, M. (1996). Loss of heterozygosity of the RB gene is a poor prognostic factor in patients with osteosarcoma. Journal of Clinical Oncology, 14(2), 467-472.

48. Goto, A., Kanda, H., Ishikawa, Y., Matsumoto, S., Kawaguchi, N., Machinami, R., ... \& Kitagawa, T. (1998). Association of loss of heterozygosity at the p53 locus with chemoresistance in osteosarcomas. Japanese journal of cancer research, 89(5), 539-547.

49. Patiño-García, A., Piñeiro, E. S., Díez, M. Z., Iturriagagoitia, L. G., Klüssmann, F. A., \& Ariznabarreta, L. S. (2003). Genetic and epigenetic alterations of the cell cycle regulators and tumor suppressor genes in pediatric osteosarcomas. Journal of Pediatric Hematology/Oncology, 25(5), 362-367.

50. Miller, C. W., Aslo, A., Tsay, C., Slamon, D., Ishizaki, K., Toguchida, J., ... \& Koeffler, H. P. (1990). Frequency and structure of p53 rearrangements in human osteosarcoma. Cancer research, 50(24), 79507954.

51. Overholtzer, M., Rao, P. H., Favis, R., Lu, X. Y., Elowitz, M. B., Barany, F., ... \& Levine, A. J. (2003). The presence of p53 mutations in human osteosarcomas correlates with high levels of genomic instability. Proceedings of the National Academy of Sciences, 100(20), 11547-11552.

52. Miller, C. W., Aslo, A., Won, A., Tan, M., Lampkin, B., \& Koefflar, H. P. (1996). Alterations of thep53, Rb andMDM2 genes in osteosarcoms. Journal of cancer research and clinical oncology, 122(9), 559-565.

53. López-Guerrero, J. A., López-Ginés, C., Pellín, A., Carda, C., \& Llombart-Bosch, A. (2004). Deregulation of the G1 to S-phase cell cycle checkpoint is involved in the pathogenesis of human osteosarcoma. Diagnostic Molecular Pathology, 13(2), 81-91.

54. Pellin, A., Boix-Ferrero, J., Carpio, D., Lopez-Terrada, D., Carda, C., Navarro, S., ... \& LlombartBosch, A. (1997). Molecular Alterations of the RBI, TP53, and MDM2 Genes in Primary and Xenografted Human Osteosarcomas. Diagnostic Molecular Pathology, 6(6), 333-341.

55. Tsuchiya, T., Sekine, K. I., Hinohara, S. I., Namiki, T., Nobori, T., \& Kaneko, Y. (2000). Analysis of the p16INK4, p14ARF, p15, TP53, and MDM2 genes and their prognostic implications in osteosarcoma and Ewing sarcoma. Cancer genetics and cytogenetics, 120(2), 91-98. 
56. Gokgoz, N., Wunder, J. S., Mousses, S., Eskandarian, S., Bell, R. S., \& Andrulis, I. L. (2001). Comparison of p53 mutations in patients with localized osteosarcoma and metastatic osteosarcoma. Cancer, 92(8), 2181-2189.

57. Henriksen, J., Aagesen, T. H., Maelandsmo, G. M., Lothe, R. A., Myklebost, O., \& Forus, A. (2003). Amplification and overexpression of COPS3 in osteosarcomas potentially target TP53 for proteasomemediated degradation. Oncogene, 22(34), 5358-5361.

58. Yan, T., Wunder, J. S., Gokgoz, N., Gill, M., Eskandarian, S., Parkes, R. K., ... \& Andrulis, I. L. (2007). COPS3 amplification and clinical outcome in osteosarcoma. Cancer, 109(9), 1870-1876.

59. Pompetti, F., Rizzo, P., Simon, R. M., Freidlin, B., Mew, D. J., Pass, H. I., ... \& Carbone, M. (1996). Oncogene alterations in primary, recurrent, and metastatic human bone tumors. Journal of cellular biochemistry, 63(1), 37-50.

60. Guo, W., Wang, X., \& Feng, C. (1996). P53 gene abnormalities in osteosarcoma. Chinese medical journal, 109(10), 752-755.

61. Walkley, C. R., Qudsi, R., Sankaran, V. G., Perry, J. A., Gostissa, M., Roth, S. I., ...

\& Alt, F. W. (2008). Conditional mouse osteosarcoma, dependent on p53 loss and potentiated by loss of $\mathrm{Rb}$, mimics the human disease. Genes \& development, 22(12), 1662-1676.

62. Berman, S. D., Calo, E., Landman, A. S., Danielian, P. S., Miller, E. S., West, J. C., ... \& Mukherjee, S. (2008). Metastatic osteosarcoma induced by inactivation of Rb and p53 in the osteoblast lineage. Proceedings of the National Academy of Sciences, 105(33), 11851-11856.

63. Chen, X., Bahrami, A., Pappo, A., Easton, J., Dalton, J., Hedlund, E., ... \& Parker, M. (2014). Recurrent somatic structural variations contribute to tumorigenesis in pediatric osteosarcoma. Cell reports, 7(1), 104-112.

64. Savage, S. A., Mirabello, L., Wang, Z., Gastier-Foster, J. M., Gorlick, R., Khanna, C., ... \& Gokgoz, N. (2013). Genome-wide association study identifies two susceptibility loci for osteosarcoma. Nature genetics, 45(7), 799-803.

65. Onda, M., Matsuda, S., Higaki, S., Iijima, T., Fukushima, J. I., Yokokura, A., ... \& Yamamoto, T. (1996). ErbB-2 expression is correlated with poor prognosis for patients with osteosarcoma. Cancer, 77(1), 71-78.

66. Akatsuka, T., Wada, T., Kokai, Y., Kawaguchi, S., Isu, K., Yamashiro, K., ... \& Ishii, S. (2002). ErbB2 expression is correlated with increased survival of patients with osteosarcoma. Cancer, 94(5), 1397-1404.

67. Gemoll, T., Epping, F., Heinrich, L., Fritzsche, B., Roblick, U. J., Szymczak, S., ... \& Lehr, S. (2015). Increased cathepsin D protein expression is a biomarker for osteosarcomas, pulmonary metastases and other bone malignancies. Oncotarget, 6(18), 16517.

68. Li, Z., Xiao, J., Hu, K., Wang, G., Li, M., Zhang, J., \& Cheng, G. (2015). FBXW7 acts as an independent prognostic marker and inhibits tumor growth in human osteosarcoma. International journal of molecular sciences, 16(2), 2294-2306.

69. Zhou, S., Wang, B., Hu, J., Zhou, Y., Jiang, M., Wu, M., ... \& Yang, X. (2016). miR- 
421 is a diagnostic and prognostic marker in patients with osteosarcoma. Tumor Biology, 1-7.

70. He, J., Zhang, P., Li, Q., Zhou, D., \& Liu, P. (2016). Expression of high mobility group box 1 protein predicts a poorer prognosis for patients with osteosarcoma. Oncology letters, 11(1), 293-298.

71. Zandueta, C., Ormazábal, C., Perurena, N., Martínez-Canarias, S., Zalacaín, M., San Julián, M., ... \& Vicent, S. (2016). Matrix-Gla protein promotes osteosarcoma lung metastasis and associates with poor prognosis. The Journal of pathology.

72. Smeland, S., Müller, C., Alvegard, T. A., Wiklund, T., Wiebe, T., Björk, O., ... \& Brosjö, O. (2003). Scandinavian Sarcoma Group Osteosarcoma Study SSG VIII: prognostic factors for outcome and the role of replacement salvage chemotherapy for poor histological responders. European Journal of Cancer, $39(4), 488-494$.

73. Pakos, E. E., Nearchou, A. D., Grimer, R. J., Koumoullis, H. D., Abudu, A., Bramer, J. A., ... \& Capanna, R. (2009). Prognostic factors and outcomes for osteosarcoma: an international collaboration. European Journal of Cancer, 45(13), 2367-2375.

74. Harting, M. T., Lally, K. P., Andrassy, R. J., Vaporciyan, A. A., Cox Jr, C. S., Hayes-Jordan, A., \& Blakely, M. L. (2010). Age as a prognostic factor for patients with osteosarcoma: an analysis of 438 patients. Journal of cancer research and clinical oncology, 136(4), 561-570.

75. Longhi, A., Pasini, A., Cicognani, A., Baronio, F., Pellacani, A., Baldini, N., \& Bacci, G. (2005). Height as a risk factor for osteosarcoma. Journal of pediatric hematology/oncology, 27(6), 314-318.

76. Pui, C. H., Boyett, J. M., Hancock, M. L., Pratt, C. B., Meyer, W. H., \& Crist, W. M. (1995). Outcome of treatment for childhood cancer in black as compared with white children: the St Jude Children's Research Hospital experience, 1962 through 1992. Jama, 273(8), 633-637.

77. Ru, G., Terracini, B., \& Glickman, L. T. (1998). Host related risk factors for canine osteosarcoma. The Veterinary Journal, 156(1), 31-39.

78. Longhi, A., Pasini, A., Cicognani, A., Baronio, F., Pellacani, A., Baldini, N., \& Bacci, G. (2005). Height as a risk factor for osteosarcoma. Journal of pediatric hematology/oncology, 27(6), 314-318.

79. Gelberg, K. H., Fitzgerald, E. F., Hwang, S., \& Dubrow, R. (1997). Growth and development and other risk factors for osteosarcoma in children and young adults. International journal of epidemiology, 26(2), 272-278.

80. Ruza, E., Sotillo, E., Sierrasesúmaga, L., Azcona, C., \& Patiño-García, A. (2003). Analysis of polymorphisms of the vitamin $\mathrm{D}$ receptor, estrogen receptor, and collagen $\mathrm{I} \alpha 1$ genes and their relationship with height in children with bone cancer. Journal of pediatric hematology/oncology, 25(10), 780-786.

81. Arora, R. S., Kontopantelis, E., Alston, R. D., Eden, T. O., Geraci, M., \& Birch, J. M. (2011). Relationship between height at diagnosis and bone tumours in young people: a meta-analysis. Cancer Causes \& Control, 22(5), 681-688.

82. Buckley, J. D., Pendergrass, T. W., Buckley, C. M., Pritchard, D. J., Nesbit, M. E., Provisor, A. J., \& Robison, L. L. (1998). Epidemiology of osteosarcoma and Ewing's sarcoma in childhood. Cancer, 83(7), 1440-1448. 
83. Subbiah, V., Madsen, V. S., Raymond, A. K., Benjamin, R. S., \& Ludwig, J. A. (2010). Of mice and men: divergent risks of teriparatide-induced osteosarcoma. Osteoporosis international, 21(6), 1041-1045.

84. Bassin, E. B., Wypij, D., Davis, R. B., \& Mittleman, M. A. (2006). Age-specific fluoride exposure in drinking water and osteosarcoma (United States). Cancer Causes \& Control, 17(4), 421-428.

85. Gandhi, D., Naoghare, P. K., Bafana, A., Kannan, K., \& Sivanesan, S. (2016). Fluoride-Induced Oxidative and Inflammatory Stress in Osteosarcoma Cells: Does It Affect Bone Development Pathway?. Biological trace element research, 1-9.

86. Andrews, E. B., Gilsenan, A. W., Midkiff, K., Sherrill, B., Wu, Y., Mann, B. H., \& Masica, D. (2012). The US postmarketing surveillance study of adult osteosarcoma and teriparatide: study design and findings from the first 7 years. Journal of Bone and Mineral Research, 27(12), 2429-2437.

87. Kim, F. M., Hayes, C., Williams, P. L., Whitford, G. M., Joshipura, K. J., Hoover, R. N., ... \& Eckardt, J. J. (2011). An assessment of bone fluoride and osteosarcoma. Journal of dental research, 90(10), 1171-1176.

88. Archer, N. P., Napier, T. S., \& Villanacci, J. F. (2016). Fluoride exposure in public drinking water and childhood and adolescent osteosarcoma in Texas. Cancer Causes \& Control, 1-6.

89. Levy, M., \& Leclerc, B. S. (2012). Fluoride in drinking water and osteosarcoma incidence rates in the continental United States among children and adolescents. Cancer epidemiology, 36(2), e83-e88.

90. Wittig, J. C., Bickels, J., Priebat, D., Jelinek, J., Kellar-Graney, K., Shmookler, B., \& Malawer, M. M. (2002). Osteosarcoma: a multidisciplinary approach to diagnosis and treatment. American family physician, 65(6).

91. Widhe, B., \& Widhe, T. (2000). Initial symptoms and clinical features in osteosarcoma and Ewing sarcoma. The Journal of Bone \& Joint Surgery, 82(5), 667-667.

92. Pan, K. L., Chan, W. H., \& Chia, Y. Y. (2010). Initial symptoms and delayed diagnosis of osteosarcoma around the knee joint. Journal of Orthopaedic Surgery, 18(1), 55.

93. Wick, M. R., McLeod, R. A., Siegal, G. P., Greditzer III, H. G., \& Unni, K. K. (1981). Sarcomas of bone complicating osteitis deformans (Paget's disease): fifty years' experience. The American journal of surgical pathology, 5(1), 47-60.

94. Grimer, R. J., Cannon, S. R., Taminiau, A. M., Bielack, S., Kempf-Bielack, B., Windhager, R., ... \& Szendroi, M. (2003). Osteosarcoma over the age of forty. European Journal of Cancer, 39(2), 157-163.

95. Liu, P. P. L., Leung, K. S., Kumta, S. M., Lee, K. M., \& Fung, K. P. (1996). Bone-specific alkaline phosphatase in plasma as tumour marker for osteosarcoma. Oncology, 53(4), 275-280.

96. Limmahakhun, S., Pothacharoen, P., Theera-Umpon, N., Arpornchayanon, O., Leerapun, T., Luevitoonvechkij, S., \& Pruksakorn, D. (2011). Relationships between serum biomarker levels and clinical presentation of human osteosarcomas. Asian Pac J Cancer Prev, 12(7), 1717-22. 
97. Bieling, P., Rehan, N., Winkler, P., Helmke, K., Maas, R., Fuchs, N., ... \& Romanowski, R. (1996). Tumor size and prognosis in aggressively treated osteosarcoma. Journal of Clinical Oncology, 14(3), 848-858.

98. Geller, D. S., \& Gorlick, R. (2010). Osteosarcoma: a review of diagnosis, management, and treatment strategies. Clin Adv Hematol Oncol, 8(10), 705-718.

99. Stokkel, M., Linthorst, M., Borm, J., Taminiau, A., \& Pauwels, E. (2002). A reassessment of bone scintigraphy and commonly tested pretreatment biochemical parameters in newly diagnosed osteosarcoma. Journal of cancer research and clinical oncology, 128(7), 393-399.

100. Bone Cancer - NCCN. (n.d.). Retrieved November 3, 2016, from

http://www.nccn.org/professionals/physician_gls/PDF/bone.pdf

101. Costelloe, C. M., Macapinlac, H. A., Madewell, J. E., Fitzgerald, N. E., Mawlawi, O. R., Rohren, E. M., ... \& Harrell, R. K. (2009). 18F-FDG PET/CT as an indicator of progression-free and overall survival in osteosarcoma. Journal of Nuclear Medicine, 50(3), 340-347.

102. Hurley, C., McCarville, M. B., Shulkin, B. L., Mao, S., Wu, J., Navid, F., ... \& Bishop, M. W. (2016). Comparison of 18F-FDG-PET-CT and Bone Scintigraphy for Evaluation of Osseous Metastases in Newly Diagnosed and Recurrent Osteosarcoma. Pediatric blood \& cancer.

103. Im, H. J., Wu, H., Yi, Z., Wu, J., Shulkin, B., \& Cho, S. (2016). Baseline Metabolic Tumor Volume Measured by FDG PET/CT Before Neoadjuvant Chemotherapy Predicts Survival in Pediatric Osteosarcoma. Journal of Nuclear Medicine, 57(supplement 2), 429-429.

104. Bajpai, J., Kumar, R., Sreenivas, V., Sharma, M. C., Khan, S. A., Rastogi, S., ... \& Bakhshi, S. (2011). Prediction of chemotherapy response by PET-CT in osteosarcoma: correlation with histologic necrosis. Journal of pediatric hematology/oncology, 33(7), e271-e278.

105. Huvos, A. G., Rosen, G. E. R. A. L. D., \& Marcove, R. C. (1977). Primary osteogenic sarcoma: pathologic aspects in 20 patients after treatment with chemotherapy en bloc resection, and prosthetic bone replacement. Archives of pathology \& laboratory medicine, 101(1), 14-18.

106. Picci, P. (2007). Osteosarcoma (osteogenic sarcoma). Orphanet journal of rare diseases, 2(1), 1.

107. Murphey, M. D., Robbin, M. R., MCRae, G. A., Flemming, D. J., Temple, H. T., \& Kransdorf, M. J. (1997). The many faces of osteosarcoma. Radiographics, 17(5), 1205-1231.

108. Bacci, G., Bertoni, F., Longhi, A., Ferrari, S., Forni, C., Biagini, R., ... \& Lari, S. (2003). Neoadjuvant chemotherapy for high-grade central osteosarcoma of the extremity. Cancer, 97(12), 30683075.

109. Enneking, W. F., Spanier, S. S., \& Goodman, M. A. (1980). A system for the surgical staging of musculoskeletal sarcoma. Clinical orthopaedics and related research, 153, 106-120.

110. American Cancer Society | Information and Resources for Cancer: Breast, Colon, Lung, Prostate, Skin. (n.d.). Retrieved July 15, 2016, from http://www.cancer.org/

111. AJCC - American Joint Committee on Cancer. (n.d.). Retrieved July 15, 2016, from https://cancerstaging.org/Pages/default.aspx 
112. Osteosarcoma. (n.d.). Retrieved July 25, 2016, from

http://www.cancer.org/cancer/osteosarcoma/index

113. Menendez, L. R. (2002). OKU, orthopaedic knowledge update. Rosemont, IL.: American Academy of Orthopaedic Surgeons.

114. Kudawara, I., Aoki, Y., Ueda, T., Araki, N., Naka, N., Nakanishi, H., ... \& Kuratsu, S. (2013). Neoadjuvant and adjuvant chemotherapy with high-dose ifosfamide, doxorubicin, cisplatin and high-dose methotrexate in non-metastatic osteosarcoma of the extremities: a phase II trial in Japan. Journal of Chemotherapy, 25(1), 41-48.

115. Bramwell, V. H., Burgers, M., Sneath, R. S. E. A., Souhami, R., van Oosterom, A. T., Voute, P. A., ... \& Somers, R. (1992). A comparison of two short intensive adjuvant chemotherapy regimens in operable osteosarcoma of limbs in children and young adults: the first study of the European Osteosarcoma Intergroup. Journal of Clinical Oncology, 10(10), 1579-1591.

116. Anninga, J. K., Gelderblom, H., Fiocco, M., Kroep, J. R., Taminiau, A. H., Hogendoorn, P. C., \& Egeler, R. M. (2011). Chemotherapeutic adjuvant treatment for osteosarcoma: where do we stand?. European journal of cancer, 47(16), 2431-2445.

117. Ferrari, S., Smeland, S., Mercuri, M., Bertoni, F., Longhi, A., Ruggieri, P., ... \& Müller, C. (2005). Neoadjuvant chemotherapy with high-dose Ifosfamide, high-dose methotrexate, cisplatin, and doxorubicin for patients with localized osteosarcoma of the extremity: a joint study by the Italian and Scandinavian Sarcoma Groups. Journal of Clinical Oncology, 23(34), 8845-8852.

118. Ferrari, S., Ruggieri, P., Cefalo, G., Tamburini, A., Capanna, R., Fagioli, F., ... \& Alberghini, M. (2012). Neoadjuvant chemotherapy with methotrexate, cisplatin, and doxorubicin with or without ifosfamide in nonmetastatic osteosarcoma of the extremity: an Italian sarcoma group trial ISG/OS-1. Journal of clinical oncology, JCO-2011.

119. Marina N, Smeland S, Bielack S. et al. Comparison of MAPIE versus MAP in patients with a poor response to pre-operative chemotherapy for newly diagnosed high-grade osteosarcoma (EURAMOS-1): an open-label, international, randomized, phase 3, controlled trial. Lancet Oncol 2016, in press.

120. Eilber, F. R., \& Rosen, G. (1989). Adjuvant chemotherapy for osteosarcoma. Semin Oncol, 16(4), 312-322.

121. Link, M. P., Goorin, A. M., Miser, A. W., Green, A. A., Pratt, C. B., Belasco, J. B., ... \& Ayala, A. G. (1986). The effect of adjuvant chemotherapy on relapse-free survival in patients with osteosarcoma of the extremity. New England Journal of Medicine, 314(25), 1600-1606.

122. Picci, P., Bacci, G., Campanacci, M., Gasparini, M., Pilotti, S., Cerasoli, S., ... \& Galletti, S. (1985). Histologic evaluation of necrosis in osteosarcoma induced by chemotherapy regional mapping of viable and nonviable tumor. Cancer, 56(7), 1515-1521.

123. Marina, N., Smeland, S., Bielack, S. S., Bernstein, M., Jovic, G., Hook, J. M., ... \& Teot, L. (2014). MAPIE vs MAP as postoperative chemotherapy in patients with a poor response to preoperative chemotherapy for newly-diagnosed osteosarcoma: results from EURAMOS-1 (Paper 032). 
124. Bertrand, T. E., Cruz, A., Binitie, O., Cheong, D., \& Letson, G. D. (2016). Do surgical margins affect local recurrence and survival in extremity, nonmetastatic, high-grade osteosarcoma?. Clinical Orthopaedics and Related Research®, 474(3), 677-683.

125. Enneking, W. F., Spanier, S. S., \& Goodman, M. A. (1980). A system for the surgical staging of musculoskeletal sarcoma. Clinical orthopaedics and related research, 153, 106-120.

126. Li, X., Moretti, V. M., Ashana, A. O., \& Lackman, R. D. (2012). Impact of close surgical margin on local recurrence and survival in osteosarcoma. International orthopaedics, 36(1), 131-137.

127. Jeon, D. G., Song, W. S., Kong, C. B., Cho, W. H., Cho, S. H., Lee, J. D., \& Lee, S. Y. (2013). Role of surgical margin on local recurrence in high risk extremity osteosarcoma: a case-controlled study.

Clinics in orthopedic surgery, 5(3), 216-224.

128. Bacci, G., Ferrari, S., Mercuri, M., Bertoni, F., Picci, P., Manfrini, M., ... \& Campanacci, M. (1998). Predictive factors for local recurrence in Osteosarcoma 540 patients with extremity tumors followed for minimum 2.5 years after neoadjuvant chemotherapy. Acta Orthopaedica Scandinavica, 69(3), 230-236.

129. Rougraff BT, Simon MA, Kneisl JS, Greenberg DB, Mankin HJ: Limb Salvage compared with amputation for osteosarcoma of the distal end of the femur: A long-term oncological, functional, and quality-of-life study. J Bone joint Surg Am1994: 76(5): 649-656.

130. Springfield, D. S., Schmidt, R. I. C. H. A. R. D., Graham-Pole, J. O. H. N., Marcus, R. B., Spanier, S. S., \& Enneking, W. F. (1988). Surgical treatment for osteosarcoma. J Bone Joint Surg Am, 70(8), 1124-1130.

131. Abudu, A. D. E. S. E. G. U. N., Sferopoulos, N. K., Tillman, R. M., Carter, S. R., \& Grimer, R. J. (1996). The surgical treatment and outcome of pathological fractures in localised osteosarcoma. Bone \& Joint Journal, 78(5), 694-698.

132. Simon, M. A., Aschliman, M. A., Thomas, N., \& Mankin, H. J. (1986). Limb-salvage treatment versus amputation for osteosarcoma of the distal end of the femur. J Bone Joint Surg Am, 68(9), 13311337.

133. Redondo, A., Cruz, J., Lopez-Pousa, A., \& Barón, F. (2013). SEOM clinical guidelines for the treatment of osteosarcoma in adults-2013. Clinical and Translational Oncology, 15(12), 1037-1043.

134. Saeter, G., Høie, J., Stenwig, A. E., Johansson, A. K., Hannisdal, E., \& Solheim, Ø. P. (1995). Systemic relapse of patients with osteogenic sarcoma. Prognostic factors for long term survival. Cancer, 75(5), 1084-1093.

135. Bacci, G., Mercuri, M., Briccoli, A., Ferrari, S., Bertoni, F., Donati, D., ... \& Manfrini, M. (1997). Osteogenic sarcoma of the extremity with detectable lung metastases at presentation. Cancer, 79(2), 245254.

136. Briccoli, A., Rocca, M., Salone, M., Bacci, G., Ferrari, S., Balladelli, A., \& Mercuri, M. (2005). Resection of recurrent pulmonary metastases in patients with osteosarcoma. Cancer, 104(8), $1721-1725$. 
137. Tabone, M. D., Kalifa, C., Rodary, C., Raquin, M., Valteau-Couanet, D., \& Lemerle, J. (1994). Osteosarcoma recurrences in pediatric patients previously treated with intensive chemotherapy. Journal of Clinical Oncology, 12(12), 2614-2620.

138. Chou, A. J., Merola, P. R., Wexler, L. H., Gorlick, R. G., Vyas, Y. M., Healey, J. H., ... \& Meyers, P. A. (2005). Treatment of osteosarcoma at first recurrence after contemporary therapy. Cancer, 104(10), 2214-2221.

139. McTiernan, A., Meyer, T., Michelagnoli, M. P., Lewis, I., \& Whelan, J. S. (2006). A phase I/II study of doxorubicin, ifosfamide, etoposide and interval methotrexate in patients with poor prognosis osteosarcoma. Pediatric blood \& cancer, 46(3), 345-350.

140. DeLaney, T. F., Park, L., Goldberg, S. I., Hug, E. B., Liebsch, N. J., Munzenrider, J. E., \& Suit, H. D. (2005). Radiotherapy for local control of osteosarcoma. International Journal of Radiation Oncology* Biology* Physics, 61(2), 492-498.

141. Longhi, A., Errani, C., De Paolis, M., Mercuri, M., \& Bacci, G. (2006). Primary bone osteosarcoma in the pediatric age: state of the art. Cancer treatment reviews, 32(6), 423-436.

142. Marina, N., Gebhardt, M., Teot, L., \& Gorlick, R. (2004). Biology and therapeutic advances for pediatric osteosarcoma. The oncologist, 9(4), 422-441.

143. Zhou, H., Randall, R. L., Brothman, A. R., Maxwell, T., Coffin, C. M., \& Goldsby, R. E. (2003). Her-2/neu expression in osteosarcoma increases risk of lung metastasis and can be associated with gene amplification. Journal of pediatric hematology/oncology, 25(1), 27-32.

144. Ebb, D., Meyers, P., Grier, H., Bernstein, M., Gorlick, R., Lipshultz, S. E., ... \& Ferguson, W. S. (2012). Phase II trial of trastuzumab in combination with cytotoxic chemotherapy for treatment of metastatic osteosarcoma with human epidermal growth factor receptor 2 overexpression: a report from the children's oncology group. Journal of clinical oncology, 30(20), 2545-2551.

145. Bell, R. S. (1998). Expression of insulin-like growth factor receptor, IGF-1, and IGF-2 in primary and metastatic osteosarcoma. J. Sur. Oncol, 69, 21-27.

146. Pappo, A. S., Vassal, G., Crowley, J. J., Bolejack, V., Hogendoorn, P. C., Chugh, R., ... \& Chawla, S. P. (2014). A phase 2 trial of R1507, a monoclonal antibody to the insulin-like growth factor-1 receptor (IGF-1R), in patients with recurrent or refractory rhabdomyosarcoma, osteosarcoma, synovial sarcoma, and other soft tissue sarcomas: Results of a Sarcoma Alliance for Research Through Collaboration study. Cancer, 120(16), 2448-2456.

147. Perry, J. A., Kiezun, A., Tonzi, P., Van Allen, E. M., Carter, S. L., Baca, S. C., ... \& Helman, E. (2014). Complementary genomic approaches highlight the PI3K/mTOR pathway as a common vulnerability in osteosarcoma. Proceedings of the National Academy of Sciences, 111(51), E5564-E5573.

148. Moriarity, B. S., Otto, G. M., Rahrmann, E. P., Rathe, S. K., Wolf, N. K., Weg, M. T., ... \& Choi, K. (2015). A Sleeping Beauty forward genetic screen identifies new genes and pathways driving osteosarcoma development and metastasis. Nature genetics, 47(6), 615-624.

149. Grignani, G., Palmerini, E., Dileo, P., Asaftei, S. D., D’Ambrosio, L., Pignochino, Y., ... \& Ferrari, S. (2011). A phase II trial of sorafenib in relapsed and unresectable high-grade osteosarcoma after failure of standard multimodal therapy: an Italian Sarcoma Group study. Annals of Oncology, mdr151. 
150. Sulzbacher, I., Birner, P., Trieb, K., Träxler, M., Lang, S., \& Chott, A. (2003). Expression of platelet-derived growth factor-AA is associated with tumor progression in osteosarcoma. Modern pathology, 16(1), 66-71.

151. Nash, G. F., Turner, L. F., Scully, M. F., \& Kakkar, A. K. (2002). Platelets and cancer. The lancet oncology, 3(7), 425-430.

152. Labelle, M., Begum, S., \& Hynes, R. O. (2011). Direct signaling between platelets and cancer cells induces an epithelial-mesenchymal-like transition and promotes metastasis. Cancer cell, 20(5), 576-590.

153. Takagi, S., Takemoto, A., Takami, M., Oh-hara, T., \& Fujita, N. (2014). Platelets promote osteosarcoma cell growth through activation of the platelet-derived growth factor receptor-Akt signaling axis. Cancer science, 105(8), 983-988.

154. Kumar, R. M. R., Arlt, M. J., Kuzmanov, A., Born, W., \& Fuchs, B. (2015). Sunitinib malate (SU11248) reduces tumour burden and lung metastasis in an intratibial human xenograft osteosarcoma mouse model. American journal of cancer research, 5(7), 2156.

155. Huang, J., Liu, K., Yu, Y., Xie, M., Kang, R., Vernon, P. J., ... \& Ni, J. (2012). Targeting HMGB1mediated autophagy as a novel therapeutic strategy for osteosarcoma. Autophagy.

156. Ouyang, Z., Li, H., Zhai, Z., Xu, J., Dass, C. R., Qin, A., \& Dai, K. (2015). Zoledronic Acid: pleiotropic anti-tumor mechanism and therapeutic outlook for osteosarcoma. Current drug targets.

157. Chang, R., Sun, L., \& Webster, T. J. (2014). Short communication: selective cytotoxicity of curcumin on osteosarcoma cells compared to healthy osteoblasts. International journal of nanomedicine, 9,461 .

158. Mori, K., Rédini, F., Gouin, F., Cherrier, B., \& Heymann, D. (2006). Osteosarcoma: current status of immunotherapy and future trends (Review). Oncology reports, 15(3), 693-700.

159. Wan, J., Zhang, X., Liu, T., \& Zhang, X. (2016). Strategies and developments of immunotherapies in osteosarcoma (Review). Oncology letters, 11(1), 511-520.

160. Ortega, R. A., Barham, W., Kumar, B., Shann, S. Y., Yull, F., \& Giorgio, T. D. (2013).

Reprogramming tumor associated macrophages toward an anti-tumor phenotype by targeting the NF- $\kappa \mathrm{B}$ pathway using novel targeted nanotherapeutics. Cancer Research, 73(8 Supplement), 3981-3981.

161. Kleinerman, E. S., Jia, S. F., Griffin, J., Seibel, N. L., Benjamin, R. S., \& Jaffe, N. (1992). Phase II study of liposomal muramyl tripeptide in osteosarcoma: the cytokine cascade and monocyte activation following administration. Journal of clinical oncology, 10(8), 1310-1316.

162. Meyers, P. A., \& Chou, A. J. (2014). Muramyl tripeptide-phosphatidyl ethanolamine encapsulated in liposomes (L-MTP-PE) in the treatment of osteosarcoma. In Current Advances in Osteosarcoma (pp. 307-321). Springer International Publishing.

163. Lammers, T. G. G. M., Hennink, W. E., \& Storm, G. (2008). Tumour-targeted nanomedicines: principles and practice. British journal of cancer, 99(3), 392-397. 
164. Fang, J. (2006). Nano-or submicron-sized liposomes as carriers for drug delivery. Chang Gung medical journal, 29(4), 358.

165. Kleinerman, E. S., Erickson, K. L., Schroit, A. J., Fogler, W. E., \& Fidler, I. J. (1983). Activation of tumoricidal properties in human blood monocytes by liposomes containing lipophilic muramyl tripeptide. Cancer Research, 43(5), 2010-2014.

166. Mantovani, A., Sozzani, S., Locati, M., Allavena, P., \& Sica, A. (2002). Macrophage polarization: tumor-associated macrophages as a paradigm for polarized M2 mononuclear phagocytes. Trends in immunology, 23(11), 549-555.

167. Buddingh, E. P., Kuijjer, M. L., Duim, R. A., Bürger, H., Agelopoulos, K., Myklebost, O., ... \& Cleton-Jansen, A. M. (2011). Tumor-infiltrating macrophages are associated with metastasis suppression in high-grade osteosarcoma: a rationale for treatment with macrophage activating agents. Clinical Cancer Research, 17(8), 2110-2119.

168. Meyers, P. A., Schwartz, C. L., Krailo, M. D., Healey, J. H., Bernstein, M. L., Betcher, D., ... \& Kleinerman, E. (2008). Osteosarcoma: the addition of muramyl tripeptide to chemotherapy improves overall survival - a report from the Children's Oncology Group. Journal of Clinical Oncology, 26(4), 633-638.

169: Chou, A. J., Kleinerman, E. S., Krailo, M. D., Chen, Z., Betcher, D. L., Healey, J. H., ... \& Womer, R. B. (2009). Addition of muramyl tripeptide to chemotherapy for patients with newly diagnosed metastatic osteosarcoma. Cancer, 115(22), 5339-5348.

170. Biteau, K., Guiho, R., Chatelais, M., Taurelle, J., Chesneau, J., Corradini, N., ... \& Redini, F. (2016). L-MTP-PE and zoledronic acid combination in osteosarcoma: preclinical evidence of positive therapeutic combination for clinical transfer. American journal of cancer research, 6(3), 677.

171. Jia, S. F., Worth, L. L., Densmore, C. L., Xu, B., Duan, X., \& Kleinerman, E. S. (2003). Aerosol gene therapy with PEI IL-12 eradicates osteosarcoma lung metastases. Clinical cancer research, 9(9), 3462-3468.

172. Strauss, S. J., McTiernan, A., \& Whelan, J. S. (2004). Late relapse of osteosarcoma: Implications for follow-up and screening. Pediatric blood \& cancer, 43(6), 692-697.

173. Chi, S. N., Conklin, L. S., Qin, J., Meyers, P. A., Huvos, A. G., Healey, J. H., \& Gorlick, R. (2004). The patterns of relapse in osteosarcoma: The memorial Sloan-Kettering experience. Pediatric blood \& cancer, 42(1), 46-51.

174. Hamilton, J. A. (2002). GM-CSF in inflammation and autoimmunity. Trends in immunology, 23(8), 403-408.

175. Arndt, C. A., Koshkina, N. V., Inwards, C. Y., Hawkins, D. S., Krailo, M. D., Villaluna, D., ... \& Bell, S. A. (2010). INHALED GM-CSF FOR FIRST PULMONARY RECURRENCE OF OSTEOSARCOMA; EFFECTS ON DISEASE FREE SURVIVAL AND IMMUNOMODULATION: A REPORT FROM THE CHILDREN'S ONCOLOGY GROUP. Clinical cancer research: an official journal of the American Association for Cancer Research, 16(15), 4024.

176. Wilson, J., \& Balkwill, F. (2002, April). The role of cytokines in the epithelial cancer microenvironment. In Seminars in cancer biology (Vol. 12, No. 2, pp. 113-120). Academic Press. 
177. Dranoff, G. (2004). Cytokines in cancer pathogenesis and cancer therapy. Nature Reviews Cancer, $4(1), 11-22$.

178. Lasfar, A., Abushahba, W., Balan, M., \& Cohen-Solal, K. A. (2011). Interferon lambda: a new sword in cancer immunotherapy. Clinical and Developmental Immunology, 2011.

179. Zeidner, J. F., Gladstone, D. E., Zahurak, M., Matsui, W. H., Gocke, C., Jones, R. J., \& Smith, B. D. (2014). Granulocyte-macrophage colony stimulating factor (GM-CSF) enhances the clinical responses to interferon- $\alpha$ (IFN) in newly diagnosed chronic myeloid leukemia (CML). Leukemia research, 38(8), 886890.

180. Wang, Z., Qiu, S. J., Ye, S. L., Tang, Z. Y., \& Xiao, X. (2001). Combined IL-12 and GM-CSF gene therapy for murine hepatocellular carcinoma. Cancer gene therapy, $8(10)$.

181. Guma, S. R., Lee, D. A., Yu, L., Gordon, N., Hughes, D., Stewart, J., ... \& Kleinerman, E. S. (2014). Natural killer cell therapy and aerosol interleukin-2 for the treatment of osteosarcoma lung metastasis. Pediatric blood \& cancer, 61(4), 618-626.

182. Buddingh, E. P., Schilham, M. W., Ruslan, S. E. N., Berghuis, D., Szuhai, K., Suurmond, J., ... \& Hogendoorn, P. C. (2011). Chemotherapy-resistant osteosarcoma is highly susceptible to IL-15-activated allogeneic and autologous NK cells. Cancer immunology, immunotherapy, 60(4), 575-586.

183. Worth, L. L., Jia, S. F., Zhou, Z., Chen, L., \& Kleinerman, E. S. (2000). Intranasal therapy with an adenoviral vector containing the murine interleukin-12 gene eradicates osteosarcoma lung metastases. Clinical cancer research, 6(9), 3713-3718.

184. Belardelli, F., Ferrantini, M., Proietti, E., \& Kirkwood, J. M. (2002). Interferon-alpha in tumor immunity and immunotherapy. Cytokine \& growth factor reviews, 13(2), 119-134.

185. Zhao, J., Wang, M., Li, Z., Chen, J., Yin, Z., Chang, J., ... \& Wang, S. (2014). Interferon- $\alpha$ suppresses invasion and enhances cisplatin-mediated apoptosis and autophagy in human osteosarcoma cells. Oncology letters, 7(3), 827-833.

186. Bielack, S. S., Smeland, S., Whelan, J. S., Marina, N., Jovic, G., Hook, J. M., ... \& Nadel, H. (2015). Methotrexate, doxorubicin, and cisplatin (MAP) plus maintenance pegylated interferon alfa-2b versus MAP alone in patients with resectable high-grade osteosarcoma and good histologic response to preoperative MAP: first results of the EURAMOS-1 good response randomized controlled trial. Journal of Clinical Oncology, 33(20), 2279-2287.

187. Uzé, G., \& Monneron, D. (2007). IL-28 and IL-29: newcomers to the interferon family. Biochimie, 89(6), 729-734.

188. Gao, D., Zhao, J., Li, X., Xia, Y., Cai, Y., Pan, J., ... \& Wen, H. (2015). Interferon- $\lambda 1$ suppresses invasion and enhances autophagy in human osteosarcoma cell. International journal of clinical and experimental medicine, 8(9), 14999.

189. Li, Z., Tang, J., \& Ye, Z. (2013). Interferon- $\gamma$ enhances human $\gamma \delta$ T cell-mediated osteosarcoma cell killing in vitro. Nan fang yi ke da xue xue bao= Journal of Southern Medical University, 33(1), 22. 
190. Zou, W. (2005). Immunosuppressive networks in the tumour environment and their therapeutic relevance. Nature Reviews Cancer, 5(4), 263-274.

191. Francisco, L. M., Salinas, V. H., Brown, K. E., Vanguri, V. K., Freeman, G. J., Kuchroo, V. K., \& Sharpe, A. H. (2009). PD-L1 regulates the development, maintenance, and function of induced regulatory $\mathrm{T}$ cells. The Journal of experimental medicine, 206(13), 3015-3029.

192. Tivol, E. A., Borriello, F., Schweitzer, A. N., Lynch, W. P., Bluestone, J. A., \& Sharpe, A. H. (1995). Loss of CTLA-4 leads to massive lymphoproliferation and fatal multiorgan tissue destruction, revealing a critical negative regulatory role of CTLA-4. Immunity, 3(5), 541-547.

193. Parry, R. V., Chemnitz, J. M., Frauwirth, K. A., Lanfranco, A. R., Braunstein, I., Kobayashi, S. V., ... \& Riley, J. L. (2005). CTLA-4 and PD-1 receptors inhibit T-cell activation by distinct mechanisms. Molecular and cellular biology, 25(21), 9543-9553.

194. Grosso, J. F., \& Jure-Kunkel, M. N. (2013). CTLA-4 blockade in tumor models: an overview of preclinical and translational research. Cancer Immunity Archive, 13(1), 5.

195. Lussier, D. M., Johnson, J. L., Hingorani, P., \& Blattman, J. N. (2015). Abstract A07: Combining CTLA4 and PD-L1 blockade leads to complete eradication of metastatic osteosarcoma. Cancer Immunology Research, 3(10 Supplement), A07-A07.

196. Lussier, D. M., Johnson, J. L., Hingorani, P., \& Blattman, J. N. (2015). Combination immunotherapy with $\alpha$-CTLA- 4 and $\alpha$-PD-L1 antibody blockade prevents immune escape and leads to complete control of metastatic osteosarcoma. Journal for immunotherapy of cancer, 3(1), 1.

197. Franzius, C., Daldrup-Link, H. E., Sciuk, J., Rummeny, E. J., Bielack, S., Jürgens, H., \& Schober, O. (2001). FDG-PET for detection of pulmonary metastases from malignant primary bone tumors: comparison with spiral CT. Annals of Oncology, 12(4), 479-486.

198. Byun, B. H., Kong, C. B., Lim, I., Kim, B. I., Choi, C. W., Song, W. S., ... \& Lim, S. M. (2013). Comparison of (18) F-FDG PET/CT and (99 m) Tc-MDP bone scintigraphy for detection of bone metastasis in osteosarcoma. Skeletal radiology, 42(12), 1673-1681.

199. Charest, M., Hickeson, M., Lisbona, R., Novales-Diaz, J. A., Derbekyan, V., \& Turcotte, R. E. (2009). FDG PET/CT imaging in primary osseous and soft tissue sarcomas: a retrospective review of 212 cases. European journal of nuclear medicine and molecular imaging, 36(12), 1944-1951. 


\section{Appendix AII}

Grisez, B. T., Ray, J. J., Bostian, P. A., Markel, J. E., \& Lindsey, B. A. (2018). Highly metastatic K7M2 cell line: a novel murine model capable of in vivo imaging via luciferase vector transfection. Journal of Orthopaedic Research®, 36(8), 2296-2304. 
Highly Metastatic K7M2 Cell Line: A Novel Murine Model Capable of In Vivo Imaging via Luciferase Vector Transfection

Brian T. Grisez, Justin J. Ray, Phillip A. Bostian, Justin E. Markel and Brock A. Lindsey

Department of Orthopaedics

West Virginia University

PO Box 9196

Morgantown, WV 26506-9196

Running Title: A Murine Model of Osteosarcoma Capable of In Vivo Imaging

Keywords: Animal cancer models; imaging of tumor progression and metastasis; immunotherapy; sarcoma; tumor microenvironment

Author Contribution Statement: The study was designed by BAL, PAB and BTG. Data were collected by BTG, PAB and JJR. Data were analyzed and processed by BTG, JJR and BAL. The manuscript was written by BTG, JJR, PAB, JEM and BAL. All authors have read and approved the final submitted manuscript.

Financial Support: Research reported in this publication was supported by the National Institute of General Medical Sciences of the National Institutes of Health under Award Number 1U54GM104942-01. The content is solely the responsibility of the authors and does not necessarily represent the official views of the National Institutes of Health.

Corresponding Author:

Brian T. Grisez, MD

West Virginia University

PO Box 9196

Morgantown, WV 26506-9196

304.293 .1168

304.293.0231

bgrisez@hsc.wvu.edu

Disclosures: The authors declare no potential conflicts of interest.

Word Count: 5375

Tables: 2

Figures: 4 


\section{Abstract}

Osteosarcoma is rare and little improvement in survival rates has occurred in the last 25 years despite modern chemotherapeutic treatment. Bioluminescent cell lines for the modeling of osteosarcoma have shown success in tracking metastases in vivo, but commonly use adenoviral vectors to transfect the native cell line with bioluminescent reporters. The purpose of this study was to develop an orthotopic model for metastatic osteosarcoma capable of in vivo monitoring of metastatic and primary tumor burden in an immunocompetent mouse and compare that model to its wild type pathogenesis. K7M2 cells were transfected using a plasmid vector and were stable after 12 weeks. Thirty-four female BALB/c mice aged four to five weeks underwent orthotopic implantation of either wild type $(n=12)$ or transfected $(n=22) \mathrm{K} 7 \mathrm{M} 2$ cells in the proximal tibia. Mice were monitored for tumor growth and weekly In Vivo Imaging System (IVIS) imaging was performed to monitor for pulmonary metastasis. Although tumors developed sooner in the wild type group, no significant differences were seen compared to Transfected Group 1 in rate of inoculation, growth rates after first detection, metastatic rate, and time between inoculation and death. This study establishes a new murine model for metastatic osteosarcoma using the K7M2wt cell line transfected with a non-viral plasmid luciferase vector. The benefits of this preclinical model include an intact immune system and orthotopically driven metastatic disease; this model appears comparable to its wild type counterpart. In the future, the model may be used to examine promising immunomodulatory therapies using bioluminescence in vivo. 


\section{Introduction}

Primary osteosarcoma is rare and has an incidence of 800 new cases diagnosed yearly, with half of cases diagnosed in children.1 Five-year survival rate for localized disease is $60-80 \%$ and a dismal $15 \%$ with a metastatic presentation.2 Complete surgical resection with reconstruction in combination with neo-adjuvant and adjuvant chemotherapy is the mainstay of treatment. However, with the addition of modern chemotherapeutic agents, little progress has been made in patient survival rates in the past 25 years.

Multiple factors contribute to the difficulty of developing new therapies, including a low incidence of disease and rapid clinical course with the onset of metastasis. However, because of the poor survival outcomes, new treatment modalities are crucial. Immunotherapy is an innovative approach to treating osteosarcoma. Spurred by the observation that patients who develop postoperative infections have increased survivorship, the goal of immunotherapy is to harness the beneficial effects of a pro-inflammatory response while limiting destructive side effects.3-5 Most current murine models of osteosarcoma are insufficient to examine immunomodulation because the models use immunodeficient animals.6-10 In an attempt to study tumor burden and metastatic progression in vivo, a model of metastatic osteosarcoma in immunocompromised mice using the luciferase vector and bioluminescent imaging was recently established.11,12. However, the use of an orthotopic driven metastatic model in an immunocompetent model is needed.

Metastatic disease is of particular interest in osteosarcoma; the ability to monitor such progression is paramount in determining the efficacy of novel treatments. Methodologies that are currently available to detect disease in vivo include the use of micro computed tomography (micro CT) and 
magnetic resonance imaging (MRI). These modalities have high sensitivity to detect metastasis in murine models, with the lowest detection limits of 600 um in the lungs.13 However, drawbacks include need for prolonged anesthesia to complete the scan partly because images are obtained between respirations to produce the best resolution. Further, machine capacity often allows for only one animal to be imaged at a time.

In osteosarcoma research, bioluminescent imaging has recently emerged as an important method for tracking both primary tumor burden and metastatic spread. It has the advantage of in vivo monitoring in live animals, precluding the need for euthanasia to survey metastasis and tumor burden. Its primary benefit is reduction in the number of animals required for in vivo studies.12,14,15 The in vivo imaging system (IVIS) quantifies flux produced by a reaction in which the luciferin substrate is oxidized by the luciferase enzyme. The gene for this enzyme can be transfected into the cancerous cell using plasmid vectors or viruses.

The purpose of this study was to develop an orthotopic model for metastatic osteosarcoma capable of in vivo monitoring of metastatic and primary tumor burden in an immunocompetent mouse and compare that model to its wild type pathogenesis. Most murine models consist of xenotransplants and have limited utility in examining the interactions among the host, the microenvironment, and the cancerous cells.16-19 In this syngeneic model, we used the highly metastatic K7M2-wt murine cell line transfected with the luciferase gene and applied cells orthotopically to the tibiae of BALB/c mice. We followed the progression of the tumor and metastasis using IVIS and performed palliative amputations of the affected extremity; at euthanasia, metastatic tumor size was 
quantified and cross referenced with IVIS detection. These data were also compared to the wild type model to determine differences in inoculation rates, tumor growth rates, and metastatic rates.

\section{Methods}

Growth and preparation of transfected and wild type K7M2 cells

The K7M2 murine osteosarcoma cell line (ATCC CRL-2836, ATCC, Manassas, VA) was kindly donated by Dr. Kurt Weiss, MD (University of Pittsburgh Medical Center, Pittsburgh, PA) in April 2014. Mycoplasma testing is not routinely performed in our lab. The K7M2 cell line is derived by harvesting pulmonary metastases of the K7 murine osteosarcoma cell line, reimplanting the metastatic cells orthotopically and repeating the harvest of a pulmonary metastatic lesion. The cell line is considered highly aggressive with a reported pulmonary metastatic rate of over $90 \%$ in mice.

Wild Type (WT) K7M2 osteosarcoma cells were cultured in Dulbecco's Modified Eagle's Medium (DMEM) containing $10 \%$ fetal bovine serum and $1 \%$ penicillin and streptomycin (Life Technologies, Carlsbad, $C A$ ) to obtain $75 \%$ confluency. The cells were washed with phosphate buffered saline $(P B S)$ without calcium and magnesium (Corning Inc., Corning, NY) and harvested with Trypsin $(0.25 \%$ Trypsin, 0.1\% EDTA in HBSS w/o Calcium, Magnesium and Sodium Bicarbonate, Corning Inc.). The cell density was adjusted to one million cells $(1.0 \times 106)$ in $25 \mu \mathrm{L}$ of PBS for inoculating mice. Passage one cells were used for orthotopic implantation.

Transfected K7M2 osteosarcoma cells were prepared by adding $1.0 \mathrm{ml}$ (approximately 3.0x105) WT cells into each well of a six-well plate containing $1.0 \mathrm{ml}$ of DMEM containing $10 \%$ fetal 
bovine serum and $1 \%$ penicillin and streptomycin. Cells were incubated at $37 \mathrm{oC}$ for 24 hours to obtain 75\% confluency. A 3:1 transfection ratio complex was created using ViaFect Transfection reagent (Promega, Madison, WI) and pGL4.51 [luc2/CMV/Neo] plasmid vector (Promega) prepared per the manufacturer's specifications and incubated with K7M2 cells for 24 hours. The cells were washed once with PBS, $2.0 \mathrm{ml}$ of DMEM media was added to each well, and the cells were incubated for 48 hours at 37oC. The selective drug Geneticin-418 (Life Technologies) was then added to kill non-transfected cells. After further propagation, the cell density was adjusted to one million cells $(1.0 \times 106)$ in $25 \mu \mathrm{L}$ of PBS for inoculating mice. Passage one transfected cells were used for orthotopic implantation.

\section{Stability of transfected cell line}

To assess if the luciferase reporter vector was stably passed to future generations, in vitro bioluminescence imaging was performed for 12 weeks. For this verification, 4.5 x 105 transfected K7M2 cells were seeded in a 12-well plate, covered with media and incubated for 24 hours which created a final concentration of $5.0 \times 105$ cells per well. The media was removed just prior to imaging and $1.0 \mathrm{ml}$ of $0.5 \mathrm{mM}$ luciferin was added to each well. The cells were imaged using the In Vivo Imaging System (IVIS) Lumina II with Living Image Software (PerkinElmer, Waltham, MA). Bioluminescent imaging was set to medium binning with an exposure time of five minutes.

\section{Surgical implantation of K7M2 cells}

All animal procedures were conducted in accordance with the Institutional Animal Care and Use Committee (IACUC). Thirty-four female BALB/c mice age four to five weeks were purchased from Jackson Laboratories (Bar Harbor, ME) and maintained in pathogen free animal housing 
facilities. A twelve-hour light/dark cycle was used, the animals were fed autoclaved chow, and had access to sterile water ad-libitum.

The Wild Type (WT) group $(\mathrm{n}=12)$ underwent inoculation with wild type K7M2 cells. Transfected Group $1(\mathrm{n}=10)$, Transfected Group $2(\mathrm{n}=10)$, and Transfected Group $3(\mathrm{n}=2)$ were all inoculated with luciferase transfected K7M2 cells. Transfected Group 1 received intraperitoneal injection of luciferin only for tumor detection. Transfected Groups 2 and 3 received both intraperitoneal and intranasal luciferin for tumor detection, along with shaving of the operative limb and chest prior to imaging.

The animal was weighed on a calibrated scale and placed into an anesthesia induction chamber. General anesthesia was induced using isofluorane (Piramal Enterprises Limited, Andhra Pradesh, India) with $100 \%$ oxygen at a rate of 2.5 liters/min until loss of the righting reflex occurred. The animal was then transferred to a heated operating stage, placed supine, and maintained under general anesthesia using a nasal cone at a rate of $2.5 \mathrm{~L} / \mathrm{min}$. Opthalamic lubricant was placed in both eyes and $0.02 \mathrm{ml}$ of $1 \mathrm{mg} / \mathrm{ml}$ buprenorphine SR (Wildlife Pharmaceuticals, Windsor, CO) was injected subcutaneously using a 25 gauge needle.

The animal's operative hind limb was prepped using betadine solution and sterilely draped. Using sterile technique, a small incision was made over the anterolateral leg under an operating microscope. Dissection was carried sharply to the tibia and directed proximally until the metaphyseal flair was identified. An 18 gauge needle was then used to create a small bore hole in the center of the tibia at the level of the proximal tibial flare. As soon as a small pilot hole was 
created, the needle was angled approximately $45^{\circ}$ in the sagittal plane and advanced until the cortex was penetrated. The needle was twisted gently which caused the anterior flare/cortex to separate from the intact tibia, creating a cortical window. The posterior cortex remained intact to avoid a bicortical fracture. Twenty-five microliters of PBS containing one million K7M2 cells was then injected into the defect. The muscle was pulled back over the bone and the skin was closed in a running fashion using a 4-0 vicryl suture. Animals were awakened and recovered in the usual fashion.

\section{Clinical monitoring of mice}

Mice were monitored weekly until a visible tumor was appreciated after which they were measured daily using digital calipers (greatest width $x$ by greatest length). Tumor volume was calculated using the formula $\mathrm{V}=($ length $x$ width $) / 2$. The mice were graded using our institution's tumor burden scoring system weekly. This scoring system ranges from 0 to 60 ( 0 = healthy animal; 60 $=$ requires euthanasia) and is based on general appearance (fur, mucus membranes, response to stimuli), body condition (well nourished, thin, cachectic), neurologic status (head tilt, bulging eyes, depression, self-mutilation, limb paralysis, seizures), tumor appearance (ulcerated, limits ability to ambulate, bleeding), and respiratory rate (normal, increased rate/effort, severe distress). After the elevation of any scoring variable, the animals were monitored daily and veterinary staff was consulted to ensure humane treatment and minimization of pain.

\section{IVIS imaging}

IVIS imaging uses a charge-coupled device camera that is able to detect light emitted from the luciferin-luciferase reaction within the tumor bed. Once a visibly detectable tumor was identified, 
animals were imaged weekly using the IVIS Lumina II with Living Image Software to monitor metastatic burden. After isoflurane induction, the animal was injected with luciferin $(150 \mathrm{mg} / \mathrm{kg})$ in the intraperitoneal cavity and placed onto the imaging platform, dorsal side up. Exposure varied between one and five minutes and bioluminescence binning was set to medium. Images were taken approximately five minutes post luciferin injection. After the first set of images was performed, the lower extremity (ifpresent, see Tissue harvest below) was shielded for the second set of images. This method improves sensitivity for small lung tumors that are below the low threshold intensity obtained when a tumor is present in the limb (Figures 1a and 1b).

\section{Intranasal luciferin}

Ten BALB/c mice (Transfected Group 2) aged four to five weeks underwent inoculation with one million K7M2 cells according to the same protocol as mentioned above. These mice received 30 microliters of intranasal luciferin $(150 \mathrm{mg} / \mathrm{kg})$ in addition to the intraperitoneal luciferin as mentioned above. The operative extremity and chest of these mice were shaved prior to imaging. Otherwise, IVIS imaging settings, exposures, and images were taken in accordance with the protocol mentioned above. The purpose of this transfected group was to show improved IVIS sensitivity and earlier detection of pulmonary metastatic disease using intranasal luciferin.

\section{Tumor luminescence over time}

Two BALB/c mice (Transfected Group 3) aged four to five weeks underwent inoculation with one million K7M2 cells according to the same protocol as mentioned above. These mice received both intranasal and intraperitoneal luciferin according to the protocol mentioned above. The operative extremity of these mice was shaved prior to imaging. IVIS imaging settings, exposures, and images 
were taken in accordance with the above protocol. The purpose of this transfected group was to monitor luminescence and primary tumor size over time. Mice were imaged twice weekly until the tumor score reached 60 , at which time the mice were euthanized.

\section{Tissue harvest}

Once the tumor reached sufficient volume (around $1.7 \mathrm{~cm} 3$ ), a palliative amputation of the affected hind limb was performed. The mouse was anesthetized and analgesic was provided as described previously. After prepping with betadine and sterile draping, a longitudinal incision was made on the medial third of the tumor bed. The tumor was dissected away from the peritoneum, taking care not to violate the peritoneal cavity. The femoral vessels were identified along the medial tumor and traced toward the pelvis. Using 4-0 vicryl suture, the vessels were ligated just distal to the inguinal ligament. The hip was disarticulated from the pelvis by sharply incising the capsule, exposing the femoral head and dislocating it from the acetabulum with the tip of the scalpel. The limb was amputated by circumferentially dividing any remaining soft tissue attachments in line with the original incision. The skin edges were approximated and closed using a 4-0 vicryl suture in a running fashion. The animal was then awakened and recovered in the usual fashion.

Weekly IVIS imaging was continued (per above) until the tumor score reached 60 or at 16 weeks post-inoculation. At this time, the mice were euthanized using carbon dioxide asphyxiation. The lungs were harvested en bloc and placed into a conical tube containing $10 \%$ neutral buffered formalin $(N B F)$. The specimens were delivered to the Pathology Laboratory for Translational Medicine, processed, and embedded per their protocol. The paraffin blocks were mounted on a microtome and $5 \mu \mathrm{m}$ sections were obtained every $25 \mu \mathrm{m}$ of sectioning until the entire specimen 
was exhausted. The slides were stained with hematoxylin and eosin. Slides were analyzed by one author $(B G)$ as well as a board certified pathologist to confirm the presence of metastatic disease. The number of metastatic lesions was counted as well as the dimensions of the single largest tumor identified in the specimen.

\section{Results}

Stability of transfected cell line

There were no significant differences in radiance of the transfected cells at baseline and at 12 weeks $\left(3.93 \times 106 \mathrm{p} / \mathrm{sec} / \mathrm{cm}_{2}\right.$ versus $\left.9.71 \times 106 \mathrm{p} / \mathrm{sec} / \mathrm{cm}_{2}\right)$. Linear regression modeling did not reveal significant differences in radiance of the transfected cells over time $\left(R_{2}=0.019, p=0.65\right)$.

\section{Inoculation data of the wild type group}

Twelve mice were inoculated ( 6 left limbs, 6 right limbs) with wild type K7M2 cells. The average mouse weight was $18.73 \mathrm{~g}$ (range $17.5-21.0$ ) at time of inoculation. Eleven of twelve mice grew primary tumors at an average of 16.82 days (range $15-26$ ) after cell implantation. One mouse died 15 days after the inoculation; no tumor growth was noted in this mouse. Eight mice underwent palliative amputation of their limb at an average of 12 days (range 10 - 16) after first tumor sighting. Seven of eight survived the palliative amputation; one mouse died due to uncontrollable hemorrhage from the femoral vessels. Three mice did not undergo palliative amputation of their hind limb; these mice were euthanized at the time of primary tumor harvest. Average mouse weight was $23.58 \mathrm{~g}$ (range $21.0-26.5$ ) at time of amputation or early euthanasia and average tumor weight was $2.72 \mathrm{~g}$ (range $1.90-4.53$ ). A primary recurrence of the tumor was noted in six of the eight mice. Five mice were euthanized at an average of 74 days (range 68 - 89) after inoculation 
for tumor scores of 60. All five of these mice had noted recurrent primary disease. Two mice were euthanized at 16 weeks post-inoculation in accordance with the study protocol; neither mouse demonstrated primary recurrence and both had a tumor score of 0 at time of euthanasia. Three mice were euthanized at an average of 10.3 days (range 10-12) after first tumor sighting. At the time of euthanasia, lung specimens were collected on 10 mice (all eight that underwent palliative amputation and two mice euthanized at the time of primary tumor harvest) and processed for metastatic evaluation (see below).

\section{Inoculation data for Transfected Group 1}

Ten mice were inoculated ( 5 left limbs, 5 right limbs) with the luciferase transfected K7M2 cells. The average mouse weight was $18.46 \mathrm{~g}$ (range $16.5-20.8$ ) at the time of inoculation. Nine of the ten mice grew a primary tumor at an average of 35 days (range 27 - 55) after cell implantation. One mouse failed to develop a tumor; this mouse was euthanized eight weeks after inoculation. Six mice underwent palliative amputation of the affected hind limb an average of 11 days (range $9-18)$ after first tumor sighting. All mice survived the palliative amputation. Three mice did not undergo a palliative amputation of their affected limb; one mouse died during IVIS imaging prior to the procedure and two mice were euthanized at the time of primary tumor harvest rather than undergoing an amputation. The average mouse weight at time of amputation or early euthanasia was $25.2 \mathrm{~g}$ (range $23.5-26.5$ ) and the average tumor weight was $3.06 \mathrm{~g}$ (range 2.06 - 4.34). Four of the six mice that underwent palliative amputation demonstrated a recurrence of tumor growth at the amputation site. Four mice were euthanized at an average of 87 days (range $77-132)$ after inoculation due to a tumor score of 60 . One mouse was euthanized at 16 weeks post-inoculation in accordance with the study protocol and one mouse died in the IVIS system 91 days after 
inoculation (41 days after palliative amputation). The lungs of seven mice (all six that underwent palliative amputation and the one mouse that did not develop a primary tumor) were harvested at the time of euthanasia for histological analysis (see below).

\section{Inoculation data for Transfected Group 2}

Ten mice were inoculated (5 left limbs, 5 right limbs) with the luciferase transfected K7M2 cells to study IVIS sensitivity and detection of pulmonary metastasis using intranasal luciferin. The average mouse weight was $18.6 \mathrm{~g}$ (range $17.0-20.0$ ) at the time of inoculation. Eight of the ten mice grew a primary tumor at an average of 23 days (range $21-26$ ) after cell implantation. Two mice failed to develop a tumor; these mice were euthanized sixteen weeks after inoculation. Eight mice underwent palliative amputation of the affected hind limb an average of 12 days (range 8 16) after first tumor sighting. Seven mice survived the palliative amputation; one mouse died due

to uncontrollable hemorrhage from the femoral vessels. The average mouse weight at time of amputation was $23.0 \mathrm{~g}$ (range $20.0-24.0$ ) and the average tumor weight was $2.13 \mathrm{~g}$ (range 1.13 -3.0). Seven mice were euthanized at an average of 57 days (range 48 - 79) after inoculation due to a tumor score of 60 . Two mice were euthanized at 16 weeks post-inoculation in accordance with the study protocol and one mouse died during palliative amputation. The lungs of seven mice (all seven that survived palliative amputation) were harvested at the time of euthanasia for histological analysis (see below).

\section{Inoculation data of Transfected Group 3}

Two mice were inoculated (1 left limb, 1 right limb) with the luciferase transfected K7M2 cells to monitor luminescence and primary tumor size over time. The average mouse weight was $17.0 \mathrm{~g}$ at 
the time of inoculation. Both mice grew a primary tumor at an average of 11 days after cell implantation. These mice were imaged twice weekly until reaching a tumor score of 60, at which time the mice were euthanized. The lungs were not harvested for histological analysis.

\section{Analysis}

There were no significant differences between the WT and Transfected Group 1 with regard to inoculation rate, time from tumor detection to removal, tumor weight, mouse weight at death, or time from inoculation to time of death (any cause). There was a significant difference between the time of inoculation to time of tumor growth and weight of the mouse at time of primary tumor harvest, however. On average, it took 17.85 days longer for a primary tumor to develop in the transfected mice compared to the wild type strain $(p<0.0001)$ and the transfected mice weighed $1.63 \mathrm{~g}$ more $(p=0.0175)$ (Table 1).

There were no significant differences between Transfected Group 1 and Transfected Group 2 with regard to inoculation rate, time from tumor detection to removal, time from inoculation to death (days), metastatic rate, or IVIS positivity in histologically-confirmed metastasis. There was a significant difference noted between the time from inoculation to primary tumor detection, mouse weight at time of tumor harvest, and average primary tumor weight. On average, it took 12.2 days longer for a primary tumor to develop in Transfected Group 1 compared to Group 2 ( $p$ $=0.0032)$. Tumors for Transfected Group 1 weighed $0.87 \mathrm{~g}$ more than tumors for Group $2(p=$ 0.0269) (Table 2).

\section{Histology results}


Lungs were collected on ten of the twelve WT specimens. Lungs were not collected on one mouse that died prior to developing a primary tumor and one mouse euthanized at the time of primary tumor harvest. Nine of ten specimens showed evidence of metastatic disease when analyzed microscopically. The number and size of metastatic nodules varied per mouse with a range of 1 to $>10$ lesions measuring $0.1 \mathrm{~mm}$ to $5.8 \mathrm{~mm}$.

Lungs were collected on seven of ten Transfected Group 1 mice. Lungs were not collected on one mouse that died during IVIS imaging (prior to palliative amputation) and two mice euthanized at the time of primary tumor collection. Six of seven mice showed evidence of metastatic disease by microscopy. Again, size and number of lesions ranged from 1 to $>10$ and measured $1.3 \mathrm{~mm}$ to 20 $\mathrm{mm}$ in greatest single dimension. One mouse (T17) showed nearly complete obliteration of native lung tissue with tumor; tumor was seen within the lymph nodes as well as invading the diaphragm (Figures 2a-c).

Lungs were collected on seven of ten Transfected Group 2 mice. Lungs were not collected on one mouse that died during palliative amputation and two mice that failed to develop a primary tumor. Six of seven mice showed evidence of metastatic disease by microscopy. Size and number of lesions ranged from 1 to $>10$ and measured $0.1 \mathrm{~mm}$ to $4.0 \mathrm{~mm}$ in greatest single dimension. Lungs were not collected for Transfected Group 3 mice.

Histologically, specimens revealed confluent sheets of poorly differentiated cells. There was a spectrum of cells from those with a spindle shaped appearance, prominent chromatin, and eosinophilic cytoplasm to those with a purely anaplastic appearance. High numbers of mitotic 
figures were appreciated. There was no statistical difference between the rate of metastasis between the WT and Transfected Group 1 (90\% WT versus 86\% transfected $\mathrm{p}=1.0000$ ).

\section{IVIS data}

Seven of ten mice in Transfected Group 1 were imaged routinely by IVIS. All primary tumors were confirmed by presence of a bioluminescent signal from the leg region that was inoculated. Three mice showed evidence of a pulmonary metastatic lesion by IVIS. These lesions were seen on average 85.3 days (range 51 - 128) after inoculation with tumor cells. The smallest pulmonary lesion that was IVIS positive measured $5.3 \mathrm{~mm}$ microscopically. This mouse did not show a pulmonary lesion until 128 days after inoculation and was euthanized four days later (Figures $3 a$ and $3 b$ ). The largest non-IVIS positive lesion measured $2.2 \mathrm{~mm}$ microscopically.

Seven of ten mice in Transfected Group 2 were imaged routinely by IVIS. These mice received intranasal and intraperitoneal luciferin, along with shaving of the operative limb and chest. All primary tumors were confirmed by presence of a bioluminescent signal from the leg region that was inoculated. Six mice showed evidence of a pulmonary metastatic lesion by IVIS. These lesions were seen at an average of 50 days (range $44-62$ ) after inoculation with tumor cells. The smallest pulmonary lesion that was IVIS positive measured $1.4 \mathrm{~mm}$ microscopically. The largest non-IVIS positive lesion measured $3.8 \mathrm{~mm}$ microscopically.

The two mice in Transfected Group 3 were imaged routinely by IVIS to monitor luminescence and primary tumor size over time. Primary tumor was first detected on day 12 via IVIS imaging for 
both mice. Primary tumor luminescent intensity increased linearly over time as the size of the tumor increased. There was a slight drop in intensity from day 21 to day 33 (Figures 4a-4c).

\section{Discussion}

Prior to 1975 , osteosarcoma treatment was limited in scope and often ended in amputation. Advances in chemotherapeutic agents led to the widespread use of adjuvant and neo-adjuvant therapy. Concurrent refinement of surgical technique and a paradigm shift from amputation to limb preserving therapy has led to an improved prognosis. Despite these advances, overall prognosis for osteosarcoma remains grave. The most significant challenge surrounding osteosarcoma is its potential for early metastasis and the nature of metastatic lesions that remain elusive to early detection. Indeed, it is estimated that patients presenting with pulmonary metastases have a fiveyear survival rate of less than $20 \%$. Whereas chemotherapeutic agent expansion in the last 20 years has shown only minimal gains with regard to mortality, new avenues for treatment are actively being sought, including immunotherapy.

Relevant animal models are needed to examine the response of pulmonary metastases to various treatment agents and the primacy of an immunocompetent model cannot be understated. Further, being orthotopically driven allows cell selection, self-seeding, and other processes to occur in vivo that may have an effect on the pathogenesis of this disease and, in turn, on treatment success. The resulting metastatic lesions have a unique subset of cellular characteristics secondary to all of these

processes that take part in an orthotopic model. Studies recapitulated in immunocompromised animals,8-10,21-23 by inducing tumorigenesis via intravenous or subcutaneous cell injection, or use of a transfection method with antigenic vectors do not exploit these essential principles.24-25 
Comparing the results of the WT cell line to Transfected Group 1, the only clinically significant difference between the groups was the time from inoculation to detection of tumor growth. On average, the transfected tumors took about twice as long to present; thereafter, growth rates were similar. It is not clear why this pattern occurs, but it is consistent with other studies our lab is conducting. We have speculated that it is likely resulting from the additional genetic material that must be transcribed due to insertion of the luciferase vector. In vitro analysis of the transfected cell line did not demonstrate a loss of cell viability with propagation. In vivo, inoculation rates as well as metastatic rates appear to be similar between the two groups and consistent with previously reported rates for this cell line.20 The smallest detectable lesion by IVIS measured $5.3 \mathrm{~mm}$, which is larger than the detection limit for MRI or micro CT. However, the specificity of the lesions is $100 \%$ in this model because tumors from this cell line will fluoresce, whereas pulmonary nodules could be caused by factors other than cancer. The other advantage is the much shorter length of time under anesthesia as well as the anesthesia being less deep. These issues become much more important for survivability during testing once the animal becomes metastatic. In addition, IVIS is a more cost effective tool and logistically easier to perform compared to MRI.

With the addition of intranasal luciferin and shaving of the chest, the sensitivity of IVIS imaging to detect pulmonary metastasis improved. For Transfected Group 1 that did not receive intranasal luciferin, IVIS imaging detected a signal intensity in three out of six mice with histologicallypositive pulmonary metastasis. With the addition of intranasal luciferin to Transfected Group 2, IVIS imaging detected a signal intensity in six out of seven mice with histologically-positive pulmonary metastasis. The sensitivity to detect pulmonary metastasis was $50 \%$ in Transfected 
Group 1 compared to an improved sensitivity of $85.7 \%$ in Transfected Group 2 with the addition of intranasal luciferin and shaving of the chest.

Pulmonary metastasis was also detected much earlier on IVIS with the addition of intranasal luciferin and shaving of the chest. On average, pulmonary metastasis was detected on IVIS imaging 50 days after inoculation in Transfected Group 2 compared to 85 days after inoculation in Transfected Group 1 that did not receive intranasal luciferin $(\mathrm{p}=0.0568)$. The smallest pulmonary lesion that was IVIS positive measured $5.3 \mathrm{~mm}$ microscopically in Transfected Group 1 compared to $1.4 \mathrm{~mm}$ microscopically in Transfected Group 2. With the addition of intranasal luciferin and shaving, pulmonary metastasis was detected much earlier and with much smaller histologically sized lesions. The increased sensitivity of intranasal luciferin has been confirmed in previous studies, as one study showed a nearly ten-fold increase in sensitivity on bioluminescent imaging when comparing intranasal to intraperitoneal delivery of luciferin.26

The utility of IVIS imaging can also be extended to monitor temporal increases in tumor intensity as the size of the tumor increases. Temporal increases in tumor intensity was demonstrated by the data from Transfected Group 3, which showed that the tumor intensity (photons/second) increased linearly over time as the size of the tumor increased. There was a slight drop in intensity from day 21 to day 33, which likely corresponded to central tumor necrosis or hypoxic areas of the tumor bed as the tumor continued to grow in size. A depiction of potential tumor necrosis or hypoxia is well visualized in Figure 4a for mouse T41 at day 33. Based on these data, it seems reasonable to extend the application of IVIS imaging to monitor metastatic luminescence over time. 
Khanna et al. reported the results of orthotopically implanted K7M2 cells in BALB/c mice.20 They found a tumor take rate of $95 \%$ and a pulmonary metastatic rate of $93.3 \%$ with an average of 5.4 metastatic lesions per mouse. All mice underwent a palliative amputation of their affected hind limb once tumor volume reached approximately $450 \mathrm{~mm}$. Tumor latency (time from implantation to detection) was $14+/-3.1$ days which is consistent with the WT mice in our study; however, in our study, tumors were larger and were removed, on average, ten days sooner. Metastases in the Khanna study were enumerated using India ink via intratracheal injection at the time of euthanasia instead of histology which is the clinical standard. In addition, they did not use a luciferase reporter making it impossible to perform in vivo imaging.

Sottnik and colleagues developed a luciferase transfected orthotopic model of mouse osteosarcoma in $\mathrm{C} 3 \mathrm{H}$ mice using the DLM8 cell line.15 A plasmid transfection process was utilized. All mice underwent palliative amputation of their hind limbs at various intervals following implantation. They did find that amputations performed at seven days post implantation did not develop metastases indicating that peripheral organ seeding does not occur at the time of inoculation and metastatic potential requires maturation of the primary tumor bed. All mice amputated after day seven in their study developed metastatic disease by 22 days post-implantation. They did not report the rate of IVIS positive pulmonary lesions and did not have a non-metastatic line for comparison (the K12 cell line for the K7M2).

Miretti et al. developed an orthotopic lentiviral luciferase transfected K5 osteosarcoma model in BALB/c mice.14 They reported all mice had IVIS detectable metastatic lesions by 45 days posttumor implantation. The wild type and transfected cell lines had similar rates of primary tumor 
and spontaneous pulmonary disease development. They did not correlate tumor size by histology to the IVIS results. A potential drawback to this study is the use of a lentivirus transfection process which may have an effect on tumor infiltrating lymphocytes moreso than a plasmid vector, which was used in our study.

There are some limitations to our model. First, the K7M2 cell line is murine in origin and not human derived. However, the utility of murine derived tumor models is well established. The use of xenograft models is an alternative; however, it is known that tumor characteristics are influenced by the surrounding microenvironment. Thus, invasion of murine cells may affect the tumorigenicity. The development of metastatic disease does take time following orthotopic implantation. However, the use of orthotopic models most closely mimics the clinical course of cancer progression in this disease.

This study establishes a new murine model for metastatic osteosarcoma in an immunocompetent mouse using the K7M2-wt highly metastatic cell line transfected with a non-viral plasmid luciferase vector. Bioluminescence and histopathology confirmed the establishment of a primary tumor and metastasis to the lungs. The sensitivity of IVIS imaging was determined by comparison of histopathological analysis of the lungs. To our knowledge, this is the first syngeneic osteosarcoma using the K7M2 cell line that uses bioluminescence to survey and quantify metastatic burden. The primary benefit of the preclinical model is that the immune system is intact, metastatic disease is orthotopically driven, and it appears to have a comparable pathogenesis to its wild type counterpart. The model can be used to examine promising immunomodulatory therapies in the future using bioluminescence in vivo. 


\section{Acknowledgments}

The authors thank Jabeen Noore, PhD for culturing and preparation of the K7M2 cells. The authors also thank Gerald R. Hobbs, PhD for his assistance with the statistical analysis and James E. Coad, MD for histopathological assistance.

Small animal imaging and image analysis were performed in the West Virginia University Animal Models \& Imaging Facility, which has been supported by the West Virginia University Cancer Institute and NIH grants P20 RR016440, P30 GM103488, and P30RR032138, and U54GM104942. 


\section{Figure Legends}

Figure la demonstrates extensive recurrent tumor growth after palliative amputation. No pulmonary lesions appear to be present, although 113 (middle) had evidence of a pulmonary lesion a week prior (not shown). Figure $1 \mathrm{~b}$ shows bioluminescent signal consistent with pulmonary metastasis after shielding of the lower extremities. The images were taken sequentially the same day.

Figure 2 a illustrates extensive pulmonary metastatic disease by IVIS. At necropsy, widespread disease was found with near total obliteration of the chest cavity with tumor (Figure 2b). Histologically, the classic spindle cell morphology is appreciated, with tumor invading the diaphragm (Figure 2c).

Figure 3 a show that, at 128 days post-inoculation, a bioluminescent signal was detected in the pulmonary region of mouse T16. This lesion (Figure 3b) measured $5.3 \mathrm{~mm}$ histologically and was the smallest IVIS positive lesion.

Figure 4 a illustrates temporal changes in luminescent intensity over time in transfected mice T41 and T42. Figure $4 b$ shows a linear representation of the primary tumor luminescence and tumor size over time for mice T41 and T42. 


\section{References}

1. Osteosarcoma. American Cancer Society [Available Updated February 24, 2017. Accessed April 4, 2017] From: https://www.cancer.org/cancer/osteosarcoma.html

2. Aljubran AH, Griffin A, Pintilie M, Blackstein M Osteosarcoma in adolescents and adults: survival analysis with and without lung metastases. Ann Oncol 2009; 20:1136-1141

3. Chen YU, Xu SF, Xu M, Yu XC Postoperative infection and survival in osteosarcoma patients: Reconsideration of immunotherapy for osteosarcoma. Mol Clin Oncol 2015; 3:495-500 4. Jeys LM, Grimer RJ, Carter SR, Tillman RM, Abudu A Post operative infection and increased survival in osteosarcoma patients: are they associated? Ann Surg Oncol 2007; 14:28872895

5. Lascelles BD, Dernell WS, Correa MT, Lafferty M, Devitt CM, Kuntz CA, et al Improved survival associated with postoperative wound infection in dogs treated with limb-salvage surgery for osteosarcoma. Ann Surg Oncol 2005; 12:1073-1083

6. Berlin O, Samid D, Donthineni-Rao R, Akeson W, Amiel D, Woods VL, Jr. Development of a novel spontaneous metastasis model of human osteosarcoma transplanted orthotopically into bone of athymic mice. Cancer Res 1993; 53:4890-4895

7. Jia SF, Worth LL, Kleinerman ES A nude mouse model of human osteosarcoma lung metastases for evaluating new therapeutic strategies. Clin Exp Metastasis 1999; 17:501-506

8. Kjonniksen I, Winderen M, Bruland O, Fodstad O Validity and usefulness of human tumor models established by intratibial cell inoculation in nude rats. Cancer Res 1994; 54:1715-1719

9. Miwa S, Hiroshima, Y, Yano S, Zhang Y, Matsumoto Y, Uehara F, et al. FluorescenceGuided Surgery Improves Outcome in an Orthotopic Osteosarcoma Nude-Mouse Model. J Orthop Res 2014; 32:1596-1601 
10. Hayashi K, Zhao M, Yamauchi K, Yamamoto N, Tsuchiya H, Tomita K, et al. Systemic targeting of primary bone tumor lung metastasis of high-grade osteosarcoma in nude mice with a tumor-selective strain of Salmonella typhimurium. Cell Cycle 2009; 8:6, 870-875

11. Comstock KE, Hall CL, Daignault S, Mandlebaum SA, Yu C, Keller ET A bioluminescent orthotopic mouse model of human osteosarcoma that allows sensitive and rapid evaluation of new therapeutic agents In vivo. In Vivo 2009; 23:661-66810.

12. Garimella R, Eskew J, Bhamidi P, Vielhauer G, Hong Y, Anderson HC, et al Biological characterization of preclinical Bioluminescent Osteosarcoma Orthotopic Mouse (BOOM) model: A multi-modality approach. J Bone Oncol 2013; 2:11-21

13. Martiniova L, Kotys MS, Thomasson D, Schimel D, Lai EW, Bernardo M, et al Noninvasive monitoring of a murine model of metastatic pheochromocytoma: a comparison of contrast-enhanced microCT and nonenhanced MRI. J Magn Reson Imaging 2009; 29:685-691

14. Miretti S, Roato I, Taulli R, Ponzetto C, Cilli M, Olivero M, et al A mouse model of pulmonary metastasis from spontaneous osteosarcoma monitored in vivo by Luciferase imaging. PLoS One 2008; 3:e1828

15. Sottnik JL, Duval DL, Ehrhart EJ, Thamm DH An orthotopic, postsurgical model of luciferase transfected murine osteosarcoma with spontaneous metastasis. Clin Exp Metastasis 2010; $27: 151-160$

16. Cooper CR, Chay CH, Gendernalik JD, Lee HL, Bhatia J, Taichman RS, et al Stromal factors involved in prostate carcinoma metastasis to bone. Cancer 2003; 97:739-747

17. De Wever, O. and M. Mareel. Role of tissue stroma in cancer cell invasion. J Pathol 2003; 200(4): 429-47

18. Khanna C, Hunter K Modeling metastasis in vivo. Carcinogenesis 2005; 26:513-523 
19. Schmidt-Hansen B, Klingelhofer J, Grum-Schwensen B, Christensen A, Andresen S, Kruse C, et al Functional significance of metastasis-inducing S100A4(Mts1) in tumor-stroma interplay. J Biol Chem 2004; 279:24498-24504

20. Khanna C, Prehn J, Yeung C, Caylor J, Tsokos M, Helman L An orthotopic model of murine osteosarcoma with clonally related variants differing in pulmonary metastatic potential. Clin Exp Metastasis 2000; 18:261-271

21. Berlin O, Samid D, Donthineni-Rao R, Akeson W, Amiel D, Woods VL, Jr. Development of a novel spontaneous metastasis model of human osteosarcoma transplanted orthotopically into bone of athymic mice. Cancer Res 1993; 53:4890-4895

22. Jia SF, Worth LL, Kleinerman ES A nude mouse model of human osteosarcoma lung metastases for evaluating new therapeutic strategies. Clin Exp Metastasis 1999; 17:501-506

23. Luu HH, Kang Q, Park JK, Si W, Luo Q, Jiang W, et al An orthotopic model of human osteosarcoma growth and spontaneous pulmonary metastasis. Clin Exp Metastasis 2005; 22:319329

24. Asai T, Ueda T, Itoh K, Yoshioka K, Aoki Y, Mori S, et al Establishment and characterization of a murine osteosarcoma cell line (LM8) with high metastatic potential to the lung. Int J Cancer 1998; 76:418-422

25. Lussier DM, Johnson JL, Hingorani P, Blattman JN Combination immunotherapy with alpha-CTLA-4 and alpha-PD-L1 antibody blockade prevents immune escape and leads to complete control of metastatic osteosarcoma. J Immunother Cancer 2015; 3:21

26. Buckley S, Howe S, Rahim A, Buning H, McIntosh J, Wong S, et al. Luciferin detection after intranasal vector delivery is improved by intranasal rather than intraperitoneal luciferin administration. Human Gene Therapy 2008; 19, 1050-1056. 
Figure 1
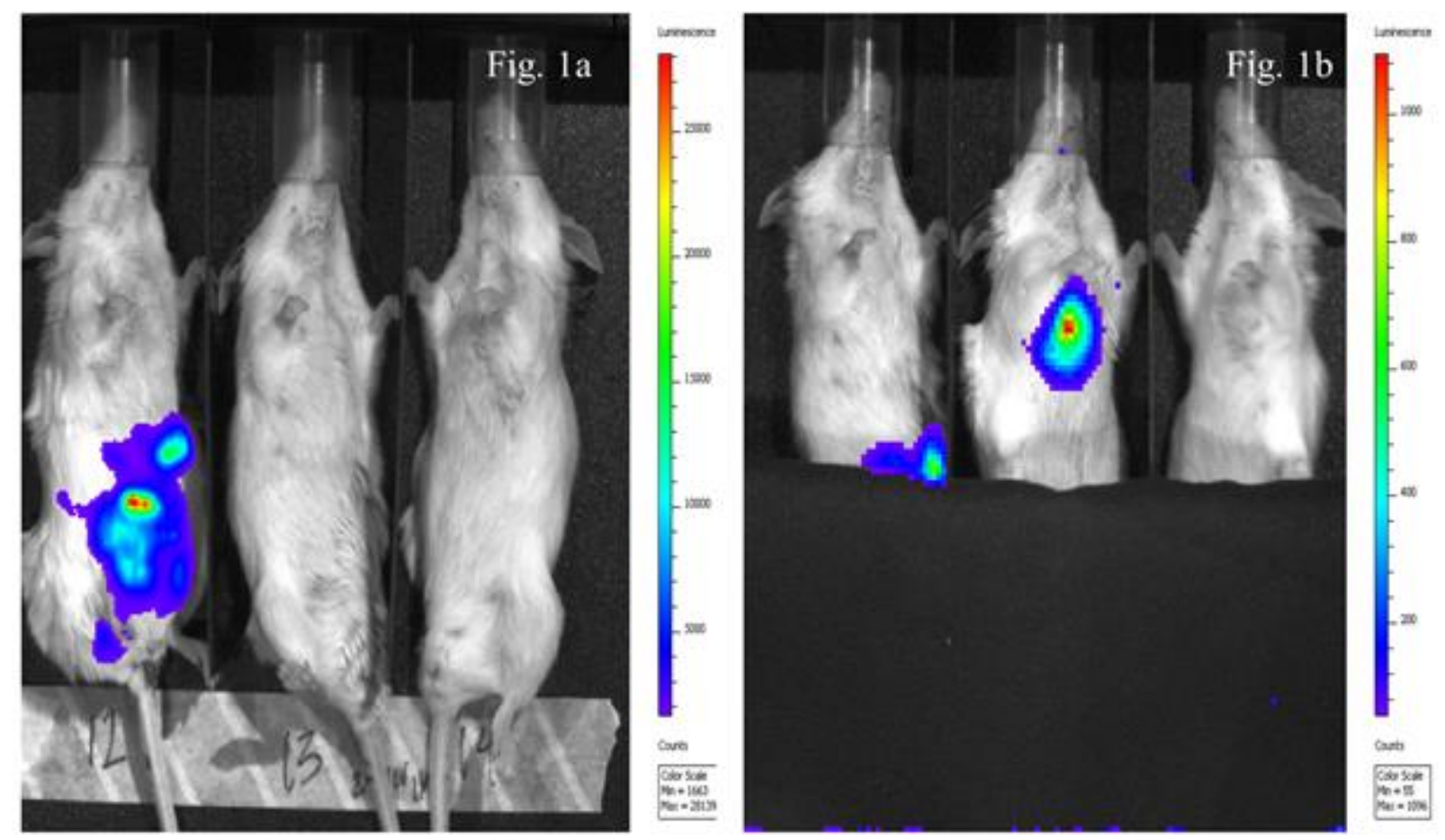

Figure 1a demonstrates extensive recurrent tumor growth after palliative amputation. No pulmonary lesions appear to be present, although T13 (middle) had evidence of a pulmonary lesion a week prior (not shown). Figure 1b shows bioluminescent signal consistent with pulmonary metastasis after shielding of the lower extremeties. The images were taken sequentialy the same day. 
Figure 2
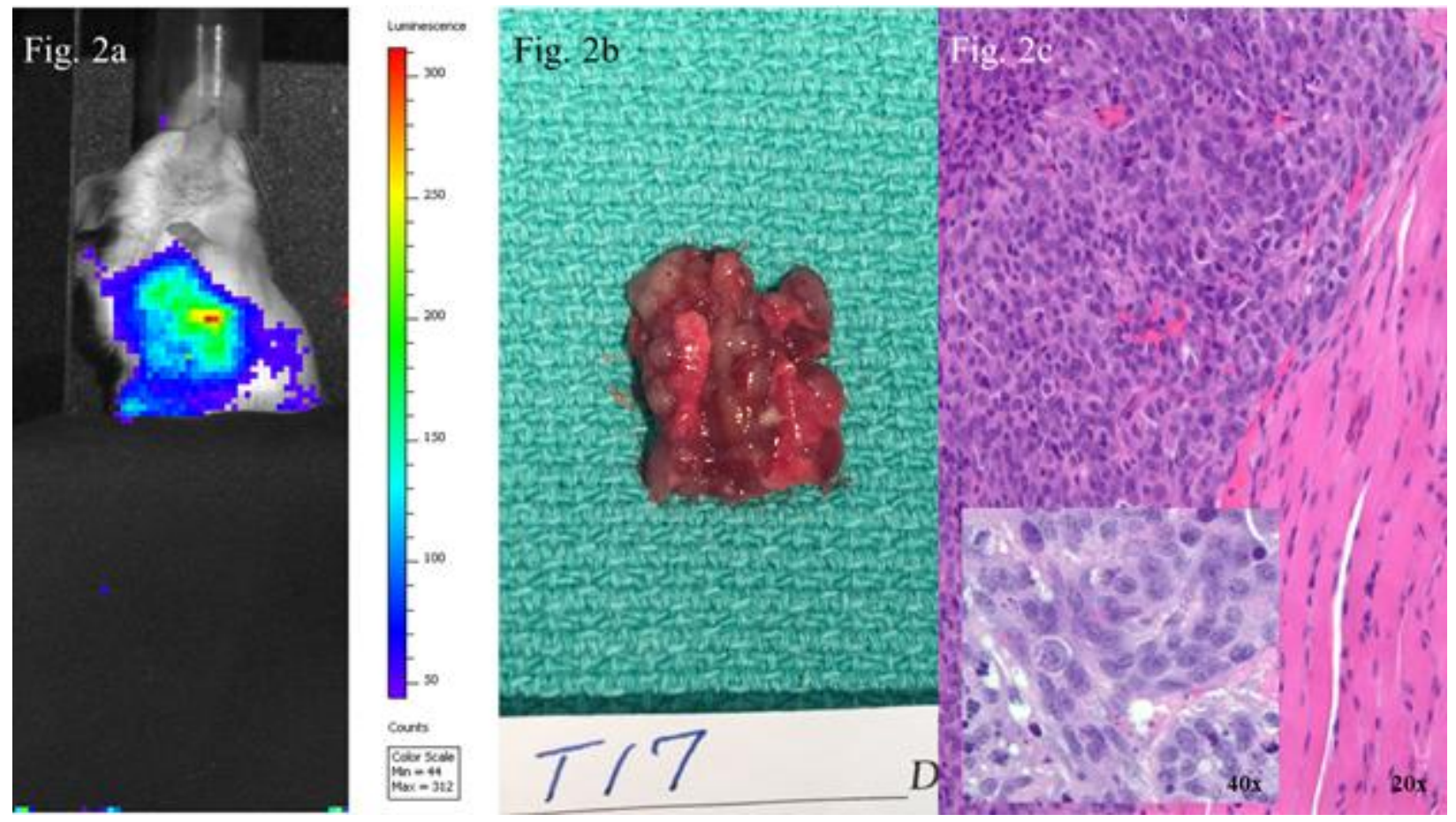

Figure 2a illustrates extensive pulmonary metastatic disease by IVIS. At necroscopy, widespread disease was found with near total obliteration of the chest cavity with tumor (Fig. 2b).

Histologically, the classic spindle cell morphoplogy is appreciated, with tumor invading the diaphragm (Fig. 2c). 
Figure 3
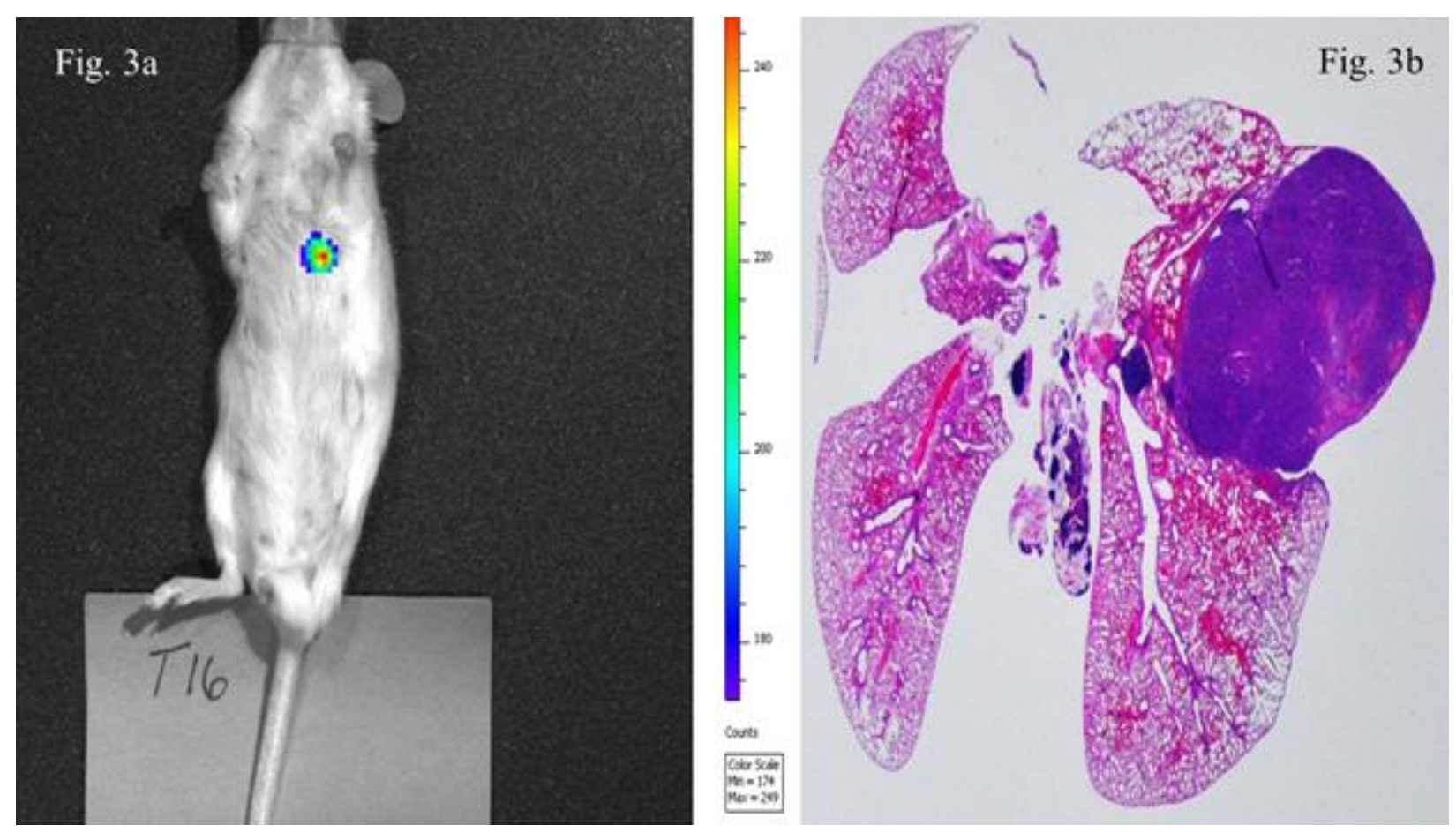

Figure 3 a shows that, at 128 days post-inoculation, a bioluminescent signal was detected in the pulmonary region of mouse T16. This lesion (Fig. 3b) measured $5.3 \mathrm{~mm}$ histologically and was the smallest IVIS positive lesion. 
Figure 4

Fig 4a

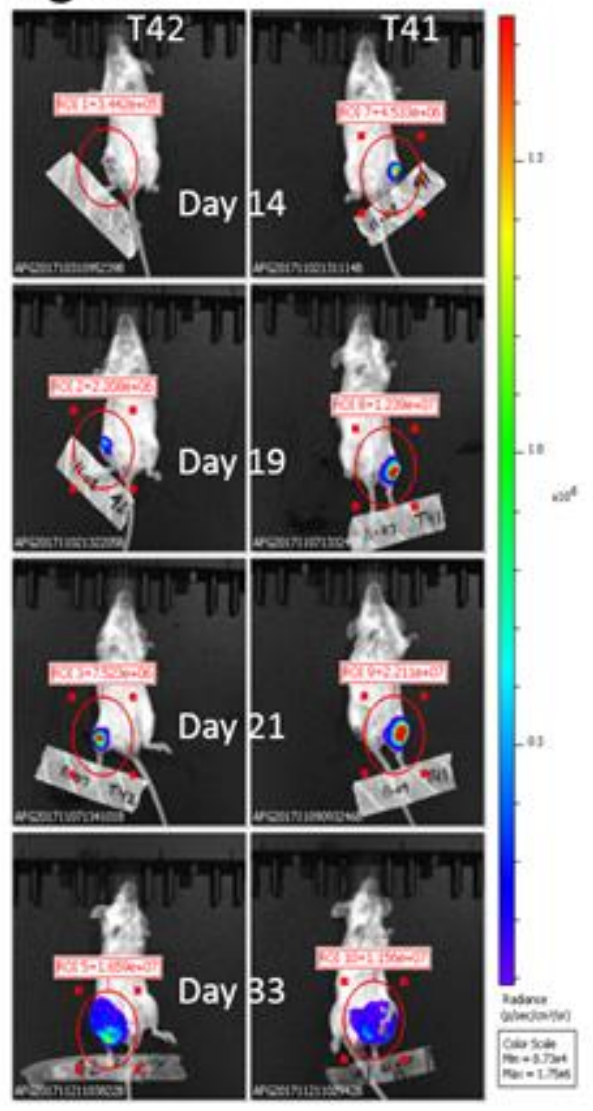

\section{Fig $4 \mathrm{~b}$}

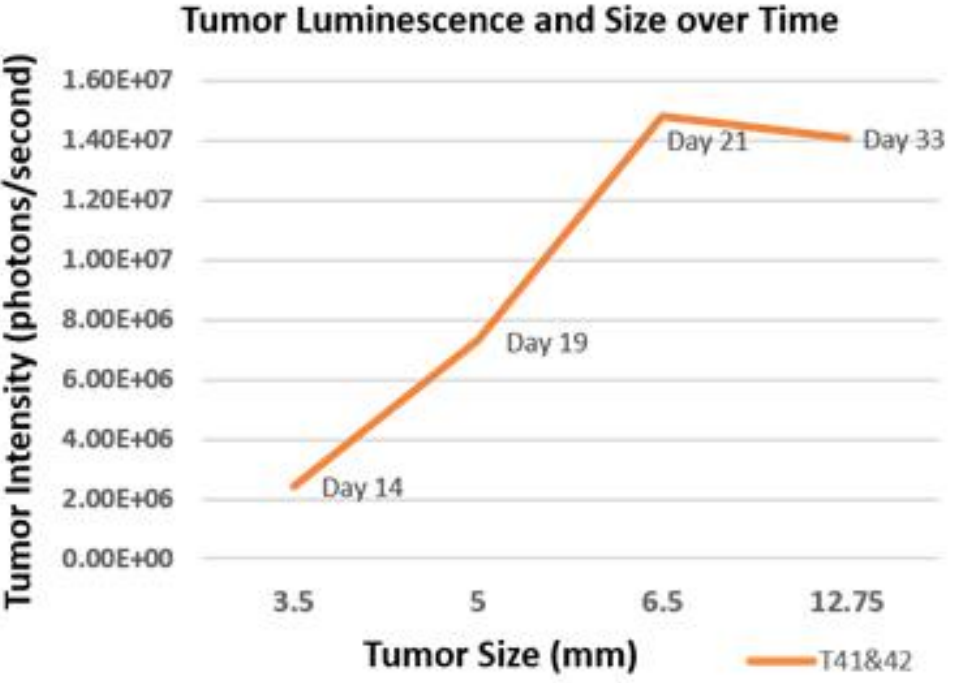

Figure 4a illustrates temporal changes in luminescent intensity over time in transfected mice T41 and T42. Figure $4 \mathrm{~b}$ shows a linear representation of the primary tumor luminescence and tumor size over time for mice T41 and T42. 
Table 1. Summary of tumor characteristics comparing the wild type and transfected cell lines.

\begin{tabular}{|c|c|c|c|}
\hline & Wild Type & Transfected & P-Value \\
\hline Inoculation Rate & $11 / 12$ & $9 / 10$ & 1.0000 \\
\hline $\begin{array}{l}\text { Time from Inoculation to Primary Tumor } \\
\text { Detection (Days) }\end{array}$ & $16.8 \pm 2.0$ & $34.7 \pm 2.2$ & $<0.0001 *$ \\
\hline $\begin{array}{l}\text { Time from Detection of Primary Tumor to Tumor } \\
\text { Removal (Days) }\end{array}$ & $11.91 \pm 0.8$ & $12.5 \pm 1.0$ & 0.6526 \\
\hline Mouse Weight at Time of Tumor Harvest ( $g$ ) & $23.6 \pm 0.4$ & $25.2 \pm 0.5$ & $0.0175 *$ \\
\hline Average Primary Tumor Weight $(g)$ & $2.7 \pm 0.3$ & $3.1 \pm 0.3$ & 0.4490 \\
\hline Time from Inoculation to Death (Days) & $67.5 \pm 10.2$ & $81.33 \pm 11.2$ & 0.3748 \\
\hline Average Weight at Death & $24.35 \pm 0.9$ & $24.19 \pm 1.0$ & 0.9053 \\
\hline Metastatic Rate & $9 / 10$ & $6 / 7$ & 1.0000 \\
\hline
\end{tabular}

* Statistically significant difference, $p<0.05$. 
Table 2. Summary of Tumor Characteristics Comparing Transfected Groups 1 and 2

\begin{tabular}{|l|l|l|l|}
\hline & $\begin{array}{l}\text { Transfected } \\
\text { Group 1 }\end{array}$ & $\begin{array}{l}\text { Transfected } \\
\text { Group 2 }\end{array}$ & P-value \\
\hline Inoculation Rate & $9 / 10$ & $8 / 10$ & 0.5921 \\
\hline $\begin{array}{l}\text { Time from Inoculation to Primary Tumor } \\
\text { Detection (Days) }\end{array}$ & $34.7 \pm 2.2$ & $22.5 \pm 2.6$ & $0.0032 *$ \\
\hline $\begin{array}{l}\text { Time from Detection of Primary Tumor to Tumor } \\
\text { Removal (Days) }\end{array}$ & $12.5 \pm 1.0$ & $12.1 \pm 1.2$ & 0.8299 \\
\hline Mouse Weight at Time of Tumor Harvest (g) & $25.2 \pm 0.5$ & $22.6 \pm 0.5$ & $0.0037 *$ \\
\hline Average Primary Tumor Weight (g) & $3.06 \pm 0.26$ & $2.19 \pm 0.24$ & $0.0269 *$ \\
\hline Time from Inoculation to Death (Days) & $85.33 \pm 12.6$ & $50.17 \pm 8.9$ & 0.0568 \\
\hline Metastatic Rate & $6 / 7$ & $6 / 7$ & 1.0000 \\
\hline IVIS positivity in histologically-confirmed mets & $3 / 6$ & $6 / 7$ & 0.2127 \\
\hline
\end{tabular}

* Statistically significant difference, $p<0.05$. 


\section{Appendix AIII}

Markel, J. E., Lacinski, R. A., Lindsey, B. A (in publication). Nanocapsule Delivery of IL-12.

In Kleinerman, E. S., Gorlick, R. (Eds.), Clinical Advances in Osteosarcoma. Springer Nature 


\title{
Chapter 8 Nanocapsule Delivery of IL-12
}

\author{
Justin E. Markelı, Ryan A. Lacinskiı, and Brock A. Lindsey ${ }^{4}$
}

\begin{abstract}
Interleukin(IL)-12 is a protein that activates $\mathrm{T}$ cells and macrophages to kill tumor cells. However, despite this cytokine showing strong antitumor activity in preclinical settings, translation to patients has been slowed by toxic side effects, poor distribution to peripheral tissues, and improper dosing regimens. Osteosarcoma (OS) is an aggressive primary tumor of bone that has shown particular responsiveness to recombinant (r)IL-12 in preclinical models. Poly(lactic-co-glycolic) acid (PLGA) nanospheres, an FDA-approved drug delivery vector, may be viable delivery vector for transporting biologically active IL-12 to tissues without disturbing normal homeostasis. In this chapter, we explore the potential for using IL-12-loaded nanospheres (IL-12-NS, $<1 \mu \mathrm{m}$ in diameter) to treat cancer, describe the synthesis process, and examine a typical protein release profile while providing insight and future directions of nanoscale tumor immunotherapeutics.
\end{abstract}

\section{Section 1 Interleukin-12 immunotherapy - the past, present and future}

\section{A. Why IL-12?}

Interleukin(IL)-12, a member of the IL-12 family of cytokines, has a long history of antitumor activity against a wide range of preclinical cancer models[1,2]. Originally characterized as a potent inducer of Natural Killer (NK) cell cytotoxic activity, IL-12 is now recognized as a key regulator of the cell-mediated immune response and a bridge between innate and adaptive immunity [3]. Although the immunostimulatory functions of IL-12 can be induced locally with intratumoral (i.t.) injections, preclinical models suggest that more complete antitumor responses are achieved via systemic administration [4]. By influencing the differentiation of CD4+ T helper (Тн) cells into T helper type 1 (Тн1) cells, IL-12 coordinates antitumor immunity through pro-inflammatory M1 activation of macrophages and stimulation of cytotoxic T (Tc) cells [5-7]. Signaling through IL-12 is also crucial for the survival and reactivation of circulating memory cells, making it an important component of long-term cancer remission [8]. Upon binding of the heterodimeric $\mathrm{p} 35 \mathrm{p} 40$ ( $\mathrm{p} 70$ ) IL-12 protein to its type 1 cytokine receptor on the surface of T cells, Janus kinase-signal transducer and activator of transcription protein (JAK-STAT) signaling pathways are propagated through the downstream effector molecule STAT4 [9]. In addition to its effects on the immune system, IL-12 has strong antiangiogenic properties, making it an extremely attractive protein candidate for cancer therapies targeting metastasis and/or highly vascularized tumors [10].

Osteosarcoma (OS) is characterized by extensive disruption of the genome [11] resulting in a plethora of neoantigens that can stimulate an immune reaction if given the proper co-stimulatory signals [12], and evidence of IL-12's importance to OS immunology stems from a variety of sources: at the cytokine level, it was found that $I L-12$ gene polymorphisms resulting in low serum IL-12 levels were associated with increased OS risk in a cohort of Chinese patients, with affected individuals having significantly lower levels of circulating IL-12 compared to healthy controls [13]. Moreover, IL-12 has been shown to upregulate Fas expression on OS tumor cells, an occurrence that inversely correlates with metastatic potential by increasing the likelihood of Fas-Fas ligand (Fas L)-induced apoptosis [14-16]. In a preclinical murine model, specific targeting of lung metastases through aerosol gene therapy with a

4 West Virginia University School of Medicine Department of Orthopaedics

PO Box 9196 Morgantown, WV 26507-9196

No table of contents entries found. 
polyethyleneimine-transported plasmid increased parenchymal IL-12 mRNA expression while decreasing metastatic burden [17].

At the cellular level, the relative abundance of IL-12-associated immune events in both the primary tumor microenvironment (pTMic) and systemic tumor macroenvironment (sTMac) can influence the progression of disease. Macrophages account for a large portion of the cellular content of OS primaries, and adopt anti- or pro-tumorigenic phenotypes depending on activation status, namely classical (M1) or alternative (M2), respectively. Classical activation of macrophages stimulates IL-12 production, $\mathrm{T}_{\mathrm{H}} 1$ polarization of $\mathrm{T}_{\mathrm{H}}$ cells, and the release of Type 1 interferons (IFNs) like IFN- $\gamma$ that in turn stimulate additional inflammation and further M1 activation [18]. The drug mifurmatide, a synthetic derivative of a cell wall component of Mycobacterium species, is approved in Europe for the treatment of nonmetastatic OS and is an M1-activator of macrophages [19, 20]. Still, immunosuppressive influences from the tumor can override IL-12 signaling cascades and cause macrophages to adopt alternative M2 phenotypes, bestowing actions that are mainly pro-tumorigenic. These cellular changes are then reflected in both the pTMic and the sTMac, where the immune cell composition (A.K.A., the "immunophenotype," for more information see Chapter "X") can be assembled to yield insights regarding prognosis. Indeed, the presence of IL-12-secreting M1-activated macrophages (as determined by inducible nitric oxide synthase [INOS] protein expression) in the OS pTMic has been shown to correlate negatively with metastasis [21]. Figure 1 displays a visual summary of the major effects of IL-12 signaling.

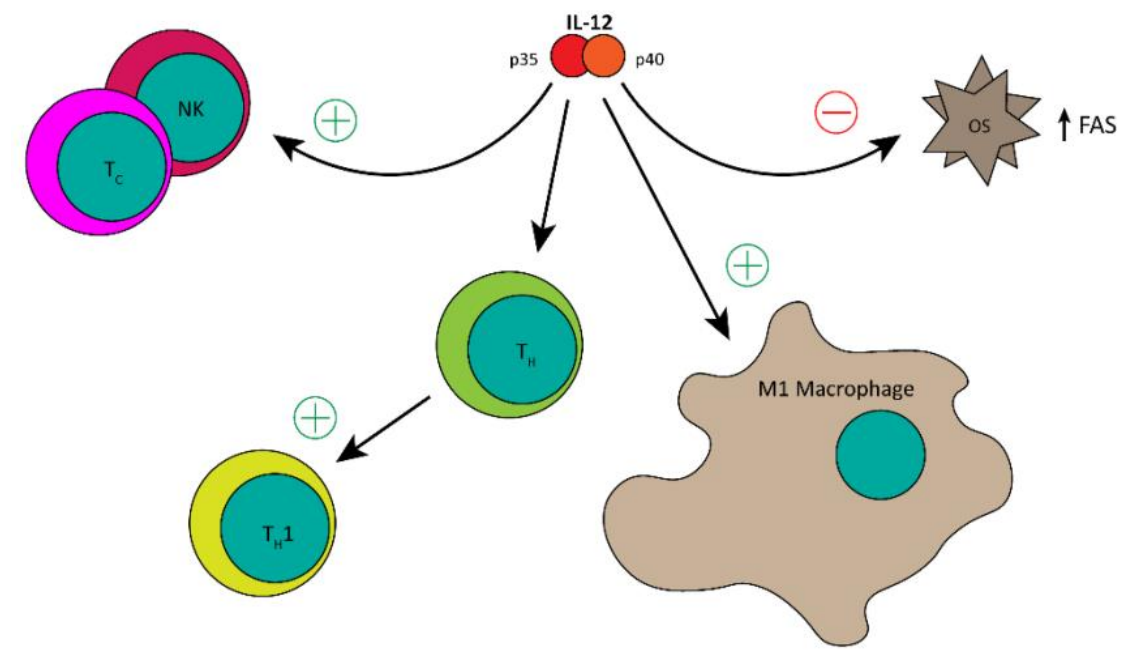

Fig. 1: Visual Summary of the Major Effects of IL-12 Signaling

\section{B. The Past and Present (1994-2019)}

Building upon a plethora of exciting preclinical data from multiple tumor types, the first Phase I clinical trial examining the clinical utility of intravenous (i.v.) recombinant human IL-12 (rhIL-12) for human cancer began in the spring of 1994 [22]. Upon enrollment in the study, all subjects were administered a preliminary rhIL-12 test dose and, if tolerated, were given a subsequent series of six high-intensity 5-day treatment cycles every fifteen days. Here, objective tumor responses were observed in only two of the 40 subjects, with the majority of patients experiencing lymphopenia, elevated liver enzymes (occasionally dose-limiting), flu-like symptoms, and transiently increased serum IFN- $\gamma$ levels that paradoxically diminished as treatment continued. Nevertheless, data from this study prompted a Phase II trial in renal cell carcinoma (RCC) that ended prematurely due to dose-limiting toxicities (DLTs); it was later realized that the test doses given to patients in the Phase I study produced an immunoprotective effect of unknown etiology that improved drug tolerability [23]; further studies investigating the clinical utility of rhIL-12 were thus motivated to construct more conservative regimens with designated rhIL-12 acclimation periods.

In an attempt to mitigate systemic toxicities, most subsequent studies began to move away from i.v. rhIL-12 [24] and focus instead on subcutaneous (s.c.) and locoregional routes of delivery. One notable exception includes a 2004 Phase II clinical trial that compared the efficacy of i.v. to s.c. delivery of rhIL-12 in relapsed and refractory non-Hodgkin's lymphoma (NHL). In this study, although the objective response rates were suboptimal, patients receiving i.v. administration showed higher response rates (40\%) than those dosed subcutaneously (7\%); importantly, the inclusion of a rhIL-12 primer dose coupled with less protein per treatment cycle allowed for improved patient tolerability in the 
i.v. administration group [25]. A concurrent Phase II trial conducted for advanced cervical cancer using a similar regimen observed increased cell-mediated immune responses that did not associate with increased survival [26]. Other studies investigating i.v. therapy used dose titration to decrease toxicity and increase the longevity of IFN- $\gamma$ responses (an effect that was shown to associate with clinical response) [27].

In the late 1990s, two Phase I clinical trials of s.c. rhIL-12 therapy in patients with advanced RCC and metastatic melanoma showed positive responses while markedly reducing the frequency of DLTs [28, 29]. Unfortunately, future investigations in RCC were stopped once a multicenter Phase II trial comparing rhIL-12 to IFN- $\alpha 2$ a reported no increased efficacy [30]. Intraperitoneal (i.p.) and intravesicular (i.vs.) injections of rhIL-12-containing solutions for metastatic ovarian and bladder cancer, respectively, have also shown satisfactory patient tolerability without appreciably improving survival [31-33]. Of note, one Phase II study carrying particular importance to OS cytokine immunotherapy followed relapsed follicular NHL patients treated with s.c. rhIL-12 in combination with the anti-CD20 monoclonal antibody rituximab. Here, researchers observed decreased survival rates in the combination group versus rituximab alone [34]; these findings were later attributed to a life-threatening state of immune system over-activation known as T cell exhaustion (TCE) [35], characterized by lymphocyte anergy, systemic inflammatory response syndrome (SIRS), and ultimately multiple organ failure (MOF). As TCE is a phenomenon inherently associated with OS disease progression [36], the exhaustive side effects of IL-12 administration must be adequately addressed to prevent further exacerbation of this deadly condition; these studies highlight both the importance of proper dosing strategies as well as the value of an adequate immune monitoring platform (see Chapter "X") when administering potentially immune-exhaustive experimental therapeutics.

Although they have presented a mixed bag of clinical findings, studies investigating locoregional IL-12 delivery in combination with other immunostimulatory cytokines, monoclonal antibodies, and radiation have also been investigated. Direct modifications to the IL-12 protein itself, such as fusion to necrosis-targeting antibodies for enhanced delivery to irradiated sites [37] and peptide truncation to decrease toxicity [38] have been published, as well as the effectiveness of IL-12 administration as an adjuvant for cancer vaccination [39]. For OS, one study showed that IL-18, a member of the IL-1 family of cytokines with similar properties to IL-12, has particular efficacy against OS when given in combination with IL-12 [40].

Another method of IL-12 delivery include the use of genetically-engineered cell types to increase i.t. IL-12 levels upon introduction into the pTMic. Fibroblasts, dendritic cells (DCs), mesenchymal stem cells, T lymphocytes (e.g., CAR T cells), and even tumor cells themselves have been engineered to release supra-physiological levels of IL-12 within the pTMic for the purposes of increasing the immunogenicity of tumor antigens [41-46]. Viruses and DNA vaccines carrying IL-12-coding genes to metastatic sites have also been studied; in 2003, Shu-Fang Jia et al. eliminated OS pulmonary metastases using aerosol therapy to direct an IL-12-coding polycationic DNA carrier directly to the lungs of mice [17]. Although this method of IL-12 delivery did not translate to the clinic, it was one of the first instances displaying the in vivo efficacy of IL-12 against metastatic OS. In 2007, a Phase I trial for pediatric cancer (including seven OS patients) was published combining cancer vaccines with genetically-engineered IL-12 secreting DCs; while intranodal administration resulted in antigen-specific IFN release in vitro, no OS patients displayed noticeable improvement following vaccination [47].

Without toxic loading doses, s.c. and i.v. administration of rIL-12 does not ensure adequate distribution to peripheral tissues. To solve this issue, IL-12 can be encapsulated within biodegradable organic polymers that release their contents in a slow and controlled manner, thereby reducing side effects and prolonging antitumor activity. In 1999, Kuriakose et al. showed that tumor regression with a single i.t. dose of rIL-12-loaded polylactic (PLA) microspheres (IL-12-MS, > $1 \mu \mathrm{m}$ in diameter) was superior to multiple doses of free rIL-12 in a head and neck tumor xenograft model following the transfer of human peripheral blood lymphocytes [48]. These data were further supported a year later when Egilmez et al. discovered that tumor regression from IL-12-MS was superior not only to i.t., but also i.p. free rIL-12 in a Line-1/BALB/c murine alveolar lung adenocarcinoma model [49]. Shortly thereafter, i.t. injection of rmIL-12-loaded PLA microspheres was found to regress primary MT-901 breast cancer tumors while simultaneously inducing systemic antitumor immunity in the form of circulating, antigen-primed memory $\mathrm{T}$ cells that prevented tumor growth following rechallenge [50].

One possible answer to many of the above problems could be rIL-12-loaded poly(lactic-co-glycolic) acid (PLGA) nanospheres (IL-12-NS, $<1 \mu \mathrm{m}$ ) for i.v. delivery. PLGA colloidal systems provide protection for and enhance the stability of the entrapped compound while providing enhanced delivery to target tissues. Indeed, while many other researchers have moved towards i.t. formulations, other literature continues to support the notion that cancers (including OS) induce systemic immune dysfunctions unrelated to the immunophenotype of the primary lesion. By using flow cytometry to assess splenic immunophenotypes, systemic immunotherapies like anti-programmed death ligand 1 (PD-L1) can reverse malignancy-induced immunosuppression [36]; therefore, the long-term goal is to use the PLGA capsule as a barrier to allow safe transit of IL-12-NS through the blood to the peripheral tissues, where they 
can release their protein slowly over extended periods of time. Much of the remainder of this chapter will describe the process of synthesizing IL-12-NS for systemic administration.

\section{The Future}

Analogous to human immunodeficiency virus (HIV) anti-retroviral therapy (ART), rIL-12 will most likely represent one component of an immunotherapeutic "cocktail" tailored to the specific immunological needs of each patient. That said, there are certain themes that have arisen from three decades of IL-12 research that must be considered, of which PLGA encapsulation may help solve:

1. Large bolus loading doses of rIL-12 are required to ensure adequate tissue distribution when delivered systemically, often resulting in DLTs that can be somewhat attenuated by dose escalation. Upon administration of IL-12-NS, PLGA encapsulation delays the release of entrapped IL-12 thereby allowing for increased delivery to peripheral tissues.

2. I.v. rIL-12 dosing regimens require multiple injections including dose titrations to prevent unwanted proinflammatory reactions (DLTs). IL-12-NS have intrinsic dose titration properties, as encapsulated protein is released slowly and continually as the PLGA coating hydrolyzes in aqueous environments. Additionally, preliminary in vitro data show that one sample of IL-12-NS continues to elute protein up to 14 days, which may drastically reduce the number of injections needed per patient.

3. I.v. rIL-12 cannot be targeted to specific areas of interest without direct modification of the protein itself, which may lead to decreased biological function. Due to the presence of free carboxylic acid moieties, PLGA nanospheres can undergo a number of different surface modifications that allow for control over their in vivo biodistribution properties; some common conjugates that have been tested include monoclonal antibodies for specific cell targeting, albumins to increase the enhanced permeation and retention (EPR) effect, and chitosan for enhanced lung delivery via the formation of transient microaggregates in pulmonary capillaries [51-53].

To illustrate the concept of using IL-12-NS for the treatment of OS, consider the following scenario. OS induces systemic immune suppression in mice (Figure 2A) that can be reversed by checkpoint blockade using anti-PD-L1 (Figure 2B); that is, their systemic immunophenotype returns to baseline status (see Chapter " $X$ " for more details). However, baseline status does not provide enough immune stimulation to effectively reduce tumor burden in mice with advanced disease. On the other end of the spectrum, when patients in the aforementioned Phase II study of rituximab/rhIL-12 combination therapy for relapsed and refractory NHL succumbed to DLTs, their immunophenotypes were pushed past stimulation and into the realm of overstimulation and TCE (Figure 2D). However, in a background of anti-PD-L1 checkpoint blockade providing disinhibition of activated T cells, slow and sustained delivery of low dose rIL-12 (which is generally considered safe [54]) from hydrolyzing PLGA nanospheres to the sTMac may provide the systemic stimulation necessary to effectively reduce disease burden while still remaining beneath the exhaustion threshold (Figure 2C); from this example, it is clear that a real-time immunophenotypes monitoring platform, like the one described in Chapter " $X$ ", would be of considerable value for this application.

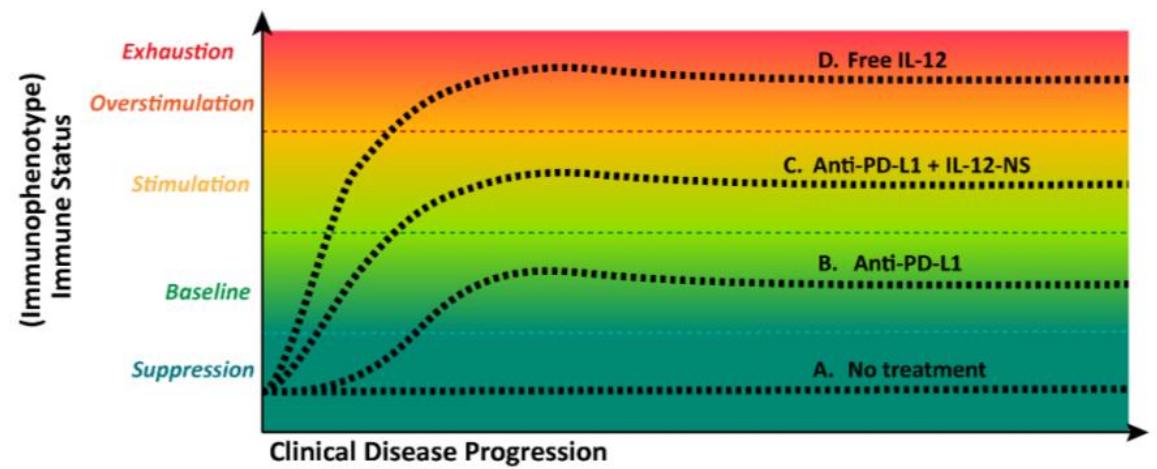


Fig. 2. Hypothetical application of IL-12-NS for the treatment of metastatic OS. A) Pathologic immune suppression observed in patients with OS. B) Systemic treatment of metastatic OS with the immune checkpoint blocker anti-PD-L1 reverses malignancy-induced immunosuppression back to baseline but does not improve survival. C) Systemic treatment of metastatic OS with IL-12-NS in the background of anti-PD-L1 T cell disinhibition provides adequate immune stimulation to reduce disease burden without falling into the realm of exhaustion. D) Toxic loading doses of free IL-12, which are necessary to ensure adequate distribution to peripheral tissues, push the immune system into a state of exhaustion leading to SIRS, MOF, and ultimately death.

\section{Section 2 Production of IL-12-loaded PLGA nanospheres}

\section{A. Double solvent emulsion-evaporation method: the basics}

Synthesizing protein-loaded PLGA nanospheres via the double solvent emulsion-evaporation (DSEE) method (AKA, the water-in-oil-in-water [w1/o/w2] method) involves three main steps: 1) primary emulsion (w1/o), 2) double emulsion, and 3) nanosphere isolation and purification. First, the drug of choice is suspended in an aqueous solution to create the internal aqueous phase (w1). The internal aqueous phase (w1) is then added to a solution of organic solvent (o) containing polymer and agitated briefly (usually via homogenization or sonication) to create the primary emulsion (w1/o, Figure 3A); some commonly used organic solvents include dichloromethane (DCM), acetone (AC), and ethyl acetate (EA). During this step, the emulsion can be kept in an ice bath to counteract the rise in temperature caused by agitation. Next, the primary emulsion ( $\left.\mathrm{w}_{1} / \mathrm{o}\right)$ is added to the external aqueous phase (w2) and agitated again to form the double emulsion (w1/o/w2, Figure 3B). The w2 phase contains a stabilizer (usually polyvinyl alcohol [PVA]) to prevent coalescence of the resulting emulsion droplets [55]. As the agitation/shear force increases, the droplets and hence resulting particles become smaller [56]. To solidify the droplets and form particles, the organic solvent is evaporated at the water/air interface with stirring. As the organic solvent evaporates, the PLGA precipitates thereby encapsulating the internal aqueous phase in a spherical matrix (Figure 3C). The last step involves removal of excess PLGA and non-encapsulated protein via a series of washes, flash-freezing, and lyophilization for long-term storage. By minimizing contact between the internal aqueous phase and organic solvent, this method of synthesis is suitable for the encapsulation of bioactive proteins [57].

A.

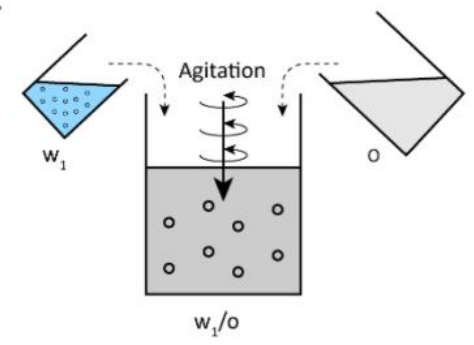

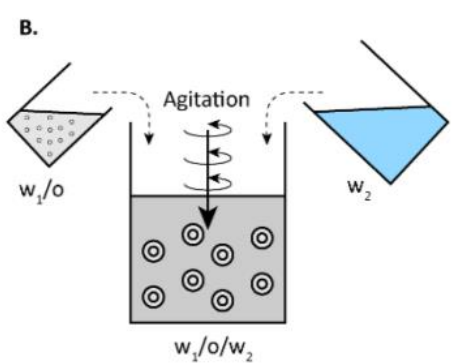

c.

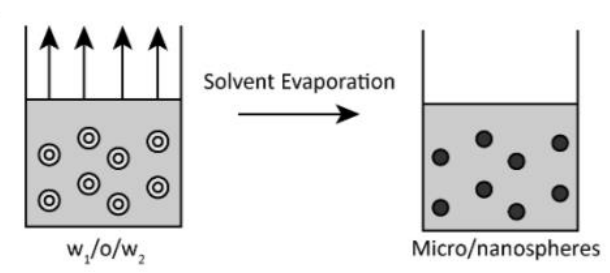

Fig. 3. Basic process for the creation of biodegradable micro/nanospheres using the double solvent emulsion-evaporation technique. A) The internal aqueous phase (w1), which contains the bioactive compound of interest, is combined with polymer dissolved in an organic solvent (o) and agitated to create the primary emulsion ( $\left.\mathrm{w}_{1} / \mathrm{o}\right)$. B) The primary emulsion is then dispersed in the external aqueous phase (w2) and agitated to form the double emulsion (w1/o/w2). C) The double emulsion is stirred to remove the organic solvent via evaporation which solidifies the droplets into micro/nanospheres. 


\section{B. Double solvent emulsion-evaporation method of encapsulating rIL-12 w/ homogenization}

The primary emulsion is formed by adding aqueous rIL-12 to a small beaker containing PLGA in DCM and agitating on ice (Figure 4A). The double emulsion is formed by combining the primary emulsion with an aqueous solution of $\mathrm{PVA} / \mathrm{NaCl}$ and agitating for eight minutes on ice (Figure 4B); the addition of salt to the external aqueous phase increases protein encapsulation while decreasing the surface area of the resulting product [58]. Both agitation steps in the homogenization method are done with a standard tissue homogenizer tip set at medium speed ( 175000 RPM). The double emulsion is then stirred overnight at room temperature (RT) to evaporate off the DCM (Figure 4C), and the resulting product is a mixture of both microspheres and nanospheres; the morphology of the resulting product prior to centrifugation can be seen in Figure 4D. To remove the microspheres, the colloid is centrifuged at low g $(<1000$ $\mathrm{x} \mathrm{g}$ ) and the resulting supernatant aspirated and stored on ice. Once microspheres are removed, the nanospheres in the supernatant are harvested by ultracentrifuging ( $>50,000 \mathrm{xg}$ ), washing to remove non-encapsulated protein and excess PLGA, flash-frozen, and lyophilized for long-term storage (Figure 4E). Figure 4F displays the resulting product once the microspheres and excess protein and PLGA are removed. Zeta potential $(\zeta)$ is a physical property that determines colloidal stability and hence propensity of suspended particles to flocculate; IL-12-NS made via the homogenization method (IL-12-HNS) have a $\zeta$ of $-15.1 \pm 1.25 \mathrm{mV}$.

A.

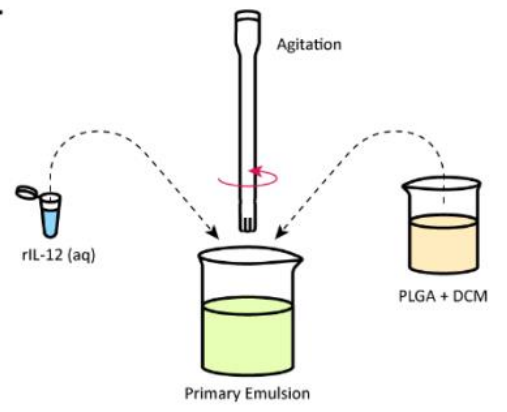

c.

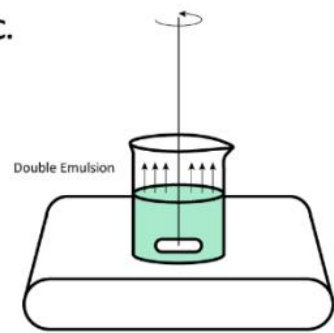

B.

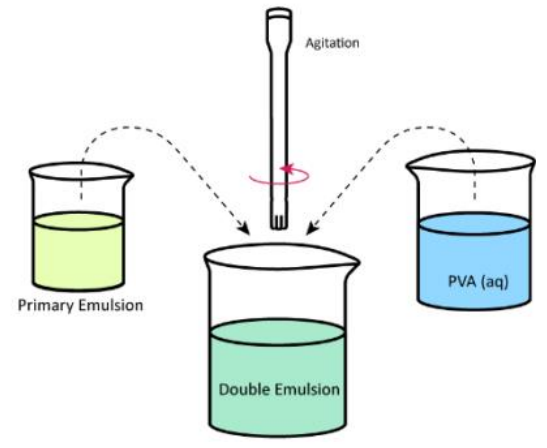

D.

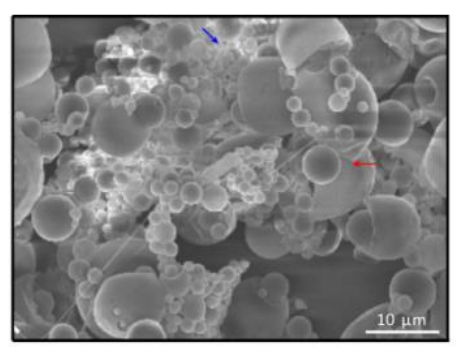

F.

E.
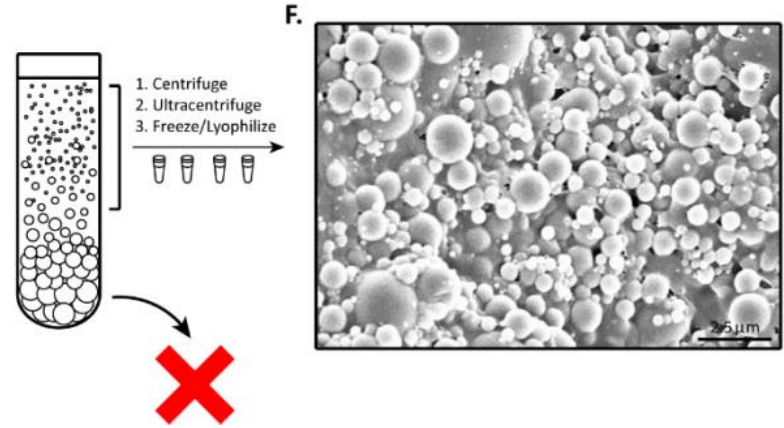

Fig. 4. Stepwise procedure for double solvent emulsion-evaporation method of encapsulating rIL-12 w/ homogenization. A) To form the primary emulsion, aqueous rIL-12 is added to PLGA dissolved in DCM and homogenized for six minutes at 17500 RPM. B) To form the double emulsion, the primary emulsion is dispersed in an aqueous solution of $2 \% \mathrm{PVA} / 0.8 \% \mathrm{NaCl}$ and homogenized for eight minutes at 17500 RPM. C) The double emulsion is stirred overnight to evaporate off the DCM, forming the products shown by scanning electron microscopy (SEM) in (D), a mix of microspheres and nanospheres. The red arrow is pointing to a microspheres, and the blue 
arrow is pointing to a next of nanospheres. E) The microspheres are excluded and the nanospheres harvested via centrifugation $(<1000 \mathrm{x}$ g) and ultracentriguation (> 50,000 x g), respectively. Following a series of washes, flash-freezing, and lyophilization, the final nanosphere product. (F). Scanning electron micrograph of the nanosphere product formed via the homogenization method of PLGA nanosphere synthesis.

\section{Double solvent emulsion-evaporation method w/ ultrasonication}

First, the primary emulsion is formed by using a microprobe sonicator set at 50W to agitate aqueous rIL-12 in PLGA dissolved in DCM in an ice bath (Figure 5A). For this application, each emulsion is formed in a glass test tube to minimize time needed to create a homogeneous mixture. The primary emulsion is then transferred to another test tube containing $1 \%$ PVA in water, and the solution is sonicated on ice for another 10 seconds at $50 \mathrm{~W}$ power to create the double emulsion (Figure 5B). The double emulsion is then stirred for at least three hours to evaporate the organic solvent, washed, flash-frozen, and lyophilized for long-term storage (Figure 5C); a scanning electron micrograph of the final nanosphere product is shown in Figure 5D. Due to the type of agitation used, the ultrasonication method does not require an extra isolation step as in the homogenization method. The zeta potential of IL-12-NS made via the ultrasonication method (IL-12-SNS) have a $\zeta$ of $-36.35 \pm 2.85 \mathrm{mV}$.

A.

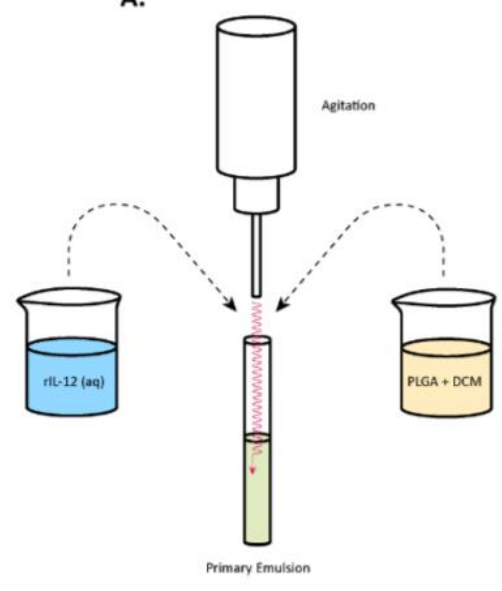

C.

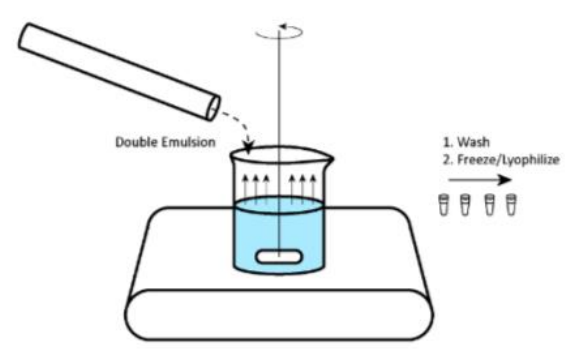

B.

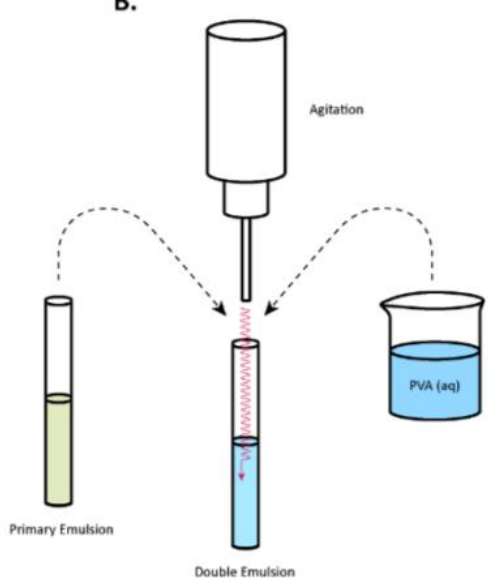

D.

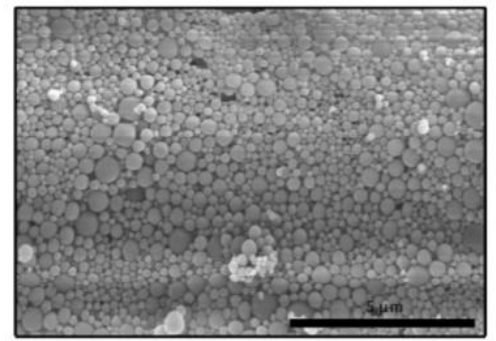

Fig. 5. Stepwise procedure for double solvent emulsion-evaporation method of encapsulating rIL-12 w/ ultrasonication. A) To form the primary emulsion, aqueous rIL-12 is added to PLGA dissolved in DCM and sonicated at 50W for 10 seconds. B) To form the double emulsion, the primary emulsion is dispersed in an aqueous solution of $1 \%$ PVA and sonicated again at 50W for 10 seconds. C) The double emulsion is stirred for three hours to evaporate off the DCM, washed, flash-frozen, and lyophilized for long-term storage. (D) Scanning electron micrograph of the nanosphere product formed via the ultrasonication method of PLGA nanosphere synthesis. 


\section{Section 3 In vitro IL-12 release characterization - Elution Study}

\section{A. Characterizing nanosphere protein release profile via an in vitro elution study}

Once a batch of IL-12-NS is synthesized, the next step is to characterize how the protein is released over time, called protein elution. To conduct an in vitro elution profile, a known quantity of suspended nanospheres is shaken vigorously at $37{ }^{\circ} \mathrm{C}$ and sampled once every 24 hours. At the end of the study, the amount of biologically-active protein eluted each day can be measured using enzyme-linked immunosorbent assays (ELISA) and expressed as a percent of total protein eluted throughout the entire release profile.

To understand how the encapsulated protein is released, it is important to consider the ultrastructure of a PLGA nanosphere. When the second emulsion is formed, protein becomes wound up in tiny strands of PLGA, (much like a ball of string), which coalesces and precipitates into a sphere as the organic solvent is removed. During this process, protein becomes both entrapped within the polymer matrix (Figure 6A, top right panel, blue arrow) and adsorbed to the outer surface (Figure 6A, top left panel, red arrow), producing a characteristic biphasic elution curve shown in Figure 6B. The burst phase, which normally occurs between baseline and two days, is due to the adsorbed protein on the surface of the nanospheres being released upon resuspension in aqueous medium (Figure 6A, bottom panel). The controlled release phase is due to entrapped protein (Figure 6A, bottom panel), and is released slowly over time as the PLGA hydrolyzes via the reaction in Figure 6C. The elution characteristics can be altered by varying a number each synthesis parameter, however, an in-depth discussion of nanosphere modification is beyond the scope of this book.

A.
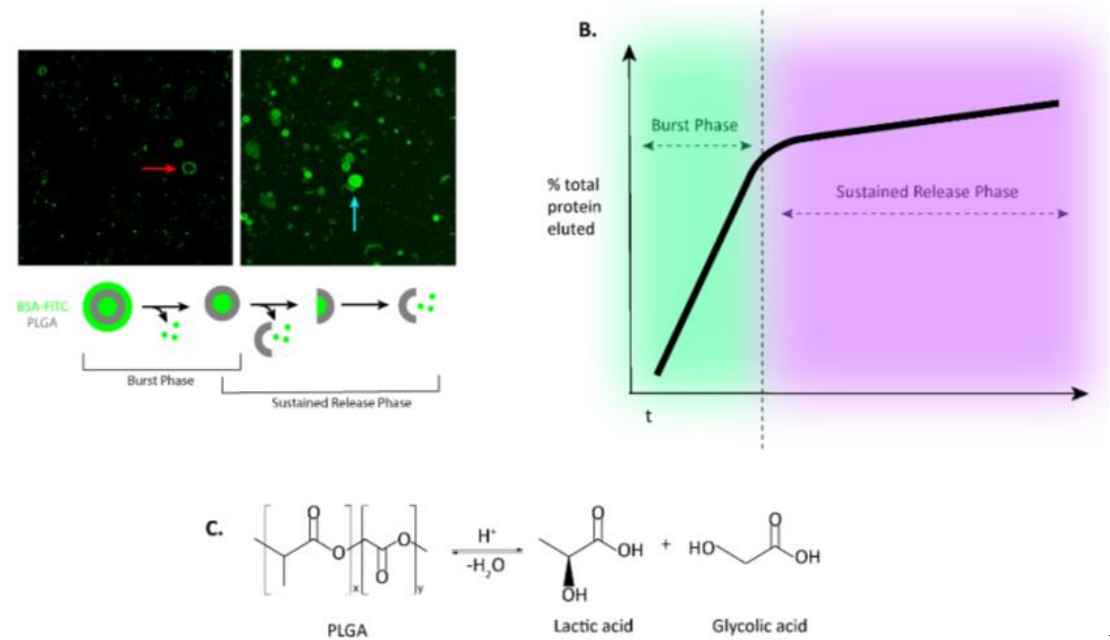

Fig. 6. Release characteristics of a

typical protein-loaded PLGA nanosphere elution profile. A) Top two panels: PLGA spheres were loaded with FITC-tagged bovine serum albumin (BSA) and imaged via confocal microscopy to show the distribution of protein both adhered to the surface (left) and entrapped within the matrix (right). Bottom panel: Schematic showing the mechanism of protein release from PLGA nanospheres creating a biphasic release profile, shown in (B) as a percent of total protein released. C) Schematic showing the hydrolysis of PLGA into lactic and glycolic acid.

\section{B. Elution Profiles: Homogenization and Ultrasonication Methods}

We will now compare the release profiles of IL-12-HNS and IL-12-SNS loaded with recombinant mouse (rm)IL-12. At eight days, rmIL-12-loaded-HNS at a concentration of 1 billion particles $/ \mathrm{mL}$ released $\sim 3500$ pg of p70 rmIL-12, or 0.7 pg per 100,000 particles. SNS, which have higher solubility and less tendency to flocculate, were suspended at a concentration of $100 \mathrm{billion} / \mathrm{mL}$ and released a total of $~ 230,000 \mathrm{pg}$ of rmIL-12 over 14 days, averaging 0.46 pg per 100,000 particles. Therefore, although IL-12-HNS have a more efficient protein release profile per capsule, it is likely that SNS will carry more clinical impact due to their superior solubility properties and extended release profile. Figures 7A and 7B shows the release profiles as a function of cumulative protein eluted per day/total protein eluted for both HNS and SNS, 
respectively. Approximately $70 \%$ of the total protein for both methods is released over the first 48 hours during the burst phase, where the remaining 30\% is spread over 6 (7A) and 12 (7B) days during the sustained release phase for HNS and SNS, respectively.
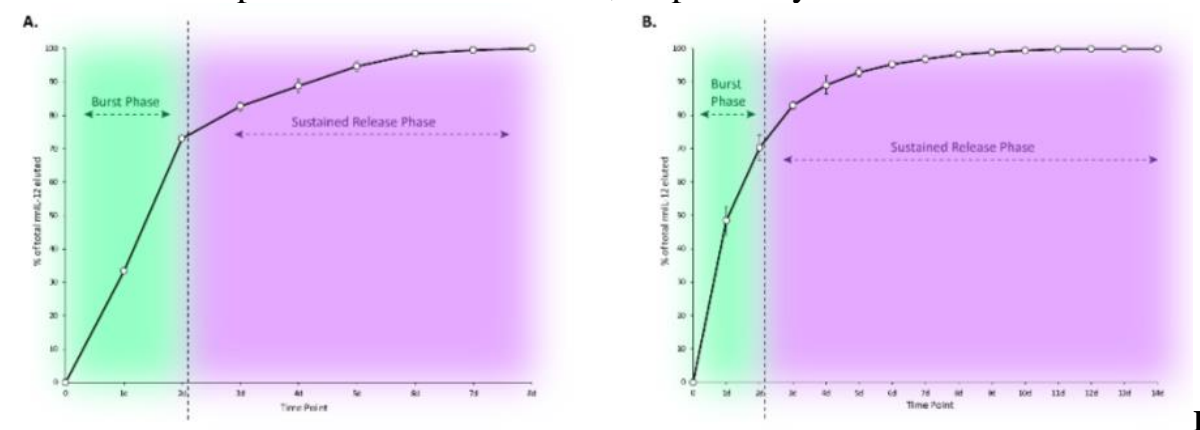

Fig. 7. Comparative analysis of rmIL-12-HNS and rmIL-12SNS elution profiles. A) Elution of 1 billion rmIL-12-HNS/mL over the span over eight days showing the characteristic biphasic release profile, with $\sim 70 \%$ total IL-12 being released within the burst phase (Day 0 - Day 2) and the remainder during the sustained release phase (Day 2- Day 8). B) Elution of 100 billion rmIL-12-SNS/mL over the span over 14 days showing the characteristic biphasic release profile, with $\sim 70 \%$ total IL-12 being released within the burst phase (Day 0 - Day 2 ) and the remainder during the sustained release phase (Day 2- Day 14). Y values display the cumulative protein eluted per day/total protein eluted; each value is an average of $n=2$ biological replicates.

\section{Section 4 Conclusions and Future Perspectives}

IL-12 has a long and controversial history as an agent for cancer immunotherapy. However, one central theme that has emerged over the years is that proper dosing is a key factor determining both safety and therapeutic efficacy. As of yet, the line separating what is helpful from harmful has not been established, and the number of variables with potential immunological influences (e.g., tumor type, co-morbidities, prior therapies, age, sex, etc.) further complicates the scenario. Therefore, the next step will be to conduct extensive dose escalation trials that evaluate the effects of rIL-12-NS on the systemic immunophenotype, paying careful attention to the warning signs of overstimulation. Additionally, for future attempts at IL-12 tumor immunotherapy to be successful, a reliable and efficient means of assessing immune status must be developed; as such, this project has evolved alongside that discussed in Chapter " $X$ ".

The importance of establishing systemic immunity to a malignancy is supported by earlier observations that suggest that the antitumor efficacy of rIL-12 is maximized upon systemic administration. Indeed, studies have shown that tumor-induced immunosuppression can be reversed with immunotherapy, and that immunotherapy is only successful following the activation of secondary immune organs [59]. However, systemic rIL-12 delivery in humans has thus far proven difficult due to toxic loading doses being needed to ensure adequate tissue delivery. PLGA encapsulation, which effectively reduces the amount of available rIL-12 at one time, may be a promising method for delivering controlled doses of IL-12 to tissues without massive disruption of normal homeostatic processes. In theory, unlike the destructive inflammatory syndromes induced by introducing free rIL-12 into the bloodstream, IL-12-NS-laden tissues would slowly and continually influence the sTMac to favor pathways that support cell-mediated immunity while shifting the macrophage polarization status towards an M1 bias. In turn, priming tissues for a cell-mediated immune response may increase the chances that circulating effector cells can respond appropriately when encountering tumor antigen. 
1. Nastala, C. L., Edington, H. D., McKinney, T. G., Tahara, H., Nalesnik, M. A., Brunda, M. J., ... \& Storkus, W. J. (1994). Recombinant IL-12 administration induces tumor regression in association with IFN-gamma production. The Journal of Immunology, 153(4), 16971706.

2. Brunda, M. J., Luistro, L., Warrier, R. R., Wright, R. B., Hubbard, B. R., Murphy, M., ... \& Gately, M. K. (1993). Antitumor and antimetastatic activity of interleukin 12 against murine tumors. Journal of Experimental Medicine, 178(4), 1223-1230.

3. Trinchieri, G. (1995). Interleukin-12: a proinflammatory cytokine with immunoregulatory functions that bridge innate resistance and antigen-specific adaptive immunity. Annual review of immunology, 13(1), 251-276.

4. Cavallo, F., Di Carlo, E., Butera, M., Verrua, R., Colombo, M. P., Musiani, P., \& Forni, G. (1999). Immune events associated with the cure of established tumors and spontaneous metastases by local and systemic interleukin 12. Cancer research, 59(2), 414-421.

5. Manetti, R., Parronchi, P., Giudizi, M. G., Piccinni, M. P., Maggi, E., Trinchieri, G., \& Romagnani, S. (1993). Natural killer cell stimulatory factor (interleukin 12 [IL-12]) induces T helper type 1 (Th1)-specific immune responses and inhibits the development of IL4-producing Th cells. Journal of Experimental Medicine, 177(4), 1199-1204.

6. Martinez, F. O., Sica, A., Mantovani, A., \& Locati, M. (2008). Macrophage activation and polarization. Front Biosci, $13(1), 453-461$.

7. Gately, M. K., Warrier, R. R., Honasoge, S., Carvajal, D. M., Faherty, D. A., Connaughton, S. E., ... \& Murphy, M. (1994). Administration of recombinant IL-12 to normal mice enhances cytolytic lymphocyte activity and induces production of IFN- $\gamma$ in vivo. International immunology, 6(1), 157-167.

8. Pearce, E. L., \& Shen, H. (2007). Generation of CD8 T cell memory is regulated by IL-12. The Journal of Immunology, 179(4), 20742081.

9. Jacobson, N. G., Szabo, S. J., Weber-Nordt, R. M., Zhong, Z., Schreiber, R. D., Darnell, J. E., \& Murphy, K. M. (1995). Interleukin 12 signaling in T helper type 1 (Th1) cells involves tyrosine phosphorylation of signal transducer and activator of transcription (Stat) 3 and Stat4. Journal of Experimental Medicine, 181(5), 1755-1762.

10. Voest, E. E., Kenyon, B. M., O'Reilly, M. S., Truitt, G., D'Amato, R. J., \& Folkman, J. (1995). Inhibition of angiogenesis in vivo by interleukin 12. JNCI: Journal of the National Cancer Institute, 87(8), 581-586.

11. Kuijjer, M. L., Hogendoorn, P. C., \& Cleton-Jansen, A. M. (2013). Genome-wide analyses on high-grade osteosarcoma: Making sense of a genomically most unstable tumor. International journal of cancer, 133(11), 2512-2521.

12. Rathe, S. K., Popescu, F. E., Johnson, J. E., Watson, A. L., Marko, T. A., Moriarity, B. S., ... \& Largaespada, D. A. (2019). Identification of candidate neoantigens produced by fusion transcripts in human osteosarcomas. Scientific reports, 9(1), 358.

13. Wang, J., Nong, L., Wei, Y., Qin, S., Zhou, Y., \& Tang, Y. (2013). Association of interleukin-12 polymorphisms and serum IL-12p40 levels with osteosarcoma risk. DNA and cell biology, 32(10), 605-610.

14. Lafleur, E. A., Jia, S. F., Worth, L. L., Zhou, Z., Owen-Schaub, L. B., \& Kleinerman, E. S. (2001). Interleukin (IL)-12 and IL-12 gene transfer up-regulate Fas expression in human osteosarcoma and breast cancer cells. Cancer Research, 61(10), 4066-4071.

15. Worth, L. L., Lafleur, E. A., Jia, S. F., \& Kleinerman, E. S. (2002). Fas expression inversely correlates with metastatic potential in osteosarcoma cells. Oncology reports, 9(4), 823-827.

16. Lafleur, E. A., Koshkina, N. V., Stewart, J., Jia, S. F., Worth, L. L., Duan, X., \& Kleinerman, E. S. (2004). Increased Fas expression reduces the metastatic potential of human osteosarcoma cells. Clinical cancer research, 10(23), 8114-8119.

17. Jia, S. F., Worth, L. L., Densmore, C. L., Xu, B., Duan, X., \& Kleinerman, E. S. (2003). Aerosol gene therapy with PEI: IL-12 eradicates osteosarcoma lung metastases. Clinical cancer research, 9(9), 3462-3468.

18. Biswas, S. K., \& Mantovani, A. (2010). Macrophage plasticity and interaction with lymphocyte subsets: cancer as a paradigm. Nature immunology, 11(10), 889.

19. Sousa, S., \& Määttä, J. (2016). The role of tumour-associated macrophages in bone metastasis. Journal of bone oncology, 5(3), 135138.

20. Ando, K., Mori, K., Corradini, N., Redini, F., \& Heymann, D. (2011). Mifamurtide for the treatment of nonmetastatic osteosarcoma. Expert opinion on pharmacotherapy, 12(2), 285-292.

21. Dumars, C., Ngyuen, J. M., Gaultier, A., Lanel, R., Corradini, N., Gouin, F., ... \& Heymann, M. F. (2016). Dysregulation of macrophage polarization is associated with the metastatic process in osteosarcoma. Oncotarget, 7(48), 78343.

22. Atkins, M. B., Robertson, M. J., Gordon, M., Lotze, M. T., DeCoste, M., DuBois, J. S., ... \& Mier, J. W. (1997). Phase I evaluation of intravenous recombinant human interleukin 12 in patients with advanced malignancies. Clinical Cancer Research, 3(3), $409-417$.

23. Leonard, J. P., Sherman, M. L., Fisher, G. L., Buchanan, L. J., Larsen, G., Atkins, M. B., ... \& Ryan, J. L. (1997). Effects of singledose interleukin-12 exposure on interleukin-12-associated toxicity and interferon- $\gamma$ production. Blood, 90(7), 2541-2548.

24. Del Vecchio, M., Bajetta, E., Canova, S., Lotze, M. T., Wesa, A., Parmiani, G., \& Anichini, A. (2007). Interleukin-12: biological properties and clinical application. Clinical Cancer Research, 13(16), 4677-4685.

25. Younes, A., Pro, B., Robertson, M. J., Flinn, I. W., Romaguera, J. E., Hagemeister, F., .. \& McLaughlin, P. W. (2004). Phase II clinical trial of interleukin-12 in patients with relapsed and refractory non-Hodgkin's lymphoma and Hodgkin's disease. Clinical Cancer Research, 10(16), 5432-5438.

26. Wadler, S., Levy, D., Frederickson, H. L., Falkson, C. I., Wang, Y., Weller, E., ... \& Kadish, A. S. (2004). A phase II trial of interleukin12 in patients with advanced cervical cancer: clinical and immunologic correlates: Eastern Cooperative Oncology Group study E1E96. Gynecologic oncology, 92(3), 957-964.

27. Gollob, J. A., Mier, J. W., Veenstra, K., McDermott, D. F., Clancy, D., Clancy, M., \& Atkins, M. B. (2000). Phase I trial of twiceweekly intravenous interleukin 12 in patients with metastatic renal cell cancer or malignant melanoma: ability to maintain IFN- $\gamma$ induction is associated with clinical response. Clinical Cancer Research, 6(5), 1678-1692.

28. Motzer, R. J., Rakhit, A., Schwartz, L. H., Olencki, T., Malone, T. M., Sandstrom, K., ... \& Bukowski, R. (1998). Phase I trial of subcutaneous recombinant human interleukin-12 in patients with advanced renal cell carcinoma. Clinical Cancer Research, 4(5), 11831191.

29. Bajetta, E., Del Vecchio, M., Mortarini, R., Nadeau, R., Rakhit, A., Rimassa, L., ... \& Parmiani, G. (1998). Pilot study of subcutaneous recombinant human interleukin 12 in metastatic melanoma. Clinical Cancer Research, 4(1), 75-85. 
30. Motzer, R. J., Rakhit, A., Thompson, J. A., Nemunaitis, J., Murphy, B. A., Ellerhorst, J., ... \& Bukowski, R. M. (2001). Randomized multicenter phase II trial of subcutaneous recombinant human interleukin-12 versus interferon- $\alpha 2$ a for patients with advanced renal cell carcinoma. Journal of Interferon \& Cytokine Research, 21(4), 257-263.

31. Lenzi, R., Rosenblum, M., Verschraegen, C., Kudelka, A. P., Kavanagh, J. J., Hicks, M. E., ... \& Platsoucas, C. D. (2002). Phase I study of intraperitoneal recombinant human interleukin 12 in patients with Müllerian carcinoma, gastrointestinal primary malignancies, and mesothelioma. Clinical cancer research, 8(12), 3686-3695.

32. Weiss, G. R., O'Donnell, M. A., Loughlin, K., Zonno, K., Laliberte, R. J., \& Sherman, M. L. (2003). Phase 1 study of the intravesical administration of recombinant human interleukin-12 in patients with recurrent superficial transitional cell carcinoma of the bladder. Journal of Immunotherapy, 26(4), 343-348.

33. Lenzi, R., Edwards, R., June, C., Seiden, M. V., Garcia, M. E., Rosenblum, M., \& Freedman, R. S. (2007). Phase II study of intraperitoneal recombinant interleukin-12 (rhIL-12) in patients with peritoneal carcinomatosis (residual disease $<1 \mathrm{~cm}$ ) associated with ovarian cancer or primary peritoneal carcinoma. Journal of translational medicine, 5(1), 66.

34. Ansell, S. M., Geyer, S. M., Maurer, M. J., Kurtin, P. J., Micallef, I. N., Stella, P., ... \& Witzig, T. E. (2006). Randomized phase II study of interleukin-12 in combination with rituximab in previously treated non-Hodgkin's lymphoma patients. Clinical Cancer Research, 12(20), 6056-6063.

35. Yang, Z. Z., Grote, D. M., Ziesmer, S. C., Niki, T., Hirashima, M., Novak, A. J., ... \& Ansell, S. M. (2012). IL-12 upregulates TIM-3 expression and induces $\mathrm{T}$ cell exhaustion in patients with follicular B cell non-Hodgkin lymphoma. The Journal of clinical investigation, 122(4), 1271-1282.

36. Markel, J. E., Noore, J., Emery, E. J., Bobnar, H. J., Kleinerman, E. S., \& Lindsey, B. A. (2018). Using the Spleen as an In Vivo Systemic Immune Barometer Alongside Osteosarcoma Disease Progression and Immunotherapy with a-PD-L1. Sarcoma, 2018.

37. Eckert, F., Jelas, I., Oehme, M., Huber, S. M., Sonntag, K., Welker, C., ... \& Schilbach, K. (2017). Tumor-targeted IL-12 combined with local irradiation leads to systemic tumor control via abscopal effects in vivo. Oncoimmunology, 6(6), e1323161.

38. Wang, P., Li, X., Wang, J., Gao, D., Li, Y., Li, H., ... \& Cheng, Z. (2017). Re-designing Interleukin-12 to enhance its safety and potential as an anti-tumor immunotherapeutic agent. Nature communications, 8(1), 1395.

39. Afonso, L. C., Scharton, T. M., Vieira, L. Q., Wysocka, M., Trinchieri, G., \& Scott, P. (1994). The adjuvant effect of interleukin-12 in a vaccine against Leishmania major. Science, 263(5144), 235-237.

40. Liebau, C., Baltzer, A. W., Schmidt, S., Roesel, C., Karreman, C., Prisack, J. B., ... \& Merk, H. (2002). Interleukin-12 and interleukin18 induce indoleamine 2, 3-dioxygenase (IDO) activity in human osteosarcoma cell lines independently from interferongamma. Anticancer research, 22(2A), 931-936.

41. Zitvogel, L., Tahara, H., Robbins, P. D., Storkus, W. J., Clarke, M. R., Nalesnik, M. A., \& Lotze, M. T. (1995). Cancer immunotherapy of established tumors with IL-12. Effective delivery by genetically engineered fibroblasts. The Journal of Immunology, 155(3), $1393-1403$. 42. Nishioka, Y., Hirao, M., Robbins, P. D., Lotze, M. T., \& Tahara, H. (1999). Induction of systemic and therapeutic antitumor immunity using intratumoral injection of dendritic cells genetically modified to express interleukin 12. Cancer research, 59(16), 4035-4041.

43. Ryu, C. H., Park, S. H., Park, S. A., Kim, S. M., Lim, J. Y., Jeong, C. H., ... \& Jeun, S. S. (2011). Gene therapy of intracranial glioma using interleukin 12-secreting human umbilical cord blood-derived mesenchymal stem cells. Human gene therapy, 22(6), 733-743.

44. Kerkar, S. P., Muranski, P., Kaiser, A., Boni, A., Sanchez-Perez, L., Yu, Z., ... \& Morgan, R. A. (2010). Tumor-specific CD8+ T cells expressing interleukin-12 eradicate established cancers in lymphodepleted hosts. Cancer research, 70(17), 6725-6734.

45. Chmielewski, M., \& Abken, H. (2012). CAR T cells transform to trucks: chimeric antigen receptor-redirected T cells engineered to deliver inducible IL-12 modulate the tumour stroma to combat cancer. Cancer Immunology, Immunotherapy, 61(8), $1269-1277$.

46. Liebau, C., Roesel, C., Schmidt, S., Karreman, C., Prisack, J. B., Bojar, H., ... \& Baltzer, A. W. (2004). Immunotherapy by gene transfer with plasmids encoding IL-12/IL-18 is superior to IL-23/IL-18 gene transfer in a rat osteosarcoma model. Anticancer research, 24(5A), 2861-2867.

47. Dohnal, A. M., Witt, V., Hügel, H., Holter, W., Gadner, H., \& Felzmann, T. (2007). Phase I study of tumor Ag-loaded IL-12 secreting semi-mature DC for the treatment of pediatric cancer. Cytotherapy, 9(8), 755-770.

48. Kuriakose, M. A., Chen, F. A., Egilmez, N. K., Jong, Y. S., Mathiowitz, E., DeLacure, M. D., ... \& Bankert, R. B. (2000). Interleukin12 delivered by biodegradable microspheres promotes the antitumor activity of human peripheral blood lymphocytes in a human head and neck tumor xenograft/SCID mouse model. Head \& Neck: Journal for the Sciences and Specialties of the Head and Neck, 22(1), 57-63.

49. Egilmez, N. K., Jong, Y. S., Sabel, M. S., Jacob, J. S., Mathiowitz, E., \& Bankert, R. B. (2000). In situ tumor vaccination with interleukin-12-encapsulated biodegradable microspheres: induction of tumor regression and potent antitumor immunity. Cancer research, 60(14), 3832-3837.

50. Sabel, M. S., Skitzki, J., Stoolman, L., Egilmez, N. K., Mathiowitz, E., Bailey, N., ... \& Chang, A. E. (2004). Intratumoral IL-12 and TNF- $\alpha$-loaded microspheres lead to regression of breast cancer and systemic antitumor immunity. Annals of surgical oncology, 11(2), 147-156.

51. Kocbek, P., Obermajer, N., Cegnar, M., Kos, J., \& Kristl, J. (2007). Targeting cancer cells using PLGA nanoparticles surface modified with monoclonal antibody. Journal of controlled release, 120(1-2), 18-26.

52. Manoochehri, S., Darvishi, B., Kamalinia, G., Amini, M., Fallah, M., Ostad, S. N., ... \& Dinarvand, R. (2013). Surface modification of PLGA nanoparticles via human serum albumin conjugation for controlled delivery of docetaxel. DARU Journal of Pharmaceutical Sciences, 21(1), 58.

53. Yang, R., Yang, S. G., Shim, W. S., Cui, F., Cheng, G., Kim, I. W., ... \& Shim, C. K. (2009). Lung-specific delivery of paclitaxel by chitosan-modified PLGA nanoparticles via transient formation of microaggregates. Journal of pharmaceutical sciences, 98(3), 970-984. 54. Cohen, J. (1995). IL-12 deaths: explanation and a puzzle. Science, 270(5238), 908-909.

55. Mao, S., Xu, J., Cai, C., Germershaus, O., Schaper, A., \& Kissel, T. (2007). Effect of WOW process parameters on morphology and burst release of FITC-dextran loaded PLGA microspheres. International journal of pharmaceutics, 334(1-2), 137-148.

56. Gasparini, G., Kosvintsev, S. R., Stillwell, M. T., \& Holdich, R. G. (2008). Preparation and characterization of PLGA particles for subcutaneous controlled drug release by membrane emulsification. Colloids and surfaces B: biointerfaces, 61(2), 199-207. 
57. Ravi, S., Peh, K. K., Darwis, Y., Murthy, B. K., Singh, T. R. R., \& Mallikarjun, C. (2008). Development and characterization of polymeric microspheres for controlled release protein loaded drug delivery system. Indian journal of pharmaceutical sciences, $70(3)$, 303.

58. Pistel, K. F., \& Kissel, T. (2000). Effects of salt addition on the microencapsulation of proteins using W/O/W double emulsion technique. Journal of microencapsulation, 17(4), 467-483.

59. Spitzer, M. H., Carmi, Y., Reticker-Flynn, N. E., Kwek, S. S., Madhireddy, D., Martins, M. M., ... \& Fong, L. (2017). Systemic immunity is required for effective cancer immunotherapy. Cell, 168(3), 487-502. 


\section{Appendix AIV}

Pratt, H. G., Markel, J.E., Lindsey, B. A. (in publication). Applying Osteosarcoma Immunology to Understand Disease Progression and Assess Immunotherapeutic Response. In Kleinerman, E. S., Gorlick, R. (Eds.), Clinical Advances in Osteosarcoma. Springer Nature 


\title{
Chapter 17: Applying Osteosarcoma Immunology to Understand Disease Progression and Assess Immunotherapeutic Response
}

\author{
Brock A. Lindsey, Hillary G. Pratt, Justin, E. Markel \\ West Virginia University School of Medicine
}

Keywords: Immunology - immunopathology - immunotherapy - myeloid - granulocyte - lymphoid - lymphocyte cytokine

\begin{abstract}
Osteosarcoma, the most common malignant bone tumor in children and adolescents, remains a complicated disease to treat; no new treatments have been developed in more than three decades. Due to the importance of the immune system in osteosarcoma disease progression, immunotherapeutic strategies have been explored to potentially improve long-term survival. Understanding the immune system in osteosarcoma will be key to optimizing treatments and improving patient outcomes. Many subtypes of white blood cells have been studied in association with osteosarcoma disease progression. In particular, neutrophil-lymphocyte ratios are correlated with a poor prognosis. Macrophage polarization can be altered late in osteosarcoma progression to an M2 immunosuppressive phenotype, which promotes tumorigenesis and metastasis. This relationship is likely due to the association between M2 macrophages and $\mathrm{T}_{\mathrm{H}} 2$ cells, which are also immunosuppressive. $\mathrm{T}$ cells in osteosarcoma express markers of dysfunction, including PD-1, CTLA-4, and Tim-3. Furthermore, Tregs are an important cell type in osteosarcoma that prevent an appropriate immune response to the malignantly transformed cells. Similarly, myeloid derived suppressor cells (MDSCs), which are upregulated in osteosarcoma, cause immunosuppression and have specifically been shown to inhibit NK cells. Utilizing this knowledge about the immune changes in osteosarcoma, antibodies targeted against PD-1, PD-L1, and CTLA-4 have been employed in osteosarcoma treatment, termed checkpoint blockade. Additionally, the immune system has been stimulated and primed for an immune response with cytokines, CAR-T cells, dendritic cells, and peptide vaccines. Tracking the immune system throughout disease and treatment of patients with osteosarcoma could allow for a personalized approach to immunotherapy.
\end{abstract}

\section{Introduction}

Despite being the most common malignant bone tumor in children and adolescents, osteosarcoma remains a difficult tumor to treat. Over the past three decades, no new treatments for osteosarcoma have been developed; thus, improvements in long term survival have remained stagnant since the first addition of chemotherapy for the disease. Nonetheless, although osteosarcoma has been deemed a highly pleomorphic, immunologically cold tumor historically speaking, more recent data has suggested immunotherapy may be successful.1 Recent studies have shown osteosarcoma to be an immunologically "hot" cancer as both the innate and adaptive immune systems are affects not only in the local tumor microenvironment but also systemically. In order to overcome non-response and immunoresistance, both of which have been problematic in immunotherapy, it logically follows that we should also attempt to understand the impact of osteosarcoma on the immune system. One mechanism by which these alterations can be assessed involves immunophenotyping various tissue samples from the patient, including tumor biopsies, metastatic tumor biopsies, splenic biopsies, and serum samples. Immunophenotyping, commonly performed by immunohistochemistry (IHC) or flow cytometry, allows us to quantify particular subsets of white blood cells using highly specific fluorescent antibodies. Furthermore, the cell surface epitopes marked by these antibodies can also be quantified to assess the status of the immune cell in question. Thus, this chapter outlines the known immunological alterations that occur with osteosarcoma disease progression and subsequently discusses the immunotherapies that have been explored to combat this malignancy. 


\title{
II. Assessing Osteosarcoma Disease Progression
}

\author{
A. Osteosarcoma Immunology: Innate Immune System
}

\section{Myeloid Lineage}

\section{Granulocytes}

The most prominent granulocyte in osteosarcoma immunopathology is the neutrophil, which is one of the main players in the acute inflammatory response. Following injury or infection, the affected tissue releases interleukin(IL)-8, which is a neutrophil chemoattractant and degranulation agent. Upon arrival to the site of the insult, they release inflammatory mediators and phagocytose cellular debris. As neutrophils are one of the first immune cells to arrive in infected tissues, it is likely that they contribute to the tumor microenvironment although the details of this are not well defined. Indeed, little work has been done to examine neutrophils in the context of osteosarcoma disease progression; however, there are a few studies with some indirect evidence to support a role for neutrophils in tumor progression. Postoperative infections have been associated with significantly increased event-free survival and 5-year overall survival (100\% and $100 \%$ with infection versus $54 \%$ and $43 \%$ without, respectively; $\mathrm{P}=0.012$ ), while high neutrophillymphocyte ratios (NLR) are associated with poor prognoses $(\mathrm{P}<0.05)$; furthermore, multivariate Cox regression has shown that NLR is a top risk factor associated with death in osteosarcoma patients.3, Osteosarcoma patients have also been found to have high serum levels of IL-8, as well as polymorphisms in its coding gene that are linked to increased likelihood of metastasis.4,5 High expression levels of another neutrophil chemoattractant, CXCL5, has also been linked to advanced clinical stage and metastasis. 6 Interestingly, both IL-8 and CXCL5 have been found to have direct effects on osteosarcoma tumor cells themselves by inducing migration and invasion. Anther cell type, related to neutrophils, polymononuclear myeloid derived suppressor cells (PMN-MDSCs) will be discussed further in the MDSC section below.

\section{Mononucelar Phagocytic System: Monocytes, Macrophages, and Dendritic Cells}

\author{
a. Monocytes
}

Monocytes are antigen-presenting cells (APCs) that are one of the main means of communication between the innate and adaptive arms of the immune system. They are produced in the bone marrow and migrate to areas of inflammation where they differentiate into macrophages or dendritic cells. Early in vitro studies found that monocytes were cytotoxic to osteosarcoma tumor cells leading to the belief that they contributed to tumor immunosurveillance 7,8 ; however, multiple in vivo studies have suggested a more immunosuppressive role for monocytes in the context of a developing malignancy. Notably, it was discovered that in osteosarcoma patients, circulating T cell immunoglobulin and mucindomain containing-3 (Tim-3) positive monocytes suppress antitumor T helper type 1 (TH1) responses by interacting with Tim-3+ T cells, naïve CD4+ T cells, and galectin-9 (Gal-9)-expressing T regulatory cells (Treg). Additionally, a group in 2017 found clinical relevance in the ratio of absolute peripheral monocyte count after initial treatment divided by absolute monocyte count before initial treatment (monocyte ratio); when a patient's monocyte ratio was greater than 1 , he or she was over 5 times more likely to develop metastases compared to patients with ratios less than or equal to 1 (OR 5.367; 95\% CI, 3.083-9.343).9 Osteosarcoma cells release the cytokine IL-34 which recruits inflammatory monocytes to areas of tumorigenesis; upon arrival, they mature into tumor-associated macrophages (TAM) discussed in the subsequent section. 


\section{b. Macrophages}

Macrophages, derived from circulating monocytes, reside in many tissues throughout the body and phagocytose cellular debris, tumor cells, and pathogens. Tissue-resident macrophages can produce a diverse response to inflammation, infection, healing, and cancer based on the cytokines in the local microenvironment. Furthermore, macrophages can be delineated into a number of subsets based on their cell surface expression, including classically activated (M1) macrophages, alternatively activated (M2) macrophages, TAMs, and myeloid derived suppressor cells (MDSCs). Specifically, in tumorigenesis, TAMs of the M1 subtype have a protective effect while the M2 subtype and MDSCs suppress anti-tumor immune responses.10 The M1/M2 TAM subpopulations will be discussed here while MDSCs will be discussed in a subsequent section.

In osteosarcoma, immunohistochemical stains of resected primary tumors have shown macrophages to be the predominant infiltrating cell type.11 A number of studies have shown a correlation between TAMs and metastases in osteosarcoma. 10-12 Similar to their activity in other solid tumors, M1 macrophages have been shown to suppress tumorigenesis in osteosarcoma. One in vitro study demonstrated that IFN $\gamma$-activated CD86+HLA-DR+ M1 macrophages can inhibit osteosarcoma cell growth.13 In patients, the quantity of M1 macrophages, defined by iNOS positivity, in the primary tumor was negatively correlated with metastatic disease $(p=0.001) .11$ This observation may be related to the ability of the M1 macrophage to promote a TH1 anti-tumor response. 10 Although M1 macrophages may be the first to invade the primary tumor, in vivo studies have shown that early F4/80+CD163- M1 macrophages are replaced by F4/80+CD163+ M2 macrophages between the first and third weeks of tumor establishment.14 According to one group's in vivo study, M2 TAMs are likely recruited to the primary tumor due to IL-34 overexpression by osteosarcoma cells.15 In contrast to M1 macrophages, CD163+M2 macrophages generate a Tн2 response.16 Furthermore, in vitro studies of M2 macrophages have shown these cells enhance the migratory capacity of osteosarcoma cells.17 M2 TAMs likely promote tumorigenesis via immunosuppression, production of matrix degrading enzymes, and stimulation of angiogenesis. Notably, CD163+ M2 macrophages have been shown to correlate with increased density of CD31+ blood vessels and CD146+ vascular cells in vivo. 11 Furthermore, patients with a higher proportion of CD163+ M2 macrophages present with metastases.18 In addition to stimulating neoangiogenesis, M2 macrophages overexpress epidermal growth factor (EGF), which can bind epidermal growth factor receptor (EGFR) on osteosarcoma cells to promote growth.14

Although many studies attempt to stratify M1 and M2 populations, cancer is a complex disease process that may stimulate a more intermediate TAM phenotype. For example, Buddingh, et al. found that a higher percentage of TAMs, irrespective of M1 or M2 phenotype, was correlated with a higher overall survival rate in patients.12 Similarly, a Phase III clinical trial found a higher overall survival rate and higher rate of metastasis free survival in patients whose primary tumor samples showed $>50 \%$ CD163+ M2-like macrophages with a trend toward significance for CD68+ M2 macrophages.16 Furthermore, one group's in vivo study mimicked these observations; both M1 markers, Cxc19 and iNos, and M2 marker, Tgm2, were significantly elevated in splenic biopsies of all tumor bearing mice. Taken together, these findings suggest that macrophage polarization in malignancy is complex and requires more than one cell marker to accurately capture polarization. 19

\section{c. Dendritic cells}

Like macrophages, conventional dendritic cells (cDCs) are derived from circulating monocytes and serve to prime and activate a $\mathrm{T}$ cell response against neoantigens produced by tumor cells. $20,21 \mathrm{CDCs}$ can be divided into $\mathrm{cDC} 1$ cells and $\mathrm{cDC} 2$ cells with the $\mathrm{CD} 103+\mathrm{cDC} 1 \mathrm{~s}$ performing the roles of antigen presentation and cytotoxic $\mathrm{T}$ cell $\left(\mathrm{T}_{\mathrm{c}}\right)$ activation. In early in vitro studies, CD14+ DCs, isolated $\mathrm{cDC} 1 \mathrm{~s}$, and isolated $\mathrm{cDC} 2 \mathrm{~s}$ respectively cultured with osteosarcoma cell lines showed significantly decreased expression of maturation markers, including CD80, CD83, CD86, CD40, HLA-DR, and CCR7. 20, 22 Furthermore, in contrast to control cDC1 cells, which express IL-12, cDC1s 
cultured with osteosarcoma cells increased expression of IL-10 but did not express IL-12. Isolated cDC2s cultured with osteosarcoma cells also significantly increased IL-10 production. Elevated levels of IL-10 primes the Тн2 response and suppresses the $\mathrm{T}_{\mathrm{H}} 1$ response, which may inhibit an adequate immune response to the primary tumor.20 Although in vitro studies of cDCs have shown a possible role for DCs in osteosarcoma pathogenesis, it should be noted that a lower level of DCs have been found to infiltrate pediatric tumors, including osteosarcoma, when compared to adult onset tumors. The relative lack of infiltrating DCs and taken with the observation that osteosarcoma can suppress a cDC-induced $\mathrm{T}_{\mathrm{H}} 1$ antitumor response may suggest a role for vaccination with tumor-antigen loaded dendritic cells in treatment of osteosarcoma, which will be discussed later. 23

\section{d. Myeloid Derived Suppressor Cells}

Myeloid derived suppressor cells (MDSCs) constitute a population of myeloid cells that have failed to achieve maturation to dendritic cells, monocytes, or granulocytes. Associated with pathology, especially chronic inflammation and cancer, MDSCs can be categorized as CD11b+HLA-DR-CD14+CD15- monocytic MDSCs (M-MDSCs) or CD66b+CD15+CD14-/dimCD33dimHLA-DR-polymononuclear MDSCs (PMN-MDSCs) in humans.24 While M-MDSCs suppress $\mathrm{T}$ cell activity via both antigen dependent and independent mechanisms, including production of reactive oxygen species through arginase (Arg) and nitric oxide synthase (iNOS), the PMN-MDSCs suppress T cell activity mainly by an antigen dependent pathway.25 For patients with solid tumors, increased levels of MDSCs have been correlated with lower overall survival.26 In vivo studies of MDSCs in murine osteosarcoma models show elevated levels of both PMN-MDSCs and M-MDSCs in peripheral blood and spleen (Unpublished data). 27 Although elevated early in the disease process, the M-MDSC cell levels trended downward as the disease progressed while the PMNMDSC population remained stable as shown in Figure 1. (Unpublished data) Similarly, in pediatric sarcoma patients, CD14+HLA-DR 10 - M-MDSCs were found to be increased compared to healthy controls.28 Regarding HLA-DR, a marker for antigen presenting cells of the myeloid lineage, a decrease has been shown on monocytes of patients with aggressive sarcoma, which is postulated to be one of the initial steps in development of M-MDSCs from monocytes. 28,29 Studies done in vivo have shown that HLA-DR expression decreases on myeloid-type cells as PD-L1 expression increases. Specifically, the ratio of PD-L1 to HLA-DR (P[1]/M[II]) in peripheral blood increased with osteosarcoma and trended upward in both peripheral blood and spleen as the disease progressed. Moreover, the systemic M-MDSC $\mathrm{P}[1] / \mathrm{M}[\mathrm{II}]$, including blood, bone marrow, and spleen tissues, showed a positive correlation with the lung M-MDSC ratio that was significant in blood (Figure 2: $\mathrm{R}=0.972 ; \mathrm{p}<0.01$ ) and spleen (Figure 2: $\mathrm{R}=0.998 ; \mathrm{p}<0.01$ ) and trending toward significance in bone marrow. (Unpublished data).
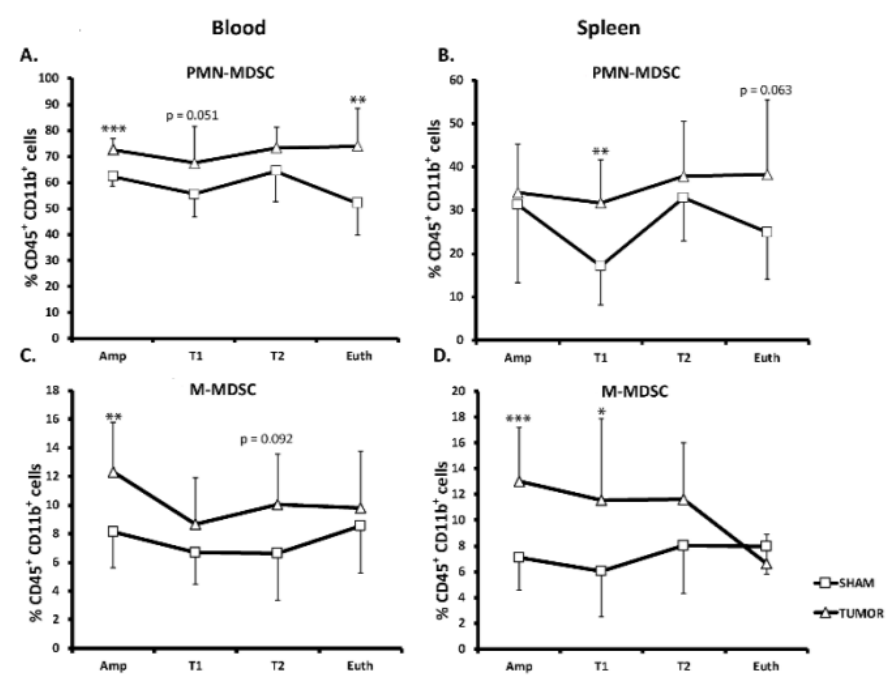
Fig. 1. Systemic PMN-MDSCs remain relatively constant while M-MDSCs decrease during disease progression in vivo. $* * * p=0.001$, $* * \mathrm{p}=0.01, * \mathrm{p}=0.05$.

IL-18 expression has also been shown to be elevated in vivo on MDSCs with osteosarcoma disease progression. As inhibition of IL-18 decreased levels of MDSCs in both peripheral blood and the primary tumor, this cytokine may play a role in the development and recruitment of MDSCs.27 A more immature population of MDSCs has also been identified as Lin-(including CD3, CD14, CD15, CD19, and CD56) HLA-DR-CD33+ but was not found to be elevated in pediatric sarcoma and has not been studied further.25,28 Certainly, due to the apparent role of MDSCs in malignancy, these cell populations may be targeted to improve treatment strategies, as will be discussed with treatment strategies.

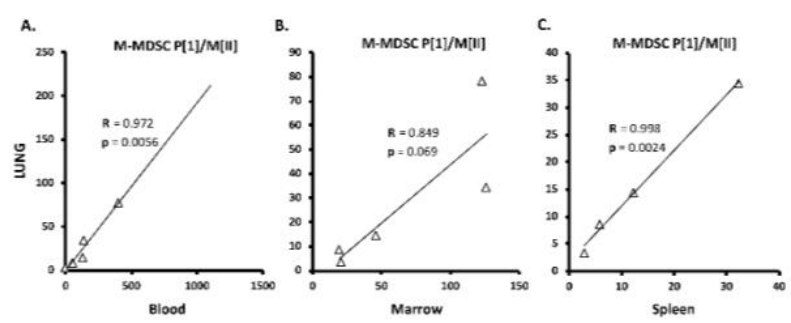

Fig. 2. Ratio of PD-L1 to HLA-DR (P[1]/M[II]) on M-MDSCs correlated with ratio of M-MDSCs in the lung in vivo.

\section{B. Osteosarcoma Immunology: Adaptive Immune System}

\section{Lymphoid Lineage}

\section{T Lymphocytes}

\section{a. Helper T Cells}

Helper $\mathrm{T}(\mathrm{TH})$ cells, commonly defined as being $\mathrm{CD} 4+$, can generate several different types of immune response: Тн1 or Тн 2 being the most common. Tн1 cells primarily secrete IFN $\gamma$ and TNF $\alpha$ cytokines enhancing both the effector and memory functions of CD8+ cytotoxic T cells (CTLs). 30-31 CD4+ TH cells can also enhance the antitumor response by optimizing the $\mathrm{cDC}$ response, which in turn stimulates the CTLs.32 Regarding tumor rejection in particular, Тн1 cells can recruit NK cells and macrophages to form an antitumor response. Often considered to be in opposition of $\mathrm{T}_{\mathrm{H}} 1$ cells, $\mathrm{TH}_{\mathrm{H}}$ response involves IL-4, IL-5, and IL-13, which together activate a humoral response. 30 Although IL-4 recruits macrophages and eosinophils in murine models, both IL-4 and IL-13 have been shown to activate macrophages toward an M2-like immunosuppressive phenotype.30,33,34 The role of another distinct CD4+ TH cell type, $\mathrm{T}_{\mathrm{H}} 17$, has been debated for its potential antitumor function.35 One in vitro study found that IL-22, commonly produced by Тн17 cells in vivo, stimulated the proliferation and invasion of two different osteosarcoma cell lines.36 Thus, an abundance of $\mathrm{CD} 4+\mathrm{T} 17$ cells in the primary tumor might suggest a poor prognosis. 
While numerous studies have shown that $\mathrm{CD} 4+\mathrm{T}_{\mathrm{H}}$ can improve the antitumor response, one group has shown that CD8+ CTLs can have an antitumor effect in osteosarcoma without CD4+ Тн aid.31 Nonetheless, for adult osteosarcoma, CD4 $+\mathrm{T}_{\mathrm{H}}$ cells likely play a role in the immune process as this cell type represented the predominant TIL and demonstrated lytic activity against several osteosarcoma cell lines.37 Furthermore, an in vivo study of the immunologic changes during osteosarcoma pathogenesis showed that percentages of CD45+CD4+CD8- $\mathrm{TH}$ trended downward in the peripheral blood and spleen as the disease progressed, which is shown in Figure 3 (Unpublished data). Similarly, for peripheral blood samples of patients with osteosarcoma, there was a decreased overall numbers of CD4+ T cells. 28 In addition to the overall decrease in CD4+ T cells, patients with osteosarcoma exhibited increased expression of PD-1 and Tim-3, markers of $\mathrm{T}$ cell dysfunction and exhaustion, respectively, in CD4+ TH cells compared to patients without osteosarcoma. 38-40 Furthermore, elevated levels of Tim-3+CD4+ T cells in the peripheral blood was shown to be correlated with later stage of disease and increased expression of IFN $\gamma$ and IL-2, which stimulate a T cell response. Elevated levels of peripheral Tim-3+CD4+ T cells also correlated with decreased overall survival in osteosarcoma patients. 41
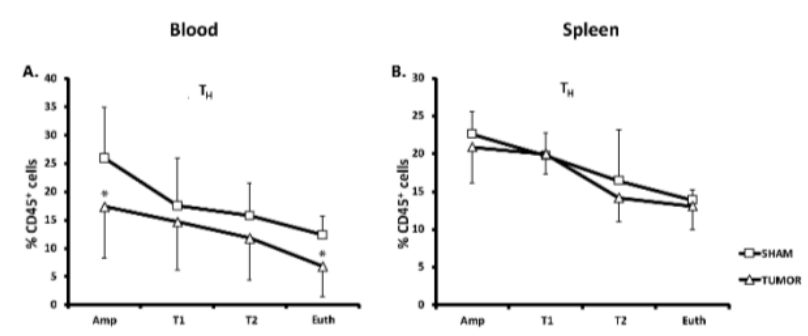

Fig. 3. Helper $\mathrm{T}$ cells decrease systemically during disease progression in vivo. * $\mathrm{p}=0.05$.

In addition to traditional $\mathrm{T}_{\mathrm{H}}$ cells, $\mathrm{CD} 4+\mathrm{CXCR} 5+$ follicular $\mathrm{T}$ helper $\left(\mathrm{T}_{\mathrm{FH}}\right)$ cells, which promote a $\mathrm{B}$ cell response and maintain germinal centers in secondary lymphoid organs have been found to be of importance in osteosarcoma. 42-43 Indeed, levels of CXCR3-CCR6- Тн2-type cells and CXCR3+CCR6+ Тн17-type cells were found to be nearly two times greater in osteosarcoma patients while levels of CXCR3+CCR6- Tн1-type cells were lower. Furthermore, TFH levels were found to be elevated peripherally in patients with both metastatic and high grade osteosarcoma, specifically the $\mathrm{T}_{\mathrm{H}} 1$ and $\mathrm{T}_{\mathrm{H}} 17$ types but not the $\mathrm{TH}_{\mathrm{H}}$ subtypes.42 Although $\mathrm{CD}_{4} \mathrm{~T}_{\mathrm{H}}$ and $\mathrm{T}_{\mathrm{F}}$ have been shown to play a role in the disease process of osteosarcoma, these cells have received less attention than CTLs.

\section{b. Cytotoxic T cells}

Data accumulated from a number of human cancers have shown that the number, type, and location of tumor infiltrating lymphocytes (TILs) in the primary tumor can be prognostic for overall survival. In particular, an increased number of CD8+ TILs has been shown to correlate with increased survival rates and decreased rates of recurrence. 44 CD8+ TILs are also prominent in osteosarcoma as they represent the major infiltrating cell type in pediatric osteosarcoma and make up $15.5 \%$ of infiltrating cells in adult osteosarcoma.45, 37 An early study of primary tumor samples from patients with high-grade osteosarcoma found CD3+CD8+ TILs to predominate infiltrating inflammatory cells. As the pattern of CD3+CD8+CTL infiltration did not differ significantly from benign bone tumor samples, CD8+ TILs in osteosarcoma were not thought to have any prognostic significance.46 Later, in a retrospective study, it was found that higher CD8+ TIL density correlated with improved survival in osteosarcoma. Additionally, in this study, a positive relationship between the CD8/Foxp3 TIL ratio and overall survival was observed. 47 This relationship between CD8+ cells and Foxp3+ cells has been mirrored in other cancer types as CTLs have been shown to upregulate Treg cells. 47, 48 In addition to demonstrating a relationship with overall survival, levels of CD8+ TILs have also been found to correlate with pretreatment metastases; patients presenting with metastases had lower levels of CD8+ TILs.16 Further studies of primary tumors in patients with poor disease outcome showed that higher density of CD3+CD8+ TILs correlated with increased expression of PD-L1 on tumor cells and immune cells, which may be a mechanism by which 
the tumor evades the immune system and causes T cell exhaustion.49,50,19 Furthermore, patients with high expression of PD-L1 had a median overall survival of 28 months while median survival for patients with low PD-L1 expression was 89 months.50 PD-1 has also been shown to be upregulated on CD8+ T cells in patients with osteosarcoma.37 Thus, the PD-1/PD-L1 axis has become an attractive therapeutic target in osteosarcoma. Overall, CD8+ TILs have been shown to improve outcomes in osteosarcoma, as they have cytolytic activity, but can be subject to $\mathrm{T}$ cell exhaustion in patients with severe disease. 37

Peripheral CD8+ CTLs have also been shown to have significant role in the pathogenesis of osteosarcoma. One in vivo study demonstrated that the percentages of CD45+CD4-CD8+CTLs decreased in the peripheral blood and spleen as the disease progressed as demonstrated in Figure 4 (Unpublished data). Nevertheless, the amount of circulating CD8+ CTLs was found to be higher than levels of CD8+ T cells within the primary tumor in patients. 37 Some of these cells were found to express Tim-3, a marker of T cell exhaustion, and were particularly prevalent in patients at later stages of disease. Furthermore, increased levels of Tim-3+CD8+ T cells in the periphery negatively correlated with serum levels of IFN $\gamma$ and TNF $\alpha$. Since these cytokines activate the Tн1 response, as discussed previously, it follows that overall survival was decreased in patients with high levels circulating Tim-3+CD8+ T cells. 39,41 For pediatric patients with osteosarcoma, cytotoxic T lymphocyte-associated protein 4 (CTLA-4), a T cell inhibitory receptor, was found to be upregulated on CD8+ cells circulating in the peripheral blood. 28 This receptor has been theorized to be another immunosuppressive immune escape mechanism that can create resistance in response to solitary immune checkpoint blockade. Therefore, since CTLA-4 has been shown to play a role in osteosarcoma progression, this receptor has become a target for immune blockade, which will be discussed in a subsequent section.
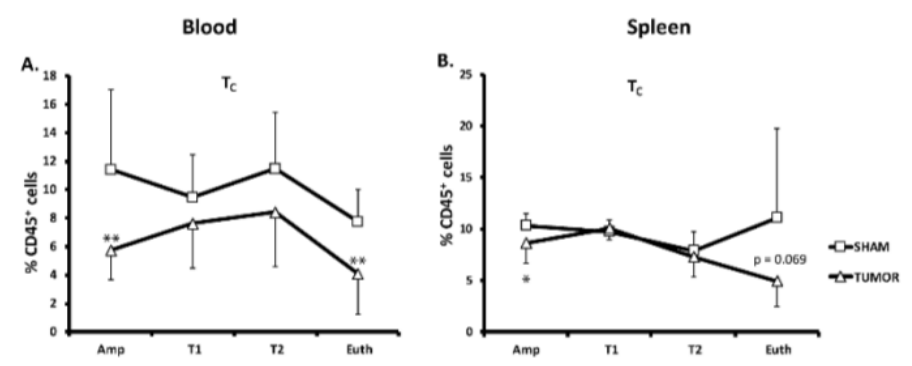

Fig. 4. In vivo osteosarcoma progression induces a decrease in percent population of systemic cytotoxic $\mathrm{T}$ cells. ${ }^{* *} \mathrm{p}=.0 .1, * \mathrm{p}=0.05$.

c. Regulatory T cells

Regulatory $\mathrm{T}$ cells ( $\mathrm{T}_{\text {regs }}$ ) play a role in peripheral self-tolerance. While these $\mathrm{CD} 4+\mathrm{CD} 25+\mathrm{T}$ cells have been shown to prevent autoimmunity, particularly in vivo, an abundance of these cells can also suppress anti-tumor immunity to tumors overexpressing self-antigens. The Treg population has been identified as expressing CD25 (IL-2 $\alpha \mathrm{R}$ ), glucocorticoid-induced TNF receptor (GITR), CTLA-4, and Foxp3 transcription factor. In vivo studies have shown that the presence of $\mathrm{T}_{\text {reg }}$ cells promotes tumor growth and inhibits CD8+CTL immunogenic response to the tumor.51 For example, when $\mathrm{CD} 4+\mathrm{CD} 25+\mathrm{T}_{\text {reg }}$ cells are cocultured with $\mathrm{CD} 8+\mathrm{T}$ cells, the $\mathrm{CD} 8+\mathrm{T}$ cells show significant suppression of proliferation and production of IFN $\gamma$, IL-2, and CD25.52 Interestingly, splenic Treg cells in vivo are increased later in disease progression (Figure 5).19 Similarly, a retrospective study of human osteosarcoma samples demonstrated that increased Foxp3+ Tregs infiltrating the tumor indicated a worse prognosis. In fact, as Foxp3+ Tregs increased relative to CD8+ CTLs, survival decreased independent of primary metastasis and chemotherapy response. This relationship is likely due to $T_{\text {reg }}$ mediated inhibition of CTLs.47 Furthermore, an increase in CD3+CD8-Foxp3+ T cells has been found in local relapses of osteosarcoma, potentially suggesting a tumor escape mechanism. 49 Importantly, while Foxp3+ cells have classically been classified as Treg cells, it should be noted that recent evidence suggests that Foxp3 can be upregulated in conventional TILs, which suggests that Foxp3 alone cannot be utilized to delineate $T_{\text {reg }}$ populations.47 Also, despite the apparent role of $T_{\text {regs }}$ in the local tumor microenvironment, these cells have not been shown to be elevated systemically in pediatric osteosarcoma. 28 


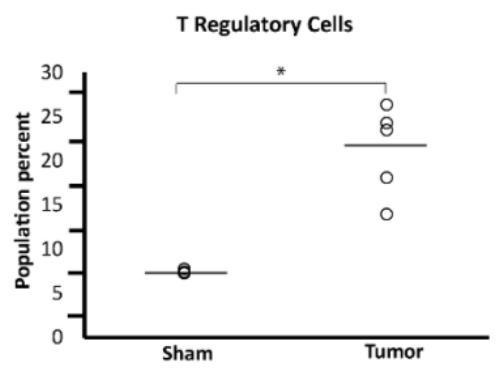

Fig. 5. Regulatory $\mathrm{T}$ cell populations $(\mathrm{CD} 45+\mathrm{CD} 4+\mathrm{Foxp3}+)$ are increased in the spleens of tumor bearing mice.

\section{B Lymphocytes}

In addition to tumor infiltrating T lymphocytes, B lymphocytes can also be found in the tumor microenvironment; these cells, however, have been studied less than their $\mathrm{T}$ cell counterparts.53 Activated B cells can generate autoantibodies that promote an antitumor response in malignancy. 53,54 Furthermore, B cells may enhance the T cell response via antigen presentation and costimulation.53-55 For several malignancies, the presence of CD20+ TILs in addition to CD4+ and CD8+ TILs has been correlated with better overall outcomes when compared to independent CD20+ B cell or CD8+ T cell tumor infiltration, respectively.53,56,57 In particular, for soft tissue non-gastrointestinal stromal tumor sarcomas, increased CD20+ TILs correlated with improved disease specific survival.58 Additionally, a study of pediatric patients with soft tissue sarcomas found increased memory class switched B cells. 28 Despite these findings, utilizing CD20+ TILs as a prognostic indicator of disease outcome and survival may be difficult as some, including immature and those producing IL-10 and TGF- $\beta$, CD20+ B cells may suppress the antitumor response. 53 Further study into the effects of CD20+ B cells should be done to elucidate the role of these cells in osteosarcoma.

\section{Natural Killer Cells}

As lymphocyte members of the innate immune system, natural killer (NK) cells play a role in immunosurveillance and are defined by CD3 negativity as well as CD56 or CD16 positivity.59 In cancer specifically, these granulocytic NK cells can release their cytotoxic granules to directly kill malignantly transformed cells. Released tumor cell antigens are then presented to B and T lymphocytes via APCs to generate an adaptive immune response. 60 NK cell mediated killing is negatively correlated to expression of HLA class I molecules, which serves to maintain selftolerance. This mechanism, however, can be exploited by malignant cells in order to evade immunosurveillance.60,61 In osteosarcoma, analysis of HLA class I expression in primary and metastatic tumors showed downregulation in 52\% and $88 \%$ of samples, respectively.62 To inhibit NK cells, HLA class I molecules on tumor cells interact with Killer Immunoglobulin-Like Receptor (KIR), which is expressed on the NK cell surface and commonly upregulated in malignancy.60,63 For patients with osteosarcoma, NK cells can express combinations of all three KIR molecules, including KIR2DL1, KIR2DL2/2DL3, and KIR3DL1, and thus display decreased activity.63

Early in osteosarcoma disease pathogenesis, peripheral blood NKG2D + NK cells do not differ in number, phenotype, or functionality.64 In general, however, circulating NK cells are commonly lower in osteosarcoma patients compared to healthy patients.65,66 These findings may be explained by in vivo studies which suggest that CD45+NKp46+ NK cells are significantly increased systemically with osteosarcoma.19 As the disease progresses, though, NK cell populations in the peripheral blood decrease. These in vivo findings may be explained by the relative increase in M-MDSCs and PMN-MDSCs in osteosarcoma, which inhibit NK cells (Unpublished data). Due to the potent anti-tumor activity of NK cells but dysregulation in osteosarcoma, these cells have become an important focus for future clinical trials.66 


\section{Assessing Immunotherapeutic Response in Osteosarcoma}

\section{A. Checkpoint Blockade}

Immune checkpoints serve to preserve self-tolerance and prevent autoimmunity and excess immune stimulation; however, these pathways can also be used by tumor cells as a means of escaping immune system detection. Due to their role in tumor immune tolerance, these $\mathrm{T}$ cell checkpoint molecules, programmed death receptor 1 (PD-1) and CTLA-4, are therapeutic targets for advanced cancers.67 Since the PD-1/PD-L1 axis has been shown to negatively impact overall survival in patients with osteosarcoma, these molecules are of particular interest.49,50,68 In vivo anti-PD1 treatment facilitated macrophage mediated decrease in both micro and macrometastases but not primary tumor growth.68,69 Specifically, Ki-67, a marker of tumor cell proliferation, expression decreased while TUNEL, a marker of cell apoptosis, expression increased in tumor tissue.69 Anti-PD-1 also increased CD4+ T cell, CD8+ T cell, NKp46+ NK cell, and F4/80+CD11b+ macrophage infiltration into lung metastases.68,69 Furthermore, anti-PD-1 shifted the macrophage polarization from CD163+ M2 to CD86+ M1 macrophages in lung metastases, which facilitates an antitumor response.69 Importantly, treatment with anti-PD-1 downregulated tumor cell expression of PD-L1, which suggests utilization of another mechanism for immune suppression. 68

Therapeutic strategies have also targeted PD-L1 in an attempt to interrupt the PD-1/PD-L1 axis. Similar to antiPD-1 treatment, anti-PD-L1 treatment downregulated expression of PD-L1 by primary tumor and metastatic osteosarcoma cells. Increased length of treatment in vivo did not enhance response to anti-PD-L1, which further suggests another pathway for immune suppression. Interestingly, following treatment with anti-PD-L1, CD8+ TILs showed decreased PD-1 expression and increased CTLA-4 expression 70,19 CTLA-4 is a membrane glycoprotein expressed by activated effector $\mathrm{T}$ cells and tumor cells which represses $\mathrm{T}$ cell proliferation, cell cycle progression, and cytokine production.71 Furthermore, in response to anti-PD-L1, the tumor cells upregulated CD80 and CD86, which bind to CTLA-4 to inhibit T cell activation.71,72 Thus, blockade of both PD-L1 and CTLA-4 in vivo completely controlled metastases and increased long-term survival to $60 \%$ from $0 \%$ shown with anti-PD-L1 treatment alone. Combination of anti-PD-L1 treatment with doxorubicin did not similarly enhance long-term survival.70 In addition to stimulating changes in tumor cells, in vivo anti-PD-L1 treatment also has an effect on systemic immune cells. Specifically, total percentage of monocytes/macrophages increased in response to anti-PD-L1. Additionally, the expression of M1 markers, Nos2 and Cxc19 and M2 marker, Tgm2, decreased resulting in a near normal M1/M2 macrophage ratio and returned the immune phenotype to that of sham status (Figure 6).19 

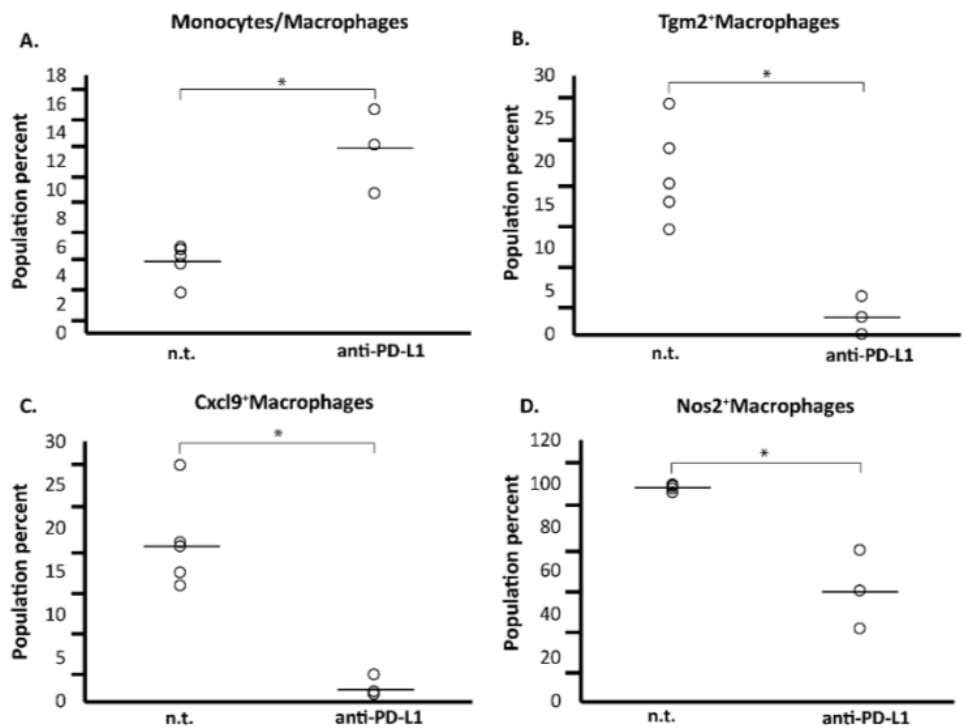

Fig. 6. In vivo treatment with anti-PD-L1 reduces macrophage markers to near normal.

Regarding the efficacy of immune blockade in humans, anti-PD-L1 has shown a durable response and prolonged disease stabilization across a variety of tumor types.72 A Phase II Trial for pembrolizumab montherapy, an anti-PD-1 antibody, showed a confirmed partial response to treatment in $5 \%$ of patients with osteosarcoma. This response, however, resulted in greater than 50\% tumor shrinkage for 6 months. Use of muramyl tripeptide phosphatidethanolamine (Mifamurtide), which will be discussed later, has been suggested to enhance immune cell infiltration into metastases thereby potentially improving the efficacy of pembrolizumab in osteosarcoma. 73 Efficacy of anti-PD-1 treatment may also be enhanced by combination therapy. For example, trabectedin, an antitumor medication approved in Europe for treatment of soft tissue sarcomas, decreases osteosarcoma tumor cell proliferation and lung metastases in vivo. Following this treatment, CD8+ TILs exhibited upregulation of PD-1 suggesting a potential role for trabectedin in immune blockade. Indeed, treatment with both trabectedin and anti-PD-1 inhibited tumor growth and enhanced $\mathrm{T}$ cell activation to a greater degree than either treatment alone. 74

\section{B. Other Immunotherapeutics}

\section{Cytokine Stimulation}

Cytokines play an important role in the coordination of immune response against tumor antigens. Due to their unique ability to modulate the immune system, these molecules have been of interest for immunotherapy and have been implemented as treatment in a number of malignancies. One such cytokine, interferon alpha (IFN $\alpha$ ), has demonstrated the ability to inhibit proliferation and induce apoptosis in tumor cells as well as inhibit angiogenesis.67 Early studies of IFN $\alpha$ showed the ability to inhibit growth of osteosarcoma cell lines in vitro and xenograft osteosarcoma tumor growth in vivo.67, 75-76 Relatively little clinical data is available regarding IFN $\alpha$ treatment in osteosarcoma. Two early clinical studies showed 5 -year recurrence free survival as well as a 10 -year metastases free survival rate and a disease specific survival rate of 39\% and 43\%, respectively. 77-80 Furthermore, with elevated dose, the disease-free survival rate increased to $63 \%$ with higher treatment dose, indicating a possible dose-response relationship that mirrors in vivo studies. 77,78,80 Combination therapy including methotrexate, doxorubicin, and cisplatin with pegylated IFN $\alpha-2 b$ demonstrated improved event free survival and overall survival; however, these differences were not significant. 81 
IL-2 is another cytokine that has been implemented as immunotherapy for malignancy, specifically osteosarcoma. Produced mostly by antigen-stimulated CD4+ T cells with some production by NK cells, CD8+ T cell, and activated DCs, IL-2 stimulates growth and differentiation in CD8+ T cells, maintains CD4+ Treg populations, and drives differentiation of CD4+ T cells into effector cells.82 Early in vitro studies of IL-2 suggest it indirectly stimulate NK cell activity by increasing IFN $\gamma .83$ Furthermore, IL-2 has been shown to stimulate lymphokine activated killer cells (LAK), which can be primed to form an anti-tumor response; thus, IL-2 has received attention as a potential therapeutic agent. 67, 82, 84-86 IL-2 combined with autologous LAK cells first demonstrated the ability to mediate tumor regression in a study of twenty-five metastatic patients that had failed standard chemotherapy. $82,84 \mathrm{In}$ patients with metastatic osteosarcoma at diagnosis, the 3-year event free survival rate and overall survival rate were $34.4 \%$ and $45.0 \%$, respectively, with IL-2 treatment. 67, 85 Despite the positive tumor response in patients, IL-2 may simultaneously stimulate high levels of $\mathrm{T}_{\text {reg }}$ cells, thereby stimulating an environment tolerant of tumorigenesis rather than stimulating an anti-tumor response. 51

\section{Dendritic Cell Vaccines}

Dendritic cells have the ability to prime the adaptive immune system, specifically a $\mathrm{T}$ cell response, in response to tumor antigens; however, in osteosarcoma and other malignancies, these DCs fail to mature and adequately stimulate a T cell response. $20,21,23,87$ Thus, priming a patient's own dendritic cells ex vivo against tumor antigens has become a potential immunotherapy for patients with advanced disease.87 In vivo studies of tumor-lysate pulsed DCs showed significantly increased CTL response and IFN $\gamma$ levels while levels of IL-4 were decreased. Increased expression of costimulatory CD86, CD83, CD205, and CD209 in the DCs correlated with the allogenic T cell response.88 Furthermore, combining the tumor-lysate DC treatment with anti-CTLA-4 antibody treatment increased the number CD8+ TILs, decreased the amount of Foxp3+ Treg cells in metastatic tumor and the spleen, and increased serum levels of IFN $\gamma$. This combined treatment reduced the metastatic tumor burden and prolonged overall survival.79 Similarly, in vivo studies combining tumor-lysate pulsed DCs with agonist anti-GITR increases the number of CD8+ TILs, increased serum IFN $\gamma$, decreased serum IL-10, and decreased numbers of CD4+Foxp3+ Treg cells in the spleen.90

In response to treatment with tumor-lysate pulsed DCs, patients with metastatic pediatric malignancy generated a specific $\mathrm{T}$ cell response allowing for tumor regression. Levels of IFN $\gamma$ levels were increased 10-fold in response to tumor antigen in these patients.91 In patients with pediatric sarcoma treated with tumor-lysate pulsed DCs, patients having an immune response had a 73\% 5-year overall survival rate while those without a response had a 37\% 5-year overall survival rate. 92 In contrast, patients with advanced soft tissue sarcoma treated with DCs pulsed with autologous tumor lysate had increased levels of IFN $\gamma$ and IL-12, which stimulate a TH1 response; nonetheless, only 1 of 35 patients achieved a partial response. These findings suggest that immune stimulation is not enough to overcome advanced malignancy. 93

\section{Genetically Modified T Cells}

Chimeric antigen receptor $\mathrm{T}$ cells consist of a specific antibody derived single-chain variable fragment ( $\mathrm{scFv}$ ) with a $\mathrm{T}$ cell signaling domain such that the effector function is released when the target cell is bound. One challenge with this therapy, particularly for osteosarcoma, is identifying a tumor antigen that is highly expressed on tumor cells with low expression in healthy tissues.94 As HER2 gene amplification has been reported in osteosarcoma, and is particularly linked with poor outcome, administration of HER2-CAR T-cells in patients with osteosarcoma resulted in stable disease for $24 \%$ of patients. $94-95$ Three of these patients had the tumor removed with one having more than $90 \%$ necrosis. IL-8 was found to be significantly elevated one week after infusion and remained elevated for 4 weeks while levels of HER2-CAR-T cells lasted for approximately 6 weeks without toxicity. 95 Pediatric sarcoma has also been shown to strongly express GD2, a glycosphingolipid.94 Although GD2-CAR T cells demonstrated lytic activity against GD2+ tumor cells in vitro, the in vivo efficacy of treatment was poor. Poor in vivo efficacy was attributed to the presence of MDSCs. However, addition of all-trans retinoic acid (ATRA) improved efficacy, likely by depleting of M-MDSCs and diminishing the suppressive capacity of PMN-MDSCs.96 Additionally, CD166, a protein that mediates communication between adjacent cells and has been implicated in tumorigenesis, has been found to be exclusively expressed in four human osteosarcoma cell lines. CD166-CAR-T cells efficiently suppressed tumor growth in tumor xenografts without significant toxicity to healthy organs. 97 
Similar to CAR-T cells, T cells can be genetically engineered to express $\mathrm{T}$ cell receptors against particular tumor antigens; however, unlike CAR-T cells, the response is dependent upon HLA haplotype.98 Genetically engineered lymphocytes reactive with NY-ESO-1, a cancer/testis antigen expressed in $80 \%$ of synovial cell sarcomas, have been shown to facilitate an objective partial response in four of six patients with metastatic synovial cell sarcoma without significant toxicity.99 The NY-ESO-1 antigen has been shown to be specific to a variety of tumors including osteosarcoma; however, the level of expression can vary between tumors. Interestingly, NY-ESO-1 expression can be upregulated in vitro in response to demethylating agent 5-aza-2'-deoxycytidine (decitabine) thus suggesting that the CAR-T cell therapy utilized against synovial cell sarcoma may be successful in osteosarcoma. Indeed, further in vivo studies showed that pre-treatment with decitabine allowed for localization of the NY-ESO-1 CAR-T cells to the tumor site. The combination treatment of decitabine and CAR-T cells decreased tumor volume when compared to controls. 100 Due to its success with B cell malignancies, genetically modified $\mathrm{T}$ cell therapies represent a promising alternative for treatment of similarly complex malignancies such as osteosarcoma.

\section{Macrophage Activation}

Mifamurtide, a nonspecific immune modulator, is a synthetic Bacille Calmette-Guerin cell wall component that can be delivered to macrophages, particularly in the lung, in the liposomal form to stimulate an anti-tumor response.101105 Liposomal muramyl tripeptide phosphatidethanolamine (L-MTP-PE) given to osteosarcoma patients increased circulating levels of TNF $\alpha$ and IL-6. Additionally, treatment with L-MTP-PE also increased circulating levels of neopterin, a marker of macrophage activation, independent of IFN $\gamma$ secretion.103 L-MTP-PE used in combination with a three or four drug chemotherapy regimen including doxorubicin, cisplatin, high dose methotrexate with or without ifosfamide (Phase III Trial INT-0133) showed improved overall 6-year survival and a trend toward improved event free survival for patients with nonmetastatic osteosarcoma; no significant difference was noted between chemotherapy regimens. 105 While it was originally determined that there was no survival benefit to the addition of L-MTP-PE for patients with metastases, a long term follow-up of those patients indicated a trend toward increased event free survival and overall survival.106 The toxicities associated with L-MTP-PE are limited to chills and fever, which are likely facilitated by the release of TNF $\alpha$ and IL-6.93 Due to the survival benefits and limited toxicity, MTP has been approved for treatment of patients with osteosarcoma by the European Medicine Agency.67,102

\section{Peptide Vaccines}

Peptide vaccines had previously gained popularity due to the potential for stimulating $\mathrm{T}$ cell response against tumorassociated antigens. 107 Vaccines involving tumor lysates, peptides, and proteins have been employed in sarcoma.67,108111 The SART3 protein was found in osteosarcoma cell lines and thus may be a useful peptide vaccine to stimulate CTLs against osteosarcoma tumors.108 Furthermore, papillomavirus binding factor protein was also found in osteosarcoma and may similarly serve as an antigenic peptide to stimulate an immunologic response against tumor tissue.109 Indeed, in a small Phase II trial in which patients with soft tissue sarcoma were treated with personalized peptide vaccines, $64.7 \%$ of patients developed an IgG response with 12 patients demonstrating epitope spreading. Furthermore, $70.6 \%$ of patients developed a CTL response following treatment. These patients with refractory sarcoma had a mean survival time of 9 months while palliative care in similar instances facilitates an 8 month survival time. 110 Due to their positive anti-tumor effects, peptide vaccines potentially represent feasible alternative for treatment of osteosarcoma; however, additional studies are required to optimize outcomes. 107

\section{Future Perspectives}

As the field of flow cytometry evolves and we improve our ability to analyze single tissue samples with more and more antibodies and fluorochromes, the field of immunology and subsequently personalized medicine is also improving. However, these enhancements come with added responsibility as the proteins studied are highly labile $e x$ vivo, which can result in poor data generation and thus false assumptions from the data. Therefore, a standardized process of tissue handling and preparation will become crucial to the process of data acquisition for this potential therapeutic tool. If done correctly, though, this tool has the potential to open many doors for the treatment of osteosarcoma using immunotherapeutics. Furthermore, previously popular treatments could be used much more 
efficiently and appropriately in dosing regiments as we can follow the patients' disease progression and recovery in real time as suggested in Figure 7.

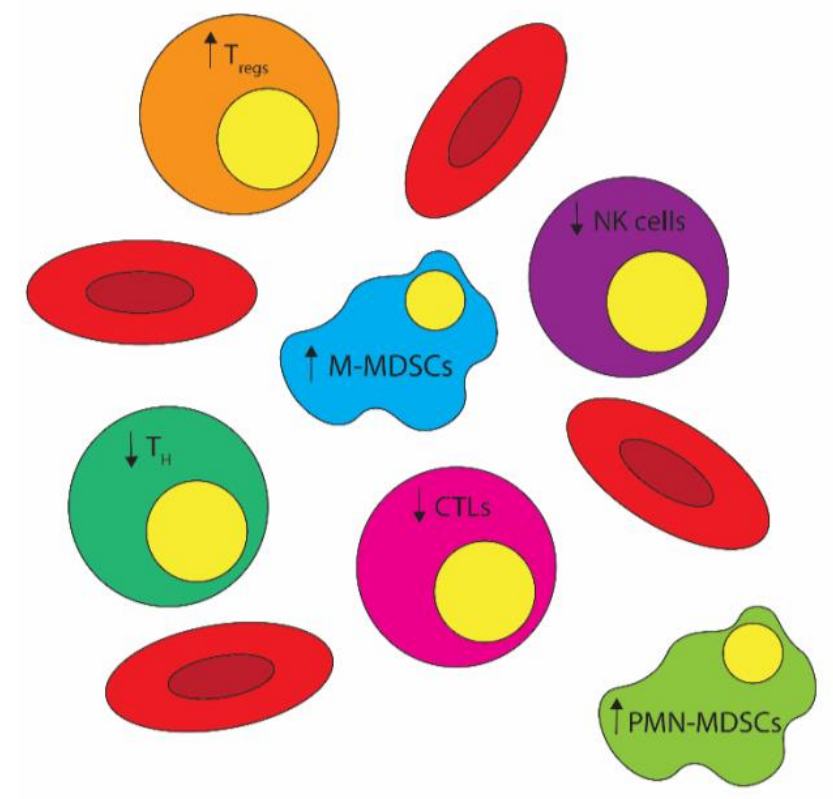

Fig. 7. Example osteosarcoma patient blood sample.

References

1. Wedekind, M. F., Wagner, L. M., Cripe, T. P. Immunotherapy for osteosarcoma: Where do we go from here? (2018). Pediatr Blood Cancer, 65(9): DOI:10.1002/pbc.27227.

2. Chen, Y. U., Xu, S. F., Xu, M., \& Yu, X. C. (2015). Postoperative infection and survival in osteosarcoma patients: Reconsideration of immunotherapy for osteosarcoma. Molecular and clinical oncology, 3(3): 495-500.

3. Liu, B., Huang, Y., Sun, Y., Zhang, J., Yao, Y., Shen, Z., ... \& He, A. (2016). Prognostic value of inflammation-based scores in patients with osteosarcoma. Scientific reports, 6: 39862 .

4. Kushlinskii, N. E., Timofeev, Y. S., Solov'ev, Y. N., Gerstein, E. S., Lyubimova, N. V., \& Bulycheva, I. V. (2014). Components of the RANK/RANKL/OPG system, IL-6, IL-8, IL-16, MMP-2, and calcitonin in the sera of patients with bone tumors. Bulletin of experimental biology and medicine, 157(4): 520-523.

5. Chen, Y., Yang, Y., Liu, S., Zhu, S., Jiang, H., \& Ding, J. (2016). Association between interleukin $8-251$ A/T and +781 C/T polymorphisms and osteosarcoma risk in Chinese population: a case-control study. Tumor Biology, 37(5): 6191-6196.

6. Dang, H., Wu, W., Wang, B., Cui, C., Niu, J., Chen, J., ... \& Liu, Y. (2017). CXCL5 plays a promoting role in osteosarcoma cell migration and invasion in autocrine-and paracrine-dependent manners. Oncology Research Featuring Preclinical and Clinical Cancer Therapeutics, 25(2): 177-186.

7. Holtermann, O. A., Djerassi, I., Lisafeld, B. A., Elias, E. G., Papermaster, B. W., \& Klein, E. (1974). In Vitro Destruction of Tumor Cells by Human Monocyte. Proceedings of the Society for Experimental Biology and Medicine, 147(2): 456-459.

8. King, G. W., File, J., \& LoBuglio, A. F. (1978). Normal human monocytes inhibit tumor cell growth in vitro. Journal of Allergy and Clinical Immunology, 62(5): 283-288.

9. Wang, S., Zheng, S., Hu, K., Sun, H., Zhang, J., Rong, G., ... \& Gui, B. (2017). A predictive model to estimate the pretest probability of metastasis in patients with osteosarcoma. Medicine. 96(3): e5909.

10. Murray, P.J., Wynn, T.A. (2017). Protective and pathogenic functions of macrophage subset. Natural Reviews Immunology, 11(11): 723-737.

11. Dumars, C., Nguyen, J., Gaultier, A., Lanel, R., Corradini, N., Gouin, F., Heymann, D., Heymann, M. (2016). Dysregulation of macrophage polarization is associated with the metastatic process in osteosarcoma. Oncotarget, 7(8): 78343-54.

12. Buddingh, E. P., Kujjer, M. L., Duim, R., Burger, H., Agelopoulous, K., Mykebost, O., ... \& Cleton-Jansen A. M. (2011). TumorInfiltrating Macrophages Are Associated with Metastasis Suppression in High-Grade Osteosarcoma: A Rationale for Treatment with Macrophage Activating Agents. Clin Cancer Res, 17(8): 2110-9.

13. Pahl, J. H., Kwappenberg, K. M. Varypataki, E. M. Satnos, S. J., Kujjer, M. L., Mohamed, S., ... \& Schilham, M. W. (2014). Macrophages inhibit human osteosarcoma cell growth after activation with the bacterial cell wall liposomal muramyl tripeptide combination with interferon- $\gamma$. Journal of Experimental \& Clinical Cancer Research. 33(27). DOI: 10.1186/1756-9966-33-27. 
14. Xiao, Q., Zhang, X., Wu, T., Yang, Y. (2014). Inhibition of macrophage polarization prohibits growth of human osteosarcoma Tumor Biol. 35: 7611-7616

15. Segaliny, A. I., Mohmadi, A. Dizier, B., Lokajczyk, A., Brion, R., Lanel, R., ... \& Heymann, D. (2015). Int. J. Cancer. $137: 73-85$.

16. Gomez-Brouchet, A., Illac, C. Gilhodes, J., Bouvier, C., Aubert, S., Guinebretiere, J., ... \& Redini, F. (2017) CD163-positive tumorassociated macrophages and CD8-positive cytotoxic lymphocytes are powerful diagnostic markers for the therapeutic stratification of osteosarcoma patients: An immunohistochemical analysis of the biopsies from a French OS2006 phase 3 trial. Oncoimmunology. 6(9): e1331193.

17. Han, Y., Guo, W., Ren, T., Huang, Y., Wang, S., Liu,. K. (2019). Tumor-associated macrophages induce epithelial-mesenchymal transition in osteosarcoma by activating the COX-2/STAT3 axis. Cancer Letters. 440-441: 116-125.

18. Heymann, M. -F., Lezot, F., Heymann, D. (2017). The contribution of immune infiltrates and the local microenvironment in the pathogenesis of osteosarcoma. Cellular Immunology: DOI: 10.1016/j.cellimm.2017.10.011.

19. Markel, J. E., Noore, J., Emery, E. J., Bobar., H. J., Kleinerman, E. S., Lindsey, B. A. (2018). Using the Spleen as an In Vivo Systemic Immune Barometer Alongside Osteosarcoma Disease Progression and Immunotherapy with $\alpha$-PD-L1. Sarcoma. 2018: Article ID 8694397.

20. Gardner, A., Ruffell, B. (2016). Dendritic Cells and Cancer Immunity. Trends in Immunology. 37(12): 855-65.

21. Chen, D. S., Mellman, I. (2013). Oncology meets immunology; the cancer-immunity cycle. Immunity. 39: 1-10.

22. Zhang, F., Zhu, Y., Fan, G., Hu, S. (2018). Photodynamic therapy reduces the inhibitory effect of osteosarcoma cells on dendritic cells by upregulating HSP70. Oncology Letters. 16: 5034-5040.

23. Vakkila, J., Jaffe, R., Michelow, M., Lotze, M. (2006). Pediatric Cancers Are Infiltrated Predominantly by Macrophages and Contain a Paucicity of Dendritic Cells: A Major Nosologic Difference with Adult Tumors. Clin Cancer Res. 12(7): 2049-54.

24. Cassetta, L., Baekkevoid, E. S., Brandau, S., Buljko, A., Cassatella, M. A., Dorhoi, A., ... \& Adema, G. J. (2019) Deciphering myeloidderived suppressor cells: isolation and markers in humans, mice, and non-human primates. Cancer Immunol Immunother. 68: 687-97.

25. Gabrilovich, D. I. (2017). Myeloid-derived suppressor cells. Cancer Immunol. Res. 5(1): 3-8.

26. Zhang, Shuo. Zhu, C., Liu, L., Wang, G., Yuan, X. (2016).The Role of Myeloid-Derived Suppressor Cells in Patients with Solid Tumors: A Meta-Analysis. PLoS ONE 11(10): e0164514.

27. Guan, Y., Zhang, R., Peng, Z., Dong, D., Wei, G., Wang, Y. (2017). Inhibition of IL-18-mediated myeloid derived suppressor cell accumulation enhances anti-PD1 efficacy against osteosarcoma cancer. Journal of Bone Oncology. 9: 59-64.

28. Hingorani, P., Maas, M. L., Gustafson, M. P., Dickman, P., Adams, R. H., Watanabe, M., ... \& Dietz, A. B. (2015). Increased CTLA4+ T cells and an increased ratio of monocytes with loss of class II (CD14+ HLA-DRlo/neg) found in aggressive pediatric sarcoma patients. Journal for ImmunoTherapy of Cancer. 3(35): 1-10.

29. Bergenfelz, C., Larsson, A. -M., von Stedingk, K., Gruvberger-Saal, S., Altonen, K., Jansson, S., ... \& Leandersson K. (2015) Systemic Monocytic-MDSCs are Generated from Monocytes and Correlate with Disease Progression in Breast Cancer Patients. PLoS ONE 10(5): e0127028.

30. Kim, H. -J. CD4 T-cell Subsets and Tumor Immunity: The Helpful and the Not-so-Helpful. (2014). Cancer Immunol Res. 2 (2): 91-8.

31. Dummer, W., Niethammer, A. G., Baccala, R., Lawson, B. R., Wagner, N., Reisfeld, R. A., Theofilopoulous, A. N. T cell homeostatic proliferation elicits effective antitumor immunity. (2002). The Journal of Clinical Investigation. 10(2): 185-92.

32. Borst, J. Ahrends, T., Babala, N. Melief, C. J. M., Kastenmüller, W. CD4+ T cell help in cancer immunology and immunotherapy. (2018). Nature Reviews. 18: 635-47.

33. Tepper, R. I., Pattengale, P. K., Leder., P. Murine Interleukin-4 Displays Potent Anti-Tumor Activity In Vivo. (1989). Cell. 57: 50312.

34. Zhou, Q., Xian, M., Xian, S., Xiang, D., Shao, X., Wang, J., ...\& He, Q. All-Trans Retinoic Acid Prevents Osteosarcoma Metastasis by Inhibiting M2 Polarization of Tumor-Associated Macrophages. (2017). Cancer Immunol Res. 5(7): 547-59.

35. Bailey, S. R., Nelson, M. H., Himes, R. A., Li, Z., Mehrotra, S., Paulos, C. M. Th17 cells in cancer: the ultimate identity crisis. (2014). Frontiers in Immunology. 5: 1-13.

36. Li, P., Shi, X., Xu, Y., Zhong, B., Lu, Y., Sun, Y. Interleukin-22 Promotes Osteosarcoma Cell Proliferation and Invasion via STAT3 Activation. (2018). Med Sci Monit. 24: 7802-8.

37. Théoleyre, S., Mori, K., Cherrier, B., Passuti, N., Gouin, F., Rédini, F., Heymann, D. Phenotypic and functional analysis of lymphocytes infiltrating osteolytic tumors: use as a possible therapeutic approach of osteosarcoma. (2005). BMC Cancer. 5(123): DOI:10.1186/14712407-5-123.

38. Zheng, W. Xiao, H., Liu, H., Zhou, Y. Expression of programmed death 1 is correlated with progression of osteosarcoma. (2014). Acta Pathologica Microbiologica et Immunologica Scandinavica. 123: 102-7.

39. Ge, W., Li, J., Fan, W., Xu, D., Sun, S. Tim-3 as a diagnostic and prognostic biomarker of osteosarcoma. (2017). Tumor Biology. 2017: 1-8.

40. Das, M., Zhu, C., Kuchroo. Tim-3 and its role in regulating anti-tumor immunity. (2017) Immunol Rev. 276(1): 97-111.

41. Liu, H., Zhi, L., Duan, N., Su, P. Abnormal expression of Tim-3 antigen on peripheral blood T cells is associated with progressive disease in osteosarcoma patients. (2016). FEBS Open Bio. 6: 807-815.

42. Xiao, H., Luo, G., Son, H., Zhou, Y., Zheng, W. Upregulation of peripheral CD4+CXCR5+ T cells in osteosarcoma. (2014). Tumor Biol. 35: 5273-79.

43. Crotty S. T follicular helper cell differentiation, function, and roles in disease. (2014). Immunity. 41(4): 529-542.

44. Galon, J., Pagès, F., Marincola, F. M., Angell, H. K., Thurin, M., Lugli, A., ...\& Fox, B. A. Cancer classification using the Immunoscore: a worldwide task force. (2012). Journal of Translational Medicine. 10(205). DOI: 10.1186/1479-5876-10-205

45. Rivoltini, L., Arienti, F., Orazi, A., Ceflo, G., Gasparini, M., Gambacorti-Passerini, C., Fossati-Bellani, F., Parmiani, G. Phenotypic and functional analysis of lymphocytes infiltrating paediatric tumours, with a characterization of the tumour phenotype. (1992). Cancer Immunol Immunother. 34(241): DOI: 10.1007/BF01741792. 
46. Trieb, K., Lechleitner, T., Lang, S., Windhager, R., Kotz, R., Dirnhofer, S. Evaluation of HLA-DR Expression and T-Lymphocyte Infiltration in Osteosarcoma. (1998) Pathol Res Pract. 194: 679-84.

47. Fritzching, B., Fellenberg, J., Moskovszky, L., Sàpi, Z., Krenacs, T., Machado, I., ...\& Kunz, P. CD8+/FOXP3+-ratio in osteosarcoma microenvironment separates survivors from non- survivors: a multicenter validated retrospective study. (2014). Oncoimmunology. 4(3). e990800.

48. Spranger, S., Gajewski, T. F. Tumor-intrinsic oncogene pathways mediating immune avoidance. (2016). Oncoimmunology. 5(3): e1086862.

49. Sundara, Y. T., Kostine, M., Cleven, A. H. G., Bovée, J. V. M. G., Schilman, M. W., Cleton-Jansen, A. -M. (2017). Increased PD-L1 and T-cell infiltration in the presence of HLA class I expression in metastatic high-grade osteosarcoma: a rationale for T-cell-based immunotherapy. Cancer Immunol Immunother. 66: 119-128.

50. Shen, J. K., Cote, G. M., Choy, E., Yang, P., Harmon, D., Schwab, J., ...\& Duan, Z. Programmed cell death 1 ligand 1 expression in osteosarcoma. (2014). Cancer Immunol Res. 2(7): 690-8.

51. Antony, P. A., Piccirillo, C. A., Akpinaril, A., Finkelstein, S. E., Speiss, P. J., Surman, D. R., ...\& Restifo, N. P. CD8+ T cell immunity against a tumor/self-antigen is augmented by CD4 T helper cells and hindered by naturally occurring T regulatory cells. (2005). $J$ Immunol. 174(5): 2591-601.

52. Piccirillo, C. A., Shevach, E. M. Cutting Edge: Control of CD8+ T Cell Activation by CD4+CD25+ Immunoregulatory Cells. (2001). J Immunol. 167(3): 1137-40.

53. Nelson, B. H. CD20+ B Cells: The Other Tumor-Infiltrating Lymphocytes. (2010). J Immunol. 185: 4977-82.

54. Fonseca, C., Dranoff, G. Capitalizing on the Immunogenicity of Dying Tumor Cells. (2008). Clin Cancer Res. 14(6): 1603-8.

55. Reuschenbach, M., M. von Knebel Doeberitz, and N. Wentzensen. 2009. A sys- tematic review of humoral immune responses against tumor antigens. Cancer Immunol Immunother. 58: 1535-1544.

56. Al-Shibli, K. I., Donnem, T., Al-Saad, S., Persson, M., Bremnes, R. M., Busund, L, -T. Prognostic Effect of Epithelial and Stromal Lymphocyte Infiltration in Non Small Cell Lung Cancer. (2008). Clin Cancer Res. 14(16): 5220-27.

57. Milne, K., Kobel, M., Kalloger, S. E., Barnes, R. O., Gao, D., Gilks, C. B., Watson, P. H., Nelson, B. H. (2009). Systematic analysis of immune infiltrates in high-grade serous ovarian cancer reveals CD20, FoxP3 and TIA-1 as positive prognostic factors. PLoS ONE. 4: e6412.

58. Sorbye, S. W., Kilvaer, T., Valkov, A., Donnem, T., Smeland, E., Al-Shibli, K., Bremnes, R. M., Busund, L. -T. Prognostic Impact of Lymphocytes in Soft Tissue Sarcomas. PLoS ONE. 6: e14611.

59. Gustafson, M. P., Lin, Y., Maas, M. L., Van Keulen, V. P., Johnston, P. B., Peikert, T., Gastineau, D. A., Dietz, A. B. A Method for Identification and Analysis of Non-Overlapping Myeloid Immunophenotypes in Humans. (2015). PLoS ONE 10(3): e0121546.

60. Souza-Fonseca-Guimaraes, F., Cursons, J., Huntington, N. D. The Emergence of Natural Killer Cells as a Major Target in Cancer Immunotherapy. (2019). Trends in Immunology. 40(2): 142-58.

61. Tarek, N., Lee, D.A. Natural Killer Cells for Osteosarcoma (2014). In: Kleinerman, M.D. E. (eds) Current Advances in Osteosarcoma. Advances in Experimental Medicine and Biology, vol 804. Springer, Cham.

62. Tsukahara, T., Kawaguchi, S., Torigoe, T., Asanuma, H., Nakazawa, E., Shimozawa, K., ...\& Sato, N. Prognostic significance of HLA class I expression in osteosarcoma defined by anti-pan HLA class I monoclonal antibody, EMR8-5. (2006). Cancer Sci. 97(12): 137480 .

63. Delgado, D., Webster, D. E., DeSantes, K. B., Durkin, E. T. Shaaban, A. F. KIR Receptor-Ligand Incompatibility Predicts Killing of Osteosarcoma Cell Lines by Allogeneic NK Cells. (2010). Pediatr Blood Cancer. 55(17): 1300-5.

64. Buddingh, E. P., Schilham, M. W., Rusaln, S. E. N., Berghuis, D., Szuhai, K., Suurmond, J., ...\& Lankester, A. C. Chemotherapyresistant osteosarcoma is highly susceptible to IL-15-activated allogeneic and autologous NK cells. (2011). Cancer Immunol Immunother. 60: 575-86.

65. Markewicz, K., Zeman, K., Kozar, A., Golebiowska-Wawrzyniak, M., Wozniak, W. Evaluation of selected parameters of cellular immunity in children with osteosarcoma at diagnosis. (2012). Medycyna wieku rozwojowego. 16: 212-21.

66. Wang, Z., Wang, Z., Li, B., Wang, S., Chen, T., Ye, Z. Innate Immune Cells: A Potential and Promising Cell Population for Treating Osteosarcoma. (2019). Front Immunol. 10: DOI: 10.3389/fimmu.2019.01114.

67. Miwa, S., Shirai, T., Yamamoto, N., Hayashi, K., Takuchi, A., Igrashi, K., Tsuchiya, H. Current and Emerging Targets in Immunotherapy for Osteosarcoma. (2019). Journal of Oncology. 2019: DOI: 10.1155/2019/7035045.

68. Zheng, B., Ren, T., Huang, Y., Sun, K., Wang, S., Bao, X., Liu, K., Guo, W. PD-1 axis expression in musculoskeletal tumors and antitumor effect of nivolumab in osteosarcoma model of humanized mouse. (2018). Journal of Hematology \& Oncology. 11(16): DOI: 10.1186/s13045-018-0560-1.

69. Dhupkar, P., Gordon, N., Stewart, J., Kleinerman, E. S. Anti-PD-1 therapy redirects macrophages from an M2 to an M1 phenotype inducing regression of OS lung metastases. (2018). Cancer Medicine. 7(6): 2654-2664.

70. Zhao, Y., Yang, W., Huang, Y., Cui, R., Li, X., Li, B. Evolving Roles for Targeting CTLA-4 in Cancer Immunotherapy. (2018). Cell Physiol Biochem. 47: 721-734.

71. Lussier, D. M., Johnson, J. L., Hingorani, P., Blattman, J. N. Combination immunotherapy with $\alpha$-CTLA-4 and $\alpha$-PD-L1 antibody blockade prevents immune escape and leads to complete control of metastatic osteosarcoma. (2015). Journal of ImmunoTherapy of Cancer. 3(21): DOI: 10.1186/s40425-015-0067-z.

72. Brahmer, J. R., Tykodi, S. S., Chow, L. Q. M., Hwu, W. -J., Topalian, S. L., Hwu, P. Safety and Activity of Anti-PD-L1 Antibody in Patients with Advanced Cancer. (2012). N Engl J Med. 366(26): 2455-65.

73. Tawbi, H. A., Burgess, M., Bolejack, V., Van Tine, B. A., Scheutze, S. M., Hu, J., ...\& Patel, S. Pembrolizumab in advanced softtissue sarcoma and bone sarcoma (SARC028): a multicentre, two-cohort, single-arm, open-label, phase 2 trial. (2017). Lancent Oncol. 18: 1493-1501. 
74. Ratti, C., Botti, L., Cancila, V., Galvan, S., Torselli, I., Garogalo, C., ...\& Chiodoni, C. Trabectedin Overrides Osteosarcoma Differentiative Block and Reprograms the Tumor Immune Environment Enabling Effective Combination with Immune Checkpoint Inhibitors. (2017). Clin Cancer Res. 23(17): 5149-61.

75. Strander, H., Einhorn, S. Effect of Human Leukocyte Interferon on the Growth of Human Osteosarcoma Cells in Tissue Culture. (1977). Int J Cancer. 19: 468-73.

76. Brosjö, O., Bauer, H. C., Broström, L. -Å. Nilsonne, U., Nilsson, O. S., Reinhold, F. P., Strander, H., Tribukait, B. Influence of Human $\alpha$-interferon on Four Human Osteosarcoma Xenografts in Nude Mice. (1985). Cancer Research. 45: 5596-602.

77. Müller, C. R., Smeland, S., Bauer, H. C. F., Sæter, G., Strander, H. Interferon- $\alpha$ as the only adjuvant treatment in high-grade osteosarcoma: Long term results of the Karolinska Hospital series. (2005). Acta Oncologica. 44: 475-80.

78. Whelan, J., Patterson, D., Perisoglou, M., Bielack, S., Marina, N., Smeland,S., Bernstein., P. The Role of Interferons in Treatment of Osteosarcoma. (2010). 54(3): 350-54.

79. Brosjö, O., Bauer, H. C., Nilsson, O. S., Halvorsen, D., Reinholt, F. P., Tribukait, B. Effect of human interferon-alpha and interferongamma on growth, histology, and DNA content of human osteosarcomas in nude mice. (1989). J Interferon Res. 9(4): 475-89.

80. Strander, H. Bauer, H. C., Brosjö, O. Fernberg, J. O., Kreichbergs, A., Nilsonne, U., ...\& Söderlund, V. Long-Term Adjuvant Interferon Treatment of Human Osteosarcoma: A pilot study. (1995). Acta Oncologica. 34(6): 877-80.

81. Bielack, S. S., Smeland, S., Whelan, J. S., Marina, N., Jovic, G., Hook, J. M. ...\& Berstein, M. Methotrexate, Doxorubicin, and Cisplatin (MAP) Plus Maintenance Pegylated Interferon Alfa-2b Versus MAP Alone in Patients With Resectable High-Grade Osteosarcoma and Good Histologic Response to Preoperative MAP: First Results of the EURAMOS-1 Good Response Randomized Controlled Trial. (2015). J Clin Oncol 33(20): 2279-87.

82. Rosenberg, S. A. Progress in human tumour immunology and immunotherapy. (2014). Nature. 411: 380-4.

83. Weigent, D. A., Stanton, G. J., Johnson, H. M. Interleukin 2 Enhances Natural Killer Cell Activity Through Induction of Gamma Interferon. (1983). Infection and Immunity. 41(3): 992-7.

84. Rosenberg, S. A., Lotze, M. T., Muul, L. M., Leitman, S. Observations on the Systemic Administration of Autologous LymphokineActivated Killer Cells and Recombinant Interleukin-2 to Patients with Metastatic Cancer. (1985). N Engl J Med. 313: 1485-92.

85. Meazza, C., Cefalo, G., Massinmino, M., Daolio, P., Pastorino, U., Scanagatta, P., ...\& Luksch, R. Primary metastatic osteosarcoma: results of a prospective study in children given chemotherapy and interleukin-2. (2017). Med Oncol. 34(12): DOI: 10.1007/s12032017-1052-9.

86. Schwinger, W., Klass, V., Benesch, M., Lackner, H., Bornbusch, H. J., Sovinz, P., Moser, A., Schwantzer, G., Urban, C. Feasibility of high-dose interleukin-2 in heavily pretreated pediatric cancer patients. (2005). Annals of Oncology. 16: 1199-1206.

87. Saxena, M. Bhardwaj, N. Re-emergence of Dendritic Cell Vaccines for Cancer Treatment. (2018). Trends Cancer. 4(2): 119-37.

88. He, Y. -T. Zhang, Q. -M., Kou, Q. -C., Tang, B. In vitro generation of cytotoxic T lymphocyte response using dendritic cell immunotherapy in osteosarcoma. (2016). Oncology Letters. 12: 1101-6.

89. Kawano, M., Itonaga, I., Iwasaki, T., Tsumura, H. Enhancement of antitumor immunity by combining anti-cytotoxic T lymphocyte antigen-4 antibodies and cryotreated tumor lysate-pulsed dendritic cells in murine osteosarcoma. (2013). Oncology Reports. 29: 10016.

90. Kawano, M., Tanaka, K., Itonaga, I., Iwasaki, T., Miyazaki, M., Ikeda, S., Tsumura, H. Dendritic cells combined with anti-GITR antibody produce antitumor effects in osteosarcoma. (2015). Oncology Reports. 34: 1995-2001.

91. Geiger, J. D., Hutchinson, R. J., Hohenkirk, L. F., McKenna, E. A., Yanik, G. A., Levine, J. E., ...\& Mulé, J. J. Vaccination of Pediatric Solid Tumor Patients with Tumor Lysate-pulsed Dendritic Cells Can Expand Specific T Cells and Mediate Tumor Regression. (2001). Cancer Research. 61: 8513-19.

92. Merchant, M. S., Bernstein, D., Amoako, M., Baird, K., Fleisher, T. A., Morre, M., ...\& Mackall, C. L. Adjuvant Immunotherapy to Improve Outcome in High-Risk Pediatric Sarcomas. (2016). Clin Cancer Res. 22(13): 3182-91.

93. Miwa, S., Nishida, H., Tanzawa, Y., Takeuchi, A., Hayashi, K., Yamamoto, N., ...\& Tsuchiya, H. Phase 1/2 Study of Immunotherapy With Dendritic Cells Pulsed With Autologous Tumor Lysate in Patients With Refractory Bone and Soft Tissue Sarcoma. (2017). Cancer. 123(9): 1576-84.

94. Köskal, H., Müller, E., Inderberg, E. M., Bruland, Ø. Wälchi, S. Treating osteosarcoma with CAR T cells. (2018). Scand J Immunol. 89: e12741.

95. Ahmed, N. Brawley, V. S., Hegde, M., Robertson, C., Ghazi, A., Gerken, C., ...\& Gottschalk, S. Human Epidermal Growth Factor Receptor 2 (HER2) -Specific Chimeric Antigen Receptor-Modified T Cells for the Immunotherapy of HER2-Positive Sarcoma. (2015). J Clin Oncol. 33(15): 1688-96.

96. Long, A. H., Highfill, S. L., Cui, ,Y., Smith, J. P., Walker, A. J., Ramakrishna, S., ...\& Mackall, C. L. Reduction of MDSCs with alltrans retinoic acid improves CAR therapy efficacy for sarcomas. (2016). Cancer Immunol Res. 4(10): 869-80.

97. Wang, Y., Yu, W., Zhu, J., Wang, J., Xia, K., Liang, C., Tao, H. Anti-CD166/4-1BB chimeric antigenmreceptor T cell therapy for the treatment of osteosarcoma. (2019). Journal of Experimental \& Clinical Cancer Research. 38(168): DOI: 10.1186/s13046-019-1147-6.

98. Wang, Z., Li, B., Ren, Y., Ye, Z. T-Cell-Based Immunotherapy for Osteosarcoma: Challenges and Opportunities. (2016). Front Immunol. 7(353): DOI: 10.3389/fimmu.2016.00353.

99. Robbins, P. F., Morgan, R. A., Feldman, S. A., Yang, J. C., Sherry, R. M., Dudley, M. E., ...\& Rosenberg, S. A. Tumor Regression in Patients With Metastatic Synovial Cell Sarcoma and Melanoma Using Genetically Engineered Lymphocytes Reactive With NY-ESO1. (2011). J Clin Oncol. 29(7): 917-24.

100. Li, B., Zhu, X., Sun, L., Yuan, L., Zhang, J., Hengyuan, L., Ye, Z. Induction of a specific CD8+ T-cell response to cancer/testis antigens by demethylating pre-treatment against osteosarcoma. (2014). Oncotarget. 5(21): 10791-802.

101. Asano, T., Kleinerman, E. S. Liposome-encapsulated MTP-PE: a novel biologic agent for cancer therapy. (1993). J Immunother Emphasis Tumor Immunol. 14(4). 286-89. Meyers, P. A. Systemic Therapy for Osteosarcoma and Ewing Sarcoma. (2015). 2015 ASCO Educational Book: E644-7. 
102. Anderson P. M., Meyers P., Kleinerman E., Venkatakrishnan, K., Hughes, D. P., Herzog, C. Mifamurtide in metastatic and recurrent osteosarcoma: a patient access study with pharmacokinetic, pharmacodynamic, and safety assessments. (2014) Pediatr Blood Cancer. 61(2): 238-244.

103. Meyers P. A., Chou A. J. Muramyl Tripeptide-Phosphatidyl Ethanolamine Encapsulated in Liposomes (L-MTP-PE) in the Treatment of Osteosarcoma. (2014). In: Kleinerman, M.D. E. (eds) Current Advances in Osteosarcoma. Advances in Experimental Medicine and Biology, vol 804. Springer, Cham.

104. Kleinerman, E. S. Jia, S. F., Griffin, J., Seibel, N. L., Benjamin R. S., Jaffe, N. Phase II study of liposomal muramyl tripeptide in osteosarcoma: the cytokine cascade and monocyte activation following administration. (1992). J Clin Oncol. 10(8): 1310-6.

105. Meyers P. A., Schwartz C. L., Krailo M. D., Healey, J.H., Bernstein, M. L. Betcher, D. ...\& Grier, H. E. Osteosarcoma: The addition of muramyl tripeptide to chemotherapy improves overall survival-A report from the Children's Oncology Group. (2008). J Clin Oncol. 26(4): 633-638.

106. Chou, A. J., Kleinerman, E. S., Krailo, M. D., Chen, Z., Betcher, D. L., Healey, J. H., ...\& Meyers, P. A. Addition of muramyl tripeptide to chemotherapy for patients with newly diagnosed metastatic osteosarcoma: a report from the Children's Oncology Group. (2009). Cancer. 1115(22): 5339-48.

107. Kumai, T., Kobayashi, H., Harabuchi, Y., Celis, E. Peptide vaccines in cancer - old concept revisited. (2017). Curr Opin Immunol. 45: $1-7$.

108. Tsuda, N., Murayama, K., Ishida, H., Matsunaga, K., Komiya, S., Itoh, K., Yamada, A. Expression of a newly defined tumor-rejection antigen SART3 in musculoskeletal tumors and induction of HLA class I-restricted cytotoxic T lymphocytes by SART3-derived peptides. (2001). Journal of Orthopaedic Research. 19: 346-51.

109. Takahashi, R., Ishibashi, Y., Hiraoka, K., Matsueda, S., Kawano, K., Kawahara, A., ...\& Sasada, T. Phase II study of personalized peptide vaccination for refractory bone and soft tissue sarcoma patients. (2013). Cancer Sci. 104(10): 1285-94.

110. Kawaguchi, S., Tsukahara, T., Ida, K., Kimura, S., Murase, M., Masanobu, K. SYT-SSX breakpoint peptide vaccines in patients with synovial sarcoma: A study from the Japanese Musculoskeletal Oncology Group. (2012). Cancer Sci. 103(9): 1625-30.

111. Tsukahara, T., Nabeta, Y., Kawaguchi, S., Ikeda, H., Sato, Y., Shimozawa, K., ...\& Sato, N. Identification of Human Autologous Cytotoxic T-Lymphocyte-Defined Osteosarcoma Gene That Encodes a Transcriptional Regulator, Papillomavirus Binding Factor. (2004). Cancer Res. 64(15): DOI: 10.1158/0008-5472.CAN-04-0522. 


\section{Appendix AV}

Lindsey, B. A., Markel, J.E., Stewart, A. B., Lacinski, R. A., Noore, J. U.S. Provisional Patent Application No.62/944, 191. Morgantown, WV: U.S. Patent and Trademark Office. 


\section{PROTEIN-CONTAINING PLGA NANOSPHERES}

\section{FIELD}

[0001] Various embodiments disclosed herein relate generally to preparation of protein-containing nanospheres.

[0002] Various embodiments disclosed herein relate to immunophenotyping for immune analysis.

\section{BACKGROUND}

[0003] Immunostimulation is an important mechanism that 1) prevents malignant transformed cells from proliferating and/or colonizing metastatic sites and 2) clears established viral and bacterial infections. Immunotherapy cancer treatment may involve monoclonal antibody blockade of specific immune checkpoints, including the Programmed Death-Ligand 1 (PD-L1) and Cytotoxic TLymphocyte-Associated Protein 4 (CTLA-4) axes.

[0004] Historically, the focus has been on the tumor microenvironment and the immune response. However, it is well documented that in order to have a lasting immunological response and cure the disease the response must be a systemic one. Many groups have looked at the immunophenotyping of treatments to help to explain what is happening immunologically to the patient in real time. This information will become key as immunotherapeutics for multiple disease become more mainstream and developing an living database of these responses will push the process forward tremendously.

[0005] Preclinical studies have shown that interleukins can induce antitumor responses against many malignancies. Interleukin-12 (IL-12), an immunostimulatory cytokine whose antitumor activity is maximized when given systemically, induces such antitumor responses. While high-dose IL-12 administration may have toxic side effects, low doses of IL-12 are considered safe.

[0006] Poly(D,L-lactic acid-co-glycolic acid) (PLGA) drug delivery vectors are FDA approved and can elute a wide variety of substances as the polymer coating breaks down. For therapeutic purposes, encapsulating peptides, potentially including immunostimulatory interleukin proteins, within PLGA nanospheres may allow for systemic delivery and tissue deposition without the need for toxic loading doses. In order to be used in systemic settings, nanospheres must be able to achieve safe and effective blood-borne travel through the circulatory system of an organism. 


\section{SUMMARY}

[0007] In light of the present need for improved methods of cytokine administration, a brief summary of various exemplary embodiments is presented. Some simplifications and omissions may be made in the following summary, which is intended to highlight and introduce aspects of certain embodiments disclosed herein, but not to limit the scope of the disclosure. Detailed descriptions of various embodiments adequate to allow those of ordinary skill in the art to make and use the concepts disclosed herein will follow in later sections.

[0008] According to various embodiments disclosed herein, a protein may be encapsulated in a nanosphere. This encapsulation process may be performed by making a double emulsion, where a first water phase is emulsified in an oil phase, and the oil phase is emulsified in a second water phase. The protein may be a cytokine, which is a small protein $(\sim 5-20 \mathrm{kDa})$ important in cell signaling. Suitable cytokines include:

interleukins, produced by T-helper cells;

lymphokines, produced by lymphocytes;

monokines, produced exclusively by monocytes;

interferons, involved in antiviral responses;

colony stimulating factors, which support the growth of cells in semisolid media; and chemokines, which mediate chemoattraction between cells.

[0009] In various embodiments, the protein encapsulated in the nanosphere may be cytokines with three-dimensional structures having a bundle of four $\alpha$-helices, which may be interferons, interleukins, e.g., interleukin-2 or interleukin-12, or non-immunological cytokines, including erythropoietin and thrombopoietin. The protein encapsulated in the nanosphere may be interleukin-12 (IL-12).

[0010] The oil phase may be prepared by dissolving from $2.5 \% \mathrm{w} / \mathrm{v}$ to $17 \% \mathrm{w} / \mathrm{v}$ of poly(lactic acid-co-glycolic acid) (PLGA) in an organic solvent. A first aqueous phase is made by suspending a protein in an aqueous medium. Finally, a second aqueous phase is made by dissolving polyvinyl alcohol (PVA) in water.

[0011] The PLGA may comprise from 50\% to 90\% lactide, and the organic solvent may be a halogenated C1-C3 organic solvent, a C2-C3 nitrile solvent, a C2-C5 alkyl ester solvent, a C3 to C5 ketone solvent, or a mixture thereof. The PLGA may comprise from $75 \%$ to $90 \%$ lactide. The PLGA may comprise from 50\% to 75\% lactide, and the organic solvent may be a halogenated C1-C3 organic 
solvent, acetonitrile, a C3 to C4 ketone solvent, or a mixture thereof. In various embodiments, the PLGA comprises from 50\% to 90\% lactide, and the organic solvent may be acetonitrile, acetone, ethyl acetate, or dichloromethane.

[0012] The protein-containing aqueous medium is added to the oil phase to form a first emulsion, with agitation. The first emulsion is added to the PVA-containing aqueous phase to form a second emulsion, with agitation. The organic solvent is then evaporated from the second emulsion to form an aqueous solution; and PLGA nanospheres containing the protein from the second aqueous phase are recovered from the aqueous solution.

[0013] In various embodiments, the protein-containing aqueous medium may be added to the oil phase to form a first emulsion, with agitation by a tissue homogenizer at a rate of 13,000 RPM to 20,000 RPM. The first emulsion may be added to the PVA-containing aqueous phase to form a second emulsion, with agitation by a tissue homogenizer at a rate of 13,000 RPM to 20,000 RPM. The organic solvent is then evaporated from the second emulsion to form an aqueous solution; and PLGA nanospheres containing the protein from the second aqueous phase are recovered from the aqueous solution.

[0014] In various embodiments, the protein in the protein-containing aqueous medium is a cytokine. Suitable cytokines include interleukins, lymphokines, monokines, interferons, colony stimulating factors, and chemokines. The protein may be a cytokines with a three-dimensional structures having a bundle of four $\alpha$-helices, e.g., interleukins, e.g., interleukin-2 or interleukin-12, or non-immunological cytokines, including erythropoietin and thrombopoietin.

[0015] In various embodiments, the protein in the protein-containing aqueous medium is IL-12. IL-12 may be incorporated into PLGA nanospheres by agitating the first and second emulsions with a tissue homogenizer at a rate of 13,000 RPM to 20,000 RPM. IL-12 is incorporated into the resulting PLGA nanospheres with an encapsulation efficiency of about $0.5 \%$ to about $2.1 \%$.

[0016] In various embodiments, the protein-containing aqueous medium is added to the oil phase to form a first emulsion, with agitation by ultrasonication. The first emulsion is added to the PVAcontaining aqueous phase to form a second emulsion, with agitation by sonication. Agitation during formation of either or both of the first and second emulsions may comprise sonication at a power level of $30 \mathrm{~W}$ to $50 \mathrm{~W}, 30 \mathrm{~W}$ to $40 \mathrm{~W}$, or $40 \mathrm{~W}$ to $50 \mathrm{~W}$, for a period of time of $5 \mathrm{sec}$ to $30 \mathrm{sec}, 10 \mathrm{sec}$ to $30 \mathrm{sec}, 10 \mathrm{sec}$ to $20 \mathrm{sec}$, or $10 \mathrm{sec}$ to $15 \mathrm{sec}$. The organic solvent is then evaporated from the second emulsion to form an aqueous solution; and PLGA nanospheres containing the protein from the second aqueous phase are recovered from the aqueous solution. 
[0017] In various embodiments, the protein-containing aqueous medium is added to the oil phase to form a first emulsion, with agitation by a tissue homogenizer at a rate of 13,000 RPM to 20,000 RPM. The first emulsion is added to the PVA-containing aqueous phase to form a second emulsion, with agitation by sonication at a power level of $30 \mathrm{~W}$ to $50 \mathrm{~W}, 30 \mathrm{~W}$ to $40 \mathrm{~W}$, or $40 \mathrm{~W}$ to $50 \mathrm{~W}$, for a period of time of $5 \mathrm{sec}$ to $30 \mathrm{sec}$. The organic solvent is then evaporated from the second emulsion to form an aqueous solution; and PLGA nanospheres containing the protein from the second aqueous phase are recovered from the aqueous solution.

[0018] In various embodiments, the protein in the protein-containing aqueous medium is IL-12. IL-12 may be incorporated into PLGA nanospheres by sonication at a power level of $30 \mathrm{~W}$ to $50 \mathrm{~W}$, for a period of time of $10 \mathrm{sec}$ to $20 \mathrm{sec}$. IL-12 is incorporated into the resulting PLGA nanospheres with an encapsulation efficiency of about $2 \%$ to about $85 \%$, about $4.5 \%$ to about $70 \%$, about $5 \%$ to $60 \%$, about $7 \%$ to about $50 \%$, about $8 \%$ to about $40 \%$, about $10 \%$ to $30 \%$, or about $5 \%$ to $10 \%$.

[0019] Various embodiments disclosed herein are directed to a nanosphere comprising a poly(lactic acid-co-glycolic acid) matrix and a protein, where a first portion of the protein is adsorbed onto a surface of the nanosphere and a second portion of the protein is incorporated into the poly(lactic acid-co-glycolic acid) matrix at a core of the nanosphere. The nanosphere is produced by making a double emulsion, where a first, protein-containing, water phase is emulsified in an oil phase, and the oil phase is then emulsified in a second water phase. The protein may be a cytokine, such as IL-12. The IL-12 may be incorporated into the nanosphere with an encapsulation efficiency of about $0.5 \%$ to about $85 \%$, about $1 \%$ to about $70 \%$, about $2 \%$ to $60 \%$, about $3 \%$ to about $50 \%$, about $4 \%$ to about $40 \%$, about $5 \%$ to $30 \%$, about $0.5 \% \%$ to $10 \%$, about $1 \%$ to $8 \%$, or about $2 \%$ to $5 \%$.

[0020] Various embodiments disclosed herein relate to a method of encapsulating a protein in a nanosphere, by preparing an oil phase by dissolving from $2.5 \% \mathrm{w} / \mathrm{v}$ to $17 \% \mathrm{w} / \mathrm{v}$ of poly(lactic acidco-glycolic acid) (PLGA) in an organic solvent containing an optional first surfactant; preparing an aqueous phase containing polyvinyl alcohol and at least one additive selected from the group consisting of mammalian serum, trehalose, and a second surfactant; and suspending the protein in an aqueous medium. The first surfactant may be a sorbitan fatty acid ester. The aqueous medium is added to the oil phase to form a first emulsion. The first emulsion is agitated, and the first emulsion is added to the aqueous phase to form a second emulsion, which is then agitated. The organic solvent is evaporated from the second emulsion to form an aqueous solution; and poly(lactic acid-co-glycolic acid) nanospheres containing the protein are recovered from the aqueous solution. The aqueous phase may contain polyvinyl alcohol and mammalian serum, e.g., fetal serum. The aqueous phase may 
contain polyvinyl alcohol and the second surfactant, where the first surfactant is a sorbitan fatty acid ester; and the second surfactant is a polyoxyethylene sorbitan fatty acid ester.

[0021] Various embodiments disclosed herein relate to a nanosphere comprising a poly(lactic acidco-glycolic acid) matrix and a protein, where a first portion of the protein is adsorbed onto a surface of the nanosphere; and a second portion of the protein is incorporated into the poly(lactic acid-coglycolic acid) matrix at a core of the nanosphere. The nanosphere further comprises at least one additive selected from the group consisting of mammalian serum albumin, trehalose, and a surfactant.. The nanosphere may comprise mammalian serum albumin and a surfactant. The nanosphere may comprise mammalian serum albumin, a first surfactant, and a second surfactant. In many disease states, e.g., cancer and autoimmune disorders, the human immune system is in constant flux. In order to treat such diseases, the medical practitioner must be able to assess the immune system of a patient in real time, and to follow the immune system status over time. When cancer, infections, and/or autoimmune disorders are being with immunomodulating agents, regardless of whether they are immunosuppressive or immunostimulating, the medical practitioner must be able to follow the impact of such agents on the immune system.

[0022] Various embodiments disclosed herein relate to a method allowing systemic analysis of the immune system from a finger stick blood draw that can be analyzed at a testing site. The response of the immune system to a disease state, e.g., cancer or an autoimmune disease, may be analyzed at a selected time, and the immune response to a treatment protocol may be followed over the course of a disease or treatment.

[0023] This diagnostic method is useful in the treatment, surveillance, and diagnosis of many diseases, including cancer, autoimmune diseases, and infections. As immunomodulating agents become more common, the method provides the medical practitioner with the ability to assess the status of the immune system at a specific time in the pathogenesis of a disease, and allows prediction as to which immomodulating treatment would be most effective in combating the disease. This will increase the overall effectiveness of treatment, and allow improved assessment of the status of the patient in the disease process. This information can also be organized in a living database of immune profiles across disease specific categories that will allow practitioners to be more informed on the treatments they will be giving based on previous experiences.

[0024] Various embodiments disclosed herein relate to a method of controlling an immunophenotype in a patient suffering from a disease which impacts the immune system, including steps of determining an initial immunophenotype, or immune status, of a patient; and either: 
administering a first drug which stimulates the immune system if the initial immunophenotype shows immunosuppression; or

administering a second drug which suppresses the immune system if the initial immunophenotype shows overstimulation of the immune system.

[0025] After administering the selected drug, the patient's immunophenotype is monitored as a function of time; and administration of the first and/or second drug may be adjusted if the patient's immunophenotype falls outside a desired range.

[0026] Various embodiments disclosed herein relate to a method of controlling an immunophenotype in a patient suffering from a disease which impacts the immune system, by determining a disease state of a patient, where the disease state includes a diagnosis and an initial immunophenotype; and comparing the disease state of the patient to a plurality of disease states within a database, where each disease state in the database includes a diagnosis, an initial immunophenotype, and a treatment protocol. Based on the comparison between the patient's disease state and disease states and treatment protocols from the database, a treatment protocol is selected from the database, where the treatment protocol involves administering an immunomodulating drug. The method may include administering the immunomodulating drug to the patient; monitoring the patient's immunophenotype as a function of time after administering the drug; and adjusting administration of the immunomodulating drug if the patient's immunophenotype falls outside a desired range.

[0027] Immunophenotyping of a patient's blood sample requires the ability to analyze and assess the data appropriately. This system will allow the appropriate dosing, treatment, and corrections to be made across several disease states. As the collection of data increases the growing database will also be able help direct medical professionals to diagnosis, treat, and dose immunotherapeutics in the broadest sense.

\section{BRIEF DESCRIPTION OF THE DRAWINGS}

[0028] The patent or application file contains at least one drawing executed in color. Copies of this patent or patent application publication with color drawings will be provided by the Office upon request and payment of the necessary fee.

[0029] In order to better understand various exemplary embodiments, reference is made to the accompanying drawings, wherein: 
[0030] FIGS. 1A and 1B show distribution of fluorescein isothiocyanate-labeled bovine serum albumin protein in a PLGA nanosphere.

[0031] FIG. 1C shows a biphasic protein elution curve from PLGA nanospheres, due to initial release of adsorbed protein on the surface of the nanospheres, followed by controlled release of protein entrapped with the PLGA nanospheres.

[0032] FIG. 1D shows the mechanism of biphasic protein release from PLGA nanospheres.

[0033] FIG. 2 shows fluorophore distribution over a 67-minute period in BALB/c mice inoculated with $1 \mathrm{mg} / \mathrm{kg}$ of Alexa Flour ${ }^{\circledR}$ 647-loaded nanospheres dissolved in sterile saline by either intraperitoneal (mouse on left) or intravenous (mouse on right) injection.

[0034] FIG. 3 shows the effect of sonication on IL-12, where IL-12 was ultrasonicated at three different wattages for 10, 20, 30, 40, or 60 seconds.

[0035] FIGS. 4A and 4B show scanning electron microscopy (SEM) images of unloaded (blank) poly(lactide-co-glycolide) acid (PLGA) nanospheres lyophilized without $25 \mathrm{mM}$ trehalose at 11,000X (FIG. 3a) and 13,000X (FIG. 3b), respectively.

[0036] FIG. 4C shows scanning electron microscopy (SEM) images of unloaded (blank) PLGA nanospheres lyophilized with $25 \mathrm{mM}$ trehalose at 5,000X magnification.

[0037] FIG. 5A shows PLGA) nanospheres loaded with FITC-conjugated bovine serum albumin visualized for protein incorporation via confocal microscopy.

[0038] FIGS. 5B and 5C show scanning electron microscopy (SEM) images of lyophilized recombinant mouse IL-12-loaded PLGA nanospheres at 8,000X and 25,000X magnification, respectively.

[0039] FIGS. 6A and 6B show size distribution analyses of unloaded (blank) PLGA acid nanospheres and recombinant mouse IL-12 (IL-12)-loaded PLGA nanospheres run at 1:50 and 1:14 dilution factors in water at 25 degrees Celsius, respectively.

[0040] FIGS. 6C and 6D show zeta potential distributions for unloaded blank and IL-12-loaded PLGA nanospheres at a 1:50 dilution factor in water at 25 degrees Celsius, respectively.

[0041] FIG. 7A and 7B shows the estimated total amount of protein eluted over time from recombinant mouse IL-12-loaded PLGA nanospheres. in terms of total protein (FIG. 7A) and protein per 100,000 particles (FIG. 7B).

[0042] FIG. 7C shows the encapsulation efficiency (EE) of recombinant mouse IL-12-loaded nanospheres calculated using the area under the curve (AUCs) of each elution profile for three 
different particle concentrations (500 million particles/mL, 750 million particles $/ \mathrm{mL}$, and 1 billion particles/mL).

[0043] FIGS. 8A to 8C show IL-12-loaded nanospheres prepared using sonication under varying conditions of sonication power and sonication time.

[0044] FIG. 9 shows the elution profiles of IL-12 from nanospheres prepared using sonication under varying conditions of sonication power and sonication time.

[0045] FIG. 10 shows the elution profile of IL-12 from PLGA nanospheres prepared using highspeed stirring, using a nanosphere concentration of 750 million particles $/ \mathrm{mL}$, as a function of stirring speed.

[0046] FIG. 11 shows application of IL-12 from PLGA nanospheres for the treatment of metastatic osteosarcoma.

[0047] FIG. 12 shows the effect of trehalose and a magnesium compound on IL-12 elution from PLGA nanospheres.

[0048] FIG. 13 shows the effect of fetal bovine serum (FBS) on IL-12 elution from PLGA nanospheres.

[0049] FIG. 14 shows the effect of surfactants, alone or in combination with FBS, on IL-12 elution from PLGA nanospheres.

\section{DETAILED DESCRIPTION: PLGA NANOSPHERES}

[0050] Poly(D,L-lactic acid-co-glycolic acid) (PLGA) drug delivery vectors are FDA approved and can elute a wide variety of substances as the polymer coating breaks down into harmless Krebs cycle intermediates. Drug solubility, bioavailability, and stability can all be altered by the organic coating allowing for large shifts in the pharmacokinetic and pharmacodynamics properties of the encapsulated substrate. For tumor and infection therapeutic purposes, encapsulating IL-12 within PLGA nanospheres may allow for systemic delivery and tissue deposition without the need for toxic loading doses. The negatively charged surface are repelled by the glycocalyx, and along with their smaller size allows for increased deposition in the interstitial space where they can elute their contents undisturbed. To date, however, the successful encapsulation of IL-12 within submicron scale PLGA particles has not been achieved. In order to be used in systemic settings, nanospheres must be able to achieve safe and effective blood-borne travel through the microvasculature of an organism (with capillaries being approximately 4-9 microns in diameter) with minimal risk of forming emboli. 
[0051] A PLGA nanosphere with an encapsulated protein may be created by: preparing an oil phase by dissolving from $2.5 \% \mathrm{w} / \mathrm{v}$ to $17 \% \mathrm{w} / \mathrm{v}$ of PLGA in an organic solvent; preparing an aqueous phase by dissolving from $1 \% \mathrm{w} / \mathrm{v}$ to $3 \% \mathrm{w} / \mathrm{v}$ of polyvinyl alcohol in an aqueous solvent; suspending a protein in an aqueous medium; adding the aqueous medium to the oil phase to form a first emulsion, and homogenizing the first emulsion;

adding the first emulsion to the aqueous phase to form a second emulsion, and homogenizing the second emulsion;

evaporating the organic solvent from the second emulsion to form an aqueous solution; and recovering PLGA nanospheres containing the protein from the aqueous solution.

[0052] In various embodiments, the protein may be a cytokine selected from the group consisting of interleukins, lymphokines, monokines, interferons, colony stimulating factors, and chemokines. The cytokine may be an interleukin or a non-immunological cytokine.

[0053] The cytokine may have an N-terminal signal sequence, a four-helix bundle comprising four helices labeled A through D, and an optional C-terminal extension following the D helix. The cytokine may lack a substantial C-terminal extension, and may be a granulocyte-macrophage colony-stimulating factor, a granulocyte colony-stimulating factor, interferon alpha-1, interferon beta, interferon gamma. interferon kappa, interferon tau-1, interferon omega-1, or an interleukin (IL) selected from the group consisting of IL-2, IL-3, IL-4, IL-5, IL-5, IL-6, IL-7, IL-9, IL-10, IL-11, the alpha chain of IL-12, IL13, IL-15, IL-19, IL-20, IL-21, IL-22, IL-23, IL-24, IL-26, and IL-27.

[0054] In various embodiments, the cytokine may be an immunological cytokine that either enhances cellular immune responses; or enhances antibody responses. If the cytokine is an immunological cytokine that enhances cellular immune responses, the cytokine may be selected from the group consisting of tumor necrosis factor-alpha (TNF $\alpha$ ), interferon-gamma (IFN- $\gamma$ ), and interleukin-12 (which stimulates the production of IFN- $\gamma$ and $T N F \alpha$ ). If the cytokine is an immunological cytokine that enhances antibody responses, the cytokine may be selected from the group consisting of transforming growth factor beta (TGF- $\beta$ ), IL-4, IL-10, and IL-13.

[0055] In various embodiments, the protein to be encapsulated in a nanosphere may be a globular protein. Suitable globular proteins may include serum albumin proteins; enzymes, e.g., esterases; hormones, e.g., insulin; and transporter proteins. 
[0056] In various embodiments, PLGA nanospheres may be used to encapsulate pharmaceutical active ingredients; vitamins; nutraceutical active ingredients, e.g., phytochemicals; and organic dyes or contrast agents. PLGA nanospheres may be used for controlled release of a variety of drugs, including poorly soluble Class III and Class IV drugs.

[0057] To create the oil phase, from $2.5 \% \mathrm{w} / \mathrm{v}$ to $17 \% \mathrm{w} / \mathrm{v}$ of PLGA is dissolved in an organic solvent. The PLGA may contain from $50 \%$ to $90 \%$ lactide, from $65 \%$ to $90 \%$ lactide, or from $75 \%$ to $90 \%$ lactide. The organic solvent may be a halogenated C1-C3 organic solvent, e.g., dichloromethane, chloroform, or 1,1,1-trichloroethane; a C2-C3 nitrile solvent, e.g., acetonitrile or propionitrile; or a C2-C5 alkyl ester solvent, e.g. ethyl acetate or butyl acetate; or a C3 to C5 ketone solvent, e.g., acetone or pentanone. The organic solvent may be a semipolar solvent with a dipole moment between 1.1 and 3.5. The oil phase may be made by dissolving PLGA in the organic solvent at room temperature (RT) with stirring, where the stirring may be at 300 to 600 RPM, 350 to 550 RPM, or 425 to 500 RPM.

[0058] In various embodiments, the solvent selection may be made based on a lactide content in the PLGA. If the PLGA contains 75\% to $90 \%$ lactide, the organic solvent may be a halogenated C1C3 organic solvent, C2-C3 nitrile solvent, or a C2-C5 alkyl ester solvent, or a C3 to C5 ketone solvent. If the PLGA contains less than 75\% lactide, the organic solvent may be a halogenated C1-C3 organic solvent, acetonitrile, or a C3 to C4 ketone solvent.

[0059] To create the aqueous phase of the emulsion, from $1 \% \mathrm{w} / \mathrm{v}$ to $3 \% \mathrm{w} / \mathrm{v}$ of polyvinyl alcohol (PVA) is dissolved in an aqueous solvent, which may be water or a buffered saline solution, e.g., phosphate buffered saline.

[0060] Next, a protein, e.g., IL-12 or bovine serum albumin, was suspended in an aqueous medium, which may be a buffered saline solution, e.g., phosphate buffered saline, and the resulting protein suspension was added to the PLGA-containing oil phase, which may be subjected to rapid stirring, e.g., 10,000 to 20,000 RPM; 12,000 to 19,500 RPM; 13,000 to 19,000 RPM; 15,000 to 18,000 RPM, or 16,000 to 17,500 RPM, to produce a first emulsion. Alternatively, the protein suspension may be added to the PLGA-containing oil phase with sonication to produce the first emulsion.

[0061] The first emulsion is then added to the PVA aqueous phase with rapid stirring, e.g., 10,000 to 20,000 RPM; 12,000 to 19,500 RPM; 13,000 to 19,000 RPM; 15,000 to 18,000 RPM, or 16,000 to 17,500 RPM; with homogenization; or with sonication to produce a second emulsion. The organic solvent is then evaporated from the second emulsion. PLGA nanoparticles containing the protein from the aqueous medium are recovered by centrifugation, and flash-frozen in liquid nitrogen and/or 
lyophilized.

[0062] When the second emulsion is formed, protein becomes wound up in strands of the PLGA matrix polymer, which coalesces and precipitates into a sphere as the organic solvent is removed. During this process, protein becomes both entrapped within the polymer matrix (Figure 1A, where the protein is fluorescein isothiocyanate-labeled bovine serum albumin [BSA-FITC]) and adsorbed to the outer surface (Figure 1B), producing a characteristic biphasic elution curve shown in FIG. 1C. The burst phase, which normally occurs between baseline and two days, is due to the adsorbed protein on the surface of the nanospheres being released upon resuspension in aqueous medium (FIG. 1D). The controlled release phase is due to entrapped protein (FIG. 1D), and is released slowly over time as the PLGA hydrolyzes.

[0063] For purposes of comparison, the above process may be carried out using a buffered saline solution instead of a protein suspension, and adding this saline solution to the PLGA-containing oil phase to produce the first emulsion. Upon adding this first emulsion to a PVA aqueous phase, proteinfree blank nanoparticles may be produced.

[0064] Blank nanospheres and IL-12-loaded PLGA nanospheres were synthesized using the above techniques. The morphology of blank and protein-loaded PLGA nanospheres was determined via scanning electron microscopy to be spherical in shape with a mean particle diameter of $50 \mathrm{~nm}$ to 500 $\mathrm{nm}, 100$ to $250 \mathrm{~nm}, 100$ to $150 \mathrm{~nm}$, or 175 to $225 \mathrm{~nm}$. In various embodiments, IL-12-loaded PLGA nanospheres may have a diameter of 100 to $150 \mathrm{~nm}$. Blank, i.e., protein-free nanospheres may have a diameter of 175 to $225 \mathrm{~nm}$.

[0065] The zeta potentials of both blank and IL-12 loaded PLGA nanospheres were also determined in a deionized water medium. The zeta potential is the potential difference between the dispersion medium and the stationary layer of fluid attached to the dispersed particle, and was determined to range from -15 to $-25 \mathrm{mV}$, respectively, following the introduction of 12.5 - $25 \mathrm{ug}$ rmIL12 for protein loading during synthesis. As the magnitude of the zeta potential increases, the stability of the nanosphere dispersion increases.

[0066] Protein was eluted from the nanospheres in a nanosphere release buffer. The amount of IL-12 (percent) encapsulated and released (encapsulation efficiency, EE) by the nanospheres was estimated using the area under the curve (AUC) of each elution profile, the particle concentration (PC; particles $/ \mathrm{mL}$ ), the total volume of synthesized particles $(\mathrm{V}, \mathrm{mL})$, and the total mass of IL-12 (mg) added during synthesis via the following equation: 


$$
E E=\left(\frac{\frac{A U C_{E P}}{100,000} * P C * V}{\text { Total } I L-12 \text { added }}\right) * 100 \%
$$

[0067] Elution may be measured by dispersing from 200 million particles/mL to 100 billion particles $/ \mathrm{mL}$, from 300 million particles $/ \mathrm{mL}$ to 50 billion particles $/ \mathrm{mL}$, from 400 million particles $/ \mathrm{mL}$ to 20 billion particles $/ \mathrm{mL}$, or from 500 million particles $/ \mathrm{mL}$ to 1 billion particles $/ \mathrm{mL}$ of protein-loaded PLGA nanospheres in a nanosphere release buffer, and analyzing the resulting dispersion for bioactive protein concentrations released over time. In the case of IL-12, the total amount of rmIL-12 eluted from the aforementioned particle concentrations was determined by area under the curve (AUC) analysis to be from 1500 to 4,000 pg. The amount of IL-12 eluted per 100,000 nanospheres was determined to be 0.3 to $0.45 \mathrm{pg} / 100,000$ nanospheres, respectively, with the most efficient elution kinetics being obtained at a concentration of 750 million particles $/ \mathrm{mL}$ and the least efficient elution kinetics being obtained at a concentration of 1 billion particles $/ \mathrm{mL}$ for the particles made via the homogenization method. Based on equation (1), the average encapsulation efficiency (EE) was determined to range from $0.4 \%$ to $0.5 \%$. The highest EE was obtained at a concentration of 750 million particles $/ \mathrm{mL}$.

[0068] To determine whether the synthesized nanospheres could indeed encapsulate protein and not merely adsorb it to the outer wall, PLGA nanospheres containing fluorescein isothiocyanatelabeled bovine serum albumin were synthesized. The resulted nanospheres were imaged via confocal microscopy to visualize the internal structure. Analysis confirmed that labeled BSA was successfully incorporated within the nanospheres.

[0069] To determine whether the contents of PLGA nanospheres distribute systemically and without causing injury following various administration routes, PLGA nanospheres loaded with the fluorescent dye Alexa Fluor ${ }^{\circledR} 647$ were injected into female mice, either intravenously through the tail vein or intraperitoneally, and monitored via in vivo imaging systems. Both routes of administration resulted in systemic distribution of nanosphere contents, as depicted in FIG. 2, without any signs of morbidity or mortality.

[0070] Similar techniques may be used to encapsulate a water-insoluble free base or salt of a drug, or a water-insoluble dye or contrast agent, in a PLGA nanosphere, where the term "water-insoluble" means that the drug or salt is less soluble in water than in the organic solvent dissolving the PLGA polymer. Similar techniques may be used to encapsulate a soluble drug, dye, or contrast agent, where controlled release by a PLGA polymer is desired to provide a therapeutically safe and effective dose 
while avoiding toxic side effects from rapid initial release.

[0071] To create the oil phase, from 2.5\% w/v to 17\% w/v of PLGA is dissolved in an organic solvent, e.g., a halogenated C1-C3 organic solvent; a C2-C3 nitrile solvent; a C2-C5 alkyl ester solvent; or a C3 to C5 ketone solvent. To create the aqueous phase of the emulsion, from $1 \% \mathrm{w} / \mathrm{v}$ to $3 \% \mathrm{w} / \mathrm{v}$ of polyvinyl alcohol (PVA) is dissolved in an aqueous solvent, which may be water or a buffered saline solution, e.g., phosphate buffered saline.

[0072] Next, a water-insoluble free base or salt of a drug, a water-insoluble dye, or a contrast agent, is suspended in an aqueous medium, and the resulting protein suspension was added to the PLGAcontaining oil phase, which may be subjected to rapid stirring or sonication to produce a first emulsion. [0073] The first emulsion is then added to the PVA aqueous phase with rapid stirring or sonication to produce a second emulsion. The organic solvent is then evaporated from the second emulsion. PLGA nanoparticles containing the drug, dye, or contrast agent from the aqueous medium are recovered by centrifugation, and may be lyophilized.

[0074] Various further modifications to the process for nanosphere preparation may increase encapsulation efficiency, and change the elution profile of the nanospheres. Preparation of nanospheres using a protein solution made by suspending 12.5 micrograms of IL-12 in 1.2 mL DPBS containing $1.5 \% \mathrm{w} / \mathrm{v}$ trehalose produces nanospheres with delayed release. The initial burst phase is delayed to second day after the start of the elution study, but encapsulation efficiency is decreased.

[0075] Preparation of nanospheres using a protein solution made by suspending 12.5 micrograms of IL-12 in $1.2 \mathrm{~mL}$ DPBS containing $2 \% \mathrm{w} / \mathrm{v} \mathrm{Mg}(\mathrm{OH})_{2}$ produces nanospheres with decreased encapsulation efficiency.

[0076] Preparation of nanospheres using a protein solution made by suspending 12.5 micrograms of IL-12 in $1.2 \mathrm{~mL}$ DPBS containing $3 \%$ to $15 \%, 5 \%$ to $12 \%, 8 \%$ to $12 \%$, or about $10 \%$ serum or fetal serum produces nanospheres with delayed release. The initial burst phase is delayed to the second day after the start of the elution study, and encapsulation efficiency is increased. If the IL-12 suspension is incubated with fetal serum for 24 hours prior to nanosphere preparation, the burst phase is delayed to the third day after the start of the elution study. For in vitro studies, any type of fetal serum may be used, e.g. fetal bovine serum or fetal murine serum. Alternatively, human serum may be used. For in vivo studies, where nanospheres are administered to a human or non-human patient, selection of serum should be specific to the species to be treated. For treatment of cattle, nanospheres made by suspending IL-12 in DPBS containing fetal bovine serum may be used. For treatment of mice, nanospheres should be made with DPBS containing fetal murine serum. For treatment of humans, 
nanospheres should be made with DPBS containing human serum. Administering nanospheres treated with serum or fetal serum from one species to a different species may cause graft vs. host disease.

[0077] The presence of surfactants during nanosphere preparation increases encapsulation efficiency. The surfactants may be incorporated into the PLGA-containing oil phase, or into the PVA/water phase. Suitable surfactants include oil-soluble sorbitan fatty acid esters (Span 20, Span 40, Span 60, and Span 80, for example), and/or water-soluble polyoxyethylene sorbitan fatty acid esters (Tween 20, Tween 40, Tween 60, and Tween 80, for example). In various embodiments, nanospheres may be prepared with a PLGA-containing oil phase containing $4 \%$ to $20 \% \mathrm{w} / \mathrm{w}$ of a Span surfactant, a PVA/water phase containing $2 \%$ to $10 \% \mathrm{w} / \mathrm{v}$ of a Tween surfactant, or both. For example, the oil phase may contain 4\% to $20 \% \mathrm{w} / \mathrm{w} \operatorname{Span} 60,10 \%$ to $16 \% \mathrm{w} / \mathrm{w}$ Span 60 , or about 14\% w/w Span 60;

the PVA/water phase may contain $2 \%$ to $10 \% \mathrm{w} / \mathrm{v}$ Tween $80,3 \%$ to $8 \% \mathrm{w} / \mathrm{v}$ Tween 80 , or $4 \%$ to $6 \% \mathrm{w} / \mathrm{v}$ Tween 80 ; or

the oil phase may contain 4\% to 20\% w/w Span 60 and the PVA/water phase may contain 2\% to $10 \% \mathrm{w} / \mathrm{v}$ Tween 80 .

[0078] The presence of both a surfactant and fetal serum greatly increases encapsulation efficiency. Preparation of nanospheres using a protein solution made by suspending 12.5 micrograms of IL-12 in $1.2 \mathrm{~mL}$ DPBS containing $10 \%$ fetal serum produces nanospheres with an IL-12 encapsulation efficiency of between $2 \%$ and $10 \%$, between $4 \%$ and $8 \%$, or between $5 \%$ and $7 \%$. Preparation of nanospheres using a protein solution made by suspending 12.5 micrograms of IL-12 in $1.2 \mathrm{~mL}$ DPBS containing 10\% fetal serum which has been incubated in the DPBS for 24 hours produces nanospheres with an IL-12 encapsulation efficiency of between $10 \%$ and $50 \%$, between $20 \%$ and $45 \%$, or between $30 \%$ and $40 \%$. Preparation of nanospheres using a protein solution made by suspending 12.5 micrograms of IL-12 in $1.2 \mathrm{~mL}$ DPBS containing both $10 \%$ fetal serum and a surfactant produces nanospheres with an IL-12 encapsulation efficiency of between $50 \%$ and $95 \%$, between $60 \%$ and $85 \%$, or between $70 \%$ and $80 \%$. In various embodiments, use of a protein solution containing a cytokine, fetal serum, and a surfactant synergistically increases encapsulation efficiency of the cytokine in PLGA nanospheres.

\section{EXAMPLES}


[0079] In the following examples, dichloromethane (DCM, \#320269), PLGA (Resomer RG 756 S, 75\% lactide, \#719927), $\mathrm{NaCl}$ (\#7647-14-5), and poly(vinyl alcohol) (\#341584) were purchased from Sigma Aldrich (St. Louis, MO). Fluorescein isothiocyanate-labeled bovine serum albumin (BSA-FITC, \#A23015), Penicillin-Streptomycin (Pen-Strep, 10,000 U/ml, \#15140122) and Alexa Fluor® 647 carboxylic acid, tris(triethylammonium) salt were purchased from Thermofisher Scientific (Waltham, MA). Recombinant mouse IL-12 (p70, rmIL-12, \#577008) and Mouse IL-12 ELISA MAX deluxe ELISA kits (\#433606) were purchased from Biolegend (San Diego, CA).

[0080] Gibco Fetal Bovine Serum Qualified Heat Inactivated US Origin (HI-FBS, \#MT35011CV) and Dulbecco's Phosphate-Buffered Salt Solution 1X (DPBS, \#21031CV) were purchased from Fisher Scientific (Pittsburgh, PA).

[0081] Female BALB/c mice (6-8 weeks of age) (\#000651) were purchased from the Jackson Laboratory (Bar Harbor, ME).

\section{PART A. IL-12 STABILITY STUDIES.}

Example 1. Stability of IL-12 in acidic and basic solutions.

[0082] To determine if IL-12 could handle the acidic nanoenvironment of a PLGA nanosphere, the recovery of bioactive recombinant mouse IL-12 following a three hour exposure to solutions of varying $\mathrm{pH}$. Specifically, IL-12 was incubated for 3 hours in a solution of $\mathrm{pH} 1$, a solution of $\mathrm{pH}$, a solution of $\mathrm{pH} 7.4$, a solution of $\mathrm{pH} 11$, and a solution of $\mathrm{pH} 13$. The results are shown in Table 1. The percentage of active IL-12 recovered, relative to an initial concentration, was determined using enzyme-linked immunosorbent assay (ELISA) to determine the amount of protein in its native, and hence biologically-active, conformation.

Table 1. IL-12 stability as a function of $\mathrm{pH}$.

\begin{tabular}{|c|c|}
\hline $\mathrm{pH}$ & IL-12 Recovery (\%) \\
\hline 1 & $0 \% \pm 0 \%$ \\
\hline 3 & $39.7 \% \pm 8.44 \%$ \\
\hline 7.4 & $100 \% \pm 0 \%$ \\
\hline 11 & $96.6 \% \pm 6.08 \%$ \\
\hline 13 & $0 \% \pm 0 \%$ \\
\hline
\end{tabular}


[0083] As seen in Table 1, IL-12 has good stability at a $\mathrm{pH}$ value of 7.4 to 11 , and is subject to denaturation outside this range.

Example 2. Stability of IL-12 in organic solvents.

[0084] As discussed above, the double emulsion method of synthesizing PLGA nanospheres requires that the PLGA be dissolved in an organic solvent. Therefore, the stability of IL-12 was tested in several aprotic solvents of varying polarity. The organic solvents tested, in order of increasing hydrophilicity, were dichloromethane (DCM), ethyl acetate (EA), and acetone (AC). While DCM dissolves PLGA the best, it is also the most hydrophobic and hence may have the greatest effect on the biological activity of IL-12. PLGA is poorly soluble in acetone, but this solvent is the most polar and therefore may affect the bioactivity of IL-12 the least.

[0085] IL-12 was added to a 1:1 solution of solvent and phosphate-buffered salt Solution, and stirred to completely remove the organic solvent; the remaining bioactive IL-12 concentrations were then determined using ELISA and expressed as a percentage, relative to an initial concentration. The results are shown in Table 2. Interestingly, AC had the most detrimental effect on the protein with only $43 \%(\mathrm{SE}=1.06 \%)$ recovered, while EA showed the highest recovery at $63 \%(\mathrm{SE}=0.77 \%$ ); DCM left approximately half of the protein in its native form $(49 \%, \mathrm{SE}=0.55)$.

Table 2. IL-12 stability as a function of organic solvent.

\begin{tabular}{|c|c|}
\hline Solvent & IL-12 Recovery (\%) \\
\hline DCM & $49 \% \pm 0.55 \%$ \\
\hline EA & $63 \% \pm 0.77 \%$ \\
\hline AC & $43 \% \pm 1.06 \%$ \\
\hline
\end{tabular}

[0086] Unless otherwise indicated, DCM was used to create nanosphere batches in further examples. Although use of DCM as a solvent led to reduced protein recovery, when compared to ethyl acetate, DCM dissolves PLGA better and results in better nanosphere morphology.

Example 3. Stability of IL-12 upon sonication.

[0087] To form emulsions when synthesizing PLGA nanospheres, IL-12 may be subjected to sonication twice. Ultrasonication during nanosphere preparation results in more uniform and smaller 
nanospheres. However, sensitive proteins like IL-12 become denatured when exposed to intense agitation.

[0088] Therefore, various sonication wattages and times were tested to determine suitable conditions for IL-12-loaded PLGA nanosphere synthesis. IL-12 was suspended in a phosphatebuffered salt solution, and sonicated at 30 Wats, 40 Watts, or 50 Watts. The duration of sonication was $10,20,30,40$, or 60 seconds. and compared to baseline. The bioactive IL-12 concentrations remaining after sonication were then determined using ELISA and expressed as a percentage, relative to an initial concentration. IL-12 was found to be more sensitive to time than wattage, as protein recovery past 30 seconds was less than $10 \%$ for all wattages, as seen in FIG. 3. The scanning electron microscopy (SEM) images of various IL-12-loaded batches are shown in FIGS. 4A to 4C. The box in FIG. 3 indicates the combination of times/wattages that recover the most protein, i.e., sonication at 30 Watts to 50 Watts for 5 to $30 \mathrm{sec}$.

[0089] Unless otherwise indicated, nanospheres made by sonication in subsequent examples were prepared using:

Sonication at $30 \mathrm{Watts}$ for 10 to $20 \mathrm{sec}$;

Sonication at 40 Watts for 10 to $15 \mathrm{sec}$; or

Sonication at 50 Watts for 10 to $15 \mathrm{sec}$.

\section{PART B. STUDIES ON PLGA NANOSPHERES.}

Example 4. Synthesis of PLGA nanospheres

[0090] To create an oil phase, $800 \mathrm{mg}$ of PLGA was dissolved in $32 \mathrm{ml} \mathrm{DCM}$ at room temperature for two hours using a magnetic stir bar at 500 RPM.

[0091] To create an aqueous phase of the emulsion, $2400 \mathrm{mg}$ PVA and $96 \mathrm{mg} \mathrm{NaCl}$ were dissolved in $120 \mathrm{ml}$ deionized water and microwaved for 10 second bursts in a standard kitchen microwave on setting HIGH until clear. The aqueous phase was then cooled on ice.

[0092] The first emulsion (w1) was made by suspending a material to be encapsulated in $1.2 \mathrm{~mL}$ DPBS and added the resulting suspension to the oil phase, which was stirred at 17,500 RPM using a tissue homogenizer for 6 minutes. The stirring was performed on ice. As a control, blank particles were made with no encapsulation material suspended in the DPBS.

[0093] The second emulsion (w2) was formed by slowly pouring the first emulsion into $120 \mathrm{ml}$ of the aqueous phase. During addition of the first emulsion, the aqueous phase was stirred with the tissue homogenizer at 17,500 RPM. Following addition of the first emulsion, stirring was continued for a 
total of 8 minutes. The resulting suspension was then stirred for 16 hours with a magnetic stir bar at 750 RPM to evaporate the organic solvent.

[0094] Once the solvent was evaporated, the resulting solution was centrifuged at 3500 RPM three times; following each centrifugation step, a pellet of nanospheres is recovered and resuspended, while the supernatant produced during centrifugation is collected and stored on ice. The nanospheres were then washed twice via ultracentrifugation at 20,000 RPM for 40 minutes at 4 degrees Celsius, flashfrozen in liquid nitrogen, and stored at -20 degrees Celsius.

[0095] The nanospheres may then be lyophilized under vacuum to remove water from the nanospheres.

[0096] The properties of nanospheres obtained are summarized in Table 3. Nanospheres containing FITC-labeled BSA, described in Table 3, were imaged via confocal microscopy to visualize the internal structure. As shown in FIG. 5A, Z-stacking analysis confirmed that FITC-labeled BSA was successfully incorporated within the nanospheres.

[0097] The morphology of both blank (FIGS. 4A to 4C) and IL-12 loaded (FIGS. 5B and 5C) PLGA nanospheres was determined via scanning electron microscopy (SEM) to be spherical in shape, with a mean particle diameter of $201.7 \pm 6.7 \mathrm{~nm}$ (FIG. 6A) and $138.1 \pm 10.8 \mathrm{~nm}$ (FIG. 6B), respectively. Zeta potentials of both blank and loaded PLGA nanospheres were also determined, with a decrease in the magnitude of the voltage from $-21.3 \pm 0.808 \mathrm{mV}$ (FIG. 6C) to $-15.1 \pm 1.249 \mathrm{mV}$ (FIG. 6D), respectively.

[0098] Nanospheres containing the Alexa 647 dye and fluorescein isothiocyanate-labeled bovine serum albumin (BSA-FITC) were also successfully prepared using the above procedure.

Table 3. Nanosphere Properties.

\begin{tabular}{|c|c|c|c|c|}
\hline $\begin{array}{c}\text { Encapsulation } \\
\text { Material }\end{array}$ & $\begin{array}{c}\text { Amount/1.2 } \\
\text { mL DPBS }\end{array}$ & $\begin{array}{c}\text { Nanosphere } \\
\text { Diameter } \\
(\mathrm{nm})\end{array}$ & $\begin{array}{c}\text { Zeta Potential } \\
(\mathrm{mV})\end{array}$ & $\begin{array}{c}\text { Nanosphere } \\
\text { Concentration } \\
(\text { Particles } / \mathrm{mL})\end{array}$ \\
\hline Nothing & -- & $201.7 \pm 6.7$ & $-21.3 \pm 0.81$ & $6.49 \times 10_{9} \pm 6.19 \times 10_{8}$ \\
\hline Alexa 647 & $5 \mathrm{mg}$ & -- & -- & -- \\
\hline BSA-FITC & $5 \mathrm{mg}$ & -- & -- & -- \\
\hline IL-12 & $0.025 \mathrm{mg}$ & $138.1 \pm 10.8$ & $-15.1 \pm 1.25$ & $1.66 \times 10_{9} \pm 4.45 \times 10_{8}$ \\
\hline
\end{tabular}

Example 5. Nanosphere IL-12 Elution Profile. 
[0099] PLGA nanospheres encapsulating IL-12 were obtained by the process of Example 4. Three different concentrations (500 million particles $/ \mathrm{mL}, 750$ million particles $/ \mathrm{mL}$, and 1 billion particles/mL) of IL-12-loaded PLGA nanospheres were prepared in $500 \mu \mathrm{l}$ of nanosphere release buffer (NRB, 10\% HI-FBS and 100 units/ml Pen-Strep in DPBS). The suspensions were then centrifuged for 15 minutes at $4^{\circ} \mathrm{C}$ to pellet the nanospheres, after which a $250 \mathrm{ul}$ aliquot of the supernatant was removed and stored at $4^{\circ} \mathrm{C}$. While on ice, $250 \mu \mathrm{l} \mathrm{NRB}$ was then added to the pellet to bring the total volume back up to $500 \mu \mathrm{l}$, which was resuspended and incubated at $37^{\circ} \mathrm{C}$ with constant agitation (750 RPM) for 24 hours. This process was repeated for a total of 12 samples over 12 days (1 time point sample/24 hours). Each aliquot was stored at 4 degrees Celsius for at least 24 hours to equilibrate with the release buffer before determining IL-12 concentration via ELISA. The aliquots were analyzed via ELISA for bioactive IL-12 concentrations released over time.

[00100] The total amount of IL-12 eluted was determined by ELISA and subsequent area under the curve (AUC) analysis to be:

$1907.66 \pm 162.00 \mathrm{pg}$ at a nanosphere concentration of 500 million particles $/ \mathrm{mL}$, $3329.77 \pm 162.67 \mathrm{pg}$ at a nanosphere concentration of 750 million particles $/ \mathrm{mL}$, and $3415.64 \pm 848.94 \mathrm{pg}$ at a nanosphere concentration of 1 billion particles $/ \mathrm{mL}$.

[00101] The amount of IL-12 eluted per 100,000 nanospheres was determined to be:

$0.3815 \pm 0.03240 \mathrm{pg} / 100,000$ particles at a nanosphere concentration of 500 million particles $/ \mathrm{mL}$,

$0.4440 \pm 0.02169 \mathrm{pg} / 100,000$ particles at a nanosphere concentration of 750 million particles/mL, and

$0.3416 \pm 0.08489 \mathrm{pg} / 100,000$ particles at a nanosphere concentration of 1 billion particles $/ \mathrm{mL}$.

[00102] The 750 million particles/mL sample reported the most efficient elution kinetics of the three concentrations tested.

[00103] As seen in FIG. 7A, IL-12 concentration eluted as a function of time at a nanosphere concentration of 750 million particles $/ \mathrm{mL}$ is significantly greater than IL-12 concentration eluted as a function of time at a nanosphere concentration of 500 million particles $/ \mathrm{mL}$. However, there is little difference between IL-12 elution as a function of time at a nanosphere concentration of 750 million particles/mL and IL-12 elution as a function of time at a nanosphere concentration of 1 billion particles/mL. Also as seen in FIG. 7A, IL-12 nanoparticles show a biphasic elution profile, with a burst phase lasting about 2 days and a sustained release phase lasting about from about day 3 to day 11. Moreover, the total amount of IL-12 eluted over time at a nanosphere concentration of 750 million 
particles $/ \mathrm{mL}$ is about $75 \%$ greater than the total amount of IL-12 eluted at a nanosphere concentration of 500 million particles/mL. However, the total amount of $I L-12$ eluted at a nanosphere concentration of 1 billion particles $/ \mathrm{mL}$ is only about $2.6 \%$ greater than the total amount of IL-12 eluted at a concentration of 750 million particles/mL. As seen in FIG. 7B, IL-12 concentration eluted per 100,000 particles, as a function of time, is greater at a nanosphere concentration of 750 million particles $/ \mathrm{mL}$ than at a concentration of either 500,000 particles $/ \mathrm{mL}$ or 1 billion particles $/ \mathrm{mL}$.

[00104] As shown in FIG. 7C, the encapsulation efficiency (EE) of recombinant mouse IL-12 (rmIL-12)-loaded nanospheres was calculated using the area under the curve (AUCs) of each elution profile for the three different particle concentrations investigated, using Equation 1. The amount of IL-12 eluted per 100,000 nanospheres was determined to be $0.44 \%$ at a nanosphere concentration of 500 million particles $/ \mathrm{mL}, 0.50 \%$ at a nanosphere concentration of 750 million particles $/ \mathrm{mL}$, and $0.39 \%$ at a nanosphere concentration of 1 billion particles $/ \mathrm{mL}$. The average encapsulation efficiency (EE) is $0.443 \% \pm 0.0551 \%$. Due to differing elution kinetics from the tested concentrations, EE was reported for all three concentrations and subsequently averaged to reflect overall EE.

[00105] Based on the above data, increasing the nanosphere concentration above 750 million particles/mL may not offer a significant increase in the elution profile of the drug, possibly due to reduced encapsulation efficiency at high particle concentrations. However, this may be due to the constraints of eluting particles in vitro in a small volume $(500 \mu \mathrm{L})$ of elution solvent. Elution in a larger volume, e.g., a larger volume of an in vitro elution solvent or an in vivo blood supply, may provide an increased elution profile as the nanosphere concentration increases above 750 million particles $/ \mathrm{mL}$.

Example 6. In vivo Alexa 647-loaded PLGA nanosphere substrate biodistribution.

[00106] PLGA nanospheres loaded with the fluorescent dye Alexa 647, described in Table 1, were injected into female BALB/c mice. In a first group of mice, the dye-loaded PLGA nanospheres were injected intravenously through the tail vein. In a second group of mice, the dye-loaded PLGA nanospheres were injected intraperitoneally. Dye distribution was monitored via IVIS imaging over a 76-minute period. At 35 minutes post-injection, both routes of administration resulted in systemic distribution of nanosphere contents, as depicted in FIG. 2. Intravenous injection resulted in generalized distribution of labeled dye through the body of the subject mouse. Intraperitoneal injection resulted in localized distribution of labeled dye within the abdominal cavity of the subject mouse, with the most intense distribution of the dye within the peritoneal cavity. Distribution occurred without any signs of morbidity or mortality. 


\section{PART C. STUDIES ON PLGA NANOSPHERES WITH SONICATION.}

Example 7. Synthesis of PLGA nanospheres with sonication.

[00107] To create an oil phase, $250 \mathrm{mg}$ of PLGA was dissolved in $1.51 \mathrm{ml}$ DCM at room temperature. $1 \mathrm{~g}$ PVA was dissolved in $100 \mathrm{ml}$ deionized water to create an aqueous phase. The aqueous phase was then cooled on ice. The first emulsion (w1) was made by suspending 12.5 micrograms of IL-12 in about $20 \pm 6$ microliters DPBS and adding the resulting suspension to the oil phase with sonication at 30 to 50 Watts for 10 to $20 \mathrm{sec}$.

[00108] The second emulsion (w2) was formed by slowly adding the first emulsion into $5 \mathrm{ml}$ of the aqueous phase. During addition of the first emulsion, the aqueous phase was subjected to sonication at 30 to 50 Watts for 10 to $30 \mathrm{sec}$. The resulting suspension was then stirred for 3 hours with a magnetic stir bar at 1,000 RPM to evaporate the organic solvent.

[00109] Once the solvent was evaporated, nanospheres were recovered from the resulting solution via three ultracentrifugation treatments at 10,000 $\mathrm{g}$ for 15 minutes at 4 degrees Celsius, flash-frozen in liquid nitrogen, and stored at -20 degrees Celsius.

[00110] The nanospheres may then be lyophilized under vacuum to remove water from the nanospheres.

[00111] FIG. 8A shows IL-12-loaded nanospheres prepared using sonication at a sonication power of 30 Watts and a sonication time ranging from $10 \mathrm{sec}$ to $20 \mathrm{sec}$ (Batches 30W10S, 30W15S, and 30W20S of Table 2).

[00112] FIG. 8B shows IL-12-loaded nanospheres prepared using sonication at a sonication power of 40 Watts and a sonication time ranging from $10 \mathrm{sec}$ to $15 \mathrm{sec}$ (Batches 40W10S and 40W15S of Table 2).

[00113] FIG. 8C shows IL-12-loaded nanospheres prepared using sonication at a sonication power of 50 Watts and a sonication time ranging from $10 \mathrm{sec}$ to $15 \mathrm{sec}$ (Batches 50W10S and 50W15S of Table 2).

[00114] As seen in Table 4, sonication with 30 Watts to 50 Watts power for 10 to 20 sec generally produces IL-12 loaded PLGA nanospheres with an encapsulation efficiency of about 5\% to about $10 \%$, and a zeta potential of between -30 and $-40 \mathrm{mV}$. 
Table 4. Impact of Sonication Conditions on Nanosphere Properties

\begin{tabular}{ccccc}
\hline $\begin{array}{c}\text { Sonication } \\
\text { Power }\end{array}$ & $\begin{array}{c}\text { Particle } \\
\text { Batch }\end{array}$ & $\begin{array}{c}\text { Sonication } \\
\text { Time }\end{array}$ & $\begin{array}{c}\text { Encapsulation } \\
\text { Efficiency (\%) }\end{array}$ & $\begin{array}{c}\text { Zeta Potential } \\
(\mathbf{m V})\end{array}$ \\
\hline $\mathbf{3 0} \mathbf{W}$ & 30W10S & $10 \mathrm{sec}$ & $9.67 \pm 4.25$ & $-36.15 \pm 2.25$ \\
& 30W15S & $15 \mathrm{sec}$ & $7.60 \pm 3.45$ & $-38.15 \pm 4.75$ \\
\cline { 2 - 5 } & 30W20S & $20 \mathrm{sec}$ & $4.84 \pm 0.80$ & $-31.75 \pm 6.35$ \\
\hline \multirow{4}{40}{$\mathbf{W}$} & 40W10S & $10 \mathrm{sec}$ & $6.23 \pm 1.29$ & $-31.55 \pm 8.35$ \\
& 40W15S & $15 \mathrm{sec}$ & $6.92 \pm 0.44$ & $-33.55 \pm 7.35$ \\
\hline $\mathbf{5 0} \mathbf{W}$ & $50 \mathrm{~W} 10 \mathrm{~S}$ & $10 \mathrm{sec}$ & $8.80 \pm 1.88$ & $-36.35 \pm 2.85$ \\
& 50W15S & $15 \mathrm{sec}$ & $5.20 \pm 3.10$ & $-35.50 \pm 4.90$ \\
\hline
\end{tabular}

Example 8. Elution of PLGA nanospheres prepared with sonication.

[00115] The IL-12-loaded nanospheres shown in Table 2 were tested for elution characteristics by the procedure of Example 5. Protein concentration per day was determined by ELISA, and the total protein released was determined using the area under the raw elution curve.

[00116] Elution curves obtained by the above procedure are shown in FIG. 9. The elution curve for each batch of Table 2 shows a biphasic curve with an initial burst phase and a sustained release phase. The burst phase is due to the adsorbed protein on the surface of the nanospheres being released upon resuspension in aqueous medium, while the controlled release phase is due to protein entrapped within the PLGA matrix. As seen in FIG. 8, for a constant sonication period, e.g., 10 sec., the initial rate of drug release during the burst phase decreases as the sonication power increases. In a batch sonicated for $10 \mathrm{sec}$ at $30 \mathrm{~W}$ power (30W10S), between $60 \%$ and $65 \%$ of the IL-12 is eluted within one day. In a batch sonicated for $10 \mathrm{sec}$ at $50 \mathrm{~W}$ power (50W10S), less than $50 \%$ of the IL-12 is eluted within one day. This suggests that the percentage of protein within the PLGA matrix in the nanosphere core increases as the sonication power increases.

\section{PART D. IMPACT OF STIRRING SPEED ON PLGA NANOSPHERES.}

Example 9.

[00117] To create an oil phase, $800 \mathrm{mg}$ of PLGA was dissolved in $32 \mathrm{ml} \mathrm{DCM}$ at room temperature for two hours using a magnetic stir bar at 500 RPM. 
[00118] To create an aqueous phase of the emulsion, $2400 \mathrm{mg}$ PVA and $96 \mathrm{mg} \mathrm{NaCl}$ were dissolved in $120 \mathrm{ml}$ deionized water and microwaved for 10 second bursts in a standard kitchen microwave on setting HIGH until clear. The aqueous phase was then cooled on ice.

[00119] An IL-12 suspension was made by suspending 25 micrograms of IL-12 in $1.2 \mathrm{~mL}$ DPBS.

[00120] Nanospheres were prepared as described in Example 4.

[00121] To observe the impact of stirring speed on encapsulation efficiency of IL-12 in a PLGA nanosphere, and elution profile of IL-12 from PLGA nanospheres, stirring during preparation of the first and second emulsions was done at speeds of 13,125 RPM; 15,312 RPM; 17,500 RPM; 19,688 RPM; 21,875 RPM; 24,063 RPM; and 26,250 RPM. Encapsulation efficiency as a function of agitation speed is shown in Table 5.

Table 5. Encapsulation Efficiency as a function of stirring speed.

\begin{tabular}{|c|c|c|}
\hline $\begin{array}{c}\text { Setting on Tissue } \\
\text { Homogenizer }\end{array}$ & Agitation Speed (RPM) & $\begin{array}{c}\text { Encapsulation Efficiency } \\
(\%)\end{array}$ \\
\hline 3 & 13,125 & 0.68 \\
\hline 3.5 & 15,312 & 0.50 \\
\hline 4 & 17,500 & 2.06 \\
\hline 4.5 & 19,688 & 0.94 \\
\hline 5 & 21,875 & 0.70 \\
\hline 5.5 & 24,063 & 0.50 \\
\hline 6 & 26,250 & 0.15 \\
\hline
\end{tabular}

[00122] As seen in FIG. 10, the elution profile of IL-12 from PLGA nanospheres prepared using high-speed stirring rather than sonication, at a nanosphere concentration of 750 million particles $/ \mathrm{mL}$, is highly dependent on stirring speed. Stirring at 17,500 RPM gives a high encapsulation efficiency, and a total drug release over a 12-day period of about $4500 \mathrm{pg}$ IL-12. Stirring at about 13,000 to about 20,000 RPM gives an acceptable drug release over a 12-day period of about 3500 pg IL-12 or greater. Stirring at greater than about 22,000 RPM gives a low encapsulation efficiency, and a total drug release over a 12-day period of less than 3,000 pg IL-12. The optimum stirring speed for producing IL-12 loaded PLGA nanospheres is about 17,500 RPM.

PART E. EFFECT OF NANOSPHERES ON RELEASE OF A SOLUBLE CYTOKINE. 
Example 10. Release of Free IL-12 vs. Nanosphere-Encapsulated IL-12.

[00123] Osteosarcoma (OS) induces systemic immune suppression in mice that can be reversed by the checkpoint blocker anti-PD-L1, as shown in FIG. 11. Using anti-PD-L1, their systemic immunophenotype returns to baseline status, relieving immunosuppression from OS. However, baseline status does not provide enough immune stimulation to effectively reduce tumor burden in mice with advanced OS. See FIG. 11, line A. However, patients in a Phase II study of a rituximab/IL12 combination therapy, where IL-12 was administered as a free peptide, for relapsed and refractory Non-Hodgkin lymphoma (NHL) showed signs of dose-limiting toxicity (DLT). Their immune status, or immunophenotype, was pushed past the level of stimulation and into the realm of a life-threatening state of immune system over-activation known as immune cell exhaustion. See FIG. 11, line C. The goal is to stimulate the immune system above baseline levels, without overstimulation or immune cell exhaustion.

[00124] However, in a background of anti-PD-L1 checkpoint blockade providing disinhibition of activated T cells, slow and sustained delivery of low dose IL-12 (which is generally considered safe) from hydrolyzing PLGA nanospheres (IL-12-NS) to the systemic tumor macroenvironment may provide the systemic stimulation necessary to effectively reduce tumor burden while still remaining beneath the exhaustion threshold, as shown in FIG. 11, line B. A real-time immunophenotype monitoring platform, like the one described herein, would be of considerable value for this application.

[00125] Thus, use of biodradable PLGA nanospheres as a delivery vehicle for a soluble drug allows administration of a therapeutically effective low dose. This is useful where the difference between an effective dosage of a drug and a toxic dose are small.

PART E. EFFECT OF NANOSPHERE ADDITIVES ON RELEASE OF A SOLUBLE CYTOKINE.

\section{Example 11.}

[00126] Seven batches of nanospheres containing the drug IL-12 were prepared. The elution profile of these batches was obtained by following the procedure of Example 5, with sonication at 50 Watts power for $10 \mathrm{sec}$.

[00127] The first batch was made by following the procedure of Example 7, using a protein solution made by suspending 12.5 micrograms of IL-12 in about $20 \pm 6$ microliters DPBS and a PVA/water phase. No additives not listed in Example 7 were included. Upon elution of the first batch by the 
method of Example 5, the drug was released with an initial burst phase lasting 1 to 2 days, and a peak IL-12 concentration of $\sim 65,000 \mathrm{pg} / \mathrm{mL}$, reached on the second day, as shown in FIG. 12.

[00128] The second batch was made by following the procedure of Example 7, except that a protein solution made by suspending 12.5 micrograms of IL-12 in about $20 \pm 6$ microliters DPBS containing $1.5 \% \mathrm{w} / \mathrm{v}$ trehalose was used. For the second batch, the drug was released with an initial burst phase lasting 3 days, and a peak IL-12 concentration of $\sim 75,000 \mathrm{pg} / \mathrm{mL}$, reached on the third day. As seen in FIG. 12, the presence of trehalose in the protein solution used to make nanospheres significantly increased the overall drug release (measured as area under the curve), compared to drug release from nanospheres made without additional additives.

[00129] The third batch was made by following the procedure of Example 7, except that a protein solution made by suspending 12.5 micrograms of IL-12 in about $20 \pm 6$ microliters DPBS containing $2 \% \mathrm{w} / \mathrm{v} \mathrm{Mg}(\mathrm{OH})_{2}$ was used. Upon elution of the third batch by the method of Example 5, the drug was released with an initial burst phase lasting 1 day, and a peak IL-12 concentration of $\sim 10,000$ $\mathrm{pg} / \mathrm{mL}$. As seen in FIG. 12, the presence of $\mathrm{Mg}(\mathrm{OH})_{2}$ in the protein solution used to make nanospheres decreased the overall drug release, compared to drug release from nanospheres made without additional additives.

[00130] For the fourth batch, nanospheres were made by following the procedure of Example 7, using a protein solution made by suspending 12.5 micrograms of IL-12 in about $20 \pm 6$ microliters DPBS containing 10\% fetal bovine serum (FBS). Upon elution by the method of Example 5, the drug was released with an initial burst phase lasting 3 days, and a peak IL-12 concentration of $\sim 115,000$ $\mathrm{pg} / \mathrm{mL}$, reached on the second day. The presence of $10 \% \mathrm{FBS}$ in the protein solution significantly increased the overall drug release (measured as area under the curve), compared to drug release from nanospheres made without additional additives, as seen in FIG. 13.

[00131] For the fifth batch, nanospheres were made by following the procedure of Example 7 , using a protein solution made by suspending 12.5 micrograms of IL-12 in about $20 \pm 6$ microliters DPBS containing 10\% fetal bovine serum (FBS), where the protein solution was incubated for 24 hours prior to production of nanospheres. Upon elution by the method of Example 5, the drug was released with an initial burst phase lasting 3 days, and a peak IL-12 concentration of from $\sim 105,000$ $\mathrm{pg} / \mathrm{mL}$ to $\sim 110,000 \mathrm{pg} / \mathrm{mL}$, reached on the third day. As seen in FIG. 13, the presence of $10 \%$ incubated FBS in the protein solution significantly increased the overall drug release from the nanospheres (measured as area under the curve), compared to drug release from either nanospheres 
made without additional additives, or nanospheres made using FBS in the absence of an incubation step.

[00132] For the sixth batch, nanospheres were made by following the procedure of Example 7 , using a PVA/water phase containing 4\% w/v Tween 80 . Upon elution by the method of Example 5, the drug was released with an initial burst phase lasting 1 day, and a peak IL-12 concentration of from $\sim 120,000 \mathrm{pg} / \mathrm{mL}$ to $\sim 125,000 \mathrm{pg} / \mathrm{mL}$. As seen in FIG. 14, the presence of Tween 80 in the protein solution increased the peak drug release from the nanospheres by about a factor of 2, compared to peak drug release from nanospheres made without additional additives. The presence of Tween 80 also substantially increased overall drug release.

[00133] For the sixth batch, nanospheres were made by following the procedure of Example 7 , using a PVA/water phase containing $10 \% \mathrm{w} / \mathrm{v}$ FBS and $4 \% \mathrm{w} / \mathrm{v}$ Tween 80 . Upon elution by the method of Example 5, the drug was released with an initial burst phase lasting 1 day, and a peak IL12 concentration of about $\sim 580,000 \mathrm{pg} / \mathrm{mL}$. As seen in FIG. 14, the presence of both FBS and Tween 80 in the protein solution increased the peak drug release from the nanospheres by about a factor of about 9, compared to a peak drug release of $\sim 65,000 \mathrm{pg} / \mathrm{mL}$ from nanospheres made without additional additives. The presence of both FBS and Tween 80 also:

increased the peak drug release from the nanospheres by about a factor of about 4.5, compared to a peak drug release of $\sim 115,000 \mathrm{pg} / \mathrm{mL}$ from nanospheres made with FBS alone; and increased the peak drug release from the nanospheres by about a factor of about 4.5, compared to a peak drug release of $\sim 120,000 \mathrm{pg} / \mathrm{mL}$ from nanospheres made with Tween 80 alone.

Table 6. Effect of Nanosphere Additives on Drug Release and Encapsulation Efficiency.

\begin{tabular}{|l|l|l|l|l|l|}
\hline \multirow{2}{*}{ Additives } & \multicolumn{3}{|c|}{ Drug Release } & EE (\%) & $\begin{array}{l}\text { Zeta } \\
\text { Potential }\end{array}$ \\
\cline { 2 - 5 } & Total (pg) & $\begin{array}{c}\text { Peak } \\
\mathbf{p g} / \mathbf{m L})\end{array}$ & $\begin{array}{l}\text { Per 100,000 } \\
\text { Particles (pg) }\end{array}$ & & \\
\hline None & -- & 65,000 & -- & 8.8 & -36.35 \\
\hline Trehalose & 207386.3905 & 75,000 & 0.414 & 5.79 & -38.95 \\
\hline Mg(OH) 2 & 26065.70647 & 10,000 & 0.0521 & 2.19 & -22.4 \\
\hline FBS & 285394.6775 & $\sim 115,000$ & 0.571 & 6.67 & -36.6 \\
\hline $\begin{array}{l}\text { FBS (incubated } \\
\text { for 24 h) }\end{array}$ & 654806.6577 & $\sim 110,000$ & 1.31 & 35.71 & -39.7 \\
\hline Tween 80 & 419307.8952 & $\sim 120.000$ & 0.839 & 17.39 & -32.5 \\
\hline Tween 80 + FBS & 1341061.532 & $\sim 580,000$ & 2.68 & 87.54 & -35.6 \\
\hline
\end{tabular}


[00134] Peak drug release, total drug release, drug release/100,000 particles, encapsulation efficiency (EE), and Zeta potential for these modified nanosphere batches are recorded in Table 6. As shown in this table, the peak drug release and the overall drug release upon elution of the drug IL-12 from nanospheres can be increased by making the nanospheres in the presence of an additive selected from the group consisting of trehalose, FBS, FBS with 24 hours incubation, a surfactant, or a mixture thereof. Also, FBS with 24 hours incubation, a surfactant, or a mixture of FBS and a surfactant lead to dramatic increases in encapsulation efficiency. The peak drug release and the overall drug release upon elution can be synergistically increased by making the nanospheres in the presence of both FBS and a surfactant.

\section{DETAILED DESCRIPTION: MONITORING IMMUNOPHENOTYPES}

[00135] Various embodiments disclosed herein relate to a technique allowing a medical professional to systemically analyze the immune system of a patient from two to three drops of blood obtained using a finger stick blood draw obtained using a lancet and Microtainer ${ }^{\circledR}$. The blood draw can be done at the office of a medical professional; in a clinic; or in a hospital.

[00136] In various embodiments, the blood draw can be done at the office of a medical professional; in a clinic; or in a hospital. The blood draw can be analyzed at a testing site or a medical facility.

[00137] The blood sample may be analyzed for the level of an immunochemical naturally present in the body of the patient, and/or for the level of an immunomodulatory drug administered to the patient. The blood sample may be analyzed for the level of an immunochemical naturally present in the body of the patient, as a function of time, allowing a medical professional to observe the effect of a treatment regimen on the immunochemical levels. Blood draws may be taken and analyzed at regular intervals, allowing a medical professional to assess changes in the immune status, or immunophenotype, over the course of treatment.

[00138] Referring to FIG. 11, there are four major immunophenotypes under consideration:

Suppression, e.g., immunosuppression induced by cancer, an autoimmune disease, or an infection;

Baseline, e.g., the immune status of a disease-free subject;

Stimulation of the immune system above baseline levels; and 
Exhaustion, a life-threatening state of immune system over-activation known as immune cell exhaustion (TCE), marked by characterized by lymphocyte anergy, systemic inflammatory response syndrome, and/or organ failure.

[00139] FIG. 11 shows the change in the immunophenotype of an osteosarcoma cancer patient during treatment with a variety of immunomodulating therapies. The patient's initial immunophenotype is Suppression, due to cancer-induced immunosuppression. Programmed cell death ligand 1 (PDL-1) is frequently expressed during osteosarcoma, so antibodies to PDL-1 (AntiPDL-1) may be administered. See FIG. 11, line A. Interleukin-12 (IL-12) has been shown to inhibit tumor growth in osteosarcoma, so free IL-12 may be administered as an immunomodulator. See FIG. 11, line C.

[00140] At Time T1, Anti-PDL-1 appear to be ineffective in changing the patient's immunophenotype, while IL-12 appears to have changed the immunophenotype to Stimulation. However, at Time T2, as the treatment progresses, IL-12 causes toxic side effects, changing the patient's immunophenotype to Exxhaustion. At Time T2, Anti-PDL-1 changes the patient's immunophenotype to baseline, combatting the cancer-induced immunosuppression without stimulating the immune system to fight the cancer.

[00141] As seen in FIG. 11, line B, combining Anti-PDL-1 with a low dose of IL-12 administered in PGLA nanospheres may stimulate the immune system to fight cancer without causing immune system over-activation. Thus, monitoring the immunophenotype over time allows a medical practitioner to adjust an immunomodulating therapy to provide immunostimulation without T-cell exhaustion.

[00142] Data relating to the immune response to a cancer therapy may be entered into a database. For example, referencing FIG. 11, the following information may be entered into the database for any given patient with a first disease which suppresses or overstimulates the immune system:

a) type of disease;

b) immunophenotype prior to treatment;

c) treatment administered for the first disease;

d) change in immunophenotype as a function of time;

e) clinical outcome;

f) medications taken by the patient for a second disease;

g) disease stage or disease level;

h) medical co-morbidities; and 
i) age of the patient.

[00143] It is important to note that changes in any one of those parameters may affect other parameters. For example, if a patient undergoing treatment for a first disease which affects the immune system is, after the initiation of treatment, diagnosed with a second disease, i.e., a medical comorbidity, either a causative agent of the second disease or a symptom of the second disease may affect:

the clinical outcome of treatment of the first disease, the change in the patient's immunophenotype over time, the stage or level of the first disease, and/or the efficacy of treatment administered for the first disease.

[00144] Similarly, a change in disease stage or disease level of the first disease, e.g., progression of a cancer from stage 2 to stage 3, may affect the patient's immunophenotype as a function of time, protocols for treatment of the first disease, and the clinical outcome of treatment of the first disease.

[00145] The database would thus include information on treatment of a variety of diseases with immunomodulating drugs, and allow predictions on how a particular patient presenting with that disease will respond to a given immunomodulating therapy.

[00146] Use of such a database allows a medical professional to predict the response of a patient's immune system to a given disease state, and to predict changes in the patient's immunophenotype over the course of a disease or treatment. The database will useful in the treatment, surveillance, and diagnosis of many diseases including cancer, autoimmune diseases, and infections. As immunomodulating agents become more common, such a database provides the ability to assess where the status of the immune system at a specific time in the pathogenesis of a disease, and to predict which immunomodulating treatments would be most effective in treating that disease.

[00147] Each blood sample obtained from a patient's finger stick blood draw may be analyzed for the patient's immunophenotype at the time of the blood draw. The results of the analysis, together with information as to the patient's disease state and current treatment, if any, may be compared to data present in the database to develop a treatment plan which is most likely to effectively modulate the patient's immunophenotype. Blood draws may be taken and analyzed at regular intervals, allowing a medical professional to assess changes in the immune status, or immunophenotype, over the course of treatment. Data from the current patient may be added to the database, potentially improving assessments of other patients. 
[00148] This has massive commercial potential for analysis of a patient's blood with complex immunophenotyping, whether the analysis is for cancer, infection, or autoimmune diseases. The database that can be created with cross referencing of diseases with immunophenotyping will be a powerful tool to treat patients in the future. The database will be a living database that generates constantly updating information on immunophenotyping and status of treatment.

[00149] Immunophenotyping of a patient's blood sample requires the ability to analyze and assess the data appropriately. This system will allow the appropriate dosing, treatment, and corrections to be made across several disease states. As the collection of data increases the growing database will also be able help direct medical professionals to diagnosis, treat, and dose immunotherapeutics in the broadest sense.

[00150] Although the various exemplary embodiments have been described in detail with particular reference to certain exemplary aspects thereof, it should be understood that the invention is capable of other embodiments and its details are capable of modifications in various obvious respects. As is readily apparent to those skilled in the art, variations and modifications can be affected while remaining within the spirit and scope of the invention. Accordingly, the foregoing disclosure, description, and figures are for illustrative purposes only and do not in any way limit the invention, which is defined only by the claims. 
What is claimed is:

1. A method of encapsulating a protein in a nanosphere, comprising:

preparing an oil phase by dissolving from $2.5 \% \mathrm{w} / \mathrm{v}$ to $17 \% \mathrm{w} / \mathrm{v}$ of poly(lactic acidco-glycolic acid) (PLGA) in an organic solvent;

preparing an aqueous phase by dissolving polyvinyl alcohol in water;

suspending the protein in an aqueous medium;

adding the aqueous medium to the oil phase to form a first emulsion, and agitating the first emulsion;

adding the first emulsion to the aqueous phase to form a second emulsion, and agitating the second emulsion;

evaporating the organic solvent from the second emulsion to form an aqueous solution; and

recovering poly(lactic acid-co-glycolic acid) nanospheres containing the protein from the aqueous solution.

2. The method of claim 1, wherein the PLGA comprises from 50\% to 90\% lactide; and the organic solvent is selected from the group consisting of halogenated C1-C3 organic solvents, C2-C3 nitrile solvents, C2-C5 alkyl ester solvents, C3 to C5 ketone solvents, and mixtures thereof.

3. The method of claim 2, wherein the organic solvent is acetonitrile, acetone, ethyl acetate, or dichloromethane.

4. The method of claim 1, wherein the PLGA comprises from $75 \%$ to $90 \%$ lactide.

5. The method of claim 1, wherein the PLGA comprises from 50\% to $75 \%$ lactide; and the organic solvent is selected from the group consisting of halogenated C1-C3 organic solvents, acetonitrile, C3 to C4 ketone solvents, and mixtures thereof. 
6. The method of claim 1, wherein the method comprises adding the aqueous medium to the oil phase to form a first emulsion, and agitating the first emulsion with a tissue homogenizer at a rate of 13,000 RPM to 20,000 RPM; and

adding the first emulsion to the aqueous phase to form a second emulsion, and agitating the second emulsion with a tissue homogenizer at a rate of 13,000 RPM to 20,000 RPM.

7. The method of claim 1, wherein the method comprises adding the aqueous medium to the oil phase to form a first emulsion, and agitating the first emulsion by sonication; and

adding the first emulsion to the aqueous phase to form a second emulsion, and agitating the second emulsion by sonication.

8. The method of claim 7 , wherein:

agitating the first emulsion comprises sonication at a power level of $30 \mathrm{~W}$ to $50 \mathrm{~W}$, for a period of time of $5 \mathrm{sec}$ to $30 \mathrm{sec}$; and agitating the second emulsion comprises sonication at a power level of $30 \mathrm{~W}$ to $50 \mathrm{~W}$, for a period of time of $5 \mathrm{sec}$ to $30 \mathrm{sec}$.

8. The method of claim 8, wherein:

agitating the first emulsion comprises sonication for a period of time of $10 \mathrm{sec}$ to 20 sec; and

agitating the second emulsion comprises sonication for a period of time of $10 \mathrm{sec}$ to $20 \mathrm{sec}$.

9. The method of claim 1, wherein the protein is a cytokine or a globular protein.

10. The method of claim 9, wherein the protein is a cytokine selected from the group consisting of interleukins, lymphokines, monokines, interferons, colony stimulating factors, and chemokines.

11. The method of claim 10, wherein the cytokine is selected from the group consisting of interleukins and non-immunological cytokines. 
12. The method of claim 9 , wherein the protein is a cytokine having an $\mathrm{N}$-terminal signal sequence, a four-helix bundle comprising four helices labeled A through D, and no C-terminal extension following the D helix.

13. The method of claim 12, wherein the cytokine is a granulocyte-macrophage colony-stimulating factor, a granulocyte colony-stimulating factor, interferon alpha-1, interferon beta, interferon gamma. interferon kappa, interferon tau-1, interferon omega-1, or an interleukin selected from the group consisting of IL-2, IL-3, IL-4, IL-5, IL-5, IL-6, IL-7, IL-9, IL-10, IL-11, the alpha chain of IL-12, IL13, IL-15, IL-19, IL-20, IL-21, IL-22, IL-23, IL-24, IL-26, and IL-27.

14. The method of claim 9, wherein the protein is an immunological cytokine that either:

a) enhances cellular immune responses; or

b) enhances antibody responses.

15. The method of claim 14, wherein the cytokine is an immunological cytokine that enhances cellular immune responses, selected from the group consisting of $\mathrm{TNF} \alpha, \mathrm{IFN}-\gamma$, and interleukin-12.

16. The method of claim 15 , wherein the cytokine is IL-12.

17. The method of claim 14, wherein the cytokine is an immunological cytokine that enhances antibody responses, selected from the group consisting of TGF- $\beta$, IL-4, IL-10, and IL-13.

18. The method of claim 16, wherein:

the first and second emulsions are each agitated with a tissue homogenizer at a rate of 13,000 RPM to 20,000 RPM; and

IL-12 is incorporated into the poly(lactic acid-co-glycolic acid) nanospheres with an encapsulation efficiency of about $0.5 \%$ to about $2.1 \%$.

19. The method of claim 16, wherein:

the first and second emulsions are each agitated with sonication at a power level of 30 $\mathrm{W}$ to $50 \mathrm{~W}$, for a period of time of $5 \mathrm{sec}$ to $30 \mathrm{sec}$; and 
IL-12 is incorporated into the poly(lactic acid-co-glycolic acid) nanospheres with an encapsulation efficiency of about $4.5 \%$ to about $10 \%$.

20. A nanosphere produced by the method of claim 1, the nanosphere comprising a poly(lactic acid-co-glycolic acid) matrix and a protein, wherein:

a first portion of the protein is adsorbed onto a surface of the nanosphere;

a second portion of the protein is incorporated into the poly(lactic acid-co-glycolic acid) matrix at a core of the nanosphere.

21. The nanosphere of claim 20, wherein:

the protein is IL-12; and

the IL-12 is incorporated into the nanosphere with an encapsulation efficiency of about $0.5 \%$ to about $10 \%$.

22. A method of encapsulating a protein in a nanosphere, comprising:

preparing an oil phase by dissolving from $2.5 \% \mathrm{w} / \mathrm{v}$ to $17 \% \mathrm{w} / \mathrm{v}$ of poly(lactic acidco-glycolic acid) (PLGA) in an organic solvent, optionally containing a first surfactant;

preparing an aqueous phase containing polyvinyl alcohol and at least one additive selected from the group consisting of mammalian serum, trehalose, and a second surfactant; suspending the protein in an aqueous medium;

adding the aqueous medium to the oil phase to form a first emulsion, and agitating the first emulsion;

adding the first emulsion to the aqueous phase to form a second emulsion, and agitating the second emulsion;

evaporating the organic solvent from the second emulsion to form an aqueous solution; and

recovering poly(lactic acid-co-glycolic acid) nanospheres containing the protein from the aqueous solution.

23. The method of claim 22, wherein the aqueous phase contains polyvinyl alcohol and mammalian serum. 
24. The method of claim 23, wherein:

the oil phase contains the first surfactant; and/or

the aqueous phase contains the second surfactant.

25. The method of claim 24, wherein:

the first surfactant is a sorbitan fatty acid ester; and/or

the second surfactant is a polyoxyethylene sorbitan fatty acid ester.

26. The method of claim 22, wherein the aqueous phase contains polyvinyl alcohol and fetal serum.

27. The method of claim 22 , wherein the first surfactant is a sorbitan fatty acid ester.

28. A nanosphere produced by the method of claim 22, the nanosphere comprising a poly(lactic acid-co-glycolic acid) matrix and a protein, wherein:

a first portion of the protein is adsorbed onto a surface of the nanosphere;

a second portion of the protein is incorporated into the poly(lactic acid-co-glycolic acid) matrix at a core of the nanosphere; and

the nanosphere comprises at least one additive selected from the group consisting of mammalian serum albumin, trehalose, the first surfactant, and the second.

29. The nanosphere of claim 28, wherein the nanosphere comprises mammalian serum albumin and a surfactant.

30. A dosage form, comprising a plurality of nanospheres produced by the method of claim 1, each nanosphere comprising a poly(lactic acid-co-glycolic acid) matrix and a protein, wherein:

a first portion of the protein is adsorbed onto a surface of the nanosphere;

a second portion of the protein is incorporated into the poly(lactic acid-co-glycolic acid) matrix at a core of the nanosphere.

31. The dosage form of claim 30, wherein:

the protein is IL-12; and 
the IL-12 is incorporated into the nanosphere with an encapsulation efficiency of about $0.5 \%$ to about $10 \%$.

32. A method of controlling an immunophenotype in a patient suffering from a disease which impacts the immune system, comprising:

a) determining an initial immunophenotype of a patient;

b) if the initial immunophenotype shows immunosuppression, administering a first drug which stimulates the immune system; or if the initial immunophenotype shows overstimulation of the immune system, administering a second drug which suppresses the immune system;

c) after step (b), monitoring the patient's immunophenotype as a function of time; and

d) adjusting administration of the first and/or second drug if the patient's immunophenotype falls outside a desired range.

33. A method of controlling an immunophenotype in a patient suffering from a disease which impacts the immune system, comprising:

a) determining a disease state of a patient, where the disease state includes a diagnosis and an initial immunophenotype;

b) comparing the disease state of the patient to a plurality of disease states within a database, where each disease state in the database includes a diagnosis, an initial immunophenotype, and a treatment protocol;

c) based on the comparing step (b), selecting a treatment protocol from the database, where the treatment protocol involves administering an immunomodulating drug.

34. The method of claim 33, further comprising:

d) administering the immunomodulating drug to the patient;

e) after step (d), monitoring the patient's immunophenotype as a function of time; and

f) adjusting administration of the immunomodulating drug if the patient's immunophenotype falls outside a desired range. 


\begin{abstract}
ABSTRAC'T
A protein may be encapsulated in a nanosphere by preparing an oil phase by dissolving from $2.5 \% \mathrm{w} / \mathrm{v}$ to $17 \% \mathrm{w} / \mathrm{v}$ of poly(lactic acid-co-glycolic acid) (PLGA) in an organic solvent; preparing an aqueous phase by dissolving polyvinyl alcohol in water; and suspending the protein in an aqueous medium. A double emulsion is produced by adding the protein-containing aqueous medium to the oil phase to form a first emulsion, and agitating the first emulsion; and then adding the first emulsion to the aqueous phase to form a second emulsion, and agitating the second emulsion. The organic solvent is evaporated from the second emulsion to form an aqueous solution; and PLGA nanospheres containing the protein are recovered from the aqueous solution. The protein may be an immunomodulating drug, and may be used with a method of controlling an immunophenotype in a patient suffering from a disease which impacts the immune system. The method of controlling an immunophenotype includes determining a disease state of a patient, where the disease state includes a diagnosis and an initial immunophenotype; comparing the disease state of the patient to a plurality of disease states within a database, where each disease state in the database includes a diagnosis, an initial immunophenotype, and a treatment protocol; and, based on the comparing step, selecting a treatment protocol from the database, where the treatment protocol involves administering an immunomodulating drug.
\end{abstract}

